\title{
Integrated Data Base Report-1994: U.S. Spent Nuclear Fuel and Radioactive Waste Inventories, Projections, and Characteristics
}

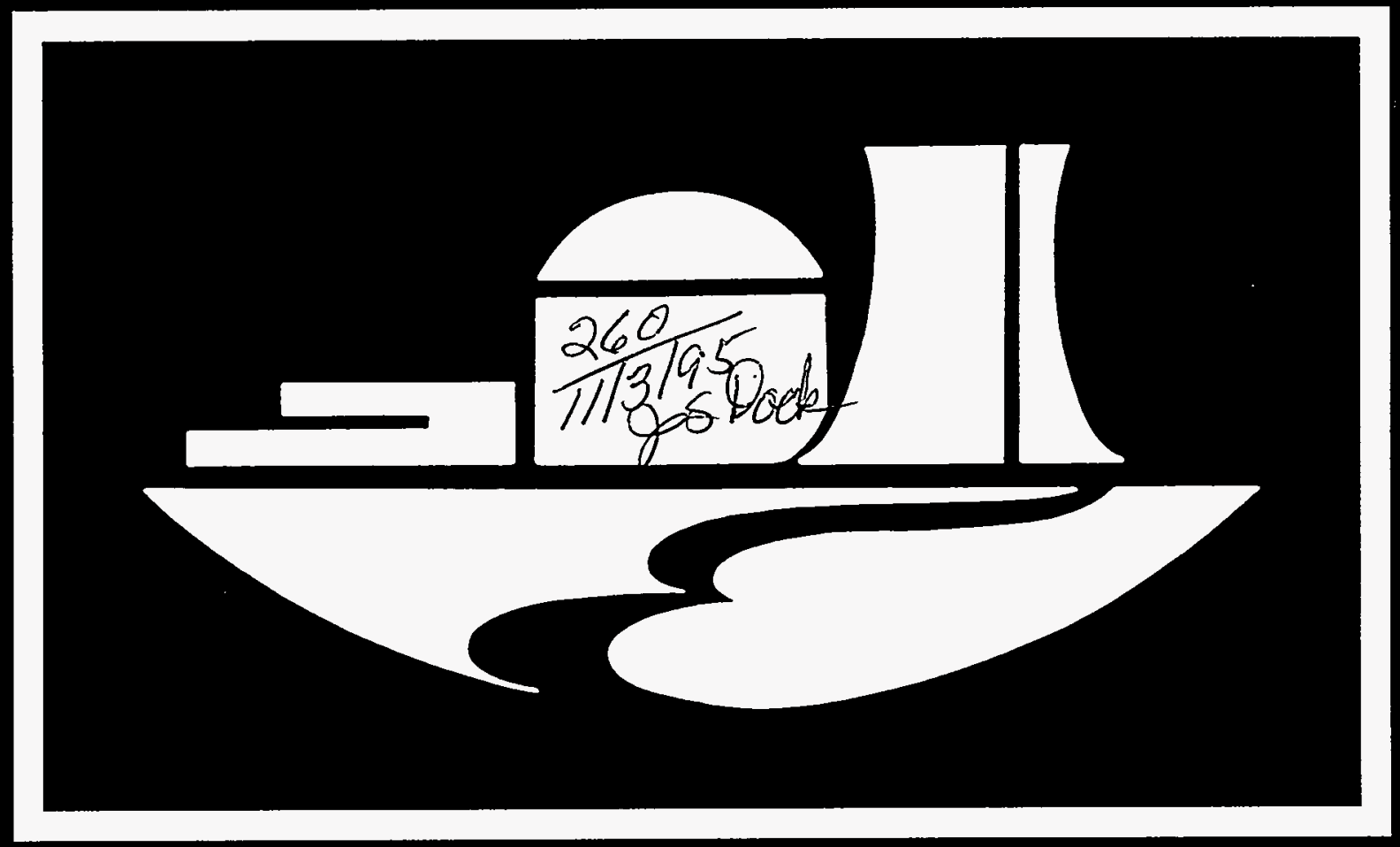

\section{Prepared for}

\section{U.S. Department of Energy}

Office of Civilian Radioactive Waste Management

Office of Environmental Management

Washington, D.C. 20585

Prepared by

Oak Ridge National Laboratory

Managed by Lockheed Martin Energy Systems, Inc., for the

U.S. Department of Energy under contract DE-AC05-84OR21400 
This report has been reproducod directly from the best available copy.

Available Irom the Office of Scientific and Technical Information, P.O. Box 62, Oak Ridge, TN 37831; Phone: (615) 576-8401.

This report was prepared as an account of work sponsored by an agency of the United States Government. Neither the United States Govemment nor any agency thereot, nor any of their employees. makes any warranty, express or implied. or assumes any legal liability or responsibility for the accuracy, com. pleteness. or uselulness of any information, apparatus. product. or process disclosed, or tepresents that its use would not infringe privately owned rights. Relerence herein to any specific commercial product, process, or service by trade name, trademark. manulacturer, or otherwise does not necessanily constitute or imply its endorsement, recommendation, or favoring by the United States Government or any agency thereot. The views and opinions of authors expressed herein do not necessarily state or rellect those of the United States Government or any agency thereot. 


\section{DISCLAIMER}

Portions of this document may be illegible in electronic image products. Images are produced from the best available original document. 
DOE/RW-0006, Rev. 11

September 1995

\section{Integrated Data Base Report-1994: U.S. Spent Nuclear Fuel and Radioactive Waste Inventories, Projections, and Characteristics}

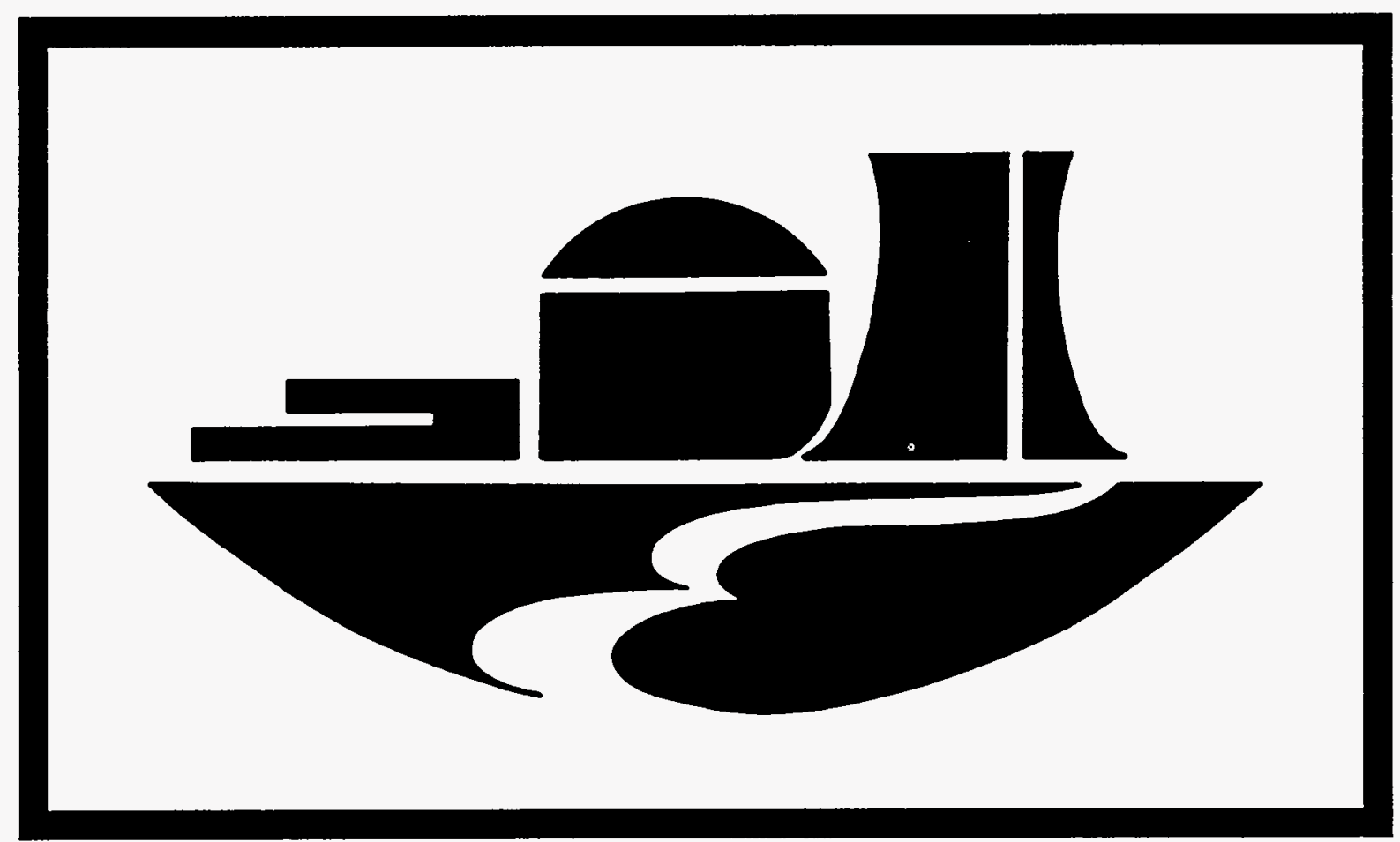

Prepared for

U.S. Department of Energy

Office of Civilian Radioactive Waste Management

Office of Environmental Management

Washington, D.C. 20585

Prepared by

Oak Ridge National Laboratory

Managed by Lockheed Martin Energy Systems, Inc., for the

U.S. Department of Energy under contract DE-AC05-84OR21400

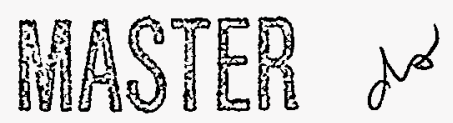




\title{
PREFACE
}

The information in this report summarizes the U.S. Department of Energy (DOE) data base for inventories, projections, and characteristics of domestic spent nuclear fuel and radioactive waste. This report is updated annually to keep abreast of continual waste inventory and projection changes in both the government and commercial sectors. Baseline information is provided for DOE program planning purposes and to support DOE program decisions. Although the primary purpose of this document is to provide background information for program planning within the DOE community, it has also been found useful by state and local governments, the academic community, and some private citizens. To sustain the objectives of this program in providing accurate and complete data in this field of operation, comments and suggestions to improve the quality and coverage are encouraged. Such comments and any general inquiries should be directed to the U.S. Department of Energy at either of the following:

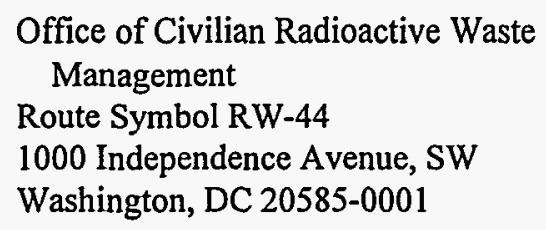

Office of Environmental Management Route Symbol EM-332 or 432 Trevion II Washington, DC 20585-0002

This report was prepared by the Integrated Data Base Program, which is jointly sponsored by the DOE Office of Civilian Radioactive Waste Management and the DOE Office of Environmental Management. Suggestions, questions, and requests for information may be directed to any of the following:

\author{
K. L. Svinicki, DOE/RW-44, Washington, DC 20585-0001 \\ Telephone: (202) 586-5684
}

\section{J. Zenkowich, DOE/EM-332, Washington, DC 20585-0002 \\ Telephone: (301) 903-7126}

M. D. Tolbert-Smith, DOE/EM-432, Washington, DC 20585-0002

Telephone: (301) 903-8121

\section{J. A. Klein, ORNL, P.O. Box 2008, Oak Ridge, TN 37831-6495 \\ Telephone: (423) 574-6823}

An important part of the Integrated Data Base Program is the Steering Committee, whose members provide both generic guidance and technical input. The membership of this committee, shown on the following page, represents all of the major DOE sites and programs for spent nuclear fuel and radioactive waste management. Each support committee member is assisted by a technical liaison as needed. The participation and assistance of these individuals are acknowledged with appreciation.

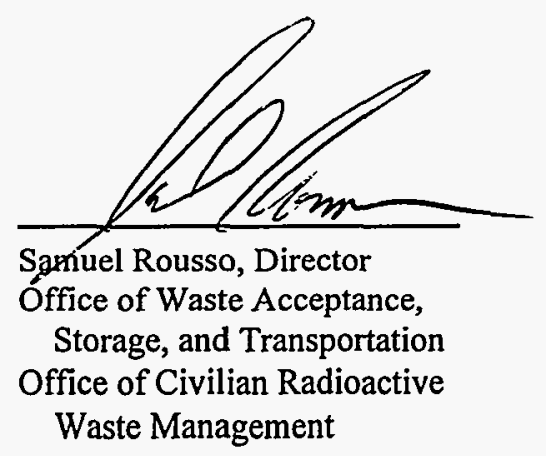

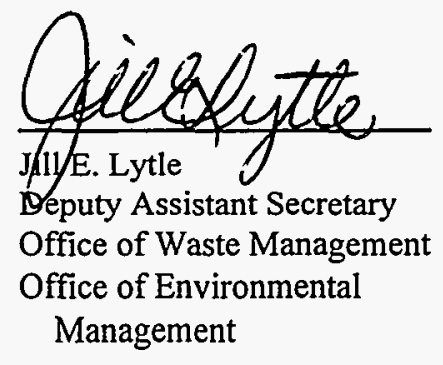

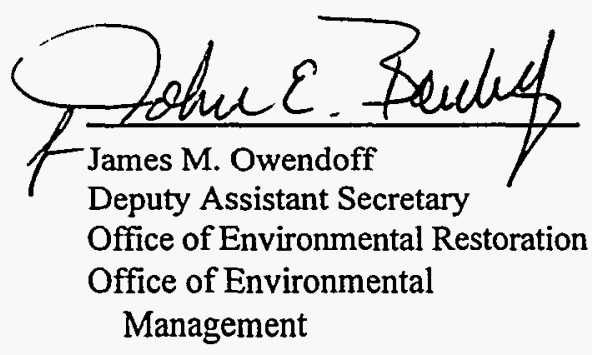


Integrated Data Base Program Steering Committee

Executive Committee

Voting members

K. L. Svinicki, DOE/RW

M. D. Tolbert-Smith, DOE/EM

M. J. Zenkowich, DOE/EM
Advisorymember

K. M. Gibbard, DOE/EIA

J. A. Klein, ORNL

\section{Support Committee}

\section{Chapter title/support area}

Spent nuclear fuel Commercial

DOE

High-level waste

Transuranic waste

Low-level waste

DOE

Commercial

Uranium mill tailings

Environmental restoration wastes

Mixed low-level waste

Waste minimization

\section{Committee member}

K. L. Svinicki, DOE/RW

J. F. Baker, DOE/EM

H. F. Walter, DOE/EM

M. L. Matthews, DOE/CAO

G. J. Duggan, DOE/EM

T. Plummer, DOE/EM

J. H. Boyd, DOE/ID

T. Chung, DOE/EIA

M. D. Tolbert-Smith, DOE/EM

J. L. Sands, DOE/EM

G. T. McBrien, DOE/EM

\section{Technical liaison}

R. R. MacDonald, CRWMSM\&O/JAI

A. R. Bringhurst, LMIT

A. Sidpara, DOE/RL

P. E. Drez, DEA

J. Hwang, CADMUS

R. L. Fuchs, LMIT

T. Chung, DOE/EIA

J. M. Peterson, ANL-E

T. D. Kirkpatrick, LMIT

G. T. McBrien, DOE/EM 
Authors and contributors providing direct support for the Integrated Data Base Program report (Rev. 11)

Chapter topic

\section{Overview}

Spent nuclear fuel

High-level waste

Transuranic waste

Low-level waste

Uranium mill tailings

Environmental restoration wastes

Commercial decommissioning wastes

Mixed low-level waste

Waste source terms

\section{Direct support service}

Clerical/DB report

Clerical/IDB Program

Computer support (data base setup and analysis)

Computer graphics

Editing

Distribution

Indexing
Author(s)

J. A. Klein and S. N. Storch

R. C. Ashline ${ }^{\mathrm{a}}$

S. L. Loghry

P. E. Drez (WTAC) ${ }^{b}$

A. S. Icenhour, S. L. Loghry, and M. L. Tharp

T. Chung (DOE/EIA)

M. Tolbert-Smith (DOE/EM-40) and J. M. Peterson (ANL-E)

S. N. Storch

S. L. Loghry and S. N. Storch ${ }^{d}$

A. S. Icenhour and S. L. Loghry

Contributor(s)

D. T. Brooksbank

S. L. McDaniel

M. L. Tharp and S. L. Loghry

S. L. McDaniel

R. W. Sharpe

S. L. McDaniel, D. T. Brooksbank, and S. N. Storch

R. W. Sharpe and S. N. Storch

${ }^{a}$ With support from DOE/EIA and DOE Office of Spent Fuel Management.

bWaste Isolation Pilot Plant Technical Assistance Contractor (WTAC). The WTAC provided the transuranic waste chapter for this report. Support for this chapter was also provided by MAC Technical Services Company.

cWith support from DOE LLW Management Program (Cadmus Group) and National Low-Level Waste Management Program.

With support from INEL Technical Support Program. 
Major DOE data sources supporting the Integrated Data Base Program report

\begin{tabular}{|c|c|c|c|}
\hline Name of data source & Waste type information provided & Supporting DOE program office & $\begin{array}{l}\text { Principal contractor and } \\
\text { mailing address } \\
\text { (Phone number) }\end{array}$ \\
\hline $\begin{array}{l}\text { DOE Spent Nuclear Fuel Management } \\
\text { Program }\end{array}$ & DOE spent nuclear fuel & $\begin{array}{l}\text { Office of Spent Fuel Management } \\
\text { (EM-37) }\end{array}$ & $\begin{array}{l}\text { Lockheed Martin Idaho Technologies } \\
\text { P.O. Box } 1625 \\
\text { Idaho Falls, ID } 83415 \\
(208 / 526-8909)\end{array}$ \\
\hline $\begin{array}{l}\text { Environmental Restoration Core } \\
\text { Data Base }\end{array}$ & $\begin{array}{l}\mathrm{DOE} / \mathrm{EM}-40 \text { contaminated } \\
\text { media/waste }\end{array}$ & $\begin{array}{l}\text { Office of Environmental Restoration } \\
(E M-40)\end{array}$ & $\begin{array}{l}\text { DOE/EM Office of Program Integration } \\
\text { (DOE/EM-43) } \\
\text { Cloverleaf Building } \\
\text { U.S. Department of Energy } \\
\text { 19901 Germantown Road } \\
\text { Germantown, MD } 20874 \\
(301 / 903-8121)\end{array}$ \\
\hline $\begin{array}{l}\text { International Nuclear Model, Personal } \\
\text { Computer Version (PCINM) }\end{array}$ & Commercial spent nuclear fuel & $\begin{array}{l}\text { Energy Information Administration } \\
\text { (DOE/EIA) }\end{array}$ & $\begin{array}{l}\text { DOE/EIA Analysis and Systems } \\
\quad \text { Division } \\
\text { Nuclear Fuel Cycle Branch } \\
\text { Route Symbol EI-531 } \\
\text { 1707 H Street, N.W. } \\
\text { Washington, DC 20585-0001 } \\
\text { (202/254-5536) }\end{array}$ \\
\hline $\begin{array}{l}\text { Manifest Information Management } \\
\text { System (MMMS) }\end{array}$ & Commercial low-level waste & Office of Waste Management (EM-30) & $\begin{array}{l}\text { Lockheed Martin Idaho Technologies } \\
\text { National Low-Level Waste } \\
\quad \text { Management Program } \\
\text { P.O. Box } 1625 \\
\text { Idaho Falls, ID } 83415 \\
\text { (208/526-9717) }\end{array}$ \\
\hline Uranium Reserve Assessment Program & Commercial uranium mill tailings & $\begin{array}{l}\text { Energy Information Administration } \\
\text { (DOE/EIA) }\end{array}$ & $\begin{array}{l}\text { DOE/EIA Analysis and Systems } \\
\quad \text { Division } \\
\text { Nuclear Fuel Cycle Branch } \\
\text { Route Symbol EI-531 } \\
\text { 1707 H Street, N.W. } \\
\text { Washington, DC 20585-0001 } \\
\text { (202/254-5556) }\end{array}$ \\
\hline
\end{tabular}


Major DOE data sources supporting the Integrated Data Base Program report (continued)

\begin{tabular}{|c|c|c|c|}
\hline Name of data source & Waste type information provided & Supporting DOE program office & $\begin{array}{l}\text { Principal contractor and } \\
\text { mailing address } \\
\text { (Phone number) }\end{array}$ \\
\hline $\begin{array}{l}\text { Waste Isolation Pilot Plant Transuranic } \\
\text { Waste Baseline Inventory Report } \\
\text { (WTWBIR) }\end{array}$ & DOE transuranic waste & $\begin{array}{l}\text { DOE Carlsbad Area Office } \\
\text { National Transuranic Program Office } \\
\text { (NTPO) }\end{array}$ & $\begin{array}{l}\text { Drez Environmental Associates } \\
8816 \text { Cherry Hills Road, N.E. } \\
\text { Albuquerque, NM } 87111 \\
\text { (505/828-9857) }\end{array}$ \\
\hline $\begin{array}{l}1995 \text { Mixed Waste Inventory Report } \\
\text { (MWIR) Data Base }\end{array}$ & $\begin{array}{l}\text { RCRA- and state-regulated DOE mixed } \\
\text { wastes (high-level, transuranic, and low- } \\
\text { level waste) }\end{array}$ & Office of Waste Management (EM-30) & $\begin{array}{l}\text { Lockheed Martin Idaho Technologies } \\
\text { P.O. Box } 1625 \\
\text { Idaho Falls, ID } 83405 \\
(208 / 526-2746)\end{array}$ \\
\hline
\end{tabular}




\section{CONTENTS}

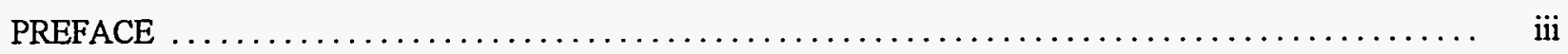

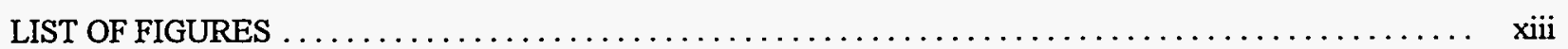

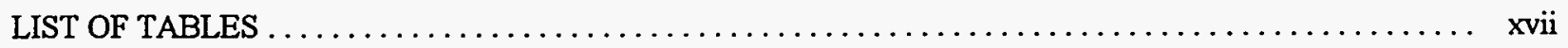

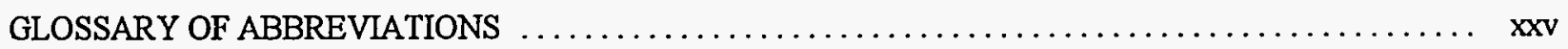

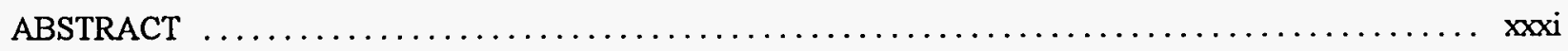

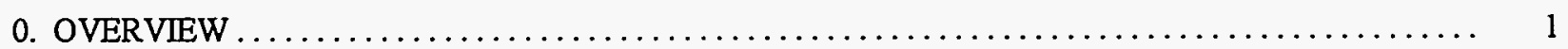

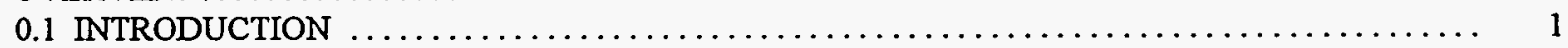

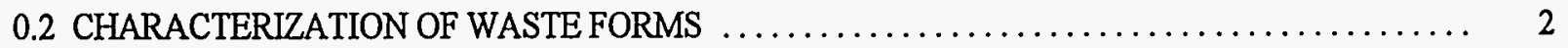

0.3 METHODS AND ASSUMPTIONS USED IN REPORT PREPARATION $\ldots \ldots \ldots \ldots \ldots \ldots \ldots \ldots$

0.4 WASTE CHARACTERISTICS AND UNITS REPORTED $\ldots \ldots \ldots \ldots \ldots \ldots \ldots \ldots \ldots \ldots \ldots \ldots \ldots \ldots \ldots$

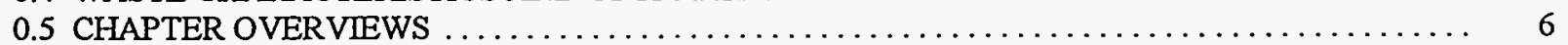

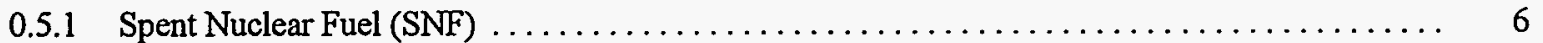

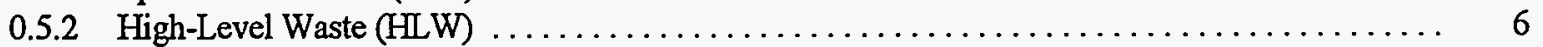

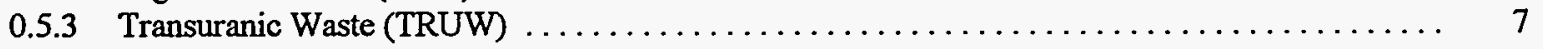

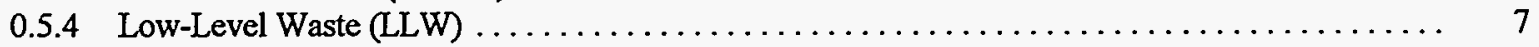

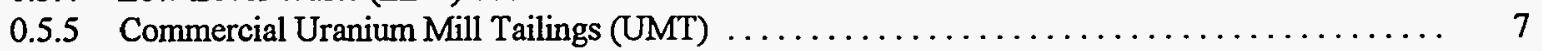

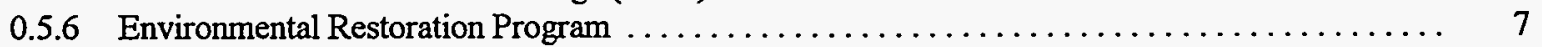

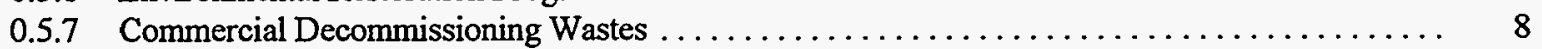

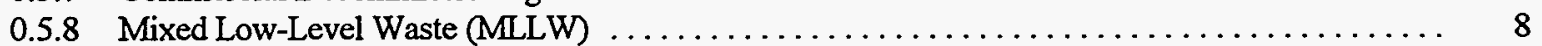

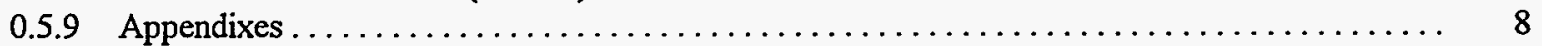

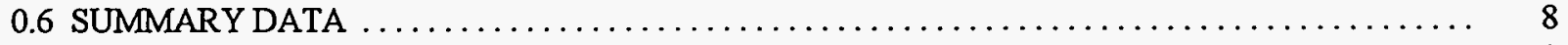

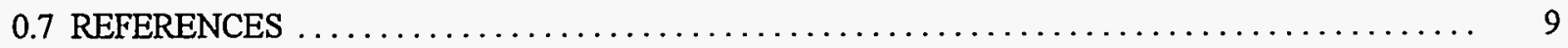

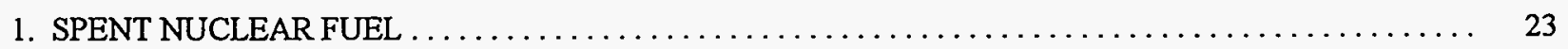

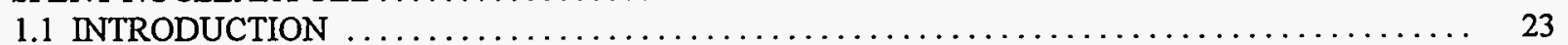

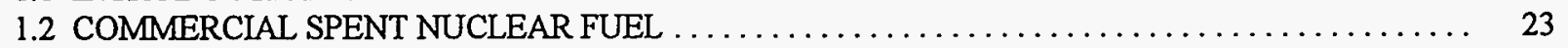

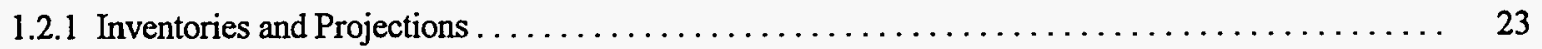

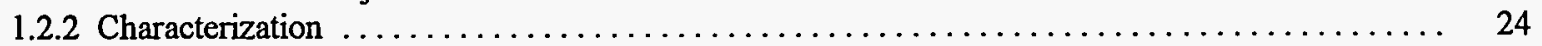

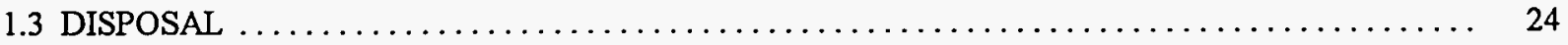

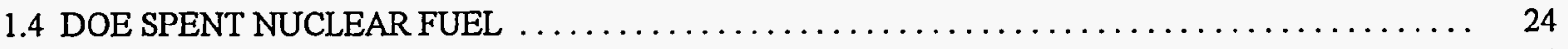

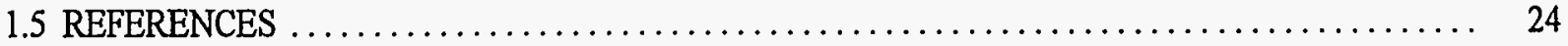

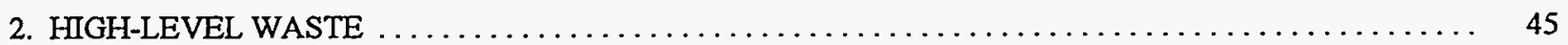

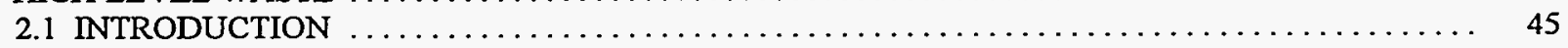

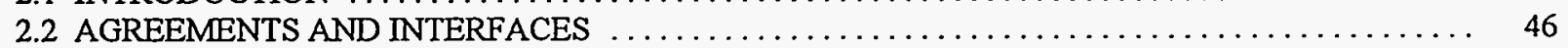

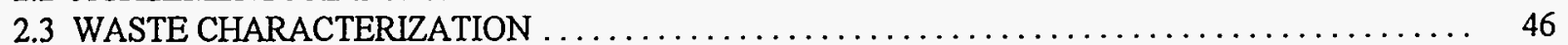

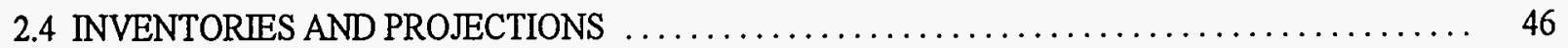

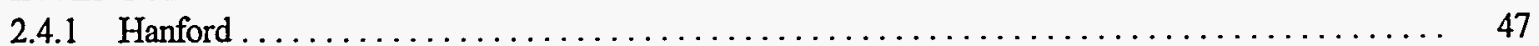

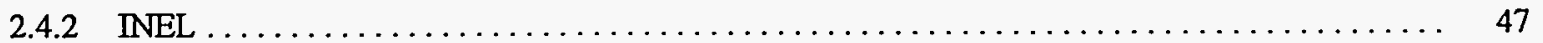




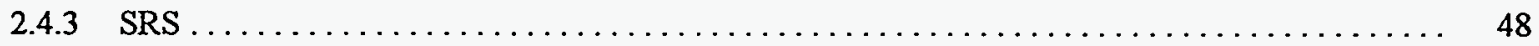

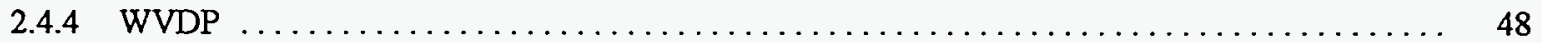

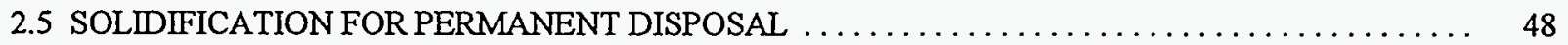

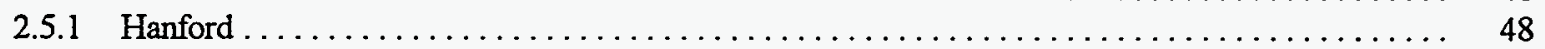

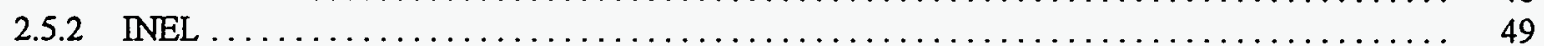

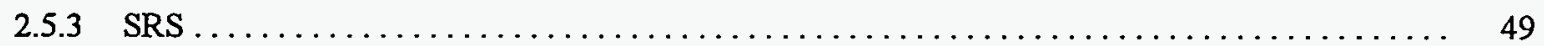

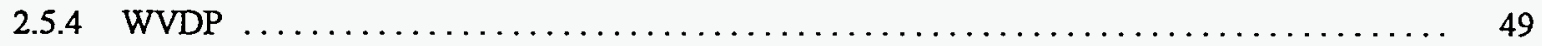

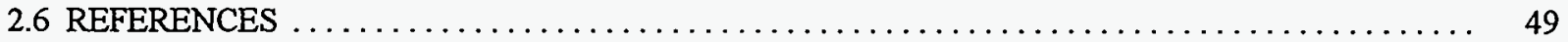

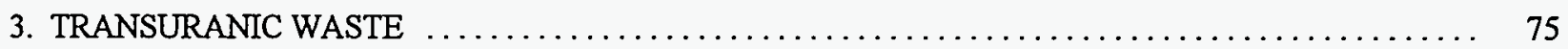

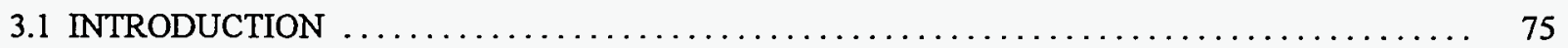

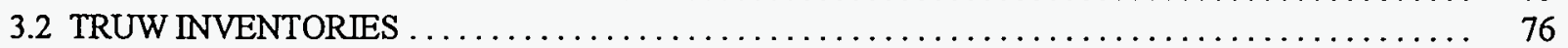

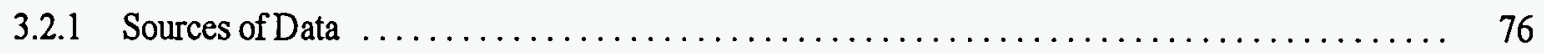

3.2.2 Site Locations-Summarized Volumes and Radioactivity $\ldots \ldots \ldots \ldots \ldots \ldots \ldots \ldots \ldots \ldots$

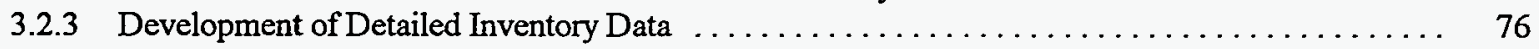

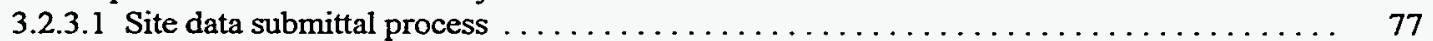

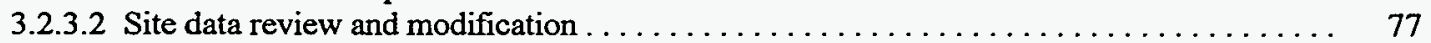

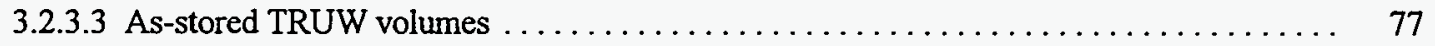

3.2.3.4 Calculation of annual decayed radioactivities $\ldots \ldots \ldots \ldots \ldots \ldots \ldots \ldots \ldots \ldots \ldots$

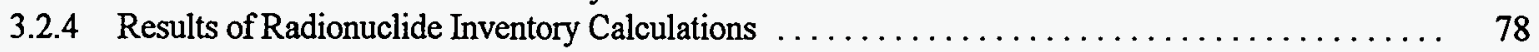

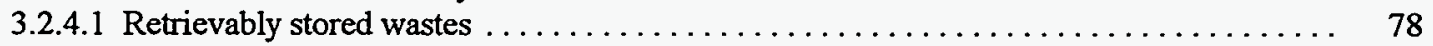

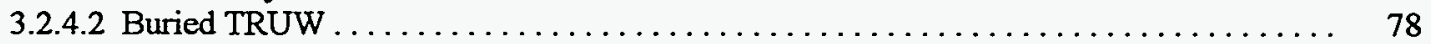

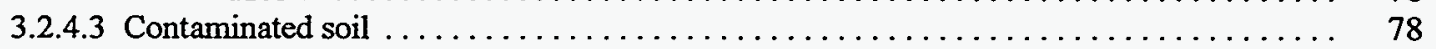

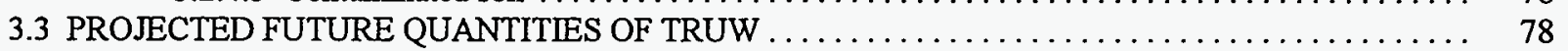

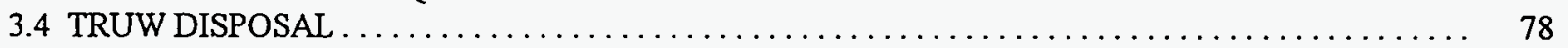

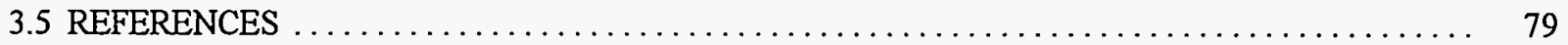

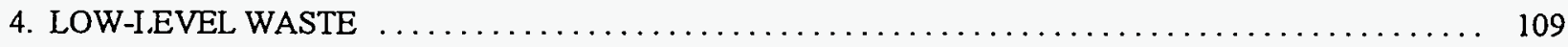

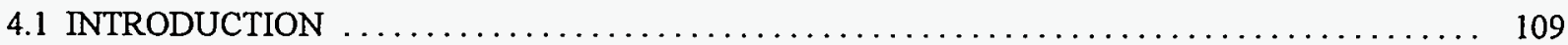

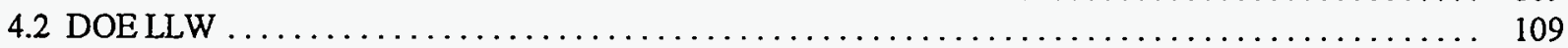

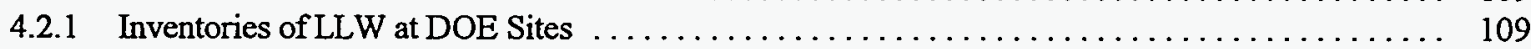

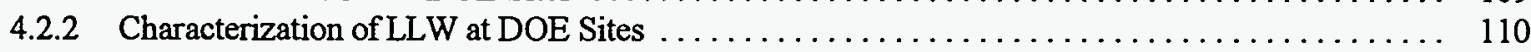

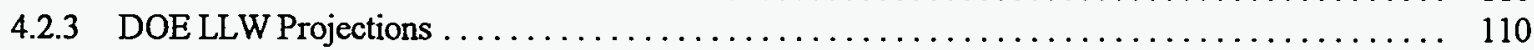

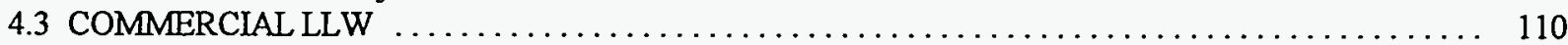

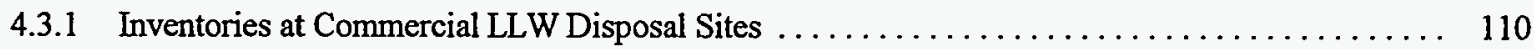

4.3.2 Characterization of LLW at Commercial Disposal Sites $\ldots \ldots \ldots \ldots \ldots \ldots \ldots \ldots \ldots \ldots \ldots \ldots \ldots \ldots$

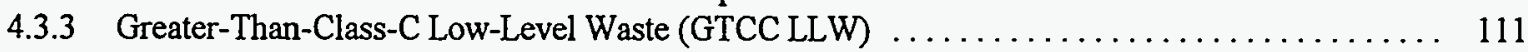

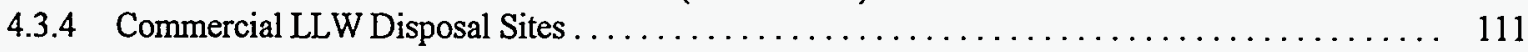

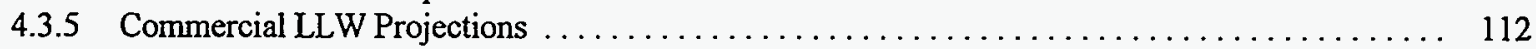

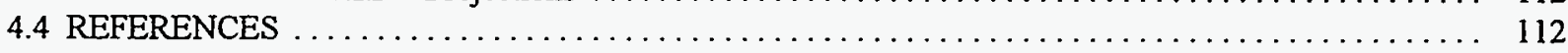

5. URANIUM MILL TAILINGS FROM COMMERCIAL OPERATIONS $\ldots \ldots \ldots \ldots \ldots \ldots \ldots \ldots \ldots \ldots$

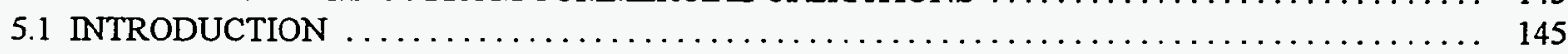

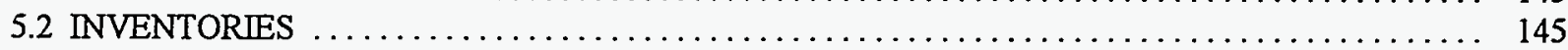

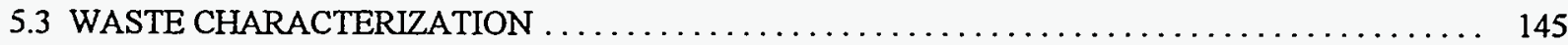

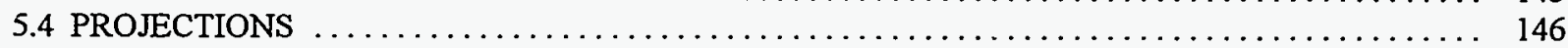

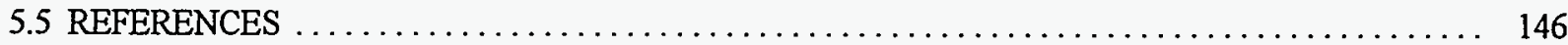

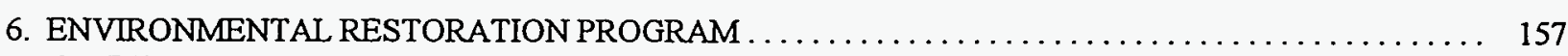

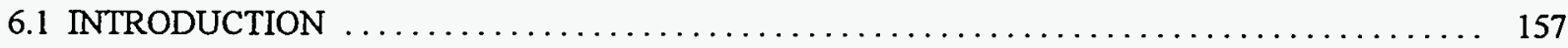


6.2 THE OFFICE OF ENVIRONMENTAL RESTORATION $\ldots \ldots \ldots \ldots \ldots \ldots \ldots \ldots \ldots \ldots \ldots \ldots \ldots \ldots$

6.3 ENVIRONMENTAL RESTORATION WASTE CHARACTERISTICS $\ldots \ldots \ldots \ldots \ldots \ldots \ldots \ldots \ldots . \ldots \ldots$

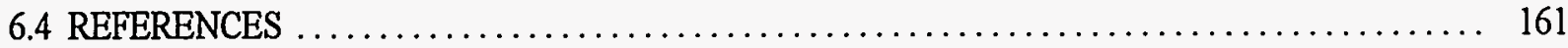

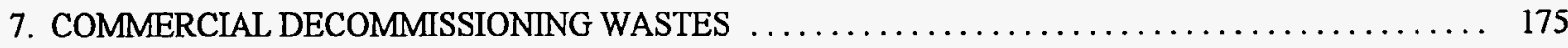

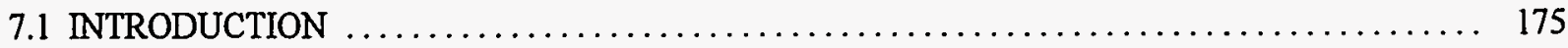

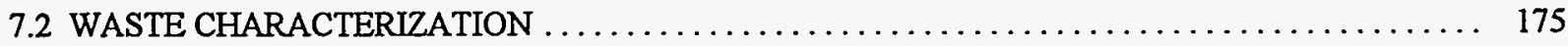

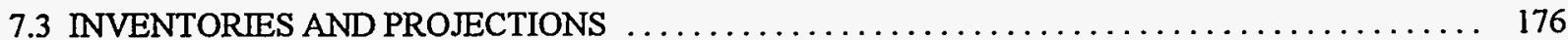

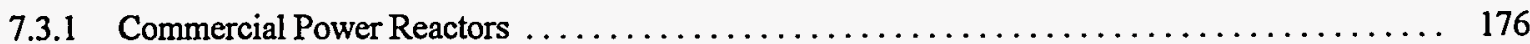

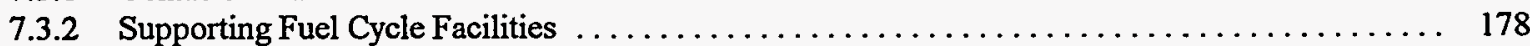

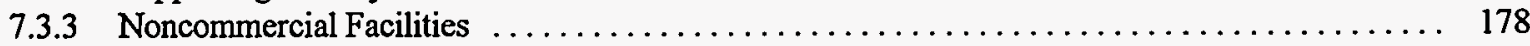

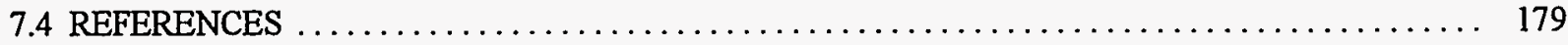

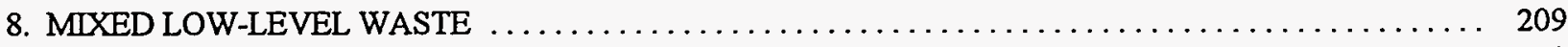

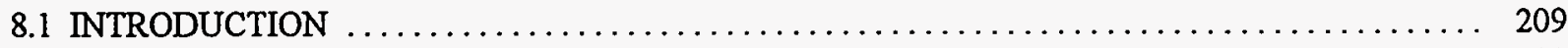

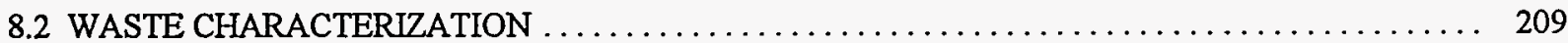

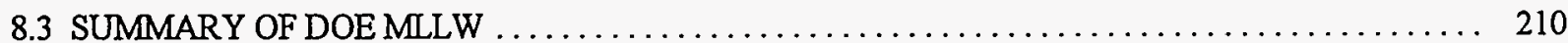

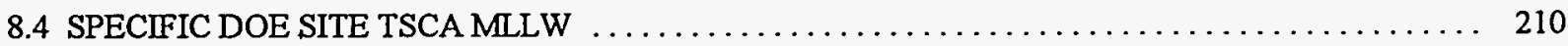

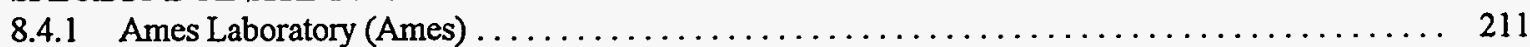

8.4.2 Argonne National Laboratory-East (ANL-E) $\ldots \ldots \ldots \ldots \ldots \ldots \ldots \ldots \ldots \ldots \ldots \ldots \ldots \ldots \ldots \ldots \ldots \ldots \ldots$

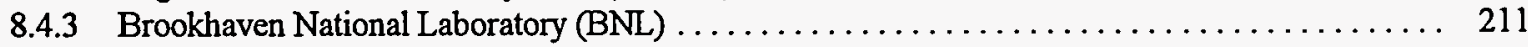

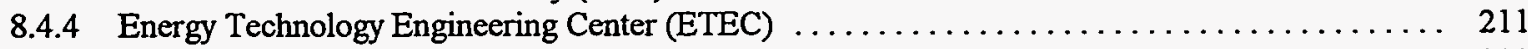

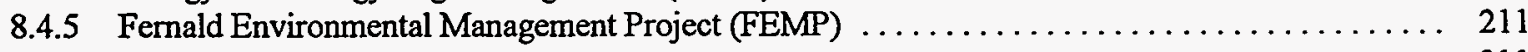

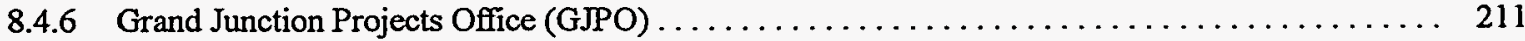

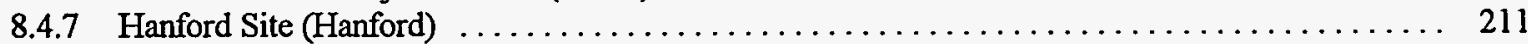

8.4.8 Idaho National Engineering Laboratory (INEL) $\ldots \ldots \ldots \ldots \ldots \ldots \ldots \ldots \ldots \ldots \ldots \ldots \ldots \ldots \ldots$

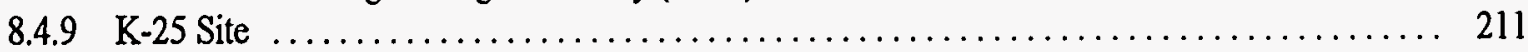

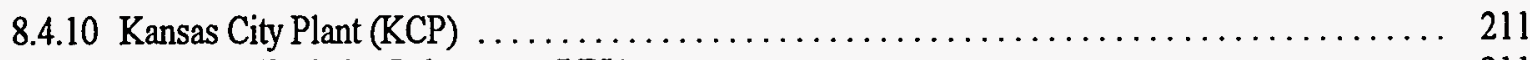

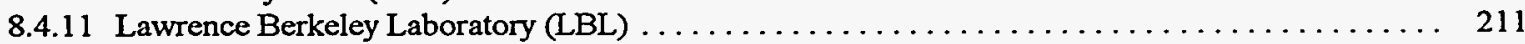

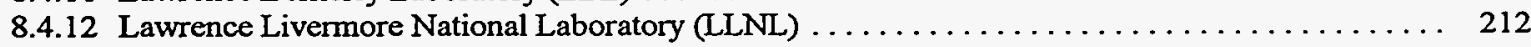

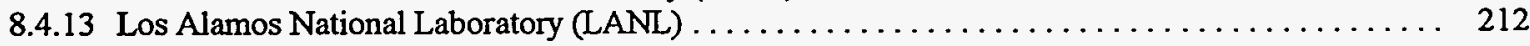

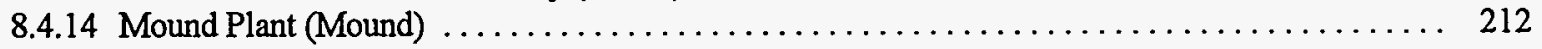

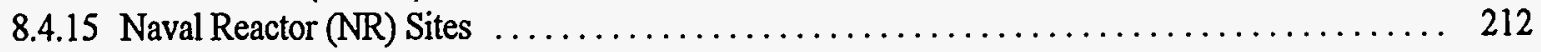

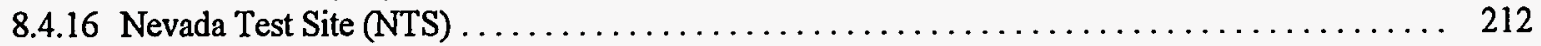

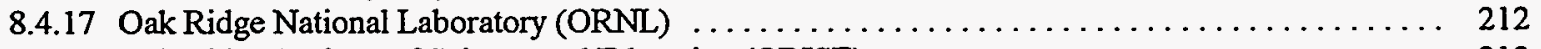

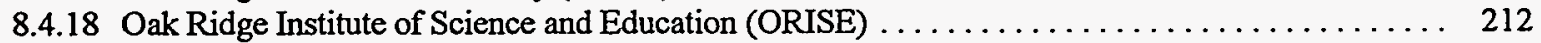

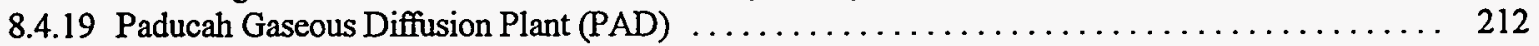

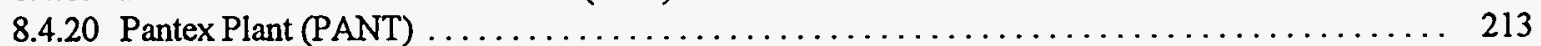

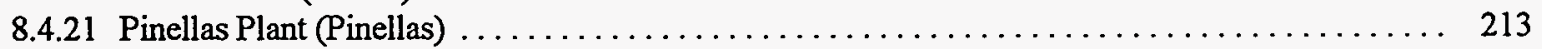

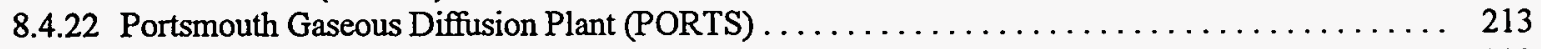

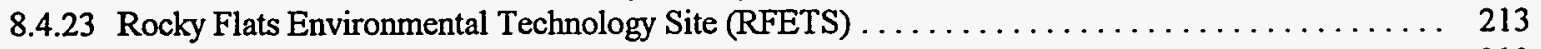

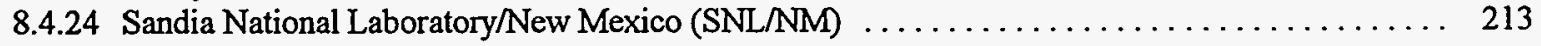

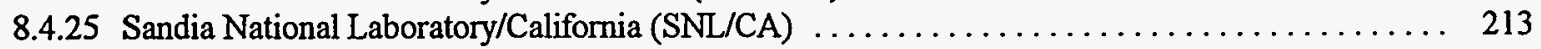

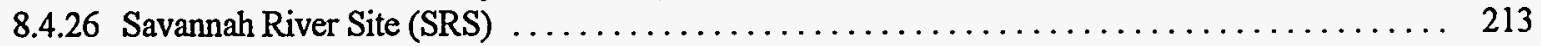

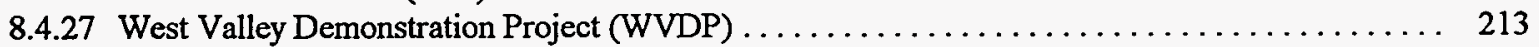

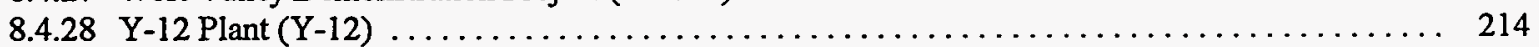

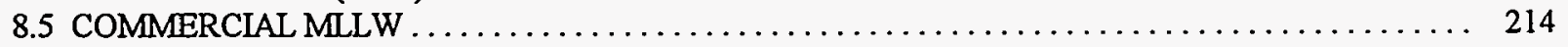

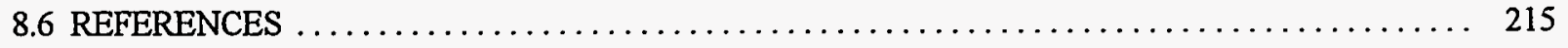

APPENDIX A. WASTE FLOWSHEETS, SOURCE TERMS, AND CHARACTERISTICS $\ldots \ldots \ldots \ldots \ldots 251$

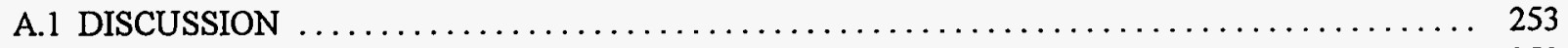

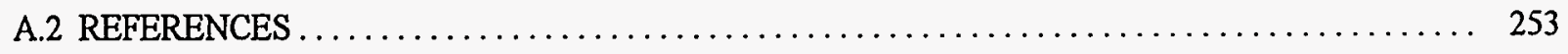


APPENDIX B. CHARACTERISTICS OF IMPORTANT RADIONUCLIDES $\ldots \ldots \ldots \ldots \ldots \ldots \ldots \ldots \ldots . \ldots \ldots$

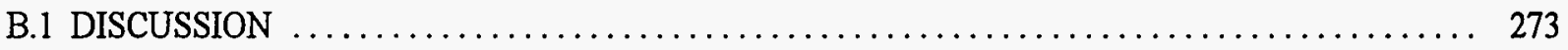

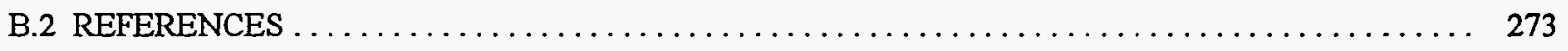

APPENDIX C. REFERENCE SITES AND FACILITIES $\ldots \ldots \ldots \ldots \ldots \ldots \ldots \ldots \ldots \ldots \ldots \ldots \ldots \ldots$

APPENDIX D. INTEGRATED DATA BASE DOCUMENT ACCESS ON THE INTERNET . . . . . . . 299

APPENDIX E. INTEGRATED DATA BASE READER COMMENT FORM $\ldots \ldots \ldots \ldots \ldots \ldots \ldots \ldots \ldots$

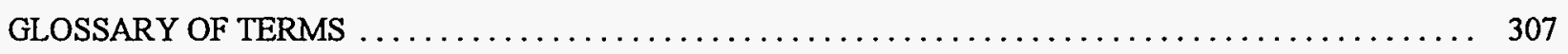

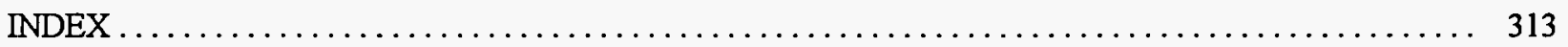




\section{LIST OF FIGURES}

\section{OVERVIEW}

0.1 Total volumes of DOE and commercial radioactive wastes and spent nuclear fuel through

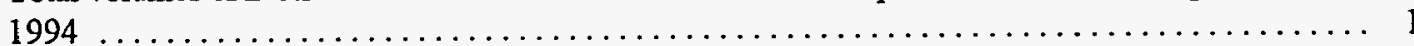

0.2 Total decayed radioactivities of $\mathrm{DOE}$ and commercial radioactive wastes and spent nuclear

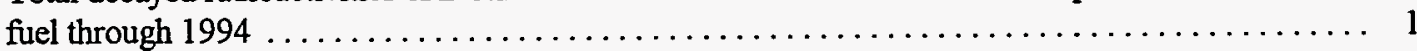

\section{SPENT NUCLEAR FUEL}

1.1 Locations of existing and planned commercial reactors as of December $31,1994 \ldots \ldots \ldots \ldots .26$

1.2 Projected mass (MTIHM) of annual commercial spent nuclear fuel discharges for the

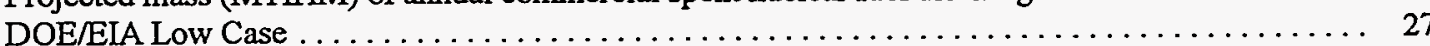

1.3 Projected cumulative radioactivity of commercial spent nuclear fuel discharges for the DOE/EIA Low Case . . . . . . . . . . . . . . . . . . . . . . . . . . . . . . . . . . 27

1.4 Projected cumulative mass (MTIHM) of commercial spent nuclear fuel discharges for the

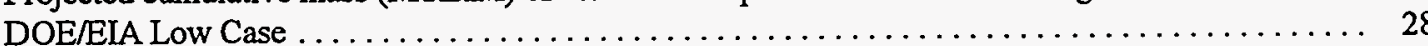

1.5 Radioactivity and thermal power of 1 MTIHM of BWR and PWR spent nuclear fuel as a function of burnup and time from reactor discharge

\section{HIGH-LEVEL WASTE}

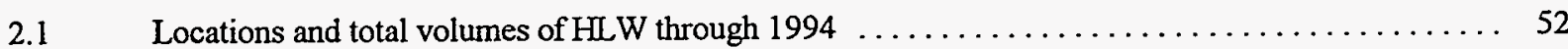

2.2 Total volume and radioactivity of solid and liquid HLW through $1994 \ldots \ldots \ldots \ldots \ldots \ldots \ldots . \ldots 2$

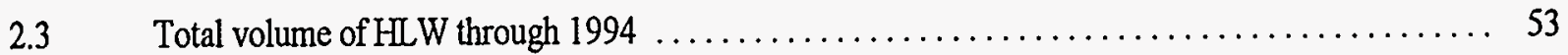

$2.4 \quad$ Total decayed radioactivity of HLW through $1994 \ldots \ldots \ldots \ldots \ldots \ldots \ldots \ldots \ldots \ldots \ldots \ldots \ldots \ldots \ldots$

2.5a Historical and projected cumulative volumes of $\mathrm{HLW}$ in storage $\ldots \ldots \ldots \ldots \ldots \ldots \ldots \ldots \ldots$

$2.5 b \quad$ Projected cumulative number of $\mathrm{HLW}$ canisters $\ldots \ldots \ldots \ldots \ldots \ldots \ldots \ldots \ldots \ldots \ldots \ldots \ldots \ldots \ldots \ldots \ldots$

2.6 Treatment methods for tank waste and capsules at Hanford $\ldots \ldots \ldots \ldots \ldots \ldots \ldots \ldots \ldots \ldots \ldots$

2.7 Treatment methods for $\mathrm{HLW}$ in tanks, bins, and canisters at INEL $\ldots \ldots \ldots \ldots \ldots \ldots \ldots \ldots$ 
$2.8 \quad$ Treatment methods for $\mathrm{HLW}$ in tanks and canisters at $\mathrm{SRS} \ldots \ldots \ldots \ldots \ldots \ldots \ldots \ldots \ldots$

$2.9 \quad$ Treatment methods for HLW in tanks and canisters at WVDP $\ldots \ldots \ldots \ldots \ldots \ldots \ldots \ldots \ldots$

\section{TRANSURANIC WASTE}

$3.1 \quad$ U.S. Department of Energy transuranic waste generation/storage sites $\ldots \ldots \ldots \ldots \ldots \ldots \ldots .83$

3.2 Retrievably stored and projected TRUW volumes by radioactivity classification $\ldots \ldots \ldots \ldots .84$

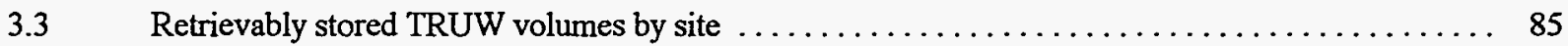

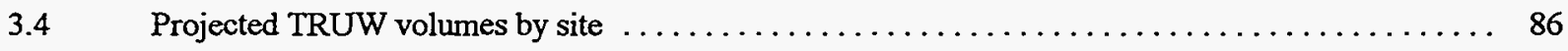

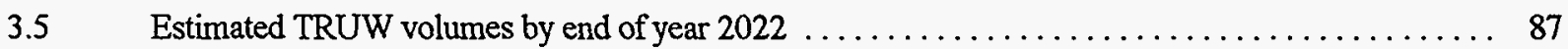

\section{LOW-LEVEL WASTE}

4.1 Volume and radioactivity of LLW disposed at commercial and DOE facilities during $1994 \ldots \ldots 116$

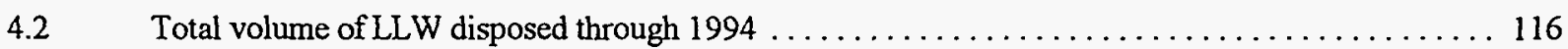

4.3 Total volume of DOE LLW disposed through $1994 \ldots \ldots \ldots \ldots \ldots \ldots \ldots \ldots \ldots \ldots \ldots \ldots$

4.4 Locations and total volumes of DOE LLW disposed through $1994 \ldots \ldots \ldots \ldots \ldots \ldots \ldots \ldots$

4.5 Total volume of commercial LLW disposed through $1994 \ldots \ldots \ldots \ldots \ldots \ldots \ldots \ldots \ldots \ldots \ldots$

4.6 Locations and total volumes of commercial LLW disposed through $1994 \ldots \ldots \ldots \ldots \ldots \ldots 118$

\section{URANIUM MILL TAILINGS FROM COMMERCIAL OPERATIONS}

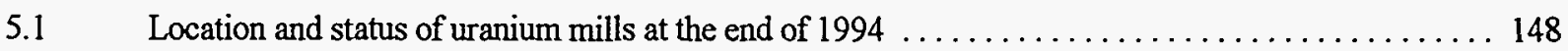

5.2 Historical and projected cumulative volume of commercial mill tailings $\ldots \ldots \ldots \ldots \ldots \ldots .149$

\section{ENVIRONMENTAL RESTORATION PROGRAM}

6.1 Locations of field offices that direct the DOE environmental restoration program $\ldots \ldots \ldots \ldots \ldots 2$

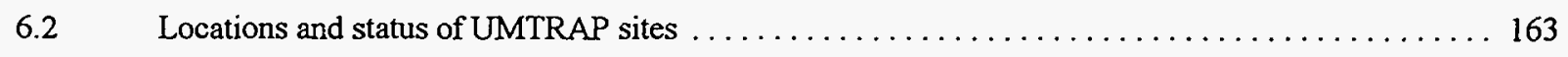

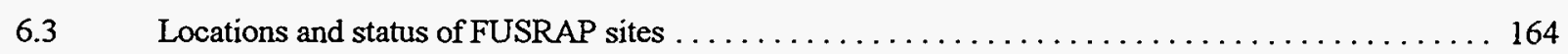

6.4 Estimated volumes of radioactively contaminated soils associated with environmental

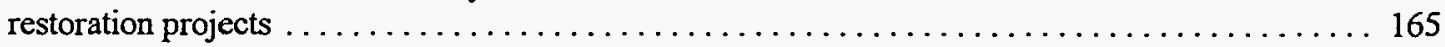

6.5 Estimated volumes of radioactively contaminated debris associated with environmental restoration projects 


\section{MIXED LOW-LEVEL WASTE}

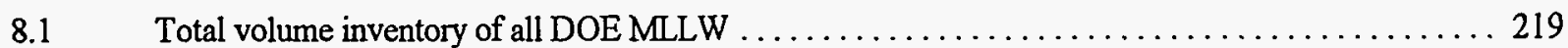

8.2 Projected cumulative volume generation of all DOE MLLW during 1994-1998 $\ldots \ldots \ldots \ldots 219$

\section{APPENDIX A. WASTE FLOWSHEETS, SOURCE TERMS, AND CHARACTERISTICS}

A.1 Boiling-water reactor decommissioning wastes per $1-\mathrm{MW}(\mathrm{e})$ capacity $\ldots \ldots \ldots \ldots \ldots \ldots \ldots \ldots$

A.2 Pressurized-water reactor decommissioning wastes per $1-\mathrm{MW}(\mathrm{e})$ capacity $\ldots \ldots \ldots \ldots \ldots \ldots 256$ 


\section{LIST OF TABLES}

\section{OVERVIEW}

$0.1 \quad$ Types of radioactive wastes managed at $\mathrm{DOE}$ sites referenced in this report $\ldots \ldots \ldots \ldots \ldots \ldots$

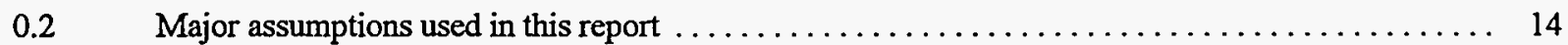

0.3 Spent nuclear fuel and radioactive waste inventories as of December $31,1994 \ldots \ldots \ldots \ldots \ldots$

0.4 Current and projected cumulative quantities of radioactive waste and spent nuclear fuel $\ldots \ldots \ldots \quad 16$

$0.5 \quad$ Volume $\left(\mathrm{m}^{3}\right)$ of DOE site wastes generated during calendar year $1994 \ldots \ldots \ldots \ldots \ldots \ldots \ldots$

0.6 Cumulative volume $\left(\mathrm{m}^{3}\right)$ of stored DOE site wastes as of December $31,1994 \ldots \ldots \ldots \ldots \ldots .18$

$0.7 \quad$ Cumulative volume $\left(\mathrm{m}^{3}\right)$ of buried DOE site wastes $\ldots \ldots \ldots \ldots \ldots \ldots \ldots \ldots \ldots \ldots \ldots \ldots \ldots \ldots \ldots$

0.8 Volume $\left(\mathrm{m}^{3}\right)$ summary of radioactive wastes disposed of at the Envirocare Facility near

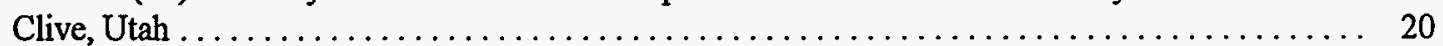

\section{SPENT NUCLEAR FUEL}

1.1 Historical and projected installed LWR electric power generating capacity for the DOE/EIA Low Case

1.2 Projected cumulative mass of commercial spent nuclear fuel discharges for the DOE/EIA

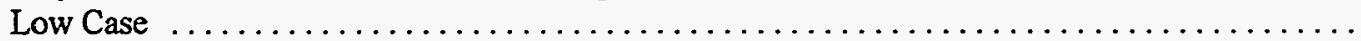

1.3 Historical and projected mass, radioactivity, and thermal power of permanently discharged spent nuclear fuel by reactor type for the DOE/EIA Low Case

1.4 Projected number of permanently discharged LWR spent nuclear fuel assemblies for the

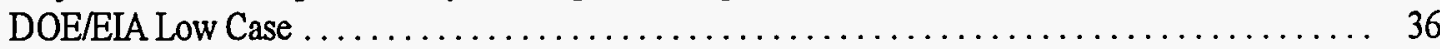

$1.5 \quad$ Spent nuclear fuel discharges from the Fort St. Vrain HTGR $\ldots \ldots \ldots \ldots \ldots \ldots \ldots \ldots \ldots \ldots \ldots \ldots \ldots$

1.6 $\mathrm{DB}$ reference characteristics of $L W R$ nuclear fuel assemblies $\ldots \ldots \ldots \ldots \ldots \ldots \ldots \ldots \ldots$

1.7 Historical mass of commercial BWR spent nuclear fuel discharged at various ranges of

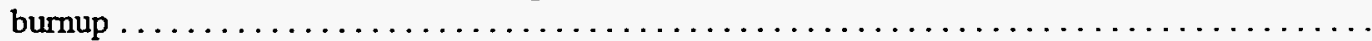

1.8 Historical mass of commercial PWR spent nuclear fuel discharged at various ranges of

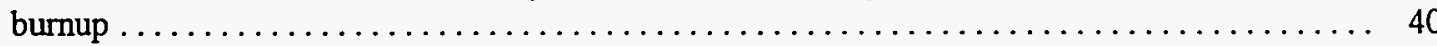

1.9 Summary inventory of DOE spent nuclear fuel for years 1995 and $2035 \ldots \ldots \ldots \ldots \ldots \ldots \ldots$ 
xviii

\section{HIGH-LEVEL WASTE}

2.1 Historical and projected cumulative volume of HLW stored in tanks, bins, and capsules, by site . . . 57

2.2 Historical and projected cumulative decayed radioactivity of $\mathrm{HLW}$ stored in tanks, bins, and

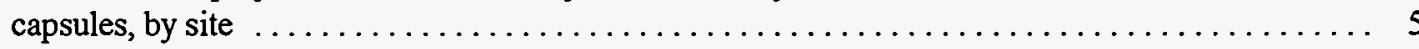

2.3 Historical and projected cumulative decayed thermal power of $\mathrm{HLW}$ stored in tanks, bins, and

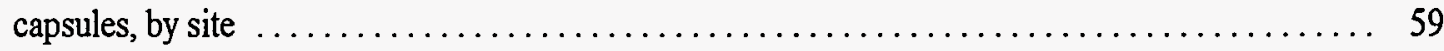

2.4 Projected annual and cumulative volume $\left(10^{3} \mathrm{~m}^{3}\right)$ of $\mathrm{HLW}$ glass stored in canisters, by site $\ldots \ldots 60$

2.5 Projected annual and cumulative decayed radioactivity $\left(10^{6} \mathrm{Ci}\right)$ of $\mathrm{HLW}$ glass stored in

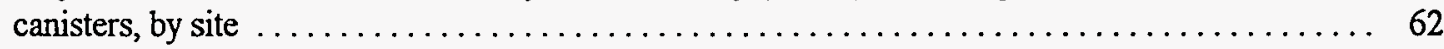

2.6 Projected annual and cumulative decayed thermal power $\left(10^{3} \mathrm{~W}\right)$ of HLW glass stored in

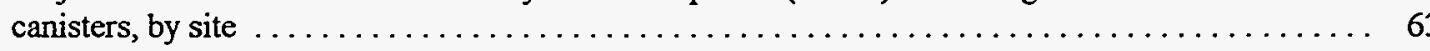

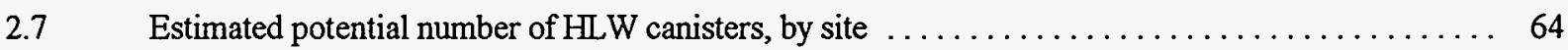

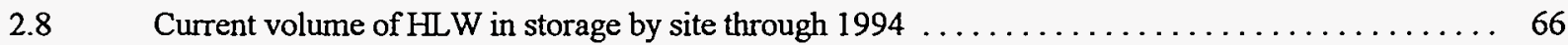

$2.9 \quad$ Current radioactivity of $H L W$ in storage by site through $1994 \ldots \ldots \ldots \ldots \ldots \ldots \ldots \ldots \ldots$

2.10 Current thermal power of HLW in storage by site through $1994 \ldots \ldots \ldots \ldots \ldots \ldots \ldots \ldots \ldots$

$2.11 \quad$ Major radionuclides comprising $\mathrm{HLW}$ and associated wastes at Hanford $\ldots \ldots \ldots \ldots \ldots \ldots$

2.12 Major radionuclides comprising $\mathrm{HLW}$ and associated wastes at INEL $\ldots \ldots \ldots \ldots \ldots \ldots \ldots$

2.13 Major radionuclides comprising $\mathrm{HLW}$ and associated wastes at SRS $\ldots \ldots \ldots \ldots \ldots \ldots$

2.14 Major radionuclides comprising $\mathrm{HLW}$ and associated wastes at WVDP $\ldots \ldots \ldots \ldots \ldots \ldots \ldots$

2.15 Significant revisions and changes in the current values for HLW compared to the values in

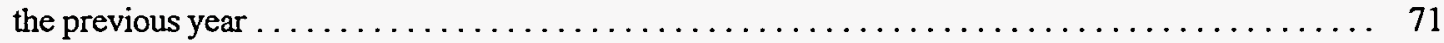

2.16 Representative chemical composition of future $\mathrm{HLW}$ glass to be generated at Hanford $\ldots \ldots \ldots 72$

2.17 Representative chemical composition of future $\mathrm{HLW}$ glass to be generated at INEL $\ldots \ldots \ldots .72$

2.18 Representative chemical composition of future $\mathrm{HLW}$ glass to be generated at SRS $\ldots \ldots \ldots \ldots 73$

2.19 Representative chemical composition of future HLW glass to be generated at WVDP ....... 73

\section{TRANSURANIC WASTE}

3.1 Comparison of IDB and WTWBIR retrievably stored volumes of TRUW $\ldots \ldots \ldots \ldots \ldots \ldots$

3.2 Summary of final waste-form volumes $\left(\mathrm{m}^{3}\right)$ of retrievably stored and projected TRUW

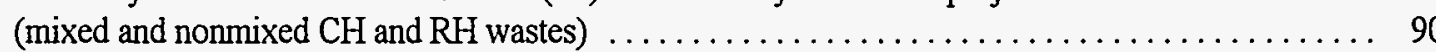


3.3 Summary of final waste-form volumes $\left(\mathrm{m}^{3}\right)$ of retrievably stored and projected mixed and

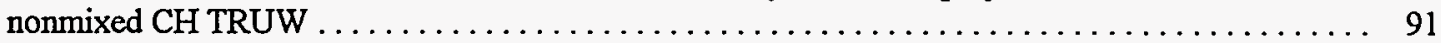

3.4 Final waste-form volumes $\left(\mathrm{m}^{3}\right)$ of retrievably stored and projected mixed CH TRUW $\ldots \ldots \ldots \ldots$

3.5 Final waste-form volumes $\left(\mathrm{m}^{3}\right)$ of retrievably stored and projected nonmixed $\mathrm{CH}$ TRUW $\ldots \ldots \ldots 94$

3.6 Summary of final waste-form volumes $\left(\mathrm{m}^{3}\right)$ of retrievably stored and projected mixed and

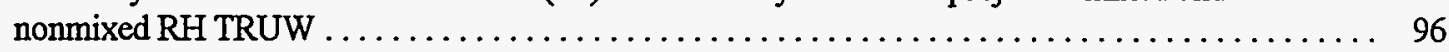

3.7 Final waste-form volumes $\left(\mathrm{m}^{3}\right)$ of retrievably stored and projected mixed RH TRUW . . . . . . . 97

3.8 Final waste-form volumes $\left(\mathrm{m}^{3}\right)$ of retrievably stored and projected nonmixed RH TRUW . . . ... 98

3.9 Estimated final-form volume $\left(\mathrm{m}^{3}\right)$ composition of retrievably stored and projected TRUW

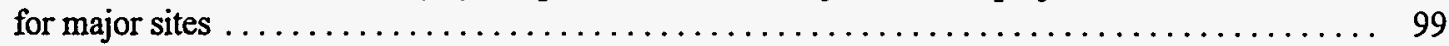

3.10 Estimated final-form volume $\left(\mathrm{m}^{3}\right)$ composition of retrievably stored and projected TRUW

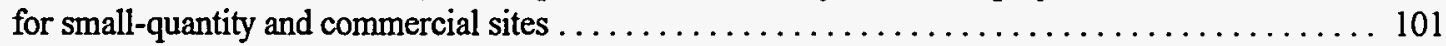

3.11 Summary of decayed radioactivity isotopic content of retrievably stored CH TRUW $\ldots \ldots \ldots \ldots 2$

3.12 Summary of decayed radioactivity (Ci) isotopic content of retrievably stored RH TRUW $\ldots \ldots \ldots 103$

3.13 Summary of decayed mass (g) isotopic content of retrievably stored $\mathrm{CH}$ TRUW $\ldots \ldots \ldots \ldots \ldots 104$

3.14 Summary of decayed mass (g) isotopic content of retrievably stored RH TRUW $\ldots \ldots \ldots \ldots \ldots 105$

3.15 Summary of buried TRUW volume and radioactivity as of December $31,1993 \ldots \ldots \ldots \ldots \ldots 106$

3.16 Volumes and radioactivities of TRU-contaminated soils $\ldots \ldots \ldots \ldots \ldots \ldots \ldots \ldots \ldots \ldots \ldots$

\section{LOW-LEVEL WASTE}

4.1 Summary of characteristics for disposed LLW as of December $31,1994 \ldots \ldots \ldots \ldots \ldots \ldots$

4.2 Historical and projected volume, radioactivity, and thermal power of disposed DOE LLW $\ldots \ldots 120$

4.3 Historical and projected volume, radioactivity, and thermal power of commercial LLW shipped for disposal

4.4 Historical annual additions and total volume of LLW disposed at DOE sites $\ldots \ldots \ldots \ldots \ldots \ldots 122$

4.5 Description of physical forms used to characterize solid LLW from DOE activities $\ldots \ldots \ldots 123$

4.6 Actual 1994 generation and projected average annual generation of LLW at DOE sites $\ldots \ldots \ldots 125$

4.7 Actual 1994 cumulative storage inventory and projected annual storage additions of LLW at DOE sites 
4.9 Breakdown by physical form of volumes of LLW generated during 1994 at DOE sites $\ldots \ldots \ldots 128$

4.10 Breakdown by physical form of radioactivity of LLW generated during 1994 at DOE sites ..... 129

4.11 Breakdown by physical form of cumulative volumes of LLW stored at DOE sites $\ldots \ldots \ldots \ldots 130$

4.12 Breakdown by physical form of total gross radioactivity of LLW stored at DOE sites . . . . . . 131

4.13 Breakdown by physical form of volumes of LLW disposed during 1994 at DOE sites $\ldots \ldots \ldots \ldots 132$

4.14 Breakdown by physical form of radioactivity of LLW disposed during 1994 at DOE sites ..... 133

4.15 DOE LLW disposed by methods other than shallow-land burial $\ldots \ldots \ldots \ldots \ldots \ldots \ldots \ldots 134$

4.16 Significant revisions and changes in the current values for LLW compared to the values in

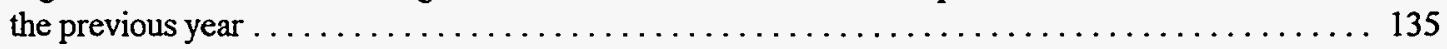

4.17 Historical and projected volume, radioactivity, and thermal power characteristics of

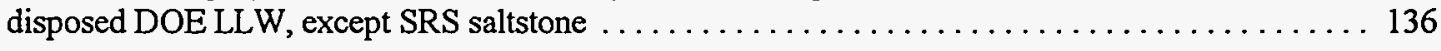

4.18 Projected volume, radioactivity, and thermal power characteristics of DOE LLW saltstone

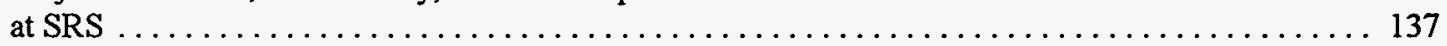

$4.19 \quad$ Historical annual additions and total volume of $L L W$ at commercial disposal sites $\ldots \ldots \ldots \ldots 138$

4.20 Historical annual additions and total undecayed radioactivity of LLW at commercial

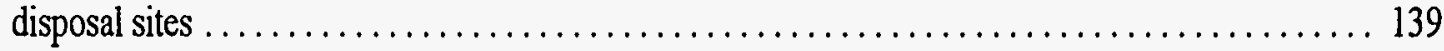

4.21 Distribution of total volume and radioactivity, by state, of LLW shipped to commercial

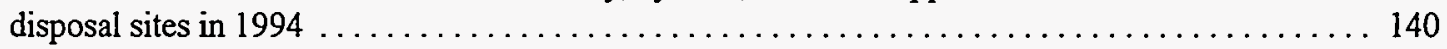

4.22 Historical and projected cumulative volume and radioactivity summary of commercial GTCC LLW

4.23 Breakdown of 1994 low-level radioactive waste by type, volume, and radioactivity received by commercial disposal sites

\section{URANIUM MILL TAILINGS FROM COMMERCIAL OPERATIONS}

$5.1 \quad$ Historical and projected volume of uranium mill tailings $\ldots \ldots \ldots \ldots \ldots \ldots \ldots \ldots \ldots$

5.2 Status of conventional uranium mill sites at the end of $1994 \ldots \ldots \ldots \ldots \ldots \ldots \ldots \ldots \ldots \ldots$

5.3 Uranium ore processed, $\mathrm{U}_{3} \mathrm{O}_{8}$ recovery rate, and tailings generated through $1994 \ldots \ldots \ldots \ldots 154$

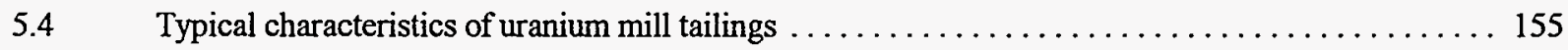




\section{ENVIRONMENTAL RESTORATION PROGRAM}

6.1 Estimated volumes of radioactively contaminated soils associated with environmental restoration projects 166

6.2 Estimated volumes of radioactively contaminated debris associated with environmental

6.3 Summary of environmental restoration wastes disposed of at the Envirocare Facility

\section{COMMERCIAL DECOMMISSIONING WASTES}

7.1 List of U.S. civilian reactors shut down or dismantled as of December $31,1994 \ldots \ldots \ldots \ldots \ldots .183$

7.2 List of U.S. nuclear fuel cycle facilities shut down or being decommissioned as of

December 31, 1994 186

7.3 Projections of radioactive wastes from decommissioning reference commercial power reactors and fuel cycle facilities

7.4 Schedule of actual and projected final shutdown dates for commercial light-water reactors $\ldots \ldots \ldots 188$

7.5 Quantities of shipped LLW from completed and ongoing reactor decommissioning projects $\ldots \ldots \ldots 189$

7.6 Revised estimates of disposal volumes for radioactive wastes from decommissioning alternatives considered for reference LWRs

7.7 Projections of cumulative volume, radioactivity, and thermal power of wastes from decommissioning commercial light-water reactors shut down during 1995-2030

7.8 Projected characteristics of radioactive wastes from Dresden-Unit 1 decommissioning activities

7.9 Actual radioactive waste disposal from the decommissioning of the Fort St. Vrain HTGR

7.10 Projected characteristics of wastes from DECON (dismantling) of the Fort St. Vrain HTGR

7.11 Projected burial volumes of radioactive wastes from SAFSTOR (mothballing/delayed dismantling) of Humboldt Bay-Unit 3 BWR

7.12 Projected volumes of wastes from Indian Point-Unit 1 PWR decommissioning activities 196

7.13 Inventories and projections of low-level radioactive wastes from La Crosse BWR

decommissioning activities .

7.14 Projected volumes of wastes from Rancho Seco PWR decommissioning activities . . . . . . . 198

7.15 Projected volumes of wastes from San Onofre-Unit 1 PWR decommissioning activities 199

7.16 Projected volumes of wastes from future Saxton PWR decommissioning activities 201 
xxii

7.17 Characteristics of wastes from Shoreham BWR decommissioning activities . . . . . . . . . 202

7.18 Characteristics of wastes from decontamination activities at the Three Mile Island-Unit 2

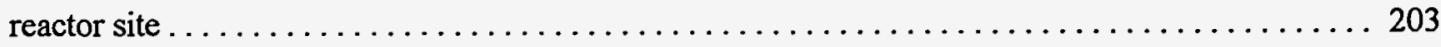

7.19 Projected classification and volumes of wastes from Trojan PWR decommissioning activities . . . . 204

7.20 Projected characteristics of wastes from Yankee Rowe PWR decommissioning activities ...... 204

7.21 Characteristics of wastes from decommissioning activities at the Cimarron Fuel

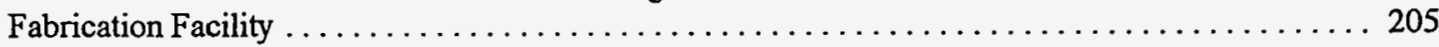

7.22 Inventories and projections of wastes from various activities at the West Valley

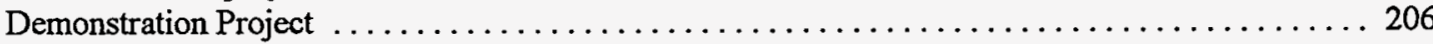

\section{MIXED LOW-LEVEL WASTE}

8.1 Thirty-eight states and territories with EPA mixed waste authorization $\ldots \ldots \ldots \ldots \ldots \ldots \ldots \ldots$

8.2 Cumulative mass $(\mathrm{kg})$ inventories of $\mathrm{MLLW}$ from $\mathrm{DOE}$ site activities $\ldots \ldots \ldots \ldots \ldots \ldots \ldots 221$

8.3 Cumulative volume $\left(\mathrm{m}^{3}\right)$ inventories of $\mathrm{MLLW}$ from $\mathrm{DOE}$ site activities $\ldots \ldots \ldots \ldots \ldots \ldots \ldots 22$

8.4 Projected 5-year (1994-1998) cumulative mass $(\mathrm{kg})$ generation of MLLW from DOE site

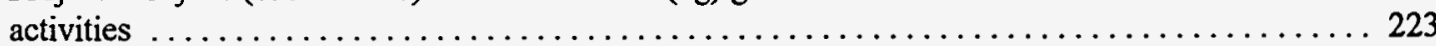

8.5 Projected 5-year (1994-1998) cumulative volume $\left(\mathrm{m}^{3}\right)$ generation of MLLW from DOE

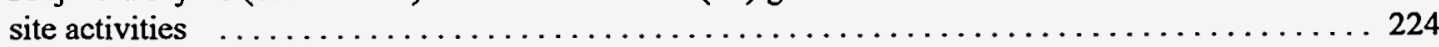

8.6 Treatability group matrix parameter categories used to characterize RCRA- and state-

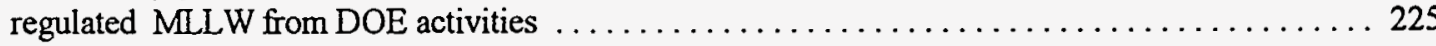

8.7 Cumulative mass and volume inventories of RCRA- and state-regulated MLLW at each

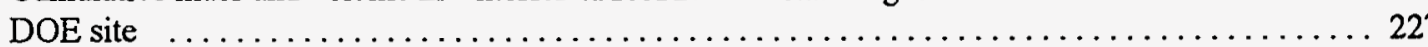

8.8 Cumulative mass and volume inventories, by physical/chemical matrix category, of RCRAand state-regulated MLLW from DOE site activities . .

8.9 Projected 5-year (1994-1998) cumulative mass and volume generation, by physical/ chemical matrix category, of RCRA- and state-regulated MLLW from DOE site activities . . ..... 236

8.10 Cumulative mass $(\mathrm{kg})$ inventories through 1994 , by physical category, of TSCA-regulated MLLW from DOE site activities

8.11 Cumulative volume $\left(\mathrm{m}^{3}\right)$ inventories through 1994 , by physical category, of TSCAregulated MLLW from DOE site activities

8.12 1994 mass $(\mathrm{kg})$ generation, by physical category, of TSCA-regulated MLLW from DOE site activities 
xxiii

8.13 1994 volume $\left(\mathrm{m}^{3}\right)$ generation, by physical category, of TSCA-regulated MLLW from DOE

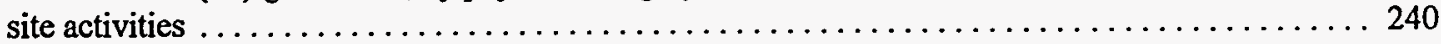

8.14 Cumulative mass $(\mathrm{kg})$ inventories through 1994, by hazard category, of TSCA-regulated MLLW from DOE site activities $\ldots \ldots \ldots \ldots \ldots \ldots \ldots \ldots \ldots \ldots \ldots \ldots \ldots \ldots \ldots \ldots \ldots \ldots \ldots \ldots \ldots, 24$

8.15 Cumulative volume $\left(\mathrm{m}^{3}\right)$ inventories through 1994, by hazard category, of TSCA-regulated

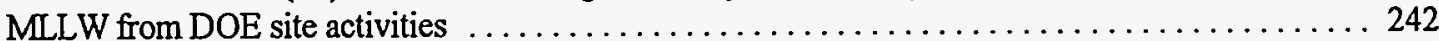

8.16 1994 mass $(\mathrm{kg})$ generation, by hazard category, of TSCA-regulated MLLW from DOE site activities ................................................... 243

8.17 1994 volume $\left(\mathrm{m}^{3}\right)$ generation, by hazard category, of TSCA-regulated MLLW from DOE site activities . . . . . . . . . . . . . . . . . . . . . . . . . . . . . . . . . . . . . . . 244

8.18 Historical and projected annual mass $(\mathrm{kg})$ generation rates for TSCA-regulated MLLW

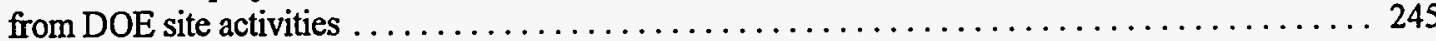

8.19 Historical and projected annual volume $\left(\mathrm{m}^{3}\right)$ generation rates for TSCA-regulated MLLW from DOE site activities . . . . . . . . . . . . . . . . . . . . . . . . . . . . . . . 246

8.20 Significant revisions and changes in historical inventory data for TSCA MLLW compared to

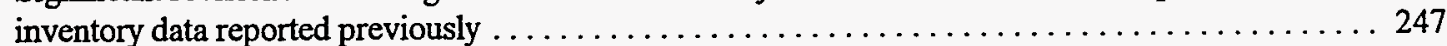

8.21 National commercially generated MLLW profile volume summary, by facility category $\ldots \ldots \ldots \ldots 248$

8.22 National commercially generated MLLW profile volume summary, by hazardous waste

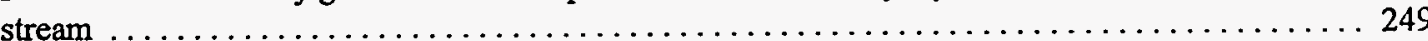

\section{APPENDIX A. WASTE FLOWSHEETS, SOURCE TERMS, AND CHARACTERISTICS}

A.1 Estimated representative unit activity and thermal power characteristics of various types of radioactive materials and wastes

A.2 Mass, radioactivity, and thermal power of nuclides in domestic commercial LWR spent

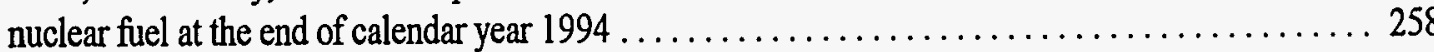

A.3 Representative DOE LLW radionuclide composition by percent activity $\ldots \ldots \ldots \ldots \ldots \ldots \ldots \ldots$

A.4 Average concentrations for representative radionuclides in LLW at commercial disposal sites

A.5 Estimated sources and characteristics of commercial Greater-Than-Class-C LLW . . . . . . . ... 269

A.6 Historical and projected number and volume of drums and classes of LLW incorporated in cement to be generated in the WVDP Low-Level Radwaste Treatment System

\section{APPENDIX B. CHARACTERISTICS OF IMPORTANT RADIONUCLIDES}

B.1 Characteristics of important radionuclides 
xxiv

\section{APPENDIX C. REFERENCE SITES AND FACILITIES}

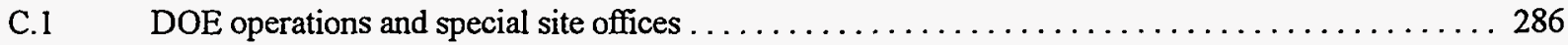

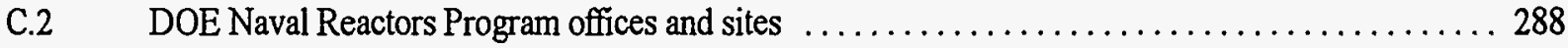

C.3 Major DOE sites and facilities referred to in this report $\ldots \ldots \ldots \ldots \ldots \ldots \ldots \ldots \ldots \ldots \ldots \ldots$

C.4 Major commercial radioactive waste disposal sites included in this report $\ldots \ldots \ldots \ldots \ldots \ldots 296$ 


\section{GLOSSARY OF ABBREVIATIONS, ACRONYMS, AND INITIALISMS}

In this report, all abbreviations, acronyms, and initialisms are defined in text before they are used. In some cases, they are redefined in text for the reader's convenience.

$\begin{array}{ll}\text { AEA } & \text { Atomic Energy Act of 1954 } \\ \text { AEC } & \text { Atomic Energy Commission } \\ \text { Ames } & \text { Ames Laboratory, Ames, Iowa } \\ \text { ANL-E } & \text { Argonne National Laboratory-East, Argonne, Illinois } \\ \text { ANL-W } & \text { Argonne National Laboratory-West, INEL, Idaho } \\ \text { APPR } & \text { Army Package Power Reactor } \\ \text { ASI } & \text { Advanced Sciences, Inc. } \\ \text { ATL 1 } & \text { Atlantic Site } 1 \text { (38'30'N, 72'06'W) } \\ \text { ATL 2 } & \text { Atlantic Site 2 (37'50'N, 70'35'W) } \\ \text { ATR } & \text { Advanced test reactor } \\ & \\ \text { BAPL } & \text { Bettis Atomic Power Laboratory, West Mifflin, Pennsylvania } \\ \text { BARN } & \text { Barnwell, South Carolina (commercial waste site) } \\ \text { BCL } & \text { Battelle Columbus Laboratories, Columbus, Ohio } \\ \text { BCLDP } & \text { Battelle Columbus Laboratories Decommissioning Project, Columbus, Ohio } \\ \text { BDM } & \text { BDM Federal, Inc., Germantown, Maryland } \\ \text { BETY } & \text { Beatty, Nevada (commercial waste site) } \\ \text { BMI } & \text { Battelle Memorial Institute, Columbus, Ohio } \\ \text { BNI } & \text { Bechtel National, Inc., Oak Ridge, Tennessee } \\ \text { BNL } & \text { Brookhaven National Laboratory, Upton, New York } \\ \text { BR } & \text { Belgium reactor } \\ \text { B\&W-NES } & \text { Babcock and Wilcox Nuclear Environmental Services, Lynchburg, Virginia } \\ \text { BWR } & \text { Boiling-water reactor } \\ & \\ \text { CADMUS } & \text { The Cadmus Group, Inc. } \\ \text { CANDU } & \text { Canadian Deuterium Reactor } \\ \text { CERCLA } & \text { Comprehensive Environmental Response, Compensation, and Liability Act of 1980 } \\ \text { CEU } & \text { Consolidated Edison Uranium } \\ \text { CFC } & \text { Chlorinated fluorocarbon } \\ \text { CH } & \text { Contact-handled (transuranic waste) } \\ \text { CISS } & \text { Colonie Interim Storage Site, Colonie, New York } \\ \text { CRWMS-M\&O } & \text { Civilian Radioactive Waste Management System-Management and Operating (contractor) } \\ \text { CY } & \text { Calendar year } \\ & \\ \text { DAW } & \text { Dry active waste } \\ \text { DOD } & \text { Department of Defense, U.S. } \\ \text { DOE } & \text { Department of Energy, U.S. } \\ \text { DOE/AL } & \text { DOE Albuquerque Operations Office, Albuquerque, New Mexico } \\ & \\ & \end{array}$


xxvi

\begin{tabular}{|c|c|}
\hline $\mathrm{DOE} / \mathrm{CAO}$ & DOE Carlsbad Area Office, Carlsbad, New Mexico \\
\hline $\mathrm{DOE} / \mathrm{CH}$ & DOE Chicago Operations Office, Argonne, Illinois \\
\hline DOE/DP & DOE/Office of Defense Programs (Headquarters), Germantown, Maryland \\
\hline DOE/EIA & DOE/Energy Information Administration, Washington, D.C. \\
\hline DOE/EM & DOE/Office of Environmental Management (Headquarters), Germantown, Maryland \\
\hline $\mathrm{DOE} / \mathrm{FN}$ & DOE Fernald Area Office, Cincinnati, Ohio \\
\hline DOE/HQ & DOE Headquarters, Washington, D.C. and Germantown, Maryland \\
\hline DOE/ID & DOE Idaho Operations Office, Idaho Falls, Idaho \\
\hline DOE/NV & DOE Nevada Operations Office, Las Vegas, Nevada \\
\hline DOE/OAK & DOE Oakland Operations Office, Oakland, California \\
\hline $\mathrm{DOE} / \mathrm{OH}$ & DOE Ohio Field Office, Miamisburg, Ohio \\
\hline DOE/OR & DOE Oak Ridge Operations Office, Oak Ridge, Tennessee \\
\hline DOE/OSTI & DOE/Office of Scientific and Technical Information, Oak Ridge, Tennessee \\
\hline $\mathrm{DOE} / \mathrm{RF}$ & DOE Rocky Flats Office, Golden, Colorado \\
\hline $\mathrm{DOE} / \mathrm{RL}$ & DOE Richland Operations Office, Richland, Washington \\
\hline DOE/RW & DOE/Office of Civilian Radioactive Waste Management (Headquarters), Washington, D.C. \\
\hline DOE/SR & DOE Savannah River Operations Office, Aiken, South Carolina \\
\hline DOE/WIPP & DOE/WIPP Project Office, Carlsbad, New Mexico \\
\hline DOE/WVAO & DOE/West Valley Area Office, West Valley, New York \\
\hline DOT & Department of Transportation, U.S. \\
\hline DRCT & Dry rod consolidation technology \\
\hline DWCS & Defueling water cleanup system \\
\hline DWMP & Defense Waste Management Plan \\
\hline DWPF & Defense Waste Processing Facility \\
\hline D\&D & Decontamination and decommissioning \\
\hline EA & Environmental assessment \\
\hline EBR & Experimental Breeder Reactor \\
\hline EBWR & Experimental Boiling-Water Reactor \\
\hline $\mathrm{EG \& G/ID}$ & $\begin{array}{l}\text { EG\&G Idaho, Inc., Idaho Falls, Idaho (former management and operating contractor for Idaho } \\
\text { National Engineering Laboratory) }\end{array}$ \\
\hline EIA & Energy Information Administration \\
\hline EIS & Environmental impact statement \\
\hline EMAD & Engine maintenance assembly and disassembly \\
\hline ENVR & Envirocare facility, Clive, Utah \\
\hline EOY & End of (calendar) year \\
\hline EPA & Environmental Protection Agency, U.S. \\
\hline EPR & Experimental power reactor \\
\hline ERR & Elk River Reactor \\
\hline ETEC & $\begin{array}{l}\text { Energy Technology Engineering Center, Canoga Park, California [also referred to as the Santa } \\
\text { Susana Field Laboratory (SSFL)] }\end{array}$ \\
\hline FEMP & Fernald Environmental Management Project, Fernald, Ohio \\
\hline FFCA & Federal Facility Compliance Act of 1992 \\
\hline FFTF & Fast Flux Test Facility, Hanford, Washington \\
\hline FIS & Farallon Islands (Pacific Ocean off Central California) \\
\hline FNAL & Fermi National Accelerator Laboratory, Batavia, Illinois \\
\hline FRR & Foreign Research Reactor \\
\hline FSVR & Fort St. Vrain Reactor, Platteville, Colorado \\
\hline FUSRAP & Formerly Utilized Sites Remedial Action Program \\
\hline FY & Fiscal year \\
\hline
\end{tabular}




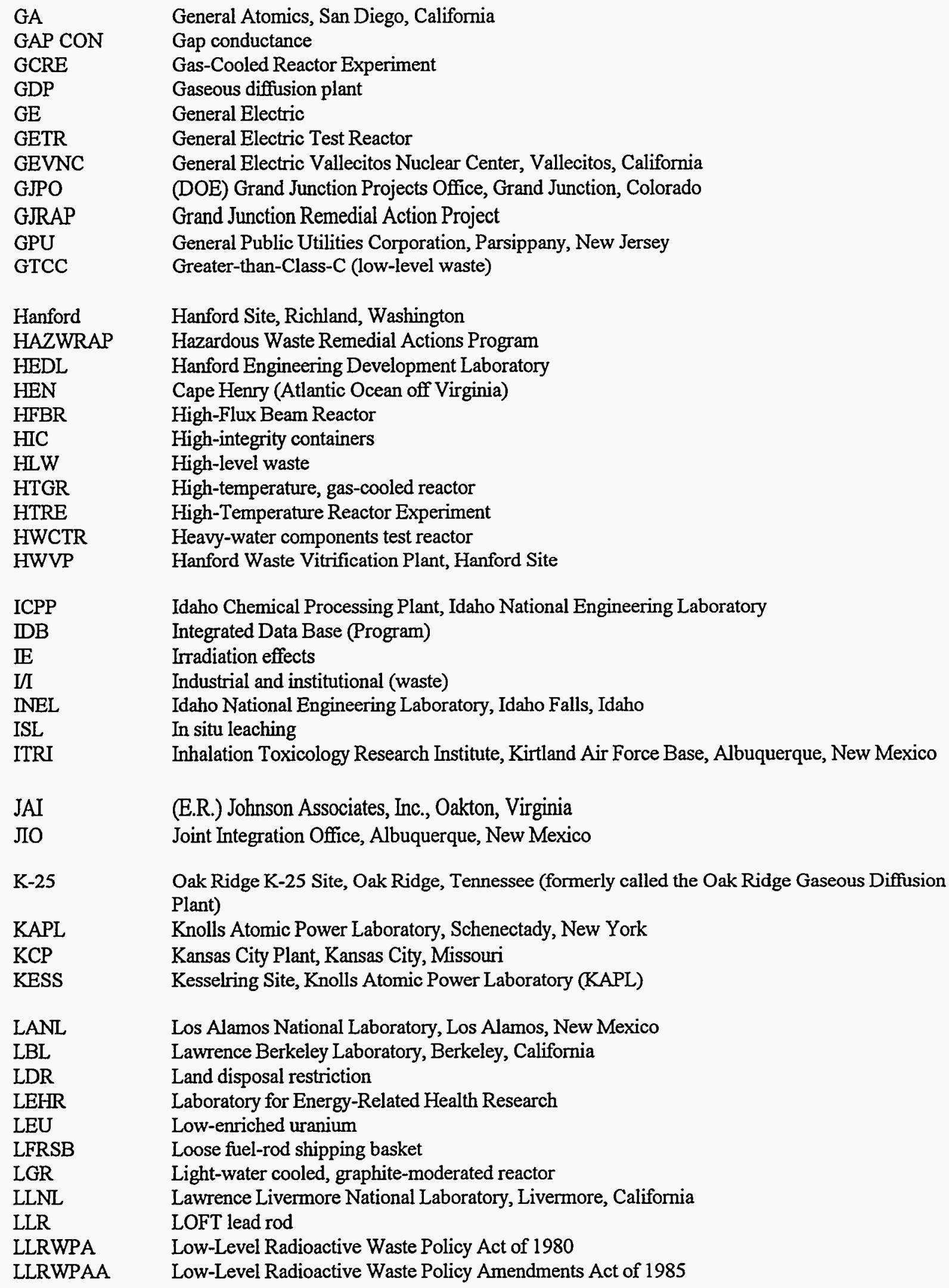


xxviii

\begin{tabular}{|c|c|}
\hline LLW & Low-level waste \\
\hline LLWMP & Low-Level Waste Management Program \\
\hline LMES & Lockheed Martin Energy Systems, Inc. \\
\hline LMFBR & Liquid Metal Fast Breeder Reactor \\
\hline LMIT & Lockheed Martin Idaho Technologies, Idaho Falls, Idaho \\
\hline LOC & Loss of coolant \\
\hline LOFT & Loss-of-fluid test \\
\hline LSA & Low specific activity \\
\hline LTC & Lynchburg Technology Center, Lynchburg, Virginia \\
\hline LWBR & Light-water breeder reactor \\
\hline LWR & Light-water reactor \\
\hline MACTEC & MAC Technical Services Company \\
\hline MAPI & Mitsubishi Atomic Power Industries \\
\hline MASS & Massachusetts Bay \\
\hline MED & Manhattan Engineer District (Manhattan Project) \\
\hline MFKY & Maxey Flats, Kentucky (commercial waste site) \\
\hline MFRP & Midwest Fuel Recovery Plant, Morris, Illinois (commercial spent fuel storage site) \\
\hline MIMS & Manifest Information Management System \\
\hline MLLW & Mixed low-level waste \\
\hline Mound & Mound Plant, Miamisburg, Ohio \\
\hline MRM & Miscellaneous radioactive material \\
\hline MSRE & Molten Salt Reactor Experiment \\
\hline MTIHM & Metric tons initial heavy metal \\
\hline MTRUW & Mixed transuranic waste \\
\hline MTU & Metric tons uranium \\
\hline MURR & Missouri University Research Reactor \\
\hline MWIR & Mixed Waste Inventory Report \\
\hline NA & Not applicable \\
\hline NARM & Naturally occurring or accelerator-produced radioactive material \\
\hline NEPA & National Environmental Policy Act of 1969 \\
\hline NFS & Nuclear Fuel Services, Erwin, Tennessee \\
\hline NORM & Naturally occurring radioactive material \\
\hline NPL & National priorities list \\
\hline NR & Naval reactors \\
\hline NRC & Nuclear Regulatory Commission \\
\hline NRF & Naval Reactors Facility, INEL, Idaho \\
\hline NTIS & National Technical Information Service, Springfield, Virginia \\
\hline NTPO & National Transuranic Waste Program Office \\
\hline NTS & Nevada Test Site, Mercury, Nevada \\
\hline NUMEC & Nuclear Uranium Materials and Equipment Corporation \\
\hline NUS & NUS Corporation, Gaithersburg, Maryland \\
\hline NWPA & Nuclear Waste Policy Act of 1982 \\
\hline NYSERDA & New York State Energy Research and Development Authority, Albany, New York \\
\hline OPTRAN & Operational transit \\
\hline OR & $\begin{array}{l}\text { Oak Ridge complex: Oak Ridge National Laboratory, K-25 Site, and Y-12 Plant, Oak Ridge, } \\
\text { Tennessee }\end{array}$ \\
\hline ORAU & Oak Ridge Associated Universities, Oak Ridge, Tennessee \\
\hline ORIGEN2 & Oak Ridge Isotope Generation and Depletion Code (Version 2) \\
\hline ORISE & Oak Ridge Institute for Science and Education, Oak Ridge, Tennessee \\
\hline
\end{tabular}


xxix

\begin{tabular}{|c|c|}
\hline ORNL & Oak Ridge National Laboratory, Oak Ridge, Tennessee \\
\hline ORR & Oak Ridge Research Reactor \\
\hline OTS & $\begin{array}{l}\text { Office of Technical Services, Roy F. Weston, Inc./H\&R Technical Associates, Inc., Germantown, } \\
\text { Maryland }\end{array}$ \\
\hline PAD & Paducah Gaseous Diffusion Plant, Paducah, Kentucky \\
\hline PANT & Pantex Plant, Amarillo, Texas \\
\hline $\mathrm{PBF}$ & Power Burst Facility \\
\hline PCB & Polychlorinated biphenyl \\
\hline $\mathrm{PCM}$ & Power coolant mismatch \\
\hline Pinellas & Pinellas Plant, Largo, Florida \\
\hline PNL & Pacific Northwest Laboratory, Richland, Washington \\
\hline PNRO & DOE Pittsburgh Naval Reactors Office, West Mifflin, Pennsylvania \\
\hline PORTS & Portsmouth Gaseous Diffusion Plant, Portsmouth, Ohio \\
\hline PPPL & Princeton Plasma Physics Laboratory, Princeton, New Jersey \\
\hline PWR & Pressurized-water reactor \\
\hline PUREX & Plutonium/uranium extraction \\
\hline RA & Remedial action \\
\hline RAP & Remedial action project \\
\hline $\mathrm{RCF}$ & RCRA (see below) facility assessment \\
\hline RCRA & Resource Conservation and Recovery Act of 1976 \\
\hline REECO & Reynolds Electrical and Engineering Co., Inc., Mercury, Nevada \\
\hline RFETS & Rocky Flats Environmental Technology Site, Golden, Colorado \\
\hline $\mathrm{RH}$ & Remote-handled (transuranic waste) \\
\hline $\mathrm{RI}$ & Rockwell International Corporation, Pittsburgh, Pennsylvania \\
\hline RIA & Reactivity initiated accident \\
\hline $\mathrm{RICH}$ & Richland, Washington (commercial waste site) \\
\hline RMI & Reactive Metals, Incorporated Titanium Company Extrusion Plant, Ashtabula, Ohio \\
\hline SCB & Santa Cruz Basin (Pacific Ocean off Santa Cruz, California) \\
\hline SDG & San Diego (Pacific Ocean off San Diego, California) \\
\hline SDS & Submerged demineralizer system \\
\hline SEG & Scientific Ecology Group, Inc., Oak Ridge, Tennessee \\
\hline SFD & Severe fuel damage \\
\hline SFMP & Surplus Facilities Management Program \\
\hline SHEF & Sheffield, Illinois (commercial waste site) \\
\hline SLAC & Stanford Linear Accelerator Center, Palo Alto, California \\
\hline SM & Stationary media \\
\hline SNAP & Space Nuclear Auxiliary Power \\
\hline SNF & Spent nuclear fuel \\
\hline SNL/CA & Sandia National Laboratories/California, Livermore, California \\
\hline SNL/NM & Sandia National Laboratories/New Mexico, Albuquerque, New Mexico \\
\hline SNM & Special nuclear material \\
\hline SNRO & DOE Schenectady Naval Reactors Office, Schenectady, New York \\
\hline SPERT & Special Power Excursion Reactor Test \\
\hline SRE & Sodium Reactor Experiment \\
\hline SRS & Savannah River Site, Aiken, South Carolina \\
\hline SS & Stainless steel \\
\hline SSFL & $\begin{array}{l}\text { Santa Susana Field Laboratory, Canoga Park, California [also referred to as the Energy Technology } \\
\text { Engineering Center (ETEC)] }\end{array}$ \\
\hline SWIMS & Solid Waste Information Management System \\
\hline
\end{tabular}


$x x x$

\begin{tabular}{|c|c|}
\hline SWU & Separative work unit \\
\hline TAN & Test Area North, Idaho National Engineering Laboratory \\
\hline TBD & To be determined \\
\hline $\mathrm{TC}$ & Thermocouple \\
\hline TCLP & Toxicity characteristic leaching procedure \\
\hline TESS & TRW Environmental Safety Systems, Inc. \\
\hline THOREX & Thorium extraction \\
\hline TMI & Three Mile Island reactor site, Middletown, Pennsylvania \\
\hline TRIGA & Training Reactor, Isotopes, General Atomic \\
\hline TRU & Transuranic \\
\hline TRUW & Transuranic waste \\
\hline TSCA & Toxic Substances Control Act of 1976 \\
\hline $\mathrm{T} / \mathrm{S} / \mathrm{D}$ & Treatment, storage, and disposal \\
\hline TVA & Tennessee Valley Authority, Knoxville, Tennessee \\
\hline UMT & Uranium mill tailings \\
\hline UMTRAP & Uranium Mill Tailings Remedial Action Program \\
\hline VBWR & Vallecitos Boiling-Water Reactor \\
\hline VEPCO & Virginia Electric Power Company \\
\hline WAC & Waste acceptance criteria \\
\hline WEC & Westinghouse Electric Corporation, Pittsburgh, Pennsylvania \\
\hline WHC & Westinghouse Hanford Company, Richland, Washington \\
\hline WHPP & (Transuranic) Waste Handling and Packaging Plant, Oak Ridge National Laboratory \\
\hline WIND & Windsor Site, Knolls Atomic Power Laboratory (KAPL) \\
\hline WIPP & Waste Isolation Pilot Plant, Carlsbad, New Mexico \\
\hline WMin & Waste minimization \\
\hline WPIO & Waste Isolation Pilot Plant Project Integration Office, Albuquerque, New Mexico \\
\hline WSSRAP & Weldon Spring Site Remedial Action Project, Weldon Spring, Missouri \\
\hline WTAC & Waste Isolation Pilot Plant Technical Assistance Contractor \\
\hline WTWBIR & Waste Isolation Pilot Plant Transuranic Waste Baseline Inventory Report \\
\hline WVDP & West Valley Demonstration Project, West Valley, New York \\
\hline WVNS & West Valley Nuclear Services Company, Inc., West Valley, New York \\
\hline WVNY & West Valley, New York (commercial waste site from 1963-1981) \\
\hline W/WIPP & Westinghouse/WIPP Project, Carlsbad, New Mexico \\
\hline$Y-12$ & Oak Ridge Y-12 Plant, Oak Ridge, Tennessee \\
\hline
\end{tabular}




\title{
INTEGRATED DATA BASE REPORT-1994: U.S. SPENT NUCLEAR FUEL AND RADIOACTIVE WASTE INVENTORIES, PROJECTIONS, AND CHARACTERISTICS
}

\begin{abstract}
The Integrated Data Base Program has compiled historic data on inventories and characteristics of both commercial and U.S. Department of Energy (DOE) spent nuclear fuel and commercial and U.S. government-owned radioactive wastes. Except for transuranic wastes, inventories of these materials are reported as of December 31, 1994. Transuranic waste inventories are reported as of December 31, 1993. All spent nuclear fuel and radioactive waste data reported are based on the most reliable information available from government sources, the open literature, technical reports, and direct contacts. The information forecasted is consistent with the latest DOE/Energy Information Administration (EIA) projections of U.S. commercial nuclear power growth and the expected DOE-related and private industrial and institutional activities.

The radioactive materials considered, on a chapter-by-chapter basis, are spent nuclear fuel, high-level waste, transuranic waste, low-level waste, commercial uranium mill tailings, DOE Environmental Restoration Program contaminated environmental media, commercial reactor and fuel-cycle facility decommissioning wastes, and mixed (hazardous and radioactive) low-level waste. For most of these categories, current and projected inventories are given through the calendar-year 2030, and the radioactivity and thermal power are calculated based on reported or estimated isotopic compositions.
\end{abstract}




\section{O. OVERVIEW}

\subsection{INTRODUCTION}

This report is an update of the previous document ${ }^{1}$ on spent nuclear fuel (SNF) and radioactive waste inventories and projections that was prepared for use in the planning and analysis of various SNF and waste management functions. Quantities of SNF and radioactive wastes produced from both commercial and U.S. Department of Energy (DOE)-sponsored activities are reported. Except for transuranic wastes, whose inventories are reported as of December 31, 1993, historical SNF and radioactive waste inventories are reported as of December 31, 1994. Projections of future SNF and radioactive wastes are generally reported for the calendar-year (CY) period 1995-2030. Such projections may change in future revisions of this report as waste minimization (WMin), environmental restoration, and decontamination and decommissioning (D\&D) programs and activities at various government and commercial sites are defined and become operative. The tables of this document report historical inventories and projection data on a CY basis. These tables use a horizontal line to mark the point in time when past history ends and future projections begin. Because historical SNF and radioactive waste inventories are generally reported as of December 31,1994 , the line is drawn between the data entries for 1994 and 1995. Data reported for 1995 in this document are regarded as projected information.

This document contains information that has been assembled as a part of the Integrated Data Base (IDB) Program at Oak Ridge National Laboratory (ORNL), which has the lead responsibility for maintaining and reporting summary files of pertinent data on current and projected inventories and characteristics of permanently discharged domestic SNF and radioactive wastes. While this report documents inventories and projections of radioactive wastes from commercial and DOE site activities, estimates for certain other waste categories are not fully reported because of the current unavailability of data. These categories include naturally occurring radioactive material (NORM) or accelerator-produced radioactive material (NARM), discussed in Sect. 0.2, and wastes from U.S. Department of Defense (DOD) activities, particularly those resulting from the cleanup of military sites contaminated with significant amounts of radioactive waste. A study ${ }^{2}$ by the U.S. General Accounting Office indicates that as many as 420 DOD locations could be contaminated with radioactive wastes. Future updates of this report will work toward reporting information and data on NARM and DOD site radioactive wastes as they become available.

This report also does not report inventories of nonradioactive materials. These include hazardous wastes, sanitary wastes, and materials not categorized as waste (MNCAW), such as uncontaminated scrap metal. Preliminary estimates of inventories of MNCAW are reported in ref. 3.

Radioactive waste originates from five major sources: (1) the commercial nuclear fuel cycle; (2) DOE-related activities; (3) institutions such as hospitals, universities, and research foundations; (4) industrial uses of radioisotopes; and (5) mining and milling of uranium ore. The waste is broadly categorized as SNF, high-level waste (HLW), transuranic waste (TRUW), low-level waste (LLW), and uranium mill tailings (UMT). Large quantities of radioactive waste also result from DOE site environmental restoration activities and the $D \& D$ programs of DOE and commercial nuclear facilities. This report also documents inventories and projections of mixed low-level waste (MLLW), which is both hazardous and radioactive.

The primary purpose of this document is to report U.S. SNF and radioactive waste inventories, projections, and characteristics. The data presented were obtained through the cooperation and assistance of the offices and programs that were established by DOE to oversee the management of the various radioactive wastes and SNFs. In addition, the recent literature was reviewed to aid in selecting the data that are presented here and to help establish a basis for many of the calculated radioactivity levels and heatgeneration rates that are included. In this report, SNF and radioactive wastes are characterized from the standpoint of their volumes (or masses) and their nuclear, physical, and chemical properties. The data reported are selected from 
more extensive information; that information is available upon request.

This annual inventory report contains summarized data of types found to be useful for programmatic planning purposes within the DOE community. The data are intended to provide a common basis for both DOE management-level planning and for more detailed analyses of the waste management system that are conducted by DOE contractors and field offices. However, this report is not intended to present the detailed types of information required as input to such analyses. The best sources of such information are the appropriate DOE operations offices, waste sites, or relevant documents previously issued, some of which may be referenced in this report.

This report does not address the programmatic implications of the data presented, such as the possible future need for interim SNF storage facilities. Discussion of the data is minimized to explain mainly what the data represent. Major DOE data sources providing information and data for this report are identified in a table following the preface. Likewise, discussions of packaging details, shielding and transportation requirements, health and environmental effects, and costs are purposely avoided. Questions regarding the information and data presented may be addressed to the $\mathrm{DDB}$ Program.

The DOE waste information and data contained in this report are furnished by the DOE contractor sites listed in Table 0.1. This table indicates also the types of radioactive waste managed at each site. The DOE site data (waste inventories, projections, and characteristics) are used by DOE-Headquarters (DOE-HQ), operations offices, and operating contractors for the management and strategic planning of various waste programs. The objective of this report is to provide waste information that is consistent, reflects current inventories and projections, and includes the types of basic data best suited to meet DOE waste program planning needs.

Information for this report is provided by a variety of sources. The DOE site waste data reported were received from DOE contractors through DOE operations offices. DOE-HQ assigns to selected organizations major responsibilities for providing information on particular topics involving SNF and radioactive waste management. Further detailed information is generally available from data bases maintained at the specific DOE and commercial sites. Additional information on the reference sites and facilities referred to in this report is provided in Appendix C.

\section{O.2 CHARACTERIZATION OF WASTE FORMS}

The major characteristics of radioactive materials and wastes are described below.

\section{- Spent Nuclear Fuel (SNF)}

SNF consists of irradiated fuel discharged from a nuclear reactor. Unless otherwise identified, all SNFs discussed in this report are assumed to be permanently discharged and eligible for repository disposal. Three categories of permanently discharged SNF are considered: (1) fuel from commercial light-water reactors (LWRs); (2) fuel from non-LWR commercial reactors [e.g., the Fort St. Vrain high-temperature, gas-cooled reactor (HTGR)]; and (3) special fuels associated with government- sponsored research and demonstration programs, universities, and private industries. This report does not track the inventories of government production reactor SNFs that have been reprocessed in the manufacture of nuclear weapons for national defense. However, the inventories of HLW resulting from the reprocessing of these fuels are reported in Chapter 2. Also, Chapter 1 reports quantities of DOE SNF.

Currently, most LWR SNF assemblies are stored in pools at the reactor sites. The bulk of the remainder is in storage at the West Valley Demonstration Project (WVDP) site at West Valley, New York; the Idaho National Engineering Laboratory (INEL) at Idaho Falls, Idaho; and the Midwest Fuel Recovery Plant (MFRP) at Morris, Illinois. The WVDP facility is currently being decommissioned. All utility-owned SNF assemblies previously stored there have been returned to the utilities, and the fuel remaining is DOE-owned material.

SNFs discharged from a variety of reactors are currently stored at the Hanford Site (Hanford) and INEL. Hanford contains inventories of fuel from the N Reactor, the Fast Flux Test Facility (FFTF), and pressurized-water reactor (PWR)-Core II fuel from Shippingport. Fuel from the damaged Three Mile Island (TMI)-Unit 2 reactor, as well as some of the SNF from the Fort St. Vrain HTGR, are stored at INEL. Some special SNFs are stored at the Savannah River Site (SRS) and at INEL. These special fuels are government owned and are not scheduled for reprocessing in support of $\mathrm{DOE}$ activities. 


\section{- High-Level Waste (HLW)}

For this report, HLW means the highly radioactive material resulting from the reprocessing of SNF. This includes mainly the liquid wastes remaining from the recovery of uranium and plutonium in a fuel reprocessing plant. This HLW may also be in the form of sludge, calcine, or other products into which such liquid wastes are converted to facilitate their handling and storage. Such waste contains fission products that result in the release of considerable decay energy. ${ }^{4}$ For this reason, heavy shielding is required to absorb penetrating radiation, and provisions (e.g., cooling systems) are needed to dissipate decay heat from $\mathrm{HLW}$.

\section{- Transuranic Waste (TRUW)}

TRUW refers to radioactive waste that contains more than $100 \mathrm{nCi} / \mathrm{g}$ of alpha-emitting isotopes with atomic numbers greater than 92 and half-lives greater than 20 years. ${ }^{5,6}$ Such waste results primarily from fuel reprocessing and from the fabrication of plutonium weapons and plutonium-bearing reactor fuel. Generally, little or no shielding is required ["contact-handled" (CH) TRUW], but energetic gamma and neutron emissions from certain transuranic (TRU) nuclides and fission-product contaminants may require shielding or remote handling ["remote-handled" (RH) TRUW].

\section{- Low-Level Waste (LLW)}

Several statutes (refs. 4, 6, and 7) define LLW not by what it is, but by what it is not. In general, LLW is radioactive waste not classified as SNF, HLW, TRUW, nor by-product materials such as UMT or thorium mill tailings. However, there are slight differences between the specific regulatory definitions of DOE-generated LLW and commercial LLW.

The definition of DOE LLW is based on DOE Order 5820.2A, ${ }^{6}$ which specifies DOE's policy for radioactive waste management. According to this order, LLW includes all radioactive waste not classified as either HLW, TRUW, SNF, or the bulk of the by-product tailings containing uranium or thorium from processed ore. The DOE policy, as stated in Order 5820.2A, allows small volumes of fissionable material to be irradiated for research and development (R\&D) only - but not for the production of power or plutonium-and small concentrations of TRU $(<100 \mathrm{nCi} / \mathrm{g}$ ) radionuclides to be managed as LLW.
The same DOE policy allows small volumes of $\mathrm{DOE}$ waste containing by-product material [specified in Sect. 11e(2) of the Atomic Energy Act of 1954 (AEA)] ${ }^{8}$ or NARM to be managed as LLW. Any LLW that also contains hazardous chemicals covered by either the Resource Conservation Recovery Act $(\text { RCRA) })^{9}$ or the Toxic Substances Control Act $(\mathrm{TSCA})^{10}$ requires management as a "mixed waste."

The definition of commercial LLW is based on two statutes, the Nuclear Waste Policy Act (NWPA) ${ }^{4}$ and the Low-Level Radioactive Waste Policy Amendments Act (LLRWPAA). ${ }^{7}$ According to both the NWPA and the LLRWPAA, commercial LLW is radioactive material that (a) is not HLW, SNF, TRUW, or byproduct material as defined in Sect. 1le(2) of the AEA; and (b) consistent with existing law, is classified by the U.S. Nuclear Regulatory Commission (NRC) as LLW.

The radiation level from LLW waste may sometimes be high enough to require shielding for handling and transport. For commercial LLWs, the NRC has defined, in ref. 11, four disposal categories of LLW that require differing degrees of confinement and/or monitoring: classes A, B, C, and Greater-ThanClass-C (GTCC). The NRC excludes NARM from the LLW category. DOE LLWs are classified by groupings of disposal categories that are site specific, yet similar to the NRC categories. This report documents only those inventories of solid LLW destined for disposal. It includes no liquid or gas waste in storage.

\section{- Commercial Uranium Mill Tailings (UMT)}

Commercial UMT are the earthen residues that remain after the extraction of uranium from ores. Tailings are generated in very large volumes and contain low concentrations of naturally occurring radioactive materials. These materials comprise a potential health hazard; the isotopes of major concern are ${ }^{226} \mathrm{Ra}$ and its daughter, ${ }^{222} \mathrm{Rn}$.

\section{- Mixed Low-Level Waste (MLLW)}

MLLW contains concentrations of both low-level radioactive materials and hazardous chemicals. The hazardous component of mixed waste has characteristics identified by any or all of the following statutes: the RCRA, as amended; ${ }^{9}$ the TSCA $;{ }^{10}$ and state regulations. Typically, MLLW from activities supporting DOE programs includes a variety of 
contaminated materials, such as air filters, cleaning solutions, engine oils and grease, paint residues, soils, construction and building materials, water-treatment chemicals, and decommissioned plant equipment. This report documents inventories and generation rates of various types of mixed wastes stored at DOE sites based on information reported in the Federal Facilities Compliance Act (FFCA) Mixed Waste Inventory Report $(M W I R)^{12}$ and TSCA waste information recently provided by $D O E$ sites.

\section{- Naturally Occurring Radioactive Material (NORM) or Accelerator-Produced Radioactive Material (NARM)}

NARM wastes include both accelerator wastes (LLW) and naturally occurring radioactive material (NORM) that contain radionuclides (e.g., ${ }^{226} \mathrm{Ra},{ }^{222} \mathrm{Rn},{ }^{232} \mathrm{Th}$, ${ }^{238} \mathrm{U}$ ) existing throughout the earth's crust. Accelerator wastes include accelerator targets, wastes from accelerator maintenance or $D \& D$, and wastes from radiopharmaceutical manufacture. NORM wastes are classified according to their specific activity as either discrete or diffuse. Discrete NORM wastes have a relatively small volume but large radioactivity and include industrial gauges, old radium watch and industrial dials, radium needles in medical equipment, and resins (filters) that remove radioactive radium and some radiopharmaceutical waste from contaminated public drinking water. Diffuse NORM wastes are characterized by a relatively large volume with small radioactivity. These materials result from industrial processes and include:

- tailings from metal (e.g., copper and uranium) mining and processing,

- fossil fuel wastes (viz., coal ash from utility electrical generation),

- phosphate mining ore wastes for fertilizer (ammonium phosphate) production,

- sludge from drinking water treatment,

- contaminated water and drilling equipment from oil and natural gas drilling, and

- wastes from geothermal energy production. ${ }^{13}$

Current inventories of domestic NARM wastes are not known. Future updates of this document will include additional information on NARM waste inventories, projections, and characteristics as they become available.
- Generated, Treated, Stored, and Disposed Wastes

It should be emphasized that all of the types of radioactive materials and wastes discussed in this report can exist either as material generated, treated, stored, or disposed. The distinctions among these various waste conditions or "states" are as follows:

- Generated waste. A material recently discharged from a facility production process or operation that can be regarded as a waste because it has no economic value. In this report, quantities of generated waste are measured in units of volume [cubic meters $\left(\mathrm{m}^{3}\right)$ ] or mass $(\mathrm{kg})$ produced during a calendar year.

- Treated waste. A waste that, following generation, has been altered chemically or physically to reduce its toxicity or prepare it for storage or disposal on- or off-site. Waste treatment can include volume-reduction activities, such as incineration or compaction, which may be done prior to either storage or disposal or both (discussed next).

- Stored waste. A waste that, following generation (and usually some treatment), is being (temporarily) retained and monitored in a retrievable manner pending disposal. In this report, inventories and projections of stored radioactive materials or wastes are reported in volume $\left(\mathrm{m}^{3}\right)$ or mass $(\mathrm{kg})$ units or both.

- Disposed waste. A waste that has been put in final emplacement to ensure its isolation from the biosphere, with no intention of retrieval. Deliberate action is required to regain access to the waste. Disposed waste includes materials placed in a geologic repository, buried underground in shallow pits, dumped at sea, or discarded by hydrofracture injection. The latter two techniques were past practices and are no longer performed.

Throughout this report, the reader is urged to note the distinctions among these waste conditions. Such conditions have a great impact on the regulatory status of the waste materials considered in this report. 


\subsection{METHODS AND ASSUMPTIONS USED IN REPORT PREPARATION}

This report consolidates a large amount of information from many sources. Some of these data are historical in nature, some are current, and some are projected; some have been calculated or estimated, and some have been measured. Over the years, waste regulations have been revised, waste category definitions have changed, measurement instruments and calibration methods have been improved, and record-keeping has been upgraded at all waste-generating and -receiving sites. In preparing this report a major effort has been made to integrate waste data from many sources and to strive for a consistent and technically rational approach for the entire scope of coverage. Our primary sources of data are referenced, and, for calculated values (e.g., decayed radioactivity and thermal power), the bases for the calculations are identified. To achieve adequate integration of data, numerous factors had to be considered; these are cited in footnotes that generally accompany the tables and figures of this report. In some cases, a more thorough explanation is provided in the text.

Each chapter details the assumptions on which its waste inventories and projections are based. The broader assumptions are mentioned here and are listed in Table 0.2. These include the projected time frame and specific assumptions used for estimating commercial and government (DOE) waste projections. For the commercial fuel cycle, the SNF and waste projections depend upon the nuclear power growth scenario. The commercial fuel cycle waste projections reported in this document assume a reference projection of nuclear power growth and no SNF reprocessing. The reference nuclear power electrical growth projection (and associated discharged SNF schedule) used throughout this report is the 1995 DOE/Energy Information Administration (EIA) Low Case (No New Orders) ${ }^{14}$ Throughout this report this case will be referred to as the Low Case. The Low Case SNF and power-capacity projection cases are based on a set of assumptions involving nuclear electricity generation growth, reactor fuel burnup levels, reactor construction schedules, and reactor operating lifetimes and capacity factors. These assumptions are documented by DOE/EIA in ref. 14. In particular, the Low Case assumes that no new advanced LWRs will become operational before the year 2015 and that all current nuclear units are retired on the dates when their initial license-terms expire.

Detailed information about reactors already built, being built, or planned in the United States for domestic use or export as of December 31,1994, is provided in report DOE/OSTI-8200-R58 (ref. 15), which contains a comprehensive listing of all domestic reactors categorized by primary function or purpose: viz., civilian, production, military, export, and critical assembly.

The data for total waste inventories (which comprise historical data) are obviously less accurate than the values recorded for recent waste additions. The number of digits used in reporting these values is generally greater than justified in terms of numerical significance, but this proves useful and necessary for bookkeeping purposes. In some cases, the values cited are significantly different from those previously reported. This is generally a result of improved estimates, new measurements, or redefinition of terms. Explanations are given in such cases. Many of the comments received during the final review stage of this report deal with changes that have occurred after December 31, 1994-some as recently as September 1995. These changes are generally cited in footnotes.

For the sake of brevity, many of the figures and tables of this report use the exponential (E) notation. As examples of this notation, the constant $1.234 \mathrm{E}+2$ means $1.234 \times 10^{2}$, or 123.4 ; and $1.234 \mathrm{E}-4$ means $1.234 \times 10^{-4}$, which is 0.0001234 .

\section{O.4 WASTE CHARACTERISTICS AND UNITS REPORTED}

Principal characteristics reported for most radioactive wastes discussed in this report include volume, radioactivity, and thermal power. All characteristics are reported in metric units and, depending on the waste form, can be significant considerations in meeting the requirements for waste treatment, storage, and disposal. Waste volume is reported in cubic meters $\left(\mathrm{m}^{3}\right)$ and generally reflects the amount of space occupied by the waste and its container. Radioactivity represents the rate of spontaneous disintegration of the radionuclides comprising the waste. In this report, radioactivity is measured by a unit called a curie $(\mathrm{Ci})$, which is $3.7 \times 10^{10}$ nuclear disintegrations per second. Over time, radionuclides decay to nonradioactive, stable isotopes. As an example, the short-lived radionuclides found in SNF rapidly decay during the first few years after the fuel is removed from a reactor.

It should be noted that while waste volumes accumulate with time by conventional addition, total radioactivity does not. Because of radioactive decay, cumulative activity cannot be based on reported annual additions; rather it must be estimated from knowledge of the waste composition, which includes the radionuclides comprising the waste, their concentrations, and decay attributes (e.g., half-lives and decay schemes). In this report, decayed radioactivity is generally estimated for various wastes by an abridged version of the ORIGEN2 
code (ref. 16). Annual levels of radioactivity (Ci) reported in this document include contributions from both parent and daughter decay products.

Thermal power is a measure of the rate of heat-energy emission resulting from the decay of radionuclides in a waste. Like radioactivity, thermal power is not cumulative by conventional addition because of radioactive decay. Information on thermal power is needed in the design of shipping casks, storage facilities, and repositories where temperature rise, especially with regard to SNF and $\mathrm{HLW}$, is an important concern. Thermal energy generation rates are highest for SNF, HLW, and RH TRUW. They may also be important for certain types of LLW. The unit of thermal power used in this report is the watt $(W)$, which represents 1 joule (J) of thermal energy emitted per second. Estimates of thermal power are based on radionuclide composition as well as total activity. While levels of thermal power may not be significant for certain waste forms (particularly some types of LLW), they are nevertheless reported for the major radioactive waste categories referenced in this report to provide a standard for comparison.

For SNF and TRUW, mass is reported to provide better assurances of accountability. SNF is reported in units of metric tons of initial heavy metal (MTHM) to avoid difficulties and confusion arising from the need to estimate ranges of varied heavy-metal content (MTHM) that result from different levels of enrichment and reactor fuel burnup. Mass is reported in kilograms ( $\mathrm{kg}$ ) for the TRU radionuclides comprising TRUWs.

In this report, quantities of generated wastes are expressed in terms of either the amount of mass $(\mathrm{kg})$ or volume $\left(\mathrm{m}^{3}\right)$ produced in a given $C Y$. Thus, generation rates for wastes are expressed in either kilograms per year (kg/year) or cubic meters per year ( $\left.\mathrm{m}^{3} / y e a r\right)$, depending on the availability of site information. Annual generation rates are reported in this document for SNF, TRUW, LLW, and MLLW. Annual generation rates are not reported for HLW in part because of security restrictions for the DOE nuclear weapon production activities that produce these wastes. Additionally, there are problems in accurately estimating HLW generation levels. One major difficulty is accounting for net waste-quantity changes due to the combined effects of various modes of site waste management operations such as evaporation and calcination.

Quantities of wastes can also be reported in terms of the number and types of waste containers. LWR SNF inventories and projections can be expressed in terms of the number of permanently discharged boiling-water reactor (BWR) and PWR fuel assemblies. HLW will be immobilized in either borosilicate glass or a glass/ceramic matrix solidified in stainless steel canisters. Estimates of the quantities of HLW to be disposed of in a geologic repository are based on the number and types of these canisters. Quantities of LLW and stored TRUW can be based on the number and types of drums, boxes, or containers used or scheduled for use.

Waste characteristics are also identified by waste composition. Throughout this report, waste composition is expressed in terms of the following:

- radioactivity $(\mathrm{Ci})$ or specific-activity $\left(\mathrm{Ci} / \mathrm{m}^{3}\right)$ breakdown by radionuclide (with accompanying daughter products) and

- physical form (solid, liquid, gas, or sludge) or chemical content (by chemical component), expressed in terms of either volume $\left(\mathrm{m}^{3}\right)$ or mass $(\mathrm{kg})$ or as a percentage of total weight (wt \%), volume (vol \%), or activity (act \%).

\subsection{CHAPTER OVERVIEWS}

A brief summary of each chapter in this report is presented in the following paragraphs.

\subsubsection{Spent Nuclear Fuel (SNF)}

Chapter 1 of this report presents national data on the quantities of permanently discharged SNF from commercial nuclear power reactors. Historical data on commercial SNF inventories ${ }^{17}$ are reported along with 1995 DOE/EIA projections for the Low Case. ${ }^{14}$ The Low Case is the baseline commercial scenario used throughout this report to make waste projections. For the projection period considered in this report (CYs 1995-2030), the Low Case assumes that no new reactors will be ordered.

DOE SNF inventories are also reported in Chapter 1. These include various types of research reactor SNFs which are stored at the SRS and the INEL.

In this report the mass of discharged SNF is generally measured in MTIFM. The term "initial heavy metal" refers to the original mass of the actinide elements of the fuel, most of which is uranium. (Elements of the actinide group are those with atomic numbers greater than 89 .)

\subsubsection{High-Level Waste (HLW)}

The inventories of $\mathrm{HLW}$ in storage at the end of CY 1994 and projected through CY 2030 are given in Chapter 2. The waste forms include liquid, sludge, salt cake, slurry, calcine, precipitate, zeolite, glass, and capsules of separated strontium and cesium. Vitrified defense HLW is projected after the startup of the Defense Waste Processing Facility (DWPF) at SRS in 1996, and 
projections of vitrified $\mathrm{HLW}$ from commercial reprocessing activities are given for the WVDP. Projections recently made of the number of canisters containing the final immobilized form for the DOE HLW at Hanford and the INEL are also reported. In addition, Chapter 2 gives the locations, volumes, and radioactivities of HLW.

In 1992, DOE decided to phase out the reprocessing of its production reactor SNFs. Until then, the reprocessing activities recovered enriched uranium and plutonium which were used to support nuclear weapons production. As a consequence of ceasing to reprocess reactor SNFs, little additional HLW is expected to be generated at DOE sites in the future. However, DOE site D\&D activities may generate some wastes with radioactivity levels high enough to require disposal in a deeply mined geologic repository.

\subsubsection{Transuranic Waste (TRUW)}

The locations, inventories, and projections of TRUW buried and stored at DOE sites are presented in Chapter 3. Inventories of TRUW are reported as of December 31, 1993, and are virtually all derived from government operations. The inventories documented in this report are based on data provided by the sites and include waste volumes and the masses and radioactivities of contained radionuclides. Projected future TRUW volumes through CY 2022 were also requested from the sites, but the sites were not able to make such estimates in all cases. Projections are reported through CY 2022 for those sites that provided estimates.

In. 1984, DOE (with input from other federal agencies) revised the minimum radioactivity concentration level for defining TRUW from greater than $10 \mathrm{nCi} / g$ to greater than $100 \mathrm{nCi} / \mathrm{g} .{ }^{18}$ Consequently, some waste currently in the inventory may contain wastes stored under both criteria. This redefinition, as well as the development of instrumentation to detect these low levels of radioactivity, may reduce the volume of TRUW. As the waste is assayed, that portion of it which is greater than $10 \mathrm{nCi} / \mathrm{g}$ and less than $100 \mathrm{nCi} / \mathrm{g}$ will be reclassified to other waste categories.

\subsubsection{Low-Level Waste (LLW)}

Data for LLW from commercial and government activities are given in Chapter 4 and Appendix A. Commercial fuel-cycle LLW is generated from the conversion of yellowcake to uranium hexafluoride $\left(\mathrm{UF}_{6}\right)$, enrichment, fuel fabrication, and reactor operation. LLW also results from commercial operations by private organizations that are licensed to use radioactive materials.
These include institutions and industries engaged in research and various medical and industrial activities. DOELLW is similar in nature to the commercial industrial and institutional $(I / I)$ waste and the commercial fuel cycle LLW.

A wide variety of radionuclides are found in LLW. Uranium isotopes and their daughters dominate in the conversion, enrichment, and fuel-fabrication steps of the nuclear fuel cycle. Reactor operations produce LLW containing mostly activation products and fission products. A significant fraction of institutional LLW that is shipped to disposal sites is contaminated with small quantities of ${ }^{3} \mathrm{H}$ and ${ }^{14} \mathrm{C}$.

By the end of 1994, approximately $66 \%$ of the total cumulative volume of disposed LLW resulted from various DOE activities. The remaining 34\% resulted from domestic commercial activities. About $32 \%$ of the volume of LLW disposed during 1994 resulted from commercial activities.

\subsubsection{Commercial Uranium Mill Tailings (UMT)}

Current inventories and projections of tailings from commercial uranium mill operations are summarized in Chapter 5. Twenty-six licensed uranium mills have accumulated tailings from their operations. Half of these mills have accumulated both commercial and government tailings. By the end of 1994, only two of the NRC-licensed mills were still active. To date, almost all domestic uranium has been produced by conventional mining and milling methods from which these tailings derive. A small portion has been obtained via in situ leaching, recovery from mine water, recovery from copper/vanadium dump leach liquor, and recovery from wet-process phosphoric acid effluents. Projections of uranium mill tailings are based on commercial fuel-cycle requirements, adjusted for foreign imports, as specified by the DOE/EIA Low Case projection of commercial reactor power growth. Tailings from the now inactive mills that produced uranium only for government operations are classified as Environmental Restoration Program wastes (see Chapter 6).

\subsubsection{Environmental Restoration Program}

An overview of DOE environmental restoration projects and activities is given below. Further details are provided in Chapter 6. The scope of Chapter 6 is limited to radioactive and mixed (radioactive and chemically hazardous) wastes that could be generated by environmental restoration activities. Nonradioactive hazardous and sanitary wastes are outside the scope of this report. 
The major objective for DOE environmental restoration activities is to ensure that risks to the environment and to human health and safety posed by inactive and surplus facilities and sites contaminated by radioactive and chemically hazardous materials are either eliminated or reduced to prescribed, safe levels. Projects within the Office of Environmental Restoration (EM-40) are comprised of remedial action (RA) and decommissioning activities. RA involves the assessment and cleanup of inactive sites and deals mainly with contaminated environmental media such as soil, sediment, and ground water. Decommissioning activities are primarily concerned with the safe caretaking of surplus nuclear facilities following shutdown and for either their ensuing decontamination for reuse or their complete dismantlement. About 500 contaminated facilities are currently included in the EM-40 inventory for future action.

An intensive effort is currently under way to better define waste management requirements for implementing the DOE environmental restoration program. This information will assist $\mathrm{DOE}$ in planning, constructing, and operating appropriate treatment, storage, and disposal facilities to manage the wastes resulting from implementation of the EM-40 program. Waste projections are currently available for only a few sites. Hence, environmental restoration waste volumes are not provided in this report. However, the volumes of contaminated solid media, such as soils and debris from which environmental restoration wastes will be generated, are known at most EM-40 sites. Estimates of the volumes of such contaminated media are reported in Chapter 6.

\subsubsection{Commercial Decommissioning Wastes}

Chapter 7 presents waste projections for the decommissioning of commercial power reactors and fuel cycle facilities. The D\&D activities at such installations may result in very large volumes of LLW, depending on the methods selected. The major LLW volumes will result from the decommissioning of power reactors, which will also produce a small volume of high-activity waste. Unlike that for other waste generation activities, the timing of decommissioning operations is very uncertain, since facilities may be either decommissioned upon shutdown or put into a mothballed or protective storage condition to allow for sufficient radioactive decay before decommissioning. Chapter 7 reports a set of projected characteristics for wastes from commercial LWR decommissioning activities. These projections are based on the assumption that each power reactor is decommissioned soon after it is shut down. To date, only a few commercial reactors have been fully decommissioned, and several have been placed in protective storage. Wastes from completed decommissioning actions have been included with existing inventories discussed in other chapters. Because of timing uncertainties, projected commercial decommissioning wastes are not included in the projections of LLW (Chapter 4). Rather, commercial decommissioning waste projections are reported separately in Chapter 7.

\subsubsection{Mixed Low-Level Waste (MLLW)}

Current inventories and generation rates of MLLW from both DOE and commercial sources are summarized in Chapter 8. These wastes are contaminated with both low-level radioactivity and chemically hazardous substances. The radioactive components are defined by the $\mathrm{AEA},{ }^{8}$ while the hazardous components are defined by the RCRA, ${ }^{9}$ the TSCA,${ }^{10}$ and pertinent state regulations. As of the end of 1994, inventories of MLLW at DOE sites totaled about $162,400 \mathrm{~m}^{3}$. It is estimated that about $97,100 \mathrm{~m}^{3}$ of MLLW will be generated during the period 1994-1998.

\subsubsection{Appendixes}

Several appendixes are included in this report. Appendix A is a compilation of waste flowsheets, source terms, and characteristics used for waste projections. Source terms include both quantitative and descriptive characteristics used to describe radioactive wastes. As developed and used in the IDB Program, the source term for a particular waste is comprised of two components unique to that waste: (1) the number of curies of radioactivity, expressed either per unit of facility production or per unit of waste volume or mass, and (2) a listing of the relative contributions of component radioisotopes per curie of radioactivity of the waste. A tabulation of the properties of important radionuclides is given in Appendix B. Appendix C lists the sites and facilities referred to in this report. Appendix $D$ describes how to get computer access to this document via the Internet, and Appendix $\mathrm{E}$ describes a reader comment form, which is provided in the front of this report.

\subsection{SUMMARY DATA}

A few graphical presentations and summary tables are included in this chapter to provide a broad overview. Figures 0.1 and 0.2 , respectively, show the volumes and decayed radioactivities of commercial and DOE wastes and SNF accumulated through 1994. 
Summaries of SNF and radioactive waste inventories and projections are provided in Tables 0.3 and 0.4 . In general, material to be sent to $R \& D$ facilities or to the proposed national geologic repository for SNF and HLW is still listed in each individual site's inventory.

DOE waste volume summaries for specific sites are given in Tables 0.5-0.7. Waste generation for 1994 is presented in Table 0.5, and cumulative 1994 inventories for stored and buried wastes are provided in Tables 0.6 and 0.7 , respectively.

In recent years, various types of $\mathrm{DOE}$ and commercial wastes have been disposed of at a facility operated by Envirocare of Utah, Inc., near Clive, Utah. A summary of 1994 disposed waste volume inventories at the Envirocare Facility is given in Table 0.8 (data from ref. 19).

\subsection{REFERENCES}

1. U.S. Department of Energy, Integrated Data Base Report-1993: U.S. Spent Nuclear Fuel and Radioactive Waste Inventories, Projections, and Characteristics, DOE/RW-0006, Rev. 10, Oak Ridge National Laboratory, Oak Ridge, Tennessee (December 1994).

2. U.S. General Accounting Office, Environmental Cleanup-Better Data Needed for Radioactively Contaminated Defense Sites, GAO/NSIAD-94-168, Washington, D.C. (August 1994).

3. Coreen Casey and Beth A. Heath, EG\&G Idaho, Inc., Radioactive Waste Technical Support Program, Material Not Categorized as Waste (MNCAW) Data Report, DOE/LLW-93, predecisional draft, Idaho National Engineering Laboratory, Idaho Falls, Idaho (November 1993).

4. U.S. Congress, Nuclear Waste Policy Act of 1982, Pub. L. 97-425, Jan. 7, 1983, as amended by the Budget Reconciliation Act for Fiscal Year 1988, Title V-Energy and Environment Programs, Pub. L. 100-203, Dec. 22, 1987.

5. U.S. Environmental Protection Agency, "Environmental Protection Radiation Standards for Management and Disposal of Spent Nuclear Fuel, High-Level and Transuranic Radioactive Wastes," Code of Federal Regulations, 40 CFR Part 191 (1993).

6. U.S. Department of Energy, DOE Order 5820.2A, Radioactive Waste Management, Washington, D.C., Sept. 26, 1988.

7. U.S. Congress, The Low-Level Radioactive Waste Policy Amendments Act of 1985, Pub. L. 99-240, Jan. 15, 1986.

8. U.S. Congress, Atomic Energy Act of 1954, Pub. L. 83-703, Aug. 15, 1954.

9. U.S. Congress, Resource Conservation and Recovery Act of 1976, Pub. L. 94-580, Oct. 21, 1976, as amended by the Hazardous and Solid Waste Amendments Acts of 1984, Pub. L. 98-616, Nov. 9, 1984.

10. U.S. Congress, Toxic Substances Control Act of 1976, Pub. L. 94-469, Oct. 11, 1976.

11. U.S. Nuclear Regulatory Commission, "Licensing Requirements for Land Disposal of Radioactive Waste-Waste Classification," Code of Federal Regulations, 10 CFR Part 61, Sect. 61.55 (Jan. 1, 1995).

12. U.S. Department of Energy, Office of Environmental Management, 1995 Mixed Waste Inventory Report (to be issued).

13. The League of Women Voters Education Fund, The Nuclear Waste Primer, Lyons \& Burford, New York, 1993.

14. U.S. Department of Energy, Energy Information Administration, World Nuclear Outlook 1995, DOE/EIA-0436(95), Washington, D.C. (October 1995). 
15. U.S. Department of Energy, Office of Scientific and Technical Information, Nuclear Reactors Built, Being Built, or Planned: 1994, DOE/OSTI-8200-R58, Oak Ridge, Tennessee (August 1995).

16. A. G. Croff, ORIGEN2-A Revised and Updated Version of the Oak Ridge Isotope Generation and Depletion Code, ORNL-5621, Oak Ridge National Laboratory, Oak Ridge, Tennessee (July 1980).

17. U.S. Department of Energy, Energy Information Administration, Nuclear Fuel Data Form RW-859 (data as of Dec. 31, 1994), Washington, D.C.

18. U.S. Department of Energy, DOE Order 5820.2, Radioactive Waste Management, Washington, D.C., Feb. 6, 1984 [updated by DOE Order 5820.2A (ref. 6)].

19. Al Rafati, Envirocare of Utah, Inc., correspondence to Stephen N. Storch, IDB Program, ORNL, Oak Ridge, Tennessee, dated May 24, 1995. 


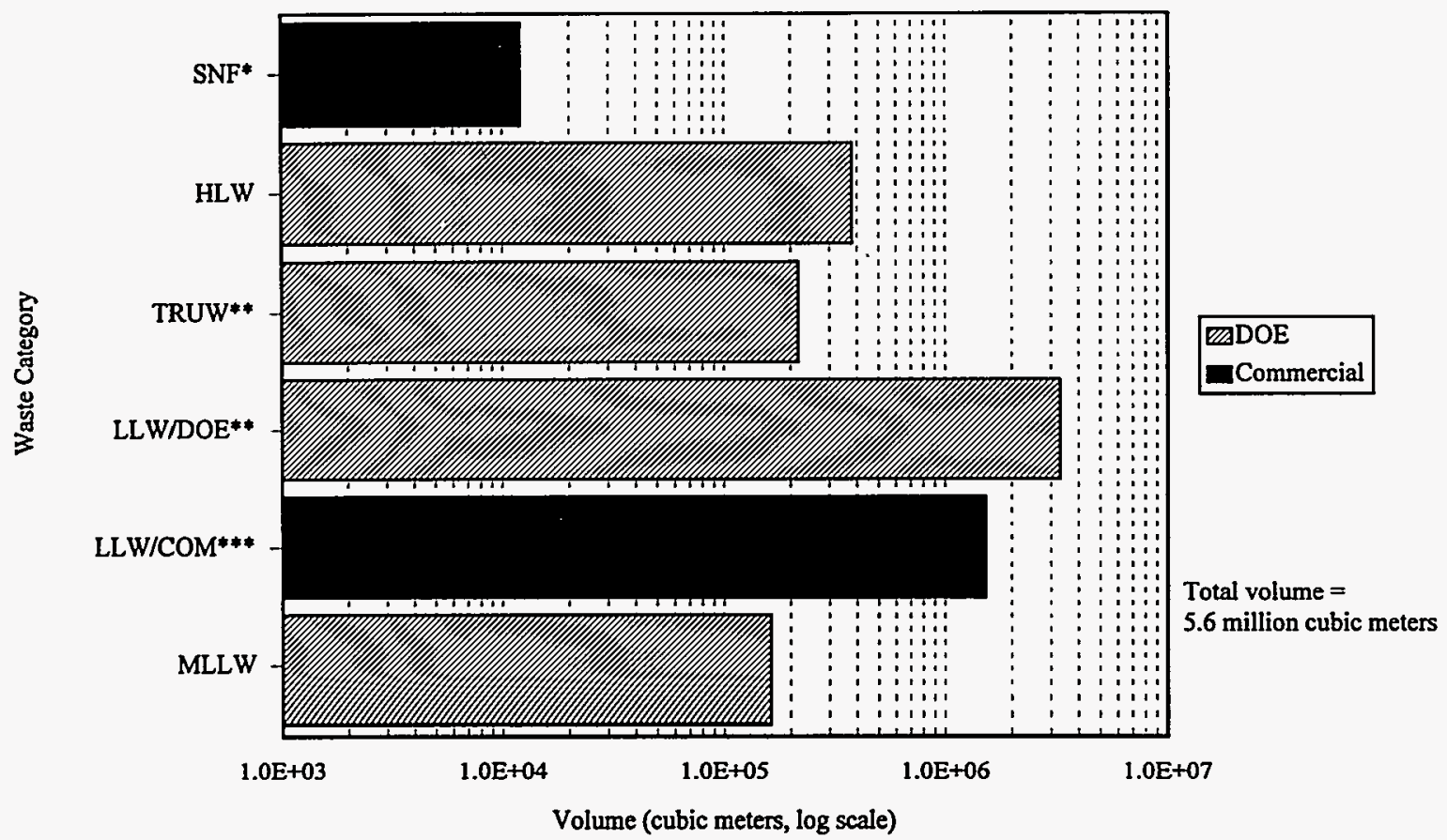

* Commercial LWR fuel permanently discharged. Includes spacing between fuel assembly rods.

**DOE wastes include both retrievably stored and buried materials. Excludes LLW disposed of at Envirocare facility.

*** Includes contributions from disposed wastes only. Excludes LLW disposed of at the Envirocare facility. 1994.

Fig. 0.1. Total volumes of DOE and commercial radioactive wastes and spent nuclear fuel through

ORNL DWG 95-8850R

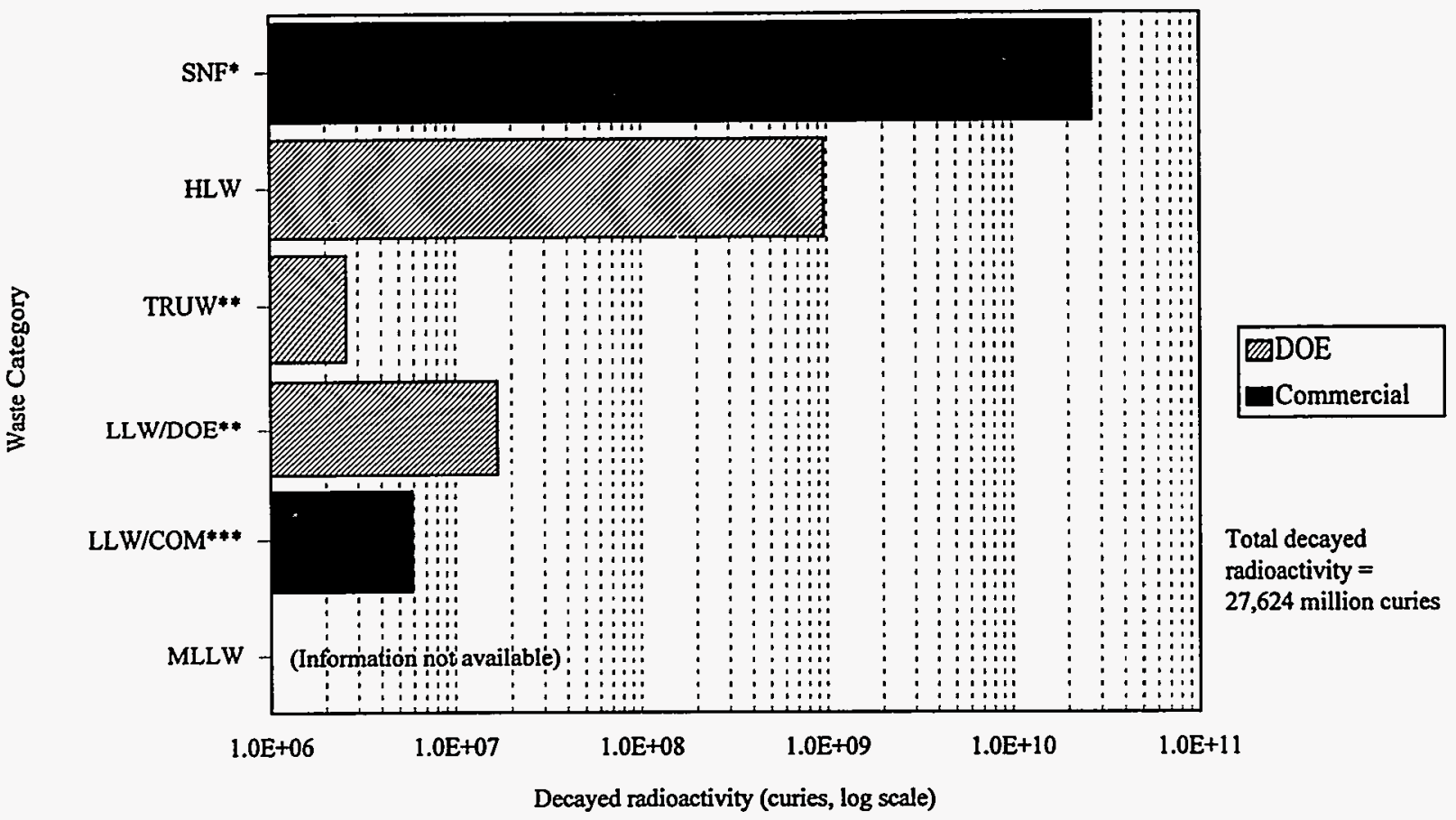

- Commercial LWR fuel permanently discharged.

** DOE wastes include both retrievably stored and buried materials. Excludes LLW disposed of at Envirocare facility.

*** Includes contributions from disposed wastes only. Excludes LLW disposed of at Envirocare facility.

Fig. 0.2. Total decayed radioactivities of $D O E$ and commercial radioactive wastes and spent nuclear fuel through 1994. 


\begin{tabular}{|c|c|c|c|c|c|c|c|}
\hline Site(s) & Symbol/label & SNF & HIW & TRUW & LLW & MLLW & IMT \\
\hline Ames Laboratory & Ames & & & $\mathrm{X}$ & $\mathrm{X}$ & $\mathrm{X}$ & \\
\hline Argonne National Laboratory-East & ANL-E & $\mathrm{X}$ & & $\mathrm{X}$ & $\mathrm{X}$ & $\mathrm{X}$ & \\
\hline Argonne National Laboratory-West & ANL-W & $\mathrm{X}$ & & $\mathrm{X}$ & $X$ & $\mathrm{X}$ & \\
\hline Battelle Columbus Laboratories & $\mathrm{BCL}$ & & & $\mathrm{X}$ & $\mathrm{X}$ & & \\
\hline Brookhaven National Laboratory & $\mathrm{BNL}$ & $\mathrm{X}$ & & & $\mathrm{X}$ & $\mathrm{X}$ & \\
\hline Energy Technology Engineering Center & ETEC & & & $\mathrm{X}$ & $\mathrm{X}$ & $\mathrm{X}$ & \\
\hline Fermi National Accelerator Laboratory & FNAL & & & & $\mathrm{X}$ & & \\
\hline Fernald Environmental Management Project & FEMP & & & & $\mathrm{X}$ & $\mathrm{X}$ & \\
\hline Hanford Site ${ }^{\mathrm{b}}$ & Hanford & $\mathrm{X}$ & $\mathrm{X}$ & $\mathrm{X}$ & $\mathrm{X}$ & $\mathrm{X}$ & \\
\hline Idaho National Engineering Laboratoryc & INEL & $\mathrm{X}$ & $\mathrm{X}$ & $\mathrm{X}$ & $\mathrm{X}$ & $\mathrm{X}$ & \\
\hline Inhalation Toxicology Research Institute & ITRI & & & & $X$ & $X$ & \\
\hline K-25 Site (Oak Ridge) & $\mathrm{K}-25$ & & & & $\mathrm{X}$ & $\mathrm{X}$ & \\
\hline Kansas City Plant & $\mathrm{KCP}$ & & & & $\mathrm{X}$ & $\mathrm{X}$ & \\
\hline Lawrence Berkeley Laboratory & LBL & & & $\mathrm{X}$ & $\mathrm{X}$ & $\mathrm{X}$ & \\
\hline Lawrence Livermore National Laboratory & LLNL & & & $\mathrm{X}$ & $\mathrm{X}$ & $\mathrm{X}$ & \\
\hline Los Alamos National Laboratory & LANL & $\mathrm{X}$ & & $\mathrm{X}$ & $\mathrm{X}$ & $\mathrm{X}$ & \\
\hline Missouri (Univ. of) Research Reactor & MURR & & & $\mathrm{X}$ & & & \\
\hline Mound Plant & Mound & & & $\mathrm{X}$ & $\mathrm{X}$ & $X$ & \\
\hline Naval Reactors Facilities and Shipyards ${ }^{\mathrm{d}}$ & NR Sites & & & $\mathrm{X}$ & $\underline{X}$ & $\mathrm{X}$ & \\
\hline Nevada Test Site & NTS & & & $\mathrm{X}$ & $\mathrm{X}$ & $\mathrm{X}$ & \\
\hline Oak Ridge Institute of Science and Education & ORISE & & & & $\mathrm{X}$ & & \\
\hline Oak Ridge National Laboratory & ORNL & $\mathrm{X}$ & & $\mathrm{X}$ & $\mathrm{X}$ & $\mathrm{X}$ & \\
\hline Paducah Gaseous Diffusion Plant & $\mathrm{PAD}$ & & & $\mathrm{X}$ & $\mathrm{X}$ & $\mathrm{X}$ & \\
\hline Pantex Plant & PANT & & & $\mathrm{X}$ & $\mathrm{X}$ & $\mathrm{X}$ & \\
\hline Pinellas Plant & Pinellas & & & & $\mathrm{X}$ & $\mathrm{X}$ & \\
\hline Portsmouth Gaseous Diffusion Plant & PORTS & & & & $\mathrm{X}$ & $\mathrm{X}$ & \\
\hline Princeton Plasma Physics Laboratory & PPPL & & & & $\mathrm{X}$ & $\mathrm{X}$ & \\
\hline Reactive Metals, Inc., Extrusion Plant & RMI & & & & $\mathrm{X}$ & $\mathrm{X}$ & \\
\hline Remedial Action Program Sites ${ }^{\mathrm{e}}$ & RAP Sites & & & $\mathrm{X}$ & $\mathrm{X}$ & $\mathrm{X}$ & $\mathrm{X}$ \\
\hline Rocky Flats Environmental Technology Site & RFETS & & & $\mathrm{X}$ & $\mathrm{X}$ & $\mathrm{X}$ & \\
\hline Sandia National Laboratory/California & $\mathrm{SNL} / \mathrm{CA}$ & & & & $X$ & $\mathrm{X}$ & \\
\hline
\end{tabular}




\begin{tabular}{|c|c|c|c|c|c|c|c|}
\hline \multicolumn{8}{|c|}{ Table 0.1 (continued) } \\
\hline Site(s) & Symbol/abel & SNF & $\mathrm{HLW}$ & TRUW & LLW & MLLW & UMT \\
\hline Sandia National Laboratory/New Mexico & SNLANM & $\mathrm{X}$ & & $\mathrm{X}$ & $\mathrm{x}$ & $\mathrm{X}$ & \\
\hline Savannah River Site & SRS & $\mathrm{x}$ & $\mathrm{X}$ & $\mathrm{x}$ & $\mathrm{X}$ & $\mathrm{x}$ & \\
\hline Stanford Linear Accelerator Center & SLAC & & & & $\mathrm{X}$ & & \\
\hline West Valley Demonstration Project & WVDP & $\mathrm{x}$ & $\mathrm{X}$ & $\mathrm{x}$ & $\mathrm{X}$ & $\mathrm{x}$ & \\
\hline Y-12 Plant (Oak Ridge) & $\mathrm{Y}-12$ & & & & $\mathrm{X}$ & $\mathrm{x}$ & \\
\hline $\begin{array}{l}\text { aAn "X" entry in this table indicate } \\
\text { bIncludes Pacific Northwest Labor } \\
\text { "Includes the Idaho Chemical Proc } \\
\text { dThese sites are listed in Table C.2 } \\
\text { eThese sites are listed in Chapter } 6\end{array}$ & $\begin{array}{l}\text { listed site mana } \\
\text { L). } \\
\text { int (ICPP); excl } \\
\text { dix C. }\end{array}$ & $\begin{array}{l}\text { waste } 0 \\
\text { ANL- }\end{array}$ & & & ite in & is is $\mathrm{g}_{1}$ & opendix \\
\hline
\end{tabular}


Table 0.2. Major assumptions used in this report

Inventory/projection basis

- Inventories are reported as of December 31, 1994

- Projections are made for the CYs 1995-2030

\section{HLW solidification activities}

- For WVDP, HLW solidification (glass production) starts in 1996 and is completed in 1998

- For SRS, HLW solidification [glass production at the Defense Waste Processing Facility (DWPF)] starts in 1996 and continues through 2021

- For INEL, HLW solidification (immobilization) starts in 2019 and continues through 2045

- For Hanford, HLW solidification (borosilicate glass production at the Hanford Waste Vitrification Plant) starts in 2010 and continues through 2028

\section{Commercial activities}

- DOE/EIA projections of installed net LWR electrical capacity for the Low Case ${ }^{a}$ of ref. 14:

\begin{tabular}{lrrrrrrrr}
\multicolumn{8}{c}{ Low Case } \\
Year & 1995 & 2000 & 2005 & 2010 & 2015 & 2020 & 2025 & 2030 \\
GW(e) & 99 & 100 & 100 & 91 & 61 & 47 & 22 & 2
\end{tabular}

- DOE/EIA assumptions for LWR fuel enrichment and design burnup:

\begin{tabular}{cccc} 
LWR fuel & $\begin{array}{c}\text { CY fuel is } \\
\text { loaded }\end{array}$ & $\begin{array}{c}\text { Fuel enrichment } \\
\left(\%{ }^{235} \mathrm{U}\right)\end{array}$ & $\begin{array}{c}\text { Design burnup } \\
\text { BWR }\end{array}$ \\
\cline { 2 - 3 } & 1993 & 3.20 & 36,000 \\
& 1998 & 3.35 & 40,000 \\
& 2006 & 3.50 & 43,000 \\
& 2018 & 3.67 & 46,000 \\
PWR & 1993 & 3.81 & 42,000 \\
& 1998 & 4.06 & 46,000 \\
& 2004 & 4.38 & 50,000 \\
& 2008 & 4.72 & 55,000 \\
& 2018 & 5.03 & 60,000
\end{tabular}

- SNF from commercial reactors is not reprocessed. Thus, a fuel cycle without reprocessing is assumed for all commercial projections

${ }^{a}$ This case assumes that each reactor will be retired when the expiration date specified in its operating license is reached. Previously, this case was referred to as the No New Orders Case. 
Table 0.3. Spent nuclear fuel and radioactive waste inventories as of December 31, 1994

\begin{tabular}{|c|c|c|c|c|c|}
\hline Waste category & $\begin{array}{c}\text { TRU } \\
\text { isotopes } \\
(\mathrm{kg})\end{array}$ & $\begin{array}{c}\text { Mass } \\
\text { (MTIHM) }\end{array}$ & $\begin{array}{l}\text { Volume } \\
\qquad\left(\mathrm{m}^{3}\right)\end{array}$ & $\begin{array}{l}\text { Radioactivity }^{a} \\
\qquad\left(10^{6} \mathrm{Ci}\right)\end{array}$ & $\begin{array}{c}\text { Thermal } \\
\text { power } \\
\left(10^{3} \mathrm{~W}\right)\end{array}$ \\
\hline \multicolumn{6}{|l|}{ Spent nuclear fuel } \\
\hline \multicolumn{6}{|l|}{ Commercial } \\
\hline BWRs & & 10,788 & $4,349^{b}$ & 7,540 & 27,800 \\
\hline PWRs & & 19,024 & $7,669^{b}$ & 19,100 & 72,500 \\
\hline DOE & & $>2,643$ & 1,213 & c & c \\
\hline \multicolumn{6}{|l|}{ High-level waste } \\
\hline Savannah River (DOE) & & & 126,300 & 534.5 & $1,497.3$ \\
\hline Idaho (DOE) & & & 11,000 & 51.6 & 150.8 \\
\hline Hanford (DOE) ${ }^{d}$ & & & 238,900 & 348.0 & 999.8 \\
\hline West Valley (commercial) & & & 2,180 & 24.7 & 78.1 \\
\hline \multicolumn{6}{|l|}{ Transuranic waste $(\mathrm{DOE})^{\mathrm{e}}$} \\
\hline Buried TRUW & c & & 141,100 & $>0.75$ & c \\
\hline Potentially contaminated soil & c & & $>32,000$ & $>0.08$ & c \\
\hline Stored TRUW & c & & 74,200 & 1.84 & c \\
\hline \multicolumn{6}{|l|}{ Low-level waste } \\
\hline \multicolumn{6}{|l|}{ DOE sites } \\
\hline Generated & & & 37,985 & 0.9 & c \\
\hline Cumulative stored & & & $125,890^{f}$ & 3.9 & $\mathrm{c}$ \\
\hline Cumulative disposed & & & $2,963,349$ & 12.9 & 21.5 \\
\hline \multicolumn{6}{|l|}{ Commercial sites } \\
\hline Cumulative disposed & & & $1,518,878$ & 5.8 & 22.7 \\
\hline Envirocare disposal facility & & & 40,461 & c & c \\
\hline \multicolumn{6}{|l|}{ Uranium mill tailings (commercial) } \\
\hline Licensed mill sitesg & & & $118,600,000$ & c & c \\
\hline $\begin{array}{l}\text { Environmental restoration program } \\
\text { wastes }(\mathrm{DOE})^{\mathrm{h}}\end{array}$ & & & c & c & c \\
\hline Commercial reactor decommissioning & & & $27,156^{i}$ & c & $\mathbf{c}$ \\
\hline \multicolumn{6}{|l|}{ MLLW (cumulative stored) } \\
\hline DOE & & $105,666^{j}$ & 162,377 & c & c \\
\hline Commercial & & c & c & c & c \\
\hline Envirocare disposal facility & & c & 3,621 & c & c \\
\hline
\end{tabular}

aExcept for transuranic wastes, radioactivity data are calculated decayed values as of December 31, 1994.

$\mathrm{b}$ Includes volume of spacing between the fuel rods of each assembly.

Information not available.

${ }^{d}$ Hanford tank wastes consist of HLW, TRUW, and LLW. However, in the interim storage mode, the tank wastes are managed as if they contain HLW and, therefore, are included in the HLW inventory.

Data as of December 31, 1993.

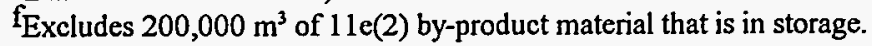

Includes contributions from 26 NRC-licensed mills.

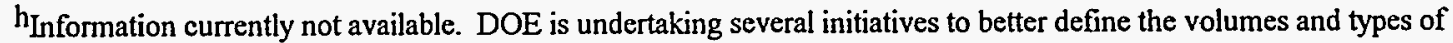
wastes currently in storage at environmental restoration sites and those which may be generated during future remediation activities across the entire DOE complex. This information, which should become available in a few years, will be included in future revisions of this report.

'Quantity of LLW shipped from completed and ongoing reactor decommissioning projects. This inventory is included in the commercial LLW inventory listed above.

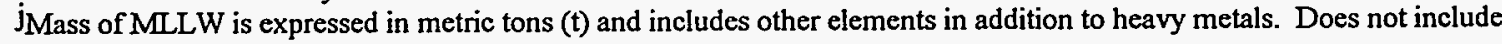
contributions from some sites that reported volume data. 
Table 0.4. Current and projected cumulative quantities of radioactive waste and spent nuclear fuel [Quantities are expressed as volume $\left(10^{3} \mathrm{~m}^{3}\right)$ unless otherwise indicated]

\begin{tabular}{|c|c|c|c|c|c|}
\hline \multirow{2}{*}{ Source and type of material } & \multicolumn{5}{|c|}{ End of calendar year } \\
\hline & 1994 & 2000 & 2010 & 2020 & 2030 \\
\hline \multicolumn{6}{|l|}{ DOE sites } \\
\hline SNF, mass, MTIHM ${ }^{\mathrm{a}}$ & $>2,643$ & $\mathbf{b}$ & $\mathrm{b}$ & $\mathrm{b}$ & $\mathbf{b}$ \\
\hline \multicolumn{6}{|l|}{ HLW } \\
\hline Interim storage & 378.4 & 341 & 259 & 83 & 7 \\
\hline Glass or glass/ceramic $c$ & 0 & 0.3 & 2.0 & 8.7 & 12.8 \\
\hline \multicolumn{6}{|l|}{ TRUW } \\
\hline Buried & $141^{d}$ & 141 & 141 & 141 & 141 \\
\hline Stored & $75.4^{\mathrm{d}}$ & b & b & b & b \\
\hline LLW (buried) ${ }^{\mathrm{e}}$ & 2,963 & 3,572 & 4,534 & 5,494 & 5,926 \\
\hline $\begin{array}{l}\text { Environmental restoration } \\
\text { program wastes } \mathrm{f}\end{array}$ & b & b & b & $b$ & b \\
\hline MLLW & 162.4 & $\mathrm{~b}$ & $\mathrm{~b}$ & b & $\mathbf{b}$ \\
\hline \multicolumn{6}{|l|}{ Commercial sites } \\
\hline \multicolumn{6}{|l|}{$\begin{array}{l}\text { LWR SNF, mass, MTIHMM } \\
\text { (no reprocessing) }\end{array}$} \\
\hline Low Case & 29,812 & 42,300 & 61,800 & 77,100 & 84,100 \\
\hline \multicolumn{6}{|l|}{ HLW (WVDP) } \\
\hline Interim storage & 2.180 & 0.0 & 0.0 & 0.0 & 0.0 \\
\hline Glass & 0.0 & 0.24 & 0.24 & 0.24 & 0.24 \\
\hline LLW (no reprocessing) & 1,519 & 1,665 & b & $\mathrm{b}$ & b \\
\hline \multicolumn{6}{|l|}{ D\&D (LLW $)^{\mathrm{h}}$} \\
\hline Classes A, B, and C LLW & i & 0.00 & 0.83 & 124.23 & 453.22 \\
\hline Greater-than-Class-C LLW & i & 0.00 & 0.00 & 0.10 & 0.45 \\
\hline Mill tailings (no reprocessing) & 118,600 & 118,800 & 120,500 & $b$ & $b$ \\
\hline MLLW & $\mathrm{b}$ & b & b & $\mathrm{b}$ & $\mathrm{b}$ \\
\hline \multicolumn{6}{|l|}{ Envirocare disposal facility } \\
\hline LLW & 40.5 & b & $\mathbf{b}$ & $\mathrm{b}$ & b \\
\hline MLLW & 3.6 & b & b & $\mathrm{b}$ & $\mathrm{b}$ \\
\hline NARM/NORM & 3.3 & b & b & $\mathrm{b}$ & $\mathrm{b}$ \\
\hline $11 \mathrm{e}(2)$ by-product & 16.8 & b & b & $\mathrm{b}$ & $\mathrm{b}$ \\
\hline
\end{tabular}

aHistorically, spent nuclear fuel has been measured in units of mass (MTIHM) rather than units of volume.

binformation not available.

CIncludes projections for glass at SRS and glass/ceramic at INEL.

dProjected inventory level for end of CY 1994.

EProjections include contributions from SRS saltstone.

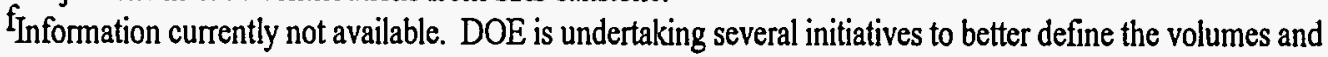
types of wastes currently in storage at environmental restoration sites and those which may be generated during future remediation activities across the entire DOE complex. This information, which should be available in a few years, will be included in future revisions of this report.

BThe 1994 discharged spent nuclear fuel mass is a BWR and PWR mass sum rounded to the nearest metric ton. Such rounding may result in slight differences between the spent nuclear fuel inventories and projections reported in this document and those reported by DOE/EIA.

hProjected D\&D wastes from light-water reactors shut down after 1994. Wastes collected from historical $D \& D$ of reactors are included in the LLW inventories listed above.

information not applicable. 
Table 0.5. Volume $\left(\mathrm{m}^{3}\right)$ of DOE site wastes generated during calendar year 1994

\begin{tabular}{|c|c|c|c|c|c|c|c|}
\hline \multirow{2}{*}{ Site(s) } & \multirow{2}{*}{ HLW } & \multicolumn{2}{|c|}{ TRUW } & \multirow{2}{*}{ LLW $^{\mathrm{b}}$} & \multicolumn{2}{|c|}{ MLLW } & \multirow{2}{*}{ Total (site) } \\
\hline & & $\mathrm{CH}^{\mathrm{a}}$ & $\mathrm{RH}^{\mathrm{a}}$ & & $\mathrm{RCRA}^{\mathrm{c}}$ & TSCA & \\
\hline Ames & & & & 5 & $\mathrm{~d}$ & & 5 \\
\hline ANL-E & & 2 & & 669 & 14 & 52 & 737 \\
\hline ANL-W & & 2 & d & & 3 & e & 5 \\
\hline BNL & & & & 254 & $<1$ & d & 255 \\
\hline ETEC & & & & & e & 8 & 8 \\
\hline FEMP & & & & & e & e & e \\
\hline FNAL & & & & 61 & e & & 61 \\
\hline GJPO & & & & & d & 2 & 2 \\
\hline Hanford & & 169 & 2 & 4,498 & 1,312 & 3 & 5,984 \\
\hline INEL & & & & 2,665 & 160 & 1 & 2,826 \\
\hline ITRI & & & & 31 & 15 & & 46 \\
\hline $\mathrm{K}-25$ & & & & 8,135 & 215 & 5,587 & 13,937 \\
\hline $\mathrm{KCP}$ & & & & $<1$ & e & & $<1$ \\
\hline LANL & & 254 & 12 & 1,898 & 17 & 167 & 2,348 \\
\hline LBL & & & & 15 & 1 & 3 & 19 \\
\hline LLNL & & 21 & & 53 & 282 & 1 & 357 \\
\hline Mound & & & & 909 & e & 7 & 916 \\
\hline NR sites & & & 1 & 1,568 & 224 & 344 & 2,137 \\
\hline NTS & & & & 71 & 507 & & 578 \\
\hline ORISE & & & & 12 & e & & 12 \\
\hline ORNL & & 9 & 12 & 1,322 & 115 & 13 & 1,471 \\
\hline PAD & & & & & e & 1,061 & 1,061 \\
\hline PANT & & & & 122 & 177 & 3 & 302 \\
\hline Pinellas & & & & 52 & e & & 52 \\
\hline PORTS & & & & & 330 & 260 & 590 \\
\hline PPPL & & & & 42 & 2 & & 44 \\
\hline RFETS & & 66 & & 503 & 725 & 36 & 1,330 \\
\hline SLAC & & & & 81 & e & & 81 \\
\hline SNL/CA & & & & 24 & 1 & d & 25 \\
\hline SNL/NM & & & & & 6 & e & 6 \\
\hline SRS & & 509 & 4 & 10,491 & 1,846 & 12 & 12,862 \\
\hline WVDP & & 55 & & 1,372 & 6 & 4 & 1,437 \\
\hline$Y-12$ & & & & 3,131 & 310 & 21 & 3,462 \\
\hline Others $\mathrm{f}$ & & & & & 6,078 & & 6,078 \\
\hline $\begin{array}{l}\text { Total } \\
\text { (complex) }\end{array}$ & 0 & 1,087 & 31 & 37,985 & 12,347 & 7,585 & 59,035 \\
\hline
\end{tabular}

aProjected annual addition to stored inventory during 1994. Includes contributions from both mixed and nonmixed wastes.

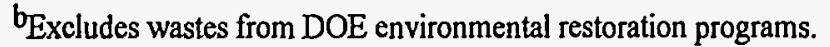

cAnnual average estimated for the period 1994-1998.

drace amount.

EInformation unavailable or unknown.

Includes contributions from 14 sites. 
Table 0.6. Cumulative volume $\left(\mathrm{m}^{3}\right)$ of stored DOE site wastes as of December 31, 1994

\begin{tabular}{|c|c|c|c|c|c|c|c|}
\hline \multirow{2}{*}{ Site(s) } & \multirow{2}{*}{ HLW } & \multicolumn{2}{|c|}{ TRUW ${ }^{a}$} & \multirow{2}{*}{ LLW } & \multicolumn{2}{|c|}{ MLLW } & \multirow{2}{*}{ Total (site) } \\
\hline & & $\mathrm{CH}$ & $\mathrm{RH}$ & & RCRA & TSCA & \\
\hline Ames & & & & 16 & $b$ & 3 & 19 \\
\hline ANL-E & & 31 & & 284 & 34 & 52 & 401 \\
\hline ANL-W & & 2 & 9 & & 391 & c & 402 \\
\hline BNL & & & & 556 & 27 & 2 & 585 \\
\hline ETEC & & & & 565 & 1 & 11 & 577 \\
\hline FEMP & & & & $27,000^{d}$ & 2,151 & c & 29,151 \\
\hline FNAL & & & & 83 & c & & 83 \\
\hline GJPO & & & & & $<1$ & 47 & 48 \\
\hline Hanford & 238,900 & 9,559 & 35 & 51 & 6,331 & 96 & 254,972 \\
\hline INEL & 11,000 & 34,700 & 31 & 14,080 & 25,440 & 2 & 85,253 \\
\hline ITRI & & & & 51 & & & 51 \\
\hline $\mathrm{K}-25$ & & & & 14,586 & 29,473 & 9,493 & 53,552 \\
\hline $\mathrm{KCP}$ & & & & 9 & c & & 9 \\
\hline LANL & & 11,154 & 103 & & 609 & 5,993 & 17,859 \\
\hline LBL & & & & 19 & 6 & b & 25 \\
\hline LLNL & & 245 & & 606 & 457 & 2 & 1,310 \\
\hline Mound & & 263 & & 8,860 & 98 & 16 & 9,237 \\
\hline NR sites & & & 12 & & 48 & 22 & 82 \\
\hline NTS & & 620 & & 270 & 908 & & 1,798 \\
\hline ORISE & & & & & $\mathrm{c}$ & & c \\
\hline ORNL & & 792 & 1,006 & 3,538 & 3,020 & 15 & 8,371 \\
\hline PAD & & & & 5,400 & 1,028 & 4,359 & 10,787 \\
\hline PANT & & & & 209 & 128 & 3 & 340 \\
\hline Pinellas & & & & 66 & & & 66 \\
\hline PORTS & & & & 25,000 & 7,514 & 3,670 & 36,184 \\
\hline PPPL & & & & 3 & & & 3 \\
\hline RFETS & & 1,196 & & 5,315 & 13,550 & 112 & 20,173 \\
\hline SLAC & & & & 242 & 6 & & 248 \\
\hline SNL/CA & & & & 26 & $<1$ & b & 27 \\
\hline SNL/NM & & & & & 75 & c & 75 \\
\hline SRS & 126,300 & 15,109 & 4 & 1,655 & 7,200 & 142 & 150,410 \\
\hline WVDP & 2,200 & & 427 & 14,274 & 86 & 44 & 17,031 \\
\hline$Y-12$ & & & & 825 & 13,465 & 150 & 14,440 \\
\hline Others $^{e}$ & & & & $2,301^{\mathrm{f}}$ & 26,706 & & 29,007 \\
\hline $\begin{array}{l}\text { Total } \\
\text { (complex) }\end{array}$ & 378,400 & 73,671 & 1,627 & 125,890 & 138,754 & 24,234 & 742,576 \\
\hline
\end{tabular}

aProjected for the end of CY 1994. Includes both mixed and nonmixed wastes.

$\mathrm{b}_{\text {Trace amount. }}$

CInformation unavailable or unknown.

$\mathrm{d}_{\text {Excludes }} 10,000 \mathrm{~m}^{3}$ of $11 \mathrm{e}(2)$ by-product material that is in storage.

Includes contributions from 14 sites.

$f_{\text {Excludes }} 190,000 \mathrm{~m}^{3}$ of $11 \mathrm{e}(2)$ by-product material that is in storage at the Weldon Spring Site Remedial Action Project. 
Table 0.7. Cumulative volume $\left(\mathrm{m}^{3}\right)$ of buried DOE site wastes $\mathrm{a}, \mathrm{b}, \mathrm{c}, \mathrm{d}$

\begin{tabular}{|c|c|c|c|}
\hline Site(s) & TRUWe & LLWf $^{\mathrm{f}}$ & Total (site) \\
\hline $\begin{array}{l}\text { Ames } \\
\text { ANL-E } \\
\text { ANL-W }\end{array}$ & & 200 & 200 \\
\hline BNL & & 839 & 839 \\
\hline ETEC & & 343,218 & 343,218 \\
\hline FEMP & & & \\
\hline $\begin{array}{l}\text { FNAL } \\
\text { GJPO }\end{array}$ & & & \\
\hline Hanford & 63,600 & 615,283 & 678,883 \\
\hline $\begin{array}{l}\text { INEL } \\
\text { ITRI }\end{array}$ & 57,100 & 148,990 & 206,090 \\
\hline $\begin{array}{l}\mathrm{K}-25 \\
\mathrm{KCP}\end{array}$ & & 81,048 & 81,048 \\
\hline $\begin{array}{l}\text { LANL } \\
\text { LBL }\end{array}$ & 14,000 & 222,610 & 236,610 \\
\hline $\begin{array}{l}\text { LLNL } \\
\text { Mound } \\
\text { NR sites }\end{array}$ & & 9,102 & 9,102 \\
\hline $\begin{array}{l}\text { NTS } \\
\text { ORISE }\end{array}$ & & 481,308 & 481,308 \\
\hline ORNL & 176 & 209,665 & 209,841 \\
\hline PAD & & 7,613 & 7,613 \\
\hline PANT & & 134 & 134 \\
\hline Pinellas & & & \\
\hline PORTS & & 12,110 & 12,110 \\
\hline PPPL & & & \\
\hline RFETS & & & \\
\hline SLAC & & & \\
\hline SNL/CA & & & \\
\hline SNL/NM & 1 & 3,218 & 3,219 \\
\hline SRS & 4,870 & 676,668 & 681,538 \\
\hline WVDP & 1,350 & $\mathrm{~g}$ & 1,350 \\
\hline$Y-12$ & & 151,343 & 151,343 \\
\hline $\begin{array}{c}\text { Total } \\
\text { (complex) }\end{array}$ & 141,097 & $2,963,349$ & $3,104,446$ \\
\hline
\end{tabular}

aExcludes 89,472 containers $(94,273 \mathrm{Ci}$, undecayed) of LLW in 50 - and 80-gal drums disposed of by sea dumping.

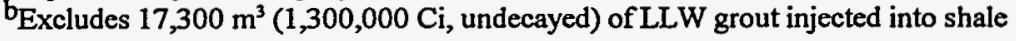
underlying the ORNL site.

${ }^{C}$ Excludes wastes from environmental restoration activities.

dExcludes contributions from the following DOE wastes disposed of at the Envirocare Facility as of the end of 1994: $\operatorname{LLW}\left(1,759 \mathrm{~m}^{3}\right), \operatorname{MLLW}\left(1,356 \mathrm{~m}^{3}\right)$, and $11 \mathrm{e}(2)$ by-product material $\left(3,822 \mathrm{~m}^{3}\right)$.

As of December 31, 1993.

$f_{A s}$ of December 31, 1994. The data listed for LLW represent disposed inventories and include materials that are not buried.

gonly commercial LLW is buried at WVDP. 
Table 0.8. Volume $\left(\mathrm{m}^{3}\right)$ summary of radioactive wastes disposed of at the Envirocare Facility near Clive, Utah $^{\mathrm{a}}$

\begin{tabular}{|c|c|c|c|c|}
\hline \multirow{2}{*}{ Waste type } & \multicolumn{2}{|c|}{ DOE wastes } & \multicolumn{2}{|c|}{ Commercial wastes } \\
\hline & 1994 & Cumulative $^{\mathrm{b}}$ & 1994 & Cumulative $^{\mathrm{c}}$ \\
\hline LLW & 1,427 & 1,759 & 11,957 & 38,702 \\
\hline MLLW & 1,288 & 1,356 & 851 & 2,265 \\
\hline NARM/NORMd & 0 & 0 & 811 & 3,320 \\
\hline $11 \mathrm{e}(2)^{\mathrm{e}}$ & 3,822 & 3,822 & 13,005 & 13,005 \\
\hline Total & 6,537 & 6,937 & 26,624 & 57,292 \\
\hline
\end{tabular}

aBased on ref. 19.

b Covers wastes disposed during 1992-94.

${ }^{c}$ Covers wastes disposed during 1988-94.

$\mathrm{d}^{\mathrm{d}}$ Soil contaminated with radium and building debris.

e By-product material as defined in Section 1 1e(2) of the Atomic Energy Act of 1954 (P.L. 83-703). 


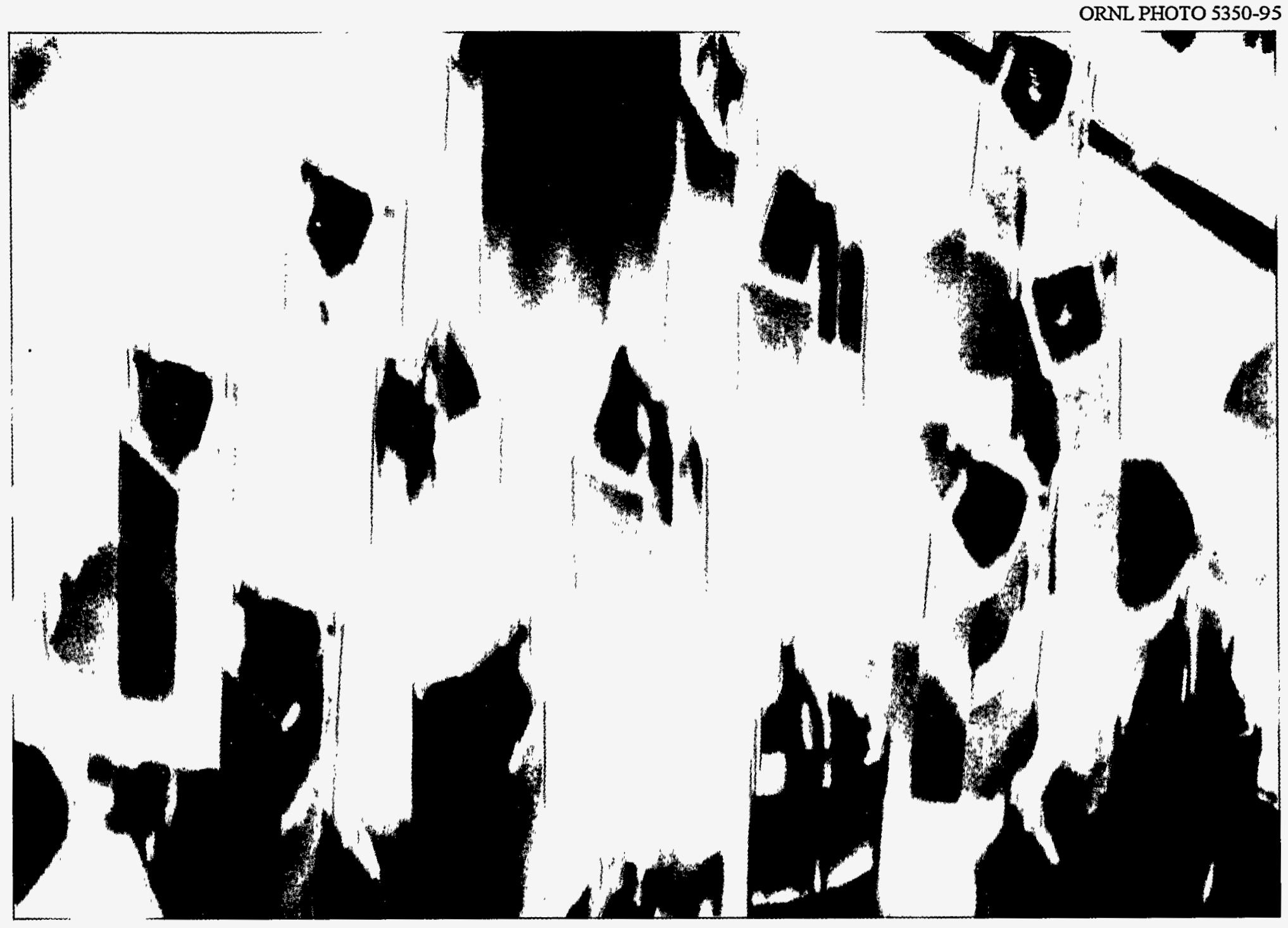

Spent nuclear fuel being generated in the assemblies of the core of the Advanced Test Reactor at the Idaho National Engineering Laboratory, Idaho Falls, Idaho. Courtesy of Lockheed Martin Idaho Technologies, Idaho Falls, Idaho. 


\section{SPENT NUCLEAR FUEL}

\subsection{INTRODUCTION}

This chapter reports the quantities and characteristics of spent nuclear fuel (SNF) that has been permanently discharged from commercial LWRs and one-of-a-kind reactors. In addition, this chapter contains a mass summary report of DOE SNF (see Sect. 1.4) which is not scheduled for reprocessing. Though currently in storage at numerous commercial and DOE sites, this fuel will ultimately require geologic disposal.

Some commercial SNF in inventory will be reinserted into reactors for further irradiation. However, this amount is relatively small, and the schedules for reinsertion are not always predictable. Therefore, for the purposes of this report, all SNF is considered permanently discharged from the reactors.

Historical inventories of LWR SNF have been updated through December 31, 1994.' The data reported in this chapter include the inventories of SNF stored at the West Valley Demonstration Project (WVDP), the Midwest Fuel Recovery Plant (MFRP), and the Idaho National Engineering Laboratory (INEL) sites in addition to those stored at the various reactor sites. The map in Fig. 1.1 shows the locations of existing and planned power reactor sites and commercial LWR SNF storage facilities. A list of commercial reactors is given also in report DOE/ OSTI-8200-R58 (ref. 2).

Projections of nuclear capacity and SNF discharges are given for the years 1995-2030 for the DOE/ELA Low Case (No New Orders) forecast schedule reported in ref. 3. The Low Case (No New Orders) forecast, hereafter referred to simply as the Low Case, projects installed capacity to increase from $99.1 \mathrm{GW}(\mathrm{e})$ at the end of 1994 to $100.3 \mathrm{GW}(\mathrm{e})$ by the year 2000 , ultimately decreasing to $2.3 \mathrm{GW}(\mathrm{e})$ by 2030 .

The Low Case reference scenario considered for projecting accumulated SNF assumes a fuel cycle with no reprocessing. Commercial SNF projections developed for the DOE/EIA Low Case are illustrated, along with historical discharge data, in Figs. 1.2-1.4. SNF discharge projections, in terms of annual mass discharged and accumulated radioactivity, are graphically illustrated in Figs. 1.2 and 1.3, respectively. A graph showing the increase in the cumulative mass of discharged SNF for the DOE/EIA Low Case is shown in Fig. 1.4. This plot also shows both the age and mass distribution for SNF from 1970 to 2030.

DOE/EIA projections for the Low Case assume that burnup levels of discharged SNF will increase from their current average levels of 33,065 and $39,989 \mathrm{MWd} /$ MTIHM for BWR and PWR fuel, respectively, at the rate of about $0.5 \%$ year for BWR fuel and about $1.0 \%$ per year for PWR fuel. This increase in burnup is projected to occur from 1994 to approximately 2020, at which time the equilibrium cycle bumup is projected to be roughly 42,000 and 54,000 MWd/MTIFM (ref. 3) for BWR and PWR fuel, respectively. The final cycle discharges will be somewhat lower because most of the final cycle cores will not have achieved the projected design burnups. Figure 1.5 graphically illustrates how the activity and thermal power of BWR and PWR SNF vary with bumup and time from discharge. ${ }^{4}$

\subsection{COMMERCIAL SPENT NUCLEAR FUEL}

\subsubsection{Inventories and projections}

The total inventory of commercial LWR SNF in storage at the WVDP site, the MFRP, INEL, and the reactor sites as of December 31, 1994, amounted to 29,812 MTIHM. Of this total amount, 27 MTIHM are in storage at the WVDP site, ${ }^{5} 674$ MTIHM are in storage at the MFRP, ${ }^{\prime}$ and 43 MTIHM are in storage at INEL.' The remainder is stored at the reactor sites. These inventories do not include the SNF reprocessed at the WVDP site when the facility was operated as a fuel reprocessing plant. Additional information on WVDP SNF inventories is given in Chapter 7, Table 7.22. Details concerning the SNF reprocessed at West Valley may be obtained from ref. 6.

A BWR/PWR breakdown of the electric power generating capacity for the Low Case forecast, along with historical reactor capacity data, is given in Table 1.1. Table 1.2 gives the projected cumulative mass of 
commercial SNF discharges associated with the DOE/EIA capacity-growth forecast given in Table 1.1. The historical and projected buildups of permanently discharged BWR and PWR SNF mass, radioactivity, and thermal power are given for the DOE/ELA Low Case in Table 1.3. Projections of the number of permanently discharged BWR and PWR SNF assemblies for the DOE/EIA Low Case are given in Table 1.4.

The historical and projected mass of SNF discharged from a one-of-a-kind reactor, the Fort St. Vrain HTGR, ${ }^{7}$ is given in Table 1.5. All of the discharged nuclear fuel from the Fort St. Vrain reactor that has been shipped off-site is located at the ICPP. The Fort St. Vrain reactor was permanently shut down in 1989.

\subsubsection{Characterization}

Reference characteristics of BWR and PWR fuel assemblies, obtained from refs. 8 and 9, were used for this report These characteristics are summarized in Table 1.6. Fuel assembly structural material masses and compositions, nonactinide fuel impurities, and other physical and irradiation characteristics of LWR SNF are discussed in ref. 10. More detailed information on SNF characteristics may be found in ref. 11. The BWR and PWR SNF annually discharged has a broad range of burnup levels, as illustrated in Tables 1.7 and 1.8, respectively. The mass, radioactivity, and thermal power of the nuclides contained in all stored domestic commercial LWR SNF as of December 31, 1994, are listed in Table A.2 in Appendix A.

\subsection{DISPOSAL}

Through the first half of 1995, the Office of Civilian Radioactive Waste Management (OCRWM) met regulatory milestones by completing the first Technical Basis Report on surface processes and by submitting the Annotated Outline for the License Application. Extensive surface-based and underground investigation is being conducted. This investigation is essential to evaluate the suitability of the Yucca Mountain site before a license application can be submitted to the Nuclear Regulatory Commission (NRC). This work is well underway and will lead to a determination of the suitability of the Yucca Mountain site in 1998.

Tunnel excavation is proceeding well despite ground conditions that have been more difficult than anticipated. The assembly of the 25- $\mathrm{ft}$ diameter Tunnel Boring Machine (TBM) was completed, and the TBM was moved into the Starter Tunnel in early August 1994. The TBM had advanced to $3,733 \mathrm{ft}$ by the end of June 1995, when it was shut down for a scheduled maintenance service and the installation of a muck conveyer.

As of this writing (late summer, 1995), the Congress was involved in a broad debate regarding the future direction of the Repository Program. A number of bills were introduced, and hearings were held. The discussion focused on the interim storage issue and the related aspects of the budget.

\subsection{DOE SPENT NUCLEAR FUEL}

Summary inventory characteristics of DOE SNF as of July 1995 are given in Table 1.9 (based on ref. 12). Projected site inventories for the year 2035 are also included.

For purposes of clarification, the quantities of SNF reported in Table 1.9 include contributions from other nuclear fuels besides those permanently discharged from production reactors. SNFs reported in this table also include DOE-owned nuclear fuel that has been withdrawn from or resides for storage in a nuclear reactor following irradiation, the constituent elements of which have not been separated by processing. In addition to intact fuel, reactor-irradiated fuel materials requiring special handling (e.g., defective fuel and special fuel forms) are also considered SNF and are eligible for inclusion in Table 1.9. This table also lists some commercially generated nuclear fuels and nuclear fuels from foreign reactors and university research reactors which are stored at DOE sites. More detailed information on these special nuclear fuels will be included in future updates of this report.

\subsection{REFERENCES}

1. U.S. Department of Energy, Energy Information Administration, Nuclear Fuel Data Form RW-859, Washington, D.C. (data as of December 31, 1994).

2. U.S. Department of Energy, Office of Scientific and Technical Information, Nuclear Reactors Built, Being Built, or Planned: 1994, DOE/OSTI-8200-R58, Oak Ridge, Tennessee (August 1995). 
3. U.S. Department of Energy, Energy Information Administration, World Nuclear Outlook 1995, DOE/EIA-0436(95), Washington, D.C. (October 1995).

4. J. W. Roddy et al., Physical and Decay Characteristics of Commercial LWR Spent Fuel, ORNL/TM-9591/V1\&R1, Oak Ridge National Laboratory, Oak Ridge, Tennessee (January 1986).

5. John J. Hollinden, West Valley Nuclear Services Company, Inc., West Valley, New York, correspondence to Steven N. Storch, IDB Program, ORNL, Oak Ridge, Tennessee, attachment to "Submittal of the Department of Energy Site Toxic Substance Control Act Mixed Low-Level Waste Information Request for the 1995 Integrated Data Base Report (DOE/RW-0006, Rev. 11),"WZ:95:0095, dated June 15, 1995.

6. E. R. Johnson Associates, Inc., Review of the Operating History of the Nuclear Fuel Services, Inc., West Valley, New York, Irradiated Fuel Reprocessing Plant, ORNL/Sub-81/31066/1, Oak Ridge National Laboratory, Oak Ridge, Tennessee (September 1981).

7. M. J. Fisher, Public Service Company of Colorado, Platteville, Colorado, correspondence to R. C. Ashline, Oak Ridge National Laboratory, Oak Ridge, Tennessee, "Update of Annual Fort St. Vrain Reactor Spent Fuel Discharges for 1995 DOE Integrated Data Base Report," dated May 23, 1995.

8. General Electric Company, General Electric Standard Safety Analysis Report, BWR/6, Docket STN 50-447, San Jose, California (1973).

9. Westinghouse Nuclear Energy Systems, Reference Safety Analysis Report, RESAR-3, Docket STN 50-480, Pittsburgh, Pennsylvania (1972).

10. A. G. Croff et al., Revised Uranium-Plutonium Cycle PWR and BWR Models for the ORIGEN Computer Code, ORNL/TM-6051, Oak Ridge National Laboratory, Oak Ridge, Tennessee (September 1978).

11. U.S. Department of Energy, Characteristics of Potential Repository Wastes, DOE/RW-0184-R1, Vols. 1-4, Oak Ridge National Laboratory, Oak Ridge, Tennessee (July 1992).

12. E. P. Stroupe, Lockheed Idaho Technologies Company, Idaho National Engineering Laboratory, Idaho Falls, Idaho, correspondence to J. A. Klein, IDB Program, Oak Ridge National Laboratory, Oak Ridge, Tennessee, "Spent Nuclear Fuel Data for Integrated Data Base-EPS-64-95," dated Aug. 22, 1995. 


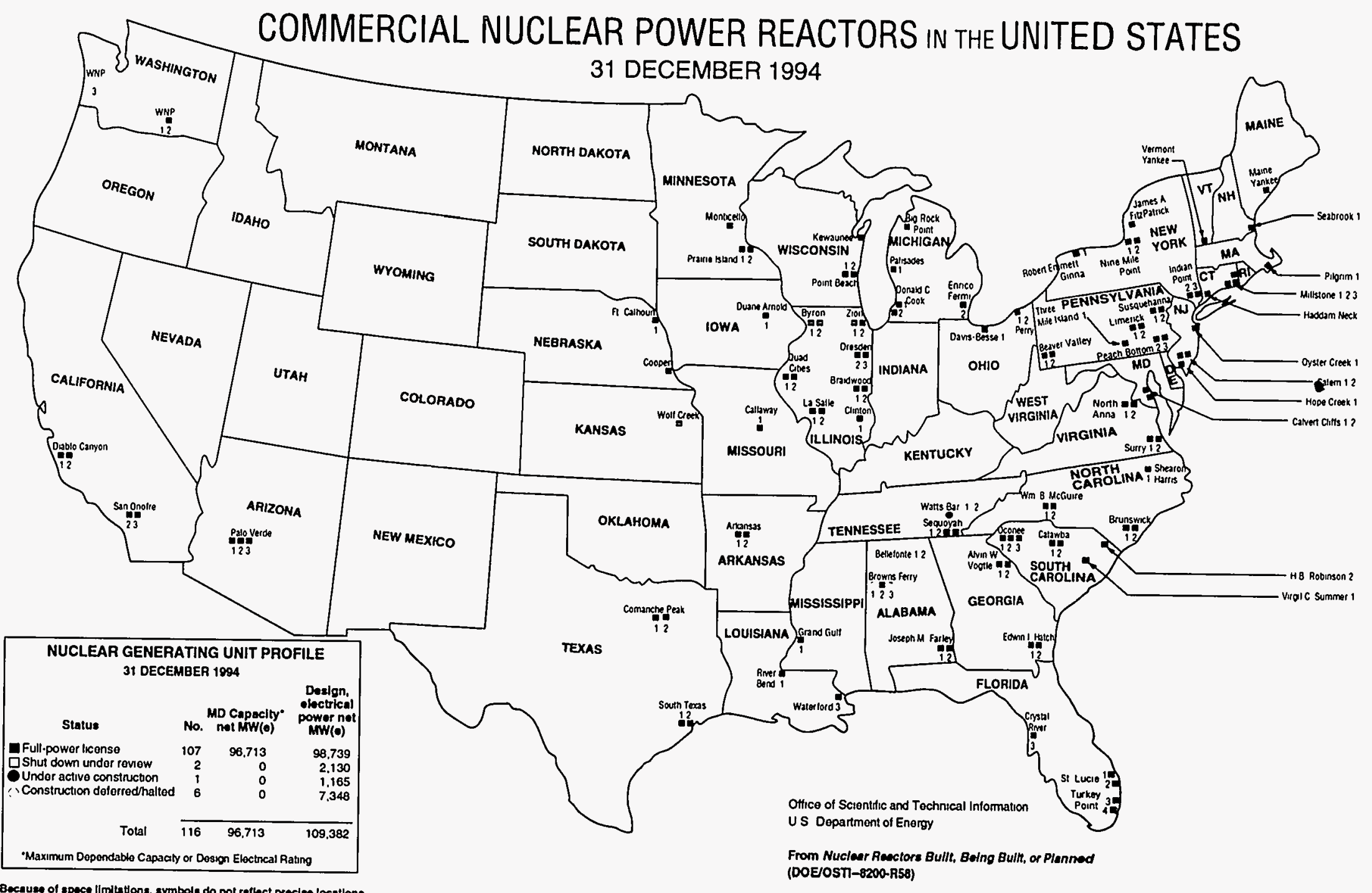

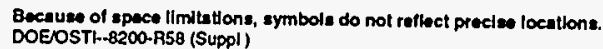

Fig. 1.1. Locations of existing and planned commercial reactors as of December 31, 1994. Courtesy of U.S. Department of Energy, Office of Scientific and Technical Information, Oak Ridge, Tennessee. 
ORNL DWG 95-8851

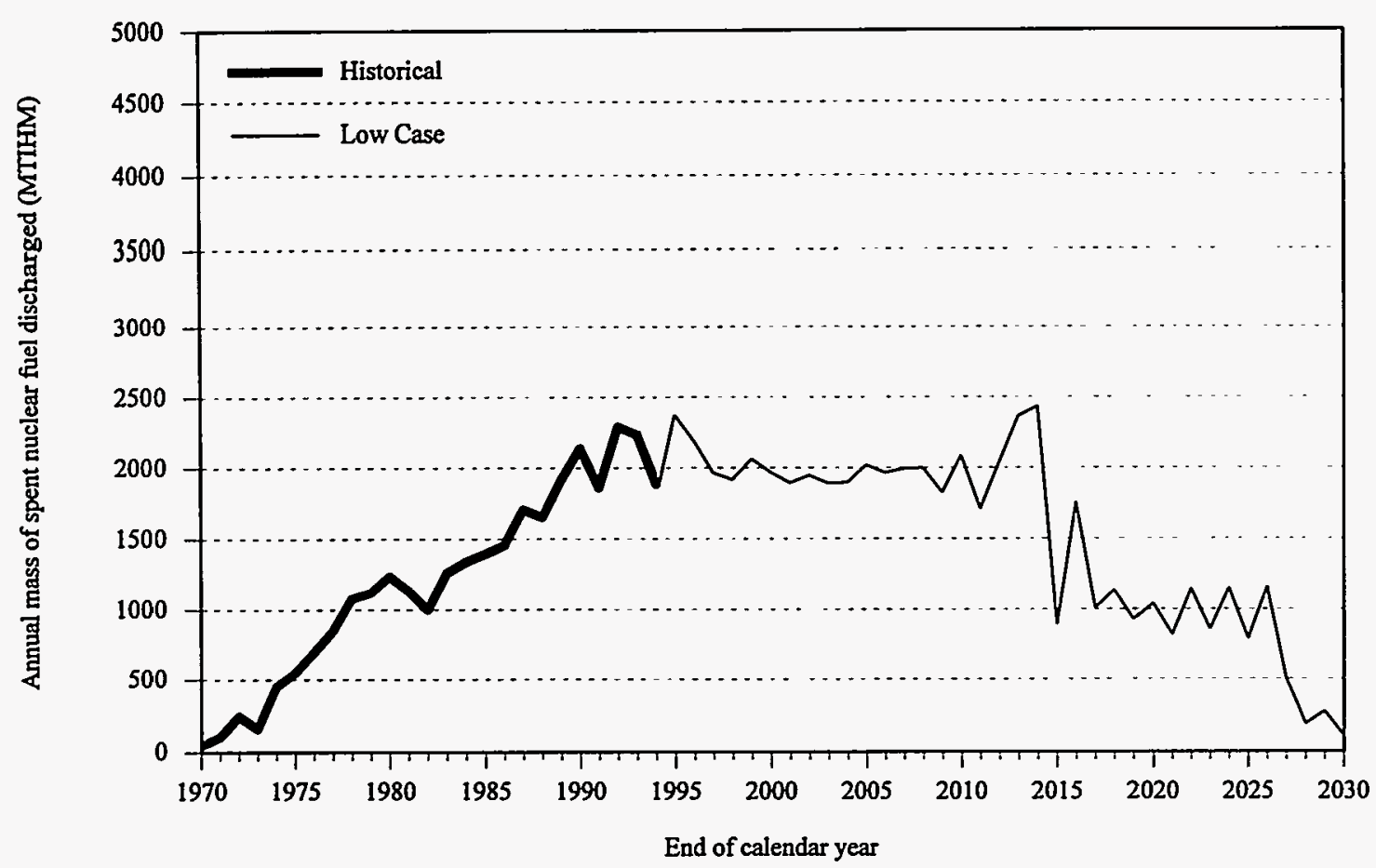

Fig. 1.2. Projected mass (MTIHM) of annual commercial spent nuclear fuel discharges for the DOE/EIA Low Case.

ORNL DWG 95-8921

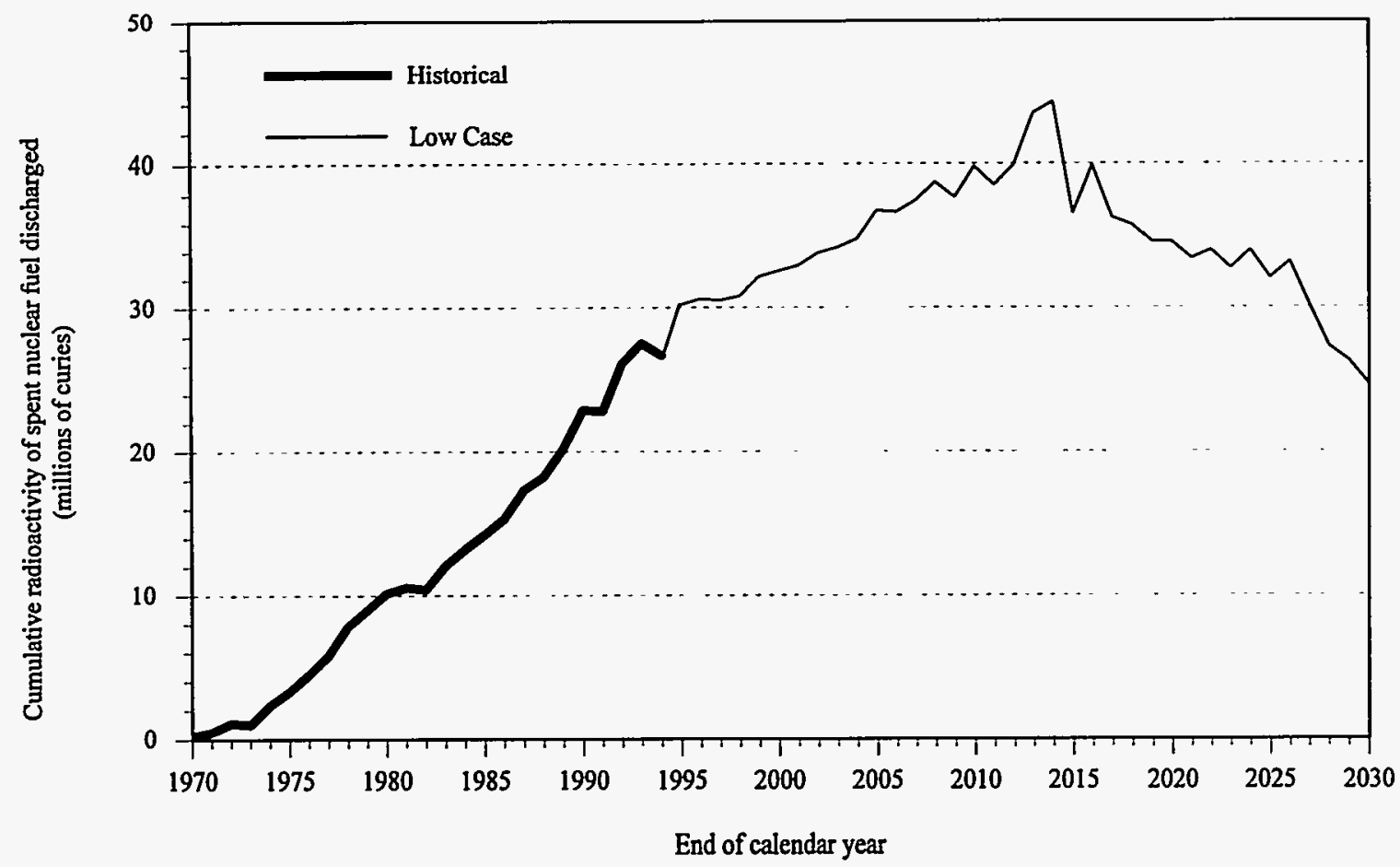

Fig. 1.3. Projected cumulative radioactivity of commercial spent nuclear fuel discharges for the DOE/EIA Low Case. 


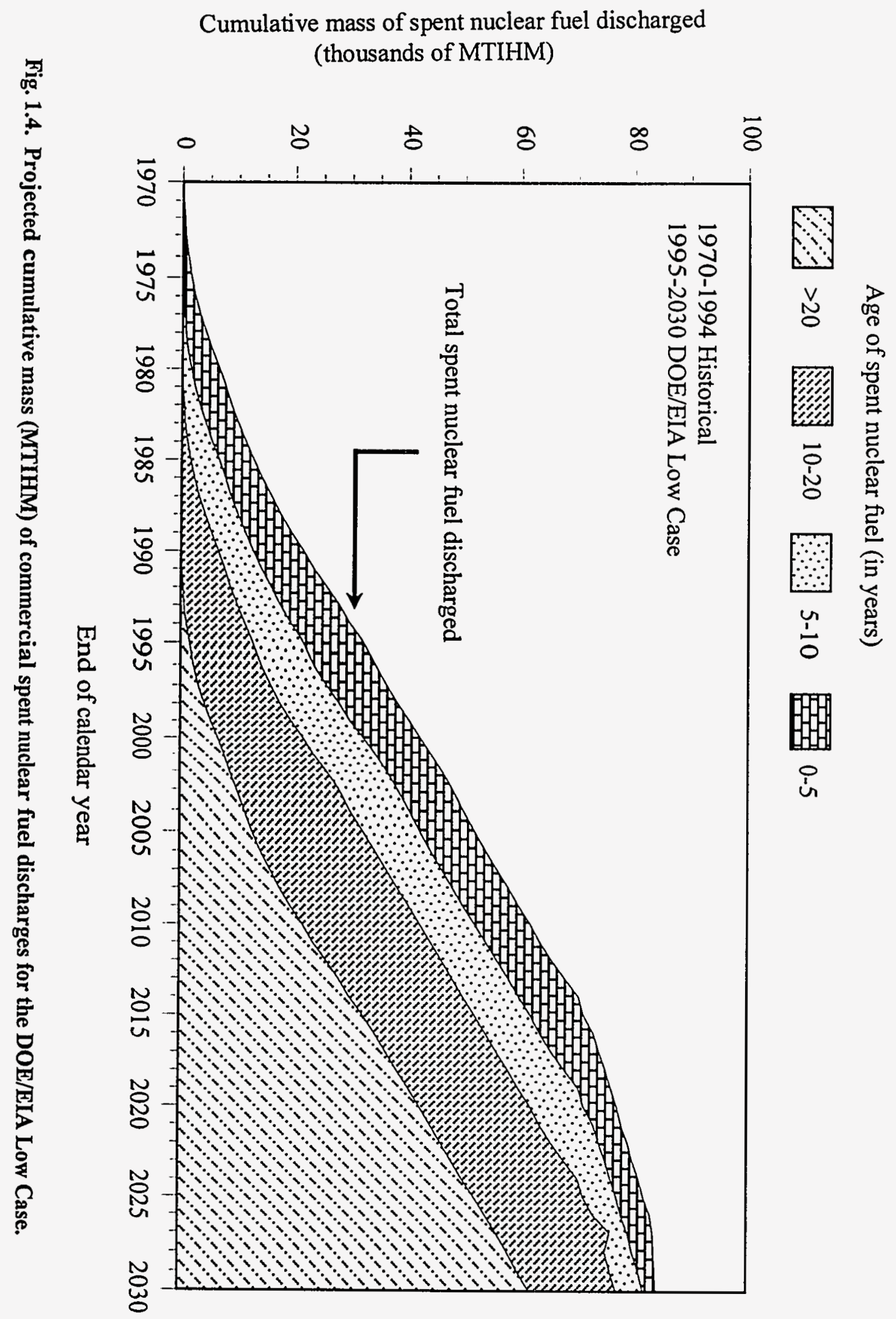

0
2
0
$\vdots$
0
0
0
0
0
$N$
$N$ 
ORNL DWG 86-332R

BOILING-WATER REACTOR SPENT NUCLEAR FUEL

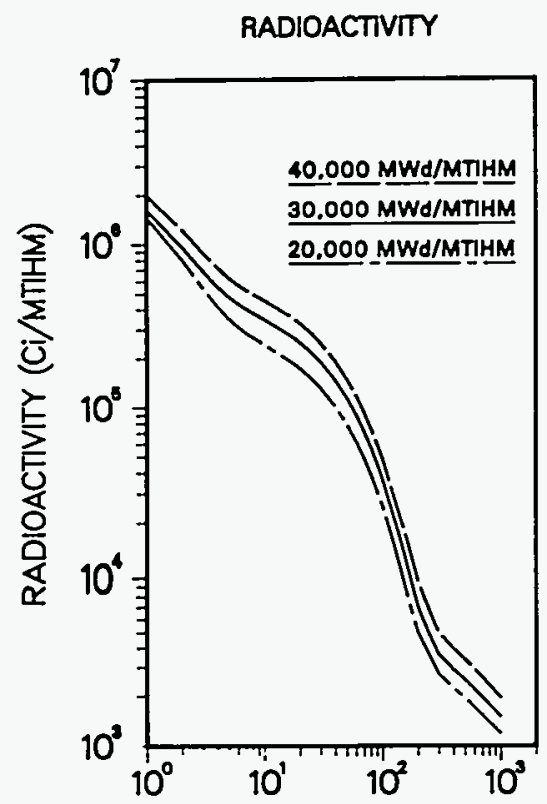

DECAY TIME AFTER DISCHARGE (yr)

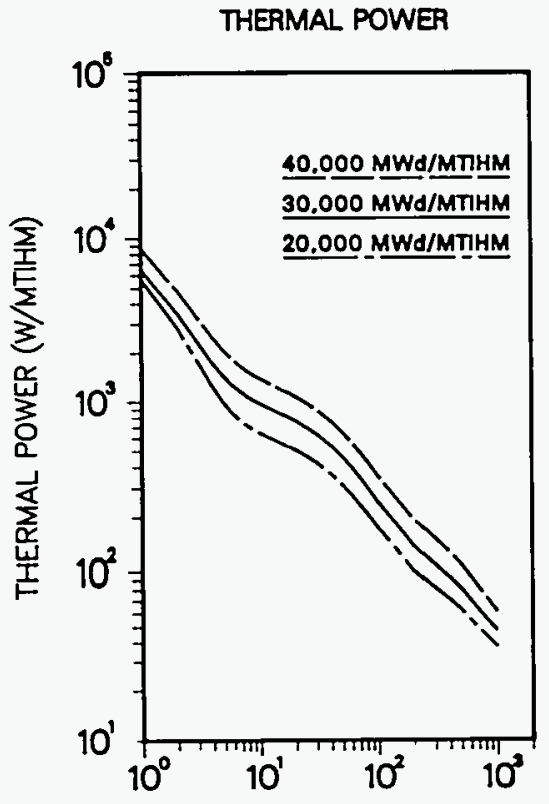

DECAY TIME AFTER DISCHARGE (yr)

\section{PRESSURIZED-WATER REACTOR SPENT NUCLEAR FUEL}
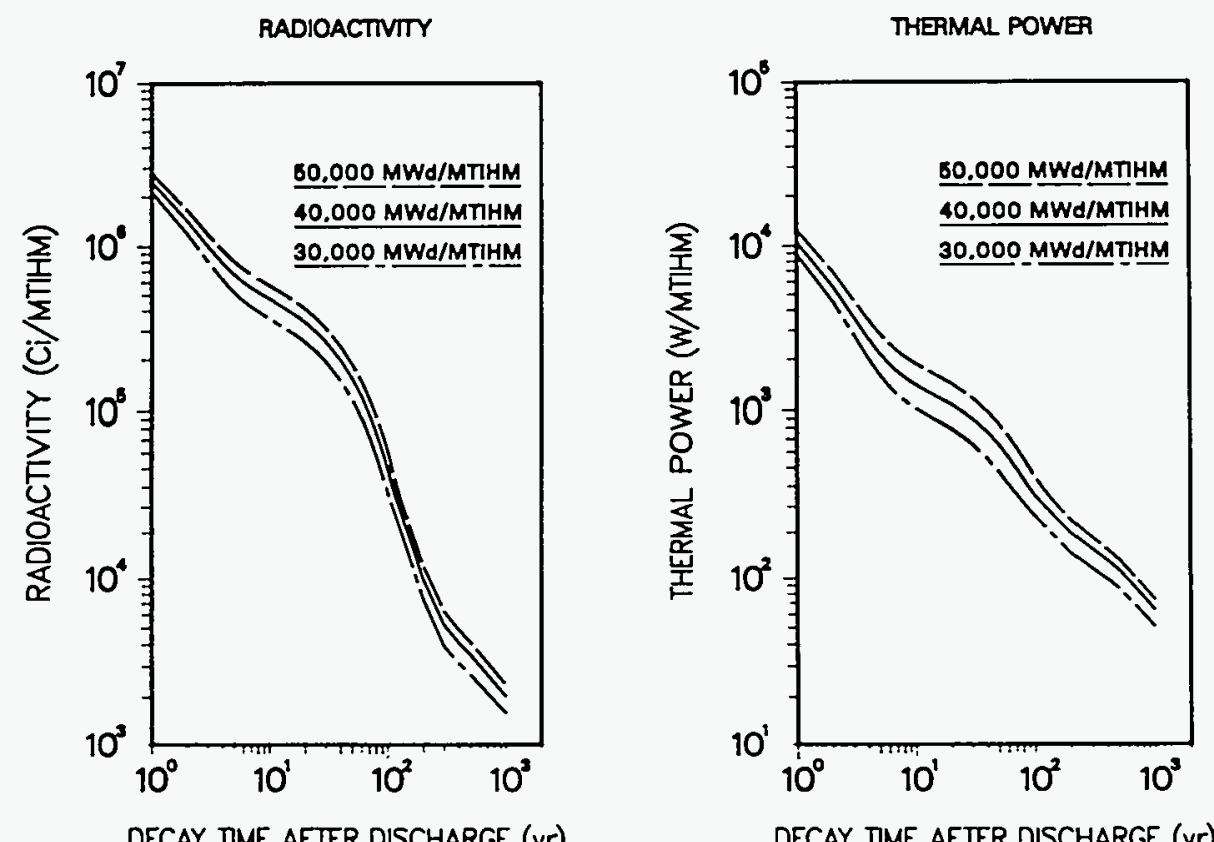

Fig. 1.5. Radioactivity and thermal power of 1 MTIHM of BWR and PWR spent nuclear fuel as a function of burnup and time from reactor discharge. 
Table 1.1. Historical and projected installed LWR electric power generating capacity for the DOE/EIA Low Case

\begin{tabular}{|c|c|c|c|c|c|c|c|}
\hline \multirow{2}{*}{$\begin{array}{l}\text { End of } \\
\text { calendar } \\
\text { year }\end{array}$} & \multicolumn{3}{|c|}{$\begin{array}{l}\text { Historical capacity }{ }^{a} \\
{[\mathrm{GW}(\mathrm{e})]}\end{array}$} & \multirow{2}{*}{$\begin{array}{l}\text { End of } \\
\text { calendar } \\
\text { year }\end{array}$} & \multicolumn{3}{|c|}{$\begin{array}{c}\text { Low Case projected capacity } \\
\text { [GW(e)] }\end{array}$} \\
\hline & BWR & PWR & Total & & BWR & PWR & Total \\
\hline 1960 & 0.1 & 0.2 & 0.3 & 1995 & 31.9 & 67.2 & 99.1 \\
\hline 1961 & 0.1 & 0.2 & 0.3 & 1996 & 31.9 & 68.4 & 100.3 \\
\hline 1962 & 0.1 & 0.2 & 0.4 & 1997 & 31.9 & 68.4 & 100.3 \\
\hline 1963 & 0.1 & 0.2 & 0.4 & 1998 & 31.9 & 68.4 & 100.3 \\
\hline 1964 & 0.1 & 0.2 & 0.4 & 1999 & 31.9 & 68.4 & 100.3 \\
\hline 1965 & 0.1 & 0.2 & 0.4 & 2000 & 31.9 & 68.4 & 100.3 \\
\hline 1966 & 0.1 & 0.2 & 0.4 & 2001 & 31.9 & 68.4 & 100.3 \\
\hline 1967 & 0.1 & 1.3 & 1.4 & 2002 & 31.9 & 68.4 & 100.3 \\
\hline 1968 & 0.2 & 1.2 & 1.4 & 2003 & 31.9 & 68.4 & 100.3 \\
\hline 1969 & 0.8 & 1.7 & 2.6 & 2004 & 31.9 & 68.4 & 100.3 \\
\hline 1970 & 2.9 & 2.9 & 5.8 & 2005 & 31.9 & 68.4 & 100.3 \\
\hline 1971 & 4.3 & 3.7 & 8.0 & 2006 & 31.1 & 68.4 & 99.5 \\
\hline 1972 & 7.0 & 6.5 & 13.5 & 2007 & 31.1 & 67.1 & 98.2 \\
\hline 1973 & 8.1 & 14.1 & 22.1 & 2008 & 31.1 & 65.1 & 96.2 \\
\hline 1974 & 13.3 & 19.4 & 32.7 & 2009 & 29.9 & 64.7 & 94.5 \\
\hline 1975 & 15.0 & 23.3 & 38.3 & 2010 & 28.7 & 62.4 & 91.1 \\
\hline 1976 & 16.8 & 27.9 & 44.7 & 2011 & 27.9 & 62.4 & 90.3 \\
\hline 1977 & 16.8 & 30.4 & 47.2 & 2012 & 25.2 & 61.0 & 86.2 \\
\hline 1978 & 17.6 & 32.2 & 49.8 & 2013 & 23.1 & 50.7 & 73.7 \\
\hline 1979 & 17.6 & 32.2 & 49.8 & 2014 & 17.4 & 45.8 & 63.2 \\
\hline 1980 & 17.6 & 34.3 & 51.9 & 2015 & 17.4 & 44.0 & 61.4 \\
\hline 1981 & 17.6 & 38.6 & 56.2 & 2016 & 15.5 & 39.6 & 55.1 \\
\hline 1982 & 18.7 & 40.5 & 59.2 & 2017 & 15.5 & 36.8 & 52.4 \\
\hline 1983 & 19.7 & 43.6 & 63.3 & 2018 & 14.8 & 35.1 & 49.8 \\
\hline 1984 & 24.2 & 45.8 & 70.0 & 2019 & 14.8 & 35.1 & 49.8 \\
\hline 1985 & 26.8 & 51.7 & 78.5 & 2020 & 14.8 & 32.0 & 46.7 \\
\hline 1986 & 28.9 & 55.2 & 84.1 & 2021 & 14.8 & 28.9 & 43.7 \\
\hline 1987 & 31.8 & 60.8 & 92.6 & 2022 & 11.5 & 28.0 & 39.6 \\
\hline 1988 & 31.8 & 63.1 & 94.9 & 2023 & 9.4 & 26.1 & 35.5 \\
\hline 1989 & 33.8 & 64.1 & 97.9 & 2024 & 7.2 & 20.4 & 27.6 \\
\hline 1990 & 32.9 & 66.7 & 99.6 & 2025 & 5.2 & 16.8 & 22.0 \\
\hline 1991 & 32.0 & 67.7 & 99.6 & 2026 & 1.1 & 11.5 & 12.5 \\
\hline 1992 & 31.8 & 67.1 & 98.9 & 2027 & 1.1 & 5.9 & 6.9 \\
\hline 1993 & 31.8 & 67.2 & 99.0 & 2028 & 1.1 & 4.6 & 5.7 \\
\hline \multirow[t]{2}{*}{1994} & 31.9 & 67.2 & 99.1 & 2029 & 0.0 & 3.5 & 3.5 \\
\hline & & & & 2030 & 0.0 & 2.3 & 2.3 \\
\hline
\end{tabular}

aBased on ref. 1 .

b Data from ref. 3 . Assumes that no new reactors will be ordered. 
Table 1.2. Projected cumulative mass of commercial spent nuclear fuel discharges for the DOE/ELA Low Case

\begin{tabular}{lccc}
\hline $\begin{array}{c}\text { End of } \\
\text { calendar } \\
\text { year }\end{array}$ & $\begin{array}{c}\text { Mass } \\
\left(10^{3} \text { MTIrMM }\right.\end{array}$ & $\begin{array}{c}\text { End of } \\
\text { calendar } \\
\text { year }\end{array}$ & $\begin{array}{c}\text { Mass } \\
\left(10^{3} \text { MTIHM }\right)\end{array}$ \\
\hline $1994^{\mathrm{a}}$ & 29.8 & 2013 & 67.9 \\
$1995^{\mathrm{b}}$ & 32.2 & 2014 & 70.4 \\
1996 & 34.4 & 2015 & 71.3 \\
1997 & 36.3 & 2016 & 73.0 \\
1998 & 38.2 & 2017 & 74.0 \\
1999 & 40.3 & 2018 & 75.2 \\
2000 & 42.3 & 2019 & 76.1 \\
2001 & 44.2 & 2020 & 77.1 \\
2002 & 46.1 & 2021 & 78.0 \\
2003 & 48.0 & 2022 & 89.1 \\
2004 & 49.9 & 2023 & 81.1 \\
2005 & 52.0 & 2024 & 81.9 \\
2006 & 53.9 & 2025 & 83.1 \\
2007 & 55.9 & 2026 & 83.6 \\
2008 & 57.9 & 2027 & 83.8 \\
2009 & 59.7 & 2028 & 84.0 \\
2010 & 61.8 & 2029 & 84.1 \\
2011 & 63.5 & 2030 & \\
2012 & 65.6 & & \\
\hline
\end{tabular}

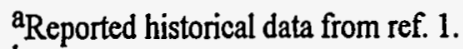

bData for years 1995-2030 from ref. 3 . 
Table 1.3. Historical and projected mass, radioactivity, and thermal power of permanently discharged spent nuclear fuel by reactor type for the DOE/EIA Low Case

\begin{tabular}{|c|c|c|c|c|c|c|}
\hline \multirow{2}{*}{$\begin{array}{l}\text { End of } \\
\text { calendar } \\
\text { year }\end{array}$} & \multicolumn{2}{|c|}{ Mass, a,b MTIHM } & \multicolumn{2}{|c|}{ Radioactivity, $10^{6} \mathrm{Ci}$} & \multicolumn{2}{|c|}{ Thermal power, $10^{6} \mathrm{~W}$} \\
\hline & Annual & Cumulative & Annual & Cumulative & Annual & Cumulative \\
\hline \multicolumn{7}{|c|}{ Boiling-water reactor } \\
\hline $1968-1970$ & & 16 & & 11 & & 0.0 \\
\hline 1971 & 65 & 81 & 193 & 199 & 0.8 & 0.8 \\
\hline 1972 & 146 & 226 & 447 & 481 & 1.7 & 1.9 \\
\hline 1973 & 94 & 320 & 344 & 439 & 1.4 & 1.7 \\
\hline 1974 & 242 & 562 & 898 & 1,033 & 3.5 & 4.0 \\
\hline 1975 & 226 & 787 & 921 & 1,217 & 3.7 & 4.7 \\
\hline 1976 & 298 & 1,085 & 1,154 & 1,584 & 4.6 & 6.1 \\
\hline 1977 & 383 & 1,469 & 1,567 & 2,131 & 6.2 & 8.2 \\
\hline 1978 & 384 & 1,852 & 1,620 & 2,414 & 6.5 & 9.3 \\
\hline 1979 & 400 & 2,252 & 1,735 & 2,730 & 7.1 & 10.5 \\
\hline 1980 & 620 & 2,872 & 2,685 & 3,889 & 10.9 & 15.1 \\
\hline 1981 & 459 & 3,331 & 2,014 & 3,664 & 8.2 & 14.0 \\
\hline 1982 & 357 & 3,688 & 1,582 & 3,362 & 6.5 & 12.6 \\
\hline 1983 & 491 & 4,179 & 2,218 & 4,016 & 9.1 & 15.1 \\
\hline 1984 & 498 & 4,677 & 2,210 & 4,283 & 9.0 & 16.0 \\
\hline 1985 & 532 & 5,209 & 2,317 & 4,590 & 9.4 & 17.0 \\
\hline 1986 & 458 & 5,667 & 1,963 & 4,424 & 8.0 & 16.1 \\
\hline 1987 & 597 & 6,264 & 2,584 & 5,088 & 10.5 & 18.7 \\
\hline 1988 & 536 & 6,799 & 2,363 & 5,134 & 9.7 & 18.7 \\
\hline 1989 & 698 & 7,497 & 3,023 & 5,953 & 12.3 & 21.8 \\
\hline 1990 & 633 & 8,130 & 2,821 & 6,076 & 11.6 & 22.2 \\
\hline 1991 & 588 & 8,718 & 2,696 & 6,173 & 11.1 & 22.5 \\
\hline 1992 & 695 & 9,413 & 3,212 & 6,881 & 13.3 & 25.3 \\
\hline 1993 & 700 & 10,113 & 3,255 & 7,285 & 13.5 & 26.8 \\
\hline 1994 & 675 & 10,788 & 3,200 & 7,542 & 13.4 & 27.8 \\
\hline 1995 & 700 & 11,500 & 3,500 & 8,100 & 14.7 & 30.0 \\
\hline 1996 & 800 & 12,300 & 3,800 & 8,800 & 16.2 & 32.8 \\
\hline 1997 & 600 & 12,900 & 2,900 & 8,300 & 12.1 & 30.4 \\
\hline 1998 & 600 & 13,500 & 2,900 & 8,300 & 12.1 & 30.3 \\
\hline 1999 & 700 & 14,200 & 3,600 & 9,200 & 15.3 & 33.8 \\
\hline 2000 & 600 & 14,900 & 3,100 & 9,100 & 13.0 & 33.1 \\
\hline 2001 & 600 & 15,400 & 2,900 & 9,100 & 12.4 & 33.0 \\
\hline 2002 & 600 & 16,100 & 3,200 & 9,500 & 13.7 & 34.7 \\
\hline 2003 & 600 & 16,700 & 2,900 & 9,500 & 12.4 & 34.4 \\
\hline 2004 & 700 & 17,300 & 3,300 & 10,000 & 13.9 & 36.4 \\
\hline 2005 & 600 & 17,900 & 2,800 & 9,800 & 11.9 & 35.4 \\
\hline 2006 & 800 & 18,700 & 3,900 & 11,000 & 16.6 & 40.3 \\
\hline 2007 & 700 & 19,400 & 3,500 & 11,000 & 14.9 & 40.4 \\
\hline 2008 & 500 & 19,900 & 2,500 & 10,300 & 10.6 & 37.0 \\
\hline 2009 & 800 & 20,700 & 4,000 & 11,700 & 16.8 & 42.6 \\
\hline 2010 & 700 & 21,400 & 3,500 & 11,700 & 14.9 & 42.5 \\
\hline 2011 & 600 & 22,000 & 2,800 & 11,200 & 11.8 & 40.1 \\
\hline 2012 & 1,000 & 23,000 & 4,800 & 13,100 & 20.1 & 48.0 \\
\hline 2013 & 600 & 23,600 & 2,800 & 11,800 & 11.6 & 42.2 \\
\hline 2014 & 1,200 & 24,800 & 5,600 & 14,400 & 23.1 & 52.7 \\
\hline 2015 & 200 & 25,000 & 1,200 & 10,900 & 5.2 & 38.1 \\
\hline 2016 & 600 & 25,600 & 3,000 & 11,900 & 12.7 & 42.2 \\
\hline 2017 & 200 & 25,900 & 1,100 & 10,200 & 4.8 & 35.1 \\
\hline 2018 & 400 & 26,300 & 2,100 & 10,600 & 8.8 & 37.1 \\
\hline 2019 & 300 & 26,500 & 1,300 & 9,800 & 5.5 & 34.0 \\
\hline 2020 & 400 & 26,900 & 1,900 & 10,300 & 8.4 & 36.0 \\
\hline
\end{tabular}


Table 1.3 (continued)

\begin{tabular}{|c|c|c|c|c|c|c|}
\hline \multirow{2}{*}{$\begin{array}{l}\text { End of } \\
\text { calendar } \\
\text { year }\end{array}$} & \multicolumn{2}{|c|}{ Mass, ${ }^{a, b}$ MTIHM } & \multicolumn{2}{|c|}{ Radioactivity, $10^{6} \mathrm{Ci}$} & \multicolumn{2}{|c|}{ Thermal power, $10^{6} \mathrm{~W}$} \\
\hline & Annual & Cumulative & Annual & Cumulative & Annual & Cumulative \\
\hline \multicolumn{7}{|c|}{ Boiling-water reactor (continued) } \\
\hline 2021 & 200 & 27,100 & 800 & 9,200 & 3.4 & 31.5 \\
\hline 2022 & 700 & 27,800 & 3,200 & 11,300 & 13.4 & 40.2 \\
\hline 2023 & 400 & 28,100 & 1,700 & 10,300 & 7.2 & 36.3 \\
\hline 2024 & 400 & 28,600 & 2,000 & 10,400 & 8.4 & 36.8 \\
\hline 2025 & 300 & 28,900 & 1,600 & 10,000 & 6.6 & 35.1 \\
\hline 2026 & 600 & 29,500 & 2,600 & 10,800 & 10.4 & 38.4 \\
\hline 2027 & 0 & 29,500 & 200 & 8,700 & 0.8 & 29.6 \\
\hline 2028 & 0 & 29,500 & 0 & 7,800 & 0.0 & 26.4 \\
\hline 2029 & 100 & 29,600 & 600 & 8,000 & 2.5 & 27.3 \\
\hline 2030 & 0 & 29,600 & 0 & 7,200 & 0.0 & 24.5 \\
\hline \multicolumn{7}{|c|}{ Pressurized-water reactor } \\
\hline 1970 & 39 & 39 & 204 & 204 & 0.8 & 0.8 \\
\hline 1971 & 44 & 83 & 247 & 296 & 1.0 & 1.2 \\
\hline 1972 & 100 & 183 & 545 & 638 & 2.2 & 2.5 \\
\hline 1973 & 67 & 250 & 374 & 571 & 1.5 & 2.2 \\
\hline 1974 & 208 & 458 & 1,097 & 1,320 & 4.4 & 5.2 \\
\hline 1975 & 322 & 780 & 1,683 & 2,098 & 6.7 & 8.2 \\
\hline 1976 & 401 & 1,181 & 2,222 & 2,894 & 8.9 & 11.3 \\
\hline 1977 & 467 & 1,648 & 2,659 & 3,676 & 10.8 & 14.5 \\
\hline 1978 & 699 & 2,346 & 4,028 & 5,426 & 16.4 & 21.5 \\
\hline 1979 & 721 & 3,068 & 4,185 & 6,253 & 17.1 & 24.7 \\
\hline 1980 & 618 & 3,686 & 3,667 & 6,248 & 15.0 & 24.5 \\
\hline 1981 & 676 & 4,362 & 4,025 & 6,887 & 16.5 & 26.9 \\
\hline 1982 & 640 & 5,002 & 3,797 & 7,037 & 15.6 & 27.2 \\
\hline 1983 & 771 & 5,773 & 4,585 & 8,072 & 18.8 & 31.1 \\
\hline 1984 & 841 & 6,614 & 4,975 & 8,939 & 20.4 & 34.4 \\
\hline 1985 & 861 & 7,475 & 5,195 & 9,639 & 21.4 & 37.0 \\
\hline 1986 & 996 & 8,472 & 5,944 & 10,884 & 24.4 & 41.7 \\
\hline 1987 & 1,109 & 9,581 & 6,657 & 12,204 & 27.4 & 46.8 \\
\hline 1988 & 1,117 & 10,697 & 6,819 & 13,073 & 28.1 & 50.1 \\
\hline 1989 & 1,215 & 11,913 & 7,353 & 14,256 & 30.3 & 54.4 \\
\hline 1990 & 1,504 & 13,417 & 9,247 & 16,833 & 38.2 & 64.7 \\
\hline 1991 & 1,271 & 14,688 & 7,889 & 16,652 & 32.7 & 63.5 \\
\hline 1992 & 1,596 & 16,284 & 10,006 & 19,255 & 41.6 & 73.8 \\
\hline 1993 & 1,532 & 17,816 & 9,813 & 20,231 & 41.0 & 77.6 \\
\hline 1994 & 1.207 & 19,024 & 7,809 & 19,120 & 32.7 & 72.5 \\
\hline 1995 & 1,600 & 20,700 & 10,600 & 22,100 & 44.5 & 84.4 \\
\hline 1996 & 1,400 & 22,100 & 9,100 & 21,800 & 38.2 & 82.8 \\
\hline 1997 & 1,400 & 23,400 & 9,000 & 22,200 & 37.7 & 83.8 \\
\hline 1998 & 1,300 & 24,800 & 8,700 & 22,400 & 36.7 & 84.5 \\
\hline 1999 & 1,300 & 26,100 & 8,800 & 23,000 & 37.1 & 86.4 \\
\hline 2000 & 1,300 & 27,400 & 8,800 & 23,600 & 37.4 & 88.6 \\
\hline 2001 & 1,300 & 28,700 & 8,600 & 23,900 & 36.4 & 89.5 \\
\hline 2002 & 1,300 & 30,000 & 8,600 & 24,400 & 36.6 & 91.3 \\
\hline 2003 & 1,300 & 31,300 & 8,600 & 24,900 & 36.4 & 92.7 \\
\hline 2004 & 1,200 & 32,600 & 8,200 & 24,900 & 34.7 & 92.6 \\
\hline 2005 & 1,500 & 34,100 & 10,100 & 27,100 & 42.8 & 101.8 \\
\hline 2006 & 1,200 & 35,200 & 7,700 & 25,800 & 32.9 & 95.8 \\
\hline 2007 & 1,300 & 36,500 & 8,500 & 26,600 & 35.9 & 98.7 \\
\hline 2008 & 1,500 & 38,000 & 10,000 & 28,500 & 42.4 & 106.6 \\
\hline
\end{tabular}


Table 1.3 (continued)

\begin{tabular}{|c|c|c|c|c|c|c|}
\hline \multirow{2}{*}{$\begin{array}{l}\text { End of } \\
\text { calendar } \\
\text { year }\end{array}$} & \multicolumn{2}{|c|}{ Mass, ${ }^{\text {a,b }}$ MTIHM } & \multicolumn{2}{|c|}{ Radioactivity, $10^{6} \mathrm{Ci}$} & \multicolumn{2}{|c|}{ Thermal power, $10^{6} \mathrm{~W}$} \\
\hline & Annual & Cumulative & Annual & Cumulative & Annual & Cumulative \\
\hline \multicolumn{7}{|c|}{ Pressurized-water reactor (continued) } \\
\hline 2009 & 1,000 & 39,000 & 6,700 & 26,100 & 28.5 & 96.3 \\
\hline 2010 & 1,400 & 40,400 & 9,100 & 28,200 & 38.5 & 104.6 \\
\hline 2011 & 1,100 & 41,500 & 7,600 & 27,400 & 32.3 & 101.3 \\
\hline 2012 & 1,000 & 42,600 & 6,900 & 26,800 & 29.2 & 98.6 \\
\hline 2013 & 1,800 & 44,300 & 11,600 & 31,500 & 48.8 & 117.7 \\
\hline 2014 & 1,300 & 45,600 & 8,300 & 29,700 & 35.0 & 110.0 \\
\hline 2015 & 600 & 46,200 & 4,400 & 25,800 & 18.5 & 93.3 \\
\hline 2016 & 1,100 & 47,400 & 7,600 & 28,000 & 32.3 & 102.5 \\
\hline 2017 & 800 & 48,200 & 5,300 & 26,200 & 22.5 & 95.0 \\
\hline 2018 & 700 & 48,900 & 4,700 & 25,300 & 20.2 & 91.1 \\
\hline 2019 & 700 & 49,600 & 4,600 & 24,800 & 19.7 & 89.4 \\
\hline 2020 & 700 & 50,200 & 4,400 & 24,500 & 18.8 & 88.0 \\
\hline 2021 & 700 & 50,900 & 4,500 & 24,400 & 19.1 & 87.7 \\
\hline 2022 & 500 & 51,300 & 3,100 & 22,900 & 13.3 & 81.7 \\
\hline 2023 & 500 & 51,800 & 3,300 & 22,500 & 13.8 & 80.3 \\
\hline 2024 & 700 & 52,500 & 4,700 & 23,700 & 19.6 & 85.1 \\
\hline 2025 & 500 & 53,000 & 3,000 & 22,100 & 12.4 & 78.8 \\
\hline 2026 & 600 & 53,600 & 3,800 & 22,400 & 15.6 & 80.2 \\
\hline 2027 & 500 & 54,100 & 3,000 & 21,500 & 12.2 & 76.6 \\
\hline 2028 & 200 & 54,300 & 1,200 & 19,400 & 5.2 & 68.2 \\
\hline 2029 & 100 & 54,400 & 900 & 18,300 & 3.9 & 64.0 \\
\hline 2030 & 100 & 54,500 & 700 & 17,400 & 2.9 & 60.8 \\
\hline \multicolumn{7}{|c|}{ Total } \\
\hline $1968-1970$ & & 55 & & 215 & & 0.8 \\
\hline 1971 & 109 & 164 & 440 & 494 & 1.7 & 1.9 \\
\hline 1972 & 246 & 410 & 991 & 1,120 & 3.9 & 4.4 \\
\hline 1973 & 161 & 570 & 718 & 1,011 & 2.9 & 3.9 \\
\hline 1974 & 449 & 1,020 & 1,995 & 2,353 & 7.9 & 9.2 \\
\hline 1975 & 548 & 1,567 & 2,604 & 3,315 & 10.3 & 12.9 \\
\hline 1976 & 699 & 2,266 & 3,376 & 4,478 & 13.5 & 17.4 \\
\hline 1977 & 850 & 3,116 & 4,226 & 5,807 & 17.0 & 22.7 \\
\hline 1978 & 1,082 & 4,199 & 5,648 & 7,841 & 22.9 & 30.8 \\
\hline 1979 & 1,121 & 5,320 & 5,920 & 8,983 & 24.1 & 35.2 \\
\hline 1980 & 1,238 & 6,558 & 6,351 & 10,137 & 26.0 & 39.6 \\
\hline 1981 & 1,135 & 7,692 & 6,039 & 10,552 & 24.7 & 40.9 \\
\hline 1982 & 998 & 8,690 & 5,378 & 10,400 & 22.0 & 39.8 \\
\hline 1983 & 1,263 & 9,952 & 6,804 & 12,088 & 27.9 & 46.3 \\
\hline 1984 & 1,339 & 11,291 & 7,185 & 13,222 & 29.4 & 50.4 \\
\hline 1985 & 1,393 & 12,684 & 7,512 & 14,228 & 30.8 & 54.0 \\
\hline 1986 & 1,454 & 14,139 & 7,907 & 15,308 & 32.4 & 57.9 \\
\hline 1987 & 1,706 & 15,844 & 9,241 & 17,292 & 37.9 & 65.5 \\
\hline 1988 & 1,652 & 17,497 & 9,182 & 18,207 & 37.8 & 68.8 \\
\hline 1989 & 1,913 & 19,410 & 10,376 & 20,209 & 42.6 & 76.3 \\
\hline 1990 & 2,137 & 21,547 & 12,068 & 22,910 & 49.8 & 86.9 \\
\hline 1991 & 1,859 & 23,406 & 10,584 & 22,825 & 43.8 & 86.0 \\
\hline 1992 & 2,291 & 25,697 & 13,218 & 26,136 & 54.9 & 99.2 \\
\hline 1993 & 2,232 & 27,929 & 13,068 & 27,516 & 54.5 & 104.4 \\
\hline 1994 & 1,882 & 29.811 & 11,009 & 26,661 & 46.1 & 100.2 \\
\hline 1995 & 2,400 & 32,200 & 14,100 & 30,200 & 59.2 & 114.5 \\
\hline 1996 & 2,200 & 34,400 & 12,900 & 30,600 & 54.4 & 115.7 \\
\hline
\end{tabular}


Table 1.3 (continued)

\begin{tabular}{|c|c|c|c|c|c|c|}
\hline \multirow{2}{*}{$\begin{array}{l}\text { End of } \\
\text { calendar } \\
\text { year }\end{array}$} & \multicolumn{2}{|c|}{ Mass, ${ }^{\mathrm{a}, \mathrm{b}}$ MTIFM } & \multicolumn{2}{|c|}{ Radioactivity, $10^{6} \mathrm{Ci}$} & \multicolumn{2}{|c|}{ Thermal power, $10^{6} \mathrm{~W}$} \\
\hline & Annual & Cumulative & Annual & Cumulative & Annual & Cumulative \\
\hline \multicolumn{7}{|c|}{ Total (continued) } \\
\hline 1997 & 2,000 & 36,300 & 11,800 & 30,500 & 49.8 & 114.2 \\
\hline 1998 & 1,900 & 38,200 & 11,600 & 30,800 & 48.8 & 114.8 \\
\hline 1999 & 2,100 & 40,300 & 12,400 & 32,200 & 52.4 & 120.3 \\
\hline 2000 & 2,000 & 42,300 & 11,900 & 32,600 & 50.3 & 121.7 \\
\hline 2001 & 1,900 & 44,200 & 11,500 & 33,000 & 48.8 & 122.5 \\
\hline 2002 & 1,900 & 46,100 & 11,900 & 33,900 & 50.4 & 126.0 \\
\hline 2003 & 1,900 & 48,000 & 11,500 & 34,300 & 48.8 & 127.2 \\
\hline 2004 & 1,900 & 49,900 & 11,500 & 34,900 & 48.6 & 129.0 \\
\hline 2005 & 2,100 & 52,000 & 12,900 & 36,900 & 54.7 & 137.2 \\
\hline 2006 & 2,000 & 53,900 & 11,600 & 36,800 & 49.5 & 136.1 \\
\hline 2007 & 2,000 & 55,900 & 12,000 & 37,600 & 50.8 & 139.1 \\
\hline 2008 & 2,000 & 57,900 & 12,500 & 38,800 & 53.1 & 143.6 \\
\hline 2009 & 1,800 & 59,700 & 10,700 & 37,800 & 45.2 & 138.9 \\
\hline 2010 & 2,100 & 61,800 & 12,600 & 39,800 & 53.4 & 147.1 \\
\hline 2011 & 1,700 & 63,500 & 10,400 & 38,600 & 44.1 & 141.4 \\
\hline 2012 & 2,000 & 65,600 & 11,700 & 39,900 & 49.3 & 146.5 \\
\hline 2013 & 2,400 & 67,900 & 14,400 & 43,300 & 60.4 & 159.9 \\
\hline 2014 & 2,400 & 70,400 & 13,900 & 44,100 & 58.1 & 162.7 \\
\hline 2015 & 900 & 71,300 & 5,600 & 36,700 & 23.8 & 131.5 \\
\hline 2016 & 1,800 & 73,000 & 10,600 & 39,900 & 45.0 & 144.7 \\
\hline 2017 & 1,000 & 74,000 & 6,400 & 36,400 & 27.3 & 130.1 \\
\hline 2018 & 1,100 & 75,200 & 6,800 & 35,900 & 29.0 & 128.2 \\
\hline 2019 & 900 & 76,100 & 5,900 & 34,700 & 25.2 & 123.4 \\
\hline 2020 & 1,000 & 77,100 & 6,400 & 34,700 & 27.2 & 123.9 \\
\hline 2021 & 800 & 78,000 & 5,300 & 33,500 & 22.5 & 119.2 \\
\hline 2022 & 1,100 & 79,100 & 6,300 & 34,100 & 26.7 & 121.9 \\
\hline 2023 & 900 & 80,000 & 5,000 & 32,800 & 21.0 & 116.7 \\
\hline 2024 & 1,200 & 81,100 & 6,700 & 34,100 & 28.0 & 122.0 \\
\hline 2025 & 800 & 81,900 & 4,600 & 32,100 & 19.0 & 114.0 \\
\hline 2026 & 1,200 & 83,100 & 6,300 & 33,300 & 26.0 & 118.7 \\
\hline 2027 & 500 & 83,600 & 3,200 & 30,200 & 13.1 & 106.2 \\
\hline 2028 & 200 & 83,700 & 1,200 & 27,300 & 5.2 & 94.7 \\
\hline 2029 & 300 & 84,000 & 1,500 & 26,300 & 6.3 & 91.3 \\
\hline 2030 & 100 & 84,100 & 700 & 24,700 & 2.9 & 85.3 \\
\hline
\end{tabular}

${ }^{a}$ Ref. 1 (1968-1994).

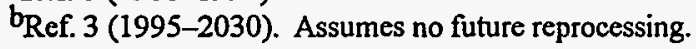


Table 1.4. Projected number of permanently discharged $L W R$ spent nuclear fuel assemblies for the DOE/ELA Low Case

\begin{tabular}{|c|c|c|c|c|c|c|}
\hline \multirow{2}{*}{$\begin{array}{l}\text { End of } \\
\text { calendar } \\
\text { year }\end{array}$} & \multicolumn{2}{|c|}{ BWR } & \multicolumn{2}{|c|}{ PWR } & \multicolumn{2}{|c|}{ Total } \\
\hline & Annual & Cumulative & Annual & Cumulative & Annual & Cumulative \\
\hline $1994^{\mathrm{a}}$ & 3,781 & 59,518 & 2,824 & 44,426 & 6,605 & 103,944 \\
\hline $1995^{b}$ & 4,100 & 63,600 & 3,800 & 48,200 & 7,900 & 111,800 \\
\hline 1996 & 4,500 & 68,100 & 3,200 & 51,400 & 7,700 & 119,500 \\
\hline 1997 & 3,400 & 71,500 & 3,100 & 54,600 & 6,500 & 126,000 \\
\hline 1998 & 3,400 & 74,800 & 3,100 & 57,700 & 6,500 & 132,500 \\
\hline 1999 & 4,200 & 79,000 & 3,000 & 60,700 & 7,200 & 139,800 \\
\hline 2000 & 3,600 & 82,600 & 3,100 & 63,900 & 6,700 & 146,500 \\
\hline 2001 & 3,400 & 86,000 & 2,900 & 66,800 & 6,300 & 152,800 \\
\hline 2002 & 3,700 & 89,700 & 3,100 & 69,900 & 6,800 & 159,600 \\
\hline 2003 & 3,300 & 93,000 & 3,000 & 72,900 & 6,300 & 165,900 \\
\hline 2004 & 3,800 & 96,800 & 2,800 & 75,700 & 6,600 & 172,500 \\
\hline 2005 & 3,200 & 100,000 & 3,500 & 79,200 & 6,800 & 179,300 \\
\hline 2006 & 4,600 & 104,700 & 2,700 & 81,900 & 7,300 & 186,600 \\
\hline 2007 & 4,000 & 108,700 & 3,000 & 84,900 & 7,000 & 193,600 \\
\hline 2008 & 2,800 & 111,600 & 3,500 & 88,400 & 6,300 & 200,000 \\
\hline 2009 & 4,700 & 116,300 & 2,300 & 90,700 & 7,000 & 207,000 \\
\hline 2010 & 4,200 & 120,400 & 3,100 & 93,800 & 7,300 & 214,300 \\
\hline 2011 & 3,400 & 123,800 & 2,600 & 96,500 & 6,000 & 220,200 \\
\hline 2012 & 5,700 & 129,500 & 2,300 & 98,800 & 8,000 & 228,300 \\
\hline 2013 & 3,300 & 132,800 & 4,200 & 102,900 & 7,400 & 235,700 \\
\hline 2014 & 6,600 & 139,400 & 2,900 & 105,800 & 9,500 & 245,200 \\
\hline 2015 & 1,400 & 140,800 & 1,500 & 107,300 & 2,900 & 248,100 \\
\hline 2016 & 3,400 & 144,200 & 2,600 & 109,900 & 6,100 & 254,200 \\
\hline 2017 & 1,300 & 145,500 & 1,800 & 111,700 & 3,100 & 257,200 \\
\hline 2018 & 2,400 & 147,900 & 1,600 & 113,300 & 4,100 & 261,300 \\
\hline 2019 & 1,400 & 149,400 & 1,500 & 114,900 & 3,000 & 264,200 \\
\hline 2020 & 2,200 & 151,600 & 1,500 & 116,400 & 3,700 & 267,900 \\
\hline 2021 & 900 & 152,500 & 1,500 & 117,900 & 2,400 & 270,300 \\
\hline 2022 & 3,900 & 156,400 & 1,000 & 118,900 & 4,900 & 275,300 \\
\hline 2023 & 2,100 & 158,400 & 1,100 & 120,000 & 3,200 & 278,400 \\
\hline 2024 & 2,400 & 160,900 & 1,700 & 121,700 & 4,200 & 282,600 \\
\hline 2025 & 1,900 & 162,700 & 1,000 & 122,700 & 2,900 & 285,500 \\
\hline 2026 & 3,100 & 165,900 & 1,400 & 124,100 & 4,500 & 290,000 \\
\hline 2027 & 200 & 166,100 & 1,000 & 125,100 & 1,200 & 291,200 \\
\hline 2028 & 0 & 166,100 & 400 & 125,500 & 400 & 291,600 \\
\hline 2029 & 800 & 166,800 & 300 & 125,800 & 1,100 & 292,700 \\
\hline 2030 & 0 & 166,800 & 300 & 126,100 & 300 & 293,000 \\
\hline
\end{tabular}

${ }^{a}$ Reported historical data (ref. 1).

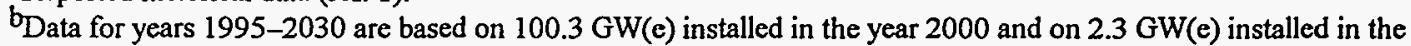
year 2030 (ref. 3). Number of projected fuel assemblies reported has been rounded to the nearest 100. 
Table 1.5. Spent nuclear fuel discharges from the Fort St. Vrain HTGR

\begin{tabular}{cccccc}
\hline \multirow{2}{*}{$\begin{array}{c}\text { End of } \\
\text { calendar } \\
\text { year }\end{array}$} & $\begin{array}{c}\text { Number of fuel assemblies } \\
\text { discharged }\end{array}$ & & \multicolumn{2}{c}{$\begin{array}{c}\text { Mass of fuel discharged } \\
\text { (MTHM) }\end{array}$} \\
\cline { 2 - 3 } \cline { 5 - 6 } & Annual & Cumulative & & Annual & Cumulative \\
\hline 1979 & $246^{\mathrm{b}}$ & 246 & & 2.80 & 2.80 \\
1980 & 0 & 246 & & 0.00 & 2.80 \\
1981 & 240 & 486 & & 2.77 & 5.57 \\
1982 & 0 & 486 & & 0.00 & 5.57 \\
1983 & 0 & 486 & & 0.00 & 5.57 \\
1984 & 240 & 726 & & 2.85 & 8.42 \\
1985 & 0 & 726 & & 0.00 & 8.42 \\
1986 & 0 & 726 & & 0.00 & 8.42 \\
1987 & 0 & 726 & & 0.00 & 8.42 \\
1988 & 0 & $726^{\mathrm{c}}$ & & 0.00 & 8.42 \\
$1989^{\mathrm{d}, \mathrm{e}}$ & 126 & 852 & & 1.32 & 9.74 \\
$1990^{\mathrm{d}}$ & 332 & 1,184 & 3.49 & 13.23 \\
$1991^{\mathrm{f}}$ & 42 & 1,226 & & 0.48 & 13.71 \\
$1992^{\mathrm{g}}$ & 982 & 2,208 & 10.29 & 24.00 \\
1993 & 0 & 2,208 & 0.00 & 24.00 \\
1994 & 0 & 2,208 & 0.00 & 24.00 \\
$1995-1998^{\mathrm{h}}$ & 0 & 2,208 & 0.00 & 24.00 \\
\hline
\end{tabular}

a Data obtained from ref. 7. Discharges identified in this table are those made directly from the reactor.

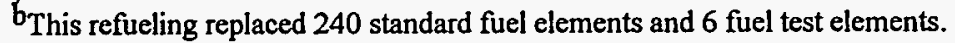

${ }^{c}$ All spent nuclear fuel discharged prior to December 31,1988 , is located at the ICPP.

dFuel removed from the reactor in 1989 and 1990 was temporarily stored in on-site storage wells.

'Power operations effectively ceased on August 18, 1989.

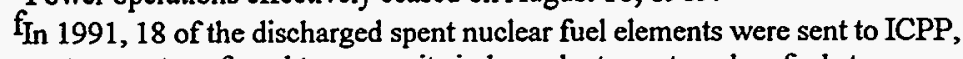
18 elements were transferred to an on-site independent spent nuclear fuel storage installation (ISFSI), and 6 elements were temporarily stored in on-site storage wells.

gAll spent nuclear fuel elements have been discharged from the reactor and transferred to the ISFSI. All spent nuclear fuel elements in temporary on-site storage wells have been relocated to the ISFSI.

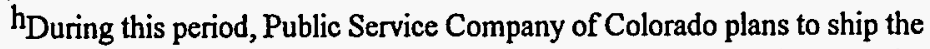
1,464 elements currently in the ISFSI to ICPP once legal issues have been resolved. 
Table 1.6. IDB reference characteristics of $L W R$ nuclear fuel assemblies

\begin{tabular}{|c|c|c|}
\hline Characteristics & $\mathrm{BWR}^{\mathrm{a}}$ & $\mathrm{PWR}^{\mathrm{b}}$ \\
\hline Overall assembly length, $\mathrm{m}$ & 4.470 & 4.059 \\
\hline Cross section, $\mathrm{cm}$ & $13.9 \times 13.9$ & $21.4 \times 21.4$ \\
\hline Fuel rod length, m & 4.064 & 3.851 \\
\hline Active fuel height, $m$ & 3.759 & 3.658 \\
\hline Fuel rod outer diameter, $\mathrm{cm}$ & 1.252 & 0.950 \\
\hline Fuel rod array & $8 \times 8$ & $17 \times 17$ \\
\hline Fuel rods per assembly & 63 & 264 \\
\hline Assembly total weight, $\mathrm{kg}$ & 319.9 & 657.9 \\
\hline Uranium/assembly, $\mathrm{kg}$ & 183.3 & 461.4 \\
\hline $\mathrm{UO}_{2} /$ assembly, $\mathrm{kg}$ & 208.0 & 523.4 \\
\hline Zircaloy/assembly, kg & $103.3^{\mathrm{c}}$ & $108.4^{\mathrm{d}}$ \\
\hline Hardware/assembly, kg & $8.6^{\mathrm{e}}$ & $26.1^{\mathrm{f}}$ \\
\hline Total metal/assembly, kg & 111.9 & 134.5 \\
\hline Nominal volume/assembly, $\mathrm{m}^{3}$ & $0.0864 \mathrm{~g}$ & $0.186 \mathrm{~g}$ \\
\hline
\end{tabular}

${ }^{\mathrm{a}}$ Ref. 8.

${ }^{\text {Ref. } 9 .}$

CIncludes Zircaloy fuel-rod spacers and fuel channel.

Includes Zircaloy control-rod guide thimbles.

Tncludes stainless steel tic-plates, Inconel springs, and plenum springs.

Includes stainless steel nozzles and Inconel-718 grids.

BBased on overall outside dimension. Includes spacing between the stacked fuel rods of an assembly. 
Table 1.7. Historical mass of commercial BWR spent nuclear fuel discharged at various ranges of burnup ${ }^{\mathrm{a}, \mathrm{b}}$

\begin{tabular}{|c|c|c|c|c|c|c|c|c|c|c|c|}
\hline \multirow{2}{*}{$\begin{array}{l}\text { End of } \\
\text { calendar } \\
\text { year }\end{array}$} & \multicolumn{10}{|c|}{ Annual mass of discharged spent nuclear fuel for various burnup ranges, MTIHM } & \multirow{2}{*}{$\begin{array}{c}\text { Total annual } \\
\text { mass over all } \\
\text { burnup ranges } \\
\text { (MTIHMD) }\end{array}$} \\
\hline & $\begin{array}{c}0- \\
4,999^{c}\end{array}$ & $\begin{array}{l}5,000- \\
9,999\end{array}$ & $\begin{array}{l}10,000 \\
14,999\end{array}$ & $\begin{array}{l}15,000- \\
19,999\end{array}$ & $\begin{array}{l}20,000- \\
24,999\end{array}$ & $\begin{array}{l}25,000 \\
29,999\end{array}$ & $\begin{array}{l}30,000- \\
34,999\end{array}$ & $\begin{array}{l}35,000- \\
39,999\end{array}$ & $\begin{array}{l}40,000 \\
44,999\end{array}$ & $\begin{array}{l}45,000- \\
49,999\end{array}$ & \\
\hline 1968 & 0.6 & & & & & & & & & & 0.6 \\
\hline 1969 & & 1.2 & 1.0 & 7.3 & 0.2 & 0.1 & & & & & 9.8 \\
\hline 1970 & 5.6 & & & & & & & & & & 5.6 \\
\hline 1971 & 41.7 & 8.6 & 2.8 & 10.0 & 1.6 & & & & & & 64.7 \\
\hline 1972 & 98.1 & 11.9 & 31.1 & 4.2 & 0.5 & & & & & & 145.8 \\
\hline 1973 & 9.7 & 17.2 & 28.8 & 36.7 & 1.0 & 0.1 & & & & & 93.5 \\
\hline 1974 & & 78.6 & 114.4 & 44.8 & 3.8 & & & & & & 241.6 \\
\hline 1975 & 0.3 & 1.7 & 62.2 & 136.4 & 25.3 & & & & & & 225.8 \\
\hline 1976 & 0.9 & 67.1 & 108.7 & 119.1 & 2.3 & & & & & & 298.1 \\
\hline 1977 & & 48.0 & 40.3 & 235.2 & 58.9 & 0.7 & & & & & 383.2 \\
\hline 1978 & 6.3 & 32.4 & 13.1 & 84.2 & 232.4 & 15.2 & & & & & 383.6 \\
\hline 1979 & & & 18.6 & 108.7 & 149.2 & 123.0 & 0.3 & & & & 399.8 \\
\hline 1980 & 14.0 & 0.4 & 0.6 & 93.3 & 413.3 & 87.6 & 10.7 & & & & 619.8 \\
\hline 1981 & & 0.2 & 0.2 & 58.1 & 265.4 & 133.3 & 0.7 & 0.7 & & & 458.7 \\
\hline 1982 & & 0.2 & 4.6 & 22.6 & 141.5 & 173.6 & 13.8 & 0.6 & 0.4 & & 357.2 \\
\hline 1983 & & & 0.9 & 2.9 & 113.5 & 337.8 & 35.7 & 0.4 & & & 491.3 \\
\hline 1984 & & 7.9 & 43.0 & 0.3 & 136.0 & 239.5 & 70.8 & & 0.4 & & 497.9 \\
\hline 1985 & 16.9 & 42.5 & 18.5 & 39.5 & 106.3 & 297.4 & 10.2 & & 0.2 & & 531.6 \\
\hline 1986 & 50.8 & 32.4 & 42.5 & 66.6 & 43.1 & 180.8 & 41.7 & 0.4 & & & 458.3 \\
\hline 1987 & 31.0 & 36.1 & 68.8 & 40.8 & 24.7 & 352.5 & 42.9 & & 0.4 & & 597.0 \\
\hline 1988 & 17.0 & 24.5 & 1.8 & 42.9 & 168.3 & 192.4 & 88.7 & & & & 535.6 \\
\hline 1989 & 30.9 & 16.9 & 68.4 & 71.8 & 193.2 & 227.7 & 85.5 & 3.6 & & & 698.0 \\
\hline 1990 & 17.0 & & 34.0 & 67.6 & 106.2 & 247.5 & 158.9 & 1.6 & & & 632.8 \\
\hline 1991 & 17.8 & 24.6 & & 7.2 & 23.9 & 215.1 & 287.2 & 12.1 & & & 588.0 \\
\hline 1992 & & & 7.6 & 75.7 & 62.5 & 83.9 & 361.8 & 103.4 & & & 694.8 \\
\hline 1993 & & & & 31.0 & 47.4 & 144.8 & 353.8 & 122.7 & & & 699.7 \\
\hline 1994 & & & 0.8 & 1.1 & 19.0 & 113.5 & 275.4 & 256.8 & 7.6 & 0.7 & 675.0 \\
\hline
\end{tabular}

aBased on ref. 1 .

boes not include commercial spent nuclear fuel reprocessed at WVDP.

${ }^{\mathrm{C}}$ Burnup range is given in units of MWd/MTHM. 
Table 1.8. Historical mass of commercial PWR spent nuclear fuel discharged at various ranges of burnup ${ }^{\mathrm{a}, \mathrm{b}}$

\begin{tabular}{|c|c|c|c|c|c|c|c|c|c|c|c|c|c|}
\hline \multirow{2}{*}{$\begin{array}{l}\text { End of } \\
\text { calendar } \\
\text { year }\end{array}$} & \multicolumn{12}{|c|}{ Annual mass of discharged spent nuclear fuel for various burnup ranges, MTIFM } & \multirow{2}{*}{$\begin{array}{c}\text { Total annual } \\
\text { mass over all } \\
\text { burnup ranges } \\
\text { (MTIHM) }\end{array}$} \\
\hline & $\begin{array}{c}0- \\
4,999^{c}\end{array}$ & $\begin{array}{l}5,000 \\
9,999\end{array}$ & $\begin{array}{l}10,000- \\
14,999\end{array}$ & $\begin{array}{l}15,000 \\
19,999\end{array}$ & $\begin{array}{l}20,000 \\
24,999\end{array}$ & $\begin{array}{l}25,000- \\
29,999\end{array}$ & $\begin{array}{l}30,000- \\
34,999\end{array}$ & $\begin{array}{l}35,000- \\
39,999\end{array}$ & $\begin{array}{l}40,000- \\
44,999\end{array}$ & $\begin{array}{l}45,000- \\
49,999\end{array}$ & $\begin{array}{l}50,000 \\
54,999\end{array}$ & $\begin{array}{l}55,000 \\
59,999\end{array}$ & \\
\hline 1970 & & & 1.7 & 37.3 & & & & & & & & & 39.0 \\
\hline 1971 & & 4.6 & & & 6.2 & 33.7 & & & & & & & 44.5 \\
\hline 1972 & & & 11.9 & 29.3 & 27.8 & 8.9 & 22.1 & & & & & & 99.9 \\
\hline 1973 & & & & 26.1 & & 33.3 & 7.6 & & & & & & 67.1 \\
\hline 1974 & 7.4 & 1.5 & 86.4 & 13.6 & 40.5 & 57.2 & 1.1 & & & & & & 207.7 \\
\hline 1975 & 2.7 & 42.6 & 95.0 & 53.6 & 79.3 & 25.3 & 23.1 & & & & & & 321.8 \\
\hline 1976 & & & 5.6 & 194.2 & 82.4 & 63.3 & 55.4 & & & & & & 401.0 \\
\hline 1977 & & & 2.8 & 108.3 & 113.0 & 140.3 & 87.1 & 15.4 & & & & & 466.9 \\
\hline 1978 & & 0.9 & 47.9 & 89.8 & 45.1 & 329.0 & 125.0 & 60.4 & 0.4 & & & & 698.6 \\
\hline 1979 & & & 30.6 & 109.4 & 64.0 & 227.6 & 239.1 & 50.1 & 0.5 & & & & 721.2 \\
\hline 1980 & & & 0.4 & & 66.8 & 246.1 & 276.4 & 26.3 & 2.0 & & & & 618.1 \\
\hline 1981 & & & 17.2 & 1.9 & 25.8 & 228.5 & 351.6 & 49.6 & 1.3 & & & & 675.9 \\
\hline 1982 & & & 1.8 & 81.2 & 80.4 & 61.4 & 290.6 & 119.7 & 2.7 & 0.4 & 1.3 & 0.9 & 640.4 \\
\hline 1983 & & 5.5 & 3.1 & 80.6 & 44.7 & 166.7 & 327.6 & 137.3 & 5.4 & & 0.5 & & 771.3 \\
\hline 1984 & & & 58.0 & 45.2 & 56.3 & 195.3 & 365.0 & 117.4 & 4.1 & & & & 841.3 \\
\hline 1985 & & & & 49.0 & 13.6 & 218.7 & 317.8 & 237.5 & 24.1 & 0.4 & & & 861.0 \\
\hline 1986 & & 0.8 & 27.6 & 131.1 & 15.6 & 181.2 & 336.3 & 265.6 & 35.5 & 1.3 & 1.3 & & 996.2 \\
\hline 1987 & & & 27.2 & 77.7 & 53.4 & 175.3 & 411.9 & 311.7 & 51.8 & & & & $1,108.9$ \\
\hline 1988 & & & & 79.7 & 14.7 & 138.4 & 345.1 & 428.9 & 103.1 & 4.6 & 0.4 & 2.0 & $1,116.9$ \\
\hline 1989 & & & 47.1 & 91.4 & 68.6 & 112.1 & 283.0 & 408.4 & 189.1 & 15.2 & & 0.4 & $1,215.3$ \\
\hline 1990 & & & 22.3 & 83.4 & 16.2 & 121.2 & 393.8 & 611.0 & 249.2 & 7.0 & 0.3 & & $1,504.3$ \\
\hline 1991 & & 9.2 & 53.2 & 1.4 & 79.0 & 52.5 & 148.8 & 606.2 & 253.1 & 64.2 & 3.4 & & $1,271.0$ \\
\hline 1992 & & 19.8 & 14.8 & 43.7 & 12.3 & 115.5 & 287.7 & 463.9 & 504.9 & 118.9 & 14.8 & & $1,596.4$ \\
\hline 1993 & & & 0.5 & 2.3 & 22.4 & 67.4 & 167.3 & 444.6 & 627.4 & 197.8 & 2.1 & & $1,531.9$ \\
\hline 1994 & 3.2 & & 27.8 & 9.8 & 6.5 & 7.5 & 107.5 & 292.9 & 506.1 & 225.5 & 19.4 & 1.3 & $1,207.5$ \\
\hline
\end{tabular}

a Based on ref. 1.

${ }^{b}$ Does not include commercial spent nuclear fuel reprocessed at WVDP.

${ }^{\text {Burnup range is given in units of MWd/MTIHM. }}$ 
Table 1.9. Summary inventory of DOE spent nuclear fuel for years 1995 and $2035^{a}$

\begin{tabular}{|c|c|c|c|c|c|}
\hline \multirow{2}{*}{ Site } & \multirow{2}{*}{ Facilityb } & \multirow{2}{*}{$\begin{array}{c}1995 \\
\text { total mass } \\
(\mathrm{kg})\end{array}$} & \multirow{2}{*}{$\begin{array}{c}1995 \\
\text { volume } \\
\left(\mathrm{m}^{3}\right)\end{array}$} & \multicolumn{2}{|c|}{$\begin{array}{c}\text { Discharged heavy metal } \\
\text { (MTHM) }\end{array}$} \\
\hline & & & & 1995 & 2035 \\
\hline \multirow[t]{3}{*}{ ANL-E } & Alpha-gamma hot cell & 257.10 & 0.10 & 0.08 & 0.08 \\
\hline & $\mathrm{CP}-5$ & 2.00 & 0.02 & 0.00 & 0.00 \\
\hline & Subtotal & 259.10 & 0.12 & 0.08 & 0.08 \\
\hline \multirow[t]{6}{*}{ ANLLW } & EBR-II & $90,000.00$ & 100.00 & 14.31 & 14.31 \\
\hline & HFEF & $4,999.99$ & 5.00 & 2.04 & 2.04 \\
\hline & NRAD & 193.80 & 0.04 & 0.01 & 0.01 \\
\hline & RSWF & $15,000.00$ & 32.99 & 11.91 & 11.91 \\
\hline & TREAT & 15.00 & 11.00 & 0.02 & 0.02 \\
\hline & Subtotal & $110,208.79$ & 149.03 & 28.29 & 28.29 \\
\hline \multirow[t]{3}{*}{ BNL } & BMRR & 160.99 & 0.13 & 0.00 & 0.01 \\
\hline & HFBR & $4,136.61$ & 3.41 & 0.25 & 1.70 \\
\hline & Subtotal & $4,297.60$ & 3.54 & 0.25 & 1.71 \\
\hline \multirow[t]{4}{*}{ FRR } & FRR-East & - & - & - & 13.24 \\
\hline & FRR-West & - & - & - & 4.76 \\
\hline & & - & - & - & - \\
\hline & Subtotal & - & - & - & 18.00 \\
\hline \multirow[t]{9}{*}{ Hanford } & Area 200 & 900.07 & 21.77 & 0.33 & 0.33 \\
\hline & Bldg. 308 & 224.52 & 0.08 & 0.02 & 0.02 \\
\hline & Bldgs. $324,325,327$ & $3,743.00$ & 1.30 & 2.30 & 2.30 \\
\hline & FFTF & $55,950.00$ & 16.51 & 11.01 & 11.01 \\
\hline & PUREX & $3,215.00$ & 0.23 & 3.14 & 3.14 \\
\hline & T-plant & $38,500.00$ & 9.45 & 15.82 & 15.82 \\
\hline & 105-K East Basin & $1,235,172.59$ & 112.05 & $1,146.34$ & $1,146.34$ \\
\hline & 105-K West Basin & $1,028,048.90$ & 93.22 & 953.89 & 953.89 \\
\hline & Subtotal & $2,365,754.08$ & 254.61 & $2,132.85$ & $2,132.85$ \\
\hline \multirow[t]{11}{*}{ INEL } & ICPP-603 & $49,590.32$ & 18.61 & 2.66 & 2.66 \\
\hline & ICPP-666 & $358,530.42$ & 114.18 & 12.36 & 12.36 \\
\hline & ICPP-749 & $210,950.00$ & 87.36 & 78.37 & 78.37 \\
\hline & ICPP-IFSF & $130,906.35$ & 85.81 & 10.03 & 10.03 \\
\hline & MTR canal & $4,204.00$ & 3.00 & 0.26 & 0.26 \\
\hline & PER-620 & $5,577.50$ & 0.84 & 0.56 & 0.56 \\
\hline & TAN-607 & $331,338.07$ & 130.94 & 85.27 & 85.27 \\
\hline & TAN-790 & $56,375.01$ & 11.78 & 38.37 & 38.37 \\
\hline & TRA-660 & 598.58 & 0.48 & 0.23 & 0.23 \\
\hline & TRA-670 & $7,197.96$ & 7.38 & 0.76 & 2.70 \\
\hline & Subtotal & $1,155,268.21$ & 460.38 & 228.87 & 230.81 \\
\hline INEL/NRF & ECF & $192,784.69$ & 44.34 & 3.06 & 3.06 \\
\hline LANL & Omega West Reactor & 507.40 & 0.60 & 0.01 & 0.01 \\
\hline \multirow[t]{2}{*}{ ORNL } & Bldg. 3525 & 1.00 & 0.10 & 0.00 & 0.00 \\
\hline & Bldg. $7823 \mathrm{~A}$ & 175.00 & 1.20 & 0.00 & 0.00 \\
\hline
\end{tabular}


Table 1.9 (continued)

\begin{tabular}{|c|c|c|c|c|c|}
\hline \multirow{2}{*}{ Site } & \multirow{2}{*}{ Facility ${ }^{b}$} & \multirow{2}{*}{$\begin{array}{c}1995 \\
\text { total mass } \\
(\mathrm{kg})\end{array}$} & \multirow{2}{*}{$\begin{array}{c}1995 \\
\text { volume } \\
\left(\mathrm{m}^{3}\right)\end{array}$} & \multicolumn{2}{|c|}{$\begin{array}{c}\text { Discharged heavy metal } \\
\text { (MTHM) }\end{array}$} \\
\hline & & & & 1995 & 2035 \\
\hline \multirow{9}{*}{$\begin{array}{l}\text { ORNL } \\
\text { (contd.) }\end{array}$} & BIdg. 7827 & $2,009.00$ & 3.68 & 0.16 & 0.16 \\
\hline & Bldg. 7829 & 552.00 & 1.15 & 0.02 & 0.02 \\
\hline & Bldg. 7920 & 996.00 & 0.10 & 0.00 & 0.00 \\
\hline & BSR & 327.22 & 0.39 & 0.06 & 0.06 \\
\hline & FIFIR & $8,673.80$ & 7.37 & 0.63 & 1.64 \\
\hline & MSR & $8,940.00$ & 3.86 & 0.04 & 0.04 \\
\hline & SWSA6 & 132.00 & 0.01 & 0.02 & 0.02 \\
\hline & TSR & 182.00 & 0.10 & 0.01 & 0.01 \\
\hline & Subtotal & $21,988.02$ & 17.96 & 0.94 & 1.95 \\
\hline \multirow[t]{6}{*}{ SNLNM } & ACRR & 151.00 & 1.10 & 0.04 & 0.04 \\
\hline & $\mathrm{HCF}$ & 20.00 & 0.01 & 0.01 & 0.01 \\
\hline & Manzano storage & 50.00 & 0.10 & 0.03 & 0.03 \\
\hline & SNM storage & 970.00 & 2.00 & 0.03 & 0.03 \\
\hline & SPR & $1,140.00$ & 1.90 & 0.26 & 0.26 \\
\hline & Subtotal & $2,331.00$ & 5.11 & 0.37 & 0.37 \\
\hline \multirow[t]{9}{*}{ SRS } & F-Canyon & $23,268.24$ & 5.20 & 22.61 & 22.61 \\
\hline & H-Canyon & 430.00 & 0.42 & 0.07 & 0.07 \\
\hline & K-Basin & $20,400.00$ & 29.14 & 3.24 & 3.24 \\
\hline & $\mathrm{K} / \mathrm{L} / \mathrm{P}-\mathrm{Basin}$ & $2,478.40$ & 3.36 & 0.06 & 0.06 \\
\hline & L-Basin & $135,986.46$ & 43.22 & 117.96 & 117.96 \\
\hline & P-Basin & $10,700.00$ & 14.51 & 1.39 & 1.39 \\
\hline & RBOF & $358,119.75$ & 29.00 & 60.84 & 60.84 \\
\hline & SRTC & 5.28 & 0.01 & 0.00 & 0.00 \\
\hline & Subtotal & $551,388.13$ & 124.86 & 206.17 & 206.17 \\
\hline U.S. Navy & U.S. Navy Shipyards & - & - & - & 54.48 \\
\hline \multirow[t]{5}{*}{ Other } & Special-case commercial- & & & & \\
\hline & B\&W, FSVR, WV FRS & $233,148.04$ & 144.56 & 40.65 & 40.76 \\
\hline & University & $10,129.00$ & 8.21 & 1.81 & 4.18 \\
\hline & Subtotal & $243,277.04$ & 152.77 & 42.46 & 44.94 \\
\hline & Grand total & $4,648,064.06$ & $1,213.32$ & $2,643.35$ & $2,722.72$ \\
\hline
\end{tabular}

a Based on ref. 12.

b Abbreviations used in this table: ACRR-Annular Core Research Reactor, BMRR-Brookhaven Medical Research Reactor, BSR-Bulk Shielding Reactor, B\&W-Babcock and Wilcox, CP-5-Chicago Pile-5, EBR-Experimental Breeder Reactor, ECF_Extended Core Facility, FFTF_Fast Flux Test Facility, FRR-Foreign Research Reactor, FSVR - Fort St. Vrain Reactor, HCF-Hot Cell Facility, HFBR - (Brookhaven) High Flux Beam (Research) Reactor, HFEF-Hot Fuel Examination Facility, HFIR_-High Flux Isotope Reactor, ICPP_Idaho Chemical Processing Plant, IFSF-Irradiated Fuel Storage Facility, MSR-Molten Salt Reactor, MTR-Materials Test Reactor, NRAD-Neutron Radiography (Reactor), PER-(Special) Power Excursion Reactor (Test), PUREX - plutonium/uranium extraction, RBOF - Receiving Basin for Off-Site Fuels, RSWF-Radioactive Scrap and Waste Facility, SNM-special nuclear material, SPR-Sandia Pulse Reactor, SRTC-Savannah River Technical Center, SWSA6-Solid Waste Storage Area (Number) 6, TAN_Test Area North, TRA-Test Reactor Area, TREAT-Transient Reactor Test Facility, TSR - Tower Shielding Reactor, WV FRS-West Valley Fuel Receiving and Storage. 


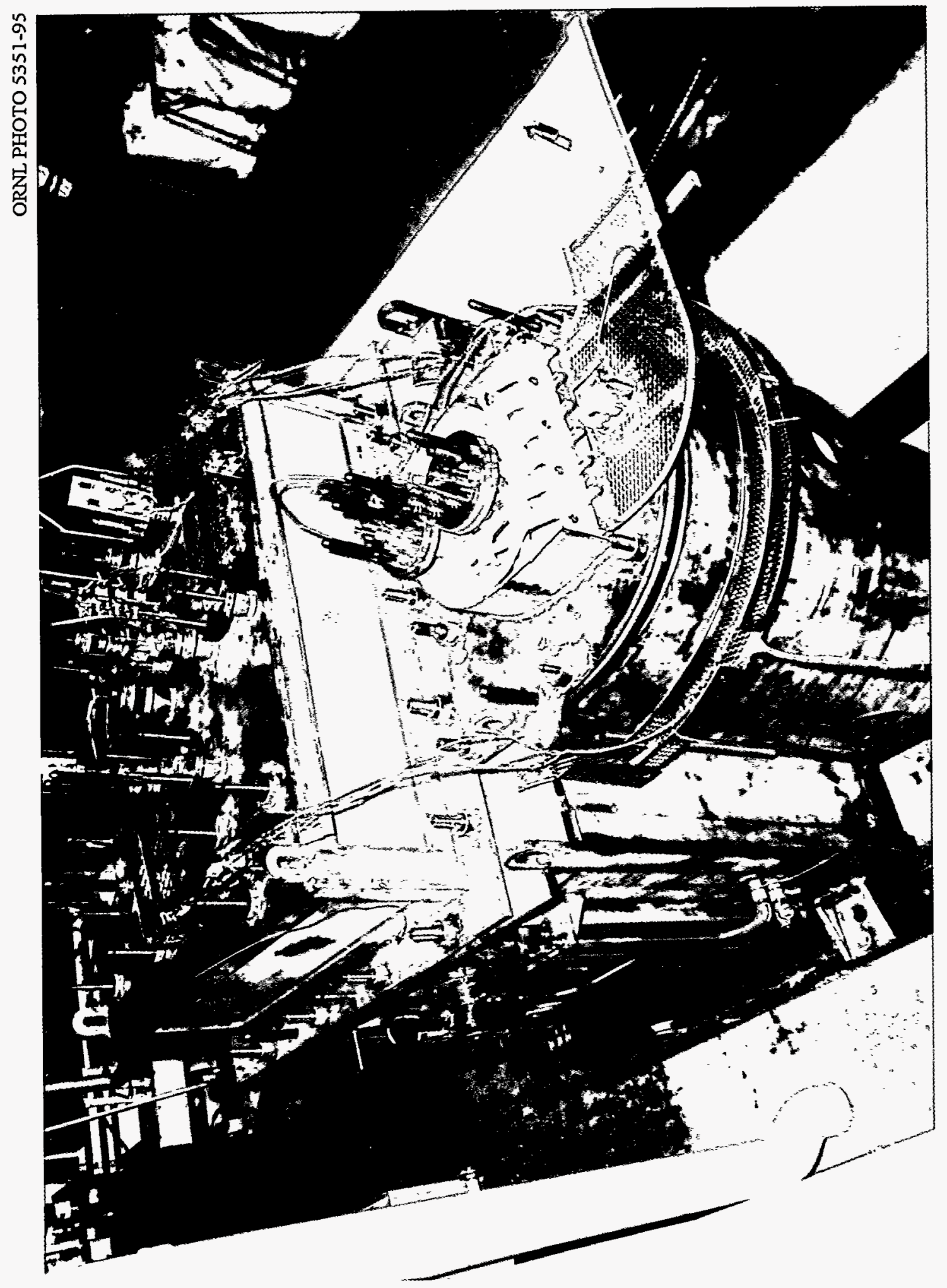

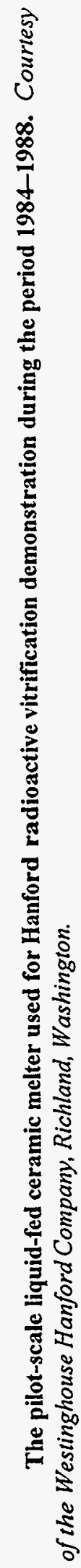




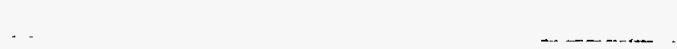




\section{HIGH-LEVEL WASTE}

\subsection{INTRODUCTION}

High-level waste (HLW) is generated by the chemical reprocessing of spent research and production reactor fuel, irradiated targets, and naval propulsion fuel. HLW generally contains more than $99 \%$ of the nonvolatile fission products produced during reactor operation. HLW from a facility that recovers both uranium and plutonium contains a residual amount of about $0.5 \%$ of those elements, while HLW from a facility that recovers only uranium contains a residual $0.5 \%$ of the uranium and essentially all of the plutonium. Most fission products have short half-lives and therefore quickly decay. HLW older than 1 year contains primarily the fission radionuclides ${ }^{137} \mathrm{Cs}$ and ${ }^{90} \mathrm{Sr}$ and very small amounts of transuranic (TRU) nuclides.

When generated, HLW is a highly radioactive, acidic liquid which generates heat and must be handled remotely behind heavy shielding in corrosion-resistant vessels, usually made of stainless steel. At the Hanford Site (Hanford), because stainless steel was in short supply, HLW was neutralized with caustic soda, and sodium nitrite was then added for corrosion control so that the HLW could be stored safely in carbon-steel tanks. This practice continued at Hanford and the Savannah River Site (SRS) even when stainless steel became more readily available. Neutralization with caustic soda forms sodium nitrate (which remains in solution) and hydrated oxides of certain radionuclides and nonradioactive chemicals (which precipitate and collect as a sludge on the floor of the tank). In addition, the ${ }^{137} \mathrm{Cs}$ remains largely in solution. At the Idaho National Engineering Laboratory (INEL), however, the waste has always been stored as an acidic liquid and then converted later into a granular solid (calcine) by thermal processing, which drives off water and decomposes nitrate salts to oxides.

The supernatant liquid resulting from neutralization may become concentrated by evaporation-either by selfboiling or in evaporators. If enough water is removed from the waste, sodium nitrate and sodium nitrite will crystallize from the solution. The crystals then will settle to the bottom of the tank liquid and on top of the sludge. If there are many crystals, a salt cake will form.
To reduce heat generation in tanks, the radionuclides ${ }^{137} \mathrm{Cs}$ and ${ }^{90} \mathrm{Sr}$ were removed from some Hanford $\mathrm{HLW}$ and stored in concentrated form as purified compounds in capsules. Some of these capsules were leased to non-DOE organizations for beneficial uses. The leased capsules are planned to be returned to DOE by 1996 .

In summary, HLW exists in a variety of physical or chemical forms (alkaline or acidic, supernatant liquid, sludge, salt cake, calcine solid, etc.), all of which must be stored behind heavy shielding and usually in underground tanks or bins.

Most of the current U.S. inventory of HLW has resulted from DOE activities. HLW is stored at SRS (Aiken, South Carolina), INEL (Idaho Falls, Idaho), and Hanford (Richland, Washington). A small amount of HLW was generated by commercial operations at the Nuclear Fuel Services (NFS) plant, near West Valley, New York, between 1966 and 1972, at a site owned by the New York State Energy Research and Development Authority (NYSERDA). After 1972, fuel reprocessing operations at this plant were discontinued. In 1980 , Congress passed the West Valley Demonstration Project (WVDP) Act (P.L. 96-368), which authorizes DOE to conduct, jointly with NYSERDA (90\% DOE, $10 \%$ NYSERDA), a demonstration of solidification of HLW for disposal and the decontamination and decommissioning (D\&D) of facilities used in the demonstration. The HLW data presented in this chapter are based on separate submittals provided by Hanford, INEL, SRS, and WVDP in ref. 1.

In 1992, DOE decided to phase out the domestic reprocessing of irradiated nuclear fuel for the recovery of enriched uranium or plutonium in support of defense activities; thus, little additional $\mathrm{HLW}$ is expected to be generated by this source for the immediate future. However, some future $D \& D$ activities may generate waste with radioactivity levels high enough such that the waste will require management in ways similar to HLW. An example of such a future activity is flushing residual wastes found in reprocessing facilities. 


\subsection{AGREEMENTS AND INTERFACES}

HLW is considered to be a mixed waste (i.e., waste containing both radioactivity and hazardous substances) unless demonstrated to the contrary. The hazardous substances of HLW are defined by the Resource Conservation and Recovery Act (RCRA). ${ }^{2}$ Liquid HLW is characteristic mixed waste (i.e., as stored, it shows the characteristic of corrosivity because of its acidity or alkalinity). Some HLW may also be listed mixed waste (i.e., it contains substances on any one of several lists regulated under RCRA). Mixed wastes must be managed according to RCRA ${ }^{2}$ and Atomic Energy Act (AEA) ${ }^{3}$ requirements.

The Federal Facility Compliance Act (FFCA) 4 of 1992 amends the Solid Waste Disposal Act to require (among other things) that DOE prepare a Site Treatment Plan (STP) for each site which generates, stores, or treats mixed waste. In effect, these STPs constitute a legally enforceable agreement between DOE and the host state that DOE must comply with certain requirements for mixed waste management. STPs must be approved by the host state for the site and must be in place by October 6, 1995 , unless another agreement was in place before the law was enacted. Two such prior agreements exist: both involve tri-party arrangements. One (for Hanford) is among DOE, the U.S. Environmental Protection Agency (EPA), and the state of Washington. This Tri-Party Agreement ${ }^{5}$ serves as an STP, is legally enforceable, and requires DOE to undertake specific actions at Hanford on a prescriptive timetable. The other tri-party agreement (for $\mathrm{SRS}$ ) involves DOE, EPA, and the state of South Carolina. However, this agreement applies only to those waste storage tanks that do not meet current DOE and regulatory criteria for secondary containment and leak detection. Consequently, an STP is being prepared for SRS. Concerning INEL, an amended Notice of Noncompliance Consent Order was issued to DOE in March 1994 by the Idaho Department of Health and Welfare. The Order includes provisions for proposing waste treatment technologies and deadlines for removing liquid waste from specific tanks. Because no prior agreements with WVDP specifically address mixed waste treatment, an STP is being prepared for that site to address such treatment.

\subsection{WASTE CHARACTERIZATION}

Generic characterization of $\mathrm{HLW}$ at some sites has been hampered over the years by the use of several different flowsheets for the processes that generated the waste or prepared the wastes for storage (e.g., nuclide separation, precipitation, and evaporation). In some instances, wastes have been blended. Information for all sites is based on historic records of reprocessing feeds and, for Hanford, SRS, and WVDP, extensive sampling of stored HLW.

Previous versions of this report have presented HLW data by physical form in some detail (e.g., liquid, sludge, slurry, salt cake, and precipitate). This revision clusters the data into just three distinct categories: solid, liquid, and process solidified (canistered) material. This approach is taken because, for example, all "liquid" materials (free tank supernatant and drainable interstitial liquid) must be stored and handled in essentially the same manner. Likewise, "solids" (sludge, salt cake, zeolite, calcine, capsules, and precipitate) and "canistered material" (the final waste form) require different handling methods, respectively. As HLW pretreatment and vitrification processes proceed, inventories of particular categories will decrease or increase.

Chemical and radionuclide compositions and inventories are given for the current and projected HLW at Hanford (Tables 2.11 and 2.16), INEL (Tables 2.12 and 2.17), SRS (Tables 2.13 and 2.18); and WVDP (Tables 2.14 and 2.19).

\subsection{INVENTORIES AND PROJECTIONS}

Tables $2.1-2.3$, respectively, present historical and projected volumes, radioactivity, and thermal power inventories of HLW currently in storage. The radioactivity and resultant thermal power of HLW decay, over time, in a manner characteristic of the constituent radionuclides, but, as previously mentioned, the volume depends significantly on the specific treatment history of the waste. When one takes into account all radionuclides in HLW, total radioactivity and thermal power each decrease about 2 to $4 \%$ per year within storage units to which no new waste has been added.

Locations of the four HLW sites and the relative volumes of $H L W$ are represented in Fig. 2.1. The total volume and radioactivity for the HLW (solid and liquid) stored at the four sites is shown graphically in Fig. 2.2. Pie charts show the relative breakdown of stored HLW volume (Fig. 2.3) and radioactivity (Fig. 2.4) as of 1994. Historical and projected cumulative volumes of HLW stored or produced at each site are graphically illustrated in Fig. 2.5a. The number of waste canisters projected to be produced by each site are depicted in Fig. $2.5 b$.

Current DOE plans are to immobilize and package HLW for disposal in a licensed, underground geologic repository. Figures 2.6-2.9 show, for each of the four sites, the general treatment processes by which the $\mathrm{HLW}$ will be immobilized to a form acceptable for disposal in a 
repository. Tables 2.4-2.6, respectively, give the projected volume, radioactivity, and thermal power for HLW immobilized as borosilicate glass. Table 2.7 gives estimates, year by year and by site, of the number of HLW canisters to be produced based on reference flowsheets. Canister estimates for SRS [Defense Waste Processing Facility (DWPF)] and WVDP are fairly well established (both projects are scheduled to begin radioactive operations in FY 1996), while canister estimates for INEL and Hanford are less certain because pretreatment and waste minimization processes have not yet been finalized. Tables 2.8-2.10 give the volume, radioactivity, and thermal power, respectively, of stored HLW by site and by physical form. Currently available summary information about the radionuclide distribution for stored HLW and projected associated wastes for each site has been given in Tables 2.11-2.14. Significant changes in any of these tables from the previous IDB report (Rev. 10) ${ }^{6}$ are presented in Table 2.15. It should be noted that the radioactivity reported in Tables $2.2,2.5$, and 2.9 include contributions from both parent and daughter products.

Projected inventories (volume, radioactivity, and thermal power) for HLW presented in Tables 2.1-2.6 have been generated by each site based on certain assumptions and therefore should be considered only as current best estimates. It is possible that new treatment methods or waste forms may be developed and may supersede current baseline projections for Hanford or INEL. All sites are moving toward shutdown; consequently, very little additional $\mathrm{HLW}$ will be generated. Major HLW activities will be (a) continued safe storage, (b) preparation for solidification, (c) solidification, and (d) interim storage pending shipment to a national repository. Thus, the inventory of liquid HLW in storage generally will decrease, and the inventory of solidified HLW in interim storage, pending shipment to a national repository, will increase. The Waste Acceptance System Requirements Document ${ }^{7}$ for the national repository states that receipt of DOE HLW will begin no sooner than 6 years after initial receipt of utility spent nuclear fuel (SNF). Thus, the canisters of solidified HLW will remain in interim storage, most likely at the production site. The current projected number of HLW canisters (Table 2.7) for Hanford, INEL, and SRS is significantly different from the value reported in the previous IDB report. For INEL, the new projections reflect recent funding and program guidance from $\mathrm{DOE}$ that effectively delays completion of the HLW solidification programs at these sites.

Summary flowsheets of the reference solidification processes are given for Hanford (Fig. 2.6), INEL (Fig. 2.7), SRS (Fig. 2.8), and WVDP (Fig. 2.9). Overall, these flowsheets are very similar; process differences reflect differences among sites in waste characteristics.

\subsubsection{Hanford}

Hanford $\mathrm{HLW}$ is stored in underground carbon-steel tanks. Current HLW inventory consists of $155,800 \mathrm{~m}^{3}$ considered to be "solid" HLW (salt cake and sludge in single- and double-shell tanks) and $83,200 \mathrm{~m}^{3}$ of "liquid" HLW (supernatant in single- and double-shell tanks and drainable interstitial liquid in single-shell tanks), for a total of $239,000 \mathrm{~m}^{3}$. At Hanford, waste in single-shell and double-shell tanks consists of HLW, TRUW, and several LLWs. However, in the interim storage mode, the tanks are managed as if they contain only HLW. Thus, their contents are included in the HLW inventory.

A total of 2,217 capsules have been manufactured at Hanford, some of which have been leased off-site for beneficial purposes. Of the 1,577 cesium and 640 strontium capsules, a total of 249 cesium capsules and 35 strontium capsules have been dismantled. The inventory of capsules that have been dismantled is not expected to be returned to Hanford for interim storage and future processing. This leaves 1,328 cesium capsules and 605 strontium capsules to be processed (overpacked) and disposed of as HLW.

The HLW projections for Hanford are based on the assumptions that (1) fuel reprocessing is not resumed, (2) irradiated fuel remains in storage, (3) pumpable liquids are transferred from single-shell tanks to double-shell tanks, (4) double-shell tanks will continue to receive any newly generated waste, and (5) volume reduction of stored wastes through evaporation will continue.

\subsubsection{INEL}

INEL HLW is currently being stored as both acidic liquid and calcined solids (calcine). Underground, highintegrity, stainless-steel tanks contain about $7,200 \mathrm{~m}^{3}$ of acidic liquid waste. (Of this waste, only $1,306 \mathrm{~m}^{3}$ is actual $\mathrm{HLW}$; the rest is sodium-bearing waste, which is managed the same way as is HLW because of site practice.) Underground stainless-steel bins currently store (retrievably) about $3,800 \mathrm{~m}^{3}$ of calcine, an interim waste form. More than $90 \%$ of the total radioactivity is in the calcine.

For INEL, HLW projections are based on the recommended option from a new systems engineering approach to integrate all DOE Office of Environmental Management waste streams at the INEL. The HLW projections include streams associated with the intermediate calcining of liquid waste, followed by separation of HLW and LLW fractions in the remaining liquid waste and redissolved calcine. No new HLW from reprocessing activities was produced after FY 1992; SNF reprocessing facilities are being placed into cold standby 
pending D\&D. In June 1995, DOE selected a waste form and a process technology to treat the sodium-bearing waste and immobilized calcine. The current reference waste form at INEL is a glass. It is assumed that INEL will begin radioactive operations and thus produce canisters in 2019 and continue this operation through 2045 (see Fig. 2.7). The projections reported in Tables 2.1-2.7 reflect this assumption.

\subsubsection{SRS}

SRS HLW is stored as alkaline liquid, sludge, salt cake, and precipitate. The current inventory of about $126,300 \mathrm{~m}^{3}$ is stored in underground, single- and doubleshelled carbon-steel tanks. This waste consists mainly of alkaline liquid, sludge, and salt cake. Although reprocessing operations are being phased out, the HLW tank farms are continuing to receive $\mathrm{HLW}$ from the canyons. The preparation of some $\mathrm{HLW}$ for vitrification during the original in-tank precipitation demonstration generated cesium precipitate, and on-going HLW sludgewashing in extended sludge processing (ESP) has generated partially washed sludge. Pretreatment of HLW salt solution is scheduled to begin at the In-Tank Precipitation Facility in August 1995. Characterization data for SRS HLW are based on sampling and process knowledge. Allowable facility design variability of feed composition is limited; therefore, the data reported in Tables 2.1-2.6 assume a uniform feed rate and composition. The current budget projections have both (a) limited the waste-blending capability to feed a uniform composition and (b) reduced the feed rate. The radiochemical streams are assumed to be processed at a uniform rate with adjustments made for radioactive decay.

\subsubsection{WVDP}

Reprocessing at the West Valley NFS plant was terminated in 1972, after which no additional HLW has been generated. HLW at WVDP is stored in underground tanks. The current $H L W$ inventory of $2,180 \mathrm{~m}^{3}$ consists of $2,040 \mathrm{~m}^{3}$ of liquid alkaline waste and $140 \mathrm{~m}^{3}$ of solid waste (composed of both alkaline sludge and inorganic zeolite ion-exchange material contaminated with radio-cesium). The alkaline waste is stored in an underground carbonsteel tank, and the zeolite is stored in an underground carbon-steel tank covered by an aqueous alkaline solution. The small amount of acidic waste remaining from reprocessing of a thorium fuel was blended into the alkaline waste in 1995.

\subsection{SOLIDIFICATION FOR PERMANENT DISPOSAL}

All HLW will be processed and solidified to a form acceptable for permanent disposal in a geologic repository. ${ }^{8-12}$ Borosilicate glass has been selected as the reference waste form for all sites. ${ }^{13}$ Projections are based on current funding guidance provided to the sites by $\mathrm{DOE}$.

\subsubsection{Hanford}

Plans are to retrieve waste from both single- and double-shell tanks, pretreat the waste as necessary to provide HLW and LLW fractions, and immobilize both fractions by vitrification (see Fig. 2.6). Waste retrieval will begin in December 2003, and pretreatment operations will begin in December 2004. Initiation of operations at an LLW vitrification facility is planned for June 2005, while operations at an HLW vitrification facility are scheduled to begin in December 2009. Vitrification of all waste from tanks is expected to be completed by December 2028.

Pretreatment enables the majority of the radioactivity of retrieved waste to be effectively routed through the HLW vitrification facility and the bulk of the tank waste material to be directed to the LLW vitrification facility. The current baseline pretreatment processes are enhanced (caustic) sludge-washing, liquid-solid separation, and cesium removal from liquids. Other pretreatment processes that will be evaluated in the Tank Waste Remediation System Program Environmental Impact Statement ${ }^{14}$ are acid dissolution, advanced separations of wastes, and organic destruction.

Hanford's reference canister for HLW disposal has a diameter of $68 \mathrm{~cm}$ and a length of $457 \mathrm{~cm}$. The nominal glass volume is estimated to be $1.26 \mathrm{~m}^{3}$ with an average waste oxide loading of $45 \%$. Capsule processing will be performed in the HLW vitrification facility concurrent with the HLW tank waste vitrification. An accelerated schedule for capsule processing (overpacking) is assumed. Eight capsules will be stacked axially along the centerline of each reference canister. Alternatively, cesium and strontium salts currently contained in capsules could be vitrified if it is decided that overpacking of existing capsules would not result in an acceptable waste form for the national repository. Because the projected volume, radioactivity, and thermal power for the LLW resulting from the production of the HLW final waste form have not been finalized, no data on the final LLW form are presented in Table 2.11. 
An interim storage facility will be built at Hanford with sufficient capacity to store the entire HLW volume of glass and the overpacked capsules produced by the HLW vitrification facility. Storage will continue until the canisters and overpacked capsules are shipped to a geologic repository. Shipment to the repository will commence no sooner than 2035. Thus, values for glass volume, curies, watts, and number of canisters given, respectively, in Tables 2.4-2.7 represent the total accumulation of Hanford's HLW disposal form, with no projected decrement for waste shipment off site.

The technical strategy for treatment and disposal of tank waste is based on the January 1994 amendments to the action plan of the Hanford Federal Facility Agreement and Consent Order. ${ }^{5}$

\subsubsection{INEL}

The preferred Full Treatment Alternative ${ }^{15}$ was selected for integrating all waste streams (at INEL) which are managed under DOE-EM. This flexible path forward emphasizes direct action, including treating and preparing waste for shipment to final disposition. In this alternative, the New Waste Calcining Facility is assumed to operate through 2008. A new separations-vitrification facility is planned to be on line after 2017. The remote-handled TRU waste (TRUW) will be processed first in the vitrification facility from 2017 to 2019. After 2019, both the liquid HLW and calcine will be processed to separate the high-activity radionuclides from the low-activity waste. The high-activity waste will be vitrified in the new facility and the separated low-activity waste will be grouted and sent to LLW disposal. The vitrified HLW will be stored until the repository is ready. All HLW is projected to be processed by 2045 under this scenario.

\subsubsection{SRS}

The plan to process SRS HLW into glass is detailed in High Level Waste System Plan Revision $5(U)^{16}$ (i.e.,
Rev. 5 baseline case, similar to Rev. 4) which was transmitted to DOE April 27, $1995 . \quad$ Briefly, Rev. 5 depicts three funding cases and the data included in this report represent the baseline case with completion of the immobilization of the current inventory of HLW in FY 2021.

For SRS, reprocessing operations are scheduled to be completed by FY 2002. Additional HLW from reprocessing activities until then will represent a maximum increase of about $14.5 \%$ of current inventory. Pretreatment (sludge-washing) of liquid HLW has been started, and the DWPF will begin producing canisters of solidified HLW in FY 1996. The HLW glass waste forms will be stored at SRS until a national repository is ready to accept them (see Fig. 2.8).

\subsubsection{WVDP}

Currently, pretreatment at the WVDP is essentially completed. In May 1988, the processing of liquid $\mathrm{HLW}$ was initiated. This alkaline liquid was decontaminated to LLW in the WVDP Supernatant Treatment System (STS) in preparation for the incorporation of all HLW at the WVDP into a glass. In the STS, an ion-exchange process that is operated in a batch mode is used to remove cesium from the alkaline liquid waste (see Fig. 2.9). The ionexchange columns are located in the underground carbonsteel tank, which was originally installed as a backup tank for the storage of alkaline HLW. The sludge in the bottom of the tank has been mixed with the residual supernatant and an alkaline solution. Both sludge-wash processing cycles were completed in 1994. The wash solutions are also treated in the STS before they are incorporated in cement. The washed sludge, the acidic waste, and the loaded zeolite will be combined and incorporated into a glass. At WVDP, vitrification of the HLW will start in FY 1996 and be completed in FY 1998. The glass will be stored on-site until it is transferred to a federal repository.

\subsection{REFERENCES}

1. DOE site HLW data submittal attachments, submitted to the IDB Program during June-July 1995 . The following HLW submittals were received and reviewed by the IDB Program before analysis and integration. Preceding each submittal is the site (in parentheses) to which it refers.

a. (Hanford) George Sanders, DOE Richland Operations Office, Richland, Washington, correspondence to Steve Loghry, IDB Program, ORNL, Oak Ridge, Tennessee, "Hanford Site High-Level Waste Information for the 1995 Integrated Data Base Report (DOE/RW-0006, Rev. 11)," dated June 1, 1995. 
b. (INEL) D. A. Knecht Lockheed Martin Idaho Technologies, Idaho Falls, Idaho, correspondence to J. G. Smook, DOE-ID, Idaho Falls, Idaho, copy to S. Loghry, IDB Program, ORNL, Oak Ridge, Tennessee, "INEL HLW Integrated Data Base Submittal for 12/31/94 Waste Quantities," DAK-28-95, dated July 20, 1995.

c. (SRS) William F. Spader, DOE Savannah River Operations Office, Aiken, South Carolina, correspondence to Stephen N. Storch, IDB Program, ORNL, Oak Ridge, Tennessee, "Savannah River Site (SRS) High Level Waste Information for the 1995 Integrated Data Base Report (DOE/RW-0006, Rev. 11),"MC-95-0043, dated June 8, 1995.

d. (SRS) Margaret Schwenker, DOE Savannah River Operations Office, Aiken, South Carolina, telefax to Steve Loghry, IDB Program, ORNL, Oak Ridge, Tennessee, "FY 95 Integrated Data Base, Chapter II, Savannah River Site Revised Input," dated Aug. 23, 1995.

e. (WVDP) P. S. Klanian, West Valley Nuclear Services Company, Inc., West Valley, New York, correspondence to Steven N. Storch, IDB Program, ORNL, Oak Ridge, Tennessee, "Submittal of High-Level Waste Information for the 1995 Integrated Data Base Report (DOE/RW-0006, Rev. 11)," WZ:95:0088, dated June 1, 1995.

2. U.S. Congress, Resource Conservation and Recovery Act of 1976, P.L. 94-580, 1976, as amended.

3. U.S. Congress, Atomic Energy Act of 1954, P. L. 83-703, Aug. 15, 1994.

4. U.S. Congress, The Federal Facility Compliance Act of 1992, P.L. 102-386, Oct. 6, 1992.

5. Washington State Department of Ecology, U.S. Environmental Protection Agency, and U.S. Department of Energy, Hanford Federal Facility Agreement and Consent Order, EPA Docket Number 1089-03-040120, Ecology Docket Number 89-54, Richland, Washington (May 1989).

6. U.S. Department of Energy, Integrated Data Base Report-1993: U.S. Spent Nuclear Fuel and Radioactive Waste Inventories, Projections, and Characteristics, DOE/RW-0006, Rev. 10, Oak Ridge National Laboratory, Oak Ridge, Tennessee (December 1994).

7. U.S. Department of Energy, Office of Civilian Radioactive Waste Management, Waste Acceptance System Requirements Document, DOE/RW-0351P, Rev. 1, Washington, D.C., (March 1994).

8. U.S. Department of Energy, Final Environmental Impact Statement, Disposal of Hanford Defense High-Level, Transuranic, and Tank Waste, Hanford Site, Richland, Washington, DOE/EIS-0113, DOE Richland Operations Office, Richland, Washington (December 1987).

9. U.S. Congress, The Nuclear Waste Policy Act of 1982, P.L. 97-425, Sect. 8, Jan. 7, 1983, as amended.

10. Ronald Reagan, President of the United States, Washington, D.C., letter to John S. Herrington, Secretary of Energy, "Disposal of Defense Waste in a Commercial Repository," dated Apr. 30, 1985.

11. U.S. Department of Energy, "Civilian Radioactive Waste Management: Calculating Nuclear Waste Fund Disposal Fees for Department of Energy Defense Program Waste; Notice," Fed. Regis. 56(161), 31508 (Aug. 20, 1987).

12. U.S. Congress, The Nuclear Waste Policy Amendments Act of 1987, P.L. 100-203, Title V, Subtitle A, Dec. 22, 1987.

13. U.S. Department of Energy, Office of Defense Waste and Transportation Management, Defense Waste and Transportation Management Program Implementation Plan, DOE/DP-0059, Washington, D.C. (August 1988).

14. U.S. Department of Energy, "Notice of Intent to Prepare the Hanford Site Tank Waste Remediation System Environmental Impact Statement," Fed. Regis. 59(19), 4052 (Jan. 28, 1994). 
15. U.S. Department of Energy, Integration of EM Activities at the INEL, Idaho National Engineering Laboratory, Idaho Falls, Idaho (March 31, 1995).

16. U.S. Department of Energy, High-Level Waste System Plan Revision 5(U), HLW-OVP-95-0031, Savannah River Site, Aiken, South Carolina (April 26, 1995). 


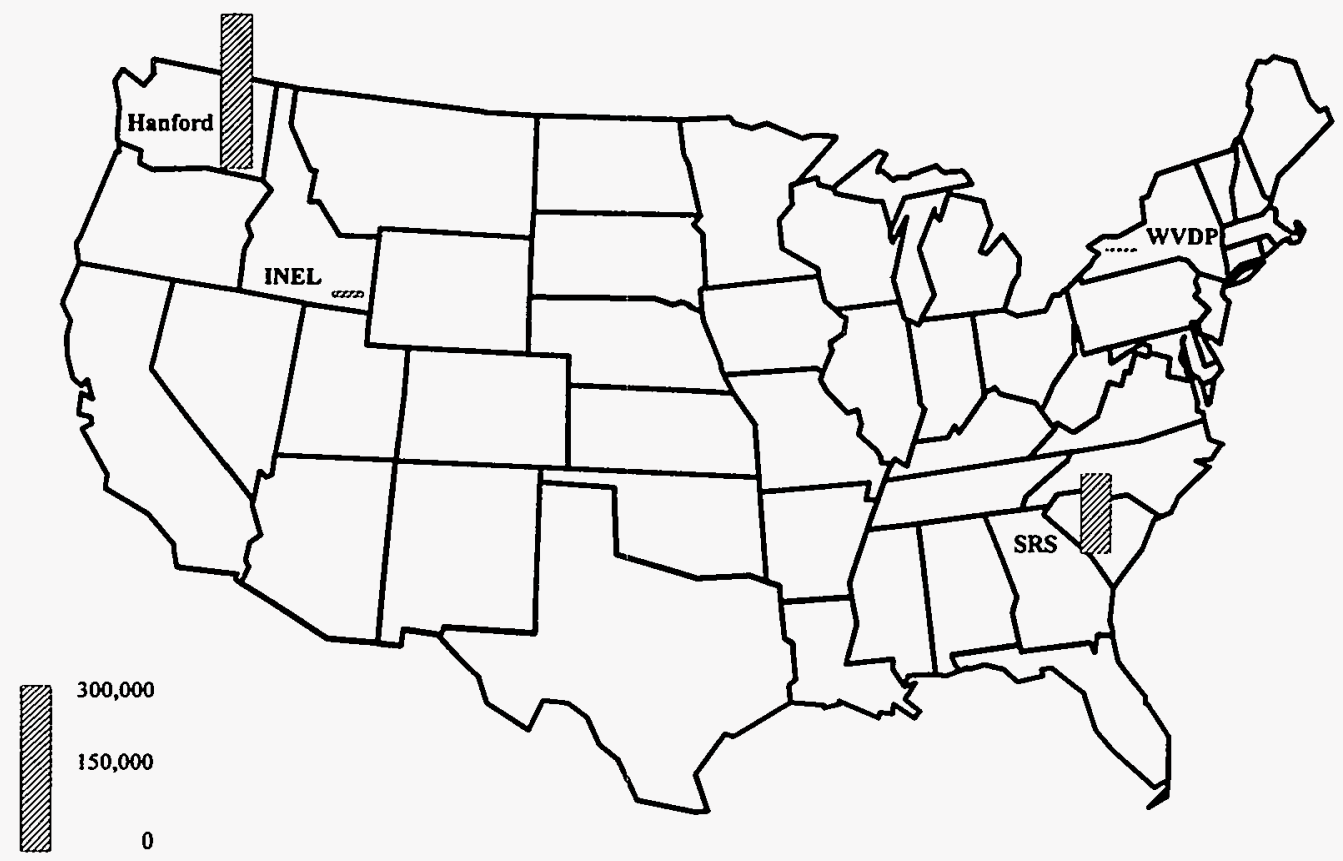

CUBIC METERS

Fig. 2.1. Locations and total volumes of HLW through 1994.

ORNL DWG 95-8223
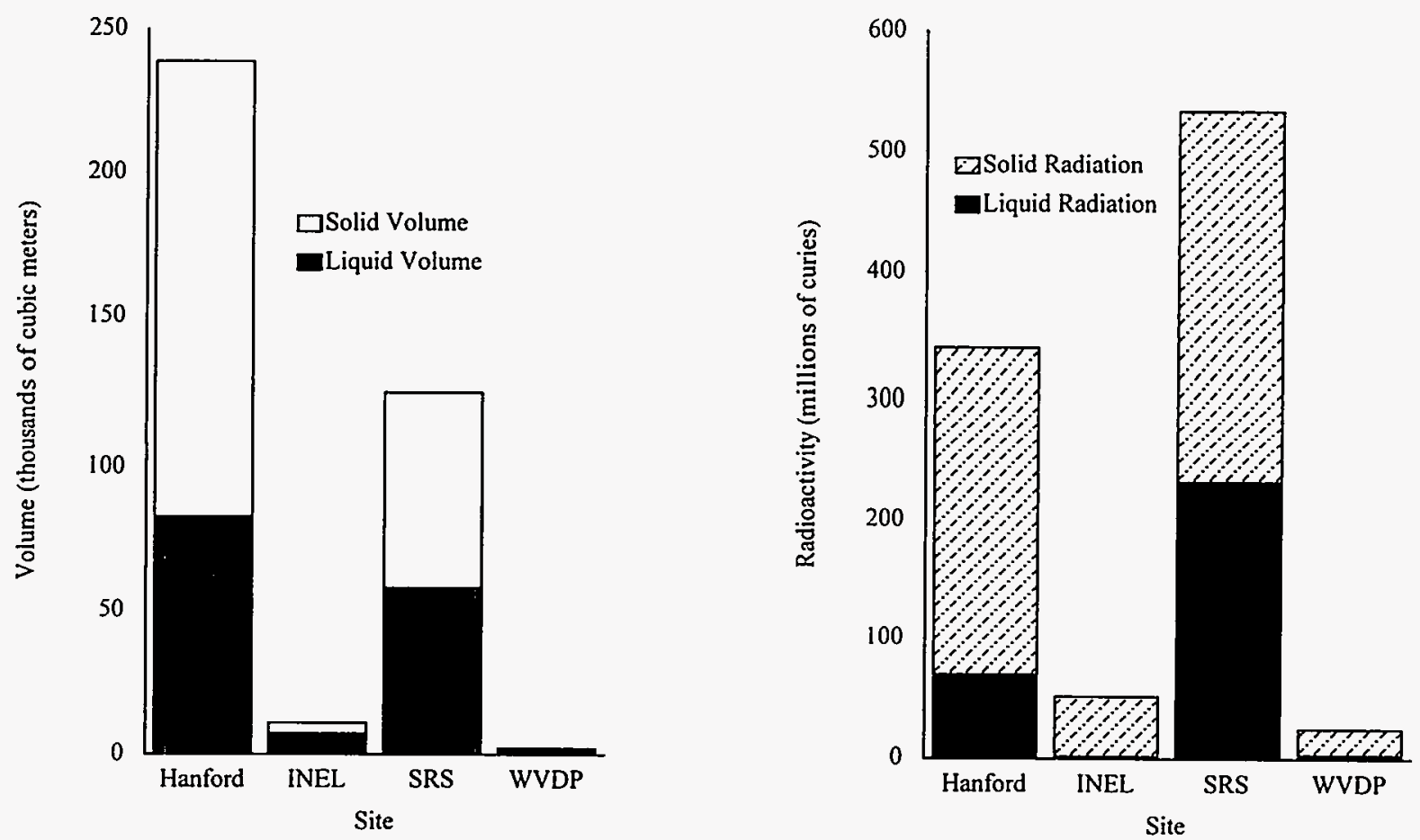

Fig. 2.2. Total volume and radioactivity of solid and liquid HLW through 1994. 
ORNL DWG 95-8224

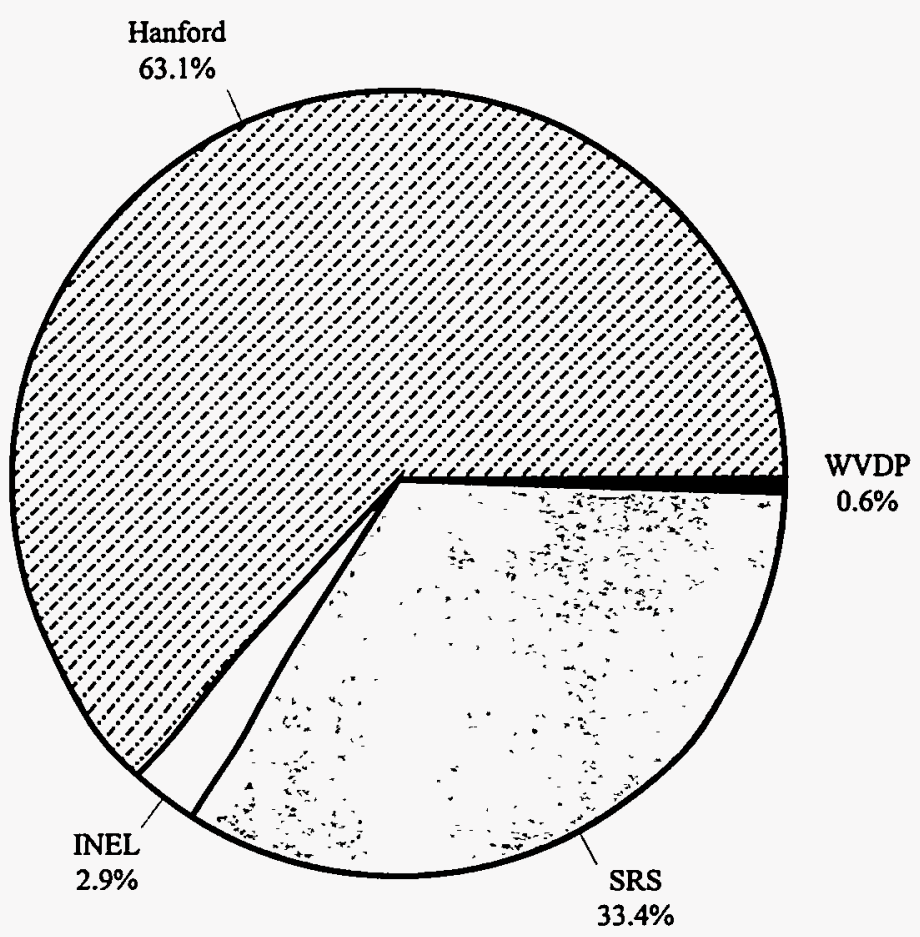

Fig. 2.3. Total volume of HLW through 1994.

ORNL DWG 95-8225

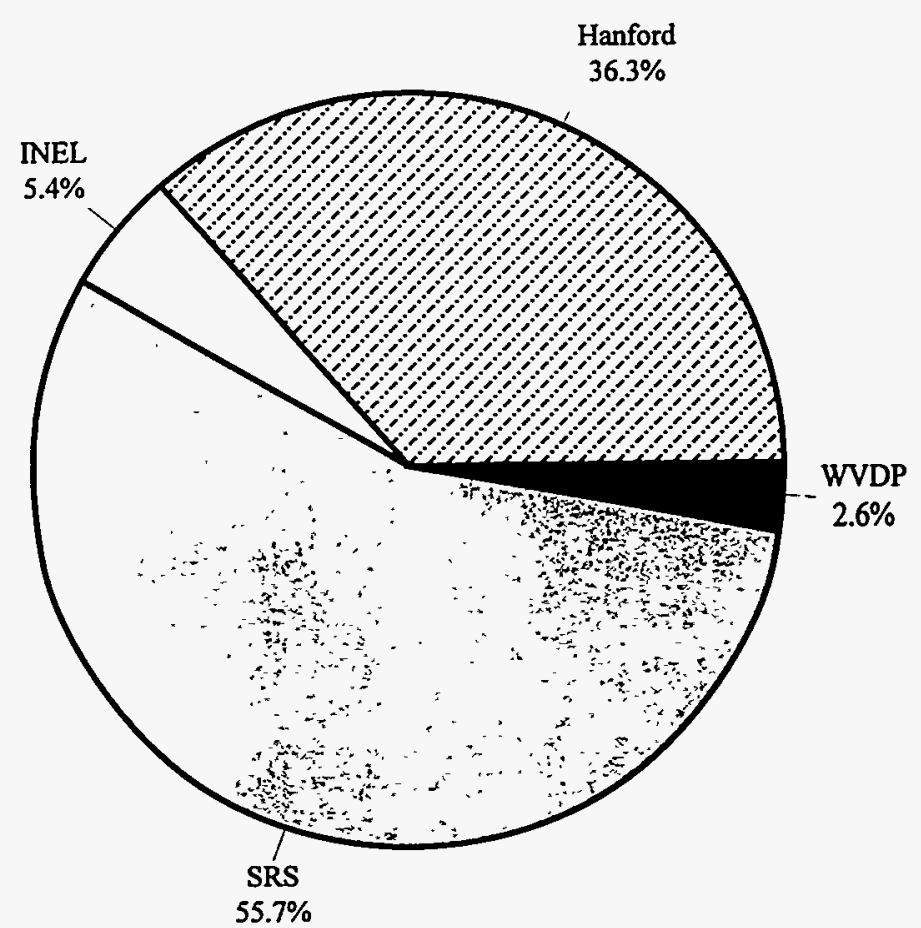

Fig. 2.4. Total decayed radioactivity of HLW through 1994. 


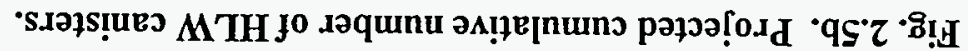

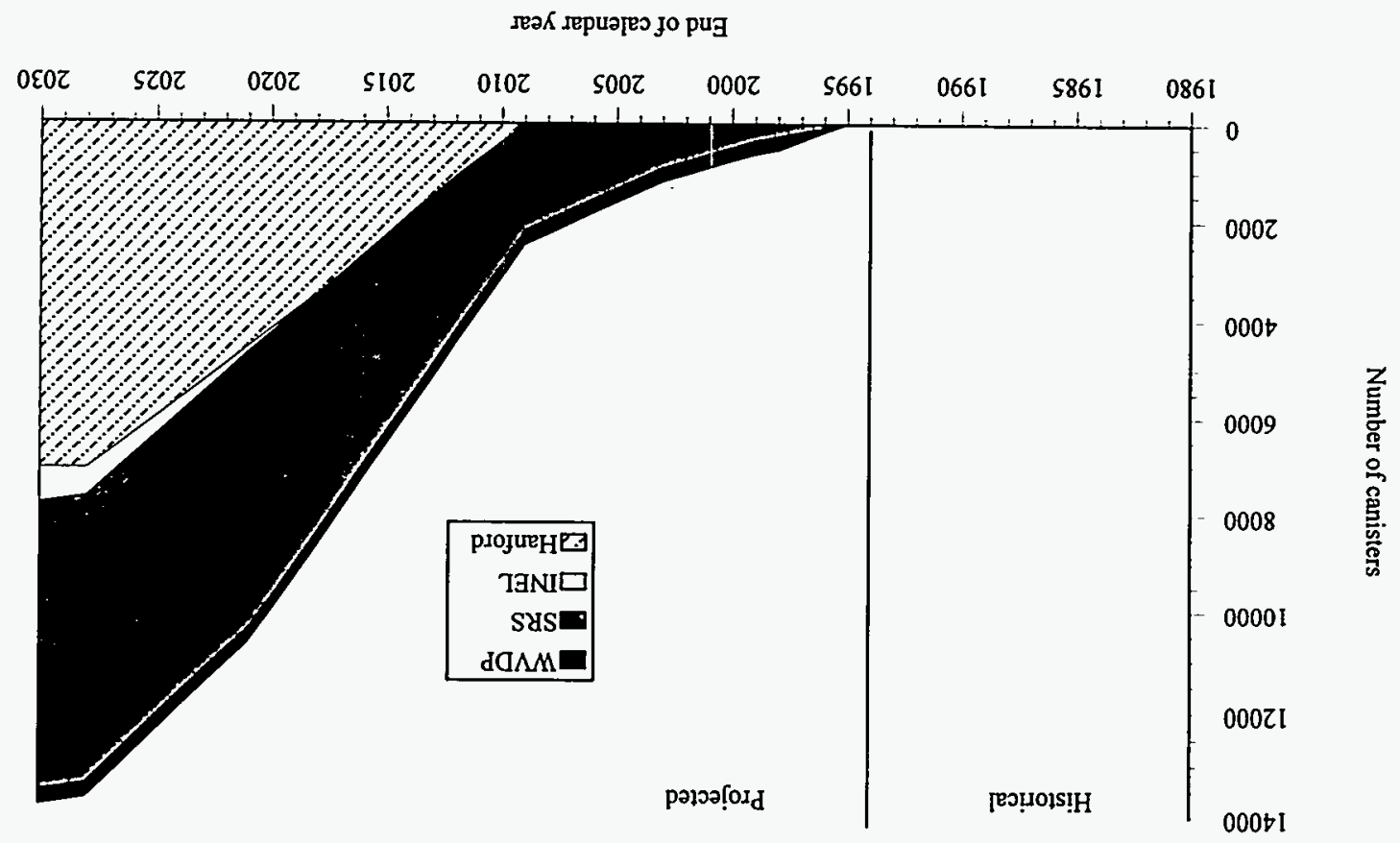

LZZ8-S6 DMA TNPO

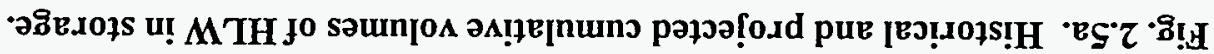
sеวК леритер jo pug

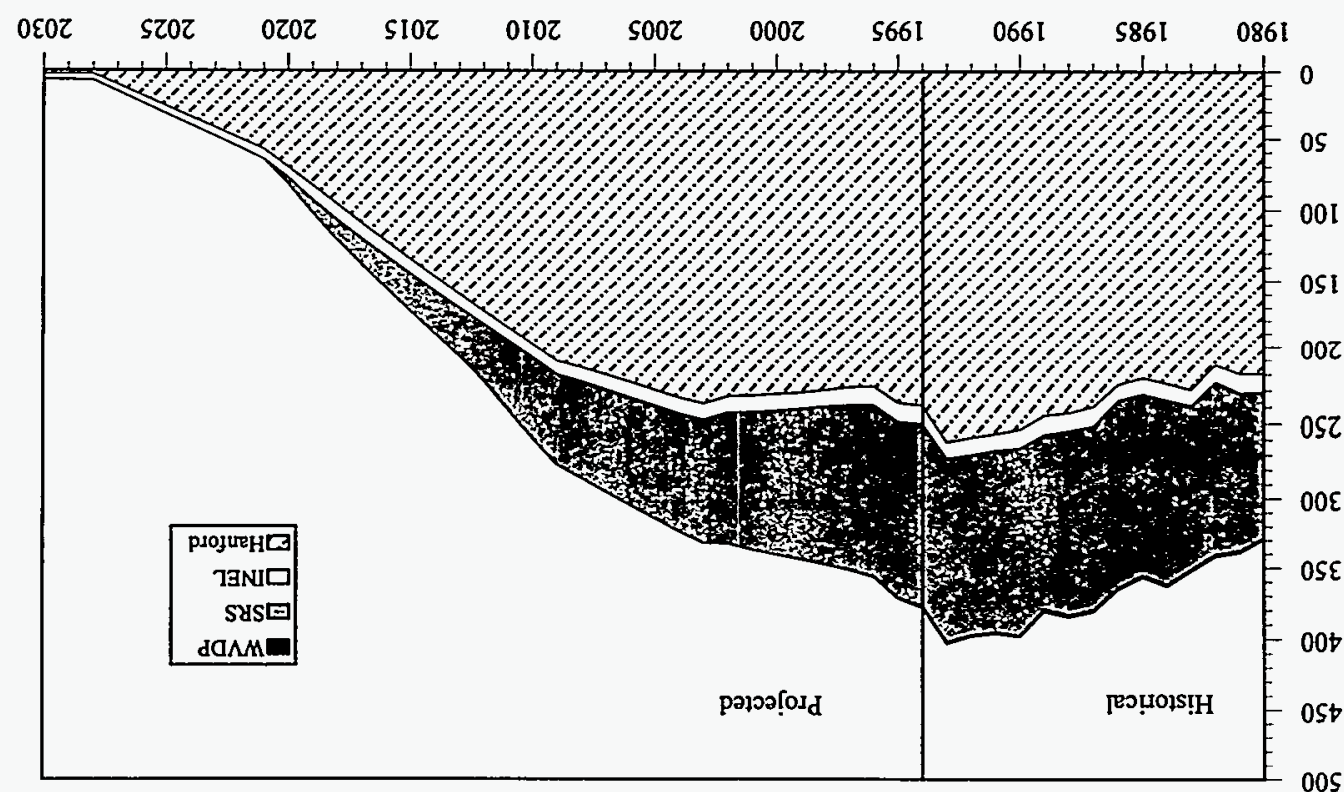

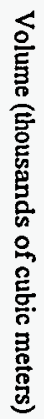

9Z28-56 DMA TN\&O 


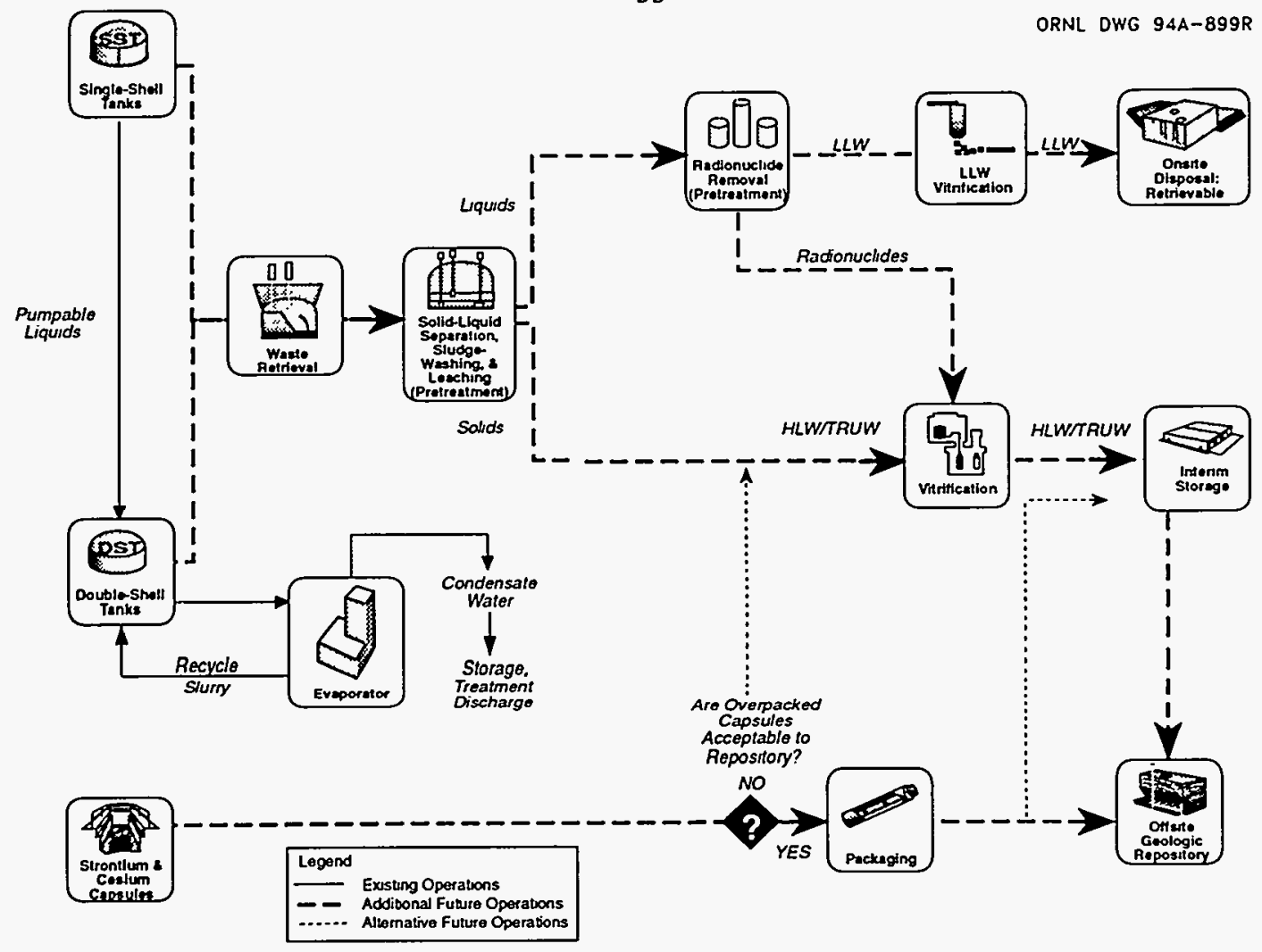

Fig. 2.6. Treatment methods for tank waste and capsules at Hanford.

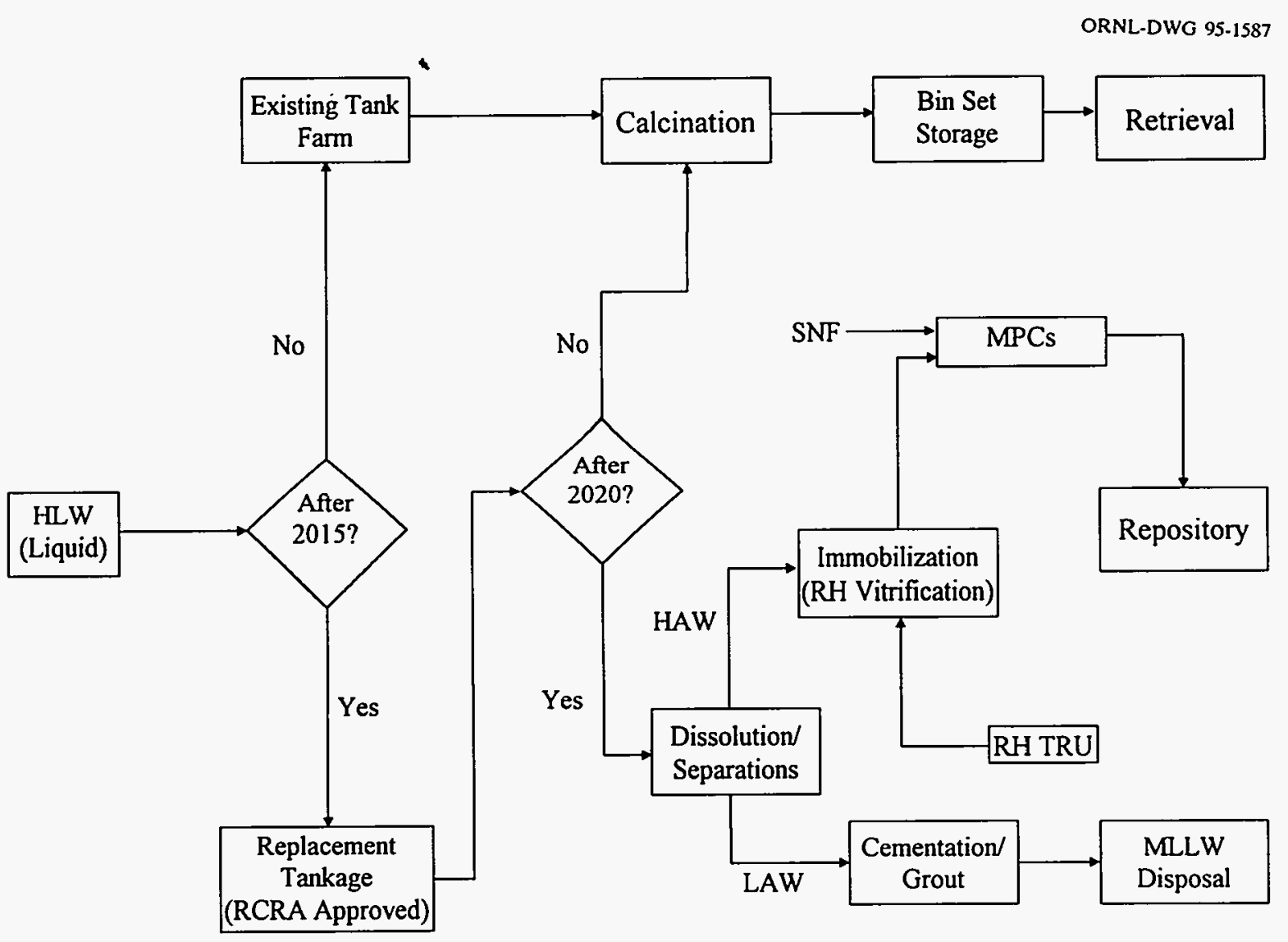

Fig. 2.7. Treatment methods for HLW in tanks, bins, and canisters at INEL. 
SRS HLW Management Process

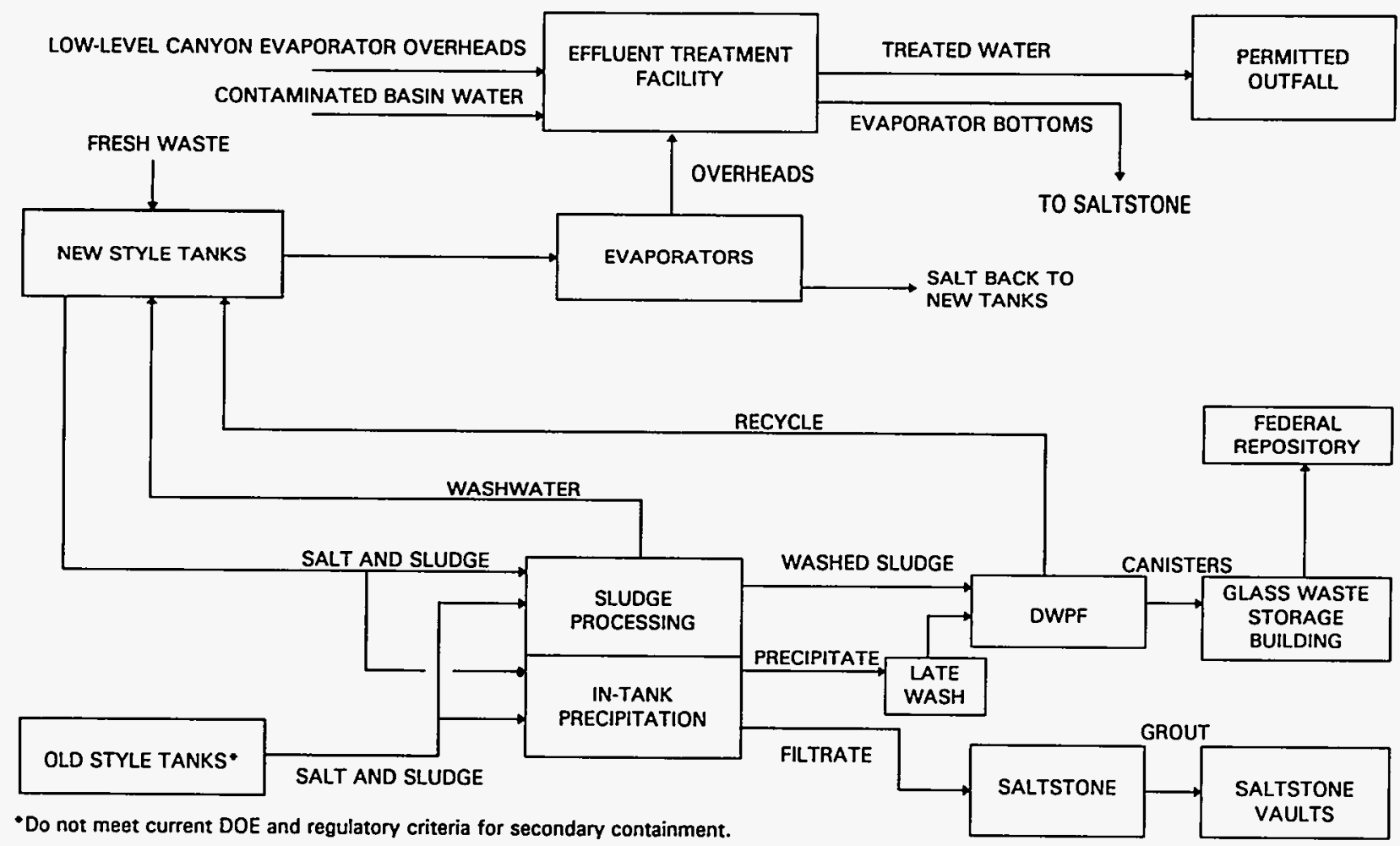

Fig. 2.8. Treatment methods HLW in tanks and canisters at SRS.

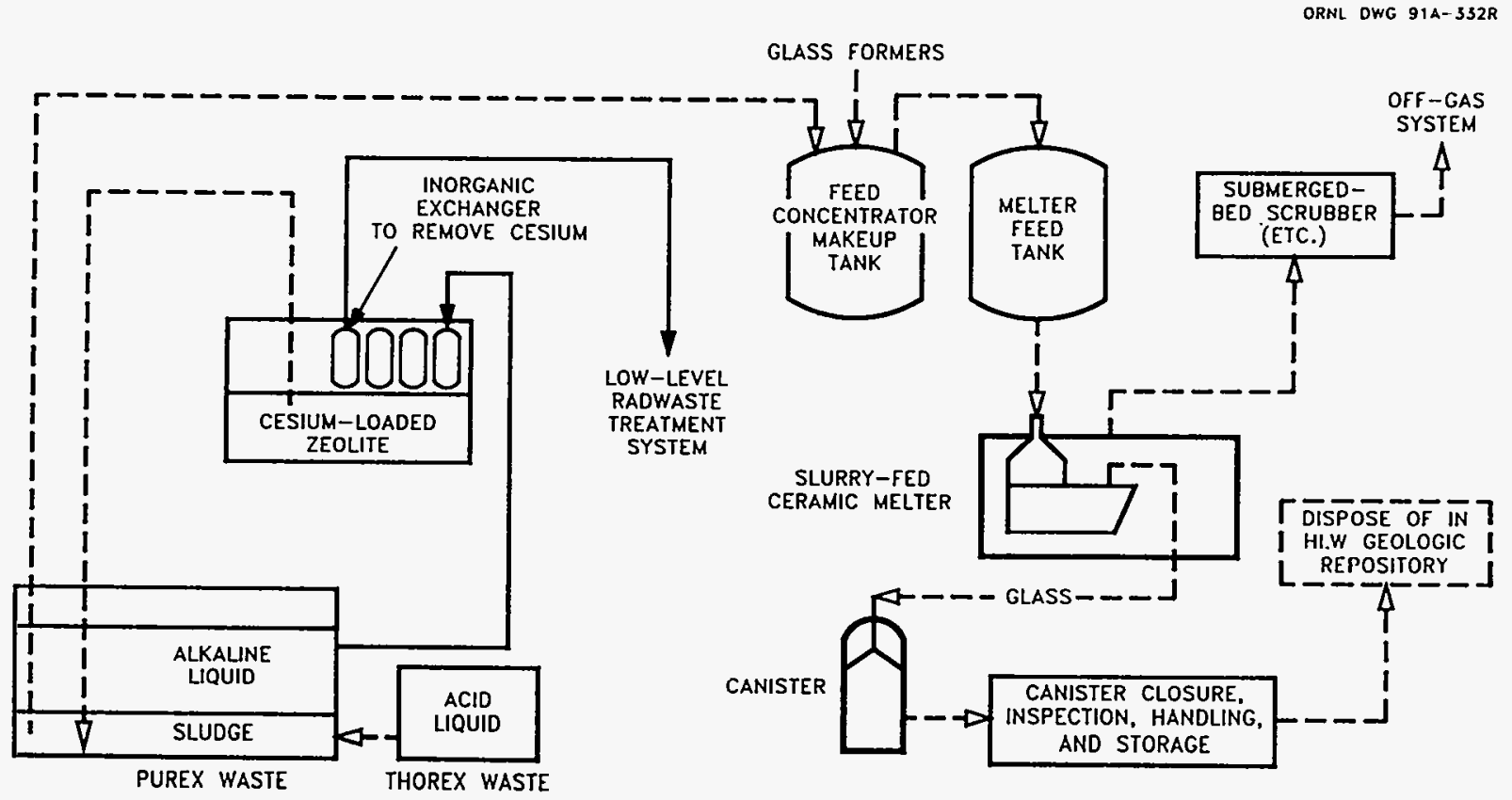

PRESENT OPERATIONS

ADDED FUTURE OPERATIONS

Fig. 2.9. Treatment methods for HLW in tanks and canisters at WVDP. 
Table 2.1. Historical and projected cumulative volume of HLW stored in tanks, bins, and capsules, by site ${ }^{\mathrm{a}}$

\begin{tabular}{|c|c|c|c|c|c|}
\hline \multirow{2}{*}{$\begin{array}{c}\text { End of } \\
\text { calendar } \\
\text { year }\end{array}$} & \multicolumn{5}{|c|}{ Volume, $10^{3} \mathrm{~m}^{3}$} \\
\hline & Hanford & INEL & SRS & WVDP & Total \\
\hline 1980 & 219.4 & 11.4 & 96.7 & 2.2 & 329.7 \\
\hline 1981 & 219.4 & 12.0 & 105.7 & 2.2 & 339.3 \\
\hline 1982 & 213.3 & 11.5 & 115.0 & 2.2 & 342.0 \\
\hline 1983 & 229.4 & 9.7 & 111.4 & 2.2 & 352.7 \\
\hline 1984 & 225.6 & 10.1 & 125.6 & 2.2 & 363.5 \\
\hline 1985 & 222.1 & 10.1 & 122.7 & 2.2 & 357.1 \\
\hline 1986 & 226.4 & 9.5 & 127.8 & 2.2 & 365.9 \\
\hline 1987 & 239.7 & 11.9 & 127.6 & 2.2 & 381.4 \\
\hline 1988 & 243.4 & 11.0 & 128.4 & 2.1 & 384.9 \\
\hline 1989 & 244.8 & 12.0 & 122.0 & 2.4 & 381.1 \\
\hline 1990 & 253.6 & 12.0 & 131.7 & 1.2 & 398.5 \\
\hline 1991 & 256.4 & 10.4 & 127.9 & 1.7 & 396.5 \\
\hline 1992 & 258.7 & 11.2 & 126.9 & 1.6 & 398.3 \\
\hline 1993 & 261.7 & 10.5 & 129.3 & 2.0 & 403.5 \\
\hline 1994 & 238.9 & 11.0 & 126.3 & 2.2 & 378.4 \\
\hline 1995 & 237.3 & 11.4 & 122.1 & 0.9 & 371.7 \\
\hline 1996 & 227.3 & 11.3 & 117.4 & 0.6 & 356.6 \\
\hline 1997 & 228.0 & 10.6 & 112.7 & 0.3 & 351.8 \\
\hline 1998 & 229.7 & 9.8 & 108.0 & & 347.5 \\
\hline 1999 & 231.2 & 9.6 & 103.3 & & 344.2 \\
\hline 2000 & 232.1 & 9.8 & 98.7 & & 340.6 \\
\hline 2001 & 233.0 & 10.0 & 94.0 & & 336.9 \\
\hline 2002 & 233.6 & 9.7 & 89.3 & & 332.6 \\
\hline 2003 & 237.8 & 9.7 & 84.6 & & 332.1 \\
\hline 2004 & 234.3 & 9.6 & 79.9 & & 323.8 \\
\hline 2005 & 229.6 & 9.5 & 75.2 & & 314.2 \\
\hline 2006 & 224.8 & 9.6 & 70.5 & & 304.9 \\
\hline 2007 & 220.1 & 9.4 & 65.8 & & 295.2 \\
\hline 2008 & 215.3 & 9.4 & 61.1 & & 285.8 \\
\hline 2009 & 210.6 & 9.6 & 56.4 & & 276.6 \\
\hline 2010 & 197.9 & 9.8 & 51.7 & & 259.4 \\
\hline 2011 & 185.2 & 10.0 & 47.0 & & 242.1 \\
\hline 2012 & 172.5 & 10.1 & 42.3 & & 224.9 \\
\hline 2013 & 159.8 & 10.2 & 37.6 & & 207.6 \\
\hline 2014 & 147.1 & 10.3 & 32.9 & & 190.3 \\
\hline 2015 & 134.4 & 10.4 & 28.2 & & 173.0 \\
\hline 2016 & 121.7 & 10.5 & 23.5 & & 155.7 \\
\hline 2017 & 109.0 & 10.6 & 18.8 & & 138.4 \\
\hline 2018 & 96.3 & 10.7 & 14.1 & & 121.1 \\
\hline 2019 & 83.6 & 9.6 & 9.4 & & 102.5 \\
\hline 2020 & 70.8 & 7.2 & 4.7 & & 82.8 \\
\hline 2021 & 58.1 & 7.1 & & & 65.2 \\
\hline 2022 & 50.2 & 6.7 & & & 56.9 \\
\hline 2023 & 42.2 & 6.4 & & & 48.6 \\
\hline 2024 & 34.2 & 6.1 & & & 40.3 \\
\hline 2025 & 26.3 & 5.8 & & & 32.0 \\
\hline 2026 & 18.3 & 5.4 & & & 23.7 \\
\hline 2027 & 10.3 & 5.1 & & & 15.4 \\
\hline 2028 & 2.4 & 4.8 & & & 7.1 \\
\hline 2029 & 2.4 & 4.4 & & & 6.8 \\
\hline 2030 & 2.4 & 4.1 & & & 6.5 \\
\hline
\end{tabular}

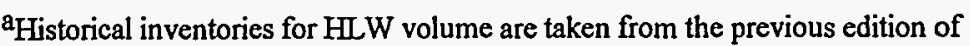
this report [i.e., DOE/RW-0006, Rev. 10 (December 1994)]. The inventories for 1994 and the projections through 2030 are taken from ref. 1. 
Table 2.2. Historical and projected cumulative decayed radioactivity of HLW stored in tanks, bins, and capsules, by site ${ }^{\mathrm{a}}$

\begin{tabular}{|c|c|c|c|c|c|}
\hline \multirow{2}{*}{$\begin{array}{l}\text { End of } \\
\text { calendar } \\
\text { year }\end{array}$} & \multicolumn{5}{|c|}{ Radioactivity, $10^{6} \mathrm{Ci}$} \\
\hline & Hanford & INEL & SRS & WVDP & Total \\
\hline 1980 & 576.7 & 53.4 & 699.0 & 33.5 & $1,362.6$ \\
\hline 1981 & 550.2 & 63.6 & 982.0 & 32.7 & $1,628.5$ \\
\hline 1982 & 437.1 & 71.6 & 828.8 & 31.9 & $1,369.4$ \\
\hline 1983 & 427.5 & 64.8 & 776.2 & 31.2 & $1,299.7$ \\
\hline 1984 & 470.2 & 58.6 & 795.9 & 30.5 & $1,355.2$ \\
\hline 1985 & 519.0 & 69.4 & 841.4 & 29.7 & $1,459.5$ \\
\hline 1986 & 534.6 & 60.6 & 794.7 & 29.1 & $1,419.0$ \\
\hline 1987 & 478.2 & 62.5 & 734.0 & 28.4 & $1,303.1$ \\
\hline 1988 & 447.4 & 67.0 & 664.4 & 27.9 & $1,206.7$ \\
\hline 1989 & 419.3 & 68.4 & 598.9 & 27.3 & $1,113.9$ \\
\hline 1990 & 399.3 & 63.2 & 561.6 & 26.7 & $1,050.8$ \\
\hline 1991 & 384.2 & 59.4 & 537.6 & 26.2 & $1,007.4$ \\
\hline 1992 & 372.1 & 50.8 & 632.4 & 25.9 & $1,081.2$ \\
\hline 1993 & 361.4 & 52.5 & 606.0 & 25.3 & $1,045.3$ \\
\hline 1994 & 348.0 & 51.6 & 534.5 & 24.7 & 958.8 \\
\hline 1995 & 339.9 & 50.5 & 502.2 & 24.1 & 916.7 \\
\hline 1996 & 332.1 & 49.1 & 461.5 & 15.7 & 858.4 \\
\hline 1997 & 324.4 & 47.7 & 432.5 & 7.6 & 812.1 \\
\hline 1998 & 316.9 & 46.5 & 404.8 & & 768.2 \\
\hline 1999 & 309.6 & 45.2 & 378.2 & & 733.0 \\
\hline 2000 & 302.4 & 44.1 & 352.7 & & 699.2 \\
\hline 2001 & 295.4 & 43.0 & 328.2 & & 666.6 \\
\hline 2002 & 288.6 & 42.0 & 304.7 & & 635.2 \\
\hline 2003 & 281.9 & 41.0 & 282.1 & & 605.0 \\
\hline 2004 & 275.4 & 39.9 & 260.4 & & 575.7 \\
\hline 2005 & 269.1 & 38.9 & 239.5 & & 547.5 \\
\hline 2006 & 262.8 & 38.1 & 219.5 & & 520.4 \\
\hline 2007 & 256.8 & 37.1 & 200.2 & & 494.1 \\
\hline 2008 & 250.8 & 36.3 & 181.8 & & 468.9 \\
\hline 2009 & 245.1 & 35.4 & 164.0 & & 444.5 \\
\hline 2010 & 232.2 & 34.6 & 147.0 & & 413.8 \\
\hline 2011 & 219.8 & 33.8 & 130.7 & & 384.3 \\
\hline 2012 & 207.9 & 33.0 & 115.0 & & 355.9 \\
\hline 2013 & 180.4 & 32.2 & 99.9 & & 312.6 \\
\hline 2014 & 154.1 & 31.5 & 85.5 & & 271.1 \\
\hline 2015 & 128.9 & 30.8 & 71.7 & & 231.3 \\
\hline 2016 & 104.7 & 30.1 & 58.4 & & 193.2 \\
\hline 2017 & 81.6 & 29.4 & 45.7 & & 156.7 \\
\hline 2018 & 59.5 & 28.7 & 33.5 & & 121.7 \\
\hline 2019 & 52.4 & 27.6 & 21.9 & & 101.9 \\
\hline 2020 & 45.5 & 26.9 & 10.7 & & 83.1 \\
\hline 2021 & 38.9 & 24.4 & & & 63.3 \\
\hline 2022 & 32.5 & 21.2 & & & 53.7 \\
\hline 2023 & 26.5 & 18.6 & & & 45.1 \\
\hline 2024 & 20.7 & 16.0 & & & 36.7 \\
\hline 2025 & 15.2 & 13.8 & & & 29.0 \\
\hline 2026 & 9.9 & 11.7 & & & 21.6 \\
\hline 2027 & 4.8 & 9.7 & & & 14.5 \\
\hline 2028 & & 7.6 & & & 7.6 \\
\hline 2029 & & 5.5 & & & 5.5 \\
\hline 2030 & & 3.9 & & & 3.9 \\
\hline
\end{tabular}

aHistorical inventories for $\mathrm{HLW}$ radioactivity are taken from the previous edition of this report [i.e., DOE/RW-0006, Rev. 10 (December 1994)]. The inventories for 1994 and the projections through 2030 are taken from ref. 1. 
Table 2.3. Historical and projected cumulative decayed thermal power of HLW stored in tanks, bins, and capsules, by site ${ }^{\mathrm{a}}$

\begin{tabular}{|c|c|c|c|c|c|}
\hline \multirow{2}{*}{$\begin{array}{l}\text { End of } \\
\text { calendar } \\
\text { year }\end{array}$} & \multicolumn{5}{|c|}{ Thermal power, $10^{3} \mathrm{~W}$} \\
\hline & Hanford & INEL & SRS & WVDP & Total \\
\hline 1980 & $1,142.7$ & 169.0 & $2,050.0$ & 96.9 & $3,458.6$ \\
\hline 1981 & $1,671.0$ & 210.0 & $2,900.0$ & 94.8 & $4,875.8$ \\
\hline 1982 & $1,261.5$ & 217.0 & $2,490.0$ & 92.6 & $4,061.1$ \\
\hline 1983 & $1,233.6$ & 190.0 & $2,279.6$ & 90.5 & $3,793.7$ \\
\hline 1984 & $1,388.9$ & 170.8 & $2,349.6$ & 88.4 & $3,997.7$ \\
\hline 1985 & $1,557.4$ & 209.9 & $2,537.2$ & 86.4 & $4,390.9$ \\
\hline 1986 & $1,605.9$ & 175.9 & $2,575.3$ & 84.5 & $4,441.6$ \\
\hline 1987 & $1,395.3$ & 182.5 & $2,151.9$ & 81.2 & $3,810.9$ \\
\hline 1988 & $1,306.4$ & 195.6 & $1,883.7$ & 80.9 & $3,466.6$ \\
\hline 1989 & $1,211.9$ & 199.2 & $1,673.7$ & 79.3 & $3,164.1$ \\
\hline 1990 & $1,150.3$ & 184.4 & $1,566.7$ & 76.9 & $2,978.3$ \\
\hline 1991 & $1,106.5$ & 172.0 & $1,509.3$ & 75.9 & $2,863.7$ \\
\hline 1992 & $1,073.1$ & 147.3 & $1,724.3$ & 79.1 & $3,023.8$ \\
\hline 1993 & $1,043.1$ & 153.7 & $1,615.3$ & 74.1 & $2,886.3$ \\
\hline 1994 & 999.8 & 150.8 & $1,497.3$ & 78.1 & $2,726.0$ \\
\hline 1995 & 976.7 & 146.6 & $1,407.4$ & 69.7 & $2,600.4$ \\
\hline 1996 & 954.1 & 142.2 & $1,300.3$ & 45.6 & $2,442.2$ \\
\hline 1997 & 932.1 & 138.3 & $1,226.7$ & 21.9 & $2,319.0$ \\
\hline 1998 & 910.5 & 135.1 & $1,155.5$ & & $2,201.1$ \\
\hline 1999 & 889.5 & 131.6 & $1,085.9$ & & $2,107.0$ \\
\hline 2000 & 868.9 & 128.1 & $1,017.8$ & & $2,014.8$ \\
\hline 2001 & 848.9 & 125.0 & 951.3 & & $1,925.2$ \\
\hline 2002 & 829.2 & 121.8 & 886.5 & & $1,837.5$ \\
\hline 2003 & 810.1 & 118.6 & 823.5 & & $1,752.2$ \\
\hline 2004 & 791.4 & 115.4 & 762.4 & & $1,669.1$ \\
\hline 2005 & 773.1 & 113.2 & 703.2 & & $1,589.4$ \\
\hline 2006 & 755.3 & 110.1 & 645.9 & & $1,511.2$ \\
\hline 2007 & 737.8 & 107.7 & 590.6 & & $1,436.2$ \\
\hline 2008 & 720.8 & 104.7 & 537.2 & & $1,362.7$ \\
\hline 2009 & 704.2 & 102.7 & 485.8 & & $1,292.6$ \\
\hline 2010 & 666.4 & 99.9 & 436.2 & & $1,202.5$ \\
\hline 2011 & 630.0 & 97.6 & 388.4 & & $1,115.9$ \\
\hline 2012 & 594.9 & 95.3 & 342.4 & & $1,032.6$ \\
\hline 2013 & 517.8 & 93.1 & 298.1 & & 909.0 \\
\hline 2014 & 443.9 & 91.0 & 255.5 & & 790.4 \\
\hline 2015 & 373.2 & 88.8 & 214.5 & & 676.5 \\
\hline 2016 & 305.5 & 86.8 & 175.1 & & 567.4 \\
\hline 2017 & 240.7 & 84.8 & 137.2 & & 462.7 \\
\hline 2018 & 178.8 & 82.8 & 100.8 & & 362.4 \\
\hline 2019 & 157.2 & 79.7 & 65.9 & & 302.8 \\
\hline 2020 & 136.5 & 77.7 & 32.3 & & 246.5 \\
\hline 2021 & 116.7 & 70.4 & & & 187.2 \\
\hline 2022 & 97.7 & 61.3 & & & 159.0 \\
\hline 2023 & 79.6 & 53.6 & & & 133.2 \\
\hline 2024 & 62.2 & 46.3 & & & 108.5 \\
\hline 2025 & 45.6 & 39.9 & & & 85.5 \\
\hline 2026 & 29.7 & 33.8 & & & 63.5 \\
\hline 2027 & 14.5 & 27.9 & & & 42.4 \\
\hline 2028 & & 21.9 & & & 21.9 \\
\hline 2029 & & 15.9 & & & 15.9 \\
\hline 2030 & & 11.1 & & & 11.1 \\
\hline
\end{tabular}

aHistorical inventories for $\mathrm{HLW}$ thermal power are taken from the previous edition of this report [i.e., DOE/RW-0006, Rev. 10 (December 1994)]. The inventories for 1994 and the projections through 2030 are taken from ref. 1. 
Table 2.4. Projected annual and cumulative volume $\left(10^{3} \mathrm{~m}^{3}\right)$ of $\mathrm{HLW}$ glass stored in canisters, by site $\mathrm{a}^{\mathrm{a}, \mathrm{b}}$

\begin{tabular}{|c|c|c|c|c|c|c|c|c|c|c|}
\hline \multirow{2}{*}{$\begin{array}{c}\text { End of } \\
\text { calendar } \\
\text { year }\end{array}$} & \multicolumn{2}{|c|}{ Hanford } & \multicolumn{2}{|c|}{$\operatorname{INEL}^{\mathrm{d}}$} & \multicolumn{2}{|c|}{ SRS $^{\mathrm{C}}$} & \multicolumn{2}{|c|}{ WVDPf } & \multicolumn{2}{|c|}{ Total } \\
\hline & Annual & Cumulative & Annual & Cumulative & Annual & Cumulative & Annual & Cumulative & Annual & Cumulative \\
\hline 1996 & & & & & 0.038 & 0.038 & 0.080 & 0.080 & 0.118 & 0.118 \\
\hline 1997 & & & & & 0.054 & 0.092 & 0.080 & 0.160 & 0.134 & 0.252 \\
\hline 1998 & & & & & 0.054 & 0.146 & 0.080 & 0.240 & 0.134 & 0.386 \\
\hline 1999 & & & & & 0.054 & 0.201 & 0.080 & 0.240 & 0.134 & 0.441 \\
\hline 2000 & & & & & 0.087 & 0.288 & & 0.240 & 0.087 & 0.528 \\
\hline 2001 & & & & & 0.089 & 0.377 & & 0.240 & 0.089 & 0.617 \\
\hline 2002 & & & & & 0.089 & 0.467 & & 0.240 & 0.089 & 0.707 \\
\hline 2003 & & & & & 0.089 & 0.556 & & 0.240 & 0.089 & 0.796 \\
\hline 2004 & & & & & 0.133 & 0.690 & & 0.240 & 0.133 & 0.930 \\
\hline 2005 & & & & & 0.133 & 0.823 & & 0.240 & 0.133 & 1.063 \\
\hline 2006 & & & & & 0.133 & 0.956 & & 0.240 & 0.133 & 1.196 \\
\hline 2007 & & & & & 0.133 & 1.090 & & 0.240 & 0.133 & 1.330 \\
\hline 2008 & & & & & 0.133 & 1.223 & & 0.240 & 0.133 & 1.463 \\
\hline 2009 & & & & & 0.130 & 1.353 & & 0.240 & 0.130 & 1.593 \\
\hline 2010 & 0.453 & 0.453 & & & 0.196 & 1.548 & & 0.240 & 0.649 & 2.241 \\
\hline 2011 & 0.453 & 0.905 & & & 0.196 & 1.744 & & 0.240 & 0.649 & 2.890 \\
\hline 2012 & 0.453 & 1.358 & & & 0.196 & 1.940 & & 0.240 & 0.649 & 3.538 \\
\hline 2013 & 0.503 & 1.861 & & & 0.196 & 2.136 & & 0.240 & 0.699 & 4.237 \\
\hline 2014 & 0.503 & 2.365 & & & 0.183 & 2.319 & & 0.240 & 0.686 & 4.924 \\
\hline 2015 & 0.503 & 2.868 & & & 0.183 & 2.502 & & 0.240 & 0.686 & 5.610 \\
\hline 2016 & 0.503 & 3.372 & & & 0.183 & 2.684 & & 0.240 & 0.686 & 6.296 \\
\hline 2017 & 0.503 & 3.875 & & & 0.188 & 2.872 & & 0.240 & 0.691 & 6.987 \\
\hline 2018 & 0.503 & 4.379 & & & 0.188 & 3.060 & & 0.240 & 0.691 & 7.678 \\
\hline 2019 & 0.453 & 4.831 & 0.012 & 0.012 & 0.188 & 3.248 & & 0.240 & 0.653 & 8.331 \\
\hline 2020 & 0.453 & 5.284 & 0.024 & 0.036 & 0.165 & 3.413 & & 0.240 & 0.641 & 8.972 \\
\hline 2021 & 0.453 & 5.736 & 0.015 & 0.050 & 0.165 & 3.578 & & 0.240 & 0.633 & 9.605 \\
\hline 2022 & 0.453 & 6.189 & 0.029 & 0.080 & & 3.578 & & 0.240 & 0.482 & 10.087 \\
\hline 2023 & 0.453 & 6.642 & 0.029 & 0.109 & & 3.578 & & 0.240 & 0.482 & 10.569 \\
\hline 2024 & 0.453 & 7.094 & 0.029 & 0.138 & & 3.578 & & 0.240 & 0.482 & 11.051 \\
\hline 2025 & 0.453 & 7.547 & 0.029 & 0.167 & & 3.578 & & 0.240 & 0.482 & 11.532 \\
\hline 2026 & 0.453 & 8.000 & 0.029 & 0.197 & & 3.578 & & 0.240 & 0.482 & 12.014 \\
\hline 2027 & 0.453 & 8.452 & 0.029 & 0.226 & & 3.578 & & 0.240 & 0.482 & 12.496 \\
\hline 2028 & 0.453 & 8.905 & 0.029 & 0.255 & & 3.578 & & 0.240 & 0.482 & 12.978 \\
\hline 2029 & & 8.905 & 0.029 & 0.284 & & 3.578 & & 0.240 & 0.029 & 13.007 \\
\hline 2030 & & 8.905 & 0.029 & 0.313 & & 3.578 & & 0.240 & 0.029 & 13.036 \\
\hline
\end{tabular}


a Taken from data given in ref. 1 . Glass may be in storage at the site, in transit to a repository, or in a repository.

bee Table 2.7 for the projected number of canisters.

'Hanford's reference canister has a diameter of $68 \mathrm{~cm}$ and is $457 \mathrm{~cm}$ long (about $2 \mathrm{ft}$ in diam by about $15 \mathrm{ft}$ in length). The nominal glass volume is expected to be $1.26 \mathrm{~m}^{3}$ with an average waste oxide loading of $45 \%$. Cesium and strontium capsules will also be disposed of in these canisters.

${ }^{d}$ At $D N E L$, each canister is assumed to contain nominally $0.92 \mathrm{~m}^{3}$ of a glass waste form made with $\mathrm{HLW}$ from the reprocessing of SNF.

At SRS, the DWPF canisters are $0.6 \mathrm{~m}$ in diam by $3.0 \mathrm{~m}$ in length (about $2 \mathrm{ft}$ in diam by about $10 \mathrm{ft}$ in length). Each canister is assumed to contain $0.625 \mathrm{~m}^{3}$ of glass [i.e., $85 \%$ of the usable capacity $\left(0.735 \mathrm{~m}^{3}\right)$ ] made with $\mathrm{HLW}$ from the reprocessing of SNF at SRS. The glass incorporates $36 \mathrm{wt} \%$ oxides from waste (28 wt \% from SNF and $8 \mathrm{wt} \%$ from processing chemicals) and $64 \mathrm{wt} \%$ oxides from nonradioactive glass frit. Volumes reported are for the glass waste form and not the canisters.

For WVDP, it is assumed that 300 canisters $0.6 \mathrm{~m}$ in diam by $3.0 \mathrm{~m}$ in length ( $2 \mathrm{ft}$ in diam by $10 \mathrm{ft}$ in length) are filled with waste glass during 1996-1998 and that each canister contains $0.8 \mathrm{~m}^{3}$ of glass at the filling temperature. 
Table 2.5. Projected annual and cumulative decayed radioactivity $\left(10^{6} \mathrm{Ci}\right)$ of $\mathrm{HLW}$ glass stored in canisters, by site ${ }^{a, b}$

\begin{tabular}{|c|c|c|c|c|c|c|c|c|c|c|}
\hline \multirow{2}{*}{$\begin{array}{l}\text { End of } \\
\text { calendar } \\
\text { year }\end{array}$} & \multicolumn{2}{|c|}{ Hanford $c$} & \multicolumn{2}{|c|}{ INEL } & \multicolumn{2}{|c|}{ SRS } & \multicolumn{2}{|c|}{ WVDP } & \multicolumn{2}{|c|}{ Total } \\
\hline & Annual & Cumulative & Annual & Cumulative & Annual & Cumulative & Annual & Cumulative & Annual & Cumulative \\
\hline 1996 & & & & & 18.31 & 18.31 & 7.75 & 7.75 & 26.06 & 26.06 \\
\hline 1997 & & & & & 17.88 & 35.75 & 7.62 & 15.37 & 25.50 & 51.12 \\
\hline 1998 & & & & & 17.47 & 52.37 & 7.03 & 22.40 & 24.50 & 74.77 \\
\hline 1999 & & & & & 17.07 & 68.20 & & 21.88 & 17.07 & 90.08 \\
\hline 2000 & & & & & 16.68 & 83.29 & & 21.37 & 16.68 & 104.66 \\
\hline 2001 & & & & & 16.30 & 97.66 & & 20.87 & 16.30 & 118.53 \\
\hline 2002 & & & & & 15.94 & 111.35 & & 20.39 & 15.94 & 131.74 \\
\hline 2003 & & & & & 15.58 & 124.36 & & 19.91 & 15.58 & 144.27 \\
\hline 2004 & & & & & 15.23 & 136.74 & & 19.45 & 15.23 & 156.19 \\
\hline 2005 & & & & & 14.89 & 148.50 & & 18.99 & 14.89 & 167.49 \\
\hline 2006 & & & & & 14.56 & 159.67 & & 18.55 & 14.56 & 178.22 \\
\hline 2007 & & & & & 14.24 & 170.27 & & 18.12 & 14.24 & 188.39 \\
\hline 2008 & & & & & 13.92 & 180.32 & & 17.69 & 13.92 & 198.01 \\
\hline 2009 & & & & & 13.61 & 189.84 & & 17.28 & 13.61 & 207.12 \\
\hline 2010 & 7.18 & 7.18 & & & 13.31 & 198.86 & & 16.88 & 20.49 & 222.91 \\
\hline 2011 & 7.01 & 14.02 & & & 13.02 & 207.38 & & 16.48 & 20.03 & 237.88 \\
\hline 2012 & 6.85 & 20.55 & & & 12.73 & 215.42 & & 16.10 & 19.58 & 252.07 \\
\hline 2013 & 22.70 & 42.77 & & & 12.45 & 223.02 & & 15.73 & 35.15 & 281.52 \\
\hline 2014 & 22.17 & 63.96 & & & 12.18 & 230.18 & & 15.36 & 34.35 & 309.49 \\
\hline 2015 & 21.66 & 84.14 & & & 11.91 & 236.91 & & 15.00 & 33.57 & 336.06 \\
\hline 2016 & 21.16 & 103.37 & & & 11.65 & 243.25 & & 14.65 & 32.82 & 361.26 \\
\hline 2017 & 20.67 & 121.65 & & & 11.40 & 249.19 & & 14.31 & 32.07 & 385.16 \\
\hline 2018 & 20.20 & 139.05 & & & 11.15 & 254.76 & & 13.98 & 31.35 & 407.79 \\
\hline 2019 & 5.82 & 141.65 & 0.42 & 0.42 & 10.91 & 259.98 & & 13.65 & 17.15 & 415.71 \\
\hline 2020 & 5.68 & 144.07 & 0.03 & 0.45 & 10.68 & 264.85 & & 13.33 & 16.39 & 422.69 \\
\hline 2021 & 5.55 & 146.30 & 1.92 & 2.36 & 10.45 & 269.39 & & 13.02 & 17.92 & 431.06 \\
\hline 2022 & 5.42 & 148.34 & 2.62 & 4.89 & & 263.39 & & 12.72 & 8.04 & 429.34 \\
\hline 2023 & 5.30 & 150.22 & 2.20 & 6.96 & & 257.53 & & 12.42 & 7.50 & 427.13 \\
\hline 2024 & 5.17 & 151.93 & 2.15 & 8.92 & & 251.81 & & 12.13 & 7.32 & 424.79 \\
\hline 2025 & 5.06 & 153.48 & 1.87 & 10.60 & & 246.23 & & 11.85 & 6.93 & 422.16 \\
\hline 2026 & 4.94 & 154.88 & 1.83 & 12.10 & & 240.78 & & 11.57 & 6.77 & 419.32 \\
\hline 2027 & 4.82 & 156.13 & 1.78 & 13.60 & & 235.45 & & 11.30 & 6.60 & 416.48 \\
\hline 2028 & 4.71 & 157.24 & 1.87 & 15.10 & & 230.25 & & 11.04 & 6.58 & 413.63 \\
\hline 2029 & & 153.62 & 1.93 & 16.70 & & 225.17 & & 10.78 & 1.93 & 406.27 \\
\hline 2030 & & 150.07 & 1.54 & 17.80 & & 220.22 & & 10.53 & 1.54 & 398.62 \\
\hline
\end{tabular}

a Taken from data given in ref. 1 .

badioactive decay is taken into account by each site by means of radioisotope generation and depletion codes.

${ }^{c}$ The significant increase in annual radioactivity for the years 2013-2018 reflects the accelerated processing schedule for the strontium and cesium capsules at Hanford (see Sect. 2.5.1). 
Table 2.6. Projected annual and cumulative decayed thermal power $\left(10^{3} \mathrm{~W}\right)$ of $\mathrm{HLW}$ glass stored in canisters, by site ${ }^{\mathrm{a}, \mathrm{b}}$

\begin{tabular}{|c|c|c|c|c|c|c|c|c|c|c|}
\hline \multirow{2}{*}{$\begin{array}{l}\text { End of } \\
\text { calendar } \\
\text { year }\end{array}$} & \multicolumn{2}{|c|}{ Hanford $^{c}$} & \multicolumn{2}{|c|}{ INEL } & \multicolumn{2}{|c|}{ SRS } & \multicolumn{2}{|c|}{ WVDP } & \multicolumn{2}{|c|}{ Total } \\
\hline & Annual & Cumulative & Annual & Cumulative & Annual & Cumulative & Annual & Cumulative & Annual & Cumulative \\
\hline 1996 & & & & & 51.59 & 51.59 & 22.46 & 22.46 & 74.05 & 74.05 \\
\hline 1997 & & & & & 50.71 & 101.39 & 22.08 & 44.54 & 72.79 & 145.93 \\
\hline 1998 & & & & & 49.87 & 149.49 & 20.38 & 64.92 & 70.25 & 214.41 \\
\hline 1999 & & & & & 49.01 & 195.85 & & 63.40 & 49.01 & 259.25 \\
\hline 2000 & & & & & 48.14 & 240.39 & & 61.92 & 48.14 & 302.31 \\
\hline 2001 & & & & & 47.26 & 283.11 & & 60.47 & 47.26 & 343.58 \\
\hline 2002 & & & & & 46.38 & 324.01 & & 59.05 & 46.38 & 383.06 \\
\hline 2003 & & & & & 45.49 & 363.09 & & 57.67 & 45.49 & 420.76 \\
\hline 2004 & & & & & 44.60 & 400.41 & & 56.32 & 44.60 & 456.73 \\
\hline 2005 & & & & & 43.72 & 436.00 & & 55.00 & 43.72 & 491.00 \\
\hline 2006 & & & & & 42.85 & 469.92 & & 53.71 & 42.85 & 523.63 \\
\hline 2007 & & & & & 41.99 & 502.23 & & 52.45 & 41.99 & 554.68 \\
\hline 2008 & & & & & 41.14 & 532.97 & & 51.22 & 41.14 & 584.19 \\
\hline 2009 & & & & & 40.31 & 562.20 & & 50.02 & 40.31 & 612.22 \\
\hline 2010 & 21.54 & 21.54 & & & 39.49 & 589.99 & & 48.85 & 61.03 & 660.37 \\
\hline 2011 & 21.04 & 42.08 & & & 38.69 & 616.38 & & 47.71 & 59.73 & 706.16 \\
\hline 2012 & 20.55 & 61.66 & & & 37.91 & 641.42 & & 46.59 & 58.46 & 749.68 \\
\hline 2013 & 63.39 & 123.63 & & & 37.14 & 665.18 & & 45.50 & 100.53 & 834.31 \\
\hline 2014 & 61.92 & 182.70 & & & 36.39 & 687.69 & & 44.43 & 98.31 & 914.83 \\
\hline 2015 & 60.50 & 238.98 & & & 35.65 & 709.02 & & 43.39 & 96.15 & 991.39 \\
\hline 2016 & 59.10 & 292.57 & & & 34.93 & 729.19 & & 42.38 & 94.03 & $1,064.15$ \\
\hline 2017 & 57.74 & 343.56 & & & 34.23 & 748.27 & & 41.39 & 91.97 & $1,133.22$ \\
\hline 2018 & 56.41 & 392.05 & & & 33.54 & 766.28 & & 40.42 & 89.95 & $1,198.75$ \\
\hline 2019 & 17.46 & 400.47 & 1.22 & 1.21 & 32.87 & 783.28 & & 39.47 & 51.55 & $1,224.43$ \\
\hline 2020 & 17.06 & 408.30 & 0.10 & 1.28 & 32.22 & 799.31 & & 38.55 & 49.38 & $1,247.43$ \\
\hline 2021 & 16.67 & 415.55 & 5.55 & 6.79 & 31.58 & 814.39 & & 37.64 & 53.80 & $1,274.38$ \\
\hline 2022 & 16.28 & 422.26 & 7.54 & 14.10 & & 797.63 & & 36.76 & 23.82 & $1,270.75$ \\
\hline 2023 & 15.91 & 428.44 & 6.34 & 20.00 & & 781.24 & & 35.90 & 22.25 & $1,265.58$ \\
\hline 2024 & 15.54 & 434.12 & 6.19 & 25.70 & & 765.22 & & 35.06 & 21.73 & $1,260.10$ \\
\hline 2025 & 15.19 & 439.31 & 5.39 & 30.40 & & 749.58 & & 34.24 & 20.58 & $1,253.53$ \\
\hline 2026 & 14.84 & 444.04 & 5.27 & 34.90 & & 734.29 & & 33.44 & 20.11 & $1,246.66$ \\
\hline 2027 & 14.50 & 448.32 & 5.15 & 39.20 & & 719.34 & & 32.66 & 19.65 & $1,239.52$ \\
\hline 2028 & 14.17 & 452.17 & 5.40 & 43.60 & & 704.74 & & 31.89 & 19.57 & $1,232.40$ \\
\hline 2029 & & 441.77 & 5.57 & 48.10 & & 690.48 & & 31.15 & 5.57 & $1,211.49$ \\
\hline 2030 & & 431.61 & 4.45 & 51.40 & & 676.54 & & 30.42 & 4.45 & $1,189.97$ \\
\hline
\end{tabular}

a Taken from data given in ref. 1.

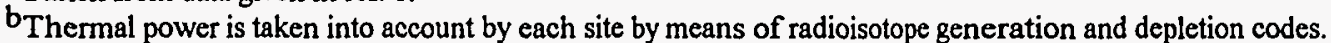

${ }^{c}$ The significant increase in annual thermal power for the years 2013-2018 reflects the accelerated processing schedule for the strontium and cesium capsules at Hanford (see Sect. 2.5.1). 
Table 2.7. Estimated potential number of HLW canisters, by site ${ }^{a}$

\begin{tabular}{|c|c|c|c|c|c|c|c|c|c|c|}
\hline \multirow{2}{*}{$\begin{array}{l}\text { End of } \\
\text { calendar } \\
\text { year }\end{array}$} & \multicolumn{2}{|c|}{ Hanford ${ }^{b}$} & \multicolumn{2}{|c|}{ INEL $^{c}$} & \multicolumn{2}{|c|}{ SRS $^{d}$} & \multicolumn{2}{|c|}{ WVDpe } & \multicolumn{2}{|c|}{ Total } \\
\hline & Annual & Cumulative & Annual & Cumulative & Annual & Cumulative & Annual & Cumulative & Annual & Cumulative \\
\hline 1996 & & & & & 60 & 60 & 100 & 100 & 160 & 160 \\
\hline 1997 & & & & & 87 & 147 & 100 & 200 & 187 & 347 \\
\hline 1998 & & & & & 87 & 234 & 100 & 300 & 187 & 534 \\
\hline 1999 & & & & & 87 & 321 & & 300 & 87 & 621 \\
\hline 2000 & & & & & 139 & 460 & & 300 & 139 & 760 \\
\hline 2001 & & & & & 143 & 603 & & 300 & 143 & 903 \\
\hline 2002 & & & & & 143 & 746 & & 300 & 143 & 1,046 \\
\hline 2003 & & & & & 143 & 889 & & 300 & 143 & 1,189 \\
\hline 2004 & & & & & 213 & 1,102 & & 300 & 213 & 1,402 \\
\hline 2005 & & & & & 213 & 1,315 & & 300 & 213 & 1,615 \\
\hline 2006 & & & & & 213 & 1,528 & & 300 & 213 & 1,828 \\
\hline 2007 & & & & & 213 & 1,741 & & 300 & 213 & 2,041 \\
\hline 2008 & & & & & 213 & 1,954 & & 300 & 213 & 2,254 \\
\hline 2009 & & & & & 207 & 2,161 & & 300 & 207 & 2,461 \\
\hline 2010 & 359 & 359 & & & 313 & 2,474 & & 300 & 672 & 3,133 \\
\hline 2011 & 359 & 718 & & & 313 & 2,787 & & 300 & 672 & 3,805 \\
\hline 2012 & 359 & 1,078 & & & 313 & 3,100 & & 300 & 672 & 4,478 \\
\hline 2013 & 400 & 1,477 & & & 313 & 3,413 & & 300 & 713 & 5,190 \\
\hline 2014 & 400 & 1,877 & & & 292 & 3,705 & & 300 & 692 & 5,882 \\
\hline 2015 & 400 & 2,276 & & & 292 & 3,997 & & 300 & 692 & 6,573 \\
\hline 2016 & 400 & 2,676 & & & 292 & 4,289 & & 300 & 692 & 7,265 \\
\hline 2017 & 400 & 3,076 & & & 300 & 4,589 & & 300 & 700 & 7,965 \\
\hline 2018 & 400 & 3,475 & & & 300 & 4,889 & & 300 & 700 & 8,664 \\
\hline 2019 & 359 & 3,834 & 27 & 27 & 300 & 5,189 & & 300 & 687 & 9,351 \\
\hline 2020 & 359 & 4,194 & 53 & 80 & 264 & 5,453 & & 300 & 676 & 10,027 \\
\hline 2021 & 359 & 4,553 & 33 & 113 & 264 & 5,717 & & 300 & 656 & 10,683 \\
\hline 2022 & 359 & 4,912 & 66 & 179 & & 5,717 & & 300 & 425 & 11,108 \\
\hline 2023 & 359 & 5,271 & 66 & 244 & & 5,717 & & 300 & 425 & 11,533 \\
\hline 2024 & 359 & 5,630 & 66 & 310 & & 5,717 & & 300 & 425 & 11,958 \\
\hline 2025 & 359 & 5,990 & 66 & 376 & & 5,717 & & 300 & 425 & 12,383 \\
\hline 2026 & 359 & 6,349 & 66 & 442 & & 5,717 & & 300 & 425 & 12,808 \\
\hline 2027 & 359 & 6,708 & 66 & 507 & & 5,717 & & 300 & 425 & 13,232 \\
\hline 2028 & 359 & 7,067 & 66 & 573 & & 5,717 & & 300 & 425 & 13,658 \\
\hline 2029 & & 7,067 & 66 & 639 & & 5,717 & & 300 & 66 & 13,723 \\
\hline 2030 & & 7,067 & 66 & 704 & & 5,717 & & 300 & 66 & 13,789 \\
\hline
\end{tabular}


a Taken from ref. 1. The projected waste volume, radioactivity, and thermal power values (Tables 2.4-2.6) are consistent with the number of canisters reported. The projections reported for Hanford, INEL, and SRS reflect major changes in the HLW solidification schedule. These changes are mainly caused by current $\mathrm{DOE}$ funding guidance.

bHanford's reference canister has a diameter of $68 \mathrm{~cm}$ and is $457 \mathrm{~cm}$ long (about $2 \mathrm{ft}$ in diam by about $15 \mathrm{ft}$ in length). The nominal glass volume is expected to be $1.26 \mathrm{~m}^{3}$ with an average waste oxide loading of $45 \%$. Cesium and strontium capsules will also be disposed of in these canisters.

${ }^{c}$ At $\mathbb{N E L}$, each canister is assumed to contain nominally $0.92 \mathrm{~m}^{3}$ of glass.

${ }^{\mathrm{d}}$ Canisters are $0.6 \mathrm{~m}$ in diam by $3.0 \mathrm{~m}$ in length (about $2 \mathrm{ft}$ in diam by about $10 \mathrm{ft}$ in length). Each canister is assumed to contain $0.625 \mathrm{~m}^{3}$ of glass made with HLW from the reprocessing of SNF at SRS. The glass incorporates $36 \mathrm{wt} \%$ oxides from waste ( $28 \mathrm{wt} \%$ from SNF and $8 \mathrm{wt} \%$ from processing chemicals) and $64 \mathrm{wt} \%$ oxides from nonradioactive glass frit.

e Canisters are $0.6 \mathrm{~m}$ in diam by $3.0 \mathrm{~m}$ in length (about $2 \mathrm{ft}$ in diam by $10 \mathrm{ft}$ in length). Each canister is assumed to contain $0.8 \mathrm{~m}^{3}$ of a borosilicate glass incorporating waste solids. 
Table 2.8. Current volume of HLW in storage by site through $1994^{\mathrm{a}}$

\begin{tabular}{|c|c|c|c|c|c|c|}
\hline \multirow{3}{*}{ Site } & \multicolumn{6}{|c|}{ Volume, $10^{3} \mathrm{~m}^{3}$} \\
\hline & \multicolumn{2}{|c|}{ Tank waste } & \multicolumn{2}{|c|}{ Capsules } & \multirow{2}{*}{$\begin{array}{l}\text { Canister } \\
\text { material }\end{array}$} & \multirow{2}{*}{ Total } \\
\hline & Liquid $b$ & Solid $\mathrm{c}^{\mathrm{S}}$ & $\mathrm{Sr}$ & Cs & & \\
\hline Hanford & 83.2 & 155.8 & 0.0010954 & 0.0024046 & & 239.0 \\
\hline INEL & 7.2 & 3.8 & & & & 11.0 \\
\hline SRS & 58.1 & 68.2 & & & & 126.3 \\
\hline WVDP & 2.0 & 0.14 & & & & 2.1 \\
\hline Total & 150.5 & 227.9 & 0.0010954 & 0.0024046 & 0 & 378.4 \\
\hline
\end{tabular}

${ }^{\text {a }}$ Taken from ref. 1.

biquid tank waste consists of free tank supernatant and drainable interstitial liquid.

${ }^{\mathrm{c}}$ Solid tank waste consists of sludge, salt cake, zeolite, calcine, and precipitate.

Table 2.9. Current radioactivity of $\mathrm{HLW}$ in storage by site through $1994^{\mathrm{a}}$

\begin{tabular}{|c|c|c|c|c|c|c|}
\hline \multirow{3}{*}{ Site } & \multicolumn{6}{|c|}{ Radioactivity, $10^{6} \mathrm{Ci}$} \\
\hline & \multicolumn{2}{|c|}{ Tank waste } & \multicolumn{2}{|c|}{ Capsules } & \multirow{2}{*}{$\begin{array}{l}\text { Canister } \\
\text { material }\end{array}$} & \multirow{2}{*}{ Total } \\
\hline & Liquid $^{b}$ & Solid ${ }^{\mathrm{c}}$ & $\mathrm{Sr}$ & Cs & & \\
\hline Hanford & 70.1 & 128.3 & 46.0 & 103.5 & & 347.9 \\
\hline INEL & 2.0 & 49.6 & & & & 51.6 \\
\hline SRS & 231.4 & 302.3 & & & & 533.7 \\
\hline WVDP & 3.5 & 21.2 & & & & 24.7 \\
\hline Total & 307.0 & 501.4 & 46.0 & 103.5 & 0 & 957.9 \\
\hline
\end{tabular}

${ }^{\text {a }}$ Taken from ref. 1.

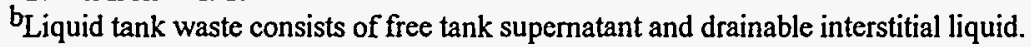

'Solid tank waste consists of sludge, salt cake, zeolite, calcine, and precipitate. 
Table 2.10. Current thermal power of HLW in storage by site through $1994^{\mathrm{a}}$

\begin{tabular}{|c|c|c|c|c|c|c|}
\hline \multirow{3}{*}{ Site } & \multicolumn{6}{|c|}{ Thermal power, $10^{3} \mathrm{~W}$} \\
\hline & \multicolumn{2}{|c|}{ Tank waste } & \multicolumn{2}{|c|}{ Capsules } & \multirow{2}{*}{$\begin{array}{l}\text { Canister } \\
\text { material }\end{array}$} & \multirow{2}{*}{ Total } \\
\hline & Liquid $^{b}$ & Solid $\mathrm{c}$ & Sr & Cs & & \\
\hline Hanford & 170.6 & 424.1 & 153.9 & 251.1 & & 999.7 \\
\hline INEL & 5.8 & 145.0 & & & & 150.8 \\
\hline SRS & 491.5 & 998.7 & & & & $1,490.2$ \\
\hline WVDP & 12.9 & 65.2 & & & & 78.1 \\
\hline Total & 680.8 & $1,633.0$ & 153.9 & 251.1 & 0 & $2,718.8$ \\
\hline
\end{tabular}

ataken from ref. 1.

biquid tank waste consists of free tank supernatant and drainable interstitial liquid.

'Solid tank waste consists of sludge, salt cake, zeolite, calcine, and precipitate.

Table 2.11. Major radionuclides comprising HLW and associated wastes at Hanford ${ }^{\mathrm{a}}$

\begin{tabular}{|c|c|c|c|c|c|c|}
\hline \multirow{3}{*}{ Radionuclides } & \multicolumn{6}{|c|}{ Radioactivity (Ci) by waste category } \\
\hline & \multicolumn{2}{|c|}{ Interim forms $b$} & \multicolumn{4}{|c|}{ Final forms $s^{c}$} \\
\hline & Tank waste & $\begin{array}{c}\text { Other } \\
\text { (capsules) }\end{array}$ & $\begin{array}{l}\text { HLW glass } \\
\text { canisters }\end{array}$ & $\begin{array}{l}\text { LLW form } \\
\text { (glass) }^{d}\end{array}$ & $\begin{array}{l}\text { Cumulative } \\
\text { out of system }\end{array}$ & $\begin{array}{c}\text { Other } \\
\text { (capsule canisters) }\end{array}$ \\
\hline${ }^{3} \mathrm{H}$ & e & e & $0.000 \mathrm{E}+00$ & & $0.000 \mathrm{E}+00$ & $0.000 \mathrm{E}+00$ \\
\hline${ }^{14} \mathrm{C}$ & $4.575 \mathrm{E}+03$ & $0.000 \mathrm{E}+00$ & $0.000 \mathrm{E}+00$ & & $4.555 \mathrm{E}+03$ & $0.000 \mathrm{E}+00$ \\
\hline${ }^{90} \mathrm{Sr}$ & $6.096 \mathrm{E}+07$ & $2.301 \mathrm{E}+07$ & $2.714 \mathrm{E}+07$ & & $0.000 \mathrm{E}+00$ & $1.024 \mathrm{E}+07$ \\
\hline${ }^{90} \mathrm{Y}$ & $6.096 \mathrm{E}+07$ & $2.301 E+07$ & $2.714 \mathrm{E}+07$ & & $0.000 \mathrm{E}+00$ & $1.024 \mathrm{E}+07$ \\
\hline${ }^{99} \mathrm{Tc}$ & $3.210 \mathrm{E}+04$ & $0.000 \mathrm{E}+00$ & $3.206 \mathrm{E}+04$ & & $4.173 \mathrm{E}+01$ & $0.000 \mathrm{E}+00$ \\
\hline${ }^{129} \mathrm{~T}$ & $2.980 \mathrm{E}-01$ & $0.000 \mathrm{E}+00$ & $0.000 \mathrm{E}+00$ & & $2.980 \mathrm{E}-01$ & $0.000 \mathrm{E}+00$ \\
\hline${ }^{137} \mathrm{Cs}$ & $3.860 \mathrm{E}+07$ & $5.318 \mathrm{E}+07$ & $1.760 \mathrm{E}+07$ & & $0.000 \mathrm{E}+00$ & $2.425 \mathrm{E}+07$ \\
\hline $137 m \mathrm{Ba}$ & $3.656 \mathrm{E}+07$ & $5.036 \mathrm{E}+07$ & $1.667 E+07$ & & $0.000 \mathrm{E}+00$ & $2.296 \mathrm{E}+07$ \\
\hline${ }^{151} \mathrm{Sm}$ & $1.066 \mathrm{E}+06$ & $0.000 \mathrm{E}+00$ & $8.206 \mathrm{E}+05$ & & $0.000 \mathrm{E}+00$ & $0.000 \mathrm{E}+00$ \\
\hline${ }^{238} \mathrm{Pu}$ & $1.427 \mathrm{E}+03$ & $0.000 \mathrm{E}+00$ & $1.091 \mathrm{E}+03$ & & $0.000 \mathrm{E}+00$ & $0.000 \mathrm{E}+00$ \\
\hline${ }^{239} \mathrm{Pu}$ & $2.635 E+04$ & $0.000 \mathrm{E}+00$ & $2.633 E+04$ & & $0.000 \mathrm{E} \div 00$ & $0.000 \mathrm{E}+00$ \\
\hline${ }^{240} \mathrm{Pu}$ & $6.692 E+03$ & $0.000 \mathrm{E}+00$ & $6.668 E+03$ & & $0.000 \mathrm{E}+00$ & $0.000 \mathrm{E}+00$ \\
\hline${ }^{24 !} \mathrm{Pu}$ & $9.775 \mathrm{E}+04$ & $0.000 \mathrm{E}+00$ & $1.903 E+04$ & & $0.000 \mathrm{E}+00$ & $0.000 E+00$ \\
\hline${ }^{242} \mathrm{Pu}$ & $2.802 \mathrm{E}-01$ & $0.000 \mathrm{E}+00$ & $2.802 \mathrm{E}-01$ & & $0.000 \mathrm{E}+00$ & $0.000 \mathrm{E}+00$ \\
\hline${ }^{241} \mathrm{Am}$ & $1.038 E+05$ & $0.000 E+00$ & $1.007 E+05$ & & $0.000 \mathrm{E}+00$ & $0.000 \mathrm{E}+00$ \\
\hline${ }^{242} \mathrm{Am}$ & $6.275 \mathrm{E}+01$ & $0.000 \mathrm{E}+00$ & $5.374 \mathrm{E}+01$ & & $0.000 \mathrm{E}+00$ & $0.000 \mathrm{E}+00$ \\
\hline Total & $1.984 E+08$ & $1.495 E+08$ & $8.955 \mathrm{E}+07$ & & $4.597 \mathrm{E}+03$ & $6.769 \mathrm{E}+07$ \\
\hline
\end{tabular}

a Data taken from ref. l(a).

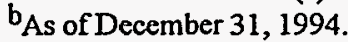

cAs of December 31, 2028.

$\mathrm{d}_{\text {Radionuclide distribution projections have not been finalized for the final } L L W \text { form. }}$

'Estimate of December 31,1994 , inventory for ${ }^{3} \mathrm{H}$ is currently unavailable. 
Table 2.12. Major radionuclides comprising HLW and associated wastes at INEL $^{a}$

\begin{tabular}{|c|c|c|c|c|}
\hline \multirow{3}{*}{ Radionuclides } & \multicolumn{4}{|c|}{ Radioactivity ( $\mathrm{Ci}$ ) by waste category } \\
\hline & \multicolumn{2}{|c|}{ Interim forms $\mathrm{s}^{\mathrm{b}}$} & \multicolumn{2}{|c|}{ Final forms ${ }^{c}$} \\
\hline & Liquid & Calcine & Glass & Grout \\
\hline${ }^{90} \mathrm{Sr}$ & $4.52 E+05$ & $1.16 \mathrm{E}+07$ & $3.54 \mathrm{E}+06$ & $7.07 \mathrm{E}+02$ \\
\hline${ }^{90} \mathrm{Y}$ & $4.52 \mathrm{E}+05$ & $1.16 E+07$ & $3.54 \mathrm{E}+06$ & $7.07 E+02$ \\
\hline${ }^{106} \mathrm{Ru}$ & $1.66 \mathrm{E}+03$ & $1.04 \mathrm{E}+04$ & $4.18 \mathrm{E}-12$ & $4.18 \mathrm{E}-12$ \\
\hline${ }^{106} \mathrm{Rh}$ & $1.66 \mathrm{E}+03$ & $1.04 \mathrm{E}+04$ & $4.18 \mathrm{E}-12$ & $4.18 E-12$ \\
\hline${ }^{134} \mathrm{Cs}$ & $1.04 \mathrm{E}+04$ & $1.43 E+05$ & $6.72 \mathrm{E}-03$ & $1.34 \mathrm{E}-06$ \\
\hline${ }^{137} \mathrm{Cs}$ & $5.33 \mathrm{E}+05$ & $1.33 E+07$ & $4.21 \mathrm{E}+06$ & $8.43 E+02$ \\
\hline${ }^{137 m} \mathrm{Ba}$ & $5.05 E+05$ & $1.26 \mathrm{E}+07$ & $3.99 \mathrm{E}+06$ & $7.99 E+02$ \\
\hline${ }^{144} \mathrm{Ce}$ & $9.27 \mathrm{E}+03$ & $5.33 E+04$ & $2.46 \mathrm{E}-15$ & $4.92 \mathrm{E}-19$ \\
\hline${ }^{144} \mathrm{Pr}$ & $9.27 \mathrm{E}+03$ & $5.33 E+04$ & $2.46 \mathrm{E}-15$ & $4.92 \mathrm{E}-19$ \\
\hline${ }^{147} \mathrm{Pm}$ & $0.00 E+00$ & $1.94 E+05$ & $3.01 \mathrm{E}-01$ & $6.02 \mathrm{E}-05$ \\
\hline${ }^{154} \mathrm{Eu}$ & $3.46 \mathrm{E}+03$ & $6.50 \mathrm{E}+04$ & $1.12 E+03$ & $2.24 \mathrm{E}-01$ \\
\hline Total & $1.98 \mathrm{E}+06$ & $4.96 E+07$ & $1.53 E+07$ & $3.06 \mathrm{E}+03$ \\
\hline
\end{tabular}

aData taken from ref. 1(b).

${ }^{b}$ As of December 31, 1994.

cAs of December 31, 2045. 
Table 2.13. Major radionuclides comprising HLW and associated wastes at SRS ${ }^{a}$

\begin{tabular}{|c|c|c|c|c|}
\hline \multirow{3}{*}{ Radionuclides } & \multicolumn{4}{|c|}{ Radioactivity ( $\mathrm{Ci}$ ) by waste category } \\
\hline & \multirow{2}{*}{$\frac{\text { Interim form }^{b}}{\text { Tank waste }}$} & \multicolumn{3}{|c|}{ Final forms ${ }^{c}$} \\
\hline & & $\begin{array}{l}\text { Canister } \\
\text { material }\end{array}$ & $\begin{array}{c}\text { Saltstone } \\
\text { (LLW) }\end{array}$ & Outfall \\
\hline${ }^{3} \mathrm{H}$ & $8.53 E+04$ & $3.25 \mathrm{E}+03$ & $8.27 \mathrm{E}-01$ & d \\
\hline${ }^{14} \mathrm{C}$ & $2.00 \mathrm{E}+01$ & $7.63 E-01$ & $1.94 \mathrm{E}-04$ & d \\
\hline${ }^{90} \mathrm{Sr}$ & $1.22 \mathrm{E}+08$ & $4.66 \mathrm{E}+06$ & $1.18 \mathrm{E}+03$ & d \\
\hline${ }^{90} \mathrm{Y}$ & $1.22 \mathrm{E}+08$ & $4.66 E+06$ & $1.18 \mathrm{E}+03$ & d \\
\hline${ }^{99} \mathrm{Tc}$ & $2.28 \mathrm{E}+04$ & $8.69 E+02$ & $2.21 \mathrm{E}-01$ & d \\
\hline${ }^{129} \mathrm{I}$ & $3.68 \mathrm{E}+01$ & $1.41 \mathrm{E}+00$ & $3.57 \mathrm{E}-04$ & d \\
\hline${ }^{137} \mathrm{Cs}$ & $1.19 \mathrm{E}+08$ & $4.53 \mathrm{E}+06$ & $1.15 \mathrm{E}+03$ & d \\
\hline $137 \mathrm{ma}$ & $1.12 \mathrm{E}+08$ & $4.28 \mathrm{E}+06$ & $1.09 E+03$ & d \\
\hline${ }^{238} \mathrm{Pu}$ & $1.73 E+06$ & $6.59 E+04$ & $1.68 \mathrm{E}+01$ & d \\
\hline${ }^{239} \mathrm{Pu}$ & $3.64 \mathrm{E}+04$ & $1.39 E+03$ & $3.54 \mathrm{E}-01$ & d \\
\hline${ }^{240} \mathrm{Pu}$ & $1.66 \mathrm{E}+04$ & $6.32 E+02$ & $1.61 \mathrm{E}-01$ & d \\
\hline${ }^{241} \mathrm{Pu}$ & $7.91 \mathrm{E}+05$ & $3.02 E+04$ & $7.67 \mathrm{E}+00$ & d \\
\hline${ }^{242} \mathrm{Pu}$ & $2.84 \mathrm{E}+01$ & $1.08 \mathrm{E}+00$ & $2.76 \mathrm{E}-04$ & d \\
\hline${ }^{241} \mathrm{Am}$ & $2.35 E+04$ & $8.97 \mathrm{E}+02$ & $2.28 \mathrm{E}-01$ & d \\
\hline${ }^{242 \mathrm{~m}} \mathrm{Am}$ & $6.41 \mathrm{E}+01$ & $2.45 E+00$ & $6.22 \mathrm{E}-04$ & d \\
\hline${ }^{232} \mathrm{Th}$ & $1.47 \mathrm{E}+00$ & $5.62 \mathrm{E}-02$ & $1.43 \mathrm{E}-05$ & d \\
\hline${ }^{233} \mathrm{U}$ & $1.08 E+02$ & $4.13 E+00$ & $1.05 E-03$ & d \\
\hline${ }^{234} \mathrm{U}$ & $3.01 \mathrm{E}+01$ & $1.15 E+00$ & $2.92 \mathrm{E}-04$ & d \\
\hline${ }^{237} \mathrm{~Np}$ & $7.04 \mathrm{E}+01$ & $2.69 \mathrm{E}+00$ & $6.83 E-04$ & d \\
\hline${ }^{244} \mathrm{Cm}$ & $2.60 \mathrm{E}+03$ & $9.92 \mathrm{E}+01$ & $2.52 \mathrm{E}-02$ & d \\
\hline Total & $4.78 E+08$ & $1.82 \mathrm{E}+07$ & $4.64 E+03$ & d \\
\hline
\end{tabular}

a Data taken from refs. 1(c) and 1(d).

${ }^{b}$ As of December 31, 1994.

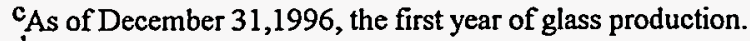

dNegligible contribution. 
Table 2.14. Major radionuclides comprising HLW and associated wastes at WVDP ${ }^{a}$

\begin{tabular}{|c|c|c|c|c|}
\hline \multirow{3}{*}{ Radionuclides } & \multicolumn{4}{|c|}{ Radioactivity, $\mathrm{Ci}$, by waste waste category } \\
\hline & \multirow{2}{*}{$\frac{\text { Interim form }^{b}}{\text { Tank waste }}$} & \multicolumn{3}{|c|}{ Final forms ${ }^{c}$} \\
\hline & & Canister material & LLW form & Emissions \\
\hline${ }^{90} \mathrm{Sr}$ & $5.93 E+06$ & & & \\
\hline${ }^{90} \mathrm{Y}$ & $5.93 E+06$ & & & \\
\hline${ }^{106} \mathrm{Ru}$ & $8.96 \mathrm{E}-01$ & & & \\
\hline${ }^{106} \mathrm{Rh}$ & $8.96 \mathrm{E}-01$ & & & \\
\hline${ }^{134} \mathrm{Cs}$ & $1.30 E+03$ & & & \\
\hline${ }^{135} \mathrm{Cs}$ & $1.56 \mathrm{E}+02$ & & & \\
\hline${ }^{137} \mathrm{Cs}$ & $6.57 \mathrm{E}+06$ & & & \\
\hline${ }^{137} \mathrm{~m} \mathrm{Ba}$ & $6.21 E+06$ & & & \\
\hline${ }^{147} \mathrm{Pm}$ & $9.00 \mathrm{E}+01$ & & & \\
\hline${ }^{238} \mathrm{Pu}$ & $7.56 \mathrm{E}+03$ & & & \\
\hline${ }^{239} \mathrm{Pu}$ & $2.11 E+00$ & & & \\
\hline${ }^{241} \mathrm{Pu}$ & $1.04 \mathrm{E}+03$ & & & \\
\hline${ }^{241} \mathrm{Am}$ & $9.10 \mathrm{E}+04$ & & & \\
\hline${ }^{244} \mathrm{Cm}$ & $1.04 E+01$ & & & \\
\hline Total & $2.47 \mathrm{E}+07$ & & & \\
\hline
\end{tabular}

a Data taken from ref. 1(e).

$b_{\text {As of December 31, } 1994 .}$

CRadionuclide information for the final forms generated from HLW treatment is currently unavailable. 
Table 2.15. Significant revisions and changes in the current values for HLW compared to the values in the previous year ${ }^{\mathrm{a}, \mathrm{b}}$

\begin{tabular}{|c|c|c|c|c|}
\hline Waste characteristics & $\begin{array}{l}\text { Previous report } \\
\text { values }^{\mathrm{a}}\end{array}$ & $\begin{array}{l}\text { Significant revisions } \\
\text { and changes }\end{array}$ & Updated values $^{b}$ & Explanation \\
\hline \multicolumn{5}{|c|}{ Hanford Site } \\
\hline Number of canisters & See Table 2.3 & $\begin{array}{l}\text { Projected number of } \\
\text { canisters significantly } \\
\text { reduced }\end{array}$ & See Table 2.7 & $\begin{array}{l}\text { Hanford selected a canister with a larger } \\
\text { volume than the canister used for previous } \\
\text { estimates }\end{array}$ \\
\hline \multicolumn{5}{|c|}{ Idaho National Engineering Laboratory } \\
\hline Number of canisters & See Table 2.3 & $\begin{array}{l}\text { Canister production } \\
\text { schedule updated }\end{array}$ & See Table 2.7 & $\begin{array}{l}\text { Based on current funding guidance from DOE } \\
\text { and the INEL Full Treatment Alternative } \\
\text { (see ref. 15) }\end{array}$ \\
\hline \multicolumn{5}{|c|}{ Savannah River Site } \\
\hline Number of canisters & See Table 2.3 & $\begin{array}{l}\text { Canister production } \\
\text { schedule updated }\end{array}$ & See Table 2.7 & $\begin{array}{l}\text { Based on current funding guidance from DOE } \\
\text { and the SRS High-Level Waste System Plan } \\
\text { Revision } 5(U) \text { (see ref. 16) }\end{array}$ \\
\hline \multicolumn{5}{|c|}{ West Valley Demonstration Project } \\
\hline $\begin{array}{l}\text { Radioactivity and thermal } \\
\text { power }\end{array}$ & $\begin{array}{l}\text { See Tables } 2.1 \text { and } \\
2.4-2.8\end{array}$ & $\begin{array}{l}\text { Radioactivity values } \\
\text { for } 1995,1996, \text { and } \\
1997 \text { were updated }\end{array}$ & $\begin{array}{l}\text { See Tables } 2.2,2.3,2.9 \text {, } \\
\text { and } 2.10\end{array}$ & $\begin{array}{l}\text { The radioactivity values did not reflect accurate } \\
\text { sludge radioactivity values for yttrium and } \\
\text { strontium in the previous report. The current } \\
\text { report reflects the updated values }\end{array}$ \\
\hline
\end{tabular}

a Data are for December 31, 1993. See tables and text cited in Chapter 2 of ref. 6 (DOE/RW-0006, Rev. 10).

b Data are for December 31, 1994, as reported in this document (DOE/RW-0006, Rev. 11). 
Table 2.16. Representative chemical composition of future HLW glass to be generated at Hanford ${ }^{a}$

\begin{tabular}{ll||lr}
\hline Component & Wt $\%$ & Component & Wt \% \\
\hline $\mathrm{Al}_{2} \mathrm{O}_{3}$ & 6.01 & $\mathrm{Na}_{2} \mathrm{O}$ & 11.28 \\
$\mathrm{~B}_{2} \mathrm{O}_{3}$ & 9.70 & $\mathrm{Na}_{2} \mathrm{SO}_{4}$ & 0.15 \\
$\mathrm{Bi}_{2} \mathrm{O}_{3}$ & 0.97 & $\mathrm{NiO}$ & 0.99 \\
$\mathrm{CaO}$ & 0.95 & $\mathrm{P}_{2} \mathrm{O}_{5}$ & 2.18 \\
$\mathrm{Ce}_{2} \mathrm{O}_{3}$ & 1.20 & $\mathrm{PbO}_{2}$ & 0.15 \\
$\mathrm{Cr}_{2} \mathrm{O}_{3}$ & 0.21 & $\mathrm{SiO}_{2}$ & 46.08 \\
$\mathrm{Fe}_{2} \mathrm{O}_{3}$ & 5.09 & $\mathrm{SrO}$ & 0.19 \\
$\mathrm{~K}_{2} \mathrm{O}$ & 0.10 & $\mathrm{ThO}_{2}$ & 0.07 \\
$\mathrm{La}_{2} \mathrm{O}_{3}$ & 0.20 & UO & 6.76 \\
$\mathrm{Li}_{2} \mathrm{O}$ & 2.69 & $\mathrm{ZrO}_{2}$ & 3.27 \\
$\mathrm{MnO}_{2}$ & 0.84 & Other & 0.37 \\
$\mathrm{NaF}$ & 0.57 & & Total \\
\hline
\end{tabular}

a Data taken from ref. 1(a).

Table 2.17. Representative chemical composition of future HLW glass to be produced at INEL ${ }^{a}$

\begin{tabular}{lccc}
\hline \multirow{2}{*}{$\begin{array}{c}\text { Chemical } \\
\text { compound }\end{array}$} & \multicolumn{2}{c}{ Glass, wt \%, formed from high-activity fraction from } \\
\cline { 2 - 4 } & $\begin{array}{c}\text { Dissolved } \\
\mathrm{Zr} \text { calcine }\end{array}$ & $\begin{array}{c}\text { Dissolved } \\
\text { Al calcine }\end{array}$ & Na-bearing-waste \\
\hline $\mathrm{Al}_{2} \mathrm{O}_{3}$ & 0.5 & 12.7 & 17.2 \\
$\mathrm{AMP}^{\mathrm{b}}$ & 0.8 & 6.6 & 11.3 \\
$\mathrm{~B}_{2} \mathrm{O}_{3}$ & 12.2 & 8.1 & \\
$\mathrm{CaF}_{2}$ & 14.5 & & \\
$\mathrm{CaO}$ & 0.4 & & 0.1 \\
$\mathrm{Cs}_{2} \mathrm{O}$ & & 0.1 & 13.8 \\
$\mathrm{Fe}_{2} \mathrm{O}_{3}$ & 12.9 & 18.0 & \\
$\mathrm{Na}_{2} \mathrm{O}$ & 0.1 & & 57.6 \\
$\mathrm{P}_{2} \mathrm{O}_{5}$ & 56.8 & 54.4 & \\
$\mathrm{SiO}_{2}$ & 1.8 & & 100.0 \\
$\mathrm{ZrO}_{2}$ & 100.0 & 100.0 & \\
$\mathrm{Total}$ & & & \\
\hline
\end{tabular}

aData taken from ref. 1(b); flowsheet estimate, not verified by laboratory tests.

bAmmoniummolybdophosphate. 
Table 2.18. Representative chemical composition of future HLW glass to be generated at SRS ${ }^{\mathrm{a}}$

\begin{tabular}{lr||cr}
\hline Component & $\mathrm{Wt} \%$ & Component & $\mathrm{Wt} \%$ \\
\hline $\mathrm{Al}_{2} \mathrm{O}_{3}$ & 3.9 & $\mathrm{MgO}$ & 2.0 \\
$\mathrm{~B}_{2} \mathrm{O}_{3}$ & 7.3 & $\mathrm{MnO}$ & 1.2 \\
$\mathrm{CaO}$ & 0.6 & $\mathrm{Na}_{2} \mathrm{O}$ & 8.7 \\
$\mathrm{Ca}_{3}\left(\mathrm{PO}_{4}\right)_{2}$ & 1.1 & $\mathrm{NiO}$ & 0.1 \\
$\mathrm{Cr}_{2} \mathrm{O}_{3}$ & 0.2 & $\mathrm{SiO}_{2}$ & 53.4 \\
$\mathrm{CuO}$ & 0.4 & $\mathrm{TiO}_{2}$ & 0.3 \\
$\mathrm{FeO}_{\mathrm{O} O}$ & 1.1 & $\mathrm{U}_{3} \mathrm{O}_{8}$ & 0.9 \\
$\mathrm{Fe}_{2} \mathrm{O}_{3}$ & 11.1 & $\mathrm{ZnO}$ & 0.1 \\
$\mathrm{~K}_{2} \mathrm{O}$ & 2.4 & Other & 0.4 \\
$\mathrm{Li}_{2} \mathrm{O}$ & 4.8 & & 100.0 \\
& & Total & \\
\hline
\end{tabular}

aData taken from ref. 1(c).

Table 2.19. Representative chemical composition of future HLW glass to be generated at WVDP ${ }^{a}$

\begin{tabular}{|c|c|c|c|}
\hline Component & Wt $\%$ & Composition & Wt \% \\
\hline $\mathrm{Al}_{2} \mathrm{O}_{3}$ & 6.00 & $\mathrm{Nd}_{2} \mathrm{O}_{3}$ & 0.14 \\
\hline $\mathrm{B}_{2} \mathrm{O}_{3}$ & 12.89 & $\mathrm{NiO}$ & 0.25 \\
\hline $\mathrm{BaO}$ & 0.16 & $\mathrm{P}_{2} \mathrm{O}_{5}$ & 1.20 \\
\hline $\mathrm{CaO}$ & 0.48 & PdO & 0.03 \\
\hline $\mathrm{Ce}_{2} \mathrm{O}_{3}$ & 0.31 & $\mathrm{Pr}_{6} \mathrm{O}_{11}$ & 0.04 \\
\hline $\mathrm{CoO}$ & 0.02 & $\mathrm{Rh}_{2} \mathrm{O}_{3}$ & 0.02 \\
\hline $\mathrm{Cr}_{2} \mathrm{O}_{3}$ & 0.14 & $\mathrm{RuO}_{2}$ & 0.08 \\
\hline $\mathrm{Cs}_{2} \mathrm{O}$ & 0.08 & $\mathrm{SO}_{3}$ & 0.23 \\
\hline $\mathrm{CuO}$ & 0.03 & $\mathrm{SiO}_{2}$ & 40.98 \\
\hline $\mathrm{Fe}_{2} \mathrm{O}_{3}$ & 12.02 & $\mathrm{Sm}_{2} \mathrm{O}_{3}$ & 0.03 \\
\hline $\mathrm{K}_{2} \mathrm{O}$ & 5.00 & $\mathrm{SrO}$ & 0.02 \\
\hline $\mathrm{La}_{2} \mathrm{O}_{3}$ & 0.04 & $\mathrm{ThO}_{2}$ & 3.56 \\
\hline $\mathrm{Li}_{2} \mathrm{O}$ & 3.71 & $\mathrm{TiO}_{2}$ & 0.80 \\
\hline $\mathrm{MgO}$ & 0.89 & $\mathrm{UO}_{3}$ & 0.63 \\
\hline $\mathrm{MnO}$ & 0.82 & $\mathrm{Y}_{2} \mathrm{O}_{3}$ & 0.02 \\
\hline $\mathrm{MoO}_{3}$ & 0.04 & $\mathrm{ZnO}$ & 0.02 \\
\hline \multirow[t]{2}{*}{$\mathrm{Na}_{2} \mathrm{O}$} & 8.00 & $\mathrm{ZrO}_{2}$ & 1.32 \\
\hline & & Total & 100.00 \\
\hline
\end{tabular}

a Data taken from ref. $1(\mathrm{e})$. 


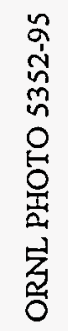

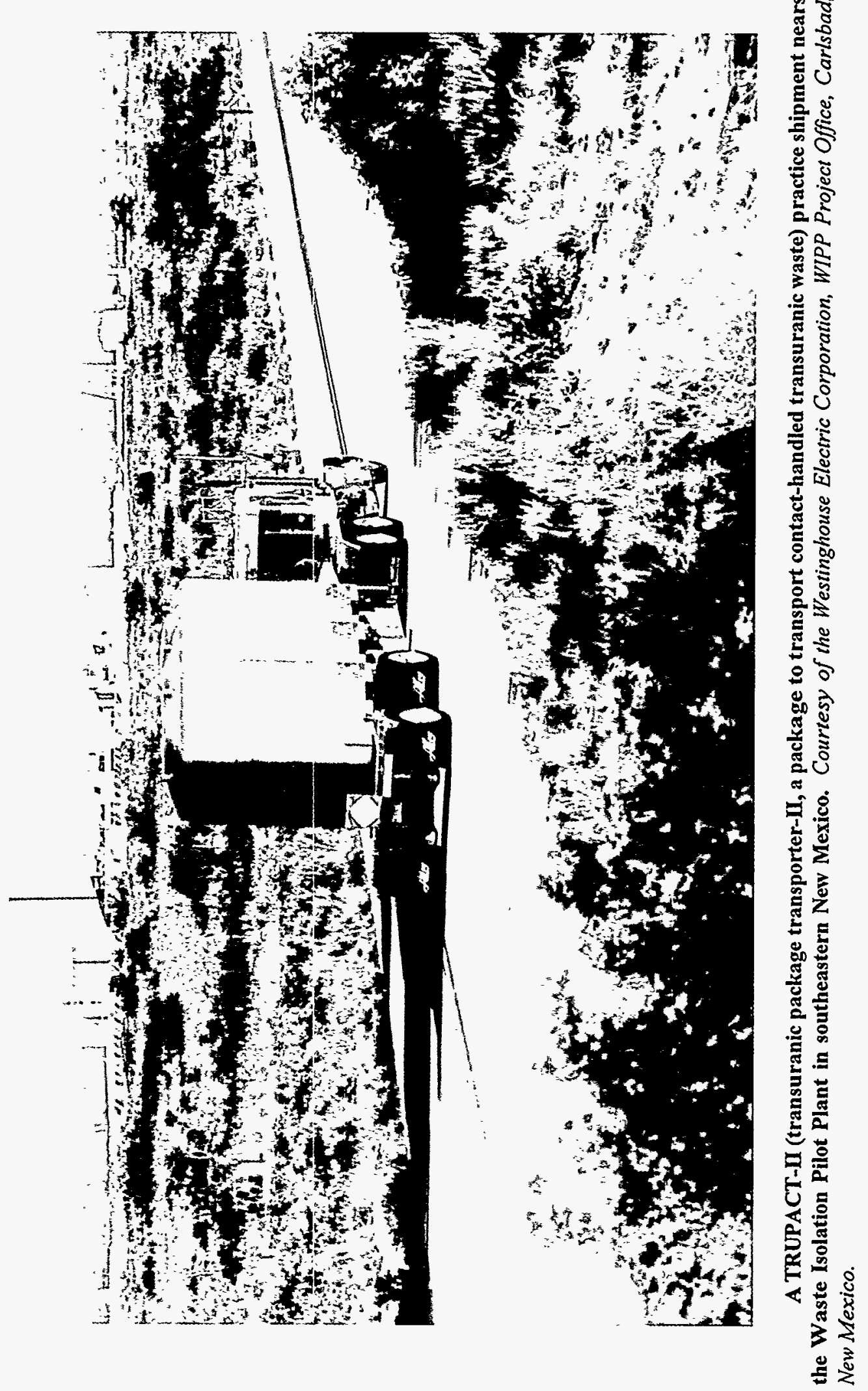




\section{TRANSURANIC WASTE}

\subsection{INTRODUCTION}

This chapter presents information on the inventories and characteristics of transuranic waste (TRUW) at various DOE waste generator and/or storage sites in the United States. TRUW is a waste category specific to DOE; it does not apply to wastes regulated by the U.S. Nuclear Regulatory Commission. DOE Order 5820.2A defines TRUW as waste that (1) is contaminated with alpha-emitting transuranic (TRU) (i.e., atomic numbers greater than 92) radionuclides with half-lives greater than 20 years and (2) contains a total concentration of such radionuclides in excess of $100 \mathrm{nCi} / \mathrm{g}$ of waste at the time of assay. ${ }^{1}$

DOE Order 5820.2A also states that heads of field elements can determine that other alpha-contaminated waste at the site(s) may be managed as TRUW. ${ }^{1}$ As a consequence of this provision, wastes containing radionuclides such as ${ }^{233} \mathrm{U}$, ${ }^{241} \mathrm{Pu}$, and ${ }^{244} \mathrm{Cm}$, which do not meet the strict definition of TRU radionuclides because of their respective atomic numbers or half-lives, may be managed as TRUW at some sites.

Most TRUW exists in solid form (e.g.; items such as protective clothing, paper, rags, glass, miscellaneous tools, and equipment that have become contaminated with TRU radionuclides). ${ }^{2}$ Some TRUW is in the form of sludges or liquids resulting from chemical processing for recovery of plutonium or other TRU elements. ${ }^{2}$ Some of the liquids have been solidified, and some sludges have been dewatered. All sludge and liquid wastes scheduled for disposal in the Waste Isolation Pilot Plant (WIPP) will be solidified prior to shipment to meet the current WIPP waste acceptance criteria. ${ }^{3}$

Before 1970, TRUW was disposed of on-site in shallow, landfill-type, DOE-owned burial sites. TRUW disposed of in this manner is referred to as "buried" TRUW. In 1970, DOE's predecessor agency, the U.S. Atomic Energy Commission, concluded that waste containing long-lived alpha-emitting radionuclides should have greater confinement from the environment. Thus, TRUW generated since the early 1970s has been segregated from other waste types and placed in retrievable storage pending shipment and final disposal in a permanent geologic repository. ${ }^{4}$ This waste is referred to as "retrievably stored" TRUW.

Retrievably stored waste is contained in a variety of packagings (e.g., metal drums and wooden and metal boxes) and is stored in various facilities such as earthmounded berms, concrete culverts, buildings, and on outdoor pads. Many of these facilities have been upgraded and are now in compliance with applicable hazardous waste storage regulations.

TRUW packages are classified as either "contact handled" ( $\mathrm{CH})$ or "remote handled" (RH), depending on the radiation level at the surface of the package at the time of packaging. If this level exceeds $200 \mathrm{mrem} / \mathrm{h}$, the package is classified as RH TRUW.

CH TRUW contains relatively small quantities of fission and activation products that produce highly penetrating radiation; typically, its emissions consists mostly of alpha particles and some neutrons and primarily low-energy gamma and X-rays. RH TRUW typically contains a greater proportion of fission and activation products that produce highly penetrating radiation and produce a higher level of radiation at the surface of the package.

TRUW waste which contains, in addition to radioactive constituents, hazardous constituents defined and regulated in accordance with the Resource Conservation and Recovery Act (RCRA) is defined as mixed TRUW. Examples of mixed TRUW are radionuclide-contaminated sludges from plutonium recovery, discarded materials contaminated with both solvents and radioactive materials, scintillation fluids, and discarded contaminated lead shielding. Mixed TRUW must be managed to comply with both the applicable hazardous waste regulations (e.g., RCRA), as well as the regulations applying only to radioactive TRUW.

Some TRUW may also be contaminated with hazardous materials defined by regulations other than RCRA (e.g., Toxic Substances Control Act and state regulations). Once identified, DOE continues to manage these wastes appropriately to meet all other hazardous waste regulations in addition to RCRA (if applicable).

Under existing arrangements, retrievably stored TRUW is the responsibility of the DOE Office of Waste 
Management (EM-30). It is planned that the retrievably stored TRUW and newly generated TRUW from routine site operational activities will be shipped to WIPP for disposal; whereas, buried TRUW and TRUW generated from site remediation activities and decontamination and decommissioning (D\&D) activities are the responsibility of the Office of Environmental Restoration (EM-40). (See Chapter 6 of this document for a discussion of environmental restoration wastes.)

\subsection{TRUW INVENTORIES}

\subsubsection{Sources of Data}

Quantitative information contained in this chapter is derived from data furnished by the DOE sites in response to the integrated data call issued for Rev. 10 of this report ${ }^{5}$ and Rev. I of the Waste Isolation Pilot Plant Transuranic Waste Baseline Inventory Report (WTWBIR) ${ }^{2}$ (Sect. 3.2.3.1). As programs and plans evolve or are changed, modifications or additions will be made to the data and other information in this chapter. It is expected that the quality and accuracy of the data will improve with each annual revision of this document, thus improving the usefulness of the data for program planning and decision purposes.

Early TRUW inventory practices were not as stringent as current ones with regard to requirements for waste identification, categorization, and segregation. Consequently, early inventory data are based largely on process knowledge and on various studies and summaries related to site-specific practices. ${ }^{6}$ As these efforts continue and TRUW is further characterized, there may be revisions in the estimated overall quantities of TRUW.

\subsubsection{Site Locations—Summarized Volumes and Radioactivity}

TRUW management activities (generation, retrievable storage, etc.) are performed at 10 major sites and 13 smallquantity sites (SQS). Figure 3.1 shows the locations of the DOE's TRUW generator and/or storage sites. Tables 3.1-3.10 and Figs. 3.2-3.5, which are discussed later in this chapter, summarize the distribution of retrievably stored and projected TRUW in its final form at various DOE generator and storage sites. Unlike previous versions of this report, the volumes reported in this revision are final-form volumes (i.e., after the sites process, treat, or repackage the waste). Tables 3.11 and 3.12 provide a breakdown of the major TRUW radionuclides by radioactivity. These tables display the decayed radioactivities that have been estimated from the data reported by the sites for retrievably stored $\mathrm{CH}$ and $\mathrm{RH}$ TRUW, as of December 31, 1993. A breakdown for $\mathrm{CH}$ and RH TRUW in terms of decayed mass is provided in Tables 3.13 and 3.14 , respectively.

\subsubsection{Development of Detailed Inventory Data}

To optimize its resources and to enhance data quality, DOE integrated the TRUW data needs of the IDB report (Rev. 10) $)^{5}$ with those of the WTWBIR, Rev. $1{ }^{2}$ in forming a joint data call to the sites. ${ }^{7}$ The information contained in this chapter is a summary of the detailed data gathered through this integrated data-call process and is based on the TRUW final-form-volume projections, as reported in the WTWBIR, Rev. $1 .^{2}$ For a complete set of the information collected, refer to the source documents.

The WTWBIR, Rev. 1, ${ }^{2}$ describes the disposal inventory of TRUW to be emplaced in WIPP, as defined by the DOE TRUW generator and/or storage sites. This information will be used to support the development of various compliance applications for the operations and postclosure phases of the WIPP repository. To meet the needs of the WTWBIR, site data were collected at the waste-stream level in both their initial and final forms. The data were grouped by similar physical and chemical properties and aggregated to produce estimated total volumes of the waste.

To maintain continuity with the previous revision of the IDB, Table 3.1 presents the changes in reported retrievably stored volumes between the IDB and the WTWBIR. The first column in this table presents the volumes at the end of 1993 that were reported in the previous revision of the IDB (Rev. 10). ${ }^{5}$ The next two columns present updated estimates of this same volume (i.e., end of 1993 data) in terms of both initial and final forms, respectively, as reported in the WTWBIR, Rev. $1{ }^{2}$ The data derived from the WTWBIR, Rev. $1,{ }^{2}$ and reported in subsequent tables in this chapter include the final-form volumes at the end of 1993, and the projected increase in these final-form volumes from 1994 through 2022 . In addition, the volumes reported in the attached tables are higher for some sites than those reported in the WTWBIR, Rev. $1,{ }^{2}$ because these volumes include some TRUW regulated under TSCA that was not included in the WIPP inventory defined in WTWBIR, Rev. $1 .^{2}$

Because the radionuclide inventory is not yet available for each waste stream, the radionuclide data in this chapter are derived from the data submittal in support of Rev. 10 of the IDB report. ${ }^{5}$ Also taken from the IDB report are the volume data for buried TRUW (Table 3.15), TRUcontaminated soil (Table 3.16), and the retrievably stored TRUW (as of the end of 1993) that is shown in Table 3.1. 
The remaining data are compiled from data presented earlier in Rev. 1 of the WTWBIR. ${ }^{2}$

\subsubsection{Site data submittal process}

As stated previously, all of the quantitative TRUW information presented in this chapter was derived from the data received from the sites in response to the integrated WTWBIR $-\mathrm{IDB}$ data call. In response to the data call, the sites provided waste stream profiles for each waste stream in their respective current and projected inventories through 2022. The waste stream profiles included such information as waste stream description, initial- and finalform volumes, specific container data, and estimates of the amount of various materials present in the waste (e.g., iron-based metals and alloys, cellulosics, plastics, etc.). In addition, the sites were provided with a list of acceptable final waste forms for disposal at WIPP and asked to assign the appropriate final waste form to each waste stream. The assignment of final waste form helps to identify the general physical and chemical properties of the final form for each waste stream.

The sites provided radionuclide compositions and curies of each radionuclide added in each year of TRUW accumulation. The annual radioactivities in the site submittals were on an as-stored basis; that is, they represent the curies of each radionuclide added each year at the end of the year in which the waste was placed in storage. Only the cumulative site radioactivities have been included in this chapter. For a complete set of references for the TRUW site data submittals, see ref. 7 of Sect. 3.5.

\subsubsection{Site data review and modification}

The site data submittals for TRUW were reviewed to make certain, to the greatest extent possible, that the data supplied met the requirements of completeness and consistency. The data review process included modifying the formats of the data so that they could be easily converted to input data files.

In support of the WTWBIR, waste streams at each TRUW generator and/or storage site that were assigned the same final waste form were grouped together to form sitespecific waste profiles. These profiles provide a condensed summary of the volume and waste material information found in the waste stream profiles for each site. Sitespecific waste profiles with the same final waste form were then combined for all the TRUW generator and/or storage sites to produce WIPP waste profiles. The summary tables and figures presented in this chapter are based on the detailed waste stream profile data reported in the WTWBIR., Rev. 1. ${ }^{2}$

\subsubsection{As-stored TRUW volumes}

Tables 3.2-3.14 and Figs. 3.2-3.5 summarize a small portion of the information in the WTWBIR site submittals. These tables show the volumes and decayed radioactivities of retrievably stored $\mathrm{CH}$ and $\mathrm{RH}$ TRUW at each site at the end of 1993, projected generation from 1994 through 2022 , and the ending volume inventory amounts at the end of 2022. Each table is constructed to highlight a different aspect of TRUW characteristics.

Table 3.2 shows the total estimated TRUW inventory in storage at the end of 1993 and projected through 2022 for each DOE site, and Tables 3.3-3.8 show the breakup of this total inventory into total CH TRUW (Table 3.3), mixed CH TRUW (Table 3.4), nonmixed $\mathrm{CH}$ TRUW (Table 3.5), total RH TRUW (Table 3.6), mixed RH TRUW (Table 3.7), and nonmixed RH TRUW (Table 3.8). The information in these tables is also presented graphically in Figs. 3.2-3.5.

Tables 3.9 and 3.10 show the volumes of TRUW at each site distributed among the 11 final waste forms (plus unknown) defined in the WTWBIR, Rev. 1. ${ }^{2}$ Table 3.9 shows the final waste form distribution for the major sites, whereas Table 3.10 presents the same for the SQS.

\subsubsection{Calculation of annual decayed radioactivities}

Decayed radioactivities shown in Rev. 10 of the IDB report ${ }^{5}$ were calculated by the ORNL computer code RADAC, using the end-of-1993 data submitted to the IDB by the sites. This code converts the annual as-stored radioactivities to annual decayed radioactivities and accumulates these quantities to produce tables showing decayed grams, curies, and watts on a year-by-year, siteby-site, and radionuclide-by-radionuclide basis. Annual added volumes and cumulative volumes are also calculated. Volumes are assumed to be unaffected by decay.

In some cases, the site data were not sufficiently detailed to permit the desired decay calculations. The difficulty most frequently encountered was that radionuclide compositions were not adequately specified on a radionuclide-by-radionuclide basis. In such cases, data conversion codes were used to convert site input data to the radionuclide-specific forms required for decay calculations. The procedures followed in making these adjustments follow accepted precedents set in previous IDB editions and are described in Rev. 9 of the IDB report. $^{8}$ 


\subsubsection{Results of Radionuclide Inventory Calculations}

For the purposes of this report, primary radionuclides are those that cumulatively contribute $98 \%$ of either the total radionuclide activity or mass.

\subsubsection{Retrievably stored wastes}

Tables 3.11-3.14 show the distribution of the primary TRUW radionuclides for all the DOE sites (decayed to the end of 1993) for both CH TRUW and RH TRUW both by radioactivity and by mass. As shown in these tables, ${ }^{238} \mathrm{Pu}$, ${ }^{241} \mathrm{Pu}{ }^{239} \mathrm{Pu},{ }^{241} \mathrm{Am}$, and ${ }^{240} \mathrm{Pu}$ cumulatively contribute more than $98 \%$ of the total activity for the CH TRUW (Table 3.11), whereas ${ }^{238} \mathrm{U},{ }^{232} \mathrm{Th},{ }^{239} \mathrm{Pu},{ }^{235} \mathrm{U}$, and ${ }^{242} \mathrm{Pu}$ contribute more than $98 \%$ of the total radionuclide mass for CH TRUW (Table 3.13). Similar information on RH TRUW and on the relative distribution of both the radioactivity and mass of the primary radionuclides for the various DOE sites can also be derived from Tables 3.12 and 3.14.

\subsubsection{Buried TRUW}

Table 3.15 summarizes the buried TRUW volumes and associated radioactivity, both as-stored and asdecayed. Buried TRUW volumes and radioactivities shown in Table 3.15 are based on data provided in the site submittals. The form of the site-submitted data for buried waste is identical to that of the retrievably stored waste except that no distinction is made between $\mathrm{CH}$ and $\mathrm{RH}$ buried wastes. Table 3.15 shows both cumulative as-stored and as-decayed radioactivities for all nuclides, as well as for TRU nuclides only.

\subsubsection{Contaminated soil}

Over the years, many of the older buried waste containers have developed leaks and contaminated the adjacent soil. Also, at some sites, soil has become contaminated by liquid spills or has been used as an ionexchange medium for dilute liquid waste streams. It is difficult to make accurate estimates of the actual quantity of the contaminated soil. The data reported by the sites are shown in Table 3.16. Additional characterization efforts will be required to reduce the uncertainties of this data.

\subsection{PROJECTED FUTURE QUANTITIES OF TRUW}

Tables 3.2-3.10 show the data submitted by the sites for estimated future volumes of TRUW generation. The sites were not requested to estimate the radioactivities or isotopic compositions of these wastes because it was believed that there would be little basis for such estimates. The estimated volumes are given in terms of average annual rates $\left(\mathrm{m}^{3} / \mathrm{year}\right)$ for four time periods from 1994 to 1997 and for two cumulative time periods (1998-2002 and 2003-2022).

\subsection{TRUW DISPOSAL}

The goals of the DOE TRUW Program are to terminate interim storage and achieve permanent disposal of DOE TRUW. As stated in Public Law 96-164, ${ }^{10}$ WIPP was to be constructed ". . a as a defense activity of the DOE for the purpose of providing a research and development facility to demonstrate the safe disposal of radioactive waste resulting from defense activities and programs of the United States." Construction of the facility is essentially complete, and WIPP is now the only facility specifically designed for isolation of TRUW. It is designed to contain about $175,000 \mathrm{~m}^{3}\left(6.2 \text { million } \mathrm{f}^{3}\right)^{11}$ of TRUW $650 \mathrm{~m}$ below ground in a mined salt formation.

Waste received at WIPP must meet the WIPP waste acceptance criteria and associated quality assurance requirements specified in WIPP/DOE-069. ${ }^{3}$ A number of other approvals remain to be completed before DOE can begin disposal operations at the facility. DOE is committed to demonstrating compliance with all applicable regulations before the permanent disposal of TRUW in the WIPP repository. These regulations are the environmental standards for management and disposal of TRU wastes, as mandated in 40 CFR Part 191 (ref. 12) and Part 194 (ref. 13), and the RCRA regulations. Compliance will be demonstrated through performance assessment calculations based on the inventory of existing and currently projected waste streams as reported by the DOE TRUW generator and/or storage sites in the WTWBIR, Rev. $1 .^{2}$

In 1992 the WIPP Legislative Land Withdrawal Act ${ }^{11}$ was passed, confirming congressional intent to have DOE continue with development and permitting of the facility. Since then, DOE has stated its intent to accelerate processes leading to the start of waste disposal operations at the WIPP. 


\subsection{REFERENCES}

1. U.S. Department of Energy, Radioactive Waste Management, DOE Order 5820.2A, Washington, D.C. (Sept. 26, 1988).

2. U.S. Department of Energy, Waste Isolation Pilot Plant Transuranic Waste Baseline Inventory Report, CAO-94-1005, Rev. 1, Carlsbad, New Mexico (February 1995).

3. U.S. Department of Energy, TRU Waste Acceptance Criteria for the Waste Isolation Pilot Plant, WIPP/DOE-069, Rev. 4, Carlsbad, New Mexico (December 1991).

4. K. S. Hollingsworth, Policy Statement Regarding Solid Waste Burial, AEC Directive IAD No. 051 1-21, Washington, D.C. (Mar. 20, 1970).

5. U.S. Department of Energy, Integrated Data Base Report-1993: U.S. Spent Nuclear Fuel and Radioactive Waste Inventories, Projections, and Characteristics, DOE/RW-0006, Rev. 10, Oak Ridge National Laboratory, Oak Ridge, Tennessee (December 1994).

6. U.S. Department of Energy, Defense Waste Management Plan for Buried Transuranic-Contaminated Waste, Transuranic-Contaminated Soil, and Difficult-to-Certify Transuranic Waste, DOE/DP-0044, Washington, D.C. (June 1987).

7. DOE site TRUW data submittal attachments, submitted to the IDB Program and WTWBIR Project. The following TRUW submittals were received and reviewed by the IDB Program and WTWBIR Project before analysis and integration. Preceding each submittal is the site (in parentheses) to which it refers.

a. (Ames) Kay M. Lampe Hannasch, Ames Laboratory, Ames, Iowa, correspondence to James E. Fletcher, DOE Chicago Operations Office, Argonne, Illinois, "Data Requests for TRU Waste, WIPP Baseline Inventory, IDB Request," dated Sept. 28, 1994.

b. (ANL-E) Michael Sodaro, Argonne National Laboratory, Argonne, Illinois, correspondence to Jeff Williams, DOE Carlsbad Area Office, Carlsbad, New Mexico, "WIPP TRU Baseline Inventory Report and Integrated Database Forecasts," dated Sept. 30, 1994.

c. (ANL-W) Nancy Stewart and Roy Grant, Argonne National Laboratory-West, Idaho Falls, Idaho, correspondence to E. W. Krieger, MAC Technical Services Company, Albuquerque, New Mexico, transmitting site data for inclusion into the IDB report (Rev. 10) and WTWBIR (Rev. 1), dated Jan. 17, 1995.

d. (BAPL) E. D. Shollenberger, DOE Pittsburgh Naval Reactors Office, West Miftlin, Pennsylvania, correspondence to Mark L. Matthews, DOE Carlsbad Area Office, Carlsbad, New Mexico, "Waste Isolation Pilot Project Transuranic Waste (TRU) Baseline Inventory Report and Updated Integrated Database TRU Information for the Bettis Atomic Power Laboratory," dated Oct. 21, 1994.

e. (BAPL) Jim Sage, DOE Pittsburgh Naval Reactors Office, West Mifflin, Pennsylvania, correspondence to E. W. Krieger, MAC Technical Services Company, Albuquerque, New Mexico, "WTWBIR Input for the Bettis Atomic Power Laboratory," dated Oct. 21, 1994.

f. (BCL) No cover letter provided with site data submittal.

g. (ETEC) G. G. Gaylord, Rockwell International Corporation, Canoga Park, California, correspondence to Mark L. Matthews, DOE Carlsbad Area Office, Carlsbad, New Mexico, "TRU Inventory at ETEC," 94ETEC-DRF-1667, dated Oct. 31, 1994. 
h. (ETEC) Alan Von Arx, Energy Technology Engineering Center, Canoga Park, California, correspondence to E. W. Krieger, MAC Technical Services Company, Albuquerque, New Mexico, "Updated Tables 2, 3, 5, 9, \& 11, , dated Nov. 17, 1994.

i. (Hanford) R. D. Wojtasek, Westinghouse Hanford Company, Richland, Washington, correspondence to Lise Wachter, Martin Marietta Energy Systems, Inc., HAZWRAP, Oliver Springs, Tennessee, "Request for Office of Waste Management, Waste Information Update," 9305688B R1, dated Aug. 30, 1993.

j. (Hanford) F. M. Coony, Westinghouse Hanford Company, Richland, Washington, correspondence to E. W. Krieger, MAC Technical Services Company, Albuquerque, New Mexico, transmitting information on TRUW added to Hanford inventory during CY 1993, dated Dec. 7, 1994.

k. (Hanford) F. M. Coony, Westinghouse Hanford Company, Richland, Washington, correspondence to Jim Teak, Advanced Sciences, Inc., Albuquerque, New Mexico, "Memorandum, Mark L. Matthews, P.E., National TRU Program Office, to Distribution, Extension of Data Package Review, dated Oct. 13, 1994," dated Nov. 1, 1994.

1. (Hanford) F. M. Coony, Westinghouse Hanford Company, Richland, Washington, correspondence to E. W. Krieger, MAC Technical Services Company, Albuquerque, New Mexico, transmitting completed data forms for the 1994 input to the IDB (Rev. 10), dated Dec. 8, 1994.

m. (INEL) Joel T. Case, DOE Idaho Operations Office, Idaho Falls, Idaho, correspondence to Mark L. Matthews, DOE Carlsbad Area Office, Carlsbad, New Mexico, "Integrated Data Base (IDB) TRU Waste 1994 Data Call," OPE-WM-94-307, dated Oct. 6, 1994.

n. (INEL) Joel T. Case, DOE Idaho Operations Office, Idaho Falls, Idaho, correspondence to Jim Teak, Advanced Sciences, Inc., Albuquerque, New Mexico, "Integrated Data Base (IDB) TRU Waste 1994 Data Call," OPE-WM 94-308, dated Oct. 6, 1994.

o. (INEL) T. L. Clements, Jr., Idaho National Engineering Laboratory, Idaho Falls, Idaho, correspondence to I. T. Case, DOE Idaho Operations Office, Idaho Falls, Idaho, "Integrated Data Base (IDB) TRU Waste 1994 Data Call," TLC-122-94, dated Sept. 29, 1994.

p. (ITR) Bruce B. Boecker, Inhalation Toxicology Research Institute, Albuquerque, New Mexico, correspondence to Jeff Williams, DOE Carlsbad Area Office, Carlsbad, New Mexico, "Response to the Waste Isolation Pilot Plant (WIPP) Transuranic Waste Baseline Inventory Report (WTWBIR) Data Package," dated Nov. 29, 1994.

q. (KAPL) M. Monaco, Knolls Atomic Power Laboratory, Schenectady, New York, correspondence to E. W. Krieger, MAC Technical Services Company, Albuquerque, New Mexico, transmitting data for inclusion into the IDB report (Rev. 10) and WTWBIR (Rev. 1), dated Jan. 16, 1995.

r. (LANL) Davis Christensen, Los Alamos National Laboratory, Los Alamos, New Mexico, correspondence to Mark L. Matthews, DOE Carlsbad Area Office, Carlsbad, New Mexico, "WIPP Transuranic Waste Baseline Inventory Report," CST14-94-061, Nov. 1, 1994.

s. (LANL) Davis Christensen, Los Alamos National Laboratory, Los Alamos, New Mexico, correspondence to E. W. Krieger, MAC Technical Services Company, Albuquerque, New Mexico, transmitting data for inclusion into the IDB report (Rev. 10) and WTWBIR (Rev. 1), dated Nov. 29, 1994 and Dec. 2, 1994.

t. (LBL) Tim Wan, University of California, Berkeley, California, correspondence to Jeff Williams, DOE Carlsbad Area Office, Carlsbad, New Mexico, "Data Package for the Waste Isolation Pilot Plant TRU Waste Baseline Inventory Report," HW-94-342, dated Sept. 13, 1994. 
u. (LLNL) Roy Kearns, DOE Oakland Operations Office, Oakland, California, correspondence to Jeff Williams, DOE Carlsbad Area Office, Carlsbad, New Mexico, "Waste Isolation Pilot Plant Transuranic Waste Baseline Inventory Report and 1994 Integrated Database for Lawrence Livermore National Laboratory," 94W399/5484.a.13, dated Oct. 18, 1994.

v. (LLNL) Kem Hainebach, Lawrence Livermore National Laboratory, Livermore, California, correspondence to Roy Kearns, DOE Oakland Operations Office, Oakland, California, "Corrections to WIPP TRU Waste Baseline Inventory Report," dated Dec. 5, 1994.

w. (Mound) Raymond J. Finney, EG\&G Mound Applied Technologies, Miamisburg, Ohio, correspondence to Robert S. Rothman, DOE Miamisburg Area Office, Miamisburg, Ohio, "Response to the Waste Isolation Pilot Plant (WIPP) Transuranic Waste Baseline Inventory Report (WTWBIR) Data Package," dated Sept. 27, 1994.

x. (Mound) Raymond J. Finney, EG\&G Mound Applied Technologies, Miamisburg, Ohio, correspondence to Robert S. Rothman, DOE Miamisburg Area Office, Miamisburg, Ohio, "Response to the Waste Isolation Pilot Plant (WIPP) Transuranic Waste Baseline Inventory Report (WTWBIR) Completed Data Package," dated Oct. 26, 1994.

y. (MURR) W. Derek Pickett, University of Missouri, Columbia, Missouri, correspondence to Jim Teak, Advanced Sciences, Inc., Albuquerque, New Mexico, transmitting MURR TRUW information and data for the IDB report (Rev. 10) and WTWBIR (Rev. 1), dated Sept. 29, 1994.

z. (NTS) Joseph M Ginanni, DOE Nevada Operations Office, Las Vegas, Nevada, correspondence to Jerry Klein, Oak Ridge National Laboratory, Oak Ridge, Tennessee; Jim Teak, Advance Sciences, Inc., Albuquerque, New Mexico; and Jeff Williams, DOE Carlsbad Area Office, Carlsbad, New Mexico, "Nevada Test Site Transuranic and Mixed Transuranic Inventory Data for the 1994 Integrated Data Base Report (DOE/RW-0006, Rev. 10)," dated Oct. 14, 1994.

aa. (ORNL) P. E. Arakawa, Oak Ridge National Laboratory, Oak Ridge, Tennessee, correspondence to Mac Roddye, DOE Oak Ridge Operations Office, Oak Ridge, Tennessee, "Complete Review and Revision of Data Package for the WIPP Transuranic Waste Baseline Inventory Report (WTWBIR)," dated Nov. 2, 1994.

ab. (ORNL) Brad Farrar, S. M. Stoller Corporation, Oak Ridge, Tennessee, correspondence to Jim Teak, Advanced Sciences, Inc., Albuquerque, New Mexico, "1993 IDB Data Call," dated Oct. 3, 1994.

ac. (ORNL) P. E. Arakawa, Oak Ridge National Laboratory, Oak Ridge, Tennessee, correspondence to M. G. Zimmerman, Consolidated Technical Services, Inc., Albuquerque, New Mexico, "Response to Comments on the ORNL WTWBIR Data Submittal, Table 11," dated Nov. 21, 1994.

ad. (ORNL) P. E. Arakawa, Oak Ridge National Laboratory, Oak Ridge, Tennessee, correspondence to E. W. Krieger, MAC Technical Services Company, Albuquerque, New Mexico, "Response to Second Set of Comments on the ORNL WTWBIR Data Submittal," dated Dec. 16, 1994.

ae. (ORNL) Kenneth P. Guay, Oak Ridge National Laboratory, Oak Ridge, Tennessee, correspondence to P. E. Arakawa, Oak Ridge National Laboratory, Oak Ridge, Tennessee, "Response to Comments on the ORNL WTWBIR Data Submittal," dated Dec. 16, 1994.

af. (PAD) Greg Shala, Paducah Gaseous Diffusion Plant, Paducah, Kentucky, correspondence to E. W. Krieger, MAC Technical Services Company, Albuquerque, New Mexico, "TRU/MTRU Data Request," dated Jan. 13, 1995. 
ag. (PANT) D. L. Heim, DOE Amarillo Area Office, Amarillo, Texas, correspondence to Jeff Williams, DOE Carlsbad Area Office, Carlsbad, New Mexico, "WIPP Transuranic (TRU) Waste Baseline Inventory Report and Integrated Database Report," dated Sept. 28, 1994.

ah. (RFETS) G. A. O'Leary, EG\&G Rocky Flats Inc., Golden, Colorado, correspondence to Paul Drez, Drez and Associates, Albuquerque, New Mexico, "Transmittal of the Rocky Flats Environmental Technology Site (RFETS) Data Forms for the Waste Isolation Pilot Plant (WIPP) Transuranic (TRU) Waste Baseline Inventory Report (WTWBIR), GAO-039-94," 94-RF-10526, dated Oct. 13, 1994.

ai. (SNL/NM) E. D. Conway, Sandia National Laboratories, Albuquerque, New Mexico, correspondence to Jeff Williams, DOE Carlsbad Area Office, Carlsbad, New Mexico, transmitting data package for WTWBIR (Rev. 1), dated Oct. 24, 1994.

aj. (SNL/NM) Joe Jones, Sandia National Laboratories, Albuquerque, New Mexico, correspondence to E. W. Krieger, MAC Technical Services Company, Albuquerque, New Mexico, "WIPP TRU Waste Comments," dated Nov. 22, 1994.

ak. (SRS) S. J. Mentrup, Westinghouse Savannah River Company, Aiken, South Carolina, correspondence to Stan Massingill, DOE Savannah River Operations Office, Aiken, South Carolina, "SRS Data Package for WIPP TRU Waste Baseline Inventory Report (U)," SWE-SWE-94-0550, dated Oct. 12, 1994.

al. (WVDP) P. S. Klanian, West Valley Nuclear Services Company, Inc., West Valley, New York, correspondence to Jeff Williams, DOE Carlsbad Area Office, Carlsbad, New Mexico, "Data Package for WIPP Transuranic Waste Baseline Inventory Report (WTWBIR)," dated Oct. 14, 1994.

am. (WVDP) Connie King, West Valley Nuclear Services Company, Inc., West Valley, New York, correspondence to Royes Salmon, Oak Ridge National Laboratory, Oak Ridge, Tennessee, transmitting input to IDB report (Rev. 10), dated Nov. 14, 1994.

an. (WVDP) Connie King, West Valley Nuclear Services Company, Inc., West Valley, New York, correspondence to E. W. Krieger, MAC Technical Services Company, Albuquerque, New Mexico, transmitting responses to WTWBIR Comments, dated Dec. 12, 1994.

8. U.S. Department of Energy, Integrated Data Base for 1993: U.S. Spent Fuel and Radioactive Waste Inventories, Projections, and Characteristics, DOE/RW-0006, Rev. 9, Oak Ridge National Laboratory, Oak Ridge, Tennessee (March 1994).

9. U.S. Department of Energy, Long Range Master Plan for Defense Transuranic Waste Program, DOE/WIPP 88-028, Carlsbad, New Mexico, (December 1988).

10. U.S. Congress, Department of Energy National Security and Military Application of Nuclear Energy Authorization Act of 1980, Pub. L. 96-164 (1980).

11. U.S. Congress, Waste Isolation Pilot Plant Land Withdrawal Act, Pub. L. 102-579 (1992).

12. U.S. Environmental Protection Agency, Environmental Radiation Protection Standards for the Management and Disposal of Spent Nuclear Fuel, High-Level and Transuranic Radioactive Wastes, 40 CFR Part 191, final rule, Fed. Regist. 58, 66398 (Dec. 20, 1993).

13. U.S. Environmental Protection Agency, Criteria for Certification of Compliance with Environmental Radiation Protection Standards for the Management and Disposal of Spent Nuclear Fuel, High-Level, and Transuranic Wastes, 40 CFR Part 194, notice of proposed rulemaking, Fed. Regist. 58, 792 (Jan. 30, 1995). 


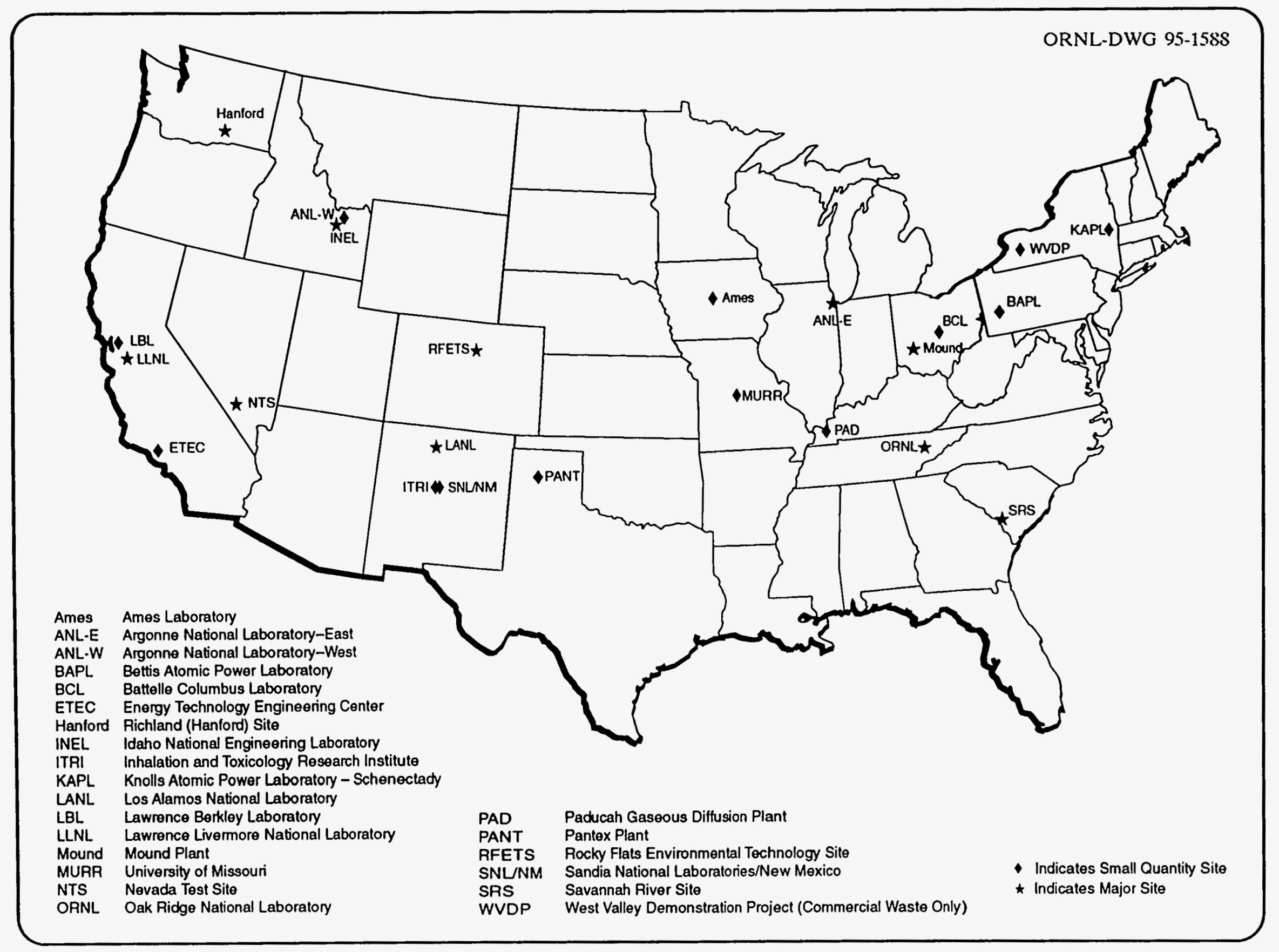

Fig. 3.1. U.S. Department of Energy transuranic waste generation/storage sites. 


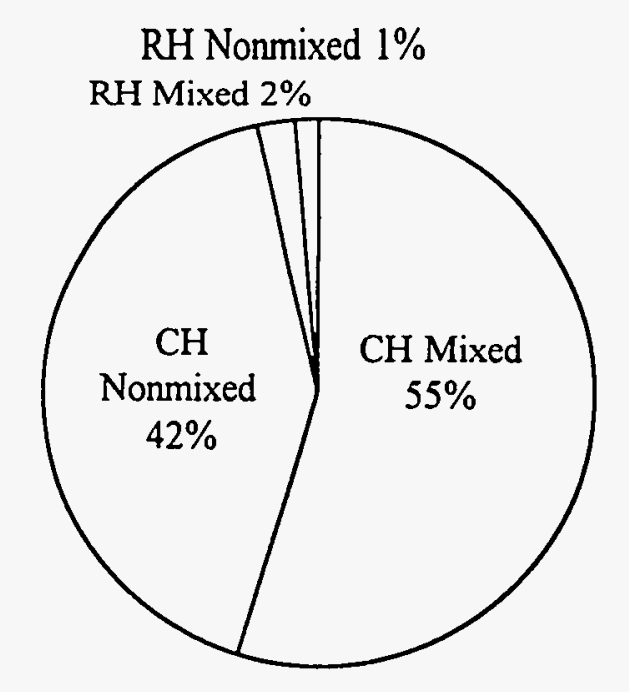

\section{Total TRUW at End of 2022}
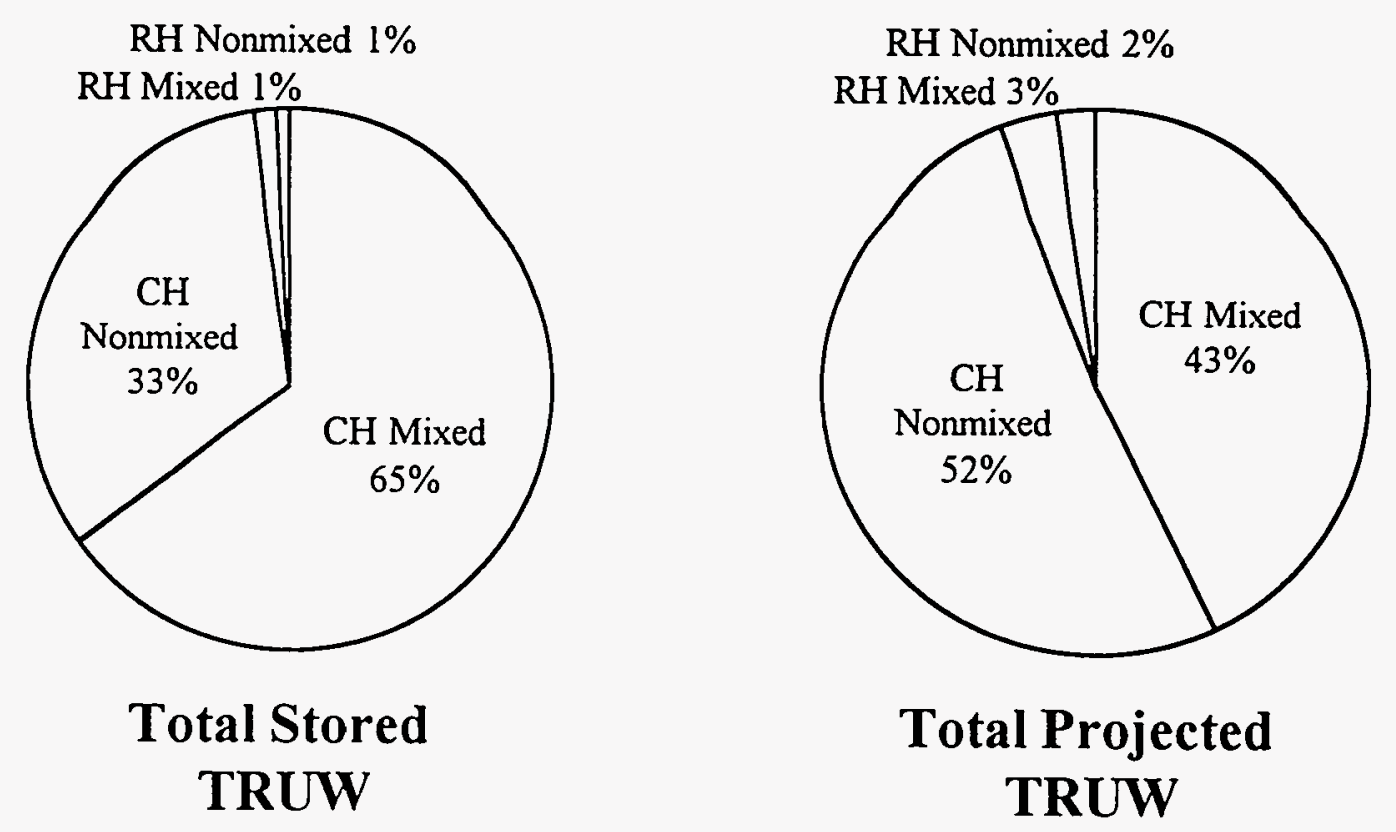

NOTE: All amounts shown are for final waste form

Fig. 3.2. Retrievably stored and projected TRUW volumes by radioactivity classification. 
ORNL DWG 95-1591R

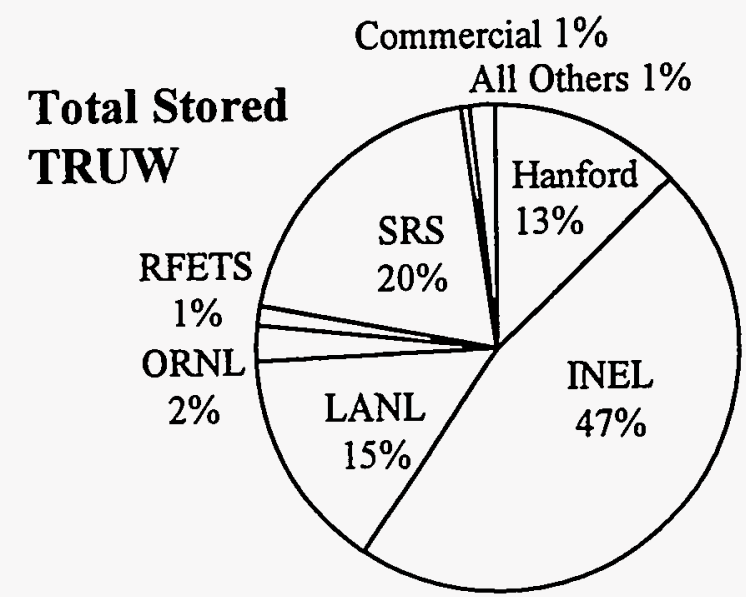

Stored

Mixed CH TRUW

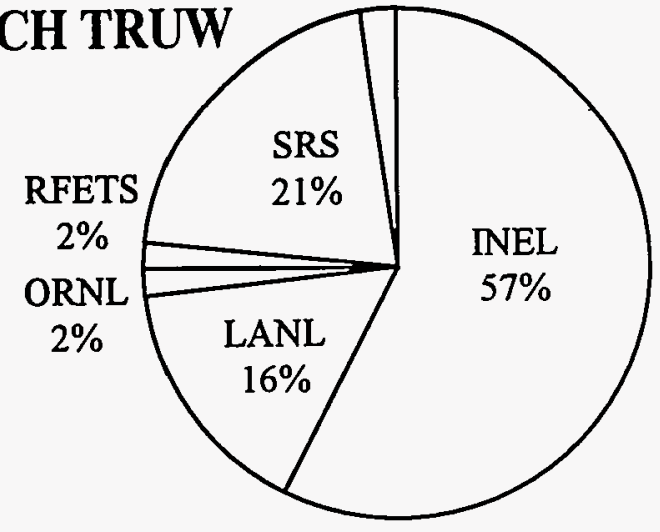

Stored

Nonmixed CH TRUW

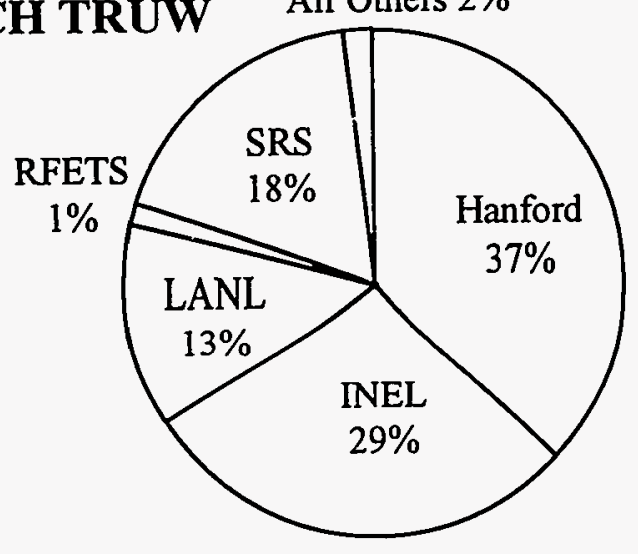

Stored

Mixed RH TRUW

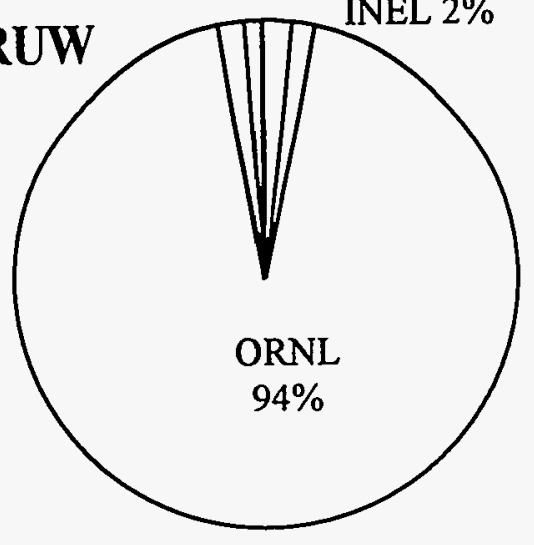

\section{Stored}

Nonmixed

Commercial $1 \%$

LANL $1 \%$ SQS $2 \%$

RH TRUW

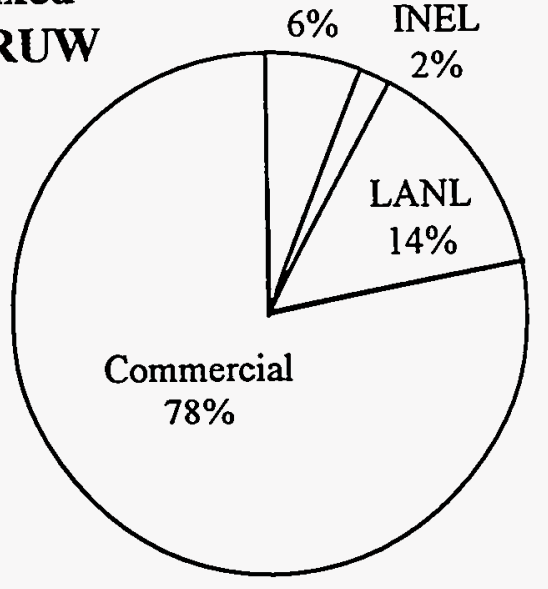

NOTE: All amounts shown are for final waste form

Fig. 3.3. Retrievably stored TRUW volumes by site. 


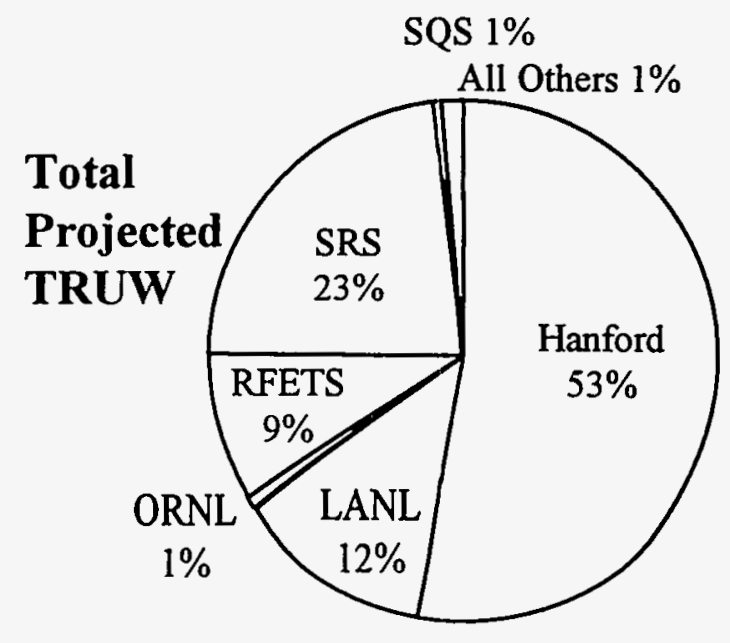

\section{Projected}

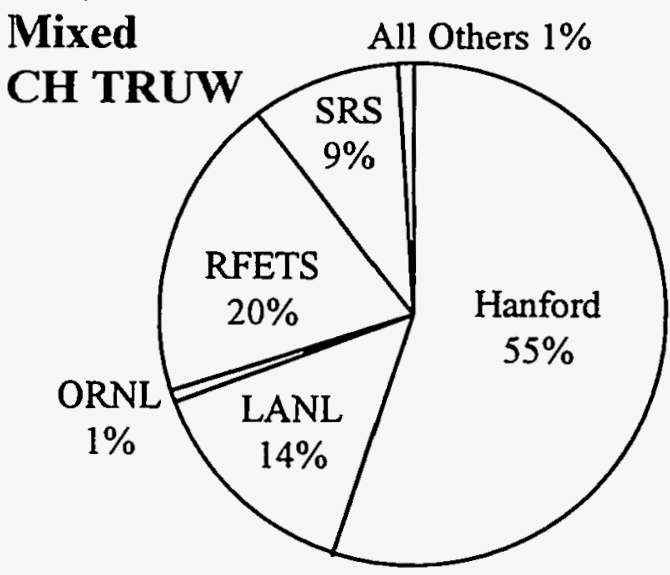

Projected

Nonmixed CH TRUW

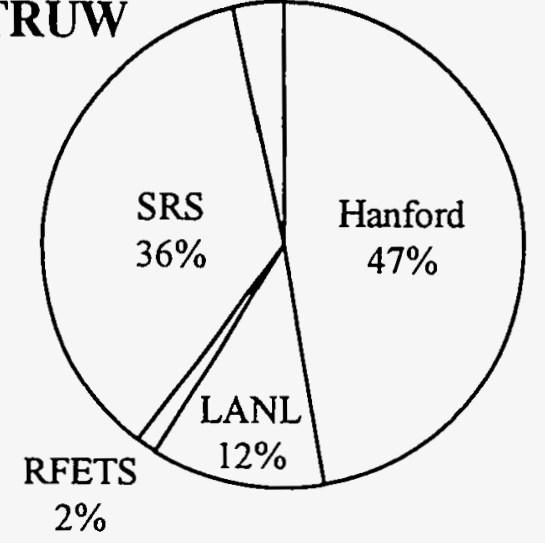

Projected

Mixed SQS 1\%

RH TRUW

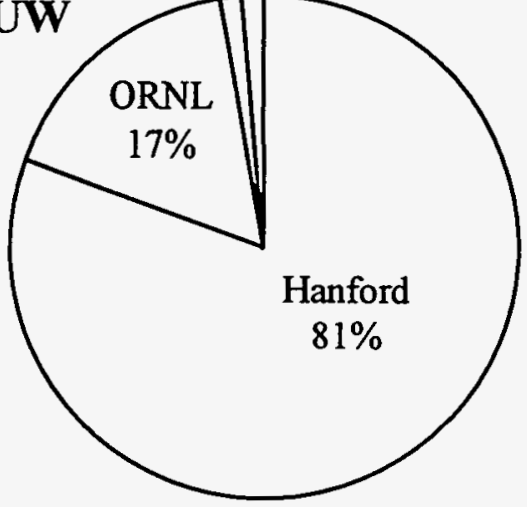

\section{Projected}

Nonmixed RH TRUW

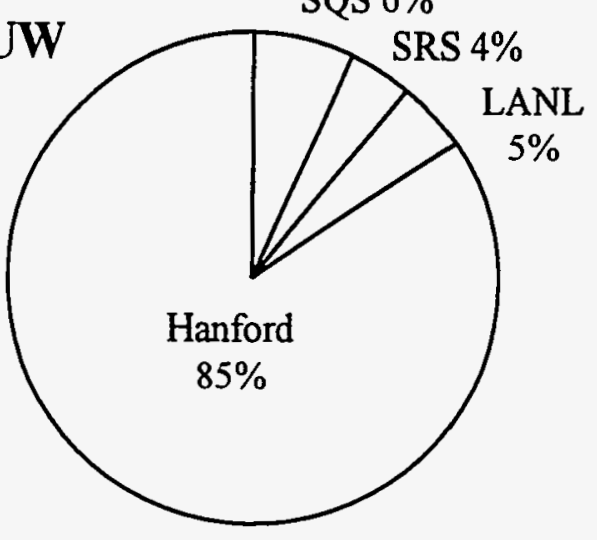

NOTE: All amounts shown are for final waste form

Fig. 3.4. Projected TRUW volumes by site. 
ORNL DWG 95-1593R

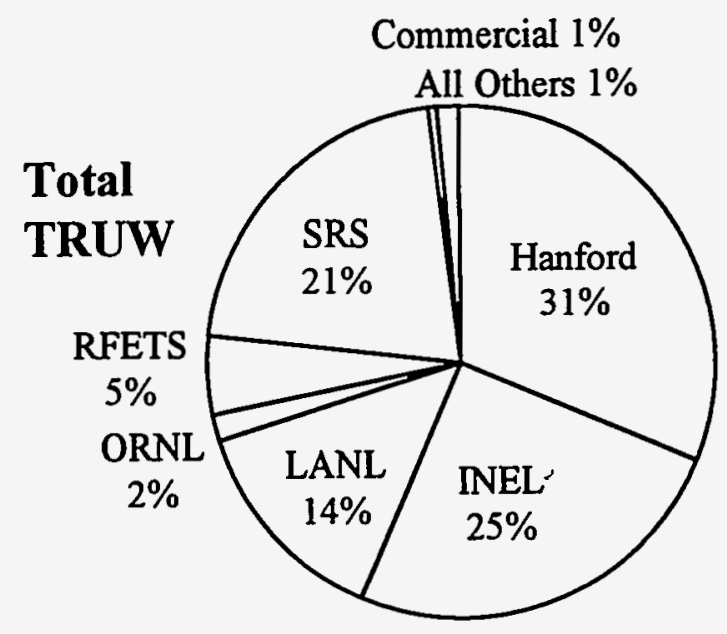

Total

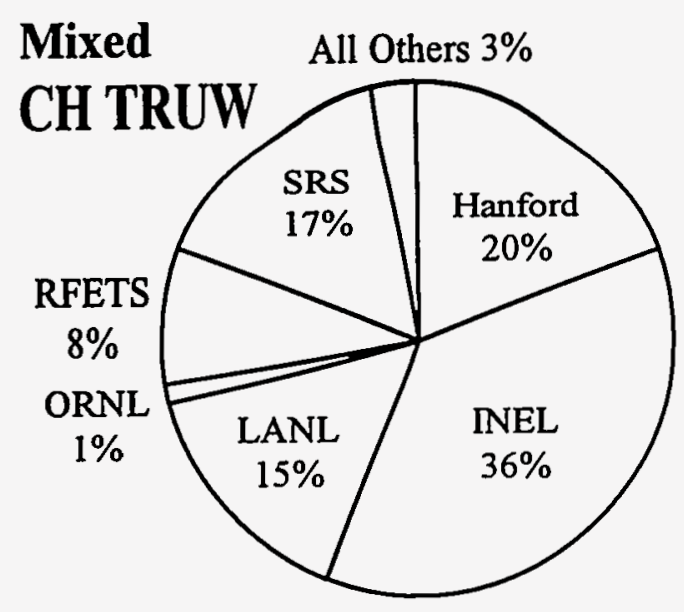

Total

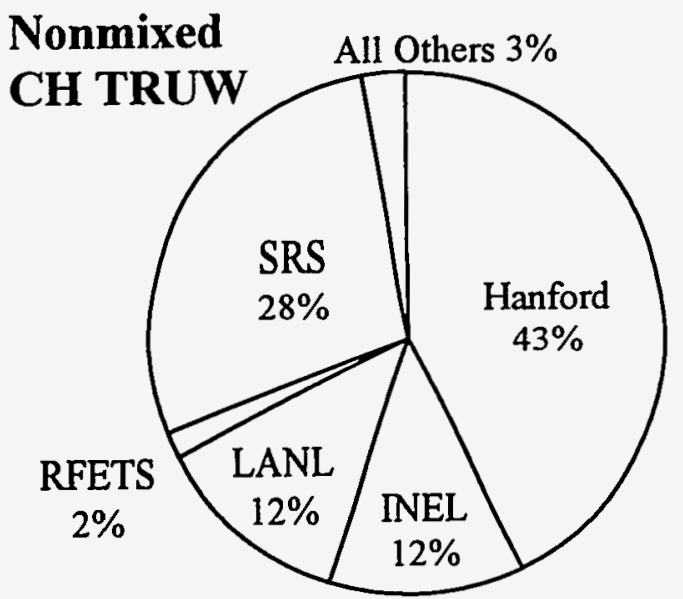

Total

Mixed RH TRUW

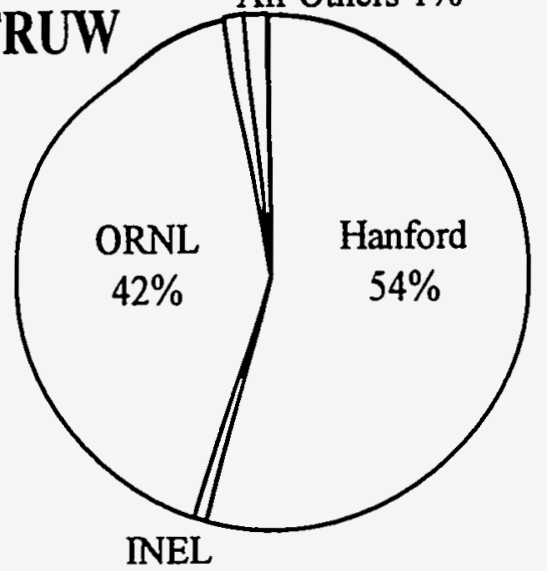

$1 \%$

Total

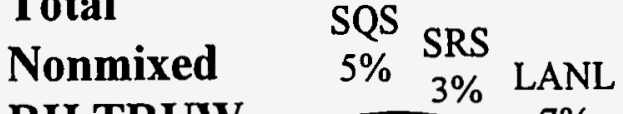

RH TRUW

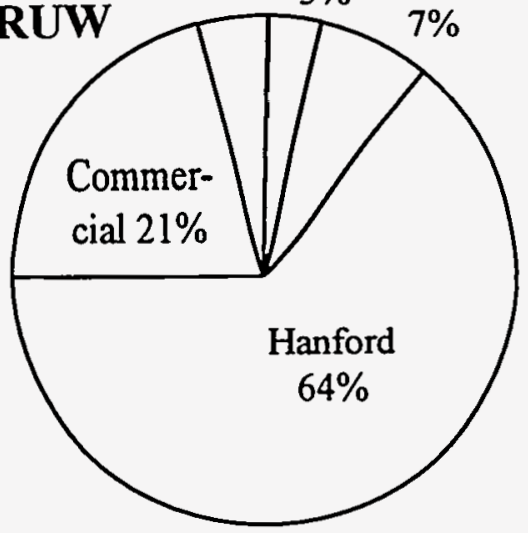

NOTE: All amounts shown are for final waste form

Fig. 3.5. Estimated TRUW volumes by end of year 2022 . 
Table 3.1. Comparison of IDB and WTWBIR retrievably stored volumes of TRUWa,b

(Volumes are expressed in $\mathrm{m}^{3}$ )

\begin{tabular}{|c|c|c|c|c|c|c|}
\hline \multirow{3}{*}{ Site } & \multicolumn{3}{|c|}{ Contact-handled TRUW } & \multicolumn{3}{|c|}{ Remote-handled TRUW } \\
\hline & \multicolumn{2}{|c|}{ As stored } & \multirow{2}{*}{$\begin{array}{l}\text { In final form } \\
1993 \text { WTWBIR }^{d}\end{array}$} & \multicolumn{2}{|c|}{ As stored } & \multirow{2}{*}{$\begin{array}{c}\text { In final form } \\
1993 W^{d}{ }^{d} I_{R}^{d}\end{array}$} \\
\hline & $1993 \mathrm{DB}^{\mathrm{c}}$ & 1993 WTWBIR $^{d}$ & & $1993 \mathrm{DB}^{\mathrm{C}}$ & 1993 WTWBIR $^{\mathrm{d}}$ & \\
\hline \multicolumn{7}{|l|}{ Major sites } \\
\hline ANL-E & $2.91 \mathrm{E}+01$ & $2.91 \mathrm{E}+01$ & $2.91 \mathrm{E}+01$ & $1.70 \mathrm{E}+00$ & $0.00 \mathrm{E}+00^{\mathrm{e}}$ & $0.00 \mathrm{E}+00$ \\
\hline Hanford & $1.56 \mathrm{E}+04$ & $8.06 E+03^{f}$ & $9.39 E+038$ & $2.01 E+02$ & $3.20 \mathrm{E}+01^{\mathrm{h}}$ & $3.32 \mathrm{E}+01 \mathrm{~g}$ \\
\hline INEL & $6.48 \mathrm{E}+04$ & $3.92 E+04^{f}$ & $3.47 E+048$ & $7.98 \mathrm{E}+01$ & $3.10 \mathrm{E}+01^{\mathrm{i}}$ & $3.10 \mathrm{E}+01$ \\
\hline LANL & $1.08 E+04$ & $1.09 \mathrm{E}+04$ & $1.09 \mathrm{E}+04$ & $9.13 \mathrm{E}+01$ & $9.13 E+01$ & $9.13 \mathrm{E}+01$ \\
\hline LLNL & $2.35 \mathrm{E}+02$ & $2.24 \mathrm{E}+02$ & $2.24 \mathrm{E}+02$ & $0.00 \mathrm{E}+00$ & $0.00 \mathrm{E}+00$ & $0.00 \mathrm{E}+00$ \\
\hline Mound & $1.19 \mathrm{E}+01$ & $2.63 \mathrm{E}+02^{\mathrm{i}}$ & $2.63 E+02$ & $0.00 \mathrm{E}+00$ & $0.00 \mathrm{E}+00$ & $0.00 \mathrm{E}+00$ \\
\hline NTS & $6.07 \mathrm{E}+02$ & $6.13 \mathrm{E}+02$ & $6.20 \mathrm{E}+02 \mathrm{~g}$ & $5.30 \mathrm{E}+00$ & $0.00 \mathrm{E}+00$ & $0.00 \mathrm{E}+00$ \\
\hline ORNL & $2.02 E+03$ & $7.83 \mathrm{E}+02^{\mathrm{i}}$ & $7.83 E+02$ & $5.64 \mathrm{E}+02$ & $9.94 E+02^{i}$ & $9.94 \mathrm{E}+02$ \\
\hline RFETS & $1.04 E+03$ & $1.06 \mathrm{E}+03$ & $1.13 E+038$ & $0.00 \mathrm{E}+00$ & $0.00 \mathrm{E}+00$ & $0.00 \mathrm{E}+00$ \\
\hline SRS & $8.93 E+03$ & $9.34 \mathrm{E}+03^{\mathrm{i}}$ & $1.46 \mathrm{E}+048$ & $0.00 \mathrm{E}+00$ & $0.00 \mathrm{E}+00$ & $0.00 \mathrm{E}+00$ \\
\hline Subtotal & $1.04 \mathrm{E}+05$ & $7.04 \mathrm{E}+04$ & $7.26 \mathrm{E}+04$ & $9.43 E+02$ & $1.15 \mathrm{E}+03$ & $1.15 E+03$ \\
\hline \multicolumn{7}{|c|}{ Small-quantity sites } \\
\hline Ames & $0.00 \mathrm{E}+00$ & $0.00 \mathrm{E}+00$ & $0.00 \mathrm{E}+00$ & $0.00 \mathrm{E}+00$ & $0.00 \mathrm{E}+00$ & $0.00 \mathrm{E}+00$ \\
\hline ANL-W & $0.00 \mathrm{E}+00$ & $2.00 \mathrm{E}-02^{\mathrm{j}}$ & $2.00 \mathrm{E}-02$ & $0.00 \mathrm{E}+00$ & $8.65 \mathrm{E}+00 \mathrm{j}$ & $8.65 \mathrm{E}+00$ \\
\hline $\mathrm{BCL}$ & $0.00 \mathrm{E}+00$ & $0.00 \mathrm{E}+00$ & $0.00 E+00$ & $0.00 \mathrm{E}+00$ & $0.00 \mathrm{E}+00$ & $0.00 \mathrm{E}+00$ \\
\hline BAPL & $0.00 \mathrm{E}+00$ & $0.00 \mathrm{E}+00$ & $0.00 \mathrm{E}+00$ & $0.00 \mathrm{E}+00$ & $0.00 \mathrm{E}+00$ & $0.00 \mathrm{E}+00$ \\
\hline ETEC & $1.90 \mathrm{E}+00$ & $1.87 \mathrm{E}+00$ & $1.87 \mathrm{E}+00$ & $0.00 E+00$ & $0.00 \mathrm{E}+00$ & $0.00 \mathrm{E}+00$ \\
\hline KAPL & $0.00 \mathrm{E}+00$ & $2.40 \mathrm{E}+00^{\mathrm{i}}$ & $2.40 \mathrm{E}+00$ & $2.40 \mathrm{E}+00$ & $1.12 \mathrm{E}+01^{\mathrm{i}}$ & $1.12 \mathrm{E}+01$ \\
\hline L.BL & $9.00 \mathrm{E}-01$ & $8.40 \mathrm{E}-01$ & $8.40 \mathrm{E}-01$ & $0.00 \mathrm{E}+00$ & $0.00 \mathrm{E}+00$ & $0.00 \mathrm{E}+00$ \\
\hline MURR & $1.00 \mathrm{E}-01$ & $6.00 \mathrm{E}-02 \mathrm{i}$ & $6.00 E-02$ & $0.00 E+00$ & $0.00 \mathrm{E}+00$ & $0.00 \mathrm{E}+00$ \\
\hline PAD & $4.30 \mathrm{E}+00$ & $2.10 \mathrm{E}+00^{\mathrm{i}}$ & $3.45 \mathrm{E}+008$ & $0.00 \mathrm{E}+00$ & $0.00 \mathrm{E}+00$ & $0.00 \mathrm{E}+00$ \\
\hline PANT & $6.00 \mathrm{E}-01$ & $6.24 \mathrm{E}-01$ & $6.24 E-01$ & $0.00 \mathrm{E}+00$ & $0.00 \mathrm{E}+00$ & $0.00 \mathrm{E}+00$ \\
\hline SNL/NM & $9.00 \mathrm{E}-01$ & $8.04 \mathrm{E}+00^{\mathrm{i}}$ & $8.04 E+00$ & $9.00 \mathrm{E}-01$ & $0.00 \mathrm{E}+00^{i}$ & $0.00 E+00$ \\
\hline Subtotal & $8.70 E+00$ & $1.60 \mathrm{E} \div 01$ & $1.73 E+01$ & $3.30 \mathrm{E}+00$ & $1.99 \mathrm{E}+01$ & $1.99 \mathrm{E}+01$ \\
\hline
\end{tabular}


Table 3.1 (continued)

\begin{tabular}{|c|c|c|c|c|c|c|}
\hline \multirow{3}{*}{ Site } & \multicolumn{3}{|c|}{ Contact-handled TRUW } & \multicolumn{3}{|c|}{ Remote-handled TRUW } \\
\hline & \multicolumn{2}{|c|}{ As stored } & \multirow{2}{*}{$\begin{array}{l}\text { In final form } \\
1993 \text { WTWBIR }^{d}\end{array}$} & \multicolumn{2}{|c|}{ As stored } & \multirow{2}{*}{$\begin{array}{l}\text { In final form } \\
1993 \text { WTWBIR }\end{array}$} \\
\hline & $1993 \mathrm{DB}^{\mathrm{C}}$ & 1993 WTWBIR $^{\mathrm{d}}$ & & $1993 \mathrm{DB}^{\mathrm{c}}$ & 1993 WTWBIR $^{d}$ & \\
\hline \multicolumn{7}{|l|}{ Commercial site } \\
\hline WVDP & $4.91 E+01$ & $5.06 \mathrm{E}+01$ & $5.06 \mathrm{E}+01$ & $4.27 \mathrm{E}+02$ & $4.27 \mathrm{E}+02$ & $4.27 \mathrm{E}+02$ \\
\hline Total & $1.04 \mathrm{E}+05$ & $7.05 E+04$ & $7.26 \mathrm{E}+04$ & $1.37 \mathrm{E}+03$ & $1.59 E+03$ & $1.60 E+03$ \\
\hline
\end{tabular}

aSmall changes between the $1993 \mathrm{DB}$ and 1993 WTWBIR (as stored) volumes are expected because of anticipated rounding methods and differences in reporting methodologies. Volumes reported by the IDB are the as-stored volumes shown by the sites in their data submittals to the IDB.

bome volumes of TSCA waste are included in this table that are not included in the WIPP inventory defined in WTWBIR, Rev. 1. See Tables 3.3, 3.4, and 3.5 for details.

cSee ref. 5.

dSee ref. 2.

eVolume reported in Hanford submittal.

$f_{\text {Adjusted to delete } \alpha-L L W}$ volume included in previous $\mathrm{DB}$ submittals.

gDifference between "as-stored" and "final-form" volumes primarily caused by volume expansion from repackaging for WIPP waste acceptance criteria (WAC) compliance. INEL shows an overall decrease in volume because of thermal processing of some waste streams.

hApproximately $200 \mathrm{~m}^{3}$ reported with CH-TRUW inventory are from waste streams containing both CH TRUW and RH TRUW (ref. 2, WTWBR, Rev. 1, Table 6-1).

iVolume differences resulting from revised inventory assessment.

jVolume previously included in INEL submittal. 
Table 3.2. Summary of final waste-form volumes $\left(m^{3}\right)$ of retrievably stored and projected TRUW (mixed and nonmixed $\mathrm{CH}$ and $\mathrm{RH}$ wastes)

\begin{tabular}{|c|c|c|c|c|c|c|c|c|c|}
\hline \multirow{2}{*}{ Site } & \multirow{2}{*}{$\begin{array}{l}\text { EOY }^{\mathrm{c}} \\
1993\end{array}$} & \multicolumn{4}{|c|}{ Annual increase } & \multicolumn{2}{|c|}{ Cumulative increase } & \multirow{2}{*}{$\begin{array}{c}\begin{array}{c}\text { Projected } \\
\text { increase }\end{array} \\
1994-2022\end{array}$} & \multirow{2}{*}{$\begin{array}{c}\text { Total } \\
\text { EOY } 2022\end{array}$} \\
\hline & & 1994 & 1995 & 1996 & 1997 & $1998-2002$ & $2003-2022$ & & \\
\hline \multicolumn{10}{|l|}{ Major sites } \\
\hline ANL-E & $2.91 \mathrm{E}+01$ & $1.68 \mathrm{E}+00$ & $0.00 \mathrm{E}+00$ & $0.00 E+00$ & $0.00 \mathrm{E}+00$ & $0.00 \mathrm{E}+00$ & $0.00 E+00$ & $1.68 \mathrm{E}+00$ & $3.08 \mathrm{E}+01$ \\
\hline Hanford & $9.42 E+03$ & $1.71 \mathrm{E}+02$ & $4.91 E+02$ & $6.19 E+02$ & $1.91 E+02$ & $1.29 \mathrm{E}+03$ & $3.11 E+04$ & $3.38 E+04$ & $4.33 E+04$ \\
\hline REL & $3.47 E+04$ & $0.00 \mathrm{E}+00$ & $6.00 \mathrm{E}-01$ & $6.00 \mathrm{E}-01$ & $1.60 \mathrm{E}+00$ & $3.00 \mathrm{E}+00$ & $1.20 \mathrm{E}+01$ & $1.78 \mathrm{E}+01$ & $3.47 E+04$ \\
\hline LANL & $1.10 \mathrm{E}+04$ & $2.66 \mathrm{E}+02$ & $2.77 \mathrm{E}+02$ & $2.77 \mathrm{E}+02$ & $2.77 \mathrm{E}+02$ & $1.34 \mathrm{E}+03$ & $5.32 \mathrm{E}+03$ & $7.76 \mathrm{E}+03$ & $1.87 \mathrm{E}+04$ \\
\hline LLNL & $2.24 \mathrm{E}+02$ & $2.11 E+01$ & $2.50 \mathrm{E}+01$ & $2.50 \mathrm{E}+01$ & $2.50 \mathrm{E}+01$ & $1.25 \mathrm{E}+02$ & $5.00 \mathrm{E}+02$ & $7.21 \mathrm{E}+02$ & $9.45 \mathrm{E}+02$ \\
\hline Mound & $2.63 E+02$ & $0.00 \mathrm{E}+00$ & $0.00 \mathrm{E}+00$ & $0.00 \mathrm{E}+00$ & $0.00 \mathrm{E}+00$ & $0.00 \mathrm{E}+00$ & $0.00 E+00$ & $0.00 \mathrm{E}+00$ & $2.63 E+02$ \\
\hline NTS & $6.20 E+02$ & $0.00 \mathrm{E}+00$ & $0.00 \mathrm{E}+00$ & $0.00 \mathrm{E}+00$ & $0.00 \mathrm{E}+00$ & $0.00 \mathrm{E}+00$ & $0.00 \mathrm{E}+00$ & $0.00 \mathrm{E}+00$ & $6.20 \mathrm{E}+02$ \\
\hline ORNL & $1.78 \mathrm{E}+03$ & $2.14 \mathrm{E}+01$ & $2.14 \mathrm{E}+01$ & $2.14 \mathrm{E}+01$ & $2.14 \mathrm{E}+01$ & $1.07 \mathrm{E}+02$ & $4.28 E+02$ & $6.21 E+02$ & $2.40 \mathrm{E}+03$ \\
\hline RFETS & $1.13 E+03$ & $6.64 \mathrm{E}+01$ & $1.22 \mathrm{E}+02$ & $9.31 \mathrm{E}+01$ & $2.58 \mathrm{E}+01$ & $1.40 E+02$ & $5.45 E+03$ & $5.90 E+03$ & $7.03 E+03$ \\
\hline SRS & $1.46 \mathrm{E}+04$ & $5.13 E+02$ & $5.11 E+02$ & $5.11 \mathrm{E}+02$ & $5.11 \mathrm{E}+02$ & $2.56 \mathrm{E}+03$ & $1.02 E+04$ & $1.48 \mathrm{E}+04$ & $2.94 \mathrm{E}+04$ \\
\hline Subtotal & $7.37 \mathrm{E}+04$ & $1.06 \mathrm{E}+03$ & $1.45 \mathrm{E}+03$ & $1.55 E+03$ & $1.05 E+03$ & $5.56 \mathrm{E}+03$ & $5.30 E+04$ & $6.37 E+04$ & $1.37 E+05$ \\
\hline \multicolumn{10}{|c|}{ Small-quantity sites } \\
\hline Ames & $0.00 \mathrm{E}+00$ & $0.00 \mathrm{E}+00$ & $0.00 \mathrm{E}+00$ & $0.00 \mathrm{E}+00$ & $0.00 \mathrm{E}+00$ & $0.00 \mathrm{E}+00$ & $1.00 \mathrm{E}-01$ & $1.00 \mathrm{E}-01$ & $1.00 \mathrm{E}-01$ \\
\hline ANL-W & $8.67 E+00$ & $2.24 \mathrm{E}+00$ & $8.60 \mathrm{E}-01$ & $1.20 \mathrm{E}+00$ & $1.76 \mathrm{E}+00$ & $7.01 E+00$ & $2.04 E+01$ & $3.35 E+01$ & $4.21 E+01$ \\
\hline $\mathrm{BCL}$ & $0.00 \mathrm{E}+00$ & $0.00 \mathrm{E}+00$ & $0.00 \mathrm{E}+00$ & $0.00 \mathrm{E}+00$ & $0.00 \mathrm{E}+00$ & $7.10 \mathrm{E}+01$ & $0.00 \mathrm{E}+00$ & $7.10 \mathrm{E}+01$ & $7.10 E+01$ \\
\hline BAPL & $0.00 \mathrm{E}+00$ & $0.00 \mathrm{E}+00$ & $7.28 \mathrm{E}-01$ & $2.68 \mathrm{E}+01$ & $1.74 \mathrm{E}+01$ & $7.65 \mathrm{E}+01$ & $3.90 \mathrm{E}+00$ & $1.25 \mathrm{E}+02$ & $1.25 \mathrm{E}+02$ \\
\hline ETEC & $1.87 \mathrm{E}+00$ & $0.00 \mathrm{E}+00$ & $5.20 E+00$ & $0.00 \mathrm{E}+00$ & $0.00 \mathrm{E}+00$ & $0.00 \mathrm{E}+00$ & $0.00 \mathrm{E}+00$ & $5.20 \mathrm{E}+00$ & $7.07 E+00$ \\
\hline KAPL & $1.36 \mathrm{E}+01$ & $8.70 \mathrm{E}-01$ & $8.70 \mathrm{E}-01$ & $8.70 E-01$ & $8.70 \mathrm{E}-01$ & $4.35 \mathrm{E}+00$ & $1.74 E+01$ & $2.52 E+01$ & $3.89 E+01$ \\
\hline LBL & $8.40 \mathrm{E}-01$ & $0.00 \mathrm{E}+00$ & $4.20 \mathrm{E}-01$ & $0.00 \mathrm{E}+00$ & $0.00 \mathrm{E}+00$ & $8.00 \mathrm{E}-01$ & $3.20 \mathrm{E}+00$ & $4.42 \mathrm{E}+00$ & $5.26 \mathrm{E}+00$ \\
\hline MURR & $6.00 \mathrm{E}-02$ & $8.00 \mathrm{E}-02$ & $8.00 E-02$ & 8.00E-02 & $1.36 \mathrm{E}+00$ & $0.00 \mathrm{E}+00$ & $0.00 \mathrm{E}+00$ & $1.60 E+00$ & $1.66 \mathrm{E}+00$ \\
\hline $\mathrm{PAD}$ & $3.45 \mathrm{E}+00$ & $0.00 \mathrm{E}+00$ & $0.00 \mathrm{E}+00$ & $0.00 \mathrm{E}+00$ & $0.00 \mathrm{E}+00$ & $0.00 \mathrm{E}+00$ & $0.00 \mathrm{E}+00$ & $0.00 \mathrm{E}+00$ & $3.45 \mathrm{E}+00$ \\
\hline PANT & $6.24 \mathrm{E}-01$ & $0.00 \mathrm{E}+00$ & $0.00 \mathrm{E}+00$ & $0.00 \mathrm{E}+00$ & $0.00 \mathrm{E}+00$ & $0.00 \mathrm{E}+00$ & $0.00 \mathrm{E}+00$ & $0.00 \mathrm{E}+00$ & $6.24 \mathrm{E}-01$ \\
\hline SNL/NM & $8.04 E+00$ & $0.00 \mathrm{E}+00$ & $5.00 E+00$ & $0.00 \mathrm{E}+00$ & $0.00 \mathrm{E}+00$ & $1.00 \mathrm{E}+00$ & $1.00 \mathrm{E}+00$ & $7.00 \mathrm{E}+00$ & $1.50 \mathrm{E}+01$ \\
\hline Subtotal & $3.72 E+01$ & $3.19 E+00$ & $1.32 \mathrm{E}+01$ & $2.90 \mathrm{E}+01$ & $2.14 \mathrm{E}+01$ & $1.61 E+02$ & $4.60 E+01$ & $2.73 E+02$ & $3.11 E+02$ \\
\hline \multicolumn{10}{|l|}{ Commercial site } \\
\hline WVDP & $4.78 \mathrm{E}+02$ & $5.46 \mathrm{E}+01$ & $2.93 \mathrm{E}+01$ & $6.33 \mathrm{E}+00$ & $6.33 E+00$ & $9.04 \mathrm{E}+01$ & $0.00 E+00$ & $1.87 \mathrm{E}+02$ & $6.64 E+02$ \\
\hline Total & $7.42 \mathrm{E}+04$ & $1.12 \mathrm{E}+03$ & $1.49 E+03$ & $1.58 \mathrm{E}+03$ & $1.08 E+03$ & $5.81 E+03$ & $5.31 E+04$ & $6.41 E+04$ & $1.38 \mathrm{E}+05$ \\
\hline
\end{tabular}

aAny small differences in volumes reported in this table and in WTWBIR, Rev. 1 (ref. 2) and in the numerical totals shown result from rounding.

b Some volumes of TSCA-contaminated wastes are included in this table that are not included in WTWBIR, Rev. 1 (ref. 2). See Tables 3.3, 3.4, and 3.5 for details.

CEOY $=$ end of (calendar) year. 
Table 3.3. Summary of final waste-form volumes $\left(\mathrm{m}^{3}\right)$ of retrievably stored and projected mixed and nonmixed CH TRUWa

\begin{tabular}{|c|c|c|c|c|c|c|c|c|c|}
\hline \multirow{2}{*}{ Site } & \multirow{2}{*}{$\begin{array}{l}\text { EOYb } \\
1993\end{array}$} & \multicolumn{4}{|c|}{ Annual increase } & \multicolumn{2}{|c|}{ Cumulative increase } & \multirow{2}{*}{$\begin{array}{c}\begin{array}{c}\text { Projected } \\
\text { increase }\end{array} \\
1994-2022\end{array}$} & \multirow{2}{*}{$\begin{array}{c}\text { Total } \\
\text { EOY } 2022\end{array}$} \\
\hline & & 1994 & 1995 & 1996 & 1997 & $1998-2002$ & $2003-2022$ & & \\
\hline \multicolumn{10}{|l|}{ Major sites } \\
\hline ANL-E & $2.91 \mathrm{E}+01$ & $1.68 \mathrm{E}+00$ & $0.00 \mathrm{E}+00$ & $0.00 E+00$ & $0.00 \mathrm{E}+00$ & $0.00 \mathrm{E}+00$ & $0.00 \mathrm{E}+00$ & $1.68 \mathrm{E}+00$ & $3.08 \mathrm{E}+01$ \\
\hline Hanford & $9.39 E+03^{c}$ & $1.69 \mathrm{E}+02$ & $3.88 \mathrm{E}+02$ & $5.80 E+02$ & $1.82 E+02$ & $1.19 E+03$ & $2.83 E+04$ & $3.09 \mathrm{E}+04^{\mathrm{d}}$ & $4.02 E+04$ \\
\hline INEL & $3.47 E+04$ & $0.00 E+00$ & $0.00 \mathrm{E}+00$ & $0.00 E+00$ & $1.00 \mathrm{E}+00$ & $0.00 \mathrm{E}+00$ & $0.00 \mathrm{E}+00$ & $1.00 \mathrm{E}+00$ & $3.47 E+04$ \\
\hline LANL & $1.09 E+04$ & $2.54 \mathrm{E}+02$ & $2.65 E+02$ & $2.65 E+02$ & $2.65 E+02$ & $1.33 E+03$ & $5.30 E+03$ & $7.67 \mathrm{E}+03$ & $1.85 \mathrm{E}+04$ \\
\hline LLNL & $2.24 E+02^{e}$ & $2.11 E+01$ & $2.50 \mathrm{E}+01$ & $2.50 \mathrm{E}+01$ & $2.50 \mathrm{E}+01$ & $1.25 \mathrm{E}+02$ & $5.00 \mathrm{E}+02$ & $7.21 E+02^{f}$ & $9.45 \mathrm{E}+02$ \\
\hline Mound & $2.63 \mathrm{E}+02 \mathrm{~g}$ & $0.00 \mathrm{E}+00$ & $0.00 \mathrm{E}+00$ & $0.00 \mathrm{E}+00$ & $0.00 \mathrm{E}+00$ & $0.00 E+00$ & $0.00 \mathrm{E}+00$ & $0.00 \mathrm{E}+00$ & $2.63 E+02$ \\
\hline NTS & $6.20 \mathrm{E}+02$ & $0.00 \mathrm{E}+00$ & $0.00 \mathrm{E}+00$ & $0.00 \mathrm{E}+00$ & $0.00 \mathrm{E}+00$ & $0.00 \mathrm{E}+00$ & $0.00 E+00$ & $0.00 \mathrm{E}+00$ & $6.20 \mathrm{E}+02$ \\
\hline ORNL & $7.83 E+02$ & $9.10 \mathrm{E}+00$ & $9.10 \mathrm{E}+00$ & $9.10 \mathrm{E}+00$ & $9.10 \mathrm{E}+00$ & $4.55 E+01$ & $1.82 E+02$ & $2.64 \mathrm{E}+02$ & $1.05 E+03$ \\
\hline RFETS & $1.13 E+03$ & $6.64 E+01$ & $1.22 \mathrm{E}+02$ & $9.31 E+01$ & $2.58 \mathrm{E}+01$ & $1.40 \mathrm{E}+02$ & $5.45 \mathrm{E}+03$ & $5.90 \mathrm{E}+03$ & $7.03 \mathrm{E}+03$ \\
\hline SRS & $1.46 \mathrm{E}+04$ & $5.09 E+02$ & $5.09 \mathrm{E}+02$ & $5.09 E+02$ & $5.09 \mathrm{E}+02$ & $2.55 E+03$ & $1.02 \mathrm{E}+04$ & $1.48 \mathrm{E}+04$ & $2.94 \mathrm{E}+04$ \\
\hline Subtotal & $7.26 \mathrm{E}+04$ & $1.03 E+03$ & $1.32 \mathrm{E}+03$ & $1.48 \mathrm{E}+03$ & $1.02 \mathrm{E}+03$ & $5.37 E+03$ & $5.00 \mathrm{E}+04$ & $6.02 E+04$ & $1.33 E+05$ \\
\hline \multicolumn{10}{|c|}{ Small-quantity sites } \\
\hline Ames & $0.00 \mathrm{E}+00$ & $0.00 \mathrm{E}+00$ & $0.00 \mathrm{E}+00$ & $0.00 E+00$ & $0.00 \mathrm{E}+00$ & $0.00 \mathrm{E}+00$ & $1.00 \mathrm{E}-01$ & $1.00 \mathrm{E}-01$ & $1.00 \mathrm{E}-01$ \\
\hline ANL-W & $2.00 \mathrm{E}-02$ & $1.92 \mathrm{E}+00$ & $4.40 \mathrm{E}-01$ & $4.40 \mathrm{E}-01$ & $4.40 \mathrm{E}-01$ & $2.20 \mathrm{E}+00$ & $4.00 \mathrm{E}-01$ & $5.84 \mathrm{E}+00$ & $5.86 E+00$ \\
\hline $\mathrm{BCL}$ & $0.00 \mathrm{E}+00$ & $0.00 \mathrm{E}+00$ & $0.00 \mathrm{E}+00$ & $0.00 \mathrm{E}+00$ & $0.00 \mathrm{E}+00$ & $0.00 \mathrm{E}+00$ & $0.00 \mathrm{E}+00$ & $0.00 \mathrm{E}+00$ & $0.00 \mathrm{E}+00$ \\
\hline BAPL & $0.00 E+00$ & $0.00 E+00$ & $4.16 \mathrm{E}-01$ & $2.66 \mathrm{E}+01$ & $1.71 E+01$ & $7.59 E+01$ & $3.80 \mathrm{E}+00$ & $1.24 \mathrm{E}+02$ & $1.24 \mathrm{E}+02$ \\
\hline ETEC & $1.87 \mathrm{E}+00$ & $0.00 \mathrm{E}+00$ & $5.20 \mathrm{E}+00$ & $0.00 \mathrm{E}+00$ & $0.00 \mathrm{E}+00$ & $0.00 \mathrm{E}+00$ & $0.00 \mathrm{E}+00$ & $5.20 \mathrm{E}+00$ & $7.07 \mathrm{E}+00$ \\
\hline KAPL & $2.40 \mathrm{E}+00$ & $0.00 E+00$ & $0.00 E+00$ & $0.00 \mathrm{E}+00$ & $0.00 \mathrm{E}+00$ & $0.00 \mathrm{E}+00$ & $0.00 \mathrm{E}+00$ & $0.00 \mathrm{E}+00$ & $2.40 \mathrm{E}+00$ \\
\hline LBL & $8.40 \mathrm{E}-01$ & $0.00 \mathrm{E}+00$ & $4.20 \mathrm{E}-01$ & $0.00 \mathrm{E}+00$ & $0.00 \mathrm{E}+00$ & $8.00 \mathrm{E}-01$ & $3.20 \mathrm{E}+00$ & $4.42 \mathrm{E}+00$ & $5.26 \mathrm{E}+00$ \\
\hline MURR & $6.00 \mathrm{E}-02$ & $8.00 E-02$ & $8.00 \mathrm{E}-02$ & $8.00 \mathrm{E}-02$ & $1.36 \mathrm{E}+00$ & $0.00 \mathrm{E}+00$ & $0.00 \mathrm{E}+00$ & $1.60 \mathrm{E}+00$ & $1.66 \mathrm{E}+00$ \\
\hline PAD & $3.45 \mathrm{E}+00$ & $0.00 E+00$ & $0.00 \mathrm{E}+00$ & $0.00 \mathrm{E}+00$ & $0.00 \mathrm{E}+00$ & $0.00 \mathrm{E}+00$ & $0.00 \mathrm{E}+00$ & $0.00 E+00$ & $3.45 E+00$ \\
\hline PANT & $6.24 \mathrm{E}-01$ & $0.00 \mathrm{E}+00$ & $0.00 \mathrm{E}+00$ & $0.00 \mathrm{E}+00$ & $0.00 \mathrm{E}+00$ & $0.00 \mathrm{E}+00$ & $0.00 \mathrm{E}+00$ & $0.00 \mathrm{E}+00$ & $6.24 \mathrm{E}-01$ \\
\hline SNL/NM & $8.04 E+00$ & $0.00 E+00$ & $5.00 \mathrm{E}+00$ & $0.00 \mathrm{E}+00$ & $0.00 \mathrm{E}+00$ & $1.00 \mathrm{E}+00$ & $1.00 \mathrm{E}+00$ & $7.00 E+00$ & $1.50 \mathrm{E}+01$ \\
\hline Subtotal & $1.73 E+01$ & $2.00 E+00$ & $1.16 \mathrm{E}+01$ & $2.71 E+01$ & $1.89 E+01$ & $7.99 \mathrm{E}+01$ & $8.50 \mathrm{E}+00$ & $1.48 \mathrm{E}+02$ & $1.65 \mathrm{E}+02$ \\
\hline
\end{tabular}


Table 3.3 (continued)

\begin{tabular}{|c|c|c|c|c|c|c|c|c|c|}
\hline \multirow{2}{*}{ Site } & \multirow{2}{*}{$\begin{array}{l}\text { EOYb } \\
1993\end{array}$} & \multicolumn{4}{|c|}{ Annual increase } & \multicolumn{2}{|c|}{ Cumulative increase } & \multirow{2}{*}{$\begin{array}{c}\begin{array}{c}\text { Projected } \\
\text { increase }\end{array} \\
1994-2022\end{array}$} & \multirow{2}{*}{$\begin{array}{c}\text { Total } \\
\text { EOY } 2022\end{array}$} \\
\hline & & 1994 & 1995 & 1996 & 1997 & 1998-2002 & $2003-2022$ & & \\
\hline \multicolumn{10}{|c|}{ Commercial site } \\
\hline WVDP & $5.06 \mathrm{E}+01$ & $5.46 E+01$ & $2.93 E+01$ & $6.33 \mathrm{E}+00$ & $6.33 E+00$ & $9.04 \mathrm{E}+01$ & $0.00 E+00$ & $1.87 E+02$ & $2.37 E+02$ \\
\hline Total & $7.26 \mathrm{E}+04$ & $1.09 E+03$ & $1.36 \mathrm{E}+03$ & $1.52 \mathrm{E}+03$ & $1.04 E+03$ & $5.54 \mathrm{E}+03$ & $5.00 \mathrm{E}+04$ & $6.05 E+04$ & $1.33 E+05$ \\
\hline
\end{tabular}

aAny small differences in volumes reported in this table and in WTWBIR, Rev. 1 (ref. 2) and in the numerical totals shown result from rounding. $b_{\mathrm{EOY}}=$ end of (calendar) year.

$c_{7.42 E}+01 \mathrm{~m}^{3}$ (approximately) of TSCA waste included from Table 3-2, WTWBIR, Rev 1 (ref 2)

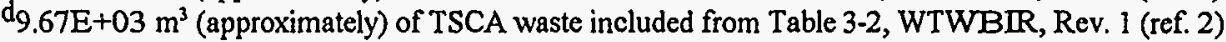

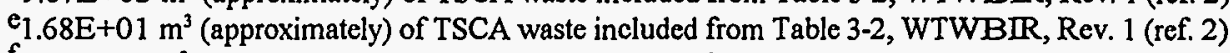

$\mathrm{f}_{3.26 \mathrm{E}}+01 \mathrm{~m}^{3}$ (approximately) of TSCA waste included from Table 3-2, WTWBIR, Rev. 1 (ref. 2)

88.00E- $01 \mathrm{~m}^{3}$ (approximately) of TSCA waste included from Table 3-2, WTWBIR, Rev. 1 (ref. 2). 
Table 3.4. Final waste-form volumes $\left(\mathrm{m}^{3}\right)$ of retrievably stored and projected mired $\mathrm{CH}$ TRUW ${ }^{\mathrm{a}}$

\begin{tabular}{|c|c|c|c|c|c|c|c|c|c|}
\hline \multirow{2}{*}{ Site } & \multirow{2}{*}{$\begin{array}{l}\text { EOY }^{b} \\
1993\end{array}$} & \multicolumn{4}{|c|}{ Annual increase } & \multicolumn{2}{|c|}{ Cumulative increase } & \multirow{2}{*}{$\begin{array}{c}\begin{array}{c}\text { Projected } \\
\text { increase }\end{array} \\
1994-2022\end{array}$} & \multirow{2}{*}{$\begin{array}{c}\text { Total } \\
\text { EOY } 2022\end{array}$} \\
\hline & & 1994 & 1995 & 1996 & 1997 & $1998-2002$ & 2003-2022 & & \\
\hline \multicolumn{10}{|l|}{ Major sites } \\
\hline ANL-E & $6.21 \mathrm{E}+00$ & $5.60 \mathrm{E}-01$ & $0.00 \mathrm{E}+00$ & $0.00 E+00$ & $0.00 \mathrm{E}+00$ & $0.00 E+00$ & $0.00 \mathrm{E}+00$ & $5.60 \mathrm{E}-01$ & $6.77 \mathrm{E}+00$ \\
\hline Hanford & $2.10 E+02^{c}$ & $1.41 E+01$ & $3.13 E+01$ & $4.60 \mathrm{E}+0 \mathrm{I}$ & $1.39 \mathrm{E}+01$ & $1.39 E+02$ & $1.49 E+04$ & $1.51 E+04^{d}$ & $1.54 E+04$ \\
\hline INEL & $2.76 \mathrm{E}+04$ & $0.00 \mathrm{E}+00$ & $0.00 \mathrm{E}+00$ & $0.00 \mathrm{E}+00$ & $1.00 \mathrm{E}+00$ & $0.00 \mathrm{E}+00$ & $0.00 \mathrm{E}+00$ & $1.00 \mathrm{E}+00$ & $2.76 \mathrm{E}+04$ \\
\hline LANL & $7.66 \mathrm{E}+03$ & $1.24 \mathrm{E}+02$ & $1.35 E+02$ & $1.35 E+02$ & $1.35 E+02$ & $6.75 E+02$ & $2.70 E+03$ & $3.90 E+03$ & $1.16 \mathrm{E}+04$ \\
\hline LLNL & $7.92 E+00$ & $6.24 \mathrm{E}-01$ & $1.57 \mathrm{E}+00$ & $1.57 \mathrm{E}+00$ & $1.57 \mathrm{E}+00$ & $7.87 \mathrm{E}+00$ & $3.15 \mathrm{E}+01$ & $4.47 \mathrm{E}+01$ & $5.26 E+01$ \\
\hline Mound & $2.70 \mathrm{E}+00^{\mathrm{e}}$ & $0.00 E+00$ & $0.00 \mathrm{E}+00$ & $0.00 E+00$ & $0.00 \mathrm{E}+00$ & $0.00 \mathrm{E}+00$ & $0.00 \mathrm{E}+00$ & $0.00 \mathrm{E}+00$ & $2.70 \mathrm{E}+00$ \\
\hline NTS & $6.20 \mathrm{E}+02$ & $0.00 E+00$ & $0.00 \mathrm{E}+00$ & $0.00 \mathrm{E}+00$ & $0.00 E+00$ & $0.00 E+00$ & $0.00 \mathrm{E}+00$ & $0.00 \mathrm{E}+00$ & $6.20 \mathrm{E}+02$ \\
\hline ORNL & $7.83 E+02$ & $9.10 E+00$ & $9.10 \mathrm{E}+00$ & $9.10 \mathrm{E}+00$ & $9.10 \mathrm{E}+00$ & $4.55 \mathrm{E}+01$ & $1.82 E+02$ & $2.64 \mathrm{E}+02$ & $1.05 E+03$ \\
\hline RFETS & $8.28 E+02$ & $4.69 \mathrm{E}+01$ & $7.49 \mathrm{E}+01$ & $6.09 \mathrm{E}+01$ & $2.21 \mathrm{E}+01$ & $1.13 E+02$ & $5.07 \mathrm{E}+03$ & $5.38 \mathrm{E}+03$ & $6.21 E+03$ \\
\hline SRS & $1.01 E+04$ & $8.84 \mathrm{E} \div 01$ & $8.84 \mathrm{E}+01$ & $8.84 E+01$ & $8.84 \mathrm{E}+01$ & $4.42 E+02$ & $1.77 \mathrm{E}+03$ & $2.56 \mathrm{E}+03$ & $1.27 \mathrm{E}+04$ \\
\hline Subtotal & $4.79 E+04$ & $2.84 \mathrm{E}+02$ & $3.40 \mathrm{E}+02$ & $3.41 \mathrm{E}+02$ & $2.71 E+02$ & $1.42 E+03$ & $2.47 \mathrm{E}+04$ & $2.73 E+04$ & $7.52 \mathrm{E}+04$ \\
\hline \multicolumn{10}{|c|}{ Small-quantity sites } \\
\hline Ames & $0.00 \mathrm{E}+00$ & $0.00 E+00$ & $0.00 \mathrm{E}+00$ & $0.00 \mathrm{E}+00$ & $0.00 E+00$ & $0.00 \mathrm{E}+00$ & $1.00 \mathrm{E}-01$ & $1.00 \mathrm{E}-01$ & $1.00 \mathrm{E}-01$ \\
\hline ANLLW & $2.00 \mathrm{E}-02$ & $1.92 \mathrm{E}+00$ & $2.00 \mathrm{E}-02$ & $2.00 \mathrm{E}-02$ & $2.00 \mathrm{E}-02$ & $1.00 \mathrm{E}-01$ & $4.00 \mathrm{E}-01$ & $2.48 \mathrm{E}+00$ & $2.50 \mathrm{E}+00$ \\
\hline $\mathrm{BCL}$ & $0.00 E+00$ & $0.00 \mathrm{E}+00$ & $0.00 \mathrm{E}+00$ & $0.00 \mathrm{E}+00$ & $0.00 E+00$ & $0.00 \mathrm{E}+00$ & $0.00 E+00$ & $0.00 \mathrm{E}+00$ & $0.00 E+00$ \\
\hline BAPL & $0.00 \mathrm{E}+00$ & $0.00 \mathrm{E}+00$ & $0.00 \mathrm{E}+00$ & $0.00 \mathrm{E}+00$ & $0.00 \mathrm{E}+00$ & $0.00 \mathrm{E}+00$ & $0.00 \mathrm{E}+00$ & $0.00 \mathrm{E}+00$ & $0.00 \mathrm{E}+00$ \\
\hline ETEC & $2.10 E-01$ & $0.00 E+00$ & $0.00 \mathrm{E}+00$ & $0.00 \mathrm{E}+00$ & $0.00 E+00$ & $0.00 \mathrm{E}+00$ & $0.00 \mathrm{E}+00$ & $0.00 E+00$ & $2.10 \mathrm{E}-01$ \\
\hline KAPL & $0.00 \mathrm{E}+00$ & $0.00 \mathrm{E}+00$ & $0.00 \mathrm{E}+00$ & $0.00 \mathrm{E}+00$ & $0.00 \mathrm{E}+00$ & $0.00 \mathrm{E}+00$ & $0.00 E+00$ & $0.00 \mathrm{E}+00$ & $0.00 E+00$ \\
\hline LBL & $0.00 E+00$ & $0.00 \mathrm{E}+00$ & $0.00 \mathrm{E}+00$ & $0.00 \mathrm{E}+00$ & $0.00 \mathrm{E}+00$ & $0.00 \mathrm{E}+00$ & $0.00 \mathrm{E}+00$ & $0.00 \mathrm{E}+00$ & $0.00 \mathrm{E}+00$ \\
\hline MURR & $6.00 \mathrm{E}-02$ & $8.00 E-02$ & $8.00 \mathrm{E}-02$ & $8.00 \mathrm{E}-02$ & $1.36 \mathrm{E}+00$ & $0.00 \mathrm{E}+00$ & $0.00 \mathrm{E}+00$ & $1.60 \mathrm{E}+00$ & $1.66 E+00$ \\
\hline PAD & $3.45 \mathrm{E}+00$ & $0.00 \mathrm{E}+00$ & $0.00 \mathrm{E}+00$ & $0.00 \mathrm{E}+00$ & $0.00 \mathrm{E}+00$ & $0.00 \mathrm{E}+00$ & $0.00 \mathrm{E}+00$ & $0.00 \mathrm{E}+00$ & $3.45 \mathrm{E}+00$ \\
\hline PANT & $0.00 E+00$ & $0.00 \mathrm{E}+00$ & $0.00 \mathrm{E}+00$ & $0.00 \mathrm{E}+00$ & $0.00 \mathrm{E}+00$ & $0.00 \mathrm{E}+00$ & $0.00 \mathrm{E}+00$ & $0.00 \mathrm{E}+00$ & $0.00 E+00$ \\
\hline SNL/NM & $1.04 \mathrm{E}+00$ & $0.00 \mathrm{E}+00$ & $0.00 E+00$ & $0.00 \mathrm{E}+00$ & $0.00 \mathrm{E}+00$ & $0.00 \mathrm{E}+00$ & $0.00 \mathrm{E}+00$ & $0.00 E+00$ & $1.04 E+00$ \\
\hline Subtotal & $4.78 \mathrm{E}+00$ & $2.00 \mathrm{E}+00$ & $1.00 \mathrm{E}-01$ & $1.00 \mathrm{E}-01$ & $1.38 \mathrm{E}+00$ & $1.00 \mathrm{E}-01$ & $5.00 \mathrm{E}-01$ & $4.18 \mathrm{E}+00$ & $8.96 E+00$ \\
\hline \multicolumn{10}{|l|}{ Commercial site } \\
\hline WVDP & $3.19 E+01$ & $4.36 \mathrm{E}+01$ & $2.38 \mathrm{E}+01$ & $7.90 \mathrm{E}-01$ & $7.90 \mathrm{E}-01$ & $6.27 \mathrm{E}+01$ & $0.00 \mathrm{E}+00$ & $1.32 \mathrm{E}+02$ & $1.64 \mathrm{E}+02$ \\
\hline Total & $4.79 \mathrm{E}+04$ & $3.29 \mathrm{E}+02$ & $3.64 \mathrm{E}+02$ & $3.42 \mathrm{E}+02$ & $2.73 E+02$ & $1.48 \mathrm{E}+03$ & $2.47 \mathrm{E}+04$ & $2.74 \mathrm{E}+04$ & $7.54 \mathrm{E}+04$ \\
\hline
\end{tabular}

a Any small differences in volumes reported in this table and in WTWBIR, Rev. 1 (ref. 2) and in the numerical totals shown result from rounding.

$\mathrm{b}_{\mathrm{EOY}}=$ end of (calendar) year.

c7.40E+01 $\mathrm{m}^{3}$ (approximately) of TSCA waste included from Table 3-2, WTWBIR, Rev. 1 (ref. 2)

d9.62E+03 $\mathrm{m}^{3}$ (approximately) of TSCA waste included from Table 3-2, WTWBIR, Rev. 1 (ref. 2)

$e_{2.00 E-01 ~ ~^{3}}$ (approximately) of TSCA waste included from Table 3-2, WTWBIR, Rev. 1 (ref. 2). 
Table 3.5. Final waste-form volumes $\left(\mathrm{m}^{3}\right)$ of retrievably stored and projected nonmixed CH TRUWa

\begin{tabular}{|c|c|c|c|c|c|c|c|c|c|}
\hline \multirow{2}{*}{ Site } & \multirow{2}{*}{$\begin{array}{l}\text { EOYb } \\
1993\end{array}$} & \multicolumn{4}{|c|}{ Annual increase } & \multicolumn{2}{|c|}{ Cumulative increase } & \multirow{2}{*}{$\begin{array}{c}\begin{array}{c}\text { Projected } \\
\text { increase }\end{array} \\
1994-2022\end{array}$} & \multirow{2}{*}{$\begin{array}{c}\text { Total } \\
\text { EOY } 2022\end{array}$} \\
\hline & & 1994 & 1995 & 1996 & 1997 & 1998-2002 & $2003-2022$ & & \\
\hline \multicolumn{10}{|l|}{ Major sites } \\
\hline ANL-E & $2.29 E+01$ & $1.12 \mathrm{E}+00$ & $0.00 \mathrm{E}+00$ & $0.00 \mathrm{E}+00$ & $0.00 E+00$ & $0.00 \mathrm{E}+00$ & $0.00 \mathrm{E}+00$ & $1.12 \mathrm{E}+00$ & $2.40 \mathrm{E}+01$ \\
\hline Hanford & $9.18 \mathrm{E}+03^{\mathrm{c}}$ & $1.55 \mathrm{E}+02$ & $3.56 E+02$ & $5.34 E+02$ & $1.68 \mathrm{E}+02$ & $1.05 E+03$ & $1.34 E+04$ & $1.57 \mathrm{E}+04^{\mathrm{d}}$ & $2.49 E+04$ \\
\hline NEL & $7.02 \mathrm{E}+03$ & $0.00 \mathrm{E}+00$ & $0.00 \mathrm{E}+00$ & $0.00 \mathrm{E}+00$ & $0.00 E+00$ & $0.00 \mathrm{E}+00$ & $0.00 E+00$ & $0.00 E+00$ & $7.02 \mathrm{E}+03$ \\
\hline LANL & $3.20 \mathrm{E}+03$ & $1.30 \mathrm{E}+02$ & $1.30 \mathrm{E}+02$ & $1.30 \mathrm{E}+02$ & $1.30 \mathrm{E}+02$ & $6.50 \mathrm{E}+02$ & $2.60 \mathrm{E}+03$ & $3.77 \mathrm{E}+03$ & $6.97 \mathrm{E}+03$ \\
\hline LLNL & $2.16 \mathrm{E}+02^{\mathrm{e}}$ & $2.05 E+01$ & $2.34 E+01$ & $2.34 \mathrm{E}+01$ & $2.34 \mathrm{E}+01$ & $1.17 E+02$ & $4.68 E+02$ & $6.76 E+02^{f}$ & $8.92 E+02$ \\
\hline Mound & $2.61 E+02 g$ & $0.00 \mathrm{E}+00$ & $0.00 E+00$ & $0.00 \mathrm{E}+00$ & $0.00 \mathrm{E}+00$ & $0.00 \mathrm{E}+00$ & $0.00 \mathrm{E}+00$ & $0.00 \mathrm{E}+00$ & $2.61 \mathrm{E}+02$ \\
\hline NTS & $0.00 E+00$ & $0.00 \mathrm{E}+00$ & $0.00 E+00$ & $0.00 \mathrm{E}+00$ & $0.00 \mathrm{E}+00$ & $0.00 \mathrm{E}+00$ & $0.00 \mathrm{E}+00$ & $0.00 \mathrm{E}+00$ & $0.00 \mathrm{E}+00$ \\
\hline ORNL & $0.00 E+00$ & $0.00 \mathrm{E}+00$ & $0.00 \mathrm{E}+00$ & $0.00 \mathrm{E}+00$ & $0.00 E+00$ & $0.00 \mathrm{E}+00$ & $0.00 \mathrm{E}+00$ & $0.00 \mathrm{E}+00$ & $0.00 E+00$ \\
\hline RFETS & $3.05 E+02$ & $1.96 \mathrm{E}+01$ & $4.67 \mathrm{E}+01$ & $3.22 E+01$ & $3.67 E+00$ & $2.70 \mathrm{E}+01$ & $3.88 E+02$ & $5.17 \mathrm{E}+02$ & $8.22 \mathrm{E}+02$ \\
\hline SRS & $4.47 \mathrm{E}+03$ & $4.21 E+02$ & $4.21 E+02$ & $4.21 \mathrm{E}+02$ & $4.21 \mathrm{E}+02$ & $2.10 \mathrm{E}+03$ & $8.42 E+03$ & $1.22 \mathrm{E}+04$ & $1.67 \mathrm{E}+04$ \\
\hline Subtotal & $2.47 E+04$ & $7.47 \mathrm{E}+02$ & $9.77 E+02$ & $1.14 \mathrm{E}+03$ & $7.46 \mathrm{E}+02$ & $3.95 E+03$ & $2.53 E+04$ & $3.29 E+04$ & $5.76 \mathrm{E}+04$ \\
\hline \multicolumn{10}{|c|}{ Small-quantity sites } \\
\hline Ames & $0.00 \mathrm{E}+00$ & $0.00 \mathrm{E}+00$ & $0.00 E+00$ & $0.00 \mathrm{E}+00$ & $0.00 \mathrm{E}+00$ & $0.00 \mathrm{E}+00$ & $0.00 \mathrm{E}+00$ & $0.00 E+00$ & $0.00 \mathrm{E}+00$ \\
\hline ANL-W & $0.00 E+00$ & $0.00 \mathrm{E}+00$ & $4.20 \mathrm{E}-01$ & $4.20 \mathrm{E}-01$ & $4.20 E-01$ & $2.10 E+00$ & $0.00 \mathrm{E}+00$ & $3.36 \mathrm{E}+00$ & $3.36 \mathrm{E}+00$ \\
\hline $\mathrm{BCL}$ & $0.00 \mathrm{E}+00$ & $0.00 \mathrm{E}+00$ & $0.00 \mathrm{E}+00$ & $0.00 \mathrm{E}+00$ & $0.00 \mathrm{E}+00$ & $0.00 \mathrm{E}+00$ & $0.00 E+00$ & $0.00 \mathrm{E}+00$ & $0.00 \mathrm{E}+00$ \\
\hline BAPL & $0.00 E+00$ & $0.00 \mathrm{E}+00$ & $4.16 \mathrm{E}-01$ & $2.66 \mathrm{E}+01$ & $1.71 \mathrm{E}+01$ & $7.59 \mathrm{E}+01$ & $3.80 \mathrm{E}+00$ & $1.24 \mathrm{E}+02$ & $1.24 \mathrm{E}+02$ \\
\hline ETEC & $1.66 \mathrm{E}+00$ & $0.00 \mathrm{E}+00$ & $5.20 E+00$ & $0.00 \mathrm{E}+00$ & $0.00 \mathrm{E}+00$ & $0.00 \mathrm{E}+00$ & $0.00 \mathrm{E}+00$ & $5.20 \mathrm{E}+00$ & $6.86 \mathrm{E}+00$ \\
\hline KAPL & $2.40 \mathrm{E}+00$ & $0.00 \mathrm{E}+00$ & $0.00 \mathrm{E}+00$ & $0.00 \mathrm{E}+00$ & $0.00 \mathrm{E}+00$ & $0.00 \mathrm{E}+00$ & $0.00 E+00$ & $0.00 \mathrm{E}+00$ & $2.40 \mathrm{E}+00$ \\
\hline LBL & $8.40 \mathrm{E}-01$ & $0.00 \mathrm{E}+00$ & $4.20 \mathrm{E}-01$ & $0.00 E+00$ & $0.00 \mathrm{E}+00$ & $8.00 \mathrm{E}-01$ & $3.20 E+00$ & $4.42 E+00$ & $5.26 \mathrm{E}+00$ \\
\hline MURR & $0.00 \mathrm{E}+00$ & $0.00 E+00$ & $0.00 \mathrm{E}+00$ & $0.00 \mathrm{E}+00$ & $0.00 \mathrm{E}+00$ & $0.00 \mathrm{E}+00$ & $0.00 E+00$ & $0.00 \mathrm{E}+00$ & $0.00 \mathrm{E}+00$ \\
\hline PAD & $0.00 E+00$ & $0.00 \mathrm{E}+00$ & $0.00 \mathrm{E}+00$ & $0.00 \mathrm{E}+00$ & $0.00 E+00$ & $0.00 E+00$ & $0.00 \mathrm{E}+00$ & $0.00 \mathrm{E}+00$ & $0.00 \mathrm{E}+00$ \\
\hline PANT & $6.24 \mathrm{E}-01$ & $0.00 \mathrm{E}+00$ & $0.00 \mathrm{E}+00$ & $0.00 \mathrm{E}+00$ & $0.00 \mathrm{E}+00$ & $0.00 \mathrm{E}+00$ & $0.00 \mathrm{E}+00$ & $0.00 \mathrm{E}+00$ & $6.24 \mathrm{E}-01$ \\
\hline SNL/NM & $7.00 \mathrm{E}+00$ & $0.00 \mathrm{E}+00$ & $5.00 \mathrm{E}+00$ & $0.00 \mathrm{E}+00$ & $0.00 \mathrm{E}+00$ & $1.00 E+00$ & $1.00 \mathrm{E}+00$ & $7.00 \mathrm{E}+00$ & $1.40 \mathrm{E}+01$ \\
\hline Subtotal & $1.25 \mathrm{E}+01$ & $0.00 \mathrm{E}+00$ & $1.15 \mathrm{E}+01$ & $2.70 \mathrm{E}+01$ & $1.75 E+01$ & $7.98 E+01$ & $8.00 E+00$ & $1.44 E+02$ & $1.56 \mathrm{E}+02$ \\
\hline
\end{tabular}


Table 3.5 (continued)

\begin{tabular}{|c|c|c|c|c|c|c|c|c|c|}
\hline \multirow{2}{*}{ Site } & \multirow{2}{*}{$\begin{array}{l}\text { EOY }^{\mathrm{b}} \\
1993\end{array}$} & \multicolumn{4}{|c|}{ Annual increase } & \multicolumn{2}{|c|}{ Cumulative increase } & \multirow{2}{*}{$\begin{array}{c}\begin{array}{c}\text { Projected } \\
\text { increase }\end{array} \\
1994-2022\end{array}$} & \multirow{2}{*}{$\begin{array}{c}\text { Total } \\
\text { EOY } 2022\end{array}$} \\
\hline & & 1994 & 1995 & 1996 & 1997 & $1998-2002$ & $2003-2022$ & & \\
\hline \multicolumn{10}{|c|}{ Commercial site } \\
\hline WVDP & $1.87 \mathrm{E}+01$ & $1.10 \mathrm{E}+01$ & $5.54 \mathrm{E}+00$ & $5.54 \mathrm{E}+00$ & $5.54 \mathrm{E}+00$ & $2.77 \mathrm{E}+01$ & $0.00 \mathrm{E}+00$ & $5.53 \mathrm{E}+01$ & $7.40 E+01$ \\
\hline Total & $2.47 \mathrm{E}+04$ & $7.58 \mathrm{E}+02$ & $9.94 E+02$ & $1.17 E+03$ & $7.69 E+02$ & $4.06 \mathrm{E}+03$ & $2.53 \mathrm{E}+04$ & $3.31 E+04$ & $5.78 \mathrm{E}+04$ \\
\hline
\end{tabular}

${ }^{a}$ Any small differences in volumes reported in this table and in WTWBIR, Rev. 1 (ref. 2 ) and in the numerical totals shown result from rounding. $\mathrm{b}_{\mathrm{EOY}}=$ end of (calendar) year.

$c_{2.00 E}-01 \mathrm{~m}^{3}$ (approximately) of TSCA waste included from Table 3-2, WTWBIR, Rev. 1 (ref. 2).

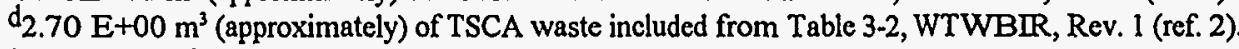

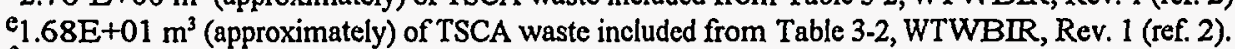

$\mathrm{f}_{3.26 \mathrm{E}}+01 \mathrm{~m}^{3}$ (approximately) of TSCA waste included from Table 3-2, WTWBIR, Rev. 1 (ref. 2).

86.00E-01 $\mathrm{m}^{3}$ (approximately) of TSCA waste included from Table 3-2, WTWBIR, Rev. 1 (ref. 2). 
Table 3.6. Summary of final waste-form volumes $\left(\mathrm{m}^{3}\right)$ of retrievably stored and projected mixed and nonmixed RH TRUWa

\begin{tabular}{|c|c|c|c|c|c|c|c|c|c|}
\hline \multirow{2}{*}{ Sitc } & \multirow{2}{*}{$\begin{array}{l}\text { EOYb } \\
1993\end{array}$} & \multicolumn{4}{|c|}{ Annual increase } & \multicolumn{2}{|c|}{ Cumulative increase } & \multirow{2}{*}{$\begin{array}{c}\begin{array}{c}\text { Projected } \\
\text { increase }\end{array} \\
1994-2022\end{array}$} & \multirow{2}{*}{$\begin{array}{c}\text { Total } \\
\text { EOY } 2022\end{array}$} \\
\hline & & 1994 & 1995 & 1996 & 1997 & $1998-2002$ & 2003-2022 & & \\
\hline \multicolumn{10}{|l|}{ Major sites } \\
\hline ANL-E & $0.00 \mathrm{E}+00$ & $0.00 \mathrm{E}+00$ & $0.00 \mathrm{E}+00$ & $0.00 \mathrm{E}+00$ & $0.00 \mathrm{E}+00$ & $0.00 \mathrm{E}+00$ & $0.00 E+00$ & $0.00 \mathrm{E}+00$ & $0.00 E+00$ \\
\hline Hanford & $3.32 E+01$ & $2.00 E+00$ & $1.03 E+02$ & $3.90 \mathrm{E}+01$ & $8.76 \mathrm{E}+00$ & $1.00 \mathrm{E}+02$ & $2.72 E+03$ & $2.97 \mathrm{E}+03$ & $3.01 \mathrm{E}+03$ \\
\hline INEL & $3.10 \mathrm{E}+01$ & $0.00 \mathrm{E}+00$ & $6.00 \mathrm{E}-01$ & $6.00 \mathrm{E}-01$ & $6.00 \mathrm{E}-01$ & $3.00 \mathrm{E}+00$ & $1.20 \mathrm{E}+01$ & $1.68 \mathrm{E}+01$ & $4.78 \mathrm{E}+01$ \\
\hline LANL & $9.13 \mathrm{E}+01$ & $1.17 \mathrm{E}+01$ & $1.17 \mathrm{E}+01$ & $1.17 \mathrm{E}+01$ & $1.17 \mathrm{E}+01$ & $1.19 \mathrm{E}+01$ & $2.40 \mathrm{E}+01$ & $8.27 E+01$ & $1.74 \mathrm{E}+02$ \\
\hline LLNL & $0.00 \mathrm{E}+00$ & $0.00 \mathrm{E}+00$ & $0.00 \mathrm{E}+00$ & $0.00 \mathrm{E}+00$ & $0.00 \mathrm{E}+00$ & $0.00 \mathrm{E}+00$ & $0.00 \mathrm{E}+00$ & $0.00 \mathrm{E}+00$ & $0.00 E+00$ \\
\hline Mound & $0.00 E+00$ & $0.00 E+00$ & $0.00 \mathrm{E}+00$ & $0.00 \mathrm{E}+00$ & $0.00 \mathrm{E}+00$ & $0.00 \mathrm{E}+00$ & $0.00 \mathrm{E}+00$ & $0.00 \mathrm{E}+00$ & $0.00 E+00$ \\
\hline NTS & $0.00 \mathrm{E}+00$ & $0.00 E+00$ & $0.00 \mathrm{E}+00$ & $0.00 E+00$ & $0.00 \mathrm{E}+00$ & $0.00 \mathrm{E}+00$ & $0.00 \mathrm{E}+00$ & $0.00 \mathrm{E}+00$ & $0.00 \mathrm{E}+00$ \\
\hline ORNL & $9.94 \mathrm{E}+02$ & $1.23 E+01$ & $1.23 E+01$ & $1.23 \mathrm{E}+01$ & $1.23 E+01$ & $6.15 \mathrm{E}+01$ & $2.46 \mathrm{E}+02$ & $3.57 \mathrm{E}+02$ & $1.35 \mathrm{E}+03$ \\
\hline RFETS & $0.00 \mathrm{E}+00$ & $0.00 E+00$ & $0.00 \mathrm{E}+00$ & $0.00 E+00$ & $0.00 \mathrm{E}+00$ & $0.00 E+00$ & $0.00 \mathrm{E}+00$ & $0.00 E+00$ & $0.00 E+00$ \\
\hline SRS & $0.00 \mathrm{E}+00$ & $4.00 E+00$ & $2.14 \mathrm{E}+00$ & $2.14 \mathrm{E}+00$ & $2.14 \mathrm{E}+00$ & $1.05 \mathrm{E}+01$ & $4.30 \mathrm{E}+01$ & $6.39 E+01$ & $6.39 \mathrm{E}+01$ \\
\hline Subtotal & $1.15 E+03$ & $3.00 \mathrm{E}+01$ & $1.30 \mathrm{E}+02$ & $6.57 E+01$ & $3.55 \mathrm{E}+01$ & $1.87 \mathrm{E}+02$ & $3.05 \mathrm{E}+03$ & $3.49 E+03$ & $4.64 \mathrm{E}+03$ \\
\hline \multicolumn{10}{|c|}{ Small-quantity sites } \\
\hline Ames & $0.00 \mathrm{E}+00$ & $0.00 E+00$ & $0.00 \mathrm{E}+00$ & $0.00 E+00$ & $0.00 \mathrm{E}+00$ & $0.00 \mathrm{E}+00$ & $0.00 \mathrm{E}+00$ & $0.00 \mathrm{E}+00$ & $0.00 E+00$ \\
\hline ANL-W & $8.65 E+00$ & $3.20 \mathrm{E}-01$ & $4.20 \mathrm{E}-01$ & $7.56 \mathrm{E}-01$ & $1.32 \mathrm{E}+00$ & $4.81 E+00$ & $2.00 \mathrm{E}+01$ & $2.76 \mathrm{E}+01$ & $3.63 E+01$ \\
\hline BCL & $0.00 \mathrm{E}+00$ & $0.00 E+00$ & $0.00 E+00$ & $0.00 \mathrm{E}+00$ & $0.00 \mathrm{E}+00$ & $7.10 \mathrm{E}+01$ & $0.00 \mathrm{E}+00$ & $7.10 \mathrm{E}+01$ & $7.10 \mathrm{E}+01$ \\
\hline BAPL & $0.00 \mathrm{E}+00$ & $0.00 E+00$ & $3.12 \mathrm{E}-01$ & $2.08 E-01$ & $3.12 \mathrm{E}-01$ & $6.25 E-01$ & $1.00 \mathrm{E}-01$ & $1.56 \mathrm{E}+00$ & $1.56 \mathrm{E}+00$ \\
\hline ETEC & $0.00 \mathrm{E}+00$ & $0.00 \mathrm{E}+00$ & $0.00 \mathrm{E}+00$ & $0.00 \mathrm{E}+00$ & $0.00 \mathrm{E}+00$ & $0.00 \mathrm{E}+00$ & $0.00 \mathrm{E}+00$ & $0.00 \mathrm{E}+00$ & $0.00 \mathrm{E}+00$ \\
\hline KAPL & $1.12 \mathrm{E}+01$ & $8.70 E-01$ & $8.70 \mathrm{E}-01$ & $8.70 \mathrm{E}-01$ & $8.70 \mathrm{E}-01$ & $4.35 \mathrm{E}+00$ & $1.74 \mathrm{E}+01$ & $2.52 \mathrm{E}+01$ & $3.65 E+01$ \\
\hline LBL & $0.00 E+00$ & $0.00 E+00$ & $0.00 \mathrm{E}+00$ & $0.00 \mathrm{E}+00$ & $0.00 \mathrm{E}+00$ & $0.00 \mathrm{E}+00$ & $0.00 \mathrm{E}+00$ & $0.00 \mathrm{E}+00$ & $0.00 \mathrm{E}+00$ \\
\hline MURR & $0.00 \mathrm{E}+00$ & $0.00 \mathrm{E}+00$ & $0.00 \mathrm{E}+00$ & $0.00 \mathrm{E}+00$ & $0.00 \mathrm{E}+00$ & $0.00 \mathrm{E}+00$ & $0.00 E+00$ & $0.00 E+00$ & $0.00 \mathrm{E}+00$ \\
\hline PAD & $0.00 \mathrm{E}+00$ & $0.00 E+00$ & $0.00 E+00$ & $0.00 E+00$ & $0.00 E+00$ & $0.00 \mathrm{E}+00$ & $0.00 \mathrm{E}+00$ & $0.00 \mathrm{E}+00$ & $0.00 E+00$ \\
\hline PANT & $0.00 \mathrm{E}+00$ & $0.00 \mathrm{E}+00$ & $0.00 \mathrm{E}+00$ & $0.00 \mathrm{E}+00$ & $0.00 \mathrm{E}+00$ & $0.00 \mathrm{E}+00$ & $0.00 \mathrm{E}+00$ & $0.00 \mathrm{E}+00$ & $0.00 \mathrm{E}+00$ \\
\hline SNL/NM & $0.00 \mathrm{E}+00$ & $0.00 E+00$ & $0.00 \mathrm{E}+00$ & $0.00 \mathrm{E}+00$ & $0.00 \mathrm{E}+00$ & $0.00 \mathrm{E}+00$ & $0.00 E+00$ & $0.00 E+00$ & $0.00 E+00$ \\
\hline Subtotal & $1.99 \mathrm{E}+01$ & $1.19 E+00$ & $1.60 \mathrm{E}+00$ & $1.83 E+00$ & $2.50 \mathrm{E}+00$ & $8.08 \mathrm{E}+01$ & $3.75 E+01$ & $1.25 \mathrm{E}+02$ & $1.45 \mathrm{E}+02$ \\
\hline \multicolumn{10}{|l|}{ Commercial site } \\
\hline WVDP & $4.27 E+02$ & $0.00 \mathrm{E}+00$ & $0.00 \mathrm{E}+00$ & $0.00 \mathrm{E}+00$ & $0.00 \mathrm{E}+00$ & $0.00 \mathrm{E}+00$ & $0.00 \mathrm{E}+00$ & $0.00 \mathrm{E}+00$ & $4.27 \mathrm{E}+02$ \\
\hline Total & $1.60 \mathrm{E}+03$ & $3.12 \mathrm{E}+01$ & $1.31 \mathrm{E}+02$ & $6.76 E+01$ & $3.80 \mathrm{E}+01$ & $2.68 \mathrm{E}+02$ & $3.08 \mathrm{E}+03$ & $3.62 \mathrm{E}+03$ & $5.22 \mathrm{E}+03$ \\
\hline
\end{tabular}

${ }^{2}$ Any small differences in volumes reported in this table and in WTWBIR, Rev. 1 (ref. 2) and in the numerical totals shown result from rounding.

$\mathrm{b}_{\mathrm{EOY}}=$ end of (calendar) year. 
Table 3.7. Final waste-form volumes $\left(\mathrm{m}^{3}\right)$ of retrievably stored and projected mired RH TRUW

\begin{tabular}{|c|c|c|c|c|c|c|c|c|c|}
\hline \multirow{2}{*}{ Site } & \multirow{2}{*}{$\begin{array}{l}\text { EOYb } \\
1993\end{array}$} & \multicolumn{4}{|c|}{ Annual increase } & \multicolumn{2}{|c|}{ Cumulative increase } & \multirow{2}{*}{$\begin{array}{c}\begin{array}{c}\text { Projected } \\
\text { increase }\end{array} \\
1994-2022\end{array}$} & \multirow{2}{*}{$\begin{array}{c}\text { Total } \\
\text { EOY } 2022\end{array}$} \\
\hline & & 1994 & 1995 & 1996 & 1997 & 1998-2002 & $2003-2022$ & & \\
\hline \multicolumn{10}{|l|}{ Major sites } \\
\hline ANL-E & $0.00 \mathrm{E}+00$ & $0.00 \mathrm{E}+00$ & $0.00 \mathrm{E}+00$ & $0.00 \mathrm{E}+00$ & $0.00 \mathrm{E}+00$ & $0.00 \mathrm{E}+00$ & $0.00 \mathrm{E}+00$ & $0.00 E+00$ & $0.00 \mathrm{E}+00$ \\
\hline Hanford & $0.00 E+00$ & $0.00 \mathrm{E}+00$ & $0.00 \mathrm{E}+00$ & $0.00 \mathrm{E}+00$ & $6.76 E+00$ & $2.02 E+01$ & $1.70 \mathrm{E}+03$ & $1.73 E+03$ & $1.73 E+03$ \\
\hline INEL & $2.12 \mathrm{E}+01$ & $0.00 \mathrm{E}+00$ & $6.00 \mathrm{E}-01$ & $6.00 \mathrm{E}-01$ & $6.00 \mathrm{E}-01$ & $3.00 \mathrm{E}+00$ & $1.20 \mathrm{E}+01$ & $1.68 \mathrm{E}+01$ & $3.80 \mathrm{E}+01$ \\
\hline LANL & $1.50 \mathrm{E}+01$ & $1.00 \mathrm{E}+00$ & $1.00 E+00$ & $1.00 \mathrm{E}+00$ & $1.00 \mathrm{E}+00$ & $1.00 \mathrm{E}+00$ & $1.00 \mathrm{E}+01$ & $1.50 E+01$ & $3.00 E+01$ \\
\hline LLNL & $0.00 \mathrm{E}+00$ & $0.00 \mathrm{E}+00$ & $0.00 \mathrm{E}+00$ & $0.00 \mathrm{E}+00$ & $0.00 \mathrm{E}+00$ & $0.00 E+00$ & $0.00 E+00$ & $0.00 \mathrm{E}+00$ & $0.00 \mathrm{E}+00$ \\
\hline Mound & $0.00 E+00$ & $0.00 E+00$ & $0.00 \mathrm{E}+00$ & $0.00 \mathrm{E}+00$ & $0.00 \mathrm{E}+00$ & $0.00 \mathrm{E}+00$ & $0.00 \mathrm{E}+00$ & $0.00 E+00$ & $0.00 E+00$ \\
\hline NTS & $0.00 \mathrm{E}+00$ & $0.00 E+00$ & $0.00 \mathrm{E}+00$ & $0.00 \mathrm{E}+00$ & $0.00 \mathrm{E}+00$ & $0.00 \mathrm{E}+00$ & $0.00 \mathrm{E}+00$ & $0.00 \mathrm{E}+00$ & $0.00 \mathrm{E}+00$ \\
\hline ORNL & $9.94 E+02$ & $1.23 E+01$ & $1.23 E+01$ & $1.23 E+01$ & $1.23 E+01$ & $6.15 E+01$ & $2.46 \mathrm{E}+02$ & $3.57 E+02$ & $1.35 E+03$ \\
\hline RFETS & $0.00 \mathrm{E}+00$ & $0.00 \mathrm{E}+00$ & $0.00 E+00$ & $0.00 \mathrm{E}+00$ & $0.00 \mathrm{E}+00$ & $0.00 \mathrm{E}+00$ & $0.00 \mathrm{E}+00$ & $0.00 \mathrm{E}+00$ & $0.00 \mathrm{E}+00$ \\
\hline SRS & $0.00 E+00$ & $0.00 \mathrm{E}+00$ & $0.00 \mathrm{E}+00$ & $0.00 E+00$ & $0.00 \mathrm{E}+00$ & $0.00 \mathrm{E}+00$ & $0.00 \mathrm{E}+00$ & $0.00 E+00$ & $0.00 \mathrm{E}+00$ \\
\hline Subtotal & $1.03 E+03$ & $1.33 E+01$ & $1.39 \mathrm{E}+01$ & $1.39 \mathrm{E}+01$ & $2.07 E+01$ & $8.57 \mathrm{E}+01$ & $1.97 E+03$ & $2.12 E+03$ & $3.15 E+03$ \\
\hline \multicolumn{10}{|c|}{ Small-quantity sites } \\
\hline Ames & $0.00 E+00$ & $0.00 E+00$ & $0.00 \mathrm{E}+00$ & $0.00 \mathrm{E}+00$ & $0.00 \mathrm{E}+00$ & $0.00 \mathrm{E}+00$ & $0.00 \mathrm{E}+00$ & $0.00 \mathrm{E}+00$ & $0.00 \mathrm{E}+00$ \\
\hline ANL-W & $8.65 E+00$ & $3.20 \mathrm{E}-01$ & $4.20 \mathrm{E}-01$ & $4.20 \mathrm{E}-01$ & $4.20 \mathrm{E}-01$ & $3.10 \mathrm{E}-01$ & $2.00 \mathrm{E}+00$ & $3.89 E+00$ & $1.25 E+01$ \\
\hline $\mathrm{BCL}$ & $0.00 \mathrm{E}+00$ & $0.00 \mathrm{E}+00$ & $0.00 \mathrm{E}+00$ & $0.00 \mathrm{E}+00$ & $0.00 \mathrm{E}+00$ & $0.00 \mathrm{E}+00$ & $0.00 \mathrm{E}+00$ & $0.00 \mathrm{E}+00$ & $0.00 \mathrm{E}+00$ \\
\hline BAPL & $0.00 E+00$ & $0.00 E+00$ & $0.00 \mathrm{E}+00$ & $0.00 E+00$ & $0.00 E+00$ & $0.00 E+00$ & $0.00 E+00$ & $0.00 E+00$ & $0.00 E+00$ \\
\hline ETEC & $0.00 \mathrm{E}+00$ & $0.00 \mathrm{E}+00$ & $0.00 \mathrm{E}+00$ & $0.00 \mathrm{E}+00$ & $0.00 \mathrm{E}+00$ & $0.00 \mathrm{E}+00$ & $0.00 \mathrm{E}+00$ & $0.00 \mathrm{E}+00$ & $0.00 E+00$ \\
\hline KAPL & $1.12 E+01$ & $8.70 \mathrm{E}-01$ & $8.70 E-01$ & $8.70 E-01$ & $8.70 E-01$ & $4.35 E+00$ & $1.74 \mathrm{E}+01$ & $2.52 \mathrm{E}+01$ & $3.65 E+01$ \\
\hline LBL & $0.00 E+00$ & $0.00 \mathrm{E}+00$ & $0.00 \mathrm{E}+00$ & $0.00 \mathrm{E}+00$ & $0.00 \mathrm{E}+00$ & $0.00 \mathrm{E}+00$ & $0.00 E+00$ & $0.00 \mathrm{E}+00$ & $0.00 \mathrm{E}+00$ \\
\hline MURR & $0.00 \mathrm{E}+00$ & $0.00 \mathrm{E}+00$ & $0.00 \mathrm{E}+00$ & $0.00 \mathrm{E}+00$ & $0.00 \mathrm{E}+00$ & $0.00 \mathrm{E}+00$ & $0.00 \mathrm{E}+00$ & $0.00 E+00$ & $0.00 \mathrm{E}+00$ \\
\hline PAD & $0.00 E+00$ & $0.00 E+00$ & $0.00 \mathrm{E}+00$ & $0.00 \mathrm{E}+00$ & $0.00 \mathrm{E}+00$ & $0.00 \mathrm{E}+00$ & $0.00 \mathrm{E}+00$ & $0.00 \mathrm{E}+00$ & $0.00 \mathrm{E}+00$ \\
\hline PANT & $0.00 E+00$ & $0.00 \mathrm{E}+00$ & $0.00 \mathrm{E}+00$ & $0.00 E+00$ & $0.00 \mathrm{E}+00$ & $0.00 \mathrm{E}+00$ & $0.00 \mathrm{E}+00$ & $0.00 \mathrm{E}+00$ & $0.00 \mathrm{E}+00$ \\
\hline SNL/NM & $0.00 \mathrm{E}+00$ & $0.00 \mathrm{E}+00$ & $0.00 \mathrm{E}+00$ & $0.00 \mathrm{E}+00$ & $0.00 \mathrm{E}+00$ & $0.00 \mathrm{E}+00$ & $0.00 E+00$ & $0.00 \mathrm{E}+00$ & $0.00 \mathrm{E}+00$ \\
\hline Subtotal & $1.99 E+01$ & $1.19 \mathrm{E}+00$ & $1.29 \mathrm{E}+00$ & $1.29 \mathrm{E}+00$ & $1.29 \mathrm{E}+00$ & $4.66 \mathrm{E}+00$ & $1.94 E+01$ & $2.91 E+01$ & $4.90 \mathrm{E}+01$ \\
\hline \multicolumn{10}{|l|}{ Commercial site } \\
\hline WVDP & $1.05 E+01$ & $0.00 E+00$ & $0.00 \mathrm{E}+00$ & $0.00 \mathrm{E}+00$ & $0.00 \mathrm{E}+00$ & $0.00 \mathrm{E}+00$ & $0.00 \mathrm{E}+00$ & $0.00 \mathrm{E}+00$ & $1.05 \mathrm{E}+01$ \\
\hline Total & $1.06 \mathrm{E}+03$ & $1.45 \mathrm{E}+01$ & $1.52 \mathrm{E}+01$ & $1.52 E+01$ & $2.20 \mathrm{E}+01$ & $9.03 E+01$ & $1.99 E+03$ & $2.15 \mathrm{E}+03$ & $3.21 \mathrm{E}+03$ \\
\hline
\end{tabular}

${ }^{a}$ Any small differences in volumes reported in this table and in WTWBIR, Rev. 1 (ref. 2) and in the numerical totals shown result from rounding. $\mathrm{b}_{\mathrm{EOY}}=$ end of (calendar) year. 
Table 3.8. Final waste-form volumes $\left(\mathrm{m}^{3}\right)$ of retrievably stored and projected nonmixed $R H$ TRUWa

\begin{tabular}{|c|c|c|c|c|c|c|c|c|c|}
\hline \multirow{2}{*}{ Site } & \multirow{2}{*}{$\begin{array}{l}\text { EOYb } \\
1993\end{array}$} & \multicolumn{4}{|c|}{ Annual increase } & \multicolumn{2}{|c|}{ Cumulative increase } & \multirow{2}{*}{$\begin{array}{c}\begin{array}{c}\text { Projected } \\
\text { increase }\end{array} \\
1994-2022\end{array}$} & \multirow{2}{*}{$\begin{array}{c}\text { Total } \\
\text { EOY } 2022\end{array}$} \\
\hline & & 1994 & 1995 & 1996 & 1997 & 1998-2002 & $2003-2022$ & & \\
\hline \multicolumn{10}{|l|}{ Major sites } \\
\hline ANL-E & $0.00 \mathrm{E}+00$ & $0.00 E+00$ & $0.00 \mathrm{E}+00$ & $0.00 \mathrm{E}+00$ & $0.00 \mathrm{E}+00$ & $0.00 \mathrm{E}+00$ & $0.00 \mathrm{E}+00$ & $0.00 \mathrm{E}+00$ & $0.00 E+00$ \\
\hline Hanford & $3.32 \mathrm{E}+01$ & $2.00 \mathrm{E}+00$ & $1.03 \mathrm{E}+02$ & $3.90 \mathrm{E}+01$ & $2.00 \mathrm{E}+00$ & $8.00 \mathrm{E}+01$ & $1.02 E+03$ & $1.25 E+03$ & $1.28 E+03$ \\
\hline INEL & $9.76 \mathrm{E}+00$ & $0.00 \mathrm{E}+00$ & $0.00 \mathrm{E}+00$ & $0.00 \mathrm{E}+00$ & $0.00 \mathrm{E}+00$ & $0.00 \mathrm{E}+00$ & $0.00 \mathrm{E}+00$ & $0.00 \mathrm{E}+00$ & $9.76 \mathrm{E}+00$ \\
\hline LANL & $7.63 \mathrm{E}+01$ & $1.07 \mathrm{E}+01$ & $1.07 \mathrm{E}+01$ & $1.07 \mathrm{E}+01$ & $1.07 \mathrm{E}+01$ & $1.09 \mathrm{E}+01$ & $1.40 \mathrm{E}+01$ & $6.77 \mathrm{E}+01$ & $1.44 E+02$ \\
\hline LLNL & $0.00 \mathrm{E}+00$ & $0.00 \mathrm{E}+00$ & $0.00 \mathrm{E}+00$ & $0.00 \mathrm{E}+00$ & $0.00 \mathrm{E}+00$ & $0.00 \mathrm{E}+00$ & $0.00 E+00$ & $0.00 E+00$ & $0.00 E+00$ \\
\hline Mound & $0.00 \mathrm{E}+00$ & $0.00 \mathrm{E}+00$ & $0.00 \mathrm{E}+00$ & $0.00 E+00$ & $0.00 \mathrm{E}+00$ & $0.00 \mathrm{E}+00$ & $0.00 \mathrm{E}+00$ & $0.00 \mathrm{E}+00$ & $0.00 \mathrm{E}+00$ \\
\hline NTS & $0.00 \mathrm{E}+00$ & $0.00 \mathrm{E}+00$ & $0.00 \mathrm{E}+00$ & $0.00 \mathrm{E}+00$ & $0.00 \mathrm{E}+00$ & $0.00 \mathrm{E}+00$ & $0.00 \mathrm{E}+00$ & $0.00 \mathrm{E}+00$ & $0.00 \mathrm{E}+00$ \\
\hline ORNL & $0.00 \mathrm{E}+00$ & $0.00 \mathrm{E}+00$ & $0.00 \mathrm{E}+00$ & $0.00 \mathrm{E}+00$ & $0.00 \mathrm{E}+00$ & $0.00 \mathrm{E}+00$ & $0.00 \mathrm{E}+00$ & $0.00 \mathrm{E}+00$ & $0.00 \mathrm{E}+00$ \\
\hline RFETS & $0.00 E+00$ & $0.00 \mathrm{E}+00$ & $0.00 \mathrm{E}+00$ & $0.00 E+00$ & $0.00 E+00$ & $0.00 \mathrm{E}+00$ & $0.00 \mathrm{E}+00$ & $0.00 \mathrm{E}+00$ & $0.00 \mathrm{E}+00$ \\
\hline SRS & $0.00 \mathrm{E}+00$ & $4.00 \mathrm{E}+00$ & $2.14 \mathrm{E}+00$ & $2.14 \mathrm{E}+00$ & $2.14 \mathrm{E}+00$ & $1.05 \mathrm{E}+01$ & $4.30 \mathrm{E}+01$ & $6.39 \mathrm{E}+01$ & $6.39 \mathrm{E}+01$ \\
\hline Subtotal & $1.19 E+02$ & $1.67 \mathrm{E}+01$ & $1.16 \mathrm{E}+02$ & $5.18 \mathrm{E}+01$ & $1.48 \mathrm{E}+01$ & $1.01 E+02$ & $1.08 E+03$ & $1.38 \mathrm{E}+03$ & $1.50 E+03$ \\
\hline \multicolumn{10}{|c|}{ Small-quantity sites } \\
\hline Ames & $0.00 E+00$ & $0.00 \mathrm{E}+00$ & $0.00 \mathrm{E}+00$ & $0.00 \mathrm{E}+00$ & $0.00 \mathrm{E}+00$ & $0.00 \mathrm{E}+00$ & $0.00 \mathrm{E}+00$ & $0.00 E+00$ & $0.00 E+00$ \\
\hline ANI $-W$ & $0.00 \mathrm{E}+00$ & $0.00 \mathrm{E}+00$ & $0.00 \mathrm{E}+00$ & $3.36 E-01$ & $9.00 \mathrm{E}-01$ & $4.50 \mathrm{E}+00$ & $1.80 \mathrm{E}+01$ & $2.37 \mathrm{E}+01$ & $2.37 \mathrm{E}+01$ \\
\hline $\mathrm{BCL}$ & $0.00 \mathrm{E}+00$ & $0.00 \mathrm{E}+00$ & $0.00 \mathrm{E}+00$ & $0.00 \mathrm{E}+00$ & $0.00 \mathrm{E}+00$ & $7.10 \mathrm{E}+01$ & $0.00 \mathrm{E}+00$ & $7.10 \mathrm{E}+01$ & $7.10 \mathrm{E}+01$ \\
\hline BAPL & $0.00 \mathrm{E}+00$ & $0.00 \mathrm{E}+00$ & $3.12 \mathrm{E}-01$ & $2.08 \mathrm{E}-01$ & $3.12 E-01$ & $6.25 \mathrm{E}-01$ & $1.00 \mathrm{E}-01$ & $1.56 \mathrm{E}+00$ & $1.56 \mathrm{E}+00$ \\
\hline ETEC & $0.00 \mathrm{E}+00$ & $0.00 \mathrm{E}+00$ & $0.00 \mathrm{E}+00$ & $0.00 \mathrm{E}+00$ & $0.00 \mathrm{E}+00$ & $0.00 \mathrm{E}+00$ & $0.00 \mathrm{E}+00$ & $0.00 \mathrm{E}+00$ & $0.00 E+00$ \\
\hline KAPL & $0.00 \mathrm{E}+00$ & $0.00 E+00$ & $0.00 \mathrm{E}+00$ & $0.00 \mathrm{E}+00$ & $0.00 \mathrm{E}+00$ & $0.00 \mathrm{E}+00$ & $0.00 \mathrm{E}+00$ & $0.00 \mathrm{E}+00$ & $0.00 E+00$ \\
\hline LBL & $0.00 \mathrm{E}+00$ & $0.00 E+00$ & $0.00 \mathrm{E}+00$ & $0.00 \mathrm{E}+00$ & $0.00 \mathrm{E}+00$ & $0.00 \mathrm{E}+00$ & $0.00 \mathrm{E}+00$ & $0.00 \mathrm{E}+00$ & $0.00 E+00$ \\
\hline MURR & $0.00 \mathrm{E}+00$ & $0.00 \mathrm{E}+00$ & $0.00 \mathrm{E}+00$ & $0.00 \mathrm{E}+00$ & $0.00 \mathrm{E}+00$ & $0.00 \mathrm{E}+00$ & $0.00 \mathrm{E}+00$ & $0.00 E+00$ & $0.00 E+00$ \\
\hline $\mathrm{PAD}$ & $0.00 \mathrm{E}+00$ & $0.00 E+00$ & $0.00 E+00$ & $0.00 E+00$ & $0.00 \mathrm{E}+00$ & $0.00 \mathrm{E}+00$ & $0.00 \mathrm{E}+00$ & $0.00 \mathrm{E}+00$ & $0.00 E+00$ \\
\hline PANT & $0.00 \mathrm{E}+00$ & $0.00 \mathrm{E}+00$ & $0.00 \mathrm{E}+00$ & $0.00 \mathrm{E}+00$ & $0.00 \mathrm{E}+00$ & $0.00 \mathrm{E}+00$ & $0.00 \mathrm{E}+00$ & $0.00 \mathrm{E}+00$ & $0.00 \mathrm{E}+00$ \\
\hline SNL/NM & $0.00 E+00$ & $0.00 \mathrm{E}+00$ & $0.00 \mathrm{E}+00$ & $0.00 \mathrm{E}+00$ & $0.00 \mathrm{E}+00$ & $0.00 \mathrm{E}+00$ & $0.00 \mathrm{E}+00$ & $0.00 \mathrm{E}+00$ & $0.00 E+00$ \\
\hline Subtotal & $0.00 \mathrm{E}+00$ & $0.00 \mathrm{E}+00$ & $3.12 \mathrm{E}-01$ & $5.44 \mathrm{E}-01$ & $1.21 \mathrm{E}+00$ & $7.61 \mathrm{E}+01$ & $1.81 \mathrm{E}+01$ & $9.63 \mathrm{E}+01$ & $9.63 E+01$ \\
\hline \multicolumn{10}{|l|}{ Commercial site } \\
\hline WVDP & $4.16 E+02$ & $0.00 \mathrm{E}+00$ & $0.00 \mathrm{E}+00$ & $0.00 \mathrm{E}+00$ & $0.00 E+00$ & $0.00 \mathrm{E}+00$ & $0.00 \mathrm{E}+00$ & $0.00 \mathrm{E}+00$ & $4.16 \mathrm{E}+02$ \\
\hline Total & $5.36 \mathrm{E}+02$ & $1.67 \mathrm{E}+01$ & $1.16 E+02$ & $5.24 \mathrm{E}+01$ & $1.61 \mathrm{E}+01$ & $1.78 \mathrm{E}+02$ & $1.10 E+03$ & $1.47 \mathrm{E}+03$ & $2.01 E+03$ \\
\hline
\end{tabular}

aAny small differences in volumes reported in this table and in WTWBIR, Rev. 1 (ref. 2) and in the numerical totals shown result from rounding. $b_{E O Y}=$ end of (calendar) year. 
Table 3.9. Estimated final-form volume $\left(\mathrm{m}^{3}\right)$ composition of retrievably stored and projected TRUW for major sites ${ }^{\mathrm{a}}$

\begin{tabular}{|c|c|c|c|c|c|c|c|}
\hline \multirow{2}{*}{$\begin{array}{l}\text { Major } \\
\text { sites }\end{array}$} & \multirow{2}{*}{ Category } & \multicolumn{3}{|c|}{ Contact-handled } & \multicolumn{3}{|c|}{ Remote-handled } \\
\hline & & Stored & Projected & Total & Stored & Projected & Total \\
\hline \multirow[t]{5}{*}{ ANL-E } & Lead/cadmium metal waste & 1.10 & 0.00 & 1.10 & 0.00 & 0.00 & 0.00 \\
\hline & Solidified inorganics & 23.05 & 1.12 & 24.17 & 0.00 & 0.00 & 0.00 \\
\hline & Solidified organics & 0.03 & 0.00 & 0.03 & 0.00 & 0.00 & 0.00 \\
\hline & Uncategorized metal & 4.96 & 0.56 & 5.52 & 0.00 & 0.00 & 0.00 \\
\hline & Subtotal & 29.14 & 1.68 & 30.82 & 0.00 & 0.00 & 0.00 \\
\hline \multirow[t]{8}{*}{ Hanford } & Combustible & $530.07^{a}$ & $12,698.57^{b}$ & $13,228.64$ & 0.00 & 0.00 & 0.00 \\
\hline & Heterogeneous & $8,568.55$ & 827.16 & $9,395.71$ & 33.16 & $2,973.71$ & $3,006.87$ \\
\hline & Lead/cadmium metal waste & 3.13 & 0.29 & 3.42 & 0.00 & 0.00 & 0.00 \\
\hline & Soils & 111.69 & 309.27 & 420.96 & 0.00 & 0.00 & 0.00 \\
\hline & Solidified inorganics & $2.93^{\mathrm{c}}$ & $3,153.57^{d}$ & $3,156.50$ & 0.00 & 0.00 & 0.00 \\
\hline & Solidified organics & $4.48^{\mathrm{e}}$ & $31.44^{f}$ & 35.92 & 0.00 & 0.00 & 0.00 \\
\hline & Uncategorized metal & 170.178 & $13,837.04^{h}$ & $14,007.21$ & 0.00 & 0.00 & 0.00 \\
\hline & Subtotal & $9,391.02$ & $30,857.34$ & $40,248.36$ & 33.16 & $2,973.71$ & $3,006.87$ \\
\hline \multirow[t]{13}{*}{ INEL } & Combustible & 670.90 & 0.00 & 670.90 & 0.00 & 0.00 & 0.00 \\
\hline & Filter & 323.56 & 0.00 & 323.56 & 0.00 & 0.00 & 0.00 \\
\hline & Graphite & 650.70 & 0.00 & 650.70 & 0.00 & 0.00 & 0.00 \\
\hline & Heterogeneous & $9,649.50$ & 1.00 & $9,650.50$ & 13.63 & 2.80 & 16.43 \\
\hline & Inorganic nonmetal & $1,052.89$ & 0.00 & $1,052.89$ & 0.00 & 0.00 & 0.00 \\
\hline & Lead/cadmium metal waste & 0.00 & 0.00 & 0.00 & 0.00 & 5.60 & 5.60 \\
\hline & Salt Waste & 22.91 & 0.00 & 22.91 & 0.00 & 2.80 & 2.80 \\
\hline & Soils & 3.80 & 0.00 & 3.80 & 0.00 & 0.00 & 0.00 \\
\hline & Solidified inorganics & $12,164.28$ & 0.00 & $12,164.28$ & 2.10 & 0.00 & 2.10 \\
\hline & Solidified organics & 912.60 & 0.00 & 912.60 & 0.00 & 0.00 & 0.00 \\
\hline & Uncategorized metal & $7,564.09$ & 0.00 & $7,564.09$ & 4.11 & 5.60 & 9.71 \\
\hline & Unknown & $1,655.91$ & 0.00 & $1,655.91$ & 11.13 & 0.00 & 11.13 \\
\hline & Subtotal & $34,671.14$ & 1.00 & $34,672.14$ & 30.97 & 16.80 & 47.77 \\
\hline \multirow[t]{5}{*}{ LANL } & Combustible & $1,768.33$ & $2,464.60$ & $4,232.93$ & 14.84 & 3.16 & 18.00 \\
\hline & Soils & 109.37 & 144.60 & 253.97 & 0.00 & 0.00 & 0.00 \\
\hline & Solidified inorganics & $4,848.38$ & $2,059.03$ & $6,907.41$ & 0.00 & 0.00 & 0.00 \\
\hline & Uncategorized metals & $4,134.80$ & $3,006.17$ & $7,140.97$ & 76.46 & 79.50 & 155.96 \\
\hline & Subtotal & $10,860.88$ & $7,674.40$ & $18,535.28$ & 91.30 & 82.66 & 173.96 \\
\hline \multirow[t]{6}{*}{ LLNL } & Combustible & 48.88 & 372.32 & 421.20 & 0.00 & 0.00 & 0.00 \\
\hline & Filter & $16.63^{\mathrm{i}}$ & $32.63^{\mathrm{j}}$ & 49.26 & 0.00 & 0.00 & 0.00 \\
\hline & Salt waste & 0.62 & 2.91 & 3.53 & 0.00 & 0.00 & 0.00 \\
\hline & Solidified inorganics & 13.30 & 66.15 & 79.45 & 0.00 & 0.00 & 0.00 \\
\hline & Uncategorized metal & 144.33 & 247.00 & 391.33 & 0.00 & 0.00 & 0.00 \\
\hline & Subtotal & 223.76 & 721.01 & 944.77 & 0.00 & 0.00 & 0.00 \\
\hline
\end{tabular}


Table 3.9 (continued)

\begin{tabular}{|c|c|c|c|c|c|c|c|}
\hline \multirow{2}{*}{$\begin{array}{l}\text { Major } \\
\text { sites }\end{array}$} & \multirow{2}{*}{ Category } & \multicolumn{3}{|c|}{ Contact-handled } & \multicolumn{3}{|c|}{ Remote-handled } \\
\hline & & Stored & Projected & Total & Stored & Projected & Total \\
\hline \multirow[t]{7}{*}{ Mound } & Combustible & 5.61 & 0.00 & 5.61 & 0.00 & 0.00 & 0.00 \\
\hline & Heterogeneous & $1.04^{\mathrm{k}}$ & 0.00 & 1.04 & 0.00 & 0.00 & 0.00 \\
\hline & Inorganic nonmetal & $0.21^{1}$ & 0.00 & 0.21 & 0.00 & 0.00 & 0.00 \\
\hline & Soils & 146.88 & 0.00 & 146.88 & 0.00 & 0.00 & 0.00 \\
\hline & Solidified inorganics & 7.28 & 0.00 & 7.28 & 0.00 & 0.00 & 0.00 \\
\hline & Uncategorized metal & 102.28 & 0.00 & 102.28 & 0.00 & 0.00 & 0.00 \\
\hline & Subtotal & 263.30 & 0.00 & 263.30 & 0.00 & 0.00 & 0.00 \\
\hline NTS & Heterogeneous & 619.50 & 0.00 & 619.50 & 0.00 & 0.00 & 0.00 \\
\hline \multirow[t]{3}{*}{ ORNL } & Heterogeneous & 672.98 & 263.90 & 936.88 & 382.81 & 182.70 & 565.51 \\
\hline & Solidified inorganics & 110.00 & 0.00 & 110.00 & 611.00 & 174.00 & 785.00 \\
\hline & Subtotal & 782.98 & 263.90 & $1,046.88$ & 993.81 & 356.70 & $1,350.51$ \\
\hline \multirow[t]{10}{*}{ RFETS } & Filter & 103.96 & $1,087.59$ & $1,191.55$ & 0.00 & 0.00 & 0.00 \\
\hline & Graphite & 18.06 & 43.40 & 61.46 & 0.00 & 0.00 & 0.00 \\
\hline & Heterogeneous & 312.86 & 804.58 & $1,117.44$ & 0.00 & 0.00 & 0.00 \\
\hline & Inorganic nonmetal & 110.68 & 318.68 & 429.36 & 0.00 & 0.00 & 0.00 \\
\hline & Lead/cadmium metal waste & 51.87 & 124.18 & 176.05 & 0.00 & 0.00 & 0.00 \\
\hline & Salt waste & 9.45 & 56.60 & 66.05 & 0.00 & 0.00 & 0.00 \\
\hline & Solidified inorganics & 228.63 & $2,988.11$ & $3,216.74$ & 0.00 & 0.00 & 0.00 \\
\hline & Solidified organics & 132.80 & 48.82 & 181.62 & 0.00 & 0.00 & 0.00 \\
\hline & Uncategorized metal & 164.82 & 429.50 & 594.32 & 0.00 & 0.00 & 0.00 \\
\hline & Subtotal & $1,133.13$ & $5,901.46$ & $7,034.59$ & 0.00 & 0.00 & 0.00 \\
\hline \multirow[t]{6}{*}{ SRS } & Combustible & $4,066.80$ & $11,962.50$ & $16,029.30$ & 0.00 & 0.00 & 0.00 \\
\hline & Heterogeneous & $10,132.20$ & $2,563.60$ & $12,695.80$ & 0.00 & 63.92 & 63.92 \\
\hline & Solidified inorganics & 0.04 & 0.00 & 0.04 & 0.00 & 0.00 & 0.00 \\
\hline & Solidified organics & 404.85 & 240.70 & 645.55 & 0.00 & 0.00 & 0.00 \\
\hline & Subtotal & $14,603.89$ & $14,766.80$ & $29,370.69$ & 0.00 & 63.92 & 63.92 \\
\hline & Major sites total & $72,578.74$ & $60,187.59$ & $132,766.33$ & $1,149.24$ & $3,493.79$ & $4,643.03$ \\
\hline
\end{tabular}

\footnotetext{
ancludes $3.59 \mathrm{~m}^{3}$ of TSCA waste not shown in the WTWBIR, Rev. 1 (ref. 2).

bIncludes $429.54 \mathrm{~m}^{3}$ of TSCA waste not shown in the WTWBIR, Rev. 1 (ref. 2).

Includes $1.47 \mathrm{~m}^{3}$ of TSCA waste not shown in the WTWBIR, Rev. 1 (ref. 2).

dincludes $228.81 \mathrm{~m}^{3}$ of TSCA waste not shown in the WTWBIR, Rev. 1 (ref. 2).

Includes $2.31 \mathrm{~m}^{3}$ of TSCA waste not shown in the WTWBIR, Rev. 1 (ref. 2).

Includes $16.19 \mathrm{~m}^{3}$ of TSCA waste not shown in the WTWBIR, Rev. 1 (ref. 2).

SIncludes $66.82 \mathrm{~m}^{3}$ of TSCA waste not shown in the WTWBIR, Rev. 1 (ref. 2).

hIncludes $8,946.09 \mathrm{~m}^{3}$ of TSCA waste not shown in the WTWBIR, Rev. 1 (ref. 2).

IIncludes $16.63 \mathrm{~m}^{3}$ of TSCA waste not shown in the WTWBIR, Rev. 1 (ref. 2).

jIncludes $32.63 \mathrm{~m}^{3}$ of TSCA waste not shown in the WTWBIR, Rev. 1 (ref. 2).

$\mathrm{k}_{\text {Includes }} 0.62 \mathrm{~m}^{3}$ of TSCA waste not shown in the WTWBIR, Rev. 1 (ref. 2).

Includes $0.21 \mathrm{~m}^{3}$ of TSCA waste not shown in the WTWBIR, Rev. 1 (ref. 2).
} 
Table 3.10. Estimated final-form volume $\left(\mathrm{m}^{3}\right)$ composition of retrievably stored and projected TRUW for small-quantity and commercial sites ${ }^{\mathrm{a}}$

\begin{tabular}{|c|c|c|c|c|c|c|c|}
\hline \multirow{2}{*}{$\begin{array}{l}\text { Major } \\
\text { sites }\end{array}$} & \multirow{2}{*}{ Category } & \multicolumn{3}{|c|}{ Contact-handled } & \multicolumn{3}{|c|}{ Remote-handled } \\
\hline & & Stored & Projected & Total & Stored & Projected & Total \\
\hline \multicolumn{8}{|c|}{ Small-quantity sites (SQS) } \\
\hline Ames & Solidified inorganics & 0.00 & 0.10 & 0.10 & 0.00 & 0.00 & 0.00 \\
\hline \multirow[t]{6}{*}{ ANL-W } & Filter & 0.00 & 0.00 & 0.00 & 0.89 & 2.09 & 2.98 \\
\hline & Heterogeneous & 0.00 & 3.36 & 3.36 & 0.59 & 0.08 & 0.67 \\
\hline & Lead/cadmium metal waste & 0.02 & 2.48 & 2.50 & 0.00 & 0.36 & 0.36 \\
\hline & Uncategorized metal & 0.00 & 0.00 & 0.00 & 7.17 & 1.36 & 8.53 \\
\hline & Unknown & 0.00 & 0.00 & 0.00 & 0.00 & 23.74 & 23.74 \\
\hline & Subtotal & 0.02 & 5.84 & 5.86 & 8.65 & 27.63 & 36.28 \\
\hline $\mathrm{BCL}$ & Heterogeneous & 0.00 & 0.00 & 0.00 & 0.00 & 71.00 & 71.00 \\
\hline BAPL & Heterogeneous & 0.00 & 123.82 & 123.82 & 0.00 & 1.56 & 1.56 \\
\hline \multirow[t]{3}{*}{ ETEC } & Heterogeneous & 1.66 & 5.20 & 6.86 & 0.00 & 0.00 & 0.00 \\
\hline & Lead/cadmium metal waste & 0.21 & 0.00 & 0.21 & 0.00 & 0.00 & 0.00 \\
\hline & Subtotal & 1.87 & 5.20 & 7.07 & 0.00 & 0.00 & 0.00 \\
\hline KAPL & Heterogeneous & 2.40 & 0.00 & 2.40 & 11.23 & 25.23 & 36.46 \\
\hline LBL & Heterogeneous & 0.84 & 4.42 & 5.26 & 0.00 & 0.00 & 0.00 \\
\hline MURR & Heterogeneous & 0.06 & 1.60 & 1.66 & 0.00 & 0.00 & 0.00 \\
\hline PAD & Solidified inorganics & 3.45 & 0.00 & 3.45 & 0.00 & 0.00 & 0.00 \\
\hline PANT & Heterogeneous & 0.62 & 0.00 & 0.62 & 0.00 & 0.00 & 0.00 \\
\hline \multirow[t]{2}{*}{ SNL/NM } & Heterogeneous & 8.04 & 7.00 & 15.04 & 0.00 & 0.00 & 0.00 \\
\hline & SQS subtotal ${ }^{a}$ & 17.30 & 147.98 & 165.28 & 19.88 & 125.41 & 145.30 \\
\hline \multicolumn{8}{|c|}{ Commercial sile } \\
\hline \multirow[t]{9}{*}{ WVDP } & Filter & 18.97 & 131.63 & 150.60 & 0.00 & 0.00 & 0.00 \\
\hline & Heterogeneous & 14.76 & 55.28 & 70.04 & 0.00 & 0.00 & 0.00 \\
\hline & Lead/cadmium metal waste & 0.21 & 0.00 & 0.21 & 0.00 & 0.00 & 0.00 \\
\hline & Solidified inorganics & 4.57 & 0.00 & 4.57 & 0.00 & 0.00 & 0.00 \\
\hline & Solidified organics & 0.42 & 0.00 & 0.42 & 0.00 & 0.00 & 0.00 \\
\hline & Uncategorized metal & 0.42 & 0.00 & 0.42 & 416.46 & 0.00 & 416.46 \\
\hline & Unknown & 11.23 & 0.00 & 11.23 & 10.49 & 0.00 & 10.49 \\
\hline & $\begin{array}{l}\text { Commercial site } \\
\text { subtotal }^{\mathbf{a}}\end{array}$ & 50.57 & 186.91 & 237.48 & 426.95 & 0.00 & 426.95 \\
\hline & Total & $72,646.61$ & $60,522.48$ & $133,169.09$ & $1,596.08$ & $3,619.20$ & $5,215.28$ \\
\hline
\end{tabular}

aAny small differences in volumes reported in this table and in WTWBIR, Rev. 1 (ref. 2) and in the numerical totals shown result from rounding. 
Table 3.11. Summary of decayed radioactivity isotopic content of retrievably stored CH TRUW

\begin{tabular}{|c|c|c|c|c|c|c|c|c|}
\hline Site & ${ }^{238} \mathrm{Pu}$ & ${ }^{241} \mathrm{Pu}$ & ${ }^{239} \mathrm{Pu}$ & ${ }^{241} \mathrm{Am}$ & ${ }^{240} \mathrm{Pu}$ & ${ }^{137} \mathrm{Cs}$ & $\begin{array}{l}\text { Sum of } \\
\text { curies }\end{array}$ & $\begin{array}{c}\text { Curies } \\
(\%)\end{array}$ \\
\hline \multicolumn{9}{|c|}{ Major sites } \\
\hline ANL-E & $2.14 \mathrm{E}+00$ & $5.98 \mathrm{E}+01$ & $3.28 E+01$ & $5.73 \mathrm{E}+00$ & $9.42 \mathrm{E}+00$ & & $1.10 \mathrm{E}+02$ & 0.01 \\
\hline Hanford & $8.18 \mathrm{E}+04$ & $9.43 E+04$ & $2.70 \mathrm{E}+04$ & $2.98 E+03$ & $6.06 \mathrm{E}+03$ & $7.03 \mathrm{E}+02$ & $2.13 E+05$ & 14.11 \\
\hline INEL & $6.07 E+04$ & $1.65 E+05$ & $4.01 E+04$ & $8.98 \mathrm{E}+04$ & $9.82 E+03$ & $6.32 E+01$ & $3.65 \mathrm{E}+05$ & 24.19 \\
\hline LANL & $1.15 \mathrm{E}+05$ & $1.77 \mathrm{E}+00$ & $7.33 E+04$ & $8.70 \mathrm{E}+03$ & $1.52 \mathrm{E}-01$ & $5.32 \mathrm{E}+01$ & $1.97 \mathrm{E}+05$ & 13.08 \\
\hline LLNL & $1.97 \mathrm{E}+01$ & Т. $67 \mathrm{E}+03$ & $1.46 \mathrm{E}+02$ & $1.26 \mathrm{E}+02$ & $6.06 E+01$ & $1.99 \mathrm{E}-06$ & $2.03 E+03$ & 0.13 \\
\hline Mound & $7.16 \mathrm{E}+02$ & & $1.99 \mathrm{E}+02$ & & & & $9.14 \mathrm{E}+02$ & 0.06 \\
\hline NTS & $1.98 E+02$ & $2.64 \mathrm{E}+02$ & $2.76 E+03$ & $2.84 E+02$ & $1.87 \mathrm{E}+01$ & $3.97 E-01$ & $3.53 E+03$ & 0.23 \\
\hline ORNL & $6.78 E+03$ & $5.52 E+04$ & $7.95 E+02$ & $2.33 E+03$ & $7.25 E+02$ & $2.17 E+03$ & $6.80 \mathrm{E}+04$ & 4.51 \\
\hline RFETS & $3.47 E+02$ & $5.72 \mathrm{E}+04$ & $9.93 E+03$ & $1.08 \mathrm{E}+04$ & $7.21 \mathrm{E}+03$ & & $8.55 E+04$ & 5.66 \\
\hline SRS & $4.71 E+05$ & $6.30 \mathrm{E}+04$ & $8.56 \mathrm{E}+03$ & $1.60 E+04$ & $2.12 E+03$ & $6.77 E-01$ & $5.61 E+05$ & 37.16 \\
\hline Subtotal & $7.37 \mathrm{E}+05$ & $4.36 \mathrm{E}+05$ & $1.63 \mathrm{E}+05$ & $1.31 E+05$ & $2.60 E+04$ & $2.99 E+03$ & $1.50 \mathrm{E} \div 06$ & 99.14 \\
\hline \multicolumn{9}{|c|}{ Small-quantity sites (SQS) } \\
\hline $\begin{array}{l}\text { Ames } \\
\text { ANL-W } \\
\text { BCL } \\
\text { BAPL }\end{array}$ & & & & & & & & \\
\hline $\begin{array}{l}\text { ETEC } \\
\text { KAPL }\end{array}$ & $1.12 \mathrm{E}-01$ & $8.06 \mathrm{E}+00$ & $1.76 E+00$ & $4.43 \mathrm{E}-01$ & $6.01 E-01$ & $2.40 \mathrm{E}-01$ & $1.12 \mathrm{E}+01$ & 0.00 \\
\hline LBL & $2.36 \mathrm{E}-04$ & $2.89 E-07$ & $7.70 E-03$ & $9.20 \mathrm{E}-02$ & $5.07 E-03$ & & $1.05 E-01$ & 0.00 \\
\hline MURR & & & $2.05 \mathrm{E}-02$ & $4.80 \mathrm{E}-02$ & & & $6.85 \mathrm{E}-02$ & 0.00 \\
\hline $\mathrm{PAD}$ & & & $5.56 \mathrm{E}+01$ & & & & $5.56 \mathrm{E}+01$ & 0.00 \\
\hline PANT & & & $5.55 E-02$ & & & & $5.55 \mathrm{E}-02$ & 0.00 \\
\hline SNL/NM & & & $2.00 \mathrm{E}-06$ & $1.00 \mathrm{E}-02$ & & & $1.00 \mathrm{E}-02$ & 0.00 \\
\hline Subtotal & $1.12 E-01$ & $8.06 \mathrm{E}+00$ & $5.75 \mathrm{E}+01$ & $5.93 \mathrm{E}-01$ & $6.06 \mathrm{E}-01$ & $2.40 \mathrm{E}-01$ & $6.71 \mathrm{E}+01$ & 0.00 \\
\hline \multicolumn{9}{|c|}{ Commercial site } \\
\hline WVDP & & $3.43 \mathrm{E}+01$ & & $2.15 \mathrm{E}+00$ & & $1.95 \mathrm{E}+00$ & $3.84 \mathrm{E}+01$ & 0.00 \\
\hline Total (all sites) & $7.37 \mathrm{E}+05$ & $4.37 \mathrm{E}+05$ & $1.63 E+05$ & $1.31 E+05$ & $2.60 E+04$ & $3.00 \mathrm{E}+03$ & $1.50 \mathrm{E}+06$ & 99.15 \\
\hline Total $\mathrm{Ci}, \%$ & 48.84 & 28.92 & 10.79 & 8.68 & 1.72 & 0.20 & & \\
\hline Cumulative, $\%$ & 48.84 & 77.76 & 88.55 & 97.23 & 98.95 & 99.15 & & \\
\hline
\end{tabular}

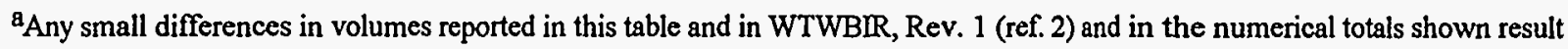
from rounding. 
Table 3.12. Summary of decayed radioactivity (Ci) isotopic content of retrievably stored RH TRUWa

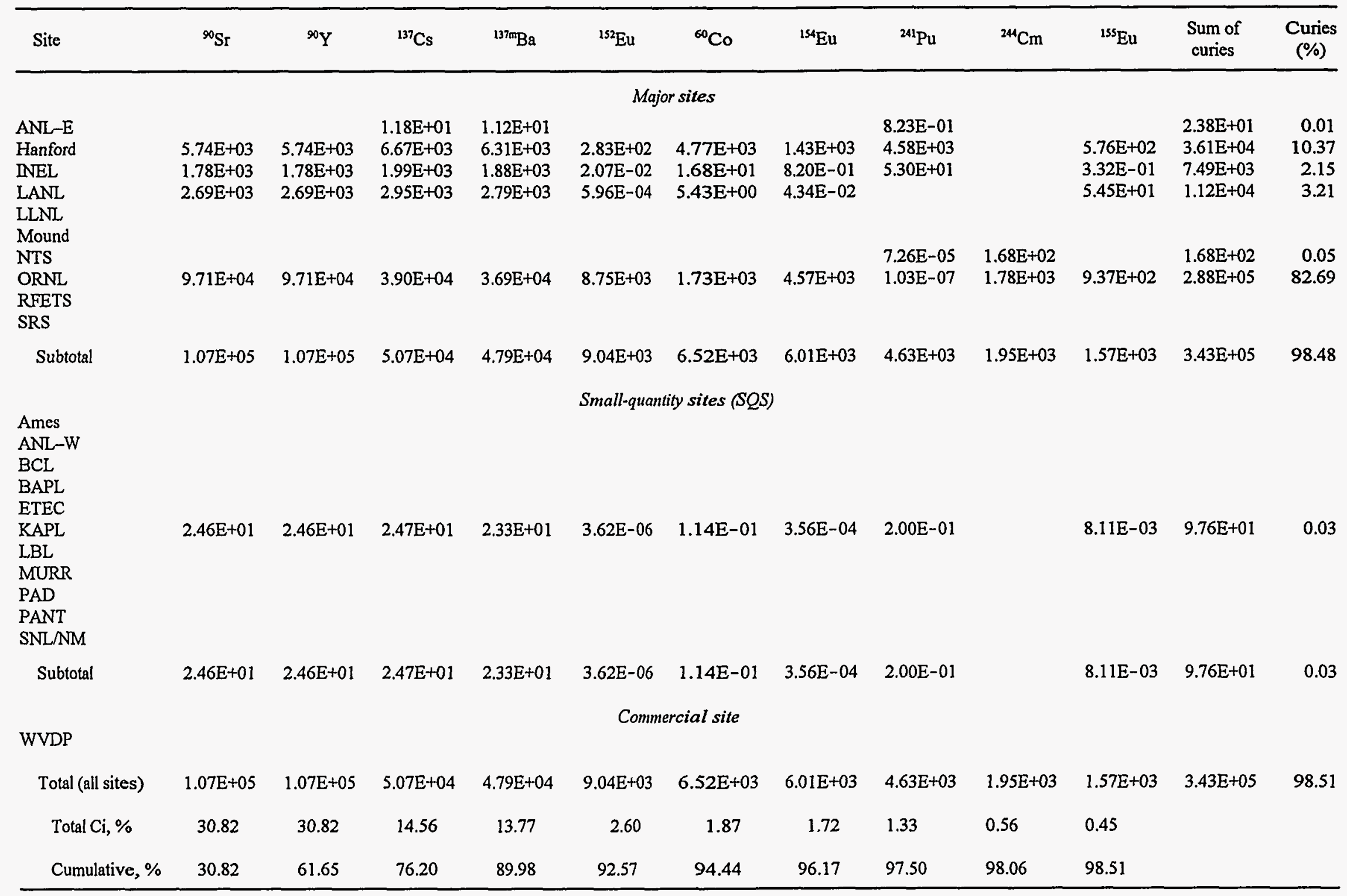

${ }^{a}$ Any small differences in volumes reported in this table and in WTWBIR, Rev. 1 (ref. 2) and in the numerical totals shown result from rounding. 
Table 3.13. Summary of decayed mass $(\mathrm{g})$ isotopic content of retrievably stored CH TRUW

\begin{tabular}{|c|c|c|c|c|c|c|c|c|c|}
\hline Site & ${ }^{238} U$ & ${ }^{232} \mathrm{Th}$ & ${ }^{239} \mathrm{Pu}$ & ${ }^{235} \mathrm{U}$ & ${ }^{242} \mathrm{Pu}$ & ${ }^{233} \mathrm{U}$ & ${ }^{240} \mathrm{Pu}$ & $\begin{array}{c}\text { Sum of } \\
\text { mass }\end{array}$ & $\begin{array}{l}\text { Mass } \\
(\%)\end{array}$ \\
\hline \multicolumn{10}{|c|}{ Major sites } \\
\hline ANL-E & $1.59 \mathrm{E}+02$ & $1.48 \mathrm{E}-09$ & $5.27 \mathrm{E}+02$ & $2.75 E+02$ & $2.62 \mathrm{E}+00$ & $3.10 \mathrm{E}+00$ & $4.13 E+01$ & $1.01 \mathrm{E}+03$ & 0.00 \\
\hline Hanford & $1.80 \mathrm{E}+07$ & $5.82 \mathrm{E}+05$ & $4.35 \mathrm{E}+05$ & $2.63 E+05$ & $9.70 \mathrm{E}+01$ & $8.18 E+03$ & $2.66 \mathrm{E}+04$ & $1.93 E+07$ & 73.79 \\
\hline INEL & $3.44 E+05$ & $3.01 \mathrm{E}+06$ & $6.45 \mathrm{E}+05$ & $2.85 E+04$ & $2.47 \mathrm{E}+02$ & $9.28 \mathrm{E}+04$ & $4.31 E+04$ & $4.16 \mathrm{E}+06$ & 15.90 \\
\hline Mound & & & $3.20 E+03$ & $9.51 \mathrm{E}-01$ & & & & $3.20 \mathrm{E}+03$ & 0.01 \\
\hline NTS & $1.66 \mathrm{E}+02$ & $4.19 E-09$ & $4.44 E+04$ & $2.60 \mathrm{E}+01$ & $2.28 \mathrm{E}+01$ & $1.87 \mathrm{E}+02$ & $8.21 \mathrm{E}+01$ & $4.49 E+04$ & 0.17 \\
\hline ORNL & $1.05 E+05$ & $3.12 \mathrm{E}+05$ & $1.28 \mathrm{E}+04$ & $3.04 E+03$ & $1.43 E+03$ & $9.22 \mathrm{E}+03$ & $3.18 \mathrm{E}+03$ & $4.47 \mathrm{E}+05$ & 1.71 \\
\hline RFETS & & $2.97 \mathrm{E}-07$ & $1.60 \mathrm{E}+05$ & $1.30 \mathrm{E}+01$ & & $7.78 \mathrm{E}-06$ & $3.16 \mathrm{E}+04$ & $1.91 E+05$ & 0.73 \\
\hline SRS & $1.56 \mathrm{E}+04$ & $9.41 \mathrm{E}+04$ & $1.38 \mathrm{E}+05$ & $2.52 \mathrm{E}+03$ & $9.82 \mathrm{E}+01$ & $9.14 \mathrm{E}-01$ & $9.29 E+03$ & $2.59 \mathrm{E}+05$ & 0.99 \\
\hline ANL-W & & & & & & & & & \\
\hline \multicolumn{10}{|l|}{$\mathrm{BCL}$} \\
\hline \multicolumn{10}{|l|}{ BAPL } \\
\hline ETEC & & & $2.83 \mathrm{E}+01$ & & $1.05 \mathrm{E}-02$ & & $2.64 \mathrm{E}+00$ & $3.10 \mathrm{E}+01$ & 0.00 \\
\hline \multicolumn{10}{|l|}{ KAPL } \\
\hline LBL & $4.55 \mathrm{E}-05$ & $3.37 \mathrm{E}-12$ & $1.24 \mathrm{E}-01$ & $1.75 \mathrm{E}-05$ & $2.64 \mathrm{E}+00$ & $4.97 \mathrm{E}-01$ & $2.23 \mathrm{E}-02$ & $3.28 \mathrm{E}+00$ & 0.00 \\
\hline MURR & $2.97 \mathrm{E}-04$ & & $3.29 \mathrm{E}-01$ & & & & & $3.30 \mathrm{E}-01$ & 0.00 \\
\hline PAD & & & $8.95 E+02$ & & & & & $8.95 E+02$ & 0.00 \\
\hline PANT & & & $8.93 \mathrm{E}-01$ & & & & & $8.93 E-01$ & 0.00 \\
\hline SNL/NM & & & $3.22 E-05$ & $2.73 E-09$ & & $5.99 \mathrm{E}-13$ & & $3.22 \mathrm{E}-05$ & 0.00 \\
\hline Subtotal & $3.43 \mathrm{E}-04$ & $3.37 \mathrm{E}-12$ & $9.24 \mathrm{E}+02$ & $1.75 \mathrm{E}-05$ & $2.65 \mathrm{E}+00$ & $4.97 \mathrm{E}-01$ & $2.66 \mathrm{E}+00$ & $9.30 \mathrm{E}+02$ & 0.00 \\
\hline
\end{tabular}

aAny small differences in volumes reported in this table and in WTWBIR, Rev. 1 (ref. 2) and in the numerical totals shown result from rounding. 
Table 3.14. Summary of decayed mass (g) isotopic content of retrievably stored RH TRUWa

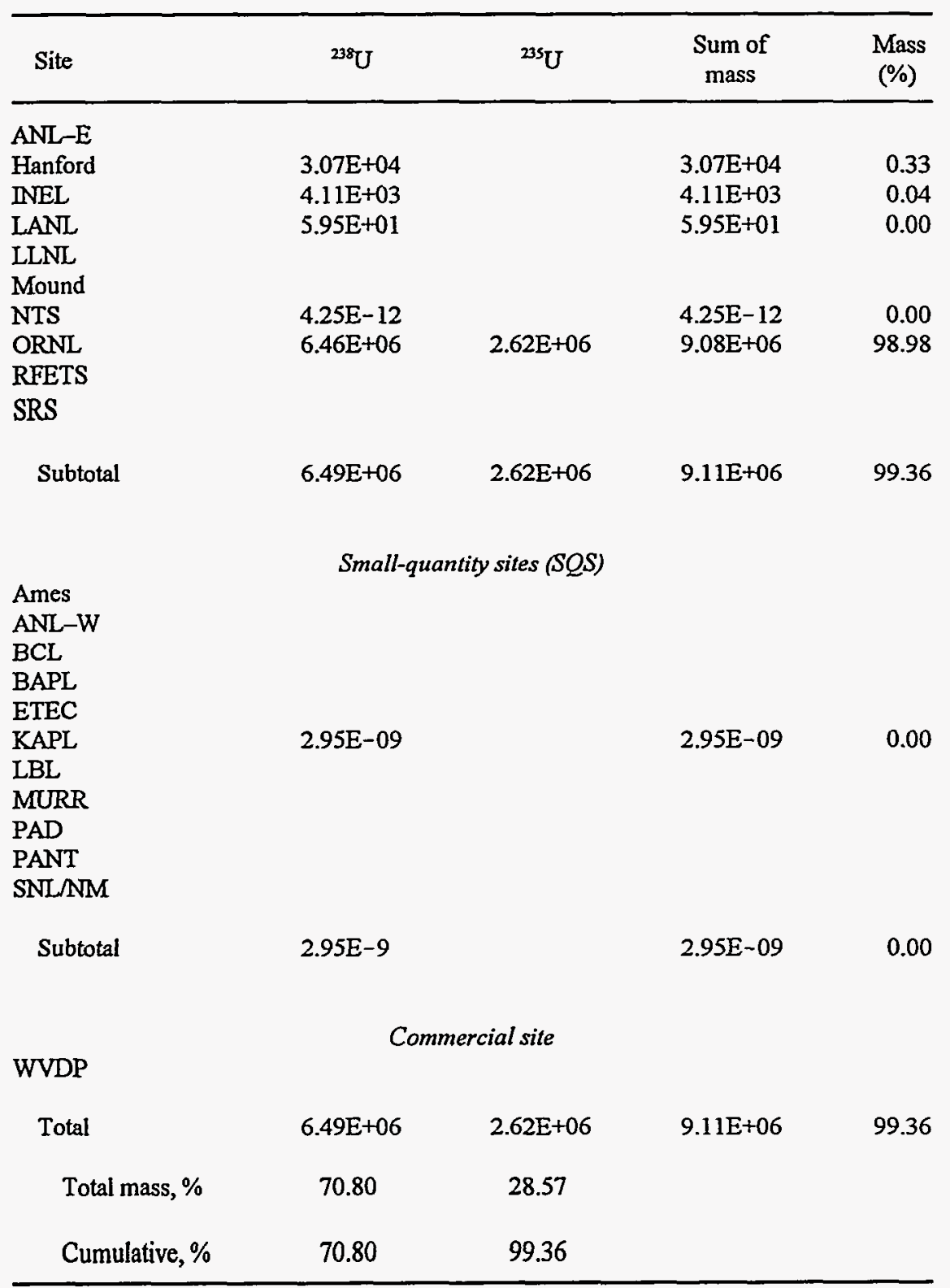

a Any small differences in volumes reported in this table and WTWBIR, Rev. 1 (ref. 2) and in the numerical totals shown result from rounding. 
Table 3.15. Summary of buried TRUW volume and radioactivity as of December 31, 1993 ${ }^{a}$

\begin{tabular}{|c|c|c|c|c|c|}
\hline \multirow[b]{2}{*}{ Site } & \multirow{2}{*}{$\begin{array}{l}\text { Cumulative } \\
\text { volume } \\
\left(\mathrm{m}^{3}\right)\end{array}$} & \multicolumn{2}{|c|}{$\begin{array}{l}\text { Cumulative as-stored } \\
\text { radioactivity }\end{array}$} & \multicolumn{2}{|c|}{ Decayed radioactivity } \\
\hline & & $\begin{array}{c}\text { All } \\
\text { nuclides } \\
\left(10^{3} \mathrm{Ci}\right)\end{array}$ & $\begin{array}{c}\text { TRU } \\
\text { only } \\
\left(10^{3} \mathrm{Ci}\right)\end{array}$ & $\begin{array}{c}\text { All } \\
\text { nuclides } \\
\left(10^{3} \mathrm{Ci}\right)\end{array}$ & $\begin{array}{c}\text { TRU } \\
\text { only } \\
\left(10^{3} \mathrm{Ci}\right)\end{array}$ \\
\hline \multicolumn{6}{|c|}{ Major sites } \\
\hline ANL-E & $0.00 E+00$ & $0.00 \mathrm{E}+00$ & $0.00 \mathrm{E}+00$ & $0.00 \mathrm{E}+00$ & $0.00 \mathrm{E}+00$ \\
\hline Hanford & $6.36 \mathrm{E}+04$ & $6.02 \mathrm{E}+02$ & $1.14 \mathrm{E}+02$ & $1.73 E+02$ & $9.38 \mathrm{E}+01$ \\
\hline INEL & $5.71 E+04$ & $2.49 E+02$ & b & b & $\mathrm{b}$ \\
\hline LANL & $1.40 \mathrm{E}+04$ & c & d & c & c \\
\hline LLNL & $0.00 E+00$ & $0.00 E+00$ & $0.00 \mathrm{E}+00$ & $0.00 E+00$ & $0.00 \mathrm{E}+00$ \\
\hline Mound & $0.00 \mathrm{E}+00$ & $0.00 \mathrm{E}+00$ & $0.00 \mathrm{E}+00$ & $0.00 \mathrm{E}+00$ & $0.00 E+00$ \\
\hline NTS & $0.00 \mathrm{E}+00$ & $0.00 \mathrm{E}+00$ & $0.00 \mathrm{E}+00$ & $0.00 E+00$ & $0.00 \mathrm{E}+00$ \\
\hline ORNL & $1.76 E+02$ & $2.40 \mathrm{E}-01$ & $1.00 \mathrm{E}-01$ & $5.43 E+02$ & $2.00 \mathrm{E}-01$ \\
\hline RFETS & $0.00 \mathrm{E}+00$ & $0.00 \mathrm{E}+00$ & $0.00 \mathrm{E}+00$ & $0.00 \mathrm{E}+00$ & $0.00 \mathrm{E}+00$ \\
\hline SRS & $4.87 E+03$ & $3.37 \mathrm{E}+01$ & $3.37 \mathrm{E}+01$ & $3.05 E+01$ & $3.05 E+01$ \\
\hline Subtotal & $1.40 E+05$ & $8.84 \mathrm{E}+02$ & $1.48 \mathrm{E}+02$ & $7.47 \mathrm{E}+02$ & $1.25 E+02$ \\
\hline
\end{tabular}

Small-quantity sites (SQS)

\begin{tabular}{llllll} 
Ames & $0.00 \mathrm{E}+00$ & $0.00 \mathrm{E}+00$ & $0.00 \mathrm{E}+00$ & $0.00 \mathrm{E}+00$ & $0.00 \mathrm{E}+00$ \\
ANL-W & $0.00 \mathrm{E}+00$ & $0.00 \mathrm{E}+00$ & $0.00 \mathrm{E}+00$ & $0.00 \mathrm{E}+00$ & $0.00 \mathrm{E}+00$ \\
BCL & $0.00 \mathrm{E}+00$ & $0.00 \mathrm{E}+00$ & $0.00 \mathrm{E}+00$ & $0.00 \mathrm{E}+00$ & $0.00 \mathrm{E}+00$ \\
BAPL & $0.00 \mathrm{E}+00$ & $0.00 \mathrm{E}+00$ & $0.00 \mathrm{E}+00$ & $0.00 \mathrm{E}+00$ & $0.00 \mathrm{E}+00$ \\
ETEC & $0.00 \mathrm{E}+00$ & $0.00 \mathrm{E}+00$ & $0.00 \mathrm{E}+00$ & $0.00 \mathrm{E}+00$ & $0.00 \mathrm{E}+00$ \\
KAPL & $0.00 \mathrm{E}+00$ & $0.00 \mathrm{E}+00$ & $0.00 \mathrm{E}+00$ & $0.00 \mathrm{E}+00$ & $0.00 \mathrm{E}+00$ \\
LBL & $0.00 \mathrm{E}+00$ & $0.00 \mathrm{E}+00$ & $0.00 \mathrm{E}+00$ & $0.00 \mathrm{E}+00$ & $0.00 \mathrm{E}+00$ \\
MURR & $0.00 \mathrm{E}+00$ & $0.00 \mathrm{E}+00$ & $0.00 \mathrm{E}+00$ & $0.00 \mathrm{E}+00$ & $0.00 \mathrm{E}+00$ \\
PAD & $0.00 \mathrm{E}+00$ & $0.00 \mathrm{E}+00$ & $0.00 \mathrm{E}+00$ & $0.00 \mathrm{E}+00$ & $0.00 \mathrm{E}+00$ \\
PANT & $0.00 \mathrm{E}+00$ & $0.00 \mathrm{E}+00$ & $0.00 \mathrm{E}+00$ & $0.00 \mathrm{E}+00$ & $0.00 \mathrm{E}+00$ \\
SNL/NM & $1.33 \mathrm{E}+00$ & $0.00 \mathrm{E}+00$ & $0.00 \mathrm{E}+00$ & $0.00 \mathrm{E}+00$ & $0.00 \mathrm{E}+00$ \\
\multicolumn{1}{l}{ Subtotal } & $1.33 \mathrm{E}+00$ & $0.00 \mathrm{E}+00$ & $0.00 \mathrm{E}+00$ & $0.00 \mathrm{E}+00$ & $0.00 \mathrm{E}+00$
\end{tabular}

Commercial site

$\begin{array}{llllll}\text { WVDP } & 1.35 \mathrm{E}+03 & 6.52 \mathrm{E}+02 & 6.52 \mathrm{E}+02 & 0.00 \mathrm{E}+00 & 0.00 \mathrm{E}+00 \\ \text { Total } & 1.41 \mathrm{E}+05 & 1.54 \mathrm{E}+03 & 8.00 \mathrm{E}+02 & 7.47 \mathrm{E}+02 & 1.25 \mathrm{E}+02\end{array}$

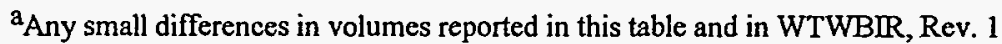
(ref. 2) and in the numerical totals shown result from rounding. See ref. 5.

DINEL data did not include any isotopic compositions, so no TRU isotope or decay calculations could be made.

CInformation not available.

$\mathrm{d}$ An estimate of $9230 \mathrm{Ci}$ was reported for $\mathrm{LANL}$ as-stored TRU alpha radioactivity (as of the end of CY 1991) in Integrated Data Base for 1992: U.S. Spent Fuel and Radioactive Waste Inventories, Projections, and Characteristics, DOE/RW-0006, Rev. 8 (October 1992). 
Table 3.16. Volumes and radioactivities of TRU-contaminated soils ${ }^{\mathrm{a}}$

\begin{tabular}{|c|c|c|c|c|}
\hline \multirow{3}{*}{ Site } & \multicolumn{4}{|c|}{ Soil contaminated with TRUW } \\
\hline & \multicolumn{2}{|c|}{ Solid } & \multicolumn{2}{|c|}{ Liquid } \\
\hline & $\begin{array}{l}\text { Volume } \\
\left(\mathrm{m}^{3}\right)\end{array}$ & $\begin{array}{l}\text { Radioactivity } \\
\text { (Ci) }\end{array}$ & $\begin{array}{l}\text { Volume } \\
\left(\mathrm{m}^{3}\right)\end{array}$ & $\begin{array}{l}\text { Radioactivity } \\
\text { (Ci) }\end{array}$ \\
\hline \multicolumn{5}{|c|}{ Major sites } \\
\hline ANL-E & 0 & 0 & 0 & 0 \\
\hline Hanford & $\mathrm{b}$ & b & 32,000 & 80,591 \\
\hline INEL & c & c & c & c \\
\hline LANL & d & e & $\mathrm{d}$ & e \\
\hline LLNL & 0 & 0 & 0 & 0 \\
\hline Mound & d & d & d & d \\
\hline NTS & d & d & c & c \\
\hline ORNL & $d$ & $d$ & $d$ & $d$ \\
\hline RFETS & 2 & 40 & c & c \\
\hline SRS & 0 & 0 & 0 & 0 \\
\hline
\end{tabular}

Small-quantity sites (SQS)

$\begin{array}{lllll}\text { Ames } & \text { f } & \text { f } & \text { f } & \text { f } \\ \text { ANL-W } & \text { f } & \text { f } & \text { f } & \text { f } \\ \text { BCL } & \text { f } & \text { f } & \text { f } & \text { f } \\ \text { BAPL } & \text { f } & \text { f } & \text { f } & \text { f } \\ \text { ETEC } & 0 & 0 & 0 & \text { f } \\ \text { ITRI } & \text { f } & \text { f } & \text { f } & \text { f } \\ \text { KAPL } & 0 & 0 & 0 & 0 \\ \text { LBL } & 0 & 0 & 0 & \text { f } \\ \text { MURR } & \text { f } & \text { f } & \text { f } & \text { f } \\ \text { PAD } & \text { c } & \text { c } & \text { c } & \text { f } \\ \text { PANT } & \text { f } & \text { f } & \text { f } & \text { f } \\ \text { SNL } N M & \text { d } & \text { d } & \text { d } & \text { d }\end{array}$

\section{Commercial site}

\begin{tabular}{lllll} 
WVDP & $\mathrm{f}$ & $\mathrm{f}$ & $\mathrm{f}$ & $\mathrm{f}$ \\
\hline
\end{tabular}

asee ref. 5 of this chapter.

Included in buried TRUW.

CListed in submittal as not applicable.

dunknown.

Eartial data submitted.

fNo data submitted. 


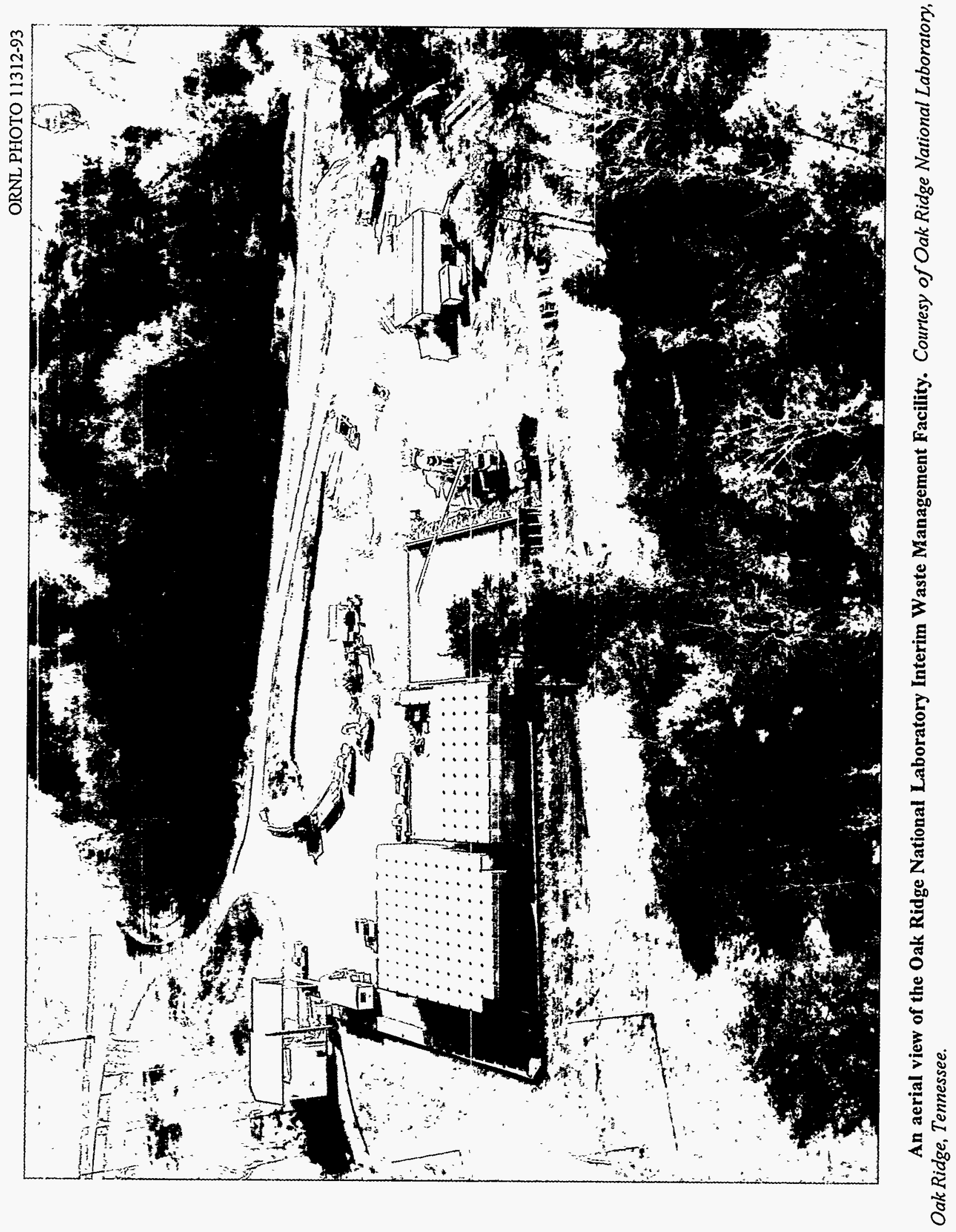




\section{LOW-LEVEL WASTE}

\subsection{INTRODUCTION}

As used in this chapter, LLW has the same meaning as in The Low-Level Waste Policy Act (Pub. L. 95-573, Dec. 22, 1980). Namely, LLW is radioactive waste not classified as high-level radioactive waste, transuranic waste (TRUW), spent nuclear fuel (SNF), or by-product material specified as uranium or thorium tailings and waste. The naturally occurring or accelerator-produced radioactive material that is disposed of at DOE or commercial disposal sites is included in the inventories given, but they are not treated as separate entities in this chapter. Tailings (viz., mill tailings) are considered in Chapters 5 and 6 . Another waste classification not delineated in this chapter is "mixed" low-level waste (MLLW), which contains both chemically hazardous and radioactive constituents (see Chapter 8 ). Specific definitions of these waste types (as defined by DOE Order $5820.2 \mathrm{~A}$ ) are given in the glossary of this report. DOE generates LLW through its defense activities, naval nuclear propulsion program, and various $R \& D$ activities. The data for DOE sites represent a summary of information obtained from each site. ${ }^{1}$

Disposal of LLW at commercial sites accounted for about 32\% of the LLW disposed during 1994 (see Fig. 4.1). Commercially disposed LLW is generally divided into five categories: academic, government, industrial, medical, and utility. ${ }^{2}$ The academic category includes university hospitals and university medical and nonmedical research facilities. The government category includes state and non-DOE federal agencies. The industrial category is comprised of private entities such as R\&D companies, manufacturers, nondestructive-testing operations, mining works, fuel fabrication facilities, and radiopharmaceutical manufacturers. The medical category includes hospitals and clinics, research facilities, and private medical offices. The utility category includes commercial nuclear reactors. In earlier revisions of the $\mathrm{IDB}$ report commercially disposed waste was reported by fuel cycle and industrial/institutional (I/) type activities. However, to achieve more consistency with other reporting agencies, the five categories previously described were used, starting with Rev. 9 of the IDB report.
Some LLW is also generated by DOE environmental restoration programs (see Chapter 6). Other LLW will be generated in future years by nonroutine $D \& D$ operations. Waste from past commercial D\&D operations is included with the commercial waste disposal portion in this chapter because it has not been reported separately. However, projections of commercial D\&D wastes are not included here but, instead, are discussed in Chapter 7.

The categorization of LLW according to DOE and commercial activities permits a comparison of the radioactivity levels and volumes of waste arising from each of these major sources (Figs. 4.1 and 4.2). Summary data on LLW disposal (DOE and commercial) are given in Table 4.1. Historical and projected data by year for DOE LLW disposal are presented in Table 4.2. In Table 4.3, similar data are shown for commercial LLW disposal.

\subsection{DOE LLW}

\subsubsection{Inventories of LLW at DOE Sites}

An abridged picture of DOE LLW inventories, projections, and characteristics through 1994 is given in Figs. 4.1-4.4, as well as Tables 4.1, 4.2, and 4.4-4.18. The data in these tables are derived from responses to questionnaires submitted to DOE sites. ${ }^{1}$ As reflected in the tables, DOE LLW data can be grouped into three major areas: generation, storage, and disposal.

Summaries of DOE site-generated LLW volumes and associated radioactivity are presented in Tables 4.6, 4.9, and 4.10 .

Tables $4.7,4.11$, and 4.12 provide summary volume and radioactivity data concerning LLW storage at DOE sites.

A summary of DOE LLW disposed volume and radioactivity is presented in Tables 4.1, 4.2, 4.4, 4.8, 4.13, 4.14, and 4.17. Before October 1979, some LLW generated by DOE contractors was shipped to commercial disposal sites. The volume and radioactivity data for DOE LLW that were shipped to commercial disposal sites are contained in the commercial LLW tables in this chapter. 
Currently, LLW generated by DOE activities is generally disposed of at DOE sites (see Figs. 4.3 and 4.4).

Small quantities of DOE LLW have been disposed of by dumping the LLW into the sea or by hydrofracture. ${ }^{3}$ Table 4.15 shows the estimated quantity and radioactivity of LLW disposed of by these methods. Dumping of LLW into the sea was halted by the United States in 1970, and hydrofracture was terminated in 1983.

\subsubsection{Characterization of LLW at DOE Sites}

Based on information reported in ref. 1, summaries of physical characteristics of DOE LLW are reported in Tables 4.6-4.14. Table 4.5 provides definitions of each of the physical forms used in the tables. This breakdown of LLW data is different from that presented in previous revisions of the $\mathrm{DB}$ report (i.e., Revs. 1-10). The new set of physical forms provides a more detailed description of the LLW that has been generated, stored, or disposed. Actual 1994 and projected generation, storage, and disposal volume and radioactivity data are presented in Tables 4.6-4.8, respectively. A more detailed breakdown of this data, by DOE site, is provided in Tables 4.9-4.14.

In addition to shallow-land burial, DOE LLW has been disposed by dumping wastes into the sea and hydrofracture (Table 4.15). Most of the DOE wastes that were dumped into the sea were incorporated into cement matrix material and packaged in steel drums (55- or 80-gal capacity). Hydrofracture was developed at ORNL for the permanent disposal of locally generated, low-level (approximately $0.25 \mathrm{Ci} / \mathrm{L}$ ) liquid waste concentrates. ${ }^{4}$ Waste was mixed with a blend of cement and other additives, and the resulting grout was injected into shale at a depth of 200 to $300 \mathrm{~m}$. The injected grout hardened into thin, horizontal sheets several hundred meters wide.

Significant changes in DOE LLW inventory and characteristics data from that reported in the 1994 edition (1993 data) of this report are summarized in Table 4.16.

\subsubsection{DOE LLW Projections}

Low-level waste generation, storage, and disposal projections reported by DOE sites are presented in Tables $4.6-4.8$, respectively. Projections are provided for three time periods: $1995,1996-2000$, and 2001-2030. The data presented in Tables 4.6-4.8 represent the average annual generation, addition to storage, or disposal, respectively, during each of the three periods.

The projected disposal data (Table 4.8), combined with actual 1994 (Tables 4.8, 4.13, and 4.14) and historical (ref. 5) disposal data, were used to calculate historical and projected volume, radioactivity, and thermal power of DOE disposed LLW. As stated in Sect. 4.2.2,
LLW data were collected by a different breakdown in previous revisions of the $\mathrm{DB}$ report. Historical disposal data through 1993 were decayed from the year of addition through 2030 using the representative radionuclide compositions given in Table A.3 of Appendix A. ${ }^{6}$ Actual 1994 and projected disposal data were decayed from the year of addition through 2030 using radionuclide compositions provided by each disposal site in ref. 1 .

Projected volume, radioactivity, and thermal power for disposed DOE LLW are presented in Tables 4.2, 4.17, and 4.18. Table 4.17 summarizes disposed DOE LLW excluding SRS saltstone. Table 4.18 summarizes projections of saltstone, an LLW by-product from the solidification of HLW at SRS. This saltstone (see Fig. 2.8 in Chapter 2) is to be stored in concrete vaults at SRS. Table 4.2 is the summation of Tables 4.17 and 4.18. Grout-immobilized LLW derived from processing doubleshell waste at Hanford (see Fig. 2.6 in Chapter 2) is excluded from the projections in Table 4.2 because the schedule and formulation for immobilization are not yet firmly defined.

\subsection{COMMERCIAL LLW}

\subsubsection{Inventories at Commercial LLW Disposal Sites}

There are six commercial shallow-land disposal sites for LLW (Figs. 4.2, 4.5, and 4.6), but only two are currently in operation: Barnwell, South Carolina; and Richland, Washington. Commercial operations at the Maxey Flats, Kentucky; West Valley, New York; Sheffield, Illinois; and Beatty, Nevada, sites have been halted. Until 1986, a second NRC-licensed burial ground at West Valley continued to receive wastes generated on-site from cleanup and water treatment operations. However, disposal operations at the WVDP have been suspended since 1986 pending the preparation of an environmental impact statement (EIS) report for the West Valley site closure. The historical data for annual additions and inventories of volume and radioactivity (undecayed) at each commercial disposal site through the end of 1994 are listed in Tables 4.19 and 4.20, respectively (compiled from refs. 5 and 7). The volumes are depicted in Figs. 4.2, 4.5, and 4.6. Sources of the historical reported data through 1984 are given in ref. 3, and those through 1993 are given in ref. 5. Quantities of LLW shipped to disposal sites during 1994 are listed in Table 4.21 on a state-by-state basis. ${ }^{7}$ Table 4.3 is a summary of historical and projected volumes, radioactivity (decayed), and thermal power for commercial LLW. Projections are made only through 2005 because of large 
uncertainties in commercial disposal facility operations. Not included in Table 4.3 are the drums of cemented LLW to be generated by the WVDP as a result of the vitrification of HLW. This LLW from the WVDP is described in Table A.6 of Appendix A. Table 4.3 also does not include $40,461 \mathrm{~m}^{3}\left(1,759 \mathrm{~m}^{3}\right.$ from DOE generators and $38,702 \mathrm{~m}^{3}$ from commercial generators) of LLW disposed of at the Envirocare disposal facility near Clive, Utah. Additional information on Envirocare disposals can be found in Table 0.8 of Chapter 0.

\subsubsection{Characterization of LLW at Commercial Disposal Sites}

All of the LLW accepted for commercial disposal is categorized as Class $\mathrm{A}, \mathrm{B}$, or $\mathrm{C}$ in compliance with NRC specifications. ${ }^{8}$ The LLW that exceeds these specifications is currently in storage at the generator site or at a DOE site which has accepted it for study (see Sect. 4.3.3). A calculated representative radionuclide composition for disposed commercial LLW is given in Table A.4 of Appendix A. This composition is periodically updated to reflect changes in waste management practices and in the regulations governing LLW disposal.

\subsubsection{Greater-Than-Class-C Low-Level Waste (GTCC LLW)}

In 1980, federal law made each state responsible for providing the disposal capacity for LLW generated within its borders, except for certain waste generated by the federal government.9 In 10 CFR Part 61 (ref. 8), the NRC codifies disposal requirements for three classes of LLW, as mentioned above, generally suitable for near-surface disposal, namely, Class $\mathrm{A}, \mathrm{B}$, and $\mathrm{C}$ (with Class $\mathrm{C}$ waste requiring the most rigorous disposal specifications). Waste with concentrations above Class $\mathrm{C}$ limits for certain short- and long-lived radionuclides (i.e., GTCC LLW) was found not generally suitable for near-surface disposal, except on a case-by-case evaluation of the waste and the proposed disposal method by NRC or state licensing agency. The Low-Level Radioactive Waste Policy Amendments Act (LLRWPAA) ${ }^{10}$ made the states responsible for the disposal of Classes $A, B$, and C LLW and made the federal government (viz., DOE) responsible for disposal of GTCC LLW. The law also required that GTCC LLW generated by licensees of NRC be disposed of in a facility licensed by NRC. The projected amounts of GTCC LLW are uncertain, both because of regulatory uncertainties affecting the definition of HLW (i.e., a clearly defined all-inclusive list of wastes considered HLW may include more than those described in Chapter 2) and because of the lack of information on the sources, volumes, and characteristics of GTCC LLW."

In May 1989, NRC promulgated a rule that requires disposal of GTCC LLW in a deep geologic repository unless disposal elsewhere has been approved by NRC. The rule as amended states: "Waste that is not generally acceptable for near-surface disposal is waste for which form and disposal methods must be different and, in general, more stringent than those specified for Class C waste. In the absence of specific requirements in this part, such waste must be disposed of in a geologic repository as defined in Part 60 of this chapter unless proposals for disposal of such waste in a disposal site licensed pursuant to this part are approved by the Commission."12 A disposal facility (other than a deep geologic repository) for GTCC LLW will probably not be available for several decades because of the complexities of siting and of NRC licensing. A generic description of estimated sources and forms of GTCC LLW is presented in Table A.5 of Appendix A.

DOE recently revised an earlier study to provide information about estimates of present and future GTCC LLW to the year 2035 (2055 in some instances). Information garnered during the study ${ }^{13}$ includes identification of generators, waste form characteristics, volumes, and radionuclide activities. The study categorizes GTCC LLW as (1) nuclear utilities waste, (2) sealed-sources waste, (3) DOE-held potential GTCC $\mathrm{LLW}$, and (4) other generator waste. Various scenarios for data projection were used: (a) unpackaged volumes; (b) packaged volumes based on the application of packaging factors to the unpackaged volumes; and (c) concentration averaging, mixing, or blending of similar materials with different radionuclide concentration values applied to the packaged volumes. Each of the three scenarios is treated for three cases: low, base, and high.

Based on base-case projections of after-concentrationaveraged-packaged waste to the year 2035, the study determined that the largest volume of GTCC wastes (approximately 53\%) is generated by nuclear power plants. The "other" generator waste category contributes approximately $31 \%$ of the total GTCC LLW volume projected to the year 2035. Sealed sources are about $16 \%$ of the total projected volume of GTCC LLW. A summary of historical and projected cumulative volume and radioactivity for GTCC LLW is presented in Table 4.22.

\subsubsection{Commercial LLW Disposal Sites}

Of the six commercial LLW disposal sites, only two remain open: Barnwell and Richland. Historical information regarding commercial LLW facilities can be 
found in previous editions of the $\mathrm{DB}$ report. Closure dates for the four closed facilities are presented in the footnotes of Tables 4.19 and 4.20 .

The Barnwell, South Carolina, disposal site is operated by Chem-Nuclear Systems, Inc. During 1994, Barnwell received about $85 \%$ of the total volume of commercial LLW shipped for disposal. Barnwell accepted LLW from all states with the exceptions of Michigan, New Hampshire, Rhode Island, and states that had access to the Richland facility. Barnwell was scheduled to restrict access to Southeast compact members starting July 1, 1995. However, Barnwell withdrew from the Southeast compact. This action allows all states to dispose of their waste at Barnwell.

The Richland, Washington, disposal site is operated by U.S. Ecology, Inc. During 1994, Richland received about $15 \%$ of the total volume of commercial LLW shipped for disposal. Richland accepted wastes only from member states of the Northwest and Rocky Mountain compacts (Alaska, Colorado, Idaho, Nevada, New Mexico, Oregon, Utah, Washington, and Wyoming).

Table 4.23 provides a breakdown of waste received at Barnwell and Richland in 1994 by category (i.e., academic, government, industrial, medical, or utility LLW).

\subsubsection{Commercial LLW Projections}

This report presents summary information for disposed commercial waste. Historical volume, radioactivity, and thermal power data through 1979 are taken from an earlier version of this report (DOE/RW-0006, Rev. 8). After 1979, the source term for commercial LLW in Table A.4 of Appendix $A$ is used to decay the annual waste additions to the commercial sites.

Projections for disposed commercial LLW are made only through 2005 because of uncertainties in current facility operations and the availability of future sites. Projections (1995-2005) are made for Barnwell and Richland and are based on ref. 7. Barnwell is projected to remain open to all states for 10 years and then to close. Richland is projected to remain open beyond 2005 to only the Northeast and Rocky Mountain compacts. Historical and projected volume, radioactivity, and thermal power for disposed commercial LLW are presented in Table 4.3.

Because of timing uncertainties, projected commercial decommissioning wastes are not included in the projections of this chapter. Rather, waste projections for such wastes are reported separately in Chapter 7 . Former DOE facilities that will be affected by environmental restoration activities are discussed in Chapter 6 and are also excluded from the projected values in this chapter.

\subsection{REFERENCES}

1. DOE site LLW data submittal attachments, submitted to the IDB Program during July-August 1995. The following LLW submittals were received and reviewed by the DB Program before analysis and integration. Preceding each submittal is the site (in parentheses) to which it refers.

a. (Ames) Kay Lampe Hannasch, Ames Laboratory, Ames, Iowa, correspondence to Jerry Klein, IDB Program, ORNL, Oak Ridge, Tennessee, "FY 1995 Low-Level Waste Management Data Call," dated July 7, 1995.

b. (ANL-E) M. A. Sodaro, Argonne National Laboratory, Argonne, Illinois, correspondence to S. N. Storch, IDB Program, ORNL, Oak Ridge, Tennessee, "Low-Level Waste Management Data Call for 1995," dated July 17, 1995.

c. (ANL-W) See reference for INEL.

d. (BNL) Carson L. Nealy, DOE Brookhaven Area Office, Upton, New York, correspondence to Steve Storch, IDB Program, ORNL, Oak Ridge, Tennessee, ‘Brookhaven National Laboratory's Response to the Low-Level Waste Management Data Call for the Integrated Data Base," dated July 14, 1995.

e. (FNAL) Andrew E. Mravca, DOE Batavia Area Office, Batavia, Illinois, correspondence to Alan Icenhour, IDB Program, ORNL, Oak Ridge, Tennessee, "Low-Level Waste Management Data Call for 1995," dated July 7, 1995.

f. (Hanford) A. K. Crowell, DOE Richland Operations Office, Richland, Washington, correspondence to Steve Storch, IDB Program, ORNL, Oak Ridge, Tennessee, "LLW Data Call," dated July 17, 1995. 
g. (INEL) R. R Piscitella, Idaho National Engineering Laboratory, Idaho Falls, Idaho, correspondence to S. Storch, IDB Program, ORNL, Oak Ridge, Tennessee, "Idaho National Engineering Laboratory (INEL) Input to Integrated Data Base (IDB)(DOE/RW-0006, Rev. 11)," RRP-16-95, dated Aug. 3, 1995.

h. (ITRD) Stephen R. Rohrer, Lovelace Biomedical and Environmental Research Institute, Inc., Albuquerque, New Mexico, correspondence to Alan Icenhour, DB Program, ORNL, Oak Ridge, Tennessee, transmitting ITRI LLW data attachment, dated July 17, 1995.

i. (K-25) A. L. Rivera, Lockheed Martin Energy Systems, Inc., Oak Ridge, Tennessee, correspondence to S. N. Storch, IDB Program, Oak Ridge, Tennessee, "Low-Level Waste (LLW) Management Data Call for 1995," dated July 31, 1995.

j. (KCP) Margaret E. Stockdale, DOE Kansas City Area Office, Kansas City, Missouri, correspondence to James Turi, DOE/EM Office of Program Integration, "Low-Level Waste Management Data Call for 1995," Washington, D.C., dated July 12, 1995.

k. (LANL) I-Li Chen, Los Alamos National Laboratory, Los Alamos, New Mexico, correspondence to Alan Icenhour and Steve Storch, DB Program, ORNL, Oak Ridge, Tennessee, "Subject Reports for 1995 LowLevel Waste," CST14-95-383, July 31, 1995.

1. (LBL) Jack Bartley, DOE Oakland Operations Office, Lawrence Berkeley Laboratory, Berkeley, California, correspondence to James A. Turi, DOE/EM Office of Program Integration (DOE/EM-33), Washington, D.C., "Low-Level Management Data Call for 1995," HW-95-138, dated July 13, 1995.

m. (LINL) Kevin Hartnett, DOE Oakland Operations Office, Oakland, California, correspondence to Steve Storch, IDB Program, ORNL, Oak Ridge, Tennessee, transmitting LLNL LLW data attachment, dated Aug. 18, 1995.

n. (MOUND) Raymond J. Finney, EG\&G Mound Applied Technologies, Miamisburg, Ohio, correspondence to Robert S. Rothman, DOE Miamisburg Area Office, Miamisburg, Ohio, "Low-level Waste Management Data Call," dated July 10, 1995.

o. (NR sites) A. R. Seepo, DOE Office of Naval Reactors (NE-60), Arlington, Virginia, correspondence to Alan Icenhour/Steve Storch, IDB Program, ORNL, Oak Ridge, Tennessee, "Low-Level Radioactive Waste Management Data Call for 1995," dated July 18, 1995.

p. (NTS) Carlos Gonzales, Reynolds Electrical \& Engineering Company, Inc., correspondence to Alan Icenhour/Steve Storch, IDB Program, ORNL, Oak Ridge, Tennessee, "NTS IDB Tables 1 through 10DRAFT," dated Aug. 16, 1995.

q. (ORISE) James E. Drewry, Director, ORISE, Oak Ridge, Tennessee, correspondence to Thomas M. Jelinek, DOE Oak Ridge Operations Office, Oak Ridge, Tennessee, "Low-Level Waste Management: Data Call (1995)," dated Aug. 22, 1995.

r. (ORNL) A. L. Rivera, Lockheed Martin Energy Systems, Inc., Oak Ridge, Tennessee, correspondence to S. N. Storch, IDB Program, ORNL, Oak Ridge, Tennessee, "Low-Level Waste (LLW) Management Data Call for 1995," dated July 31, 1995.

s. (PANT) W. A. Weinreich, Mason \& Hanger-Silas Mason Company, Inc., Amarillo, Texas, correspondence to S. Storch, IDB Program, ORNL, Oak Ridge, Tennessee, "1995 Low-Level Waste (LLW) Management Data Call," GM95-01592-791, dated July 17, 1995.

t. (Pinellas) Gary C. Schmidtke, DOE Pinellas Area Office, Largo, Florida, correspondence to Alan Icenhour, IDB Program, ORNL, Oak Ridge, Tennessee, "Pinellas Plant Response to Low-Level Waste Management Data Call for 1995," PAO:GHS:ENV081, dated July 17, 1995. 
u. (PPPL) Scott B. Larson, Princeton Plasma Physics Laboratory, Princeton University, Princeton, New Jersey, correspondence to Alan Icenhour, IDB Program, ORNL, Oak Ridge, Tennessee, "Princeton Plasma Physics Laboratory Low-Level Radioactive Waste Information for the 1994 Integrated Data Base Report," dated Aug. 18, 1995.

v. (RFETS) Eric D'Amico, Rocky Mountain Remediation Services, Golden, Colorado, correspondence to Jou Hwang, Cadmus Group, Inc., Germantown, Maryland, transmitting RFETS LLW information, dated July 19, 1995.

w. (SLAC) Michael Scharfenstein, Stanford University, Stanford, California, correspondence to IDB Program, ORNL, Oak Ridge, Tennessee, "1995 Low-Level Waste Management Data Call," dated July 21, 1995.

x. (SNL/CA) Sarah O'Connor, Lockheed Martin Sandia Corporation, Livermore, California, correspondence to Alan Icenhour and Steve Storch, IDB Program, ORNL, Oak Ridge, Tennessee, "SNL/CA 1995 LLW Data Call," dated July 13, 1995.

y. (SNL/NM) No update submittal provided.

z. (SRS) Nathaniel S. Roddy, Westinghouse Savannah River Company, Aiken, South Carolina, correspondence to Steven Storch, IDB Program, ORNL, Oak Ridge, Tennessee, "Low-Level Waste (LLW) Management Data Call for 1995," SWE-SWE-95-0357, dated July 31, 1995.

aa. (WVDP) John J. Hollinden, West Valley Nuclear Services Company, Inc., West Valley, New York, correspondence to Steven N. Storch, IDB Program, ORNL, Oak Ridge, Tennessee, "Submittal of Low-Level Waste Management Data Call for 1995," WZ:95:0103, dated July 14, 1995.

ab. (Y-12) A. L. Rivera, Lockheed Martin Energy Systems, Inc., Oak Ridge, Tennessee, correspondence to S. N. Storch, IDB Program, ORNL, Oak Ridge, Tennessee, "Low-Level Waste (LLW) Management Data Call for 1995," dated July 31, 1995.

ac. (EM-40) WSSRAP: T. Pauling, DOE; PORTS: Integrated Waste Management Plan for the Portsmouth Gaseous Diffusion Plant, Portsmouth, Ohio; PAD: Integrated Waste Management Plan for the Paducah Gaseous Diffusion Plant, Paducah, Kentucky; ETEC: M. E. Jensen, Rockwell International; all other EM-40 data: EM-40 Contaminated Media/Waste Database, M. Tolbert-Smith, DOE.

2. R. L. Fuchs, "1993 State-by-State Assessment of Low-Level Radioactive Wastes Received at Commercial Disposal Sites," DOE/LLW-205, Idaho National Engineering Laboratory, Idaho Falls, Idaho (September 1994).

3. U.S. Department of Energy, Spent Fuel and Radioactive Waste Inventories, Projections, and Characteristics, DOE/RW-0006, Rev. 1, Oak Ridge National Laboratory, Oak Ridge, Tennessee (December 1985).

4. U.S. Energy Research and Development Administration, Environmental Statement, Radioactive Waste Facilities, WASH-1532 (Draft), Oak Ridge National Laboratory, Oak Ridge, Tennessee (January 1974).

5. U.S. Department of Energy, Integrated Data Base Report-1993: U.S. Spent Nuclear Fuel and Radioactive Waste Inventories, Projections, and Characteristics, DOE/RW-0006, Rev. 10, Oak Ridge National Laboratory, Oak Ridge, Tennessee (December 1994).

6. C. W. Forsberg, W. L. Carter, and A. H. Kibbey, Flowsheets and Source Terms for Radioactive Waste Projections, ORNL/TM-8462, Oak Ridge National Laboratory, Oak Ridge, Tennessee (March 1985). 
7. R. L. Fuchs, Lockheed Idaho Technologies Company, National Low-Level Waste Management Program, Idaho Falls, Idaho, letter to A. S. Icenhour, Oak Ridge National Laboratory, Oak Ridge, Tennessee, "Transmission of Commercial Low-Level Radioactive Waste Data for the 1995 Integrated Data Base Report-RLF-25-95," dated June 13, 1995.

8. U.S. Nuclear Regulatory Commission, "Licensing Requirements for Land Disposal of Radioactive Wastes," Code of Federal Regulations, 10 CFR Part 61, Jan. 1, 1994.

9. U.S. Congress, The Low-Level Radioactive Waste Policy Act, Pub. L. 95-573, Dec. 22, 1980.

10. U.S. Congress, The Low-Level Radioactive Waste Policy Amendments Act of 1985, Pub. L. 99-240, Jan. 15, 1986.

11. U.S. Department of Energy, Recommendations for Management of Greater-than-Class-C Low-Level Radioactive Waste, DOE/NE-0077 (February 1987).

12. U.S. Nuclear Regulatory Commission, amendments to 10 CFR Part 61, "Disposal of Radioactive Wastes," Final Rule, Fed. Regist. 54(100), 22578-83 (May 25, 1989).

13. Lockheed Idaho Technologies Company, Greater-Than-Class-C Low-Level Radioactive Waste Characterization: Estimated Volumes, Radionuclide Activities, and Other Characteristics, DOE/LLW-114, Rev. 1, Idaho Falls, Idaho (September 1994). 
ORNL DWG 95-8838

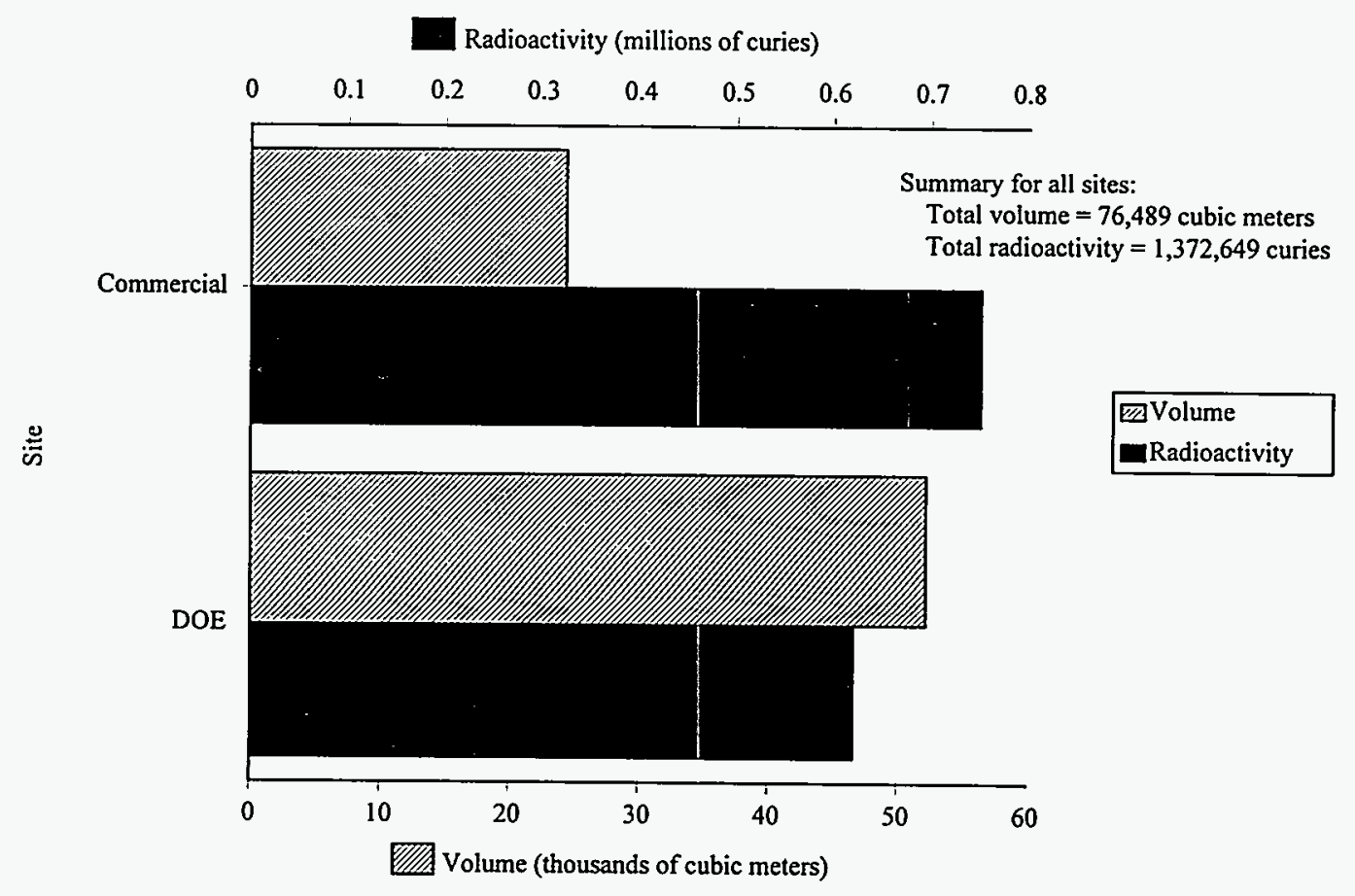

Fig. 4.1. Volume and radioactivity of LLW disposed at commercial and DOE facilities during 1994.

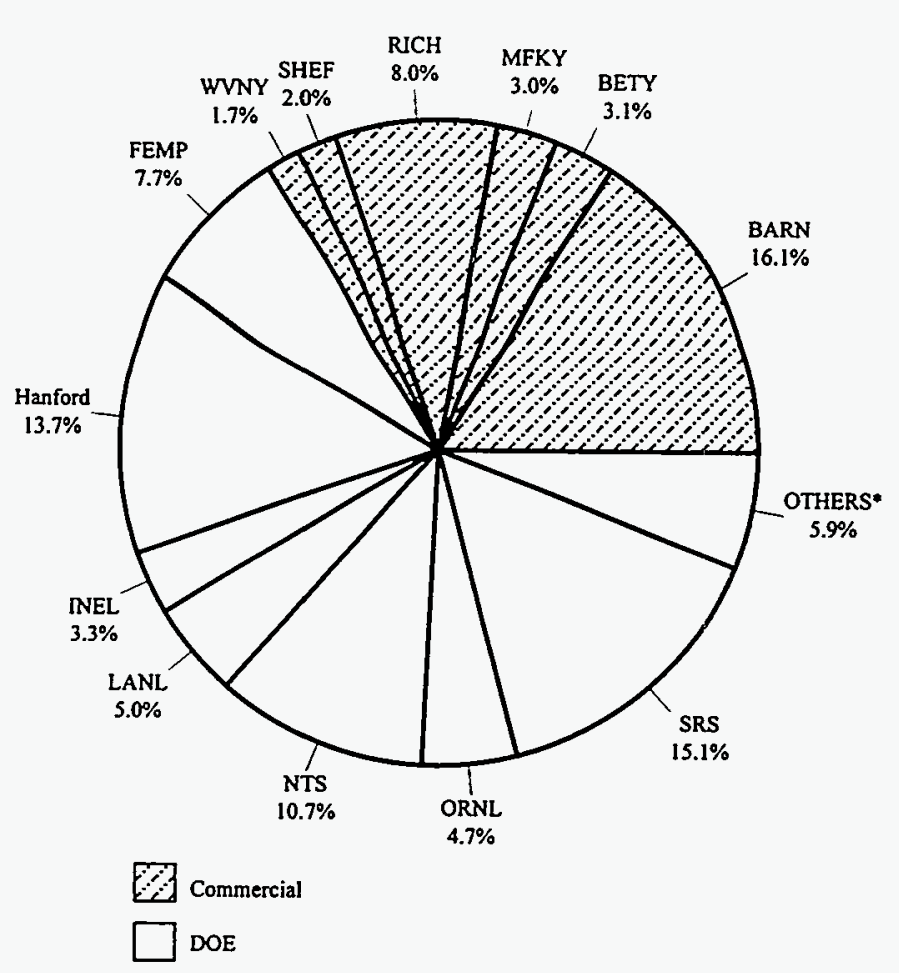

ORNL DWG 95.8839

Fig. 4.2. Total volume of LLW disposed through 1994. 
ORNL DWG 95-8845

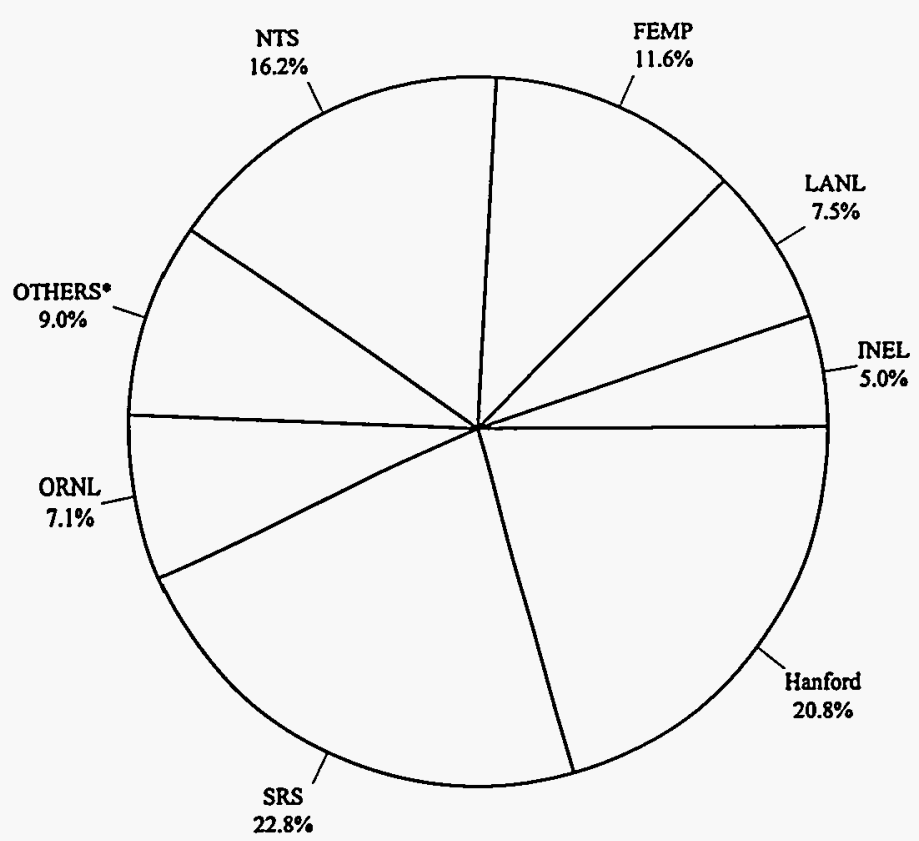

\begin{tabular}{ll} 
SITE & $\begin{array}{c}\text { CUBIC } \\
\text { METERS }\end{array}$ \\
\hline FEMP & $3.432 E+0 S$ \\
Hanford & $6.153 \mathrm{E}+05$ \\
NNEL & $1.489 \mathrm{E}+05$ \\
LANL & $2.226 \mathrm{E}+05$ \\
NTS & $4.813 \mathrm{E}+05$ \\
ORNL & $2.09 \mathrm{E}+05$ \\
SRS & $6.764 \mathrm{E}+05$ \\
OTHERS* & $2.655 \mathrm{E}+05$ \\
\hline TOTAL & $2.963 \mathrm{E}+05$
\end{tabular}

-Includes contributions from Ames,

BNL, K-25, LLNL, PANT, PAD,

PORTS, SNL/NM., and Y-12

Fig. 4.3. Total volume of DOE LLW disposed through 1994.

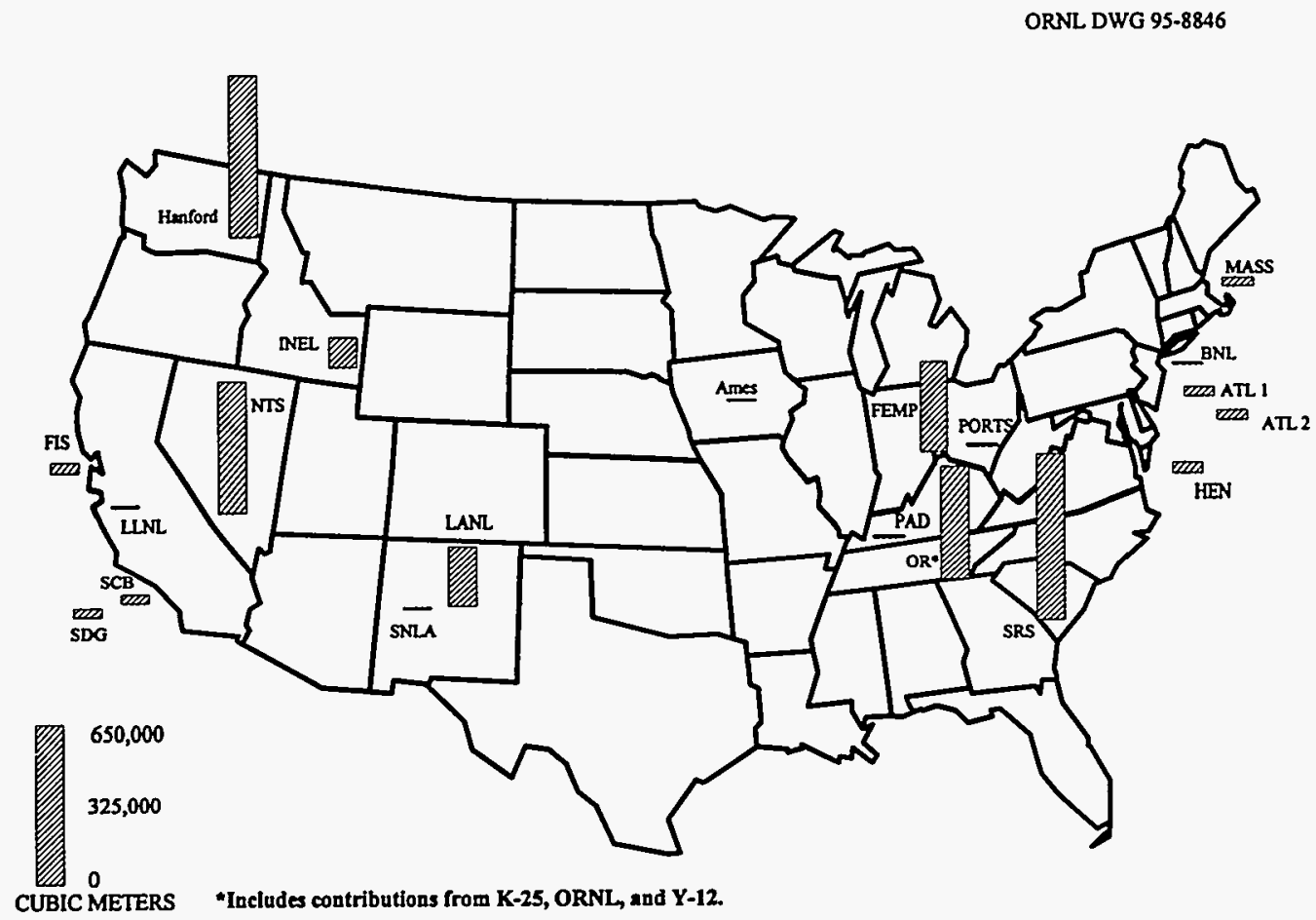

Fig. 4.4. Locations and total volumes of DOE LLW disposed through 1994. 
ORNL DWG 95-8129

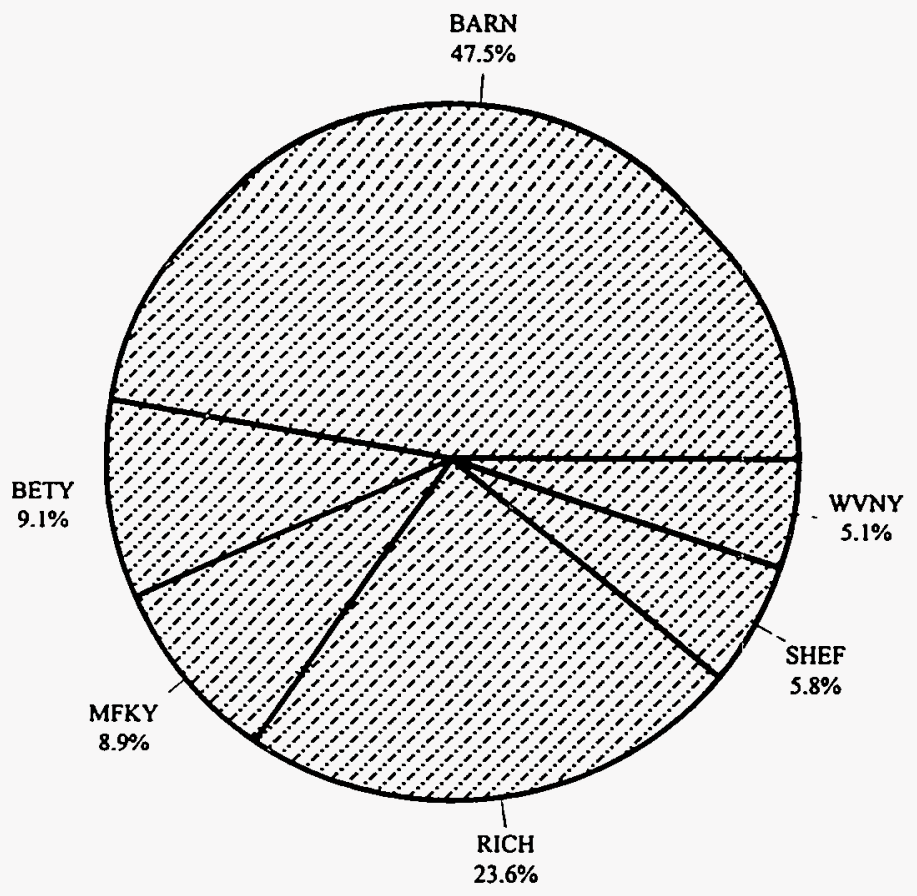

\begin{tabular}{lc}
\multicolumn{1}{c}{ SITE } & $\begin{array}{c}\text { CUBIC } \\
\text { METERS }\end{array}$ \\
\hline BARN & $7.221 \mathrm{E}+05$ \\
BETY & $1.375 \mathrm{E}+05$ \\
MFKY & $1.353 \mathrm{E}+05$ \\
RICH & $3.586 \mathrm{E}+05$ \\
SHEF & $8.833 \mathrm{E}+04$ \\
WVNY & $7.707 \mathrm{E}+04$ \\
\hline TOTAL & $1.519 \mathrm{E}+06$
\end{tabular}

Fig. 4.5. Total volume of commercial LLW disposed through 1994.

ORNL DWG 95-8130

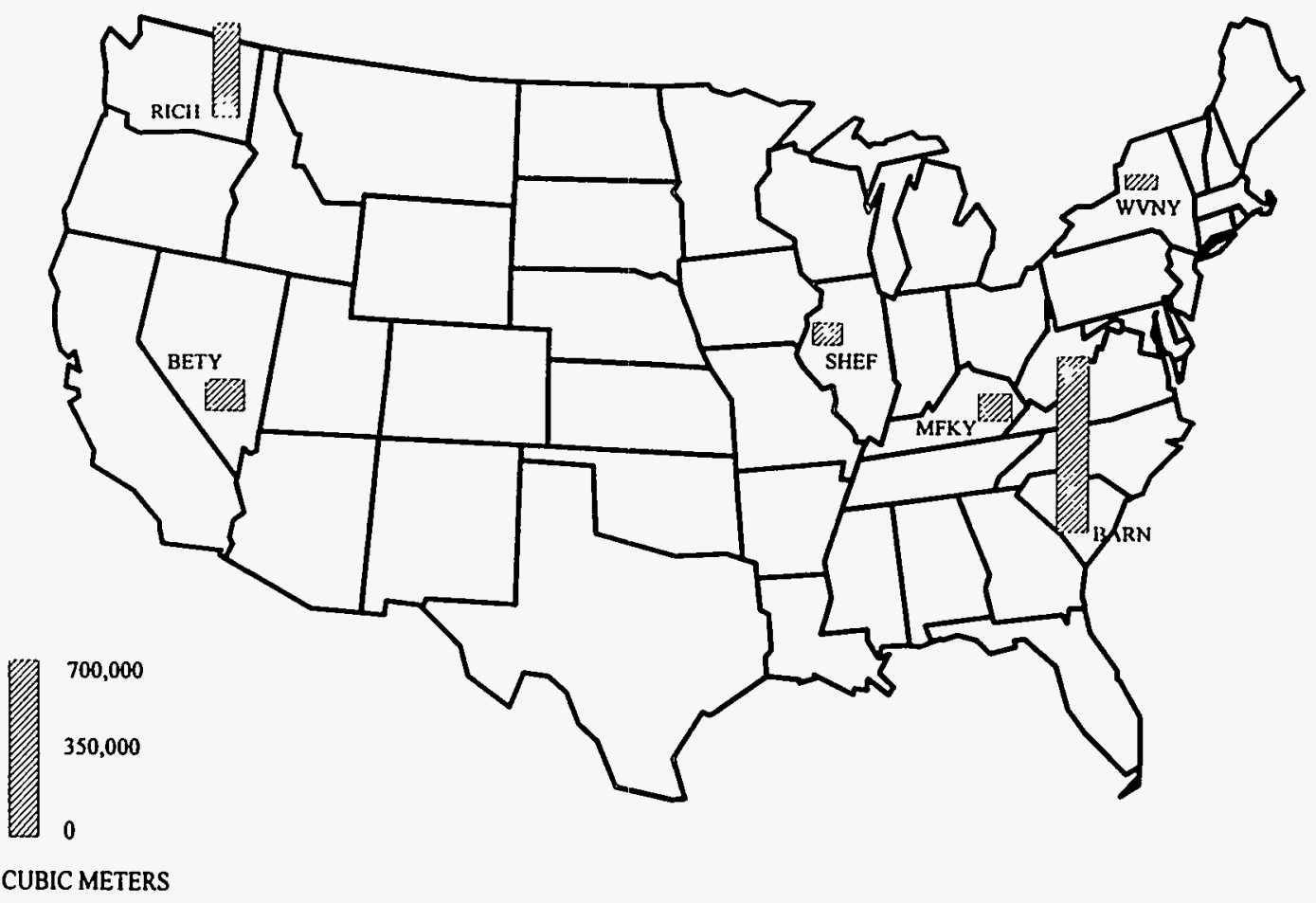

Fig. 4.6. Locations and total volumes of commercial LLW disposed through 1994. 
Table 4.1. Summary of characteristics for disposed LLW as of December 31, 1994

\begin{tabular}{|c|c|c|c|c|c|c|}
\hline \multirow{2}{*}{ Category } & \multicolumn{2}{|c|}{$\begin{array}{l}\text { Volume } \\
\left(10^{3} \mathrm{~m}^{3}\right)\end{array}$} & \multicolumn{2}{|c|}{$\begin{array}{l}\text { Radioactivity } \\
\qquad\left(10^{3} \mathrm{Ci}\right)\end{array}$} & \multicolumn{2}{|c|}{$\begin{array}{c}\text { Thermal power } \\
\text { (W) }\end{array}$} \\
\hline & Annual $^{\mathrm{a}}$ & Cumulative & Annual & Cumulative & Annual & Cumulative \\
\hline DOE sites & 52.2 & 2,963 & 621 & 12,858 & 3,463 & 21,534 \\
\hline Commercial sites & 24.3 & 1,519 & 751 & 5,841 & 3,799 & 22,746 \\
\hline Total & 76.5 & 4,482 & 1,372 & 18,699 & 7,262 & 44,280 \\
\hline
\end{tabular}

aAddition during 1994. 
Table 4.2. Historical and projected volume, radioactivity, and thermal power of disposed DOE LLWa

\begin{tabular}{|c|c|c|c|c|c|c|}
\hline \multirow{2}{*}{$\begin{array}{l}\text { End of } \\
\text { calendar } \\
\text { year }\end{array}$} & \multicolumn{2}{|c|}{$\begin{array}{l}\text { Volume } \\
\left(10^{3} \mathrm{~m}^{3}\right)\end{array}$} & \multicolumn{2}{|c|}{$\begin{array}{l}\text { Radioactivity } \\
\qquad\left(10^{3} \mathrm{Ci}\right)\end{array}$} & \multicolumn{2}{|c|}{$\begin{array}{l}\text { Thermal power } \\
\text { (W) }\end{array}$} \\
\hline & Annual & Cumulative & Annual & Cumulative $^{b}$ & Annual & Cumulative \\
\hline 1990 & 60.0 & 2,759 & 545 & 13,516 & 2,013 & 17,844 \\
\hline 1991 & 53.6 & 2,812 & 717 & 13,277 & 2,788 & 18,220 \\
\hline 1992 & 48.3 & 2,860 & 1,078 & 13,401 & 4,947 & 20,741 \\
\hline 1993 & 50.5 & 2,911 & 894 & 13,147 & 3,263 & 20,398 \\
\hline 1994 & 52.2 & 2,963 & 621 & 12,858 & 3,463 & 21,534 \\
\hline 1995 & 108.0 & 3,093 & 981 & 13,109 & 3,533 & 23,351 \\
\hline 1996 & 95.8 & 3,189 & 845 & 13,074 & 3,447 & 23,835 \\
\hline 1997 & 95.8 & 3,285 & 844 & 13,023 & 3,442 & 24,254 \\
\hline 1998 & 95.8 & 3,381 & 844 & 12,959 & 3,441 & 24,614 \\
\hline 1999 & 95.8 & 3,476 & 845 & 12,882 & 3,440 & 24,904 \\
\hline 2000 & 95.8 & 3,572 & 845 & 12,797 & 3,438 & 25,124 \\
\hline 2001 & 96.0 & 3,669 & 846 & 12,707 & 3,441 & 25,289 \\
\hline 2002 & 96.0 & 3,765 & 846 & 12,611 & 3,437 & 25,400 \\
\hline 2003 & 96.0 & 3,861 & 846 & 12,515 & 3,435 & 25,468 \\
\hline 2004 & 96.0 & 3,957 & 846 & 12,418 & 3,433 & 25,505 \\
\hline 2005 & 96.0 & 4,054 & 846 & 12,322 & 3,432 & 25,517 \\
\hline 2006 & 96.0 & 4,149 & 846 & 12,229 & 3,432 & 25,509 \\
\hline 2007 & 96.0 & 4,245 & 846 & 12,139 & 3,432 & 25,483 \\
\hline 2008 & 96.0 & 4,341 & 846 & 12,052 & 3,432 & 25,442 \\
\hline 2009 & 96.0 & 4,438 & 846 & 11,968 & 3,432 & 25,388 \\
\hline 2010 & 96.0 & 4,534 & 846 & 11,889 & 3,432 & 25,325 \\
\hline 2011 & 96.0 & 4,630 & 846 & 11,814 & 3,432 & 25,254 \\
\hline 2012 & 96.0 & 4,725 & 846 & 11,743 & 3,433 & 25,176 \\
\hline 2013 & 96.0 & 4,822 & 846 & 11,677 & 3,433 & 25,093 \\
\hline 2014 & 96.0 & 4,918 & 846 & 11,614 & 3,433 & 25,006 \\
\hline 2015 & 96.0 & 5,014 & 846 & 11,556 & 3,433 & 24,917 \\
\hline 2016 & 96.0 & 5,110 & 846 & 11,501 & 3,433 & 24,825 \\
\hline 2017 & 96.0 & 5,207 & 846 & 11,451 & 3,434 & 24,733 \\
\hline 2018 & 96.0 & 5,303 & 846 & 11,403 & 3,434 & 24,641 \\
\hline 2019 & 96.0 & 5,398 & 845 & 11,360 & 3,434 & 24,549 \\
\hline 2020 & 96.0 & 5,494 & 845 & 11,319 & 3,434 & 24,457 \\
\hline 2021 & 96.0 & 5,590 & 845 & 11,283 & 3,434 & 24,367 \\
\hline 2022 & 37.2 & 5,628 & 841 & 11,245 & 3,410 & 24,253 \\
\hline 2023 & 37.2 & 5,665 & 841 & 11,211 & 3,410 & 24,141 \\
\hline 2024 & 37.2 & 5,702 & 841 & 11,179 & 3,410 & 24,030 \\
\hline 2025 & 37.2 & 5,739 & 841 & 11,150 & 3,410 & 23,922 \\
\hline 2026 & 37.2 & 5,777 & 841 & 11,124 & 3,410 & 23,815 \\
\hline 2027 & 37.2 & 5,814 & 841 & 11,101 & 3,410 & 23,710 \\
\hline 2028 & 37.2 & 5,851 & 841 & 11,080 & 3,410 & 23,607 \\
\hline 2029 & 37.2 & 5,888 & 841 & 11,062 & 3,410 & 23,507 \\
\hline 2030 & 37.2 & 5,926 & 841 & 11,046 & 3,410 & 23,409 \\
\hline
\end{tabular}
at SRS).

a Summation of values in Tables 4.17 (buried DOE LLW, except SRS saltstone) and 4.18 (LLW saltstone

${ }^{b}$ The radioactivity added each year for each waste type is decayed as described in the footnotes of Tables 4.17 and 4.18 . 
Table 4.3. Historical and projected volume, radioactivity, and thermal power of commercial LLW shipped for disposal ${ }^{\mathrm{a}}$

\begin{tabular}{|c|c|c|c|c|c|c|}
\hline \multirow{2}{*}{$\begin{array}{l}\text { End of } \\
\text { calendar } \\
\text { year }\end{array}$} & \multicolumn{2}{|c|}{$\begin{array}{l}\text { Volume } \\
\left(10^{3} \mathrm{~m}^{3}\right)\end{array}$} & \multicolumn{2}{|c|}{$\begin{array}{l}\text { Radioactivity } \\
\qquad\left(10^{3} \mathrm{Ci}\right)\end{array}$} & \multicolumn{2}{|c|}{$\begin{array}{l}\text { Thermal power } \\
\text { (W) }\end{array}$} \\
\hline & Annual & Cumulative & Annual & Cumulative $^{\mathrm{b}}$ & Annual & Cumulative \\
\hline 1990 & 32.4 & 1,384 & 549 & 4,979 & 2,774 & 16,457 \\
\hline 1991 & 38.8 & 1,423 & 800 & 5,272 & 4,044 & 18,424 \\
\hline 1992 & 49.4 & 1,472 & 1,000 & 5,708 & 5,057 & 21,117 \\
\hline 1993 & 22.4 & 1,495 & 643 & 5,709 & 3,252 & 21,627 \\
\hline 1994 & 24.3 & 1,519 & 751 & 5.841 & 3,799 & 22,746 \\
\hline $1995^{\mathrm{c}}$ & 24.3 & 1,543 & 751 & 5,955 & 3,799 & 23,745 \\
\hline 1996 & 24.3 & 1,568 & 751 & 6,058 & 3,799 & 24,654 \\
\hline 1997 & 24.3 & 1,592 & 751 & 6,151 & 3,799 & 25,485 \\
\hline 1998 & 24.3 & 1,616 & 751 & 6,236 & 3,799 & 26,249 \\
\hline 1999 & 24.3 & 1,640 & 751 & 6,316 & 3,799 & 26,954 \\
\hline 2000 & 24.3 & 1,665 & 751 & 6,390 & 3,799 & 27,607 \\
\hline 2001 & 24.3 & 1,689 & 751 & 6,460 & 3,799 & 28,215 \\
\hline 2002 & 24.3 & 1,713 & 751 & 6,527 & 3,799 & 28,783 \\
\hline 2003 & 24.3 & 1,738 & 751 & 6,590 & 3,799 & 29,316 \\
\hline 2004 & 24.3 & 1,762 & 751 & 6,652 & 3,799 & 29,819 \\
\hline 2005 & 3.5 & 1,766 & 6 & 5,965 & 31 & 26,526 \\
\hline
\end{tabular}

aIncludes LLW disposed of at the following commercial sites: Beatty, Nevada; West Valley,

New York; Maxey Flats, Kentucky; Richland, Washington; Sheffield, Illinois; and Barnwell, South Carolina.

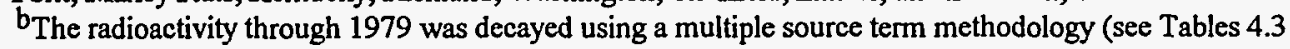
and 4.20-4.25 of Rev. 8 of this report for a description of this method). After 1979, the radioactivity is decayed from the year of addition using the representative compositions given in Table A.4 of Appendix A.

'Projections were made based on disposal operations at Richland, Washington, and Barnwell, South Carolina, as described in Sect. 4.3.5. Projections were made only through 2005 because of large uncertainties in commercial disposal facility operations. 
Table 4.4. Historical annual additions and total volume of $L L W$ disposed at DOE sites $^{a, b}$

\begin{tabular}{|c|c|c|c|c|c|c|c|c|c|c|c|}
\hline \multirow[b]{2}{*}{ Year } & \multicolumn{11}{|c|}{ Volume of waste disposed annually, $10^{3} \mathrm{~m}^{3}$} \\
\hline & FEMP & Hanford & INEL & LANL & NTS & ORNL & SRS & $Y-12^{c}$ & $\begin{array}{c}\text { All } \\
\text { others }\end{array}$ & $\begin{array}{c}\text { Total } \\
\text { annual } \\
\text { addition }\end{array}$ & $\begin{array}{c}\text { Total } \\
\text { cumulative } \\
\text { volume }\end{array}$ \\
\hline $1975^{\mathrm{e}}$ & 309.3 & 352.0 & 84.6 & 131.6 & 8.3 & 181.5 & 269.1 & 58.4 & 83.9 & $1,478.9$ & 1,479 \\
\hline 1976 & 14.4 & 4.1 & 6.2 & 8.8 & 0.0 & 3.8 & 8.1 & 2.7 & 0.9 & 49.0 & 1,528 \\
\hline 1977 & 2.8 & 10.7 & 6.6 & 3.6 & 0.5 & 2.4 & 14.7 & 1.5 & 1.1 & 43.9 & 1,572 \\
\hline 1978 & 1.9 & 9.8 & 5.9 & 7.5 & 10.0 & 2.0 & 15.5 & 1.4 & 3.2 & 57.2 & 1,629 \\
\hline 1979 & 1.6 & 17.5 & 5.3 & 4.9 & 15.8 & 2.1 & 18.2 & 1.1 & 1.1 & 67.6 & 1,697 \\
\hline 1980 & 1.3 & 10.4 & 5.1 & 4.8 & 13.3 & 2.0 & 19.6 & 1.4 & 0.7 & 58.6 & 1,755 \\
\hline 1981 & 1.5 & 12.8 & 3.1 & 5.5 & 21.1 & 1.4 & 20.1 & 1.2 & 1.6 & 68.3 & 1,824 \\
\hline 1982 & 2.8 & 11.7 & 3.2 & 4.5 & 57.0 & 1.3 & 22.4 & 2.2 & 2.0 & 107.1 & 1,931 \\
\hline 1983 & 3.4 & 17.9 & 5.5 & 3.2 & 12.1 & 1.8 & 26.7 & 3.4 & 1.7 & 75.7 & 2,006 \\
\hline 1984 & 3.5 & 18.8 & 3.9 & 5.4 & 36.0 & 2.2 & 26.1 & 7.2 & 10.6 & 113.7 & 2,120 \\
\hline 1985 & 0.7 & 17.0 & 3.1 & 6.7 & 41.7 & 2.2 & 30.5 & 18.7 & 2.1 & 122.7 & 2,243 \\
\hline 1986 & 0 & 21.1 & 3.4 & 4.5 & 27.9 & 1.8 & 30.1 & 15.0 & 1.0 & 104.8 & 2,348 \\
\hline 1987 & 0 & 20.3 & 3.0 & 3.7 & 81.1 & 0.5 & 28.2 & 16.2 & 1.0 & 154.0 & 2,501 \\
\hline 1988 & 0 & 16.8 & 2.0 & 4.3 & 39.1 & 0.6 & 30.2 & 10.6 & 1.0 & 104.6 & 2,606 \\
\hline 1989 & 0 & 13.7 & 1.3 & 6.4 & 35.0 & 1.3 & 26.8 & 5.7 & 2.3 & 92.5 & 2,699 \\
\hline 1990 & 0 & 13.4 & 1.8 & 4.5 & 9.1 & 0.3 & 26.6 & 4.4 & 0.0 & 60.1 & 2,759 \\
\hline 1991 & 0 & 10.6 & 1.3 & 5.8 & 11.6 & 0.2 & 23.8 & 0.3 & 0.0 & 53.6 & 2,812 \\
\hline 1992 & 0 & 10.9 & 0.8 & 2.3 & 20.1 & 1.1 & 13.0 & 0.0 & 0.0 & 48.2 & 2,860 \\
\hline 1993 & 0 & 12.1 & 0.9 & 2.7 & 18.6 & 0.8 & 15.3 & 0.1 & 0.0 & 50.5 & 2,911 \\
\hline 1994 & 0 & 13.7 & 1.9 & 1.9 & 22.9 & 0.4 & 11.4 & 0.0 & 0.0 & 52.2 & 2,963 \\
\hline Total & 343.2 & 615.3 & 148.9 & 222.6 & 481.3 & 209.7 & 676.4 & 151.3 & 114.2 & 2,963 & \\
\hline
\end{tabular}

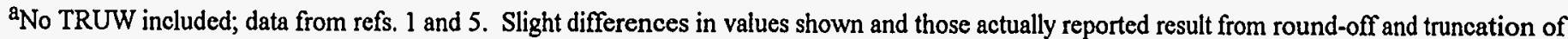
numbers.

${ }^{b}$ See Tables 4.8 and 4.13 for breakdown of disposed volumes by physical form.

cLand disposal of LLW at Y-12 was terminated July 1, 1991. A single exception was made in 1993 when waste was placed in the Bear Creek Burial Ground walk-in pits.

Includes contributions from Ames, BNL, K-25, LLNL, PAD, PANT, PORTS, and SNLANM.

EValues for 1975 are cumulative volumes to this date (ref. 3). 
Table 4.5. Description of physical forms used to characterize solid LLW from DOE activities ${ }^{\mathrm{a}}$
Physical form
Description

Debris waste

Debris-combustible

Debris-noncombustible and
compactible

Debris-noncombustible and combustible mixed

Activated metalequipment/ hardware

Contaminated metal/equipment/ hardware

Filter media

Biological waste and carcasses

Asbestos-contaminated waste

Soil/sediment/rubble

Solidified liquids/chelates/oils

Solidified sludge/resin
Waste that is at least $50 \%$ by volume solid materials that are intended for disposal and that are either manufactured objects, plant, or animal matter or that are natural geologic materials

Waste containing any material that will sustain combustion in air when exposed to an ignition source of $315^{\circ} \mathrm{C}\left(600^{\circ} \mathrm{F}\right)$ for a period of $5 \mathrm{~min}$

Waste composed of materials that are capable of undergoing volume reduction or of being compacted and that are not capable of being burned or rapidly oxidized

Waste composed of a mixture of materials that can and cannot sustain combustion

Waste materials that are contaminated with radionuclides generated through neutron activation of metal, equipment, and/or hardware. Metal waste includes slag generated from removal of dross from molten metal, activated aluminum ingots, and metal generated by the decontamination and decommissioning (D\&D) of site structures and equipment. Other examples are activated reactor hardware, glove box parts, dehumidifiers, freezers, engines, pumps, and compressors

Waste materials that are externally contaminated with radioactive material. Metal waste includes slag generated from removal of dross from molten metal, nonreusable drums, and metal generated by the D\&D of site structures and equipment

A category of solid waste that includes waste that is primarily (i.e., $\geq 50 \%$ by volume) filters constructed of one (or more than one) type of material. Examples of filter media waste are mechanical filters, cartridge-type filter media, and air filtration filters

Waste that consists of any plant or animal material such as shrubs, trees, animal carcasses, animal bedding and litter wastes, including any absorbent material and chemical agents such as lime, animal tissues in vials, and cultures

Waste that contains about $80 \%$ by volume, or more, asbestos or asbestos-based material. The asbestos contained in the waste may be friable or nonfriable asbestos. Friable asbestos is a material that, when dry, may be crumbled, pulverized, or reduced to powder by hand pressure. Nonfriable asbestos is that which is completely encapsulated or fixed in a matrix material that prevents the asbestos fibers from being released upon handling

A category of solid waste estimated to contain about $50 \%$ by volume soil and other ground media including, but not limited to, sand, silt, grass, rock, and gravel. The category also includes waste containing sediment which constitutes material in drainage and natural ponds and rubble which comprises waste material from building or demolition processes and concrete chunks and blocks from D\&D activities

Wastes that contain liquids, any polydentate ligand that will solubilize metal ions, and oils that have been immobilized or stabilized with agents, such as cement, polymers, and bitumen, and properly cured into a solidified form. The waste should not contain free liquids in excess of $1 \%$ of the volume of the waste when the waste is in a disposal container or $0.5 \%$ of the volume of the waste processed to a stable form. The waste should be in a form that meets stability requirements

Sludge is any solid, semisolid, or liquid waste generated from any of a number of airor water-treatment processes. Waste with a total suspended/settled solids (TSS) content of $>30 \%$ is a sludge. The waste may be a mixture of sludge and stabilization agents cured into a solidified form 


\section{Solidified sludge/resin (continued)}

Sources

Paint waste

Salt waste

Incinerator ash

Activated carbon (charcoal)

Other inorganic particulates

Other
Resin waste includes waste that is primarily (i.e., $\geq 50 \%$ by volume) unused or spent inorganic or organic-based resins used in wastewater treatment or other applications. An example of this waste is inorganic/organic ion-exchange resins

Waste material composed of encapsulated radioactive material whose main purpose is to generate known amounts of radiation. Sealed sources are defined as a category of special-form radioactive material in $10 \mathrm{CFR}$ Part 71.4. Special-form radioactive material is radioactive material which satisfies the following conditions: (1) it is either a single, solid piece or is contained in a sealed capsule that can be opened only by destroying the capsule; (2) the piece or capsule has at least one dimension not less than $5 \mathrm{~mm}$; and (3) it satisfies the test requirements of 10 CFR Part 71.75

Waste that is at least $50 \%$ by volume new, used, or removed paint. Examples of waste in this category are dried paint chips; containers filled with dried paint; and opened or unopened cans of heavy, viscous paint. This category does not include waste that is $50 \%$ by volume, or more, paint-related solids such as empty paint cans, depressurized spray paint cans, or other painting equipment (brushes, rollers, etc.)

Waste that is at least $50 \%$ by volume salts including interstitial liquids, if present. Chloride salts and sulfate salt wastes contain more than (i.e., $>1000 \mathrm{ppm}$ ) levels of chlorides or other halogens and of sulfur compounds, respectively. Nitrate salts are predominantly (i.e., $>50 \%$ by volume of salt) nitrates. Metal oxides/hydroxides waste is predominantly metal oxides or hydroxides

An inorganic particulate waste that is primarily (i.e., $\geq 50 \%$ by volume) bottom or fly ash resulting from incineration

An inorganic particulate waste that is primarily (i.e., $\geq 50 \%$ by volume) spent or unused activated carbon including any residual liquids. The activated carbon may be in powdered (typically 50 to $100 \mu \mathrm{m}$ ) or granular (typically 0.1 to $1 \mathrm{~mm}$ ) form

Waste that is at least $50 \%$ by volume inorganic particulates including any residual or absorbed liquids. Examples of other inorganic particulate waste are sandblasting media, inorganic particulate absorbent material (e.g., vermiculite), and ion-exchange media

This category includes solid wastes that are not characterized sufficiently or that do not meet the criteria for assignment into one of the above categories

a James A. Turi, DOE Office of Program Integration, Office of Waste Management, Environmental Management, correspondence to DOE field offices and DOE site points of contact, "Low-Level Waste Management Data Call for 1995, Attachment 1, dated May 24, 1995. 
Table 4.6. Actual 1994 generation and projected average annual generation of LLW at DOE sites

\begin{tabular}{|c|c|c|c|c|c|c|c|c|}
\hline \multirow{3}{*}{ Physical form } & \multirow{2}{*}{\multicolumn{2}{|c|}{1994 generation }} & \multicolumn{6}{|c|}{ Projections } \\
\hline & & & \multicolumn{2}{|c|}{1995} & \multicolumn{2}{|c|}{$1996-2000$} & \multicolumn{2}{|c|}{$2001-2030$} \\
\hline & $\begin{array}{c}\text { Volume } \\
\left(\mathrm{m}^{3}\right)\end{array}$ & $\begin{array}{l}\text { Activity } \\
\text { (Ci) }\end{array}$ & $\begin{array}{c}\text { Volume } \\
\left(\mathrm{m}^{3}\right)\end{array}$ & $\begin{array}{l}\text { Activity } \\
\text { (Ci) }\end{array}$ & $\begin{array}{l}\text { Volume } \\
\left(\mathrm{m}^{3} / \text { year }\right)\end{array}$ & $\begin{array}{l}\text { Activity } \\
\text { (Ci/year) }\end{array}$ & $\begin{array}{l}\text { Volume } \\
\left(\mathrm{m}^{3} / \text { year }\right)\end{array}$ & $\begin{array}{c}\text { Activity } \\
\text { (Ci/year) }\end{array}$ \\
\hline \multicolumn{9}{|l|}{ Debris } \\
\hline Combustible & 11,990 & 1,439 & 9,115 & 418 & 8,696 & 423 & 9,269 & 423 \\
\hline Noncombustible and compactible & 495 & 5 & 1,135 & 2,554 & 994 & 2,543 & 912 & 2,569 \\
\hline Noncombustible and combustible mixed & 4,709 & 17,416 & 3,551 & 27,263 & 3,712 & 27,275 & 3,032 & 27,259 \\
\hline Activated metal/equipment/hardware & 1,176 & 150,163 & 684 & 198,590 & 722 & 299,746 & 543 & 83,309 \\
\hline Contaminated metal/equipment/hardware & 6,774 & 435,858 & 2,054 & 85,257 & 1,843 & 61,688 & 1,349 & 53,668 \\
\hline Filter media (as-is, solidified, dewatered) & 291 & 4 & 146 & 5,057 & 126 & 3,419 & 124 & 3,019 \\
\hline Biological waste and carcasses (as-is, solidified) & 50 & 14 & 72 & 22 & 72 & 23 & 74 & 22 \\
\hline Asbestos-contaminated waste (as-is, solidified) & 392 & 0 & 144 & 600,001 & 147 & 600,005 & 110 & 600,000 \\
\hline Soil/sediment/rubble (as-is, solidified, dewatered) & 3,478 & 70 & 2,986 & 3 & 3,141 & 4 & 2,775 & 3 \\
\hline Solidified liquids/chelates/oils & 352 & 74,239 & 169 & 430,014 & 175 & 435,000 & 162 & 430,000 \\
\hline Solidified sludge/resin (dewatered, solidified) & 1,154 & 5 & 1,222 & 31 & 777 & 11 & 785 & 11 \\
\hline Sources (sealed sources, devices, and gauges) & $1^{\mathrm{a}}$ & $131^{\mathrm{a}}$ & $<1^{b}$ & $2,438^{b}$ & $<1^{\mathrm{c}}$ & $347^{\mathrm{c}}$ & $<1^{d}$ & $38^{d}$ \\
\hline Paint waste & 1 & 0 & 0 & 0 & 0 & 0 & 0 & 0 \\
\hline Salt waste & 138 & 4 & 1 & 0 & 1 & 0 & 1 & 0 \\
\hline Incinerator ash (solidified, as-is) & 40 & 0 & 2 & 0 & 616 & 29 & 902 & 43 \\
\hline Activated carbon (charcoal) & 45 & 0 & 177 & 2 & 177 & 2 & 175 & 2 \\
\hline Other inorganic particulates & 822 & 1,189 & 68 & 2 & 61 & 2 & 29 & 1 \\
\hline Other & 6,076 & 174,002 & 6,146 & 268,068 & 5,901 & 268,058 & 5,721 & 268,054 \\
\hline Total & 37,984 & 854,540 & 27,671 & $1,619,719$ & 27,161 & $1,698,575$ & 25,962 & $1,468,423$ \\
\hline
\end{tabular}

aEighteen sources with no volume estimate are included in radioactivity value for sources.

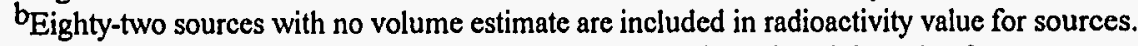

cForty-six sources with no volume estimate are included in radioactivity value for sources.

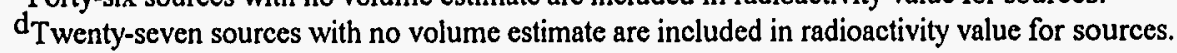


Table 4.7. Actual 1994 cumulative storage inventory and projected average annual storage additions of LLW at DOE sites

\begin{tabular}{|c|c|c|c|c|c|c|c|c|}
\hline \multirow{3}{*}{ Physical form } & & & \multicolumn{6}{|c|}{ Projected annual addition ${ }^{\mathrm{a}}$} \\
\hline & \multicolumn{2}{|c|}{ Cumulative ${ }^{\mathrm{b}}$} & \multicolumn{2}{|c|}{1995} & \multicolumn{2}{|c|}{$1996-2000$} & \multicolumn{2}{|c|}{$2001-2030$} \\
\hline & $\begin{array}{l}\text { Volume } \\
\left(\mathrm{m}^{3}\right)\end{array}$ & $\begin{array}{c}\text { Activity } \\
\text { (Ci) }\end{array}$ & $\begin{array}{l}\text { Volume } \\
\left(\mathrm{m}^{3}\right)\end{array}$ & $\begin{array}{c}\text { Activity } \\
\text { (Ci) }\end{array}$ & $\begin{array}{l}\text { Volume } \\
\left(\mathrm{m}^{3} / \text { year }\right)\end{array}$ & $\begin{array}{l}\text { Activity } \\
\text { (Ci/year) }\end{array}$ & $\begin{array}{l}\text { Volume } \\
\left(\mathrm{m}^{3} / \text { year }\right)\end{array}$ & $\begin{array}{c}\text { Activity } \\
\text { (Ci/year) }\end{array}$ \\
\hline \multicolumn{9}{|l|}{ Debris } \\
\hline Combustible & 5,699 & 43,015 & 127 & 22 & -3 & $-8,600$ & -46 & 0 \\
\hline Noncombustible and compactible & 265 & 71 & 156 & 16 & 140 & 21 & 106 & 48 \\
\hline Noncombustible and combustible mixed & 13,860 & 498,720 & 375 & 172 & -387 & $-77,016$ & 41 & 0 \\
\hline Activated metal/equipment/hardware & 1,554 & 280,028 & 188 & 45,030 & 418 & 45,100 & 204 & 0 \\
\hline Contaminated metal/equipment/hardware & 11,319 & $1,572,217$ & 243 & 31,773 & 142 & 5,210 & 300 & 83 \\
\hline Filter media (as-is, solidified, dewatered) & 692 & 13 & 8 & 3 & -12 & 6 & -1 & 19 \\
\hline Biological waste and carcasses (as-is, solidified) & 707 & 17,250 & 3 & 0 & 3 & 0 & 4 & 0 \\
\hline Asbestos-contaminated waste (as-is, solidified) & 646 & 0 & 19 & 1 & 7 & 5 & 2 & 0 \\
\hline Soil/sediment/rubble (as-is, solidified, dewatered) & 1,646 & 84 & 154 & -1 & 268 & 0 & 68 & 0 \\
\hline Solidified liquids/chelates/oils & 1,309 & 983,400 & -681 & $-969,990$ & -1 & 0 & 0 & 0 \\
\hline Solidified sludge/resin (dewatered, solidified) & 7,447 & 120 & -397 & -4 & 13 & 0 & -22 & 0 \\
\hline Sources (sealed sources, devices, and gauges) & $4^{\mathrm{c}}$ & $344,063^{c}$ & $\mathrm{o}^{\mathrm{d}}$ & $5^{d}$ & $<1^{\mathrm{e}}$ & $101^{\mathrm{e}}$ & $0^{f}$ & $<]^{f}$ \\
\hline Paint waste & 1 & 0 & 0 & 0 & 0 & 0 & 0 & 0 \\
\hline Salt waste & 161 & 4 & 0 & 0 & 0 & 0 & 0 & 0 \\
\hline Incinerator ash (solidified, as-is) & 169 & 0 & 2 & 0 & 2 & 0 & 3 & 0 \\
\hline Activated carbon (charcoal) & 82 & 0 & 2 & 0 & 3 & 0 & 1 & 0 \\
\hline Other inorganic particulates & 1,561 & 49 & 18 & 0 & 13 & -1 & 0 & 0 \\
\hline \multicolumn{9}{|l|}{ Other } \\
\hline EM-30 program waste & 11,981 & 186,397 & 49 & 0 & 54 & 0 & 120 & 0 \\
\hline EM-40 program waste & $67,000 \mathrm{~g}, \mathrm{~h}$ & $\mathrm{i}$ & $\mathrm{i}$ & $\mathrm{i}$ & $\mathrm{i}$ & $\mathrm{i}$ & $\mathrm{i}$ & $\mathrm{i}$ \\
\hline Total & $125,890^{\mathrm{j}}$ & $3,925,431$ & 268 & $-892,972$ & 661 & $-35,175$ & 782 & 151 \\
\hline
\end{tabular}

a Negative values indicate a decrease in stored volume or radioactivity.

$\mathrm{b}_{\text {Cumulative storage as of December } 31,1994 .}$

Included in radioactivity value for sources are 1,085 sources with no volume estimate.

Included in radioactivity value for sources are 58 sources with no volume estimate.

Included in radioactivity value for sources are 189 sources with no volume estimate.

Included in radioactivity value for sources are 20 sources with no volume estimate.

gFor EM-40 program LLW, data only available for cumulative storage volume for physical form "other." FUSRAP and UMTRAP sites are not included. figures.

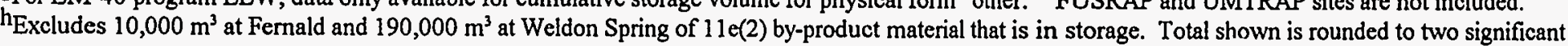

Not available.

jBased on numerical sum of separate site entries. 
Table 4.8. Actual disposal during 1994 and projected average annual disposal of LLW at DOE sites

\begin{tabular}{|c|c|c|c|c|c|c|c|c|}
\hline \multirow{3}{*}{ Physical form } & & & \multicolumn{6}{|c|}{ Projections $\mathrm{s}^{\mathrm{a}, \mathrm{b}}$} \\
\hline & \multicolumn{2}{|c|}{1994 disposal } & \multicolumn{2}{|c|}{1995} & \multicolumn{2}{|c|}{$1996-2000$} & \multicolumn{2}{|c|}{$2001-2030$} \\
\hline & $\begin{array}{c}\text { Volume } \\
\left(\mathrm{m}^{3}\right)\end{array}$ & $\begin{array}{l}\text { Activity } \\
\text { (Ci) }\end{array}$ & $\begin{array}{l}\text { Volume } \\
\left(\mathrm{m}^{3}\right)\end{array}$ & $\begin{array}{l}\text { Activity } \\
\text { (Ci) }\end{array}$ & $\begin{array}{l}\text { Volume } \\
\left(\mathrm{m}^{3} / \text { year }\right)\end{array}$ & $\begin{array}{c}\text { Activity } \\
\text { (Ci/year) }\end{array}$ & $\begin{array}{l}\text { Volume } \\
\left(\mathrm{m}^{3} / \text { year }\right)\end{array}$ & $\begin{array}{r}\text { Activity } \\
\text { (Ci/year) }\end{array}$ \\
\hline Debris-dry solids & 29,182 & 76,140 & 24,163 & 13,743 & 8,244 & 13,645 & 7,828 & 13,645 \\
\hline Activated metal/equipment/hardware & 1,115 & 47,513 & 180 & 3 & 180 & 3 & 180 & 3 \\
\hline Contaminated metal/equipment/hardware & 6,263 & 13,525 & 2,484 & 20,875 & 1,643 & 20,875 & 1,265 & 20,875 \\
\hline Filter media (as-is, solidified, dewatered) & 41 & 0 & 45 & 0 & 33 & 0 & 26 & 0 \\
\hline Biological waste and carcasses (as-is, solidified) & 45 & 0 & 68 & 9 & 68 & 9 & 68 & 8 \\
\hline Asbestos-contaminated waste (as-is, solidified) & 73 & 0 & 89 & 600,000 & 14 & 600,000 & 14 & 600,000 \\
\hline Soil/sediment/rubble (as-is, solidified, dewatered) & 2,628 & 4 & 54,409 & 2 & 17,286 & 2 & 3,691 & 2 \\
\hline Solidified liquids/chelates/oils & 6 & 0 & 0 & 0 & 0 & 0 & 0 & 0 \\
\hline Solidified sludge/resin (dewatered, solidified) & 147 & 1 & 733 & 0 & 571 & 0 & 563 & 0 \\
\hline Sources (sealed sources, devices, and gauges) & 1 & 122 & 0 & 0 & 0 & 0 & 0 & 0 \\
\hline Paint waste & 0 & 0 & 0 & 0 & 0 & 0 & 0 & 0 \\
\hline Salt waste & 16 & 0 & 0 & 0 & 0 & 0 & 0 & 0 \\
\hline Incinerator ash (solidified, as-is) & 0 & 0 & 0 & 0 & 168 & 321 & 168 & 321 \\
\hline Activated carbon (charcoal) & 5 & 0 & 172 & 2 & 172 & 2 & 172 & 2 \\
\hline Other inorganic particulates & 1,016 & 1,348 & 136 & 0 & 80 & 0 & 56 & 0 \\
\hline Other & $11,635^{\mathrm{c}}$ & $482,617^{\mathrm{c}}$ & 20,641 & 107,854 & 8,208 & 107,626 & 1,715 & 107,626 \\
\hline Total & 52,173 & 621,270 & 103,120 & 742,488 & 36,668 & 742,483 & 15,745 & 742,482 \\
\hline
\end{tabular}

a Radioactivity projections unavailable from Hanford. Volume and radioactivity projections unavailable from ORNL. Radioactivity and 2001-2030 volume projections unavailable from NTS.

bolume and radioactivity projections do not agree with those in Table 4.17. Projected values, shown in Table 4.17, were estimated for the unavailable data (from Hanford, ORNL, and NTS).

Includes $7,068 \mathrm{~m}^{3}$ and $468,597 \mathrm{Ci}$ of submarine reactor compartments at Hanford. 
Table 4.9. Breakdown by physical form of volumes of LLW generated during 1994 at DOE sites

\begin{tabular}{|c|c|c|c|c|c|c|c|c|c|c|}
\hline \multirow[b]{2}{*}{ Physical form } & \multicolumn{10}{|c|}{ Volume, $\mathrm{m}^{3}$} \\
\hline & Hanford & NEL & $K-25$ & LANL & NR sites $^{a}$ & ORNL & SRS & $\mathrm{Y}-12$ & $\begin{array}{c}\text { All } \\
\text { others }^{b}\end{array}$ & Total \\
\hline \multicolumn{11}{|l|}{ Debris } \\
\hline Combustible & 2,388 & 0 & 1,848 & 477 & 153 & 79 & 5,830 & 912 & 303 & 11,990 \\
\hline Noncombustible and compactible & 213 & 0 & 11 & 10 & 49 & 3 & 0 & 6 & 203 & 495 \\
\hline $\begin{array}{l}\text { Noncombustible and combustible } \\
\text { mixed }\end{array}$ & 83 & 330 & 793 & 417 & 334 & 1 & 422 & 1,013 & 1,315 & 4,709 \\
\hline Activated metal/equipment/hardware & 16 & 0 & 0 & 537 & 297 & 0 & 140 & 0 & 186 & 1,176 \\
\hline Contaminated metal/equipment/hardware & 1,055 & 33 & 4,186 & 1 & 203 & 9 & 177 & 804 & 307 & 6,774 \\
\hline Filter media (as-is, solidified, dewatered) & 0 & 0 & 17 & 41 & $<1$ & 0 & 0 & 61 & 171 & 291 \\
\hline $\begin{array}{l}\text { Biological waste and carcasses } \\
\text { (as-is, solidified) }\end{array}$ & 41 & 1 & 0 & 0 & 0 & 1 & 0 & 0 & 8 & 50 \\
\hline $\begin{array}{l}\text { Asbestos-contaminated waste } \\
\text { (as-is, solidified) }\end{array}$ & 8 & 0 & 143 & 51 & 36 & 3 & 12 & 122 & 17 & 392 \\
\hline $\begin{array}{l}\text { Soil/sediment/rubble (as-is, solidified, } \\
\text { dewatered) }\end{array}$ & 119 & 0 & 462 & 118 & 9 & 27 & 2,210 & 58 & 474 & 3,478 \\
\hline Solidified liquids/chelates/oils & $<1$ & 0 & 0 & 6 & 4 & 183 & 0 & 0 & 159 & 352 \\
\hline $\begin{array}{l}\text { Solidified sludge/resin (dewatered, } \\
\text { solidified) }\end{array}$ & 4 & 0 & 0 & 29 & 75 & 33 & 0 & 0 & 1,014 & 1,154 \\
\hline $\begin{array}{l}\text { Sources (sealed sources, devices, and } \\
\text { gauges) }\end{array}$ & 0 & 0 & 0 & 1 & $\ll 1$ & $\ll 1$ & 0 & 0 & 0 & 1 \\
\hline Paint waste & $<1$ & 0 & 0 & 0 & 0 & 0 & 0 & $<1$ & 1 & 1 \\
\hline Salt waste & 0 & 0 & 0 & $\ll 1$ & 0 & 136 & 0 & 1 & 1 & 138 \\
\hline Incinerator ash (solidified, as-is) & 0 & 0 & 38 & 0 & 0 & 0 & 0 & 0 & 2 & 40 \\
\hline Activated carbon (charcoal) & 0 & 0 & $<1$ & 0 & 3 & 35 & 0 & 1 & 5 & 45 \\
\hline Other inorganic particulates & 569 & 0 & 197 & 0 & 0 & 1 & 0 & 14 & 41 & 822 \\
\hline Other & 2 & 2,301 & 440 & 210 & 405 & 811 & 1,700 & 139 & 69 & 6,076 \\
\hline Total & 4,498 & 2,665 & 8,135 & 1,898 & 1,568 & 1,322 & 10,491 & 3,131 & 4,276 & 37,984 \\
\hline
\end{tabular}

aNaval Reactors (NR) sites include KAPL, BAPL, and NRF.

bIncludes Ames, ANL-E, BNL, FNAL, ITRI, KCP, LBL, LLNL, Mound, NTS, PANT, Pinellas, PPPL, RFETS, SLAC, SNL/CA, and WVDP. 
Table 4.10. Breakdown by physical form of radioactivity of LLW generated during 1994 at DOE sites

\begin{tabular}{|c|c|c|c|c|c|c|c|c|c|c|}
\hline \multirow[b]{2}{*}{ Physical form } & \multicolumn{10}{|c|}{ Radioactivity, $\mathrm{Ci}$} \\
\hline & Hanford & INEL & $\mathrm{K}-25$ & LANL & NR sites $^{a}$ & ORNL & SRS & $\mathrm{Y}-12$ & $\underset{\text { others }}{\text { All }}$ & Total \\
\hline \multicolumn{11}{|l|}{ Debris } \\
\hline Combustible & 8 & 0 & c & 1,140 & $<1$ & 0 & 266 & c & 25 & 1,439 \\
\hline Noncombustible and compactible & 1 & 0 & c & $<1$ & 1 & 0 & 0 & c & 2 & 5 \\
\hline $\begin{array}{l}\text { Noncombustible and combustible } \\
\text { mixed }\end{array}$ & $<1$ & 17,147 & c & 8 & 24 & 0 & 14 & c & 223 & 17,416 \\
\hline Activated metal/equipment/hardware & 4,840 & 0 & c & 8,420 & 136,891 & $\mathbf{0}$ & 3 & c & 9 & 150,163 \\
\hline Contaminated metal/equipment/hardware & 1 & 33,110 & c & $<1$ & 6 & 388,220 & 16 & c & 14,504 & 435,858 \\
\hline Filter media (as-is, solidified, dewatered) & 0 & 0 & c & $\ll<1$ & 1 & 0 & 0 & c & 3 & 4 \\
\hline $\begin{array}{l}\text { Biological waste and carcasses } \\
\text { (as-is, solidified) }\end{array}$ & 0 & 14 & c & 0 & 0 & 0 & 0 & c & 0 & 14 \\
\hline $\begin{array}{l}\text { Asbestos-contaminated waste } \\
\text { (as-is, solidified) }\end{array}$ & 0 & 0 & c & $<<1$ & $\ll 1$ & 0 & c & c & 0 & $\ll 1$ \\
\hline $\begin{array}{l}\text { Soil/sediment/rubble (as-is, solidified, } \\
\text { dewatered) }\end{array}$ & 3 & 0 & c & $<<1$ & 1 & 63 & c & c & 4 & 70 \\
\hline Solidified liquids/chelates/oils & 0 & 0 & $\mathbf{c}$ & $\ll<1$ & $<<1$ & 1,731 & 0 & c & 72,508 & 74,239 \\
\hline $\begin{array}{l}\text { Solidified sludge/resin (dewatered, } \\
\text { solidified) }\end{array}$ & 0 & 0 & c & 1 & 2 & $<1$ & 0 & c & 3 & 5 \\
\hline $\begin{array}{l}\text { Sources (sealed sources, devices, and } \\
\text { gauges) }\end{array}$ & 0 & 0 & c & 123 & $\ll<1$ & 0 & 0 & c & 8 & 131 \\
\hline Paint waste & 0 & 0 & c & 0 & 0 & 0 & 0 & c & 0 & 0 \\
\hline Salt waste & 0 & 0 & c & $\ll 1$ & 0 & 4 & 0 & c & 0 & 4 \\
\hline Incinerator ash (solidified, as-is) & 0 & 0 & c & 0 & 0 & 0 & 0 & c & 0 & 0 \\
\hline Activated carbon (charcoal) & 0 & 0 & c & 0 & $<<1$ & $<<1$ & 0 & c & 0 & 0 \\
\hline Other inorganic particulates & 1,189 & 0 & c & 0 & 0 & $\ll 1$ & 0 & c & 1 & 1,189 \\
\hline Other & 0 & 169,700 & $\mathrm{c}$ & $<<1$ & 11 & 3,620 & 671 & c & 0 & 174,002 \\
\hline Total & 6,042 & 219,971 & c & 9,692 & 136,937 & 393,638 & 970 & c & 87,290 & 854,540 \\
\hline
\end{tabular}

a Naval Reactors (NR) sites include KAPL, BAPL, and NRF.

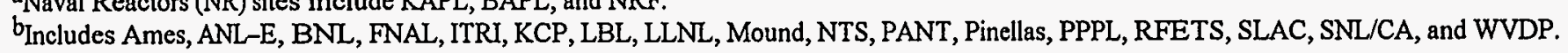

Unknown. 
Table 4.11. Breakdown by physical form of cumulative volumes of LLW stored at DOE sites ${ }^{\mathrm{a}}$

\begin{tabular}{|c|c|c|c|c|c|c|c|c|c|c|c|c|}
\hline \multirow[b]{2}{*}{ Physical form } & \multicolumn{12}{|c|}{ Volume, $\mathrm{m}^{3}$} \\
\hline & FEMP & NEL & $\mathrm{K}-25$ & Mound & ORNL & RFETS & SRS & WSSRAP & WVDP & $Y-12$ & $\begin{array}{c}\text { All } \\
\text { others } b\end{array}$ & Total \\
\hline \multicolumn{13}{|l|}{ Debris } \\
\hline Combustible & 0 & 0 & 2,293 & 235 & 110 & 2,350 & 344 & 0 & 0 & 343 & 24 & 5,699 \\
\hline Noncombustible and compactible & 0 & 0 & 12 & 0 & 0 & 54 & 0 & 0 & 0 & 0 & 199 & 265 \\
\hline $\begin{array}{l}\text { Noncombustible and combustible } \\
\text { mixed }\end{array}$ & 0 & 9,113 & 1,632 & 2,110 & 59 & 530 & 0 & 0 & 0 & 33 & 381 & 13,860 \\
\hline Activated metal/equipment/hardware & 0 & 0 & 0 & 0 & 0 & 0 & 1,240 & 0 & 0 & 0 & 314 & 1,554 \\
\hline Contaminated metal/equipment/hardware & 0 & 2,257 & 5,837 & 0 & 43 & 1,000 & 0 & 0 & 1,519 & 201 & 461 & 11,319 \\
\hline Filter media (as-is, solidified, dewatered) & 0 & 0 & 75 & 0 & 25 & 460 & 0 & 0 & 0 & $<1$ & 132 & 692 \\
\hline $\begin{array}{l}\text { Biological waste and carcasses } \\
\text { (as-is, solidified) }\end{array}$ & 0 & 685 & 8 & 0 & 2 & 0 & 0 & 0 & $<1$ & 0 & 13 & 707 \\
\hline $\begin{array}{l}\text { Asbestos-contaminated waste } \\
\text { (as-is, solidified) }\end{array}$ & 0 & 0 & 491 & 35 & 3 & 0 & 0 & 0 & 0 & 106 & 11 & 646 \\
\hline $\begin{array}{l}\text { Soil/sediment/rubble (as-is, solidified, } \\
\text { dewatered) }\end{array}$ & 0 & 0 & 1,188 & 0 & 96 & 100 & 0 & 0 & 0 & 44 & 218 & 1,646 \\
\hline Solidified liquids/chelates/oils & 0 & 0 & 0 & 680 & 628 & 0 & 0 & 0 & 0 & 0 & 1 & 1,309 \\
\hline $\begin{array}{l}\text { Solidified sludge/resin (dewatered, } \\
\text { solidified) }\end{array}$ & 0 & 0 & 1 & 900 & 37 & 720 & 0 & 0 & 5,763 & 0 & 27 & 7,447 \\
\hline $\begin{array}{l}\text { Sources (sealed sources, devices, and } \\
\text { gauges) }\end{array}$ & 0 & 0 & 0 & 0 & 1 & 1 & 0 & 0 & 0 & 0 & 2 & 4 \\
\hline Paint waste & 0 & 0 & 1 & 0 & $<1$ & 0 & 0 & 0 & 0 & $<1$ & 0 & 1 \\
\hline Salt waste & 0 & 0 & 14 & 0 & 145 & $<1$ & 0 & 0 & 0 & 1 & 1 & 161 \\
\hline Incinerator ash (solidified, as-is) & 0 & 0 & 158 & 0 & 0 & 1 & 0 & 0 & 0 & 0 & 11 & 169 \\
\hline Activated carbon (charcoal) & 0 & 0 & 49 & 0 & 31 & 0 & 0 & 0 & 0 & 1 & 0 & 82 \\
\hline Other inorganic particulates & 0 & 0 & 474 & 0 & 954 & 57 & 0 & 0 & 0 & 13 & 64 & 1,561 \\
\hline \multicolumn{13}{|l|}{ Other ${ }^{c}$} \\
\hline EM-30 program waste & 0 & 625 & 2,353 & 0 & 1,404 & 42 & 0 & 0 & 6,992 & 82 & 483 & 11,981 \\
\hline EM-40 program waste & $27,000^{d}$ & 1,400 & 0 & 4,900 & 0 & 0 & 71 & $0^{\mathrm{e}}$ & 0 & 0 & $33,416^{f}$ & $67,000 \mathrm{~g}$ \\
\hline Total & 27,000 & 14,080 & 14,586 & 8,860 & 3,538 & 5,315 & 1,655 & 0 & 14,274 & 825 & 35,757 & $125,890^{\mathrm{h}}$ \\
\hline
\end{tabular}

${ }^{\text {a C }}$ umulative storage as of December 31, 1994.

bIncludes Ames, ANL-E, BNL, ETEC, FNAL, Hanford, ITRI, KCP, LBL, LEHR, LLNL, NTS, PAD, PANT, Pinellas, PORTS, PPPL, RMI, SLAC, and SNL/CA.

cFor EM-40 program LLW, data only available for cumulative storage volume for physical form "other." FUSRAP and UMTRAP sites are not included.

${ }^{d}$ Excludes $10,000 \mathrm{~m}^{3}$ of $11 \mathrm{e}(2)$ by-product material that is in storage at Fernald (FEMP).

Excludes $190,000 \mathrm{~m}^{3}$ of $11 \mathrm{e}(2)$ by-product material that is in storage at Weldon Spring (WSSRAP)

Includes $25,000 \mathrm{~m}^{3}$ of LLW at Portsmouth (PORTS)

gRounded to two significant figures.

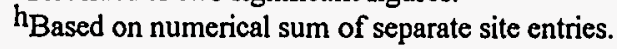


Table 4.12. Breakdown by physical form of total gross radioactivity of $L L W$ stored at DOE sites ${ }^{a}$

\begin{tabular}{|c|c|c|c|c|c|c|c|c|c|c|}
\hline \multirow[b]{2}{*}{ Physical form } & \multicolumn{10}{|c|}{ Radioactivity, Ci } \\
\hline & INEL & $\mathrm{K}-25$ & Mound & ORNL & RFETS & SRS & WVDP & $\mathrm{Y}-12$ & $\begin{array}{c}\text { All } \\
\text { others }\end{array}$ & Total \\
\hline \multicolumn{11}{|l|}{ Debris } \\
\hline Combustible & 0 & c & 43,000 & 7 & 3 & 5 & c & c & 0 & 43,015 \\
\hline Noncombustible and compactible & 0 & c & 0 & 0 & $\ll 1$ & 0 & c & c & 71 & 71 \\
\hline $\begin{array}{l}\text { Noncombustible and combustible } \\
\text { mixed }\end{array}$ & 103,488 & c & 384,000 & 36 & $\ll 1$ & 0 & c & c & 11,197 & 498,720 \\
\hline Activated metal/equipment/hardware & 0 & c & 0 & 0 & 0 & 280,000 & c & c & 28 & 280,028 \\
\hline Contaminated metal/equipment/hardware & $1,097,000$ & c & 0 & 440,463 & 3 & 0 & c & c & 34,751 & $1,572,217$ \\
\hline Filter media (as-is, solidified, dewatered) & 0 & c & 0 & $<<1$ & $<1$ & 0 & c & c & 13 & 13 \\
\hline $\begin{array}{l}\text { Biological waste and carcasses (as-is, } \\
\text { solidified) }\end{array}$ & 17,250 & c & 0 & 0 & 0 & 0 & c & c & 0 & 17,250 \\
\hline $\begin{array}{l}\text { Asbestos-contaminated waste (as-is, } \\
\text { solidified) }\end{array}$ & 0 & c & $<<1$ & 0 & 0 & 0 & c & c & 0 & 0 \\
\hline $\begin{array}{l}\text { Soil/sediment/rubble (as-is, solidified, } \\
\text { dewatered) }\end{array}$ & 0 & c & 0 & 81 & $<1$ & 0 & c & c & 2 & 84 \\
\hline Solidified liquids/chelates/oils & 0 & c & 979,000 & 4,393 & 0 & 0 & c & c & 7 & 983,400 \\
\hline $\begin{array}{l}\text { Solidified sludge/resin (dewatered, } \\
\text { solidified) }\end{array}$ & 0 & c & 3 & $<1$ & $\ll 1$ & 0 & c & c & 117 & 120 \\
\hline $\begin{array}{l}\text { Sources (sealed sources, devices, and } \\
\text { gauges) }\end{array}$ & 0 & c & 0 & 138,353 & c & 0 & c & c & 205,710 & 344,063 \\
\hline Paint waste & 0 & c & 0 & 0 & 0 & 0 & c & c & 0 & 0 \\
\hline Salt waste & 0 & c & 0 & 4 & 0 & 0 & c & c & 0 & 4 \\
\hline Incinerator ash (solidified, as-is) & 0 & c & 0 & 0 & c & 0 & c & c & 0 & 0 \\
\hline Activated carbon (charcoal) & 0 & c & 0 & $<<1$ & 0 & 0 & c & c & 0 & 0 \\
\hline Other inorganic particulates & 0 & c & 0 & 40 & 8 & 0 & c & c & 1 & 49 \\
\hline Other & 4,924 & c & 0 & 180,170 & $\ll 1$ & 0 & $\mathbf{c}$ & c & 1,303 & 186,397 \\
\hline Total & $1,222,662$ & c & $1,406,003$ & 763,547 & 14 & 280,005 & c & c & 253,200 & $3,925,431$ \\
\hline
\end{tabular}

aTotal gross radioactivity is the total radioactivity, without decay, in storage as of December 31, 1994.

bIncludes Ames, ANL-E, BNL, FNAL, Hanford, ITRI, KCP, LBL, LLNL, NTS, PANT, Pinellas, PPPL, SLAC, and SNL/CA.

CUnknown. 
Table 4.13. Breakdown by physical form of volumes of LLW disposed during 1994 at DOE sites

\begin{tabular}{|c|c|c|c|c|c|c|c|}
\hline \multirow{2}{*}{ Physical form } & \multicolumn{7}{|c|}{ Volume, $\mathrm{m}^{3}$} \\
\hline & Hanford & INEL & LANL & NTS & ORNL & SRS & Total \\
\hline Debris-dry solids & 3,863 & 333 & 901 & 18,100 & 5 & 5,980 & 29,182 \\
\hline Activated metal/equipment/hardware & 51 & 0 & 537 & 0 & 0 & 527 & 1,115 \\
\hline Contaminated metal/equipment/hardware & 1,250 & 54 & 1 & 4,770 & 8 & 180 & 6,263 \\
\hline Filter media (as-is, solidified, dewatered) & 0 & 0 & 41 & 0 & 0 & 0 & 41 \\
\hline $\begin{array}{l}\text { Biological waste and carcasses } \\
\text { (as-is, solidified) }\end{array}$ & 41 & 0 & 0 & 3 & 1 & 0 & 45 \\
\hline $\begin{array}{l}\text { Asbestos-contaminated waste } \\
\text { (as-is, solidified) }\end{array}$ & 10 & 0 & 51 & 0 & 0 & 12 & 73 \\
\hline $\begin{array}{l}\text { Soil/sediment/rubble (as-is, solidified, } \\
\text { dewatered) }\end{array}$ & 278 & 0 & 118 & 0 & 22 & 2,210 & 2,628 \\
\hline Solidified liquids/chelates/oils & $<1$ & 0 & 6 & 0 & 0 & 0 & 6 \\
\hline $\begin{array}{l}\text { Solidified sludge/resin (dewatered, } \\
\text { solidified) }\end{array}$ & 94 & 0 & 28 & 0 & 25 & 0 & 147 \\
\hline $\begin{array}{l}\text { Sources (sealed sources, devices, and } \\
\text { gauges) }\end{array}$ & 0 & 0 & 1 & 0 & 0 & 0 & 1 \\
\hline Paint waste & $<1$ & 0 & 0 & 0 & 0 & 0 & 0 \\
\hline Salt waste & 0 & 0 & $\ll 1$ & 0 & 16 & 0 & 16 \\
\hline Incinerator ash (solidified, as-is) & 0 & 0 & 0 & 0 & 0 & 0 & 0 \\
\hline Activated carbon (charcoal) & 0 & 0 & 0 & 0 & 5 & 0 & 5 \\
\hline Other inorganic particulates & 1,016 & 0 & 0 & 0 & 0 & 0 & 1,016 \\
\hline Other & $7,071^{\mathrm{a}}$ & 1,519 & 210 & 0 & 315 & 2,520 & 11,635 \\
\hline Total & 13,674 & 1,906 & 1,894 & 22,873 & 397 & 11,429 & 52,173 \\
\hline
\end{tabular}

ancludes $7,068 \mathrm{~m}^{3}$ of submarine reactor compartments. 
Table 4.14. Breakdown by physical form of radioactivity of LLW disposed during 1994 at DOE sites

\begin{tabular}{|c|c|c|c|c|c|c|c|}
\hline \multirow{2}{*}{ Physical form } & \multicolumn{7}{|c|}{ Radioactivity, Ci } \\
\hline & Hanford & NEL & LANL & NTS & ORNL & SRS & Total \\
\hline Debris-dry solids & 20 & 23,760 & 1,148 & 50,700 & 0 & 512 & 76,140 \\
\hline Activated metal/equipment/hardware & 39,067 & 0 & 8,429 & 0 & 0 & 17 & 47,513 \\
\hline Contaminated metal/equipment/hardware & 5 & 12,440 & $\ll 1$ & 1,060 & 2 & 18 & 13,525 \\
\hline Filter media (as-is, solidified, dewatered) & 0 & 0 & $\ll 1$ & 0 & 0 & 0 & 0 \\
\hline $\begin{array}{l}\text { Biological waste and carcasses } \\
\text { (as-is, solidified) }\end{array}$ & 0 & 0 & 0 & $\ll 1$ & 0 & 0 & 0 \\
\hline $\begin{array}{l}\text { Asbestos-contaminated waste } \\
\text { (as-is, solidified) }\end{array}$ & 0 & 0 & $\ll 1$ & 0 & 0 & a & 0 \\
\hline $\begin{array}{l}\text { Soil/sediment/rubble (as-is, solidified, } \\
\text { dewatered) }\end{array}$ & 3 & 0 & $\ll<1$ & 0 & $<1$ & $\mathbf{a}$ & 4 \\
\hline Solidified liquids/chelates/oils & 0 & 0 & $\ll 1$ & 0 & 0 & 0 & 0 \\
\hline $\begin{array}{l}\text { Solidified sludge/resin (dewatered, } \\
\text { solidified) }\end{array}$ & 0 & 0 & 1 & 0 & $<1$ & 0 & 1 \\
\hline $\begin{array}{l}\text { Sources (sealed sources, devices, and } \\
\text { gauges) }\end{array}$ & 0 & 0 & 122 & 0 & 0 & 0 & 122 \\
\hline Paint waste & 0 & 0 & 0 & 0 & 0 & 0 & 0 \\
\hline Salt waste & 0 & 0 & $\ll 1$ & 0 & $\ll 1$ & 0 & 0 \\
\hline Incinerator ash (solidified, as-is) & 0 & 0 & 0 & 0 & 0 & 0 & 0 \\
\hline Activated carbon (charcoal) & 0 & 0 & 0 & 0 & 0 & 0 & 0 \\
\hline Other inorganic particulates & 1,348 & 0 & 0 & 0 & 0 & 0 & 1,348 \\
\hline Other & $468,597^{b}$ & 13,320 & $<<1$ & 0 & 13 & 687 & 482,617 \\
\hline Total & 509,040 & 49,520 & 9,700 & 51,760 & 15 & 1,234 & 621,270 \\
\hline
\end{tabular}

aUnknown.

bIncludes $468,597 \mathrm{Ci}$ of submarine reactor compartments. 
Table 4.15. DOE LLW disposed by methods other than shallow-land burial ${ }^{\mathrm{a}}$

\begin{tabular}{|c|c|c|c|c|}
\hline Site & Location & $\begin{array}{l}\text { Site use } \\
\text { (year) }\end{array}$ & $\begin{array}{l}\text { Waste } \\
\text { containers } \\
\text { buried }^{b}\end{array}$ & $\begin{array}{l}\text { Undecayed } \\
\text { radioactive } \\
\text { content } \\
\text { (Ci) }\end{array}$ \\
\hline \multicolumn{5}{|c|}{ Atlantic Ocean } \\
\hline Atlantic & $\begin{array}{l}38^{\circ} 30 \mathrm{~N} \\
72^{\circ} 06^{\prime} \mathrm{W}\end{array}$ & $\begin{array}{l}\text { 1951-1956; } \\
\text { 1959-1962 }\end{array}$ & 14,300 & $74,400^{c}$ \\
\hline Atlantic & $\begin{array}{l}37^{\circ} 50 \mathrm{~N} \\
70^{\circ} 35^{\prime} \mathrm{W}\end{array}$ & $1957-1959$ & 14,500 & 2,100 \\
\hline Massachusetts Bay & $\begin{array}{l}42^{\circ} 25 \mathrm{~N} \\
70^{\circ} 35^{\prime} \mathrm{W}\end{array}$ & $1952-1959$ & 4,008 & 2,440 \\
\hline Cape Henry & $\begin{array}{l}36^{\circ} 56 \mathrm{~N} \\
74^{\circ} 23^{\circ} \mathrm{W}\end{array}$ & 1949-1967 & 843 & 87 \\
\hline Central Atlantic & $\begin{array}{l}36^{\circ} 20 \mathrm{~N} / \\
43^{\circ} 49 \mathrm{~N} \\
45^{\circ} 00^{\prime} \mathrm{W}\end{array}$ & $1959-1960$ & 432 & 480 \\
\hline Subtotal & & & 34,083 & 79,507 \\
\hline \multicolumn{5}{|c|}{ Pacific Ocean } \\
\hline $\begin{array}{l}\text { Farallon Islands } \\
\text { (Subsite A) }\end{array}$ & $\begin{array}{c}37^{\circ} 38 \mathrm{~N} \\
123^{\circ} 08^{\prime} \mathrm{W}\end{array}$ & $1951-1953$ & 3,500 & 1,100 \\
\hline $\begin{array}{l}\text { Farallon Isiands } \\
\text { (Subsite B) }\end{array}$ & $\begin{array}{r}37^{\circ} 37 \mathrm{~N} \\
123^{\circ} 17^{\prime} \mathrm{W}\end{array}$ & $\begin{array}{l}1946-1950 \\
1954-1956\end{array}$ & 44,000 & 13,400 \\
\hline Santa Cruz Basin & $\begin{array}{r}33^{\circ} 40 \mathrm{~N} \\
119^{\circ} 40^{\prime} \mathrm{W}\end{array}$ & $1946-1962$ & 3,114 & 108 \\
\hline Cape Scot & $\begin{array}{c}50^{\circ} 56^{\prime} \mathrm{N} \\
136^{\circ} 03^{\prime} \mathrm{W} \\
52^{\circ} 25 \mathrm{~N} \\
140^{\circ} 12^{\prime} \mathrm{W}\end{array}$ & $1958-1969$ & 360 & 124 \\
\hline San Diego & $\begin{array}{c}32^{\circ} 00 \mathrm{~N} \\
121^{\circ} 30^{\prime} \mathrm{W}\end{array}$ & $1959-1962$ & 4,415 & 34 \\
\hline Subtotal (oceans) & & & 55,389 & 14,766 \\
\hline Total & & & 89,472 & 94,273 \\
\hline \multicolumn{5}{|c|}{ Hydrofracture facility } \\
\hline ORNL & $\begin{array}{l}\text { Bedded Conasauga } \\
\text { shale underlying the } \\
\text { ORNL site }\end{array}$ & $\begin{array}{l}1959-1965 \\
1966-1980^{d} \\
1982^{\mathrm{e}} \\
1983^{\mathrm{e}}\end{array}$ & $\begin{array}{l}\text { Small experimental } \\
\text { amounts of grout } \\
8.0 \times 10^{3} \mathrm{~m}^{3} \\
3.8 \times 10^{3} \mathrm{~m}^{3} \\
5.5 \times 10^{3} \mathrm{~m}^{3}\end{array}$ & $\begin{array}{l}600,000 \\
200,000 \\
500,000\end{array}$ \\
\hline Total & & & $17.3 \times 10^{3} \mathrm{~m}^{3}$ & $1,300,000$ \\
\hline
\end{tabular}

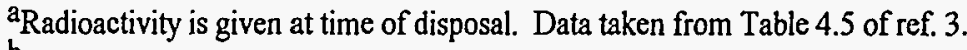

bstimated number of containers.

CIncludes approximately $33,000 \mathrm{Ci}$ of induced activity associated with the U.S.S. Seawolf reactor vessel.

$\mathrm{d}_{\text {Retired after } 18 \text { injections. }}$

ENew facility started up with four injections in 1982 and completed campaign with seven injections in 1983. 
Table 4.16. Significant revisions and changes in the current values for $L L W$ compared to the values in the previous year

\begin{tabular}{|c|c|c|c|c|}
\hline \multirow[t]{2}{*}{ Site } & \multirow{2}{*}{$\begin{array}{l}\text { DOE/RW-0006, } \\
\text { Rev. } 10(1994) \\
\text { Table No. }\end{array}$} & \multirow{2}{*}{$\begin{array}{l}\begin{array}{l}\text { DOE/RW-0006, } \\
\text { Rev. } 11 \text { (1995) }\end{array} \\
\text { Table No. }\end{array}$} & \multirow{2}{*}{$\begin{array}{l}\text { Significant revision } \\
\text { or net change }\end{array}$} & \multirow[t]{2}{*}{ Explanation } \\
\hline & & & & \\
\hline DOE & $\begin{array}{l}4.5,4.6,4.7,4.8 \\
4.9,4.10,4.11 \\
4.12, \text { and } 4.14\end{array}$ & $\mathrm{NA}^{\mathrm{a}}$ & $\begin{array}{l}\text { Tables were removed that } \\
\text { reported the breakdown of } \\
\text { LLW data by physical and } \\
\text { radionuclide characteristics }\end{array}$ & $\begin{array}{l}\text { Data required to complete } \\
\text { these tables were not collected }\end{array}$ \\
\hline DOE & $\mathrm{NA}^{\mathrm{a}}$ & $\begin{array}{l}4.5,4.6,4.7,4.8, \\
4.9,4.10,4.11 \\
4.12,4.13, \text { and } \\
4.14\end{array}$ & $\begin{array}{l}\text { Tables were added that } \\
\text { report the breakdown of } \\
\text { LLW by physical form }\end{array}$ & $\begin{array}{l}\text { Tables reflect new data } \\
\text { collection requirements }\end{array}$ \\
\hline ORNL & 4.9 & 4.11 & $\begin{array}{l}\text { Large decrease in total } \\
\text { stored inventory (from } \\
52,659 \mathrm{~m}^{3} \text { to } 3,539 \mathrm{~m}^{3} \text { ) }\end{array}$ & $\begin{array}{l}\text { Previous historical total stored } \\
\text { inventory was erroneously } \\
\text { reported in units of } \mathrm{m}^{3} \text { when } \\
\text { it was actually in units of } \mathrm{ft}^{3}\end{array}$ \\
\hline PORTS & 4.9 & 4.11 & $\begin{array}{l}\text { Large decrease in total } \\
\text { stored inventory (from } \\
49,494 \mathrm{~m}^{3} \text { to } 25,000 \mathrm{~m}^{3} \text { ) }\end{array}$ & $\begin{array}{l}\text { Major refinement by site in } \\
\text { stored LLW inventory. } \\
\text { Previous result was in error }\end{array}$ \\
\hline
\end{tabular}

a NA = not applicable. 
Table 4.17. Historical and projected volume, radioactivity, and thermal power characteristics of disposed DOE LLW, except SRS saltstone

\begin{tabular}{|c|c|c|c|c|c|c|}
\hline \multirow{2}{*}{$\begin{array}{c}\text { End of } \\
\text { calendar } \\
\text { year }\end{array}$} & \multicolumn{2}{|c|}{$\begin{array}{l}\text { Volume } a, b \\
\left(10^{3} \mathrm{~m}^{3}\right)\end{array}$} & \multicolumn{2}{|c|}{$\begin{array}{l}\text { Radioactivity } \mathrm{a}, \mathrm{b} \\
\qquad\left(10^{3} \mathrm{Ci}\right)\end{array}$} & \multicolumn{2}{|c|}{$\begin{array}{l}\text { Thermal power } \\
\text { (W) }\end{array}$} \\
\hline & Annual & Cumulative & Annual & Cumulative & Annual & Cumulative \\
\hline 1990 & 60.0 & 2,759 & 545 & 13,516 & 2,013 & 17,844 \\
\hline 1991 & 53.6 & 2,812 & 717 & 13,277 & 2,788 & 18,220 \\
\hline 1992 & 48.3 & 2,860 & 1,078 & 13,401 & 4,947 & 20,741 \\
\hline 1993 & 50.5 & 2,911 & 894 & 13,147 & 3,263 & 20,398 \\
\hline 1994 & 52.2 & 2,963 & 621 & 12,858 & 3,463 & 21,534 \\
\hline 1995 & 103.4 & 3,066 & 981 & 13,109 & 3,533 & 23,351 \\
\hline 1996 & 37.0 & 3,103 & 840 & 13,069 & 3,403 & 23,791 \\
\hline 1997 & 37.0 & 3,140 & 840 & 13,015 & 3,403 & 24,183 \\
\hline 1998 & 37.0 & 3,177 & 840 & 12,948 & 3,403 & 24,520 \\
\hline 1999 & 37.0 & 3,214 & 840 & 12,868 & 3,403 & 24,790 \\
\hline 2000 & 37.0 & 3,251 & 840 & 12,779 & 3,403 & 24,995 \\
\hline 2001 & 37.2 & 3,289 & 841 & 12,684 & 3,410 & 25,151 \\
\hline 2002 & 37.2 & 3,326 & 841 & 12,584 & 3,410 & 25,256 \\
\hline 2003 & 37.2 & 3,363 & 841 & 12,483 & 3,410 & 25,318 \\
\hline 2004 & 37.2 & 3,400 & 841 & 12,382 & 3,410 & 25,345 \\
\hline 2005 & 37.2 & 3,438 & 841 & 12,282 & 3,410 & 25,343 \\
\hline 2006 & 37.2 & 3,475 & 841 & 12,184 & 3,410 & 25,317 \\
\hline 2007 & 37.2 & 3,512 & 841 & 12,090 & 3,410 & 25,271 \\
\hline 2008 & 37.2 & 3,549 & 841 & 11,999 & 3,410 & 25,209 \\
\hline 2009 & 37.2 & 3,587 & 841 & 11,911 & 3,410 & 25,133 \\
\hline 2010 & 37.2 & 3,624 & 841 & 11,828 & 3,410 & 25,047 \\
\hline 2011 & 37.2 & 3,661 & 841 & 11,749 & 3,410 & 24,952 \\
\hline 2012 & 37.2 & 3,698 & 841 & 11,674 & 3,410 & 24,850 \\
\hline 2013 & 37.2 & 3,736 & 841 & 11,604 & 3,410 & 24,743 \\
\hline 2014 & 37.2 & 3,773 & 841 & 11,538 & 3,410 & 24,632 \\
\hline 2015 & 37.2 & 3,810 & 841 & 11,476 & 3,410 & 24,518 \\
\hline 2016 & 37.2 & 3,847 & 841 & 11,418 & 3,410 & 24,402 \\
\hline 2017 & 37.2 & 3,885 & 841 & 11,364 & 3,410 & 24,285 \\
\hline 2018 & 37.2 & 3,922 & 841 & 11,313 & 3,410 & 24,167 \\
\hline 2019 & 37.2 & 3,959 & 841 & 11,267 & 3,410 & 24,050 \\
\hline 2020 & 37.2 & 3,996 & 841 & 11,223 & 3,410 & 23,932 \\
\hline 2021 & 37.2 & 4,033 & 841 & 11,184 & 3,410 & 23,816 \\
\hline 2022 & 37.2 & 4,071 & 841 & 11,147 & 3,410 & 23,700 \\
\hline 2023 & 37.2 & 4,108 & 841 & 11,114 & 3,410 & 23,586 \\
\hline 2024 & 37.2 & 4,145 & 841 & 11,083 & 3,410 & 23,473 \\
\hline 2025 & 37.2 & 4,182 & 841 & 11,056 & 3,410 & 23,363 \\
\hline 2026 & 37.2 & 4,220 & 841 & 11,031 & 3,410 & 23,254 \\
\hline 2027 & 37.2 & 4,257 & 841 & 11,009 & 3,410 & 23,147 \\
\hline 2028 & 37.2 & 4,294 & 841 & 10,989 & 3,410 & 23,042 \\
\hline 2029 & 37.2 & 4,331 & 841 & 10,972 & 3,410 & 22,940 \\
\hline 2030 & 37.2 & 4,369 & 841 & 10,957 & 3,410 & 22,840 \\
\hline
\end{tabular}

aHistorical (beginning of operations through 1993) annual values of volume and radioactivity (by waste type) for each site are from ref. 5 . Similar values for 1994 are from ref. 1 . See Tables $4.4,4.8,4.13$, and 4.14 for more detail. For disposals prior to 1994, radioactivity (by waste type) is decayed from the year of addition using the representative compositions given in Table A.3 of Appendix A. Starting with 1994, compositions provided by the sites in ref. 1 are used to decay radioactivity.

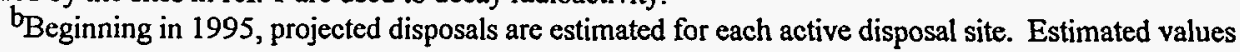
reported in this table may not agree with summary data reported in Table 4.8 because projections were reported as either unknown or unavailable by some sites. 
Table 4.18. Projected volume, radioactivity, and thermal power characteristics of DOE LLW saltstone at SRS ${ }^{a}$

\begin{tabular}{|c|c|c|c|c|c|c|}
\hline \multirow{2}{*}{$\begin{array}{l}\text { End of } \\
\text { calendar } \\
\text { year }\end{array}$} & \multicolumn{2}{|c|}{$\begin{array}{l}\text { Volume } \\
\left(10^{3} \mathrm{~m}^{3}\right)\end{array}$} & \multicolumn{2}{|c|}{$\begin{array}{l}\text { Radioactivity } \\
\qquad\left(10^{3} \mathrm{Ci}\right)\end{array}$} & \multicolumn{2}{|c|}{$\begin{array}{c}\text { Thermal power } \\
\text { (W) }\end{array}$} \\
\hline & Annual & Cumulative & Annual & Cumulative & Annual & Cumulative \\
\hline 1995 & 4.6 & $27.0^{\mathrm{b}}$ & 0.0 & 0.0 & 0.0 & 0.0 \\
\hline 1996 & 58.8 & 85.8 & 4.7 & 4.7 & 43.5 & 43.5 \\
\hline 1997 & 58.8 & 144.7 & 4.3 & 7.8 & 38.8 & 70.8 \\
\hline 1998 & 58.8 & 203.5 & 4.4 & 10.9 & 37.8 & 93.9 \\
\hline 1999 & 58.8 & 262.4 & 4.6 & 14.4 & 36.8 & 114.2 \\
\hline 2000 & 58.8 & 321.2 & 4.9 & 18.3 & 34.5 & 129.3 \\
\hline 2001 & 58.8 & 380.1 & 5.1 & 22.6 & 30.9 & 138.2 \\
\hline 2002 & 58.8 & 438.9 & 5.1 & 27.0 & 27.3 & 143.6 \\
\hline 2003 & 58.8 & 497.8 & 5.2 & 31.5 & 24.6 & 149.9 \\
\hline 2004 & 58.8 & 556.6 & 5.2 & 36.0 & 22.9 & 159.9 \\
\hline 2005 & 58.8 & 615.5 & 5.1 & 40.4 & 22.1 & 174.1 \\
\hline 2006 & 58.8 & 674.3 & 5.1 & 44.8 & 21.7 & 191.6 \\
\hline 2007 & 58.8 & 733.2 & 5.0 & 49.1 & 21.7 & 211.5 \\
\hline 2008 & 58.8 & 792.0 & 5.0 & 53.2 & 21.8 & 232.8 \\
\hline 2009 & 58.8 & 850.8 & 4.9 & 57.3 & 21.9 & 255.2 \\
\hline 2010 & 58.8 & 909.7 & 4.9 & 61.3 & 22.1 & 278.2 \\
\hline 2011 & 58.8 & 968.5 & 4.8 & 65.1 & 22.3 & 301.7 \\
\hline 2012 & 58.8 & $1,027.4$ & 4.8 & 68.9 & 22.5 & 325.5 \\
\hline 2013 & 58.8 & $1,086.2$ & 4.7 & 72.6 & 22.7 & 349.5 \\
\hline 2014 & 58.8 & $1,145.1$ & 4.7 & 76.2 & 22.9 & 373.9 \\
\hline 2015 & 58.8 & $1,203.9$ & 4.6 & 79.7 & 23.1 & 398.5 \\
\hline 2016 & 58.8 & $1,262.8$ & 4.6 & 83.2 & 23.3 & 423.3 \\
\hline 2017 & 58.8 & $1,321.6$ & 4.5 & 86.5 & 23.5 & 448.4 \\
\hline 2018 & 58.8 & $1,380.5$ & 4.5 & 89.8 & 23.6 & 473.7 \\
\hline 2019 & 58.8 & $1,439.3$ & 4.4 & 93.1 & 23.8 & 499.2 \\
\hline 2020 & 58.8 & $1,498.2$ & 4.4 & 96.2 & 24.0 & 525.1 \\
\hline 2021 & 58.8 & $1,557.0$ & 4.4 & 99.3 & 24.2 & 551.2 \\
\hline 2022 & 0.0 & $1,557.0$ & 0.0 & 98.1 & 0.0 & 553.1 \\
\hline 2023 & 0.0 & $1,557.0$ & 0.0 & 96.8 & 0.0 & 555.1 \\
\hline 2024 & 0.0 & $1,557.0$ & 0.0 & 95.6 & 0.0 & 557.0 \\
\hline 2025 & 0.0 & $1,557.0$ & 0.0 & 94.4 & 0.0 & 559.0 \\
\hline 2026 & 0.0 & $1,557.0$ & 0.0 & 93.2 & 0.0 & 560.9 \\
\hline 2027 & 0.0 & $1,557.0$ & 0.0 & 92.1 & 0.0 & 562.9 \\
\hline 2028 & 0.0 & $1,557.0$ & 0.0 & 91.0 & 0.0 & 564.8 \\
\hline 2029 & 0.0 & $1,557.0$ & 0.0 & 89.9 & 0.0 & 566.8 \\
\hline 2030 & 0.0 & $1,557.0$ & 0.0 & 88.9 & 0.0 & 568.8 \\
\hline
\end{tabular}

aSource: ref. 1 of Chapter 2 .

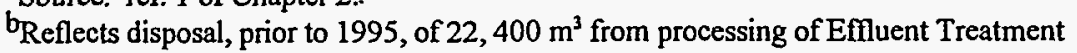
Facility concentrate. This volume was not reported in previous IDB reports. 
Table 4.19. Historical annual additions and total volume of $L L W$ at commercial disposal sites ${ }^{a}$

\begin{tabular}{|c|c|c|c|c|c|c|c|c|}
\hline \multirow{2}{*}{ Year } & \multicolumn{8}{|c|}{ Volume, $\mathrm{m}^{3}$} \\
\hline & Beatty ${ }^{b}$ & $\begin{array}{l}\text { West } \\
\text { Valleyc }\end{array}$ & $\begin{array}{l}\text { Maxey } \\
\text { Flats }^{2}\end{array}$ & Richland & Sheffield ${ }^{\mathrm{e}}$ & Barnwell & $\begin{array}{c}\text { Annual } \\
\text { total }\end{array}$ & $\begin{array}{c}\text { Cumulative } \\
\text { total }\end{array}$ \\
\hline 1962 & 1,861 & & & & & & 1,861 & 1,861 \\
\hline 1963 & 3,512 & 127 & 2,206 & & & & 5,845 & 7,706 \\
\hline 1964 & 2,836 & 5,940 & 3,872 & & & & 12,648 & 20,354 \\
\hline 1965 & 1,988 & 5,192 & 5,753 & 668 & & & 13,601 & 33,955 \\
\hline 1966 & 3,533 & 3,951 & 5,557 & 2,402 & & & 15,443 & 49,398 \\
\hline 1967 & 3,206 & 7,475 & 7,820 & 773 & 2,527 & & 21,801 & 71,199 \\
\hline 1968 & 3,576 & 3,490 & 8,178 & 1,359 & 2,713 & & 19,316 & 90,515 \\
\hline 1969 & 4,526 & 4,099 & 10,354 & 438 & 2,012 & & 21,429 & 111,944 \\
\hline 1970 & 5,152 & 4,906 & 12,521 & 423 & 2,825 & & 25,827 & 137,771 \\
\hline 1971 & 4,916 & 7,002 & 13,173 & 584 & 4,430 & 1,171 & 31,276 & 169,047 \\
\hline 1972 & 4,301 & 9,045 & 15,578 & 654 & 5,956 & 3,757 & 39,291 & 208,338 \\
\hline 1973 & 4,076 & 7,535 & 10,074 & 1,033 & 8,524 & 15,839 & 47,081 & 255,419 \\
\hline 1974 & 4,103 & 8,866 & 8,898 & 1,411 & 12,373 & 18,244 & 53,895 & 309,314 \\
\hline 1975 & 4,943 & 2,243 & 17,098 & 1,500 & 14,116 & 18,072 & 57,972 & 367,286 \\
\hline 1976 & 3,864 & 427 & 13,775 & 2,867 & 13,480 & 40,227 & 74,640 & 441,926 \\
\hline 1977 & 4,742 & 351 & 423 & 2,718 & 17,643 & 45,663 & 71,540 & 513,466 \\
\hline 1978 & 8,874 & 144 & & 7,422 & 1,735 & 61,554 & 79,729 & 593,195 \\
\hline 1979 & 6,491 & 138 & & 12,185 & & 63,861 & 82,675 & 675,870 \\
\hline 1980 & 12,717 & 141 & & 24,819 & & $54,723^{\mathrm{f}}$ & 92,400 & 768,270 \\
\hline 1981 & 3,351 & 216 & & 40,732 & & $39,427^{\mathrm{f}}$ & 83,726 & 851,996 \\
\hline 1982 & 1,505 & 632 & & 39,606 & & 34,779 & 76,522 & 928,518 \\
\hline 1983 & 1,111 & 1,284 & & 40,458 & & 35,132 & 77,985 & $1,006,503$ \\
\hline 1984 & 2,067 & 966 & & 38,481 & & 34,879 & 76,393 & $1,082,896$ \\
\hline 1985 & 1,388 & 809 & & 40,135 & & 34,389 & 76,721 & $1,159,617$ \\
\hline 1986 & 2,668 & 2,095 & & 18,833 & & 29,612 & 53,208 & $1,212,825$ \\
\hline 1987 & 9,414 & & & 15,765 & & 27,060 & 52,239 & $1,265,064$ \\
\hline 1988 & 2,645 & & & 11,430 & & 26,391 & 40,466 & $1,305,530$ \\
\hline 1989 & 3,291 & & & 11,562 & & 31,242 & 46,095 & $1,351,625$ \\
\hline 1990 & 1,684 & & & 8,362 & & 22,315 & 32,361 & $1,383,986$ \\
\hline 1991 & 4,539 & & & 11,872 & & 22,368 & 38,779 & $1,422,765$ \\
\hline 1992 & 14,575 & & & 11,271 & & 23,518 & 49,364 & $1,472,129$ \\
\hline 1993 & & & & 5,288 & & 17,145 & 22,433 & $1,494,562$ \\
\hline 1994 & & & & 3,533 & & 20,783 & 24,316 & $1,518,878$ \\
\hline Total & 137,455 & 77,074 & 135,280 & 358,584 & 88,334 & 722,151 & & $1,518,878$ \\
\hline
\end{tabular}

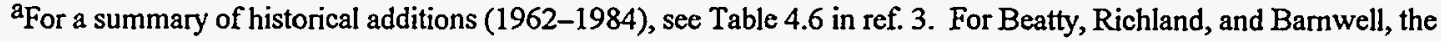
additions for 1985-1993 are from Table 4.18 in ref. 5 . Information for 1994 is taken from ref. 7.

beatty ceased accepting LLW Dec. 31, 1992.

West Valley includes a commercial state-licensed facility which opened Nov. 18, 1963, and closed Mar. 11, 1975, and an NRC-licensed facility (for on-site fuel reprocessing wastes) which opened in 1966 and continued to receive only onsite-generated LLW associated with water treatment and site cleanup until late 1986 . This license is in abeyance. Disposal operations at the West Valley Demonstration Project (WVDP) have been suspended pending the preparation of an EIS report for the West Valley site closure. The WVDP began in 1982. The LLW volumes reported for 1982 through 1986 are for the WVDP only and are taken from ref. 5. Since the beginning of 1987, LLW generated at the WVDP is stored on-site in engineered facilities pending final disposal (ref. 5).

${ }^{d}$ Closed Dec. 27, 1977. Small perturbations in waste volumes have occurred during site cleanup operations but are not included here since they are inconsequential.

elosed Apr. 8, 1978. No additional operations have taken place at the site.

These values exclude almost $19,000 \mathrm{~m}^{3}$ (approximately 14,506 in 1980 and approximately 4,279 in 1981) of very low-level-activity settling pond sludge that was not counted against the annual quota. 
Table 4.20. Historical annual additions and total undecayed radioactivity of $L L W$ at commercial disposal sites ${ }^{a}$

\begin{tabular}{|c|c|c|c|c|c|c|c|c|}
\hline \multirow{2}{*}{ Year } & \multicolumn{8}{|c|}{ Radioactivity, $\mathrm{Ci}$} \\
\hline & Beattyb & $\begin{array}{l}\text { West } \\
\text { Valleyc }\end{array}$ & $\begin{array}{l}\text { Maxey } \\
\text { Flats }^{\mathrm{d}}\end{array}$ & Richland & Sheffield ${ }^{\mathrm{e}}$ & Bamwell & $\begin{array}{c}\text { Annual } \\
\text { total }\end{array}$ & $\begin{array}{l}\text { Cumulative } \\
\text { total }\end{array}$ \\
\hline 1962 & $\mathbf{f}$ & & & & & & $\mathbf{f}$ & $\mathrm{f}$ \\
\hline 1963 & 5,690 & 100 & 22,556 & & & & 28,346 & 28,346 \\
\hline 1964 & 6,477 & 10,400 & 147,218 & & & & 164,095 & 192,441 \\
\hline 1965 & 6,377 & 22,600 & 63,828 & 144 & & & 92,949 & 285,390 \\
\hline 1966 & 11,974 & 35,400 & 52,737 & 1,606 & & & 101,717 & 387,107 \\
\hline 1967 & 10,894 & 123,100 & 23,273 & 5,378 & 3,850 & & 166,495 & 553,602 \\
\hline 1968 & 6,808 & 10,600 & 45,577 & 64,432 & 2,381 & & 129,798 & 683,400 \\
\hline 1969 & 9,761 & 36,000 & 31,028 & 55,964 & 2,192 & & 134,945 & 818,345 \\
\hline 1970 & 12,304 & 91,900 & 46,969 & 52,820 & 5,427 & & 209,420 & $1,027,765$ \\
\hline 1971 & 4,316 & 436,700 & 720,146 & 23,916 & 7,895 & 4,151 & $1,197,124$ & $2,224,889$ \\
\hline 1972 & 5,228 & 131,300 & 217,351 & 31,809 & 4,857 & 13,575 & 404,120 & $2,629,009$ \\
\hline 1973 & 5,704 & 346,000 & 118,359 & 57,037 & 2,834 & 48,212 & 578,146 & $3,207,155$ \\
\hline 1974 & 23,904 & 6,600 & 143,656 & 12,773 & 3,229 & 13,557 & 203,719 & $3,410,874$ \\
\hline 1975 & 18,388 & 11,600 & 289,570 & 113,341 & 6,103 & 17,428 & 456,430 & $3,867,304$ \\
\hline 1976 & 4,493 & 1,200 & 211,359 & 104,306 & 7,744 & 90,205 & 419,307 & $4,286,611$ \\
\hline 1977 & 23,811 & 900 & 267,063 & 7,465 & 11,147 & 390,121 & 700,507 & $4,987,118$ \\
\hline 1978 & 5,685 & 700 & & 235,548 & 2,547 & 652,061 & 896,541 & $5,883,659$ \\
\hline 1979 & 8,897 & 400 & & 164,787 & & 314,938 & 489,022 & $6,372,681$ \\
\hline 1980 & 148,312 & 300 & & 41,031 & & 143,502 & 333,145 & $6,705,826$ \\
\hline 1981 & 52,214 & 229 & & 43,905 & & 183,744 & 280,092 & $6,985,918$ \\
\hline 1982 & 80,929 & 293 & & 59,007 & & 273,962 & 414,191 & $7,400,109$ \\
\hline 1983 & 1,356 & 255 & & 120,534 & & 383,450 & 505,595 & $7,905,704$ \\
\hline 1984 & 544 & 25 & & 215,286 & & 385,079 & 600,934 & $8,506,638$ \\
\hline 1985 & 453 & 39 & & 287,849 & & 460,571 & 748,912 & $9,255,550$ \\
\hline 1986 & 672 & 13 & & 115,591 & & 116,108 & 232,384 & $9,487,934$ \\
\hline 1987 & 3,353 & 0 & & 42,734 & & 211,026 & 257,113 & $9,745,047$ \\
\hline 1988 & 8,690 & 0 & & 32,067 & & 218,901 & 259,658 & $10,004,705$ \\
\hline 1989 & 42,678 & 0 & & 99,056 & & 725,164 & 866,898 & $10,871,603$ \\
\hline 1990 & 11,323 & 0 & & 92,985 & & 444,277 & 548,585 & $11,420,188$ \\
\hline 1991 & 29,679 & 0 & & 158,784 & & 611,348 & 799,811 & $12,219,999$ \\
\hline 1992 & 90,206 & 0 & & 93,923 & & 815,974 & $1,000,103$ & $13,220,102$ \\
\hline 1993 & & & & 31,422 & & 611,785 & 643,207 & $13,863,309$ \\
\hline 1994 & & & & 6,078 & & 745,301 & 751,379 & $14,614,688$ \\
\hline Total & 641,120 & $1,266,654$ & $2,400,690$ & $2,371,578$ & 60,206 & $7,874,440$ & & $14,614,688$ \\
\hline
\end{tabular}

a For a summary of historical additions (1962-1984), see Table 4.6 in ref. 3. For Beatty, Richland, and Barnwell, the additions for 1985-1993 are from Table 4.19 in ref. 5. Information for 1994 is taken from ref. 7.

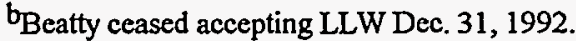

CWest Valley includes a commercial state-licensed facility which opened Nov. 18, 1963, and closed Mar. 11, 1975, and an NRC-licensed facility (for on-site fuel reprocessing wastes) which opened in 1966 and continued to receive only on-sitegenerated LLW associated with water treatment and site cleanup until late 1986. This license is in abeyance. Disposal operations at the West Valley Demonstration Project (WVDP) have been suspended pending the preparation of an EIS report for the West Valley site closure. The WVDP began in 1982. The LLW radioactivity values reported for 1982 through 1986 are for the WVDP only and are taken from ref. 5 . Since the beginning of 1987, LLW generated at the WVDP is stored on-site in engineered facilities pending final disposal (ref. 5).

dClosed Dec. 27, 1977.

Closed Apr. 8, 1978.

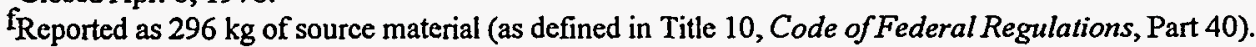


Table 4.21. Distribution of total volume and radioactivity, by state, of LLW shipped to commercial disposal sites in $1994^{\mathrm{a}}$

\begin{tabular}{|c|c|c|c|c|c|}
\hline State & $\begin{array}{l}\text { Volume } \\
\left(\mathrm{m}^{3}\right)\end{array}$ & $\begin{array}{l}\text { Radioactivity } \\
\text { (Ci) }\end{array}$ & State & $\begin{array}{l}\text { Volume } \\
\left(\mathrm{m}^{3}\right)\end{array}$ & $\begin{array}{l}\text { Radioactivity } \\
\text { (Ci) }\end{array}$ \\
\hline Alabama & 401.92 & $9,034.06$ & Nebraska & 74.60 & 991.64 \\
\hline Alaska & 1.25 & 4.69 & Nevada & 7.94 & 4.39 \\
\hline Arizona & 235.04 & 452.98 & New Hampshire & 0.00 & 0.00 \\
\hline Arkansas & 177.20 & 473.05 & New Jersey & 637.17 & $5,726.92$ \\
\hline California & $1,343.88$ & $23,132.46$ & New Mexico & 11.39 & 5.51 \\
\hline Colorado & 498.06 & $4,520.60$ & New York & $2,338.54$ & $175,843.05$ \\
\hline Connecticut & 360.67 & 888.53 & North Carolina & 869.55 & $68,213.47$ \\
\hline Delaware & 10.61 & 69.29 & North Dakota & 1.72 & 6.48 \\
\hline District of Columbia & 15.91 & 345.23 & Ohio & 330.40 & 582.68 \\
\hline Florida & 339.14 & $3,279.99$ & Oklahoma & 13.21 & 63.70 \\
\hline Georgia & 550.15 & $167,310.33$ & Oregon & 911.23 & 48.34 \\
\hline Hawaii & 53.82 & 50.07 & Pennsylvania & $1,457.36$ & $93,729.60$ \\
\hline Idaho & 0.75 & 93.76 & Puerto Rico & 0.00 & 0.00 \\
\hline Illinois & $1,733.70$ & $32,136.47$ & Rhode Island & 0.00 & 0.00 \\
\hline Indiana & 45.95 & 128.92 & South Carolina & $1,734.72$ & $2,532.01$ \\
\hline Iowa & 90.02 & $1,632.89$ & South Dakota & 0.15 & 7.05 \\
\hline Kansas & 56.21 & $2,297.20$ & Tennessee & $4,369.41$ & 194.18 \\
\hline Kentucky & 9.43 & 284.66 & Texas & 166.50 & $4,461.62$ \\
\hline Louisiana & 442.67 & $1,877.54$ & Utah & 165.63 & 80.18 \\
\hline Maine & 83.25 & 16.70 & Vermont & 81.08 & 157.85 \\
\hline Maryland & 238.46 & $1,439.69$ & Virgin Islands & 0.00 & 0.00 \\
\hline Massachusetts & 551.80 & $141,109.85$ & Virginia & $1,429.97$ & $2,402.23$ \\
\hline Michigan & 0.00 & 0.00 & Washington & $1,876.29$ & $1,263.83$ \\
\hline Minnesota & 54.72 & $1,738.82$ & West Virginia & 2.32 & 0.45 \\
\hline Mississippi & 201.85 & 660.06 & Wisconsin & 166.85 & 879.26 \\
\hline Missouri & 166.98 & $1,220.21$ & Wyoming & 5.84 & 5.65 \\
\hline \multirow[t]{2}{*}{ Montana } & 0.27 & 0.46 & & & \\
\hline & & & Total & $24,315.60$ & $751,378.59$ \\
\hline
\end{tabular}

aSource: ref. 7. 
Table 4.22. Historical and projected cumulative volume and radioactivity summary of commercial GTCC LLWa

\begin{tabular}{|c|c|c|c|c|c|c|}
\hline \multirow{3}{*}{ Category } & \multicolumn{3}{|c|}{$1993^{b}$} & \multicolumn{3}{|c|}{$2035^{c, d}$} \\
\hline & \multicolumn{2}{|c|}{$\begin{array}{l}\text { Volume } \\
\left(\mathrm{m}^{3}\right)\end{array}$} & \multirow{2}{*}{$\begin{array}{l}\text { Radioactivity } \\
\text { (Ci) }\end{array}$} & \multicolumn{2}{|c|}{$\begin{array}{c}\text { Volume } \\
\left(\mathrm{m}^{3}\right)\end{array}$} & \multirow{2}{*}{$\begin{array}{l}\text { Radioactivity } \\
\text { (Ci) }\end{array}$} \\
\hline & Unpackaged & $\begin{array}{c}\text { ACA } \\
\text { packaged }\end{array}$ & & Unpackaged & $\begin{array}{c}\text { ACA } \\
\text { packaged }\end{array}$ & \\
\hline \multicolumn{7}{|l|}{ Nuclear utility wastes } \\
\hline - BWR operations & 3.20 & 1.10 & 28,200 & 105.2 & 36.7 & $1,155,517$ \\
\hline - PWR operations & 2.82 & 0.12 & 18,300 & 77.7 & 10.0 & 573,510 \\
\hline LWR operations total & 6.02 & 1.22 & 46,500 & 182.9 & 46.7 & $1,729,027$ \\
\hline - BWR decommissioning & 6.26 & 14.48 & 757,000 & 115.4 & 188.8 & $3,270,412$ \\
\hline - PWR decommissioning & 3.98 & 10.32 & $3,086,500$ & 398.9 & 570.9 & $30,548,517$ \\
\hline LWR decommissioning total & 10.24 & 24.80 & $3,843,500$ & 514.3 & 759.7 & $33,818,929$ \\
\hline Nuclear utility total & 16.26 & 26.02 & $3,890,000$ & 697.2 & 806.4 & $35,547,956$ \\
\hline \multicolumn{7}{|l|}{ Sealed sources } \\
\hline - General license & 0.007 & 0.474 & 1,119 & 0.123 & 8.09 & 18,440 \\
\hline - Specific license & 0.125 & 38.22 & 354,000 & 0.87 & 234 & $1,560,000$ \\
\hline Sealed sources total & 0.13 & 38.69 & 355,119 & 0.99 & 242 & $1,578,440$ \\
\hline DOE-held potential GTCC waste & 0 & 0 & 0 & 0 & 0 & 0 \\
\hline Other generator waste $f^{f}$ & 46.9 & 74.2 & 2,738 & 235 & 465 & 12,680 \\
\hline Total & 63.3 & 138.9 & $4,247,857$ & 933 & 1,513 & $37,139,076$ \\
\hline
\end{tabular}

${ }_{\text {a }}$ ased on the INEL study of ref. 13. Projected data reported represent base-case scenario projections.

beported cumulative inventory as of December 31, 1993.

CProjected cumulative inventory for end of CY 2035.

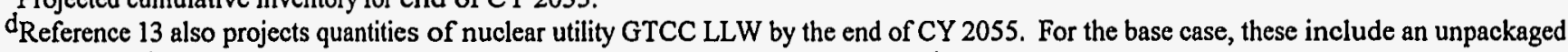
volume of $1,144 \mathrm{~m}^{3}$, an after-concentration-averaged (ACA) packaged volume of $1,347 \mathrm{~m}^{3}$, and an associated radioactivity of $88,400,000 \mathrm{Ci}$.

ACA packaged waste. This is the packaged volume of waste that is classified as GTCC LLW, after all other waste has been classified as Class $A, B$, or $C L L W$ using concentration-averaging practices.

Includes contributions from ${ }^{14} \mathrm{C}$ users, irradiation laboratories, sealed source manufacturers, a nuclear fuel fabrication facility, and a university reactor. 
Table 4.23. Breakdown of 1994 low-level radioactive waste by type, volume, and radioactivity received by commercial disposal sites ${ }^{a}$

\begin{tabular}{llrr}
\hline $\begin{array}{c}\text { Commercial } \\
\text { site }\end{array}$ & \multicolumn{1}{c}{$\begin{array}{c}\text { Type of } \\
\text { waste }\end{array}$} & $\begin{array}{c}\text { Volume } \\
\left(\mathrm{m}^{3}\right)\end{array}$ & $\begin{array}{r}\text { Radioactivity } \\
(\mathrm{Ci})\end{array}$ \\
\hline Barnwell & Academic & 394 & 410 \\
& Government & 2,849 & 32,648 \\
& Industrial & 7,759 & 31,507 \\
& Medical & 116 & 452 \\
& Utility & 9,665 & 680,284 \\
\cline { 2 - 4 } & & 20,783 & 745,301 \\
& & & \\
Richland & Academic & 111 & 11 \\
& Government & 424 & 380 \\
& Industrial & 1,932 & 20 \\
& Medical & 26 & 3,664 \\
& Utility & 1,040 & 6,078 \\
& & 3,533 &
\end{tabular}

asource: ref. 7. 


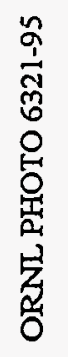

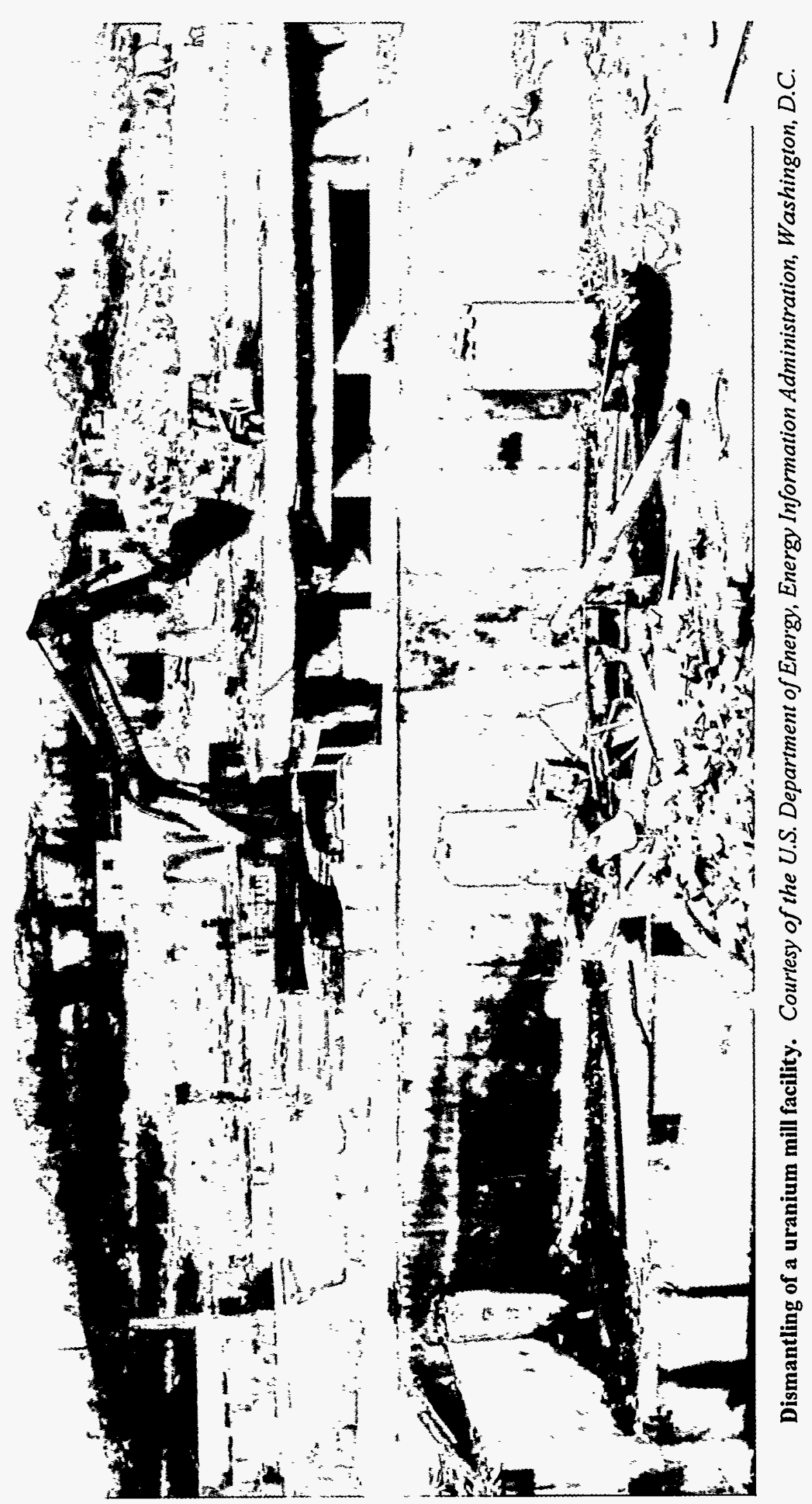




\section{URANIUM MLL TAILINGS FROM COMMERCIAL OPERATIONS}

\subsection{INTRODUCTION}

Uranium mill tailings are the residual wastes of milled ore that remain after the uranium has been recovered. Mill tailings at licensed sites and tailings that will be produced from meeting future uranium requirements are "commercial" mill tailings, the subject of this chapter. Tailings resulting from uranium milled for defense purposes are not included. Existing tailings at sites that are no longer licensed are classified as "inactive" mill tailings. Inactive tailings are administered under the remedial action projects discussed in Chapter 6.

Mill tailings are generated during the process of extracting uranium from the ore fed to the mill. Depending on the ore's chemical characteristics, uranium mills use either an acid leach or an alkaline leach process to recover uranium. Currently, all operable U.S. mills are designed to use the acid leach process. Mill tailings from both processes consist of slurries of sands and clay-like particles called slimes; the tailings slurries are pumped to tailings impoundment ponds for disposal.

U.S. uranium production from conventional milling has declined since 1980; as a consequence, the quantity of mill tailings generated each year has declined (see Table 5.1). During 1993 and 1994, there was no uranium production from conventional mills; therefore, no new mill tailings were generated. At the end of 1994, none of the U.S. mills were operational. Six of the 26 mills were on standby status, and the rest were undergoing various stages of decommissioning. The location and status of each of these mills are indicated on the map shown in Fig. 5.1 (ref. 13). The nonutilization of U.S. uranium mill capacity can be attributed, in large part, to nuclear power plant cancellations and deferments. Since the late 1970s, these have led to lower uranium demand which, in turn, has contributed to lower uranium prices and a steady decline in domestic uranium mining. In addition, cost increases for domestic uranium mining and milling have led to increased reliance on imports of less expensive uranium.

In the history of U.S. uranium production, 1993 and 1994 were the only years with no production from conventional milling of ore. Nonconventional concentrate production in 1994 increased to about $1,500 \mathrm{t} \mathrm{U}_{3} \mathrm{O}_{8}$, or $9 \%$ above 1993 production. ${ }^{1,2}$ Nonconventional concentrate production includes by-product processing resulting from the mining of phosphate ore as well as the processing of in situ leach mining solutions, heap-leach solutions, mine water, and other solutions from reclamation activities. In situ leaching (ISL) technology has been increasingly applied in recent years to mining operations. Of the total $\$ 80 / \mathrm{kg}-\mathrm{U}$ uranium reserves estimated by the Energy Information Administration (EIA), the amount for which ISL is the proposed mining method has increased from $42 \%$ in 1993 to $43 \%$ in 1994 . Because ISL mining generally is successful at lower costs compared with conventional mining methods, it could gain even wider use in the near future. ISL and by-product production methods do not generate mill tailings. Residual wastes from nonconventional methods are not considered in this chapter.

The volumes of historical and projected cumulative mill tailings through the year 2005 are shown in Fig. 5.2. This graph is based on the data reported in Table 5.1. The estimates of projected domestic tailings are based on U.S. production of uranium found in projections from ref. 3.

\subsection{INVENTORIES}

The status of the licensed mills, including their estimated commercial and government-related tailings inventories at the end of 1994, is shown in Table 5.2 (data based on refs. 1-14). For each mill, the amount of tailings generated depends on the amount of ore processed, the ore-feed grade $\left(\mathrm{U}_{3} \mathrm{O}_{8}\right.$ assay), and the percentage of $\mathrm{U}_{3} \mathrm{O}_{8}$ recovered. Table 5.3 lists the annual milling rate, ore grade, and $\mathrm{U}_{3} \mathrm{O}_{8}$ recovery. The associated mill tailings generated through 1994 are $189.5 \times 10^{6} \mathrm{t}$ $\left(118.6 \times 10^{6} \mathrm{~m}^{3}\right)$. There were no additions to the tailings pile during 1994 .

\subsection{WASTE CHARACTERIZATION}

Because the amount of uranium (by weight) extracted from the ore during milling is relatively small, the dry 
weight of the tailings produced is nearly equal to the dry weight of the ore processed. Dry tailings typically are composed of 70 to $80 \mathrm{wt} \%$ sand-sized particles and 20 to $30 \mathrm{wt} \%$ finer-sized particles. Acid leaching is preferred for ores with low lime content ( $12 \%$ or less). Those with high lime content require excessive quantities of acid for neutralization and, for economic reasons, are best treated by alkaline leaching. In either leach process, most of the uranium is dissolved, together with the other materials present in the ore (e.g., iron, aluminum, and other impurities). After the ore is leached, the uranium-laden leach liquor is removed from the tailings solids by decantation. After thorough washing, the tailings are pumped as a slurry to a tailings pond. The waste liquid accompanying the tailings solids to the disposal pond is approximately 1 to 1.5 times the weight of the processed ore. Typical characteristics of the tailings solids and liquid are outlined in Table 5.4 (ref. 8).

The tailings pile must have a cover designed to control radiological hazards for a minimum of 200 years and for 1,000 years to the greatest extent reasonably achievable. It must also limit radon $\left({ }^{222} \mathrm{Rn}\right)$ releases to $20 \mathrm{pCi} / \mathrm{m}^{2} / \mathrm{s}$ averaged over the disposal area. Radon release limitation requirements apply to any portion of the tailings disposal site unless radium concentrates do not exceed $5 \mathrm{pCi} / \mathrm{g}$ in the first $15 \mathrm{~cm}$ below the surface and $15 \mathrm{pCi} / \mathrm{g}$ in layers more than $15 \mathrm{~cm}$ below the surface. ${ }^{14}$

\subsection{PROJECTIONS}

An average tailings density of $1.6 \mathrm{t} / \mathrm{m}^{3}$ was used to calculate mill-tailings volumes resulting from the milling of uranium ore mined by open-pit and underground operations. The quantity of material produced is based on projections of uranium production as reported in the EIA publication, World Nuclear Outlook 1995, DOE/ EIA-0436(95). These projections were based on uranium requirements associated with the DOE/EIA 1995 LowCase (formerly called No-New-Orders-Case) scenario and assumed a 2-year lead time from the mining/milling of uranium to its use as a reactor fuel.

The volumes of tailings generated from 1995 through 2010 are estimated based on production schedules for conventional mining/milling operations expected to be operational in this period. Most of the U.S. production is projected to come from nonconventional extraction operations (in situ, by-product, etc.). Imports and U.S. inventory drawdowns, which are projected to make up over $80 \%$ of U.S. requirements through 2005 , will not add to U.S. tailings buildup.

\subsection{REFERENCES}

1. U.S. Department of Energy, Energy Information Administration, Form EIA-858, "Uranium Industry Annual Survey," Washington, D.C. (1994).

2. U.S. Department of Energy, Energy Information Administration, Uranium Industry Annual 1993, DOE/ EIA-0478(93), Washington, D.C. (October 1994).

3. U.S. Department of Energy, Energy Information Administration, World Nuclear Outlook 1995, DOE/ELA-0436(95), Washington, D.C. (October 1995).

4. U.S. Department of Energy, Integrated Data Base Report-1993: U.S. Spent Nuclear Fuel and Radioactive Waste Inventories, Projections, and Characteristics, DOE/RW-0006, Rev. 10, Oak Ridge National Laboratory, Oak Ridge, Tennessee (December 1994).

5. U.S. Department of Energy, Grand Junction Office, and Bendix Field Engineering Corporation, Commingled Uranium Tailings Study, DOE/DP-0011, Vol. 2, Grand Junction, Colorado (June 1982).

6. W. S. White, Directory and Profile of Licensed Uranium Recovery Facilities, NUREG/CR-2869 (ANL/ES-128), Rev. 1, U.S. Nuclear Regulatory Commission, Washington, D.C. (March 1984).

7. U.S. Environmental Protection Agency, "National Emission Standard for Radon-222 Emissions from Licensed Uranium Mill Tailings," Code of Federal Regulations, 40 CFR Part 61, Subpart W (September 1986). 
8. U.S. Nuclear Regulatory Commission, Final Generic Environmental Impact Statement on Uranium Milling, Project $M-25$, NUREG-0706, Washington, D.C. (September 1980).

9. U.S. Department of Energy, Grand Junction Office, Statistical Data of the Uranium Industry, GJ0-100(73), Grand Junction, Colorado (Jan. 1, 1973).

10. U.S. Congress, House of Representatives, Committees on Energy and Commerce; Interior and Insular Affairs; Science, Space, and Technology; and Ways and Means, Uranium Revitalization, Tailings Reclamation and Enrichment Act of 1988: Hearing on H.R. 4489, 100th Congress, 2nd sess., pp. 19-21 (Apr. 28, 1988).

11. Paul Estey, Exxon Coal and Minerals Company, Houston, Texas, personal communication to L. L. Smith, Energy Information Administration, Washington, D.C. (Aug. 22, 1988).

12. Philip S. Stoffey, Colorado Department of Public Health and the Environment, "Cotter Uranium Mill as Reported in Integrated Data Base for 1993: U.S. Spent Fuel and Radioactive Waste Inventories, Projections, and Characteristics," correspondence to M. L. Payton, U.S. Department of Energy, Office of Civilian Radioactive Waste Management, dated Aug. 31, 1994.

13. U.S. Department of Energy, Energy Information Administration, "Comparison of Uranium Mill Tailings Reclamation in the United States and Canada," Uranium Industry Annual 1994, DOE/EIA-0478(94), Washington, D.C. (July 1995).

14. U.S. Department of Energy, Energy Information Administration, Decommissioning of U.S. Uranium Production Facilities, DOE-EIA-0592, Washington, D.C. (February 1995). 


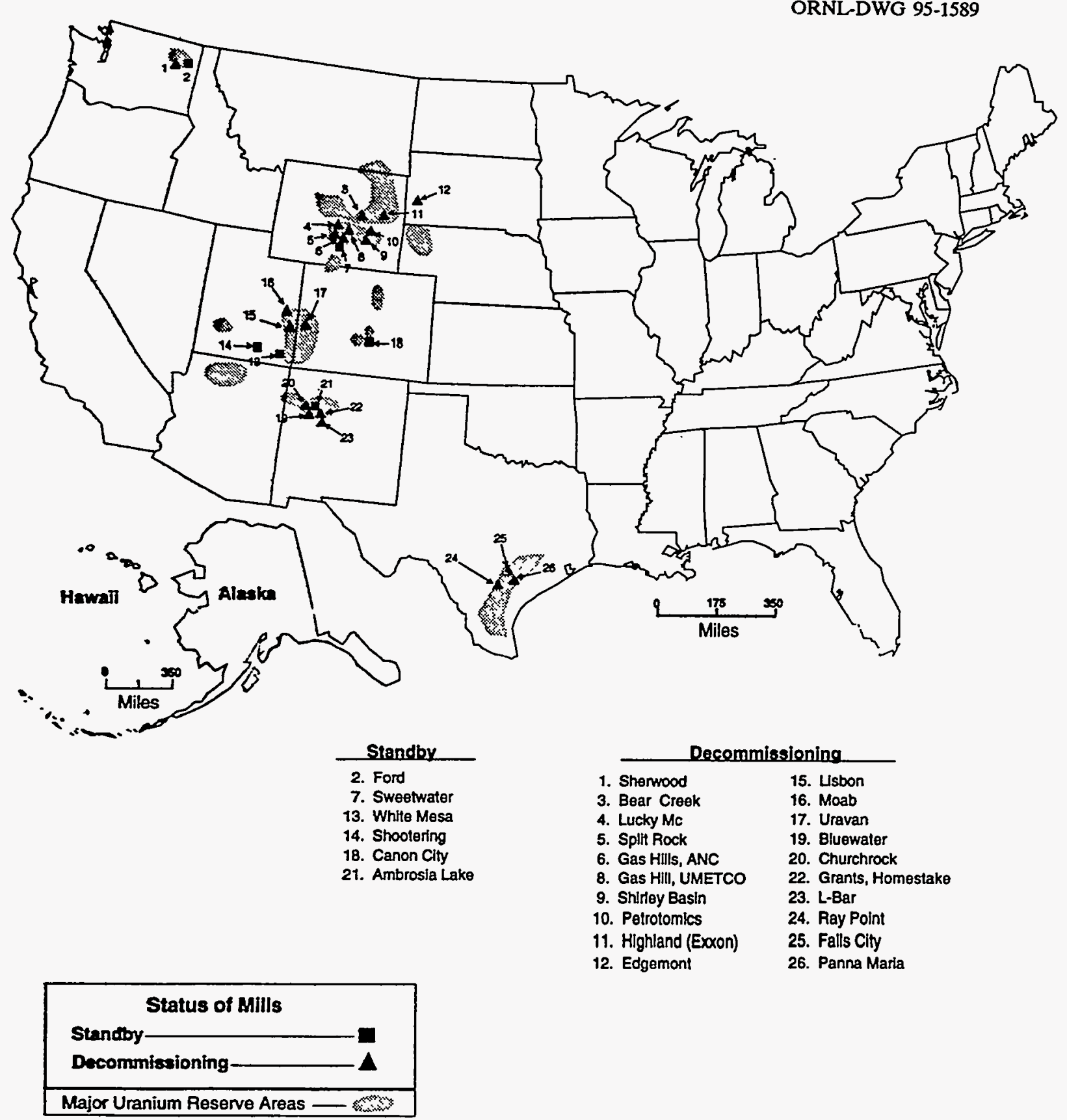

Source: Based on U.S. Department of Energy, Grand Junction Project Otfice (GJPO), National Uranlum Resources. Evaluation, Interim Report (hune 1979) Figure 3.2; G.JPO dala files; Energy Informatlon Administration, Form ElA-858, "Uranium Industry Annual Survey" (1994).

Fig. 5.1. Location and status of uranium mills at the end of 1994. 
ORNL DWG 95-8837

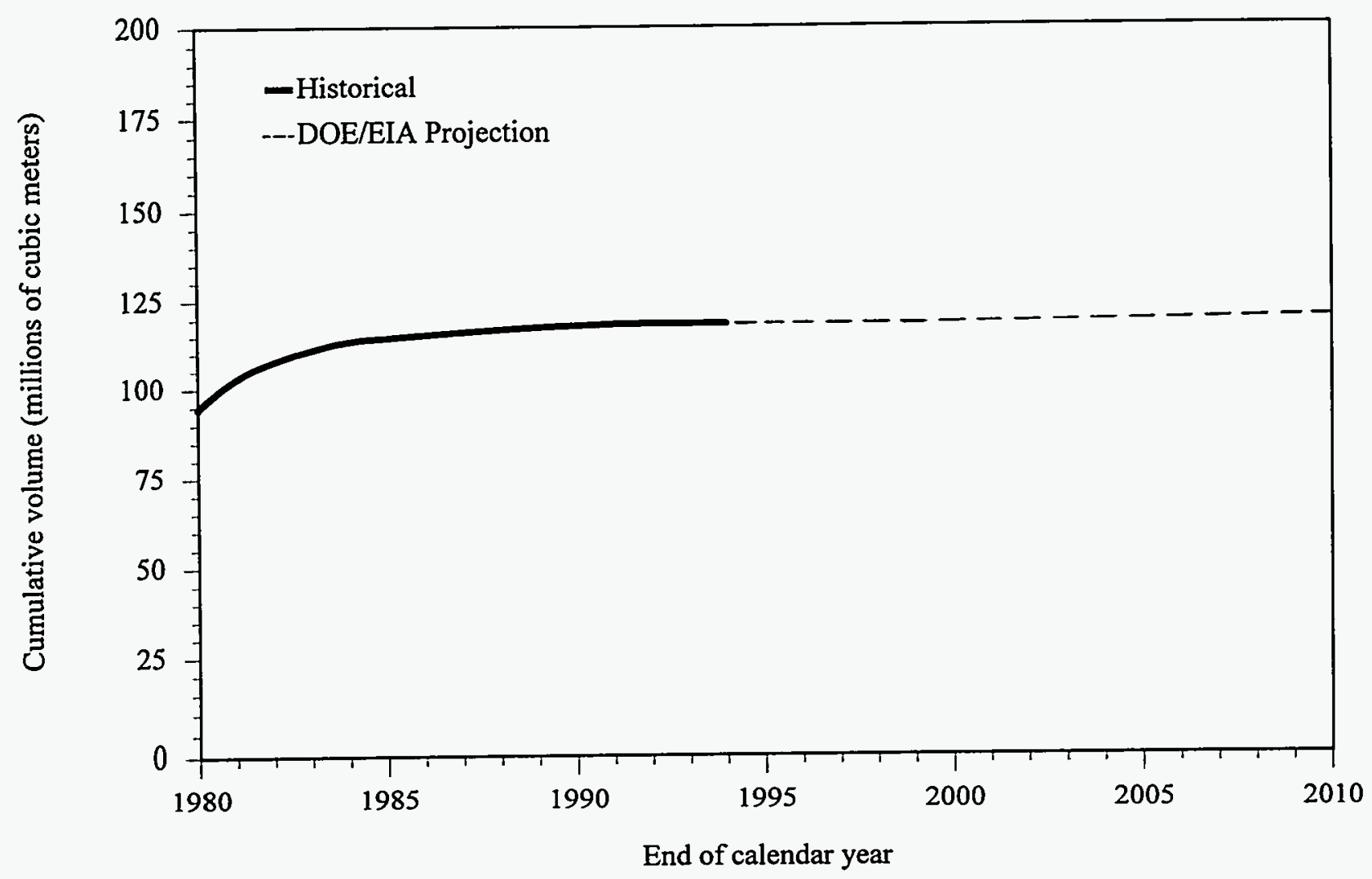

Fig. 5.2. Historical and projected cumulative volume of commercial mill tailings. 
Table 5.1. Historical and projected volume of uranium mill tailings ${ }^{a, b}$

\begin{tabular}{ccc}
\hline \multirow{2}{*}{$\begin{array}{c}\text { End of } \\
\text { calendar } \\
\text { year }\end{array}$} & \multicolumn{2}{c}{$\begin{array}{c}\text { Volume } \\
\left(10^{6} \mathrm{~m}^{3}\right)\end{array}$} \\
\cline { 2 - 3 } & Annual & Cumulative \\
\hline $\begin{array}{c}\text { Prior to } 1978 \\
1978\end{array}$ & & 68.0 \\
1979 & 7.9 & 75.8 \\
1980 & 9.1 & 84.9 \\
1981 & 9.5 & 94.4 \\
1982 & 8.2 & 102.7 \\
1983 & 5.0 & 107.7 \\
1984 & 3.4 & 111.1 \\
1985 & 2.5 & 113.6 \\
1986 & 1.0 & 114.6 \\
1987 & 0.7 & 115.4 \\
1988 & 0.8 & 116.2 \\
1989 & 0.7 & 116.9 \\
1990 & 0.7 & 117.6 \\
1991 & 0.4 & 118.0 \\
1992 & 0.4 & 118.4 \\
1993 & 0.2 & 118.6 \\
1994 & 0.0 & 118.6 \\
1995 & 0.0 & 118.6 \\
1996 & $<0.1$ & 118.6 \\
1997 & $<0.1$ & 118.6 \\
1998 & $<0.1$ & 118.6 \\
1999 & $<.1$ & 118.6 \\
2000 & $<.1$ & 118.7 \\
2001 & 0.1 & 118.8 \\
2002 & 0.1 & 118.9 \\
2003 & 0.1 & 119.0 \\
2004 & 0.2 & 119.2 \\
2005 & 0.2 & 119.4 \\
2006 & 0.2 & 119.6 \\
2007 & 0.2 & 119.8 \\
2008 & 0.2 & 120.0 \\
2009 & 0.2 & 120.2 \\
2010 & 0.2 & 120.4 \\
& 0.1 & 120.5 \\
\hline & & \\
\hline & & \\
\hline
\end{tabular}

aProjections of domestic tailings are generated from estimates of U.S. uranium production under current market conditions described in ref. 3, which is the Lower Reference Case (now referred to by DOE/EIA as the Mid Case).

bSources: Prior to 1984-U.S. Department of Energy, Grand Junction Project Office data files. From 1984 through 1994-Energy Information Administration, Form EIA-858, "Uranium Industry Annual Survey." 
Table 5.2. Status of conventional uranium mill sites at the end of $1994^{\mathrm{a}}$

\begin{tabular}{|c|c|c|c|c|c|c|c|c|}
\hline \multirow{3}{*}{ Location } & \multirow{3}{*}{ Operator } & \multirow{3}{*}{$\begin{array}{l}\text { Rated } \\
\text { capacity }^{b} \\
\text { (t/d ore) }\end{array}$} & \multirow{2}{*}{\multicolumn{2}{|c|}{ Status }} & \multirow{3}{*}{$\begin{array}{l}\text { Tailings } \\
\text { storage } \\
\text { area } \\
\text { (ha) }{ }^{d}\end{array}$} & \multicolumn{3}{|c|}{ Total tailings } \\
\hline & & & & & & & & \\
\hline & & & Operations ${ }^{b}$ & Tailings $c$ & & $\begin{array}{l}\text { Volume } \\
\left(10^{6} \mathrm{~m}^{3}\right)\end{array}$ & $\begin{array}{l}\text { Mass } \\
\left(10^{6} t\right)\end{array}$ & $\begin{array}{c}\text { portion } \\
\left(10^{6} t\right)\end{array}$ \\
\hline \multicolumn{9}{|l|}{ Colorado } \\
\hline Canon City & Cotter & 1,090 & Shut down, 1987 & & $66 \mathrm{R}^{8}$ & 1.3 & 2.0 & 0.3 \\
\hline Uravan & Umetco Minerals & $1,180^{\mathrm{h}}$ & Decommissioning & Partially stabilized & $34 \mathrm{R}$ & 5.9 & 9.5 & 5.2 \\
\hline Subtotal & & 1,090 & & & $100 R$ & 7.2 & 11.5 & 5.5 \\
\hline \multicolumn{9}{|l|}{ New Mexico } \\
\hline Ambrosia Lake & Quivira Mining & 6,350 & Shut down, 1985 & Fenced & $131 R$ & 18.8 & 30.1 & 9.1 \\
\hline Bluewater & Anaconda & $5,440^{\mathrm{h}}$ & Decommissioning & Partially stabilized & 199 & 13.6 & 21.7 & 8.0 \\
\hline Church Rock & United Nuclear & $2,720^{\mathrm{h}}$ & Decommissioning & i & $40 \mathrm{R}$ & 2.0 & 3.2 & 0 \\
\hline Grants & Homestake Mining & $3,080^{h}$ & Decommissioning & Unstabilized & $86 \mathrm{R}$ & 12.7 & 20.2 & 10.4 \\
\hline L-Bar & Sohio Western Mining & $1,450^{\mathrm{h}}$ & Decommissioning & i & $46 \mathrm{R}$ & 1.2 & 1.9 & 0 \\
\hline Marquez & Bokum Resources & $1,820^{h} \mathbf{j}$ & New (on standby) & Never operated & $\begin{array}{c}40 \pi \\
0\end{array}$ & 0 & 0 & 0 \\
\hline Subtotal & & 6,350 & & & $502 R$ & 48.3 & 77.1 & 27.5 \\
\hline \multicolumn{9}{|l|}{ South Dakota } \\
\hline Edgemont & $\begin{array}{l}\text { Tennessee Valley } \\
\text { Authority }\end{array}$ & $680^{h}$ & Decommissioning & Partially stabilized & 50 & 1.2 & 1.8 & 1.5 \\
\hline & & - & & & 一 & - & - & - \\
\hline Subtotal & & 0 & & & 50 & 1.2 & 1.8 & 1.5 \\
\hline \multicolumn{9}{|l|}{ Texas } \\
\hline Falls City & $\begin{array}{l}\text { Continental Oil/ } \\
\text { Pioneer Nuclear }\end{array}$ & $3,080^{\mathrm{h}}$ & Decommissioning & $\mathrm{i}$ & 89 & 6.5 & 10.5 & 0 \\
\hline Panna Maria & Rio Grande Resources & $2,720^{h}$ & Decommissioning & i & 101 & 3.9 & 5.9 & 0 \\
\hline $\begin{array}{l}\text { Ray Point } \\
\text { (Felder Facility) }\end{array}$ & Exxon & $1,000^{h}$ & Decommissioning & Stabilized ${ }^{k}$ & 18 & 0.2 & $0.4^{1}$ & 0 \\
\hline Subtotal & & 2,720 & & & 208 & 10.6 & 16.8 & 0 \\
\hline
\end{tabular}


Table 5.2 (continued)

\begin{tabular}{|c|c|c|c|c|c|c|c|c|}
\hline \multirow{3}{*}{ Location } & \multirow{3}{*}{ Operator } & \multirow{3}{*}{$\begin{array}{l}\text { Rated } \\
\text { capacityb } \\
\text { (t/d ore) }\end{array}$} & \multirow{2}{*}{\multicolumn{2}{|c|}{ Status }} & \multirow{3}{*}{$\begin{array}{l}\text { Tailings } \\
\text { storage } \\
\text { area } \\
(\mathrm{ha})^{\mathrm{d}}\end{array}$} & \multicolumn{3}{|c|}{ Total tailings } \\
\hline & & & & & & \multirow[b]{2}{*}{$\begin{array}{l}\text { Volume } \\
\left(10^{6} \mathrm{~m}^{3}\right)\end{array}$} & \multirow[b]{2}{*}{$\begin{array}{l}\text { Mass } \\
\left(10^{6} t\right)\end{array}$} & \multirow[b]{2}{*}{$\begin{array}{l}\text { Government } \\
\text { portion }{ }^{f} \\
\left(10^{6} \mathrm{t}\right)\end{array}$} \\
\hline & & & Operations $^{b}$ & Tailings $^{c}$ & & & & \\
\hline \multicolumn{9}{|l|}{ Utah } \\
\hline Lisbon & Rio Algom & 680 & Decommissioning & i & 14 & 2.2 & 3.5 & 0 \\
\hline Moab & Atlas & $1,270^{\mathrm{h}}$ & Decommissioning & Unstabilized & $>80$ & 6.0 & 9.6 & 5.4 \\
\hline Shootering & Plateau Resources & 910 & New (on standby) & Never operated & 28 & 0 & 0 & 0 \\
\hline White Mesa & Energy Fuels & 1,810 & Shut down, 1990 & Partially stabilized & 135 & 1.9 & 3.2 & 0 \\
\hline Subtotal & & 3,400 & & & $>257$ & 10.1 & 16.3 & 5.4 \\
\hline \multicolumn{9}{|l|}{ Washington } \\
\hline Ford & Dawn Mining & 410 & Shut down, 1982 & Wood chip covering & $53 \mathrm{R}$ & 1.8 & 2.8 & 1.1 \\
\hline Sherwood & Western Nuclear & $1,810^{\mathrm{h}}$ & Decommissioning & $\mathrm{i}^{2}$ & 17 & 1.6 & 2.6 & 0 \\
\hline Subtotal & & 410 & & & $70 \mathrm{R}$ & 3.4 & 5.4 & 1.1 \\
\hline \multicolumn{9}{|l|}{ Wyoming } \\
\hline Bear Creek & Rocky Mountain Energy & $1,810^{\mathrm{h}}$ & Decommissioning & Unstabilized & 61 & 2.7 & 4.3 & 0 \\
\hline Gas Hills & American Nuclear & $860^{\mathrm{h}}$ & Decommissioning & Unstabilized & $47 R$ & 3.3 & 5.4 & 2.0 \\
\hline Gas Hills & Umetco & $1,270^{\mathrm{h}}$ & Decommissioning & Unstabilized & $58 \mathrm{R}$ & 4.6 & 7.3 & 1.9 \\
\hline Highland & Exxon & $2,900^{\mathrm{h}}$ & Decommissioning & Partially stabilized & $116 \mathrm{R}$ & 6.4 & 10.3 & 0 \\
\hline Lucky Mc & Pathfinder & $2,540^{\mathrm{h}}$ & Decommissioning & Unstabilized & $99 \mathrm{R}$ & 6.6 & 10.6 & 2.6 \\
\hline Petrotomics & Petrotomics & $1,360^{\mathrm{h}}$ & Decommissioning & Unstabilized & 65 & 3.9 & 6.3 & 0.7 \\
\hline Shirley Basin & Pathfinder & $1,630^{\mathrm{h}}$ & Decommissioning & i & $105 \mathrm{R}$ & 4.7 & 7.4 & 0 \\
\hline Split Rock & Western Nuclear & $1,540^{\mathrm{h}}$ & Decommissioning & Interim stabilization & $67 \mathrm{R}$ & 4.4 & 7.0 & 3.0 \\
\hline Sweetwater & $\begin{array}{l}\text { Minerals Exploration/ } \\
\text { Union Energy Mining }\end{array}$ & 2,720 & Shut down, May 1983 & Partially stabilized & 121 & 1.3 & 2.1 & 0 \\
\hline \multicolumn{2}{|l|}{ Subtotal } & 4,350 & & & $739 \mathrm{R}$ & 37.9 & 60.7 & 10.2 \\
\hline \multicolumn{2}{|c|}{1994 total for all sites $b, m, n$} & $18,320^{\circ}$ & & & $>1,926 \mathrm{R}$ & 118.6 & 189.5 & $51.2 \mathrm{p}$ \\
\hline
\end{tabular}

(Footnotes on next page.) 
aData based on refs. 1-14. Note: subtotals and totals may not equal sum of components because of independent rounding. Ray Point, Texas (Felder Facility), site was stabilized during 1987 by Exxon Corporation. Historical data are revised based on detailed study of milling data from the Grand Junction Project Office and EIA files. The values shown include all tailings.

bFrom refs. 1, 5, 9, and 14. Values rounded to nearest $10 t$

Cn Aug. 15, 1986, EPA issued its final rules on ${ }^{222} \mathrm{Rn}$ emissions from tailings piles. Mill owners have 6 years (subject to certain extensions) to phase out the use of large existing tailings piles. New tailings piles may be contained in small impoundments (less than 16 ha) or disposed of continuously by dewatering and burial (i.e., no more than 4 ha are uncovered at any one time). See ref 7 .

dFrom refs. 6,12 , and $14 ; 1 \mathrm{ha}=10,000 \mathrm{~m}^{2}$ or approximately 2.5 acres.

${ }^{e}$ Calculated from reported mass using density $=1.6 \mathrm{t} / \mathrm{m}^{3}$.

fFrom ref. 5, Table 8.0. These tailings are from government contracts only and are included in the "Total tailings" column.

$\mathrm{g}_{\mathrm{R}}=$ revised. From ref. 14 .

hEstimates provided are not included in the total. See column labeled "Operations" under "Status" for reason.

i Not available.

JMill construction has not been $100 \%$ complete

kFrom ref. 11.

From ref. 10.

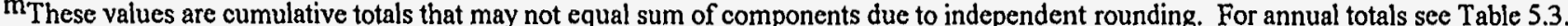

nFrom ref. 1 .

OFrom ref. 1

PTotal at the end of government-contracted deliveries in 1970 (ref. 5) 
Table 5.3. Uranium ore processed, $\mathrm{U}_{3} \mathrm{O}_{8}$ recovery rate, and tailings generated through $1994^{a, b}$

\begin{tabular}{|c|c|c|c|c|c|c|}
\hline \multirow{3}{*}{$\begin{array}{l}\text { End of } \\
\text { calendar } \\
\text { year }\end{array}$} & \multirow{2}{*}{\multicolumn{2}{|c|}{ Ore processed }} & \multicolumn{2}{|c|}{$\mathrm{U}_{3} \mathrm{O}_{8}$} & \multirow{2}{*}{\multicolumn{2}{|c|}{ Tailings generated }} \\
\hline & & & \multirow{2}{*}{$\begin{array}{l}\text { Recovery } \\
\text { from ore } \\
(\%)\end{array}$} & \multirow[b]{2}{*}{$\begin{array}{l}\text { Product } \\
\left(10^{3} t\right)\end{array}$} & & \\
\hline & $\begin{array}{l}\text { Mass } \\
\left(10^{6} t\right)\end{array}$ & $\begin{array}{c}\text { Grade } \\
\left(\% \mathrm{U}_{3} \mathrm{O}_{8}\right)\end{array}$ & & & $\begin{array}{l}\text { Masse } \\
\left(10^{6} t\right)\end{array}$ & $\begin{array}{l}\text { Volume } \\
\left(10^{6} \mathrm{~m}^{3}\right)\end{array}$ \\
\hline Prior to 1978 & $\mathrm{~g}$ & $\mathrm{~g}$ & $\mathrm{~g}$ & $\mathrm{~g}$ & 108.8 & 68.0 \\
\hline 1978 & 12.5 & 0.134 & 91 & 15.6 & 12.6 & 7.9 \\
\hline 1979 & 14.6 & 0.113 & 91 & 15.3 & 14.5 & 9.1 \\
\hline 1980 & 15.3 & 0.118 & 93 & 17.2 & 15.2 & 9.5 \\
\hline 1981 & 13.2 & 0.115 & 94 & 14.5 & 13.2 & 8.2 \\
\hline 1982 & 7.9 & 0.119 & 96 & 9.9 & 8.1 & 5.0 \\
\hline 1983 & 5.4 & 0.128 & 97 & 7.0 & 5.4 & 3.4 \\
\hline 1984 & 3.9 & 0.112 & 95 & 4.4 & 4.0 & 2.5 \\
\hline 1985 & 1.6 & 0.161 & 96 & 2.8 & 1.6 & 1.0 \\
\hline 1986 & 1.2 & 0.338 & 97 & 4.0 & 1.2 & 0.7 \\
\hline 1987 & 1.3 & 0.284 & 96 & 3.8 & 1.3 & 0.8 \\
\hline 1988 & 1.1 & 0.288 & 95 & 3.2 & 1.1 & 0.7 \\
\hline 1989 & 1.1 & 0.323 & 95 & 3.7 & 1.0 & 0.7 \\
\hline 1990 & 0.7 & 0.293 & 94 & 2.1 & 0.7 & 0.4 \\
\hline 1991 & 0.6 & 0.188 & 92 & 1.2 & 0.6 & 0.4 \\
\hline 1992 & 0.2 & 0.229 & 96 & 0.6 & 0.2 & 0.2 \\
\hline 1993 & 0.0 & 0.000 & 0 & 0.0 & 0.0 & 0.0 \\
\hline 1994 & 0.0 & 0.000 & 0 & 0.0 & 0.0 & 0.0 \\
\hline Total $^{\mathrm{h}}$ & & & & & 189.5 & 118.6 \\
\hline
\end{tabular}

aSources: Prior to 1984-U.S. Department of Energy, Grand Junction Area Office data files. 1984-1994-Energy Information Administration, Form EIA-858, "Uranium Industry Annual Survey."

${ }^{b}$ This table has been revised based on a detailed study of milling data from the Grand Junction Project Office and EIA files. The values shown include all tailings.

CBefore in-process inventory adjustments.

dConventional $\mathrm{U}_{3} \mathrm{O}_{8}$ concentrate production.

'Includes adjustments to ore-fed amounts for annual mill circuit inventory changes and uranium concentrate production.

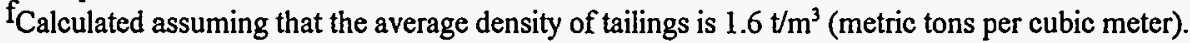

gNot available.

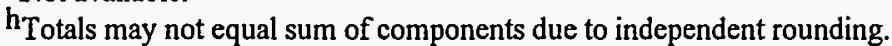


Table 5.4. Typical characteristics of uranium mill tailings ${ }^{a}$

\begin{tabular}{|c|c|c|c|}
\hline $\begin{array}{l}\text { Tailings } \\
\text { component }\end{array}$ & $\begin{array}{l}\text { Particle size } \\
\quad(\mu \mathrm{m})\end{array}$ & $\begin{array}{l}\text { Chemical } \\
\text { composition }\end{array}$ & $\begin{array}{l}\text { Radioactivity } \\
\text { characteristics }\end{array}$ \\
\hline Sands & 75 to 500 & $\begin{array}{l}\mathrm{SiO}_{2} \text { with }<1 \% \text { complex silicates } \\
\text { of } \mathrm{Al}, \mathrm{Fe}, \mathrm{Mg}, \mathrm{Ca}, \mathrm{Na}, \mathrm{K}, \mathrm{Se} \text {, } \\
\mathrm{Mn}, \mathrm{Ni}, \mathrm{Mo}, \mathrm{Zn}, \mathrm{U} \text {, and } \mathrm{V} \text {; also } \\
\text { metallic oxides }\end{array}$ & $\begin{array}{l}0.004 \text { to } 0.01 \% \mathrm{U}_{3} \mathrm{O}_{8}^{\mathrm{b}} \\
\text { Acid leaching: } \\
26 \text { to } 100 \mathrm{pCi}^{226} \mathrm{Ra} / \mathrm{g} \\
70 \text { to } 600 \mathrm{pCi}^{230} \mathrm{Th} / \mathrm{g}\end{array}$ \\
\hline Slimes & 45 to 75 & $\begin{array}{l}\text { Small amounts of } \mathrm{SiO}_{2} \text {, but mostly } \\
\text { very complex clay-like silicates } \\
\text { of } \mathrm{Na}, \mathrm{Ca}, \mathrm{Mn}, \mathrm{Mg}, \mathrm{Al} \text {, and } \mathrm{Fe} \text {; } \\
\text { also metallic oxides }\end{array}$ & $\begin{array}{l}\mathrm{U}_{3} \mathrm{O}_{8} \text { and }{ }^{226} \mathrm{Ra} \text { are almost } \\
\text { twice the concentration } \\
\text { present in the sands } \\
\text { Acid leaching: } \\
150 \text { to } 400 \mathrm{pCi}^{226} \mathrm{Ra} / \mathrm{g} \text {; } \\
70 \text { to } 600 \mathrm{pCi}^{230} \mathrm{Th} / \mathrm{g}\end{array}$ \\
\hline \multirow[t]{2}{*}{ Liquids } & $\mathrm{d}$ & $\begin{array}{l}\text { Acid leaching: } \\
\text { pH } 1.2 \text { to } 2.0^{-} \mathrm{Na}^{+}, \mathrm{NH}_{4}^{+}, \mathrm{SO}_{4}^{-2}, \\
\mathrm{Cl}^{-} \text {, and } \mathrm{PO}_{4}^{-3} ; \text { dissolved solids } \\
\text { up to } 1 \%\end{array}$ & $\begin{array}{l}\text { Acid leaching: } \\
0.001 \text { to } 0.01 \% \mathrm{U} \text {; } \\
20 \text { to } 7,500 \mathrm{pCi}^{226} \mathrm{Ra} / \mathrm{L} \text {; } \\
2,000 \text { to } 22,000 \mathrm{pCi} \\
{ }^{230} \mathrm{Th} / \mathrm{L}\end{array}$ \\
\hline & & $\begin{array}{l}\text { Alkaline leaching: } \\
\mathrm{pH} 10 \text { to } 10.5 ; \mathrm{CO}_{3}^{-2} \text { and } \mathrm{HCO}_{3}{ }^{-} \text {; } \\
\text { dissolved solids } \sim 10 \%\end{array}$ & $\begin{array}{l}\text { Alkaline leaching: } \\
200 \mathrm{pCi}{ }^{226} \mathrm{Ra} / \mathrm{L} ; \\
\text { essentially no }{ }^{230} \mathrm{Th} \\
\text { (insoluble) }\end{array}$ \\
\hline
\end{tabular}

Adapted from information in ref. 8.

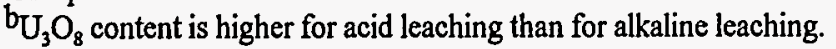

CSeparate analyses of sands and slimes from the alkaline leaching process are not available. However, total ${ }^{226} \mathrm{Ra}$ and ${ }^{230} \mathrm{Th}$ contents of up to $600 \mathrm{pCi} / \mathrm{g}$ (of each) have been reported for the combined sands and slimes.

dParticle size does not apply. Up to $70 \%$ of the liquid may be recycled. Recycle potential is greater in the alkaline process. 


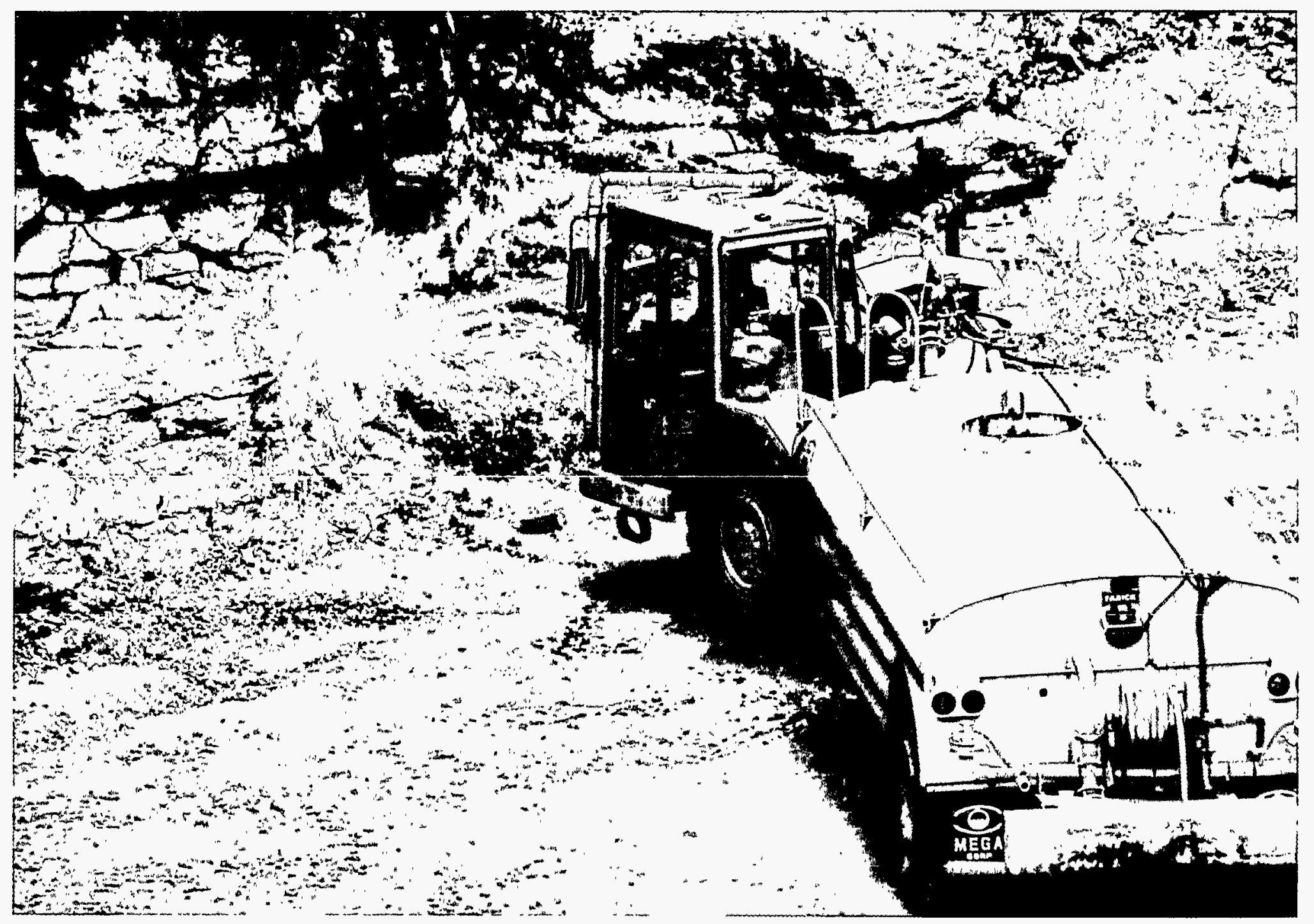

High-pressure water spraying of the Weldon Spring quarry walls following waste removal. Courtesy of the U.S. Department of Energy, Office of the Deputy Assistant Secretary for Environmental Restoration, Washington, D.C. 


\section{ENVIRONMENTAL RESTORATION PROGRAM}

\subsection{INTRODUCTION}

The fundamental goal of the DOE Office of Environmental Restoration (DOE/EM-40, or simply EM-40) is to ensure that the risks to the environment and human health and safety posed by inactive and surplus facilities and sites are either eliminated or reduced to prescribed, safe levels. These facilities contain radioactive and chemically hazardous contaminants as a result of previous activities conducted by $\mathrm{DOE}$ and its predecessor agencies. Although this goal encompasses all requirements prescribed by applicable federal, state, and local environmental statutes and regulatory requirements, it is not limited to regulatory .compliance. DOE's paramount concerns are maintaining and improving human health and safety and protecting the environment.

The DOE environmental restoration program includes a bias for action to expedite actual cleanup wherever and whenever possible. Environmental restoration efforts are proceeding in two major areas: remedial action (RA) and decommissioning. These activities include cleanup of facilities and areas that supported defense-related activities (such as nuclear weapon component fabrication) and nondefense, civilian nuclear power activities (such as the development of heat sources for the space program and the operation of small test reactors).

RAs are concerned with all aspects of the assessment and cleanup of inactive sites at which releases of radioactive and chemically hazardous substances have occurred. These actions are not only limited to those areas directly impacted by the release but also include additional areas to which contaminants may have migrated (e.g., to ground water). A number of DOE installations are on the EPA National Priorities List. RAs include site discovery, preliminary assessment, and site inspection; site characterization, analysis of cleanup alternatives, and selection of remedy; cleanup and site closure; and site compliance monitoring. Although such activities may deal with storage tanks, buildings, and structures, most are concerned with contaminated environmental media such as soil, sediment, and ground water.

The principal regulatory requirements for RAs are derived from the Resource Conservation and Recovery Act
(RCRA) and the Comprehensive Environmental Response, Compensation, and Liability Act (CERCLA). Activities may be subject further to requirements associated with compliance with the National Environmental Policy Act (NEPA) and with regulatory requirements imposed by the states. Other requirements are set forth in various DOE Orders and standards and in other guidance documents.

Decommissioning activities are primarily concerned with the safekeeping of surplus nuclear facilities following shutdown and for either their ensuing decontamination for reuse or their complete dismantlement. Such tasks include surveillance and maintenance, assessment and characterization, environmental review, engineering, specific decommissioning operations, and project closeout. Most decommissioning activities are concerned with facilities such as reactors, hot cells, processing plants, storage tanks, and other structures from which, in general, few releases to the environment have occurred. Approximately 500 contaminated facilities are currently included in the EM-40 inventory for future action. The objectives of decommissioning activities are to decontaminate these facilities and to eliminate any potential hazards to public health and the environment.

Decommissioning activities are carried out under the authority of the Atomic Energy Act (AEA) and according to requirements set forth in various DOE Orders and standards and other guidance documents. In addition, the provisions of RCRA and CERCLA may apply to those facilities from which there either has been a release or there is a potential for release to the environment. State requirements also apply in certain instances. Only those decommissioning activities at facilities currently in the EM-40 program are addressed in this chapter.

Because many RA and decommissioning sites are still in the assessment phase, it is very difficult to project the volumes and types of waste that may be generated. Moreover, detailed information on the specific cleanup activities that may be applied to various contamination problems is not yet available; therefore, the quantity of resultant waste that might be generated cannot be reliably determined. In fact, the plans for many sites are not yet to a stage at which even the broad category of response that will be taken is known. For example, the decision whether 
a given contaminated area, such as a waste pit, is to be excavated or stabilized in place is not typically made until after (1) the nature of the problem has been adequatcly defined, (2) various response alternatives and related impacts have been evaluated in considerable detail, and (3) other agencies (such as the EPA, the impacted state, and the local community) have had a chance to comment on the preferred alternative. Materials regarded as waste would be generated only if the waste pit were excavated; no waste would be generated if the pit were capped in place.

An intensive effort is currently under way to better define waste management requirements for implementing the DOE environmental restoration program. A data collection program was initiated in late 1993 to develop "cradle-to-grave" contaminated media/waste information to allow for better planning of waste treatment and disposal capacity needs.' The information provided by the field in response to this data call has been entered into the EM-40 Contaminated Media/Waste Database at Headquarters. This database is the source for most of the information provided in this chapter. In addition to this database, EM-40 developed additional information to meet the needs of the Baseline Environmental Management Report (BEMR) in late 1994. These two data sources are currently being consolidated and updated and represent the two major sources of information in the EM-40 Core Database.

Waste management information currently being compiled by EM- 40 includes data on all waste types that may result from environmental restoration activities including radioactive, sanitary, demolition, and hazardous wastes as regulated by RCRA and the Toxic Substances Control Act (TSCA). This information will assist DOE in planning, constructing, and operating appropriate treatment, storage, and disposal facilities to manage the wastes resulting from implementation of the EM-40 program. Waste projections are currently available for only a few sites. Hence, environmental restoration waste volumes are not provided in this report. However, the volumes of contaminated solid environmental media are known at most sites, and these volumes are included in Sect. 6.3.

\subsection{OFFICE OF ENVIRONMENTAL RESTORATION}

Environmental restoration activities under the auspices of EM-40 are managed in a decentralized manner. That is, much of the responsibility for program implementation rests with the various Operations and project offices. These field offices have the responsibility for determining the appropriate course of action to take at the various contaminated sites and then directing the remediation activities. The locations of the field offices responsible for directing the DOE environmental restoration program are shown in Fig. 6.1.

The Headquarters element of the EM-40 program is responsible for establishing policy and providing programmatic guidance to the field offices in implementing this program and determining appropriate priorities. In addition, Headquarters is responsible for formulating and executing the EM-40 budget. Other Headquarters responsibilities include tracking progress on environmental restoration activities and responding to information requests from Congress, other federal agencies, and public-interest groups. In addition to serving as an advocate for the EM-40 program, EM-40 Headquarters is responsible for interacting with other EM offices [such as the Office of Waste Management (EM-30), the Office of Technology Development (EM-50), and the Office of Facility Transition (EM-60)] to facilitate transfer of information.

In general, the Operations offices directing the environmental restoration program in the field are the same offices that directed activities at these sites when facilities were operational. For example, energy research and development activities at Ames Laboratory, Argonne National Laboratory, and Brookhaven National Laboratory are directed by the Chicago Operations Office. This Operations office is currently responsible for managing the environmental restoration program at these three laboratories. A listing of the field offices directing the EM-40 program and of the sites for which these offices have responsibility is given in Tables 6.1 and 6.2.

At all sites where environmental restoration activities are conducted, interim actions are used to address urgent risks and remove potential threats before the completion of detailed characterization studies. In 1994, the EM-40 program completed 119 of these actions. In 1995, DOE intends to complete another 100 interim actions, bringing the total to more than 500 since the program was initiated. ${ }^{2}$

Over half the sites in the EM- 40 program are managed under the Uranium Mill Tailings Remedial Action Program (UMTRAP) and the Formerly Utilized Sites Remedial Action Program (FUSRAP). The Albuquerque Operations Office is responsible for implementing UMTRAP, which was authorized in 1978, and involves the stabilization and control of (a) 24 inactive uraniumprocessing sites and associated vicinity properties located in 10 states and 2 Indian tribal lands and (b) vicinity properties associated with the Edgemont, South Dakota, inactive uranium mill site, which is owned by the Tennessee Valley Authority (see Fig. 6.2). All of the sites are located in the western United States, except for one in 
Canonsburg, Pennsylvania. RAs have been completed at 14 of the 24 inactive uranium processing sites. In 1995, RAs will be completed at one site, will continue at three sites, and will be initiated at four sites. RAs will be initiated at the final two sites in 1996. All surface contamination should be remediated by 1997, except for the vicinity properties at Grand Junction. ${ }^{2}$

The Oak Ridge Operations Office is responsible for implementing FUSRAP, which is primarily concerned with the cleanup of sites that were formerly used to support the activities of the Manhattan Engineer District, established for the Manhattan Project, and the Atomic Energy Commission (AEC). Private firms and institutions were contracted by the federal government in the 1940s and 1950 s to develop processes and perform research on radioactive materials. The storage and processing of uranium and thorium ores, concentrates, and residues were often involved. Although these sites were cleaned up to formerly acceptable levels, FUSRAP was established in 1974 to identify; reevaluate; and, if necessary, remediate these sites. Most FUSRAP sites are in the eastern half of the country. Currently, 46 sites have been identified in 14 states; 20 of these sites have already been remediated (see Fig. 6.3). RA at the balance of the FUSRAP sites is planned to be completed by $2016 .^{2}$

\subsection{ENVIRONMENTAL RESTORATION WASTE CHARACTERISTICS}

The volumes and types of wastes associated with $\mathrm{DOE}$ environmental restoration activities are a direct result of the remedy chosen. Waste associated with remediation of contaminated environmental media would occur only when such media are exhumed. For example, no waste would be produced at a site for which an in situ remedy was selected, such as capping an area containing contaminated soil. If a minimal remedial action were selected (e.g., pumping and treating a small pocket of contaminated ground water followed by construction of lateral barriers to minimize future migration), the site would have relatively small waste volumes. However, if large volumes of contaminated environmental media were removed, treated to provide a more suitable waste form for disposal, and then disposed of in an engineered facility, the site would have very large waste volumes.

Environmental restoration wastes are different from those associated with processing operations in that the former generally have much lower concentrations of radioactive and chemically hazardous substances. Much of the material requiring remediation is a consequence of past activities (e.g., spills, waste disposal, and environmental releases such as liquid discharges to drainage basins). In addition, operations within structures resulted in the contamination of equipment, walls, and floors from routine material-handling activities and from off-normal incidents such as spills and equipment failure. Decommissioning of these facilities will result in wastes such as wipes, concrete, metal, personal protective clothing, and decontamination solvents that have generally low concentrations of radioactive and chemical contaminants.

Environmental restoration wastes also differ from those resulting from processing operations in that the former are generally highly heterogeneous both in physical form and chemical constituency. For example, remediation of an abandoned waste pit could require the exhumation of all materials previously placed into the pit for disposal. This effort could involve any possible imaginable combination of objects ranging from small pieces of equipment and drums to entire vehicles such as trucks and forklifts. In addition, a full spectrum of contaminants could be associated with these previously disposed materials including those associated with ordnance operations, processing of uranium and thorium ores and concentrates, and the operation of nuclear reactors and associated chemical processing plants. This potential variety is in contrast to waste streams associated with processing activities that have relatively consistent chemical and physical properties.

Because most $D O E$ environmental restoration projects are in the assessment phase of the remedy-selection process, it is not possible to definitively project the wastes that will result from these projects. However, the volumes of radioactively contaminated solid media associated with the various environmental restoration sites are generally known. These volumes, which are based on historical knowledge, monitoring information, and fieldcharacterization results, are given in Table 6.1 for radioactively contaminated soil (including sediment and sludge) and in Table 6.2 for radioactively contaminated debris such as metal, concrete, brick, wood, asphalt, plastic, and rubble. The actual waste volumes that will result from cleanup of some sites may be significantly lower than those given in these two tables, especially for those sites for which minimal RAs are selected. Such minimal activities will likely occur at major DOE installations located in remote areas of the country. In addition, decontamination of metallic items such as hot-cell liners, tanks, and processing equipment could allow for unrestricted release of these items, which would reduce the volume of material requiring disposal.

The volumes of radioactively contaminated media given in Tables 6.1 and 6.2 are limited to solid materials (i.e., contaminated liquids such as surface-water impoundments and ground water are not included). These 
data are based on currently available information encompassing those projects for which characterization activities have proceeded to a point which allows for preparation of realistic volume estimates. Some projects have not yet initiated field-characterization activities, and historical records are not available in sufficient detail to allow for volume estimates to be made. The volume estimates for such sites will be larger than those shown in these two tables because not all of the potentially contaminated media would have been addressed.

Three major radioactive waste classes are associated with environmental restoration activities: LLW, TRU waste (TRUW), and $11 \mathrm{e}(2)$ by-product material. As defined in DOE Order $5820.2 \mathrm{~A}$, LLW is waste that contains radioactivity and is not classified as $\mathrm{HLW}$, TRUW, spent nuclear fuel (SNF), or $11 \mathrm{e}(2)$ by-product material. Environmental restoration activities are not expected to generate any HLW or SNF. TRUW is waste contaminated with alpha-emitting transuranium radionuclides with half-lives greater than 20 years and at concentrations greater than $100 \mathrm{nCi} / \mathrm{g}$ at the time of assay. As defined in Sect. 11e(2) of the AEA of 1954 (P.L. 83-703, as amended), 11e(2) by-product material is tailings or waste produced by the extraction or concentration of uranium or thorium from any ore processed primarily for its source material content. Materials being managed under Title 1 of the Uranium Mill Tailings Radiation Control Act of 1978 (P.L. 95-604) are defined as residual radioactive material distinct from $11 \mathrm{e}(2)$ by-product material. This residual radioactive material is largely uranium mill tailings (UMT), as well as soil and debris contaminated with UMT. Since this material has the same physical and radioactive properties as $11 \mathrm{e}(2)$ by-product material, it is reported in the accompanying tables with $11 \mathrm{e}(2)$ by-product material. In addition to radioactive contaminants, these wastes can also be contaminated with hazardous constituents as regulated by RCRA or TSCA; such wastes are considered mixed wastes. Thus, a total of six waste classes are relevant for radioactively contaminated material resulting from environmental restoration activities: LLW, mixed LLW (MLLW), TRUW, mixed TRUW (MTRUW), 11 e(2) byproduct material, and mixed $11 \mathrm{e}(2)$ by-product material.

The relative volumes of these six waste classes for contaminated soil and solid debris for environmental restoration sites are shown in Figs. 6.4 and 6.5, respectively. The two waste classes that constitute the largest volumes of radioactively contaminated soil are LLW and $11 \mathrm{e}(2)$ by-product material. The large volume of soil designated as LLW is mainly associated with three sites: NTS, LANL, and Hanford. Together, these three sites account for over $80 \%$ of the soil in this waste class. The actual amount of contaminated soil at these three sites that will eventually be handled as waste is highly uncertain because remedy- selection decisions have yet to be made. The large volume of $11 \mathrm{e}(2)$ by-product material is mainly the result of the large volume of residual radioactive material associated with UMT being remediated under UMTRAP.

The volume of radioactively contaminated debris is a very small fraction of that associated with soil. The total volume of contaminated debris is approximately $3,800,000 \mathrm{~m}^{3}$; the total volume of contaminated soil is approximately $73,000,000 \mathrm{~m}^{3}$. Most of this debris is classified as LLW (see Fig. 6.5), which accounts for about $78 \%$ of the total volume of radioactively contaminated debris. As with the volume estimates provided for soil, the total amount of debris that will be handled as waste could be lower than that given here if a significant fraction of this debris (e.g., metallic items) can be decontaminated to allow for reuse.

Because environmental restoration information is currently in the initial stages of being compiled at a number of sites, much of the information included in Tables 6.1 and 6.2 is preliminary. In addition, these tables include information only for sites currently in the EM-40 program for which data are available. That is, zero volumes may be reported for areas of a site at which characterization activities have not been initiated, thus resulting in an underestimate of the total volume of radioactively contaminated media. Conversely, overly conservative assumptions may be incorporated into some estimates to ensure the adequate sizing of treatment and disposal facilities. These uncertainties will continue to be reduced with time as characterization and engineering studies become available to refine these volume estimates.

As described earlier, RAs are currently being conducted at a number of sites, especially those associated with the FUSRAP and UMTRAP projects. Many of these RAs are small, interim actions. Wastes resulting from these activities are generally being managed at the site where the RA occurred. However, large volumes of UMT are being relocated (consolidated) for a number of UMTRAP sites. In addition, wastes resulting from RAs at some sites (such as those being remediated under FUSRAP) are being managed at commercial disposal facilities. One such commercial facility currently being utilized is the Envirocare Facility near Clive, Utah. A summary of radioactive wastes resulting from environmental restoration activities disposed of at this facility through the end of 1994 is given in Table 6.3. More than $6,100 \mathrm{~m}^{3}$ of wastes resulting from environmental restoration activities has been disposed of at that site.

The information contained in this chapter is limited to radioactively contaminated environmental media and 
wastes, consistent with the scope of this report. The volume estimates given in Tables 6.1 and 6.2 are limited to solid materials. Liquids, such as contaminated surface water and ground water and liquid wastes currently in storage, are not included. It should not be concluded that sites for which no (or minimal) volumes are given in Tables 6.1 and 6.2 have no waste management concerns. Environmental restoration activities at such sites could generate hazardous wastes as regulated by RCRA and TSCA, as well as large volumes of sanitary and demolition wastes. Also, additional characterization activities at these sites may identify areas of radioactive contamination requiring remediation in the future. As it becomes available, such information will be included in future updates of this report.

The volumes of radioactively contaminated soil and debris given in Tables 6.1 and 6.2 are limited to those sites and facilities currently in the EM-40 program. These data are summed across all elements of a site including environmental media, wastes currently in storage, and radioactively contaminated materials that could result from future decommissioning activities. At a number of sites, wastes resulting from EM-40 RA and decommissioning activities have been transferred to the Office of Waste Management (EM-30) for treatment, storage, and disposal. These wastes are no longer being managed by EM-40 and are therefore not included in this chapter. MLLW currently in storage at EM-40 sites and that projected to result from future RA and decommissioning activities are provided in Chapter 8 of this report.

The DOE Office of Facility Transition (EM-60) is currently preparing a detailed inventory of all facilities that may eventually be transferred to the Office of Environmental Management (EM-1). As facilities are transferred to EM-1, environmental restoration and waste management information will be developed and included in future updates of this report.

\subsection{REFERENCES}

1. R. P. Whitfield, U.S. Department of Energy, Deputy Assistant Secretary for Environmental Restoration, Washington, D.C., letter to distribution, “Contaminated Media/Waste Data Call Guidance," dated Oct. 8, 1993.

2. U.S. Department of Energy, Environmental Management 1995, Progress and Plans of the Environmental Management Program, DOE/EM-0228, Washington, D.C. (February 1995).

3. Al Rafati, Director, Envirocare of Utah, Inc., correspondence to Stephen N. Storch, Integrated Data Base Program, ORNL, Oak Ridge, Tennessee, dated May 24, 1995. 


\section{LOCATIONS OF FIELD OFFICES THAT DIRECT THE} DOE ENVIRONMENTAL RESTORATION PROGRAM

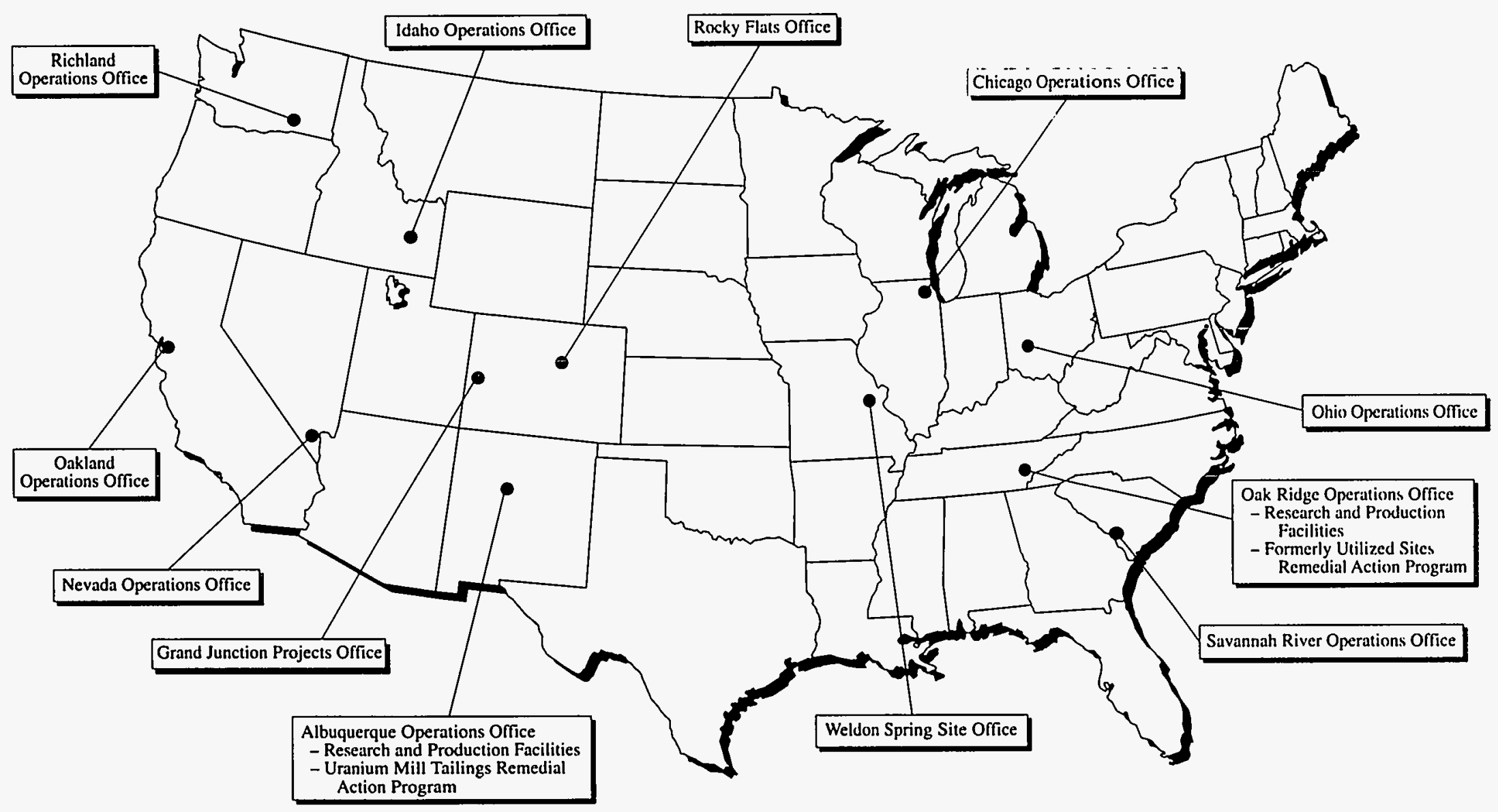

Fig. 6.1. Locations of field offices that direct the DOE environmental restoration program. 


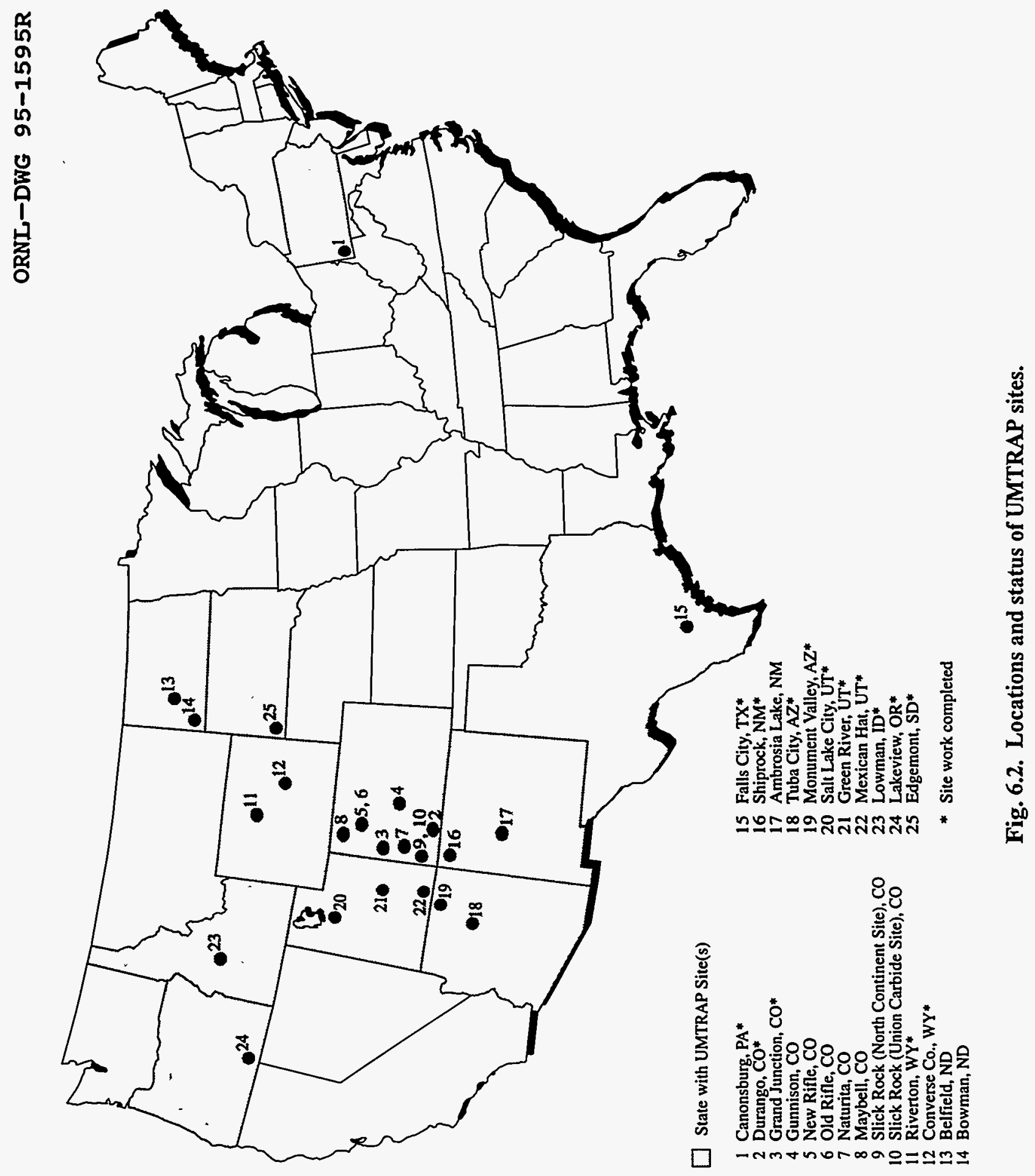




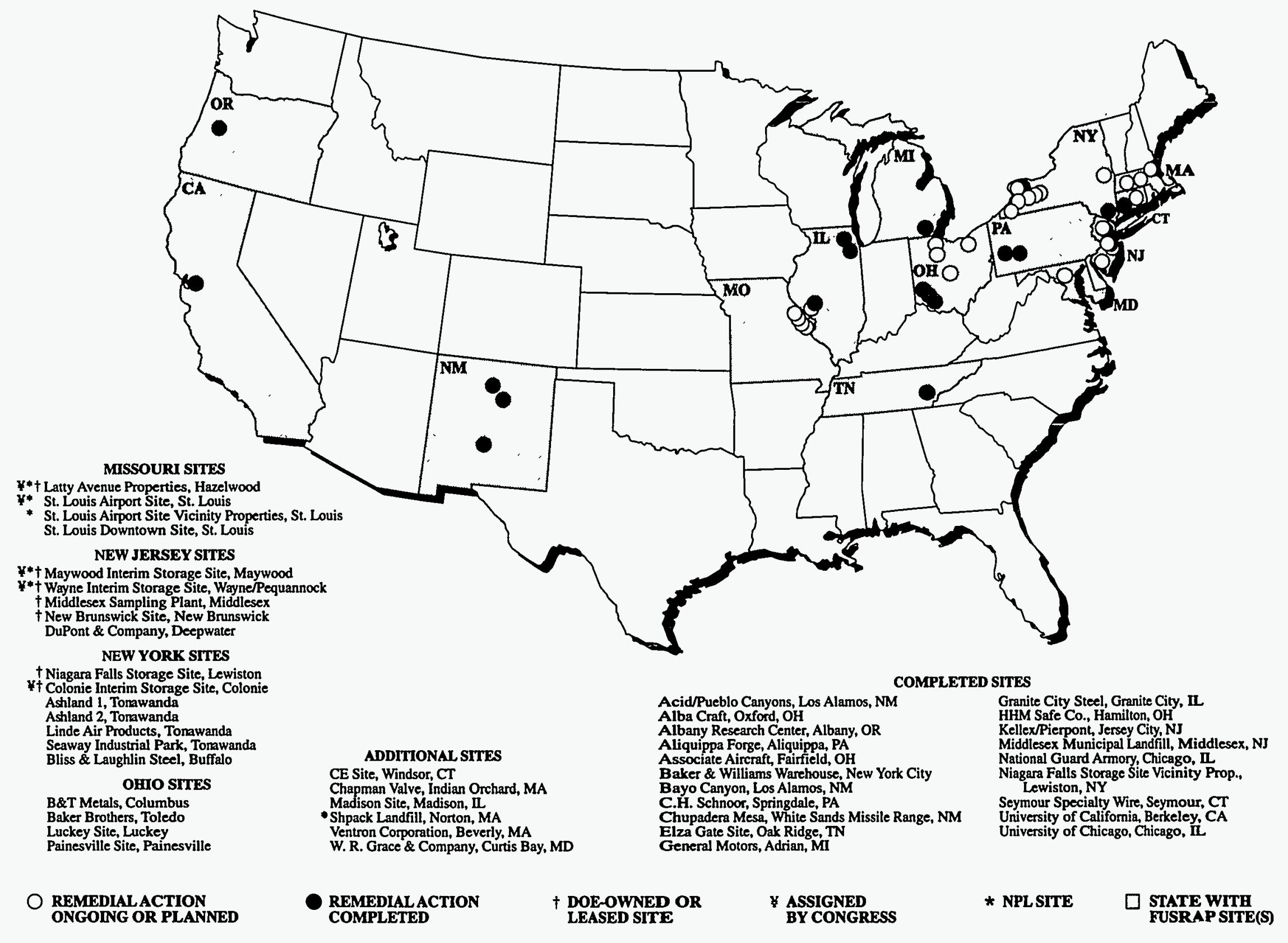

Fig. 6.3. Locations and status of FUSRAP sites. 
ORNL DWG 95-8315

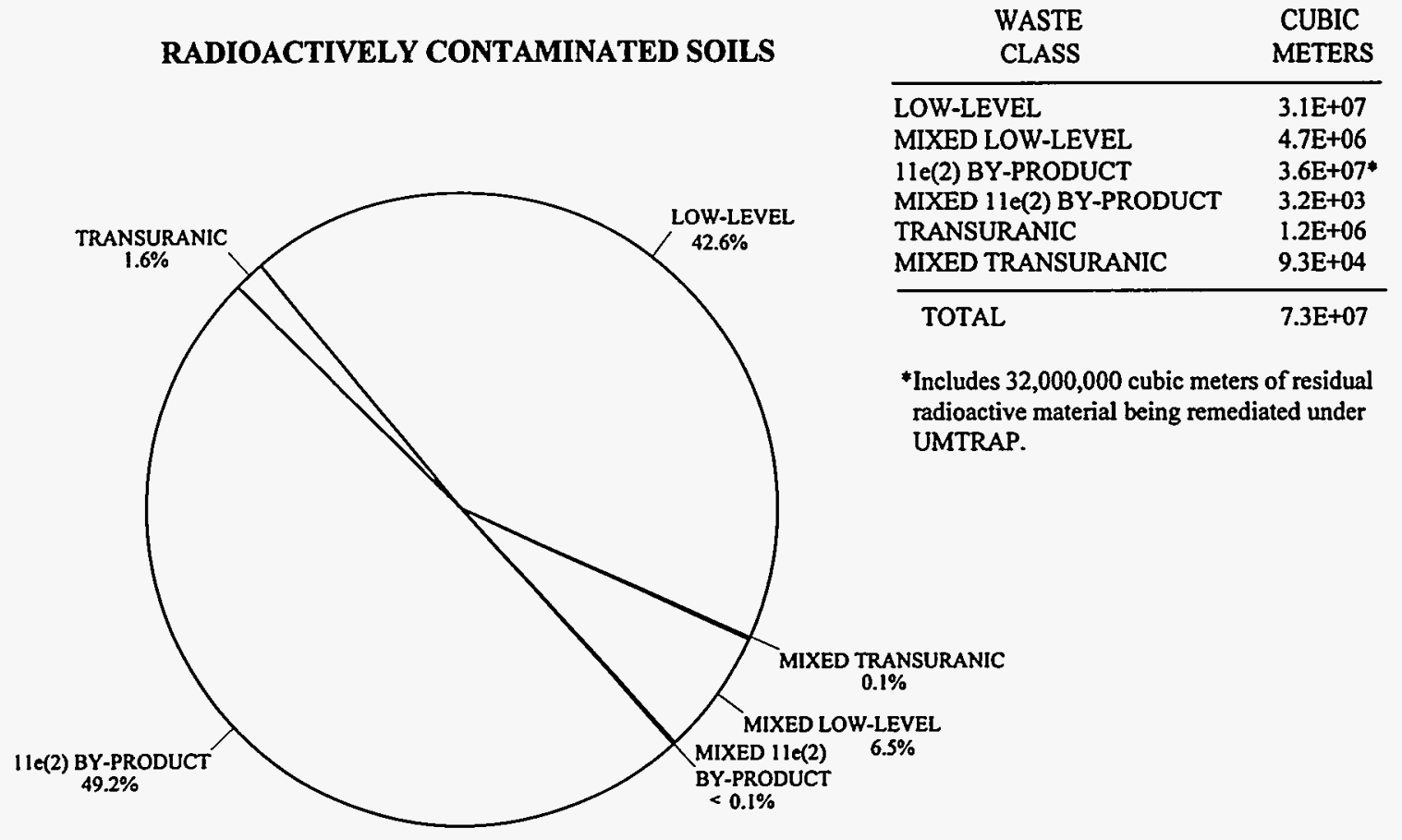

Fig. 6.4. Estimated volumes of radioactively contaminated soils associated with environmental restoration projects.

ORNL DWG 95-8316

RADIOACTIVELY CONTAMINATED DEBRIS

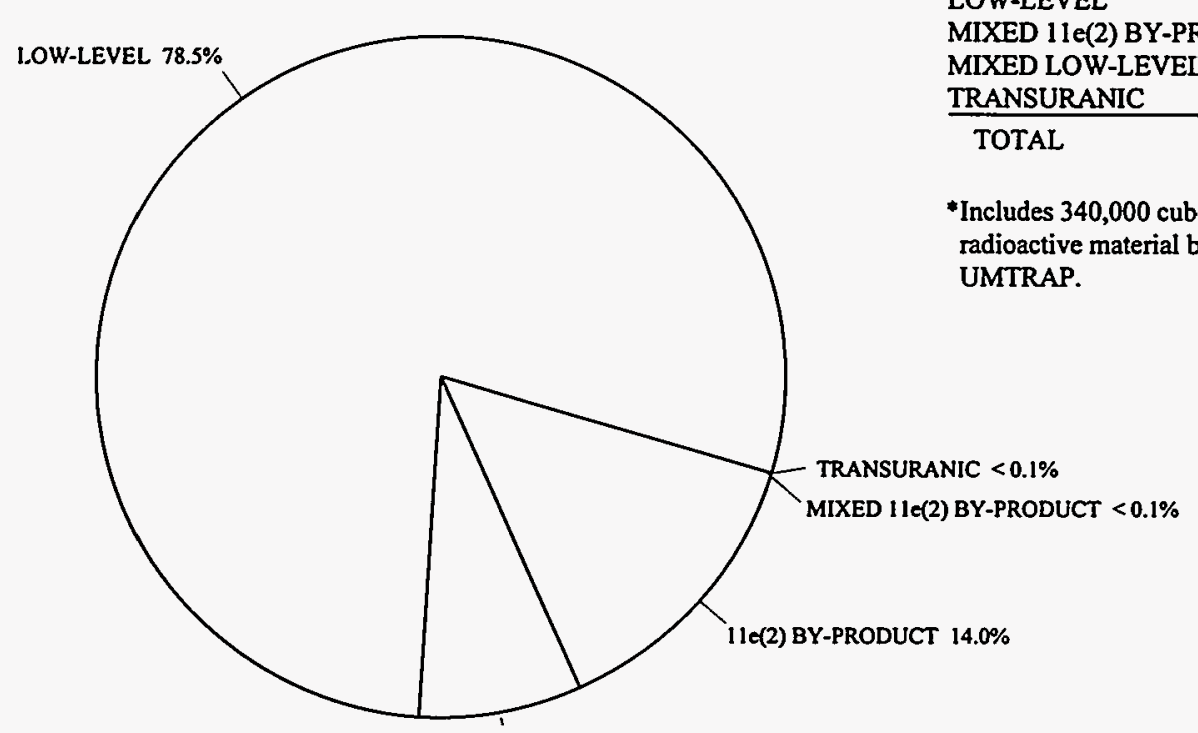

MIXED LOW-LEVEL $7.5 \%$

Fig. 6.5. Estimated volumes of radioactively contaminated debris associated with environmental restoration projects. 
Table 6.1. Estimated volumes of radioactively contaminated soils associated with environmental restoration projects $s^{\mathrm{a}, \mathrm{b}}$

\begin{tabular}{|c|c|c|c|c|c|c|}
\hline \multirow[b]{2}{*}{ Operations office } & \multicolumn{6}{|c|}{ Volume, $\mathrm{m}^{3}$} \\
\hline & LLW & MLLW & $\begin{array}{c}11 \mathrm{e}(2) \\
\text { by-product }\end{array}$ & $\begin{array}{c}\text { Mixed } 11 \mathrm{e}(2) \\
\text { by-product }\end{array}$ & TRUW & Total \\
\hline \multicolumn{7}{|l|}{ Albuquerque Operations Office } \\
\hline \multicolumn{7}{|l|}{ Research and Production Facilities } \\
\hline $\begin{array}{l}\text { Inhalation Toxicology Research Institute } \\
\text { Kansas City Plant }\end{array}$ & 9,000 & & & & & 9,000 \\
\hline Los Alamos National Laboratory & $8,300,000$ & $1,200,000$ & & & $93,000^{d}$ & $9,600,000$ \\
\hline Pantex Plant & & & & & & 0 \\
\hline Pinellas Plant & & & & & & 0 \\
\hline Sandia National Laboratories/California & & & & & & 0 \\
\hline Sandia National Laboratories/New Mexico & 15,000 & 68,000 & & & & 83,000 \\
\hline South Valley Superfund Site & & & & & & 0 \\
\hline Uranium Mill Tailings Remedial Action Programe & & & $32,000,000$ & & & $32,000,000$ \\
\hline \multicolumn{7}{|l|}{ Chicago Operations Office } \\
\hline Ames Laboratory & 1,300 & & & & & 1,300 \\
\hline Argonne National Laboratory-East & 7,700 & 17,000 & & & & 25,000 \\
\hline Argonne National Laboratory-West & 1 & & & & & 1 \\
\hline Battelle Columbus Laboratories & 640 & 24 & & & 390 & 1,000 \\
\hline Brookhaven National Laboratory & 20,000 & 3,600 & & & & 24,000 \\
\hline Hallam Site & & & & & & 0 \\
\hline Piqua Site & & & & & & 0 \\
\hline Princeton Plasma Physics Laboratory & & & & & & 0 \\
\hline Separations Process Research Unit & 14,000 & & & & 36 & 14,000 \\
\hline Site $\mathrm{A} /$ Plot $\mathrm{M}^{\mathrm{f}}$ & 4,500 & 500 & & & & 5,000 \\
\hline \multicolumn{7}{|l|}{ Grand Junction Projects Office } \\
\hline Grand Junction Projects Office Site & & & 35,000 & 46 & & 35,000 \\
\hline Monticello Remedial Action Projectg & & & $2,200,000$ & & & $2,200,000$ \\
\hline Idaho Operations Office & & & & & & \\
\hline Idaho National Engineering Laboratory & 350,000 & 170,000 & & & 170,000 & 690,000 \\
\hline \multicolumn{7}{|l|}{ Nevada Operations Office } \\
\hline Nevada Test Site & $14,000,000$ & 460 & & & & $14,000,000$ \\
\hline Nevada off-site locations $\mathrm{h}^{\mathrm{h}}$ & 9,500 & 21,000 & & & & 30,000 \\
\hline \multicolumn{7}{|l|}{ Oak Ridge Operations Office } \\
\hline \multicolumn{7}{|l|}{ Research and Production Facilities } \\
\hline K-25 Site & 3,300 & 1,700 & & & & 5,000 \\
\hline
\end{tabular}


Table 6.1 (continued)

\begin{tabular}{|c|c|c|c|c|c|c|}
\hline \multirow[b]{2}{*}{ Operations office } & \multicolumn{6}{|c|}{ Volume, $\mathrm{m}^{3}$} \\
\hline & LLW & MLLW & $\begin{array}{c}11 \mathrm{e}(2) \\
\text { by-product }\end{array}$ & $\begin{array}{c}\text { Mixed } 11 \mathrm{e}(2) \\
\text { by-product }\end{array}$ & TRUW & Total \\
\hline \multicolumn{7}{|l|}{ Oak Ridge Operations Office (continued) } \\
\hline \multicolumn{7}{|l|}{ Research and Production Facilities (continued) } \\
\hline Oak Ridge National Laboratory & 42,000 & 32,000 & & & 500 & 74,000 \\
\hline Oak Ridge Reservation (off-site) ${ }^{i}$ & 100,000 & & & & & 100,000 \\
\hline Paducah Gaseous Diffusion Plant & 1,800 & 540,000 & & & & 540,000 \\
\hline Portsmouth Gaseous Diffusion Plant & 7,700 & 2,200 & & & & 9,900 \\
\hline Y-12 Plant & 960 & 340,000 & & & & 340,000 \\
\hline \multicolumn{7}{|l|}{ Formerly Utilized Sites Remedial Action Program $j$} \\
\hline Missouri sites & 210,000 & & 500,000 & & & 720,000 \\
\hline New Jersey sites & 53,000 & $24,000^{k}$ & 390,000 & & & 460,000 \\
\hline New York sites & 9,400 & 12,000 & $460,000^{1}$ & & & 480,000 \\
\hline Other sites & 91,000 & 7,200 & 28,000 & & & 130,000 \\
\hline \multicolumn{7}{|l|}{ Oakland Operations Office } \\
\hline General Atomics Site & 240 & 65 & & & & 300 \\
\hline General Electric Vallecitos Nuclear Center & & & & & & 0 \\
\hline Laboratory for Energy-Related Health Research & 620 & & & & & 620 \\
\hline Lawrence Berkeley Laboratory & & & & & & 0 \\
\hline Lawrence Livermore National Laboratory & & & & & & 0 \\
\hline Santa Susana Field Laboratory & & & & & & 0 \\
\hline Stanford Linear Accelerator Center & & & & & & 0 \\
\hline \multicolumn{7}{|l|}{ Ohio Operations Office } \\
\hline Fernald Environmental Management Project & $2,600,000$ & 1,300 & 10,000 & & & $2,600,000$ \\
\hline Mound Plant & 140,000 & 17,000 & & & & 150,000 \\
\hline Reactive Metals, Inc., Site & 22,000 & 3,500 & & & & 25,000 \\
\hline \multicolumn{7}{|l|}{ Richland Operations Office } \\
\hline Hanford Site $\mathrm{m}$ & $3,100,000$ & 760,000 & & & & $3,900,000$ \\
\hline \multicolumn{7}{|l|}{ Rocky Flats Office } \\
\hline Rocky Flats Environmental Technology Site & 360 & 270,000 & & & & 270,000 \\
\hline \multicolumn{7}{|l|}{ Savannah River Operations Office } \\
\hline Savannah River Site & $1,400,000$ & $1,200,000$ & & & 990,000 & $3,600,000$ \\
\hline
\end{tabular}


Volume, $\mathrm{m}^{3}$

Operations office

\begin{tabular}{|c|c|c|c|c|c|}
\hline LLW & M LLW & $\begin{array}{c}11 \mathrm{e}(2) \\
\text { by-product }\end{array}$ & $\begin{array}{c}\text { Mixed } 11 \mathrm{e}(2) \\
\text { by-product }\end{array}$ & TRUW & Total \\
\hline & & 520,000 & 3,200 & & 530,000 \\
\hline $1,000,000$ & $4,700,000$ & $36,000,000$ & 3,200 & $1,300,000$ & $73,000,000$ \\
\hline
\end{tabular}

stimated as of December 31, 1994. Includes contaminated soil, sediment, and sludge. Blank entries mean there are no radioactively contaminated soils for the indicated aEstimated as of December 31, 1994. Includes contaminated soil, sediment, and sludge. Blank entries mean there are no radioactively contaminated soils for the indicated
waste class. Additional information on the quantity of mixed waste currently in storage as a result of environmental restoration activities is provided in Chapter 8 of this report.

${ }^{b}$ These volume estimates represent the quantity of in-place contaminated materials; the waste volumes resulting from environmental restoration activities may be larger or smaller depending on the selected remedy and treatment technology utilized. Waste volumes resulting from actions such as capping, monitoring, and certain in situ remedies will be quite small. All values are preliminary and are being updated as site characterization activities proceed. Values are given to two significant figures unless information was reported to only one significant figure. Some totals may not equal sum of components due to independent rounding.

'By-product material as defined in Sect. 11e(2) of the Atomic Energy Act of 1954 (P.L. 83-703), as amended. Materials being managed under Title 1 of the Uranium Mill Tailings Radiation Control Act of 1978 (P.L. 95-604) are defined as residual radioactive material. Since this material has the same physical and radioactive properties as 1 le(2) by-product material, it is reported here under $11 \mathrm{e}(2)$ by-product material.

dMixed TRUW.

${ }^{e} A$ listing of the sites being addressed under UMTRAP is given in Fig. 6.2. The volume of mill tailings associated with the 14 sites for which RAs have been completed is $21,000,000 \mathrm{~m}^{3}$, leaving $11,000,000 \mathrm{~m}^{3}$ of mill tailings for which RA is currently ongoing or planned.

Estimate is very preliminary and includes contaminated debris.

Includes contaminated debris, which will be managed in the same manner as contaminated soil.

h Consists of Amchitka Island, Alaska; the Rio Blanco and Rulison Test sites in Colorado; the Gnome and Gasbuggy Test sites in New Mcxico; the Salmon Test Site in Mississippi; and the Shoal and Central Nevada Test sites in Nevada.

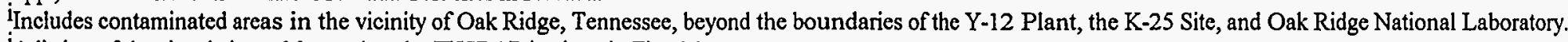

$\mathrm{j}_{\mathrm{A}}$ listing of the sites being addressed under FUSRAP is given in Fig. 6.3.

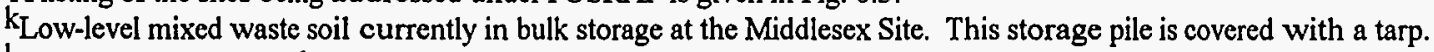

Includes the $195,000 \mathrm{~m}^{3}$ of soil and residues disposed of in a containment cell at the Niagara Falls Storage Site.

moes not include contaminated soil at the 200 Area of the Hanford Site, which is currently planned to be capped in place. A small percentage of the radioactively contaminated soil at the Hanford Site may be TRUW. 
Table 6.2. Estimated volumes of radioactively contaminated debris associated with environmental restoration projects ${ }^{\mathrm{a}, \mathrm{b}}$

Volume, $\mathrm{m}^{3}$

\begin{tabular}{|c|c|c|c|c|c|c|}
\hline \multirow{2}{*}{ Operations office } & \multirow[b]{2}{*}{ LLW } & \multirow[b]{2}{*}{ MLLW } & \multirow[b]{2}{*}{$\begin{array}{c}11 \mathrm{e}(2) \\
\text { by-product }^{\mathrm{c}}\end{array}$} & \multirow[b]{2}{*}{$\begin{array}{l}\text { Mixed } 11 \mathrm{e}(2) \\
\text { by-product }\end{array}$} & \multirow[b]{2}{*}{ TRUW } & \multirow[b]{2}{*}{ Total } \\
\hline & & & & & & \\
\hline \multicolumn{7}{|l|}{ Albuquerque Operations Office } \\
\hline \multicolumn{7}{|l|}{ Research and Production Facilities } \\
\hline Inhalation Toxicology Research Institute & 44 & & & & & 44 \\
\hline Kansas City Plant & & & & & & 0 \\
\hline Los Alamos National Laboratory & 9,000 & 1,600 & & & & 11,000 \\
\hline Pantex Plant & & & & & & 0 \\
\hline Pinellas Plant & & & & & & 0 \\
\hline Sandia National Laboratories/California & & & & & & 0 \\
\hline Sandia National Laboratories/New Mexico & 2,600 & 1,500 & & & & 4,100 \\
\hline South Valley Superfund Site & & & & & & 0 \\
\hline Uranium Mill Tailings Remedial Action Program ${ }^{d}$ & & & 340,000 & & & 340,000 \\
\hline \multicolumn{7}{|l|}{ Chicago Operations Office } \\
\hline Ames Laboratory & 58 & & & & & 58 \\
\hline Argonne National Laboratory-East & 12,000 & 120,000 & & & 190 & 140,000 \\
\hline Argonne National Laboratory-West & 150 & & & & & 150 \\
\hline Battelle Columbus Laboratories & 4,200 & & & & & 4,200 \\
\hline Brookhaven National Laboratory & 150 & 350 & & & & 490 \\
\hline Hallam Site & & & & & & 0 \\
\hline Piqua Site & & & & & & 0 \\
\hline Princeton Plasma Physics Laboratory & & & & & & 0 \\
\hline Separations Process Research Unit & 1,400 & 1.6 & & & & 1,400 \\
\hline Site $\mathrm{A} / \mathrm{Plot} \mathrm{M}^{\mathrm{e}}$ & & & & & & 0 \\
\hline \multicolumn{7}{|l|}{ Grand Junction Projects Office } \\
\hline Grand Junction Projects Office Site & & & 2,100 & 38 & & 2,200 \\
\hline Monticello Remedial Action Project ${ }^{\mathrm{f}}$ & & & & & & 0 \\
\hline \multicolumn{7}{|l|}{ Idaho Operations Office } \\
\hline Idaho National Engineering Laboratory & 24,000 & 2,200 & & & & 26,000 \\
\hline \multicolumn{7}{|l|}{ Nevada Operations Office } \\
\hline $\begin{array}{l}\text { Nevada Test Site } \\
\text { S }\end{array}$ & 3,800 & 290 & & & & 4,100 \\
\hline Nevada off-site locations $\mathrm{g}$ & & & & & & 0 \\
\hline \multicolumn{7}{|l|}{ Oak Ridge Operations Office } \\
\hline \multicolumn{7}{|l|}{ Research and Production Facilities } \\
\hline K-25 Site & 57,000 & 3,800 & & & & 60,000 \\
\hline
\end{tabular}


Table 6.2 (continued)

Volume, $\mathrm{m}^{3}$

\section{Operations office}

\begin{tabular}{|c|c|c|c|c|c|}
\hline LLW & MLLW & $\begin{array}{c}11 \mathrm{e}(2) \\
\text { by-product }\end{array}$ & $\begin{array}{c}\text { Mixed 11e(2) } \\
\text { by-product }\end{array}$ & TRUW & Total \\
\hline
\end{tabular}

Oak Ridge Operations Office (continued)

Research and Production Facilities (continued)

Oak Ridge National Laboratory

Oak Ridge Reservation (off-site) ${ }^{h}$

Paducah Gaseous Diffusion Plant

Portsmouth Gaseous Diffusion Plant

Y-12 Plant

Formerly Utilized Sites Remedial Action Program ${ }^{\mathrm{i}}$

Missouri sites

New Jersey sites

New York sites

Other sites

Oakland Operations Office

General Atomics Site

General Electric Vallecitos Nuclear Center

Laboratory for Energy-Related Health Research

Lawrence Berkeley Laboratory

Lawrence Livermore National Laboratory

Santa Susana Field Laboratory

1,700

510

23,000

$91 \quad 15,000$

$\begin{array}{rr}54,000 & 200 \\ 410 & 4,600\end{array}$

15,000

54,000

5,100

$130 \quad 17,000$

$6 \quad 65$

5,100

690

10

65
000

680

21

390

9,400

1,000

Stanford Linear Accelerator Center

Ohio Operations Office

Fernald Environmental Management Project

Mound Plant

Reactive Metals, Inc., Site

Richland Operations Office

Hanford Sitej

Rocky Flats Office

Rocky Flats Environmental Technology Site

Savannah River Operations Office

Savannah River Site

370,000

28,000

6,200

$2,300,000$

120,000 
Table 6.2 (continued)

\begin{tabular}{lcccccc}
\hline & \multicolumn{4}{c}{ Volume, $\mathrm{m}^{3}$} \\
\cline { 2 - 6 } Operations office & LLW & MLLW & $\begin{array}{c}1 \text { 1le(2) } \\
\text { by-product }\end{array}$ & $\begin{array}{c}\text { Mixed 1le(2) } \\
\text { by-product }\end{array}$ & TRUW & Total \\
\hline $\begin{array}{l}\text { Weldion Spring Site Ofice } \\
\text { Weldon Spring Site Remedial Action Project } \\
\text { Total }\end{array}$ & & 150,000 & 1,700 & 160,000 \\
\hline
\end{tabular}

${ }^{a}$ Estimated as of December 31, 1994. Includes contaminated metal, concrete, brick, wood, asphalt, plastic, rubble, and other similar materials. Blank entries mean there are no radioactively contaminated debris for the indicated waste class. Additional information on the quantity of mixed waste currently in storage as a result of environmental restoration activities is provided in Chapter 8 of this report.

${ }^{\mathrm{b}}$ These volume estimates represent the quantity of in-place contaminated materials; the waste volumes resulting from environmental restoration activities may be larger or smaller depending on the selected remedy and treatment technology utilized. Waste volumes resulting from actions such as capping, monitoring, and certain in situ remedies will be quite small. All values are preliminary and are being updated as site characterization activities proceed. Values are given to two significant figures unless information was reported to only one significant figure. Some totals may not equal sum of components due to independent rounding.

${ }^{{ }^{B} B y-p r o d u c t ~ m a t e r i a l ~ a s ~ d e f i n e d ~ i n ~ S e c t . ~ 1 l e(2) ~ o f ~ t h e ~ A t o m i c ~ E n e r g y ~ A c t ~ o f ~} 1954$ (P.L. 83-703), as amended. Materials being managed under Title 1 of the Uranium Mill Tailings Radiation Control Act of 1978 (P.L. 95-604) are defined as residual radioactive material. Since this material has the same physical and radioactive properties as 11 e(2) by-product material, it is reported here under $11 \mathrm{e}(2)$ by-product material.

$\mathrm{d}_{\mathrm{A}}$ listing of the sites being addressed under UMTRAP is given in Fig. 6.2. The volume of debris associated with the 14 sites for which RAs have been completed is $260,000 \mathrm{~m}^{3}$, leaving $78,000 \mathrm{~m}^{3}$ of debris for which RA is currently ongoing or planned.

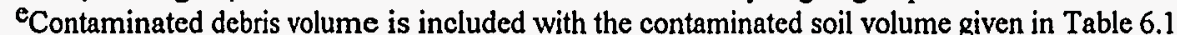

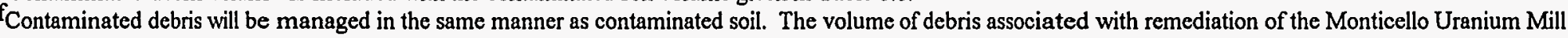
Tailings Site and vicinity properties is included with the contaminated soil volume given in Table 6.1.

8Consists of Amchitka Island, Alaska; the Rio Blanco and Rulison Test sites in Colorado; the Gnome and Gasbuggy Test sites in New Mexico; the Salmon Test Site in Mississippi; and the Shoal and Central Nevada Test sites in Nevada.

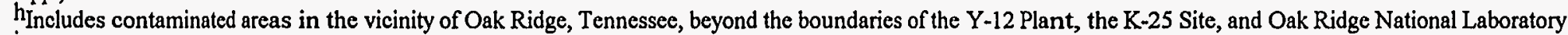

${ }^{1} \mathrm{~A}$ listing of the sites being addressed under FUSRAP is given in Fig. 6.3.

$\mathrm{j}_{\mathrm{A}}$ small percentage of the radioactively contaminated debris at the Hanford Site may be TRUW. 
Table 6.3. Summary of environmental restoration wastes disposed of at the Envirocare Facility ${ }^{a}$

\begin{tabular}{|c|c|c|}
\hline Originating site & Waste class & $\begin{array}{c}\text { Volume } \\
\left(\mathrm{m}^{3}\right)\end{array}$ \\
\hline Alba $\mathrm{Craft}^{\mathrm{b}}$ & LLW & 342 \\
\hline Aliquippa Forge ${ }^{b}$ & LLW & 310 \\
\hline Baker and Williams Warehouse ${ }^{b}$ & LLW & 8 \\
\hline Baker Brothers $^{b}$ & LLW & 582 \\
\hline Battelle Columbus Laboratories & LLW & 28 \\
\hline C. H. Schnoor ${ }^{b}$ & LLW & 159 \\
\hline \multirow[t]{2}{*}{ Colonie Interim Storage Site ${ }^{b}$} & LLW & 31 \\
\hline & MLLW & 18 \\
\hline Fernald Environmental Management Project & MLLW & 149 \\
\hline Granite City Steel ${ }^{\mathrm{b}}$ & LLW & 1 \\
\hline Maywood Interim Storage Site ${ }^{b}$ & $11 \mathrm{e}(2)$ by-product & 3,822 \\
\hline Paducah Gaseous Diffusion Plant & MLLW & 4 \\
\hline Portsmouth Gaseous Diffusion Plant & MLLW & 53 \\
\hline Reactive Metals, Inc., Site & MLLW & 3 \\
\hline Santa Susana Field Laboratory & MLLW & 337 \\
\hline Seymour Specialty Wire ${ }^{b}$ & LLW & 31 \\
\hline Wayne Interim Storage Site ${ }^{b}$ & LLW & 267 \\
\hline \multirow[t]{3}{*}{ Total } & LLW & 1,759 \\
\hline & MLLW & 564 \\
\hline & $11 \mathrm{e}(2)$ by-product & 3,822 \\
\hline Grand total & & 6,145 \\
\hline
\end{tabular}

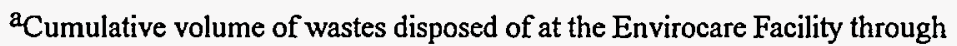
December 31, 1994; most of these wastes were disposed of in 1994. Waste volumes are limited to those originating from environmental restoration activities. Information obtained from ref. 3 .

b Sites being addressed under FUSRAP. A complete listing of FUSRAP sites is given in Fig. 6.3. 


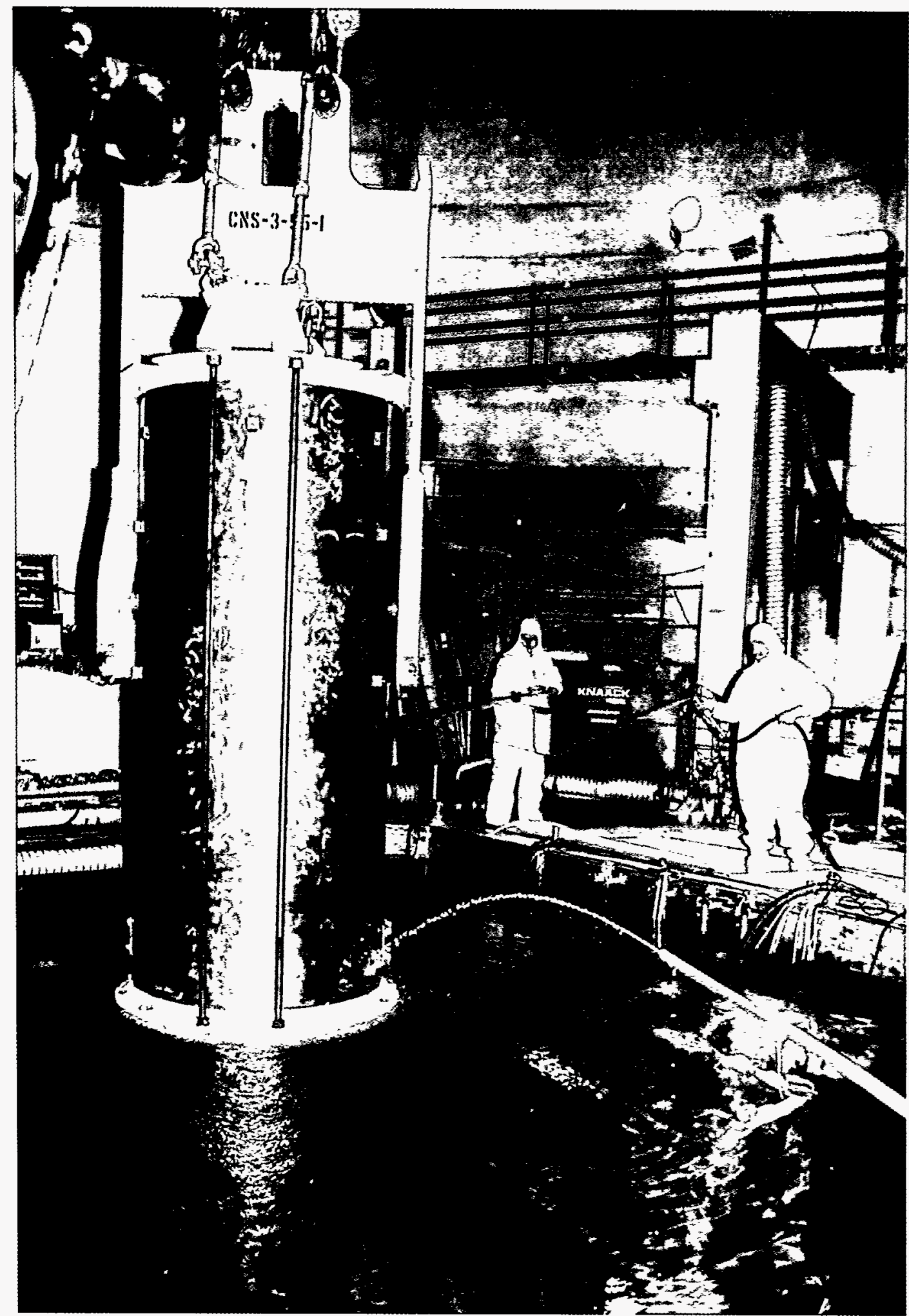

Handling activities of a cask containing highly radioactive internal components that were removed from the vessel of the Yankee Nuclear Power Station's pressurized-water reactor. Courtesy of the Yankee Atomic Electric Company, Bolton, Massachuselts. 



\section{COMMERCIAL DECOMMUSSIONING WASTES}

\subsection{INTRODUCTION}

At the end of their useful life, commercial nuclear facilities must be shut down and decommissioned. This chapter considers the radioactive wastes from the decontamination and decommissioning (D\&D) of commercial power reactors and supporting fuel cycle facilities (viz., uranium conversion, enrichment, fuel fabrication, and fuel reprocessing). As described in ref. 1 , the U.S. Nuclear Regulatory Commission (NRC) has defined the three major alternative classifications for decommissioning nuclear facilities:

- DECON- " . . the alternative in which the equipment, structures, and portions of a facility and site containing radioactive contaminants are removed or decontaminated to a level that permits the property to be released for unrestricted use shortly after cessation of operations."

- SAFSTOR - ". . . the alternative in which the nuclear facility is placed and maintained in such condition that the nuclear facility can be safely stored and subsequently decontaminated (deferred decontamination) to levels that permit release for unrestricted use."

- ENTOMB - " ... the alternative in which radioactive contaminants are encased in a structurally long-lived material, such as concrete. The entombment structure is appropriately maintained, and continued surveillance is carried out until the radioactivity decays to a level permitting the unrestricted release of property." (This alternative would be allowable for nuclear facilities contaminated with relatively short-lived radionuclides such that all contaminants would decay to levels permissible for unrestricted use within a period on the order of 100 years.)

Table 7.1 lists the major power reactors that have undergone some mode of decommissioning to date (data from refs. 2 and 3). A comprehensive listing of all types of domestic reactors that have been shut down or dismantled is provided in ref. 2. Those fuel cycle facilities that have been permanently shut down as of the end of 1993 are listed in Table 7.2 .

Several studies have been made of the D\&D of commercial LWRs and their supporting fuel cycle facilities. Most notable were those prepared and documented by Battelle Pacific Northwest Laboratory (PNL) for the NRC. These included decommissioning analyses for a reference BWR and PWR, documented in refs. 4-6 and refs. 7-9, respectively. In recent years, PNL, prepared and issued draft revised decommissioning analyses for both the reference BWR (ref. 10) and reference PWR (ref. 11). These analyses include estimates of the radioactive wastes from $D \& D$, which include lowlevel waste (LLW) (Class A, B, and C) and some greaterthan-Class-C (GTCC) wastes.

Table 7.3 summarizes projections of radioactive wastes from decommissioning reference LWRs and their supporting fuel cycle facilities (data from refs. 10-15). Most of the waste estimates reported assume a 40-year facility operating life. In practice, operating lifetimes can vary significantly, depending on the extent to which a facility's equipment is periodically upgraded or retrofitted. Not shown in Table 7.3 are the radioactive wastes that will result from the decommissioning of research, training, and test reactors. ${ }^{16,17}$ However, the quantities of these wastes are not expected to be significant because such reactors are much smaller than commercial power reactors.

\subsection{WASTE CHARACTERIZATION}

Radioactive waste from LWR decommissioning can be grouped into three major categories. ${ }^{., 8}$ (1) neutron-activated wastes, (2) contaminated wastes, and (3) miscellaneous wastes.

Neutron-activated materials include the reactor vessel and its internal components (e.g., core shroud, core support assemblies, and control-rod guide tubes) and the inner portion of the biological shield. Contaminated materials include much of the piping and equipment in the 
reactor containment, fuel-handling, and auxiliary control buildings. In addition, some concrete surfaces of the.e buildings are expected to be radioactive and will require removal. The miscellaneous radioactive waste category includes both "wet" and "dry" solids. Wet waste matcrials result from the processing of chemical decontamination solutions and contaminated water and include spent ionexchange resins, cartridge filters, and evaporator and concentrator bottoms. Dry radioactive wastes include discarded contaminated items such as rags and wipes, tools, and protective clothing.

Many reactor items with surface contamination can be decontaminated, ${ }^{18}$ thereby rendering most of the material nonradioactive and producing a smaller, more concentrated volume of waste containing the radioactivity. Waste decontamination requires the appropriate technology and a defined level of radioactivity at which the contamination level is regarded as acceptable. Depending on available technology and minimum regulatory-level specification, actual quantities of wastes from reactor and fuel cycle facility decommissioning could vary significantly from projected amounts currently reported.

LWR decommissioning operations collect LLW and a small volume of high-activity wastes from the neutron activation of internal parts of the reactor core. Under NRC rules, some of these neutron activation wastes would be classified as GTCC LLW. Some GTCC wastes contain significant concentrations of ${ }^{60} \mathrm{Co}$ and long-lived, nontransuranic radioisotopes, such as ${ }^{59} \mathrm{Ni},{ }^{63} \mathrm{Ni}$, and ${ }^{94} \mathrm{Nb}$. These isotopes are produced by the long-term irradiation (neutron activation) of the stainless steel and other alloys that comprise reactor-core structural components. Because the method of waste disposal for these reactor internals is different from LLW disposal, GTCC wastes are reported separately. Under current NRC regulations, ${ }^{19,20}$ these wastes are considered not generally acceptable for shallow-land disposal. Such wastes must be put into a federal geologic repository unless the NRC approves an alternative disposal in a licensed site. High-activity activation wastes from the DECON decommissioning of LWRs are estimated to make up less than $1 \%$ of the total radioactive waste volume, but contain more than $95 \%$ of the radioactivity. ${ }^{5,8}$ Most of this radioactivity is in a single reactor component, the stainless steel core shroud that surrounds the reactor fuel.

In recent years, analyses have been made of radioactive wastes from the Shippingport Station D\&D and of neutron-activated metal components (GTCC materials) from the internals of other reactors. Waste characterization assessments from studies such as refs. 21 and 22 have thus far indicated the following properties of reactor decommissioning materials:
- All reactor decommissioning materials, except the pressure vessel internals, have the potential for being disposed of as Class-A LLW.

- Fission products and TRU radionuclides are absent.

- Most radioactivity results from neutron-activated products, of which ${ }^{60} \mathrm{Co}$ is the principal contributor.

\subsection{INVENTORIES AND PROJECTIONS}

\subsubsection{Commercial Power Reactors}

A schedule of historical and projected commercial power LWR shutdowns, based on refs. 2 and 23, is given in Table 7.4. Projections of shutdown capacity listed are based on estimated reactor lifetimes. Actual reactor decommissioning schedules may be significantly different from those indicated in this report if any of the following are implemented:

- reactors are upgraded to extend their operating lifetimes,

- significant radioactivity decay time is allowed before D\&D operations begin, or

- the last core of spent nuclear fuel (SNF) is required to remain on-site for a minimum period (possibly several years) before shipment.

Table 7.5 reports quantities of actual wastes shipped from reactor sites that either have completed or are undergoing DECON (dismantlement). Completed DECON projects include the Elk River BWR; the Pathfinder BWR; the sodium reactor experiment at Santa Susana, California; and the Shippingport Station. Specific decommissioning waste characteristics for Elk River, Santa Susana, and Shippingport are given in Tables 7.5, 7.6, and 7.8, respectively, of the IDB Rev. 9 report. ${ }^{24}$ Table 7.14 of the IDB Rev. 10 report ${ }^{25}$ gives quantities of disposed decommissioning wastes for the Pathfinder BWR.

Projected volumes and undecayed radioactivity levels of wastes from decommissioning alternatives considered for reference model LWRs are summarized in Table 7.6. The volume estimates given in this table reflect revisions reported by PNL in updated LWR decommissioning analyses recently performed for the NRC. ${ }^{10-11}$ These revised estimates reflect significant reductions from the quantities previously reported in earlier PNL studies. ${ }^{4-9}$ The reductions are the result mainly of a large decline in the projected quantities of radioactively contaminated concrete debris resulting from DECON activities and to packaging assumptions that resulted in higher packaging densities for LLW, compared with the earlier PNL studies. 
The estimated cumulative volume, decayed radioactivity, and thermal power of radioactive wastes from decommissioning commercial power LWRs shut down during the period 1995-2030 are provided in Table 7.7. These waste projections are based on the results of the PNL reference reactor studies (Table 7.6) and represent additional quantities to those previously reported in Chapter 4 for commercial LLW. This approach is taken because the timing associated with future commercial power reactor D\&D activities is uncertain over the long term. Projected waste data shown in Table 7.7 are based on the projected LWR shutdown schedule given in Table 7.4 and decommissioning waste source terms developed from Table 7.6 and refs. 4-11. (The source terms are described in Appendix A.) The projections of Table 7.7 reflect a delayed DECON scenario, which assumes a 10-year period for decommissioning, beginning 6 years after reactor shutdown to allow sufficient preparation for $D \& D$ operations and cooling time for the last batch of SNF from the reactor's core. It is further assumed that the D\&D wastes will be shipped to a disposal site in equal volumes during each of the 4 years that the facility will be dismantled. (For comparison, it should be noted that the period for disassembly and decontamination used in the PNL studies of refs. 10 and 11 was 1.7 years following the emptying of the SNF pool, not 4 years.)

Radioactive waste inventories and projections are being compiled for several ongoing or recently completed commercial reactor decommissioning projects. Alphabetically listed, the reactors include Dresden-Unit 1 BWR, Fort St Vrain HTGR, Humboldt Bay-Unit 3 BWR, Indian Point-Unit 1 PWR, La Crosse BWR, Rancho Seco PWR, San Onofre-Unit 1 PWR, Saxton PWR, Shoreham BWR, Three Mile Island (TMI)-Unit 2 PWR, Trojan PWR, and Yankee Rowe PWR. Major highlights of the D\&D activities at each of these reactor sites follows.

A summary of projected radioactive materials from the SAFSTOR decommissioning of the Dresden-Unit $1 \mathrm{BWR}$ station is given in Table 7.8 (data from ref. 26). These estimates are based on the Dresden-Unit 1 Decommissioning Plan and Environmental Report, which was issued by the Commonwealth Edison Company. Commonwealth Edison plans to place the Unit 1 station in a SAFSTOR condition until Dresden-Units 2 and 3 are ready for decommissioning. If an extended-life program for Units 2 and 3 is not initiated, then current utility plans call for all three Dresden units to be decommissioned and dismantled; this would begin in 2017.

Actual inventories (through 1994) of wastes from D\&D activities at the Fort St. Vrain HTGR are reported in Table 7.9 (data from ref. 27). In August 1989, the 330-MW(e) Fort St. Vrain reactor was shut down to replace an inoperable control rod. During this forced outage, stress cracking of the feed-water ring headers to the steam generators was noted. Because of the potential for very large fuel costs due to the cancellation of utility orders for other HTGRs, a decision was made to permanently cease reactor operations. The DECON option was selected as the mode of decommissioning. During 1994, most remaining core internals were removed and disposed of. ${ }^{28}$ Projections of total wastes from DECON of the Fort St. Vrain reactor are reported in Table 7.10 (data from refs. 29 and 30).

Projections of wastes from decommissioning the 65-MW(e) Humboldt Bay-Unit 3 BWR are reported in Table 7.11 (data from ref. 31). Projections for the Humboldt Bay BWR include wastes from completely dismantling the reactor following a SAFSTOR period of 30 years (i.e., SAFSTOR with delayed DECON).

Table 7.12 (data from ref. 32) reports projections of wastes from the Indian Point-Unit 1 reactor. This 265-MW(e) PWR was shut down in 1974 and later placed in SAFSTOR. The projections of Table 7.12 pertain to a case of dismantlement (DECON) of the Unit 1 station upon completion of its SAFSTOR phase, which will occur when Unit 2 (PWR) is finally shut down.

The La Crosse BWR was shut down in 1987 and placed in SAFSTOR in 1991. Current plans are to dismantle the reactor after a SAFSTOR period of 30 to 50 years. Projected volumes and associated activities of annual waste shipments from this reactor during this period are given in Table 7.13 (data from ref. 33).

The 918-MW(e) Rancho Seco PWR was permanently shut down in 1989. Table 7.14 (data from ref. 34) lists projected volumes of wastes from the dismantlement of this reactor following a SAFSTOR period of about 20 years.

The San Onofre-Unit l PWR was shut down in 1992. Estimates of wastes from decommissioning this 436-MW(e) reactor are given in Table 7.15 (data from ref. 35). These projections are reported for a case of DECON following a SAFSTOR period of about 20 years. Dismantlement of San Onofre-Unit 1 is projected to start in 2013.

The Saxton Nuclear Experimental Reactor is a 23.5 MW(t) PWR that was placed in SAFSTOR following its shutdown in 1972. A three-phase decommissioning effort (DECON) was begun in 1986. Phase 1 consisted of the removal of groundwater from reactor support structures and was completed in 1987. Phase 2 involved the decontamination, final survey, and demolition of all structures except the containment vessel (reactor building); this phase was completed in 1992. The final phase will decommission the containment vessel and restore the site for unrestricted use. This phase is scheduled to be 
completed in 2000. A summary of projected waste volumes from Saxton decommissioning is provided in Table 7.16 (data from ref. 36).

The 820-MW(e) Shoreham BWR underwent lowpower tests until 1989, when the plant's owner, Long Island Lighting Company, agreed to sell the plant to the state of New York for decommissioning. A decommissioning plan $^{37}$ for the Shoreham plant was approved by the NRC in $1992 .^{38}$ That same year prompt decontamination and dismantling (DECON) of the Shoreham BWR began, and these activities were completed in 1994. Inventories of wastes from the Shoreham BWR decommissioning are reported in Table 7.17 (data from ref. 39).

Inventories of wastes shipped from the TMI-Unit 2 PWR are summarized in Table 7.18 (data from ref. 40). Removal of core debris from the damaged reactor was begun in January 1986 and completed in April 1990. This resulted in a shipment of 155.9 t of core debris to INEL for R\&D testing and storage. TMI-Unit 2 is currently in the Post Defueling Monitored Storage (PDMS) mode after completing preparation activities in December 1993. Criteria for initiating and implementing the PDMS phase were approved through an NRC review of a licensing change request. ${ }^{40}$

Table 7.19 gives projected classification and volumes of radioactive wastes from Trojan PWR decommissioning activities (data from ref. 41). Following its permanent shutdown in January 1993, the Trojan reactor was placed in a short SAFSTOR condition prior to DECON. The projections of Table 7.19 pertain to wastes from both the SAFSTOR and DECON phases of decommissioning. The Large Component Removal Project is currently in progress. As part of this project, concrete blocks were recently removed from the reactor's containment building to form an opening for the future removal of four steam generators and a pressurizer. The current Trojan PWR decommissioning plan includes the removal of these large components and their shipment by barge to the commercial Richland site for disposal. Decontamination and dismantlement of the radioactive contaminated portions of the plant are currently scheduled to be completed by $2001 .^{41}$

The 167-MW(e) Yankee Rowe PWR was shut down in 1992, and projections of wastes from its decommissioning are reported in Table 7.19 (data from ref. 42). These projections are reported for two major periods of project decommissioning: component removal (1993-1994), which includes removal of the reactor vessel internal components and steam generator; and final decommissioning (1995-1997, 1999), which includes the remainder of the reactor's components and general plant inventories. Quantities reported for the Component
Removal Project consist of waste disposed prior to the closure of the Barnwell disposal site to Massachusetts LLW generators in 1994. Recently, the final decommissioning project began after the Barnwell facility was reopened to Massachusetts generators in July 1995. Final decommissioning of the Yankee Rowe PWR is expected to be completed in two phases, the first during 1995-1997 and the second in 1999.42

Currently, the total impact of wastes from $D \& D$ activities at commercial reactor sites has been small. However, as the projected schedule of Table 7.4 suggests, the impact will become more significant after the year 2000 , when more of the older reactors complete their campaign of operation.

\subsubsection{Supporting Fuel Cycle Facilities}

Table 7.2 lists major facilities that once provided $\mathrm{UF}_{6}$ conversion, enrichment, fuel fabrication, and fuel reprocessing services for the commercial nuclear fuel cycle. Details of the radioactive waste inventories and projections associated with decommissioning each of these facilities are given below.

Inventories and projections of wastes from decommissioning activities at the Cimarron (Oklahoma) Fuel Fabrication Facility are provided in Table 7.20 (data from ref. 43). Decontamination work at this fabrication plant is scheduled to be completed during 1995. Shut down since 1975, this facility is comprised of two former fabrication plants, one for uranium fuel and one for mixedoxide fuel.

Table 7.21 (data from ref. 44) presents a summary of the waste inventories and projections from activities at the West Valley Demonstration Project (WVDP), formerly a commercial fuel reprocessing facility. Since startup of this project in 1982, more than $70 \%$ of the cell surface areas of the original process building have been decontaminated and released for project reuse.

\subsubsection{Noncommercial Facilities}

Significant quantities of radioactive wastes have and will also be produced from decommissioning noncommercial facilities. These include wastes from D\&D activities performed at various DOE installations, discussed in Chapter 6, and submarine power reactor D\&D activities performed for the U.S. Department of Defense. During a period spanning 20 to 30 years, about 100 nuclear-powered submarines of the U.S. Navy may be removed from service and consigned to permanent disposal after removal of the SNF. Current plans are to dispose of the submarine reactor compartments by land burial at the Hanford Site. Each reactor compartment 
contains about $1000 \mathrm{t}$ of metal, and it is estimated that about 4 ha (10 acres) will be required to bury 100 reactor compartments. ${ }^{45}$ At the end of 1994,78 submarines and 4 cruisers had been taken out of active service. In 43 of the submarines, the reactor compartment was first defueled, then later removed, and disposed of at Hanford. (LLW disposed from these activities is included in the DOE site inventories reported in Chapter 4.) The remaining 39 ships with reactor compartments were being held in protective storage. ${ }^{2}$

\subsection{REFERENCES}

1. U.S. Nuclear Regulatory Commission, "General Requirements for Decommissioning Nuclear Facilities," Fed. Regist. 53(123), 24018-56 (June 27, 1988).

2. U.S. Department of Energy, Office of Scientific and Technical Information, Nuclear Reactors Built, Being Built, or Planned: 1994, DOE/OSTI-8200-R58, Oak Ridge, Tennessee (August 1995).

3. J. T. A. Roberts et al., "Decommissioning of Commercial Nuclear Power Plants," Annual Reviews of Energy, Annual Reviews Inc., Palo Alto, California, 10, 251-284 (1985).

4. H. D. Oak et al., Technology, Safety and Costs of Decommissioning a Reference Boiling Water Reactor Power Station, NUREG/CR-0672, Vols. 1 and 2, U.S. Nuclear Regulatory Commission, Washington, D.C. (June 1980).

5. E. S. Murphy, Technology, Safety and Costs of Decommissioning a Reference Boiling Water Reactor Power Station-Classification of Decommissioning Wastes, NUREG/CR-0672, Addendum 2, U.S. Nuclear Regulatory Commission, Washington, D.C. (September 1984).

6. G. J. Konzek and R. I. Smith, Technology, Safety and Costs of Decommissioning a Reference Boiling Water Reactor Power Station-Technical Support for Decommissioning Matters Related to Preparation of the Final Decommissioning Rule, NUREG/CR-0672, Addendum 3, U.S. Nuclear Regulatory Commission, Washington, D.C. (July 1988).

7. R I. Smith et al., Technology, Safety and Costs of Decommissioning a Reference Pressurized Water Reactor Power Station, NUREG/CR-0130, Vols. 1 and 2, U.S. Nuclear Regulatory Commission, Washington, D.C. (June 1978).

8. E. S. Murphy, Technology, Safety and Costs of Decommissioning a Reference Pressurized Water Reactor Power Station-Classification of Decommissioning Wastes, NUREG/CR-0130, Addendum 3, U.S. Nuclear Regulatory Commission, Washington, D.C. (September 1984).

9. G. J. Konzek and R. I. Smith, Technology, Safety and Costs of Decommissioning a Reference Pressurized Water Reactor Power Station-Technical Support for Decommissioning Matters Related to Preparation of the Final Decommissioning Rule, NUREG/CR-0130, Addendum 4, U.S. Nuclear Regulatory Commission, Washington, D.C. (July 1988).

10. R. I. Smith et al., Revised Analyses of Decommissioning for the Reference Boiling Water Reactor Power Station, draft report, NUREG/CR-6174, PNL-9975, Vols. 1 and 2, U.S. Nuclear Regulatory Commission, Washington, D.C. (September 1994).

11. G. J. Konzek et al., Revised Analyses of Decommissioning for the Reference Pressurized Water Reactor Power Station, draft report, NUREG/CR-5884, PNL-8742, Vols. 1 and 2, U.S. Nuclear Regulatory Commission, Washington, D.C. (October 1993).

12. H. K. Elder, Technology, Safety and Costs of Decommissioning a Reference Uranium Hexafluoride Conversion Plant, NUREG/CR-1757, U.S. Nuclear Regulatory Commission, Washington, D.C. (October 1981). 
13. TLG Engineering, Inc., Preliminary Cost Estimate for D\&D of the Gaseous Diffusion Plants, S14-25-002, Bridgewater, Connecticut (September 1991).

14. Alfreda L. Cook, K-25 Site Environmental Restoration Division, Oak Ridge, Tennessee, correspondence to S. N. Storch, Oak Ridge National Laboratory, Oak Ridge, Tennessee, "Gaseous Diffusion Plant Decontamination and Decommissioning Waste Information for the Department of Energy's Annual Integrated Data Base Report," ERP-WM/94.144, dated Nov. 11, 1994.

15. H. K. Elder and D. E. Blahnik, Technology, Safety and Costs of Decommissioning a Reference Uranium Fuel Fabrication Plant, NUREG/CR-1266, Vols. 1 and 2, U.S. Nuclear Regulatory Commission, Washington, D.C. (October 1980).

16. G. J. Konzek et al., Technology, Safety and Costs of Decommissioning Reference Nuclear Research and Test Reactors, NUREG/CR-1756, Vols. 1 and 2, U.S. Nuclear Regulatory Commission, Washington D.C. (March 1982).

17. G. J. Konzek et al., Technology, Safety and Costs of Decommissioning Reference Nuclear Research and Test Reactors, NUREG/CR-1756, Addendum, U.S. Nuclear Regulatory Commission, Washington, D.C. (July 1983).

18. U.S. Department of Energy, Office of Environmental Restoration, Decommissioning Handbook, DOE/EM-0142P, Washington, D.C. (March 1994).

19. U.S. Nuclear Regulatory Commission, "Licensing Requirements for Land Disposal of Radioactive Wastes: 'Greaterthan-Class-C' Low-Level Radioactive Wastes," Fed. Regist. 54(100), 22578-83 (May 25, 1989).

20. U.S. Nuclear Regulatory Commission, "Licensing Requirements for Land Disposal of Radioactive Wastes," Code of Federal Regulations, 10 CFR Part 61.55(a)(2)(iv), (Jan. 1, 1995).

21. D. E. Robertson et al., Radionuclide Characterization of Reactor Decommissioning Waste and Spent Fuel Assembly Hardware-Progress Report, NUREG/CR-5343, PNL-6806, U.S. Nuclear Regulatory Commission, Washington, D.C. (January 1991).

22. Lockheed Idaho Technologies Company, Greater-Than-Class-C Low-Level Waste Management Program, GreaterThan-Class C Low-Level Radioactive Waste Characterization: Estimated Volumes, Radionuclide Activities, and Other Characteristics, DOE/LLW-114, Rev. 1, Idaho Falls, Idaho (September 1994).

23. U.S. Department of Energy, Energy Information Administration, Spent Nuclear Fuel Discharges from U.S. Reactors 1993, SR/CNEAF/95-01, Washington, D.C. (February 1995).

24. U.S. Department of Energy, Integrated Data Base for 1993: U.S. Spent Fuel and Radioactive Waste Inventories, Projections, and Characteristics, DOE/RW-0006, Rev. 9, Oak Ridge National Laboratory, Oak Ridge, Tennessee (March 1994).

25. U.S. Department of Energy, Integrated Data Base Report-1993: U.S. Spent Nuclear Fuel and Radioactive Waste Inventories, Projections, and Characteristics, DOE/RW-0006, Rev. 10, Oak Ridge National Laboratory, Oak Ridge, Tennessee (December 1994).

26. Commonwealth Edison Company, Decommissioning Program Plan for the Dresden Nuclear Power Station Unit 1, Rev. 3, Chicago (December 1991).

27. Mary J. Fisher, Public Service Company of Colorado, Denver, correspondence to S. N. Storch, Oak Ridge National Laboratory, Oak Ridge, Tennessee, P-95052, "Update of Fort St. Vrain Reactor Decommissioning Waste Data for the 1995 DOE Integrated Data Base Report,” dated May 23, 1995. 
28. A. Clegg Crawford, Public Service Company of Colorado, "Decommissioning-The New Challenge for the Nuclear Industry," view graph presentation for the opening plenary session, 1994 International Symposium on Decontamination \& Decommissioning, Knoxville, Tennessee, April 25, 1994, CONF-94046, Analysis Corp, Oak Ridge, Tennessee, 1994.

29. M. J. Fisher, Public Service Company of Colorado, Denver, Colorado, correspondence to S. N. Storch, Oak Ridge National Laboratory, Oak Ridge, Tennessee, DDO-90-0068, transmitting summary of projected wastes from dismantling the Fort St. Vrain Reactor, dated June 4, 1990.

30. F. J. Novachek, Public Service Company of Colorado, Denver, correspondence to S. N. Storch, Oak Ridge National Laboratory, Oak Ridge, Tennessee, P-92079, "Integrated Data Base Update," dated Feb. 25, 1992.

31. TLG Engineering, Inc., Decommissioning Cost Study for the Humboldt Bay Power Plant Unit 3, P01-25-006, Pacific Gas and Electric Company, San Francisco (July 1994).

32. David L. Smith, Consolidated Edison Company, Indian Point Station, Buchanan, New York, correspondence to S. N. Storch, Oak Ridge National Laboratory, Oak Ridge, Tennessee, transmitting summary of wastes from decommissioning the Indian Point-Unit 1 reactor, dated May 2, 1995.

33. S. J. Raffety, Dairyland Power Cooperative, La Crosse, Wisconsin, correspondence to S. N. Storch, Oak Ridge National Laboratory, Oak Ridge, Tennessee, transmitting summary of updated waste inventories and projections from decommissioning the La Crosse BWR, LAC-13487, dated May 25, 1995.

34. M. J. Bua, Sacramento Municipal Utility District, Rancho Seco Nuclear Generating Station, Herald, California, correspondence to S. N. Storch, Oak Ridge National Laboratory, Oak Ridge, Tennessee, RPM 91-112, transmitting data on wastes from decommissioning the Rancho Seco PWR, dated July 24, 1991.

35. E. M. Golden, Southern California Edison Company, Irvine, California, correspondence to S. N. Storch, Oak Ridge National Laboratory, Oak Ridge, Tennessee, transmitting summary of wastes from decommissioning the San Onofre-Unit 1 PWR, dated July 23, 1993.

36. R. D. Holmes, GPU Nuclear Corporation, Toms River, New Jersey, correspondence to S. N. Storch, Oak Ridge National Laboratory, Oak Ridge, Tennessee, transmitting updated summary of wastes from Saxton PWR decommissioning activities, dated Aug. 17, 1995.

37. Long Island Power Authority, Shoreham Nuclear Power Station Decommissioning Plan, NRC Docket No. 50-322, Washington, D.C. (December 1990).

38. U.S. Nuclear Regulatory Commission, Order Approving the Decommissioning Plan and Authorizing the Decommissioning of Shoreham Nuclear Power Station, Unit 1, Washington, D.C. (June 1992).

39. Siva Kumar, Long Island Power Authority, Wading River, New York, correspondence to S. N. Storch, Oak Ridge National Laboratory, Oak Ridge, Tennessee, transmitting summary of wastes from decommissioning the Shoreham Nuclear Power BWR Station, dated July 18, 1994.

40. M. E. Sweigart, GPU Nuclear Corporation, Three Mile Island Site Office, Middletown, Pennsylvania, correspondence to S. N. Storch, Oak Ridge National Laboratory, Oak Ridge, Tennessee, 3212-95-0056, transmitting updated summary of wastes from TMI-Unit 2 cleanup activities, May 16, 1995.

41. Michael B. Lackey, Portland General Electric Company, Rainier, Oregon, correspondence to Stephen N. Storch, Oak Ridge National Laboratory, Oak Ridge, Tennessee, "Trojan Reactor Decommissioning Waste Information," DEC-015-95, dated June 19, 1995. 
42. K. J. Heider, Yankee Atomic Electric Company, Bolton, Massachusetts, correspondence to Stephen N. Storch, Oak Ridge National Laboratory, Oak Ridge, Tennessee, "Yankee Nuclear Power Station Decommissioning Waste Volume Estimate," YRP 347/95, dated Aug. 1, 1995.

43. J. L. Kegin, Cimarron Fuel Fabrication Facility, Cimarron Corporation, Crescent, Oklahoma, correspondence to S. N. Storch, Oak Ridge National Laboratory, Oak Ridge, Tennessee, transmitting summary of wastes from decommissioning the Cimarron Fuel Fabrication Facility, dated June 15, 1995.

44. John J. Hollinden, West Valley Nuclear Services Company, Inc., West Valley, New York, correspondence to Steven N. Storch, Oak Ridge National Laboratory, Oak Ridge, Tennessee, "Submittal of Department of Energy Site Toxic Substance Control Act Mixed Low-Level Waste Information Request for the 1995 Integrated Data Base Report (DOE/RW-0006, Rev. 11),"WZ:95:0095, dated June 15, 1995.

45. U.S. Department of the Navy, Final Environmental Impact Statement on the Disposal of Decommissioned, Defueled Naval Submarine Reactor Plants, Vols. 1-3, Washington, D.C. (May 1984). 
Table 7.1. List of U.S. civilian reactors shut down or dismantled as of December $31,1994^{\mathrm{a}}$

[Reactors of 10-MW(th) capacity or greater]

\begin{tabular}{|c|c|c|c|c|c|c|c|}
\hline \multirow{2}{*}{ Reactor facility } & \multirow{2}{*}{ Location } & \multirow{2}{*}{ Reactor type } & \multicolumn{2}{|c|}{ Capacity rating } & \multirow{2}{*}{$\begin{array}{l}\text { Year of } \\
\text { permanent } \\
\text { shutdown }\end{array}$} & \multirow{2}{*}{$\begin{array}{l}\text { Decommissioning } \\
\text { alternative } \\
\text { selected }\end{array}$} & \multirow{2}{*}{$\begin{array}{l}\text { Present status of } \\
\text { decommissioning } \\
\text { alternative }\end{array}$} \\
\hline & & & $M W(e)$ & MW(th) & & & \\
\hline $\begin{array}{l}\text { Boiling Nuclear Superheater } \\
\text { Power Station (BONUS) }\end{array}$ & Punta Higuera, PR & Boiling-water & 37 & 50 & 1968 & ENTOMB & ENTOMB \\
\hline $\begin{array}{l}\text { Carolinas-Virginia Tube Reactor } \\
\text { (CVTR) }\end{array}$ & Parr, SC & $\begin{array}{l}\text { Pressure-tube, } \\
\text { heavy-water }\end{array}$ & 17 & 64 & 1967 & SAFSTOR & SAFSTOR \\
\hline $\begin{array}{l}\text { Dresden Nuclear Power Station- } \\
\text { Unit } 1\end{array}$ & Morris, $\mathbb{I L}$ & Boiling-water & 200 & 700 & 1978 & SAFSTOR & SAFSTOR preparation ${ }^{b}$ \\
\hline Elk River Power Station & Elk River, $\mathrm{MN}$ & Boiling-water & 22 & 58 & 1968 & DECON & DECON completed ${ }^{c}$ \\
\hline Enrico Fermi-Unit 1 & Lagoona Beach, MI & Sodium-cooled, fast & 61 & 200 & 1972 & SAFSTOR & SAFSTOR \\
\hline $\begin{array}{l}\text { ESADA/GE Vallecitos } \\
\text { Experimental Superheat } \\
\text { Reactor (Empire States } \\
\text { Atomic Development } \\
\text { Associates and General } \\
\text { Electric Company) }\end{array}$ & Pleasanton, $\mathrm{CA}$ & $\begin{array}{l}\text { Light-water, } \\
\text { moderated }\end{array}$ & $N E^{d}$ & 17 & 1967 & SAFSTOR & SAFSTOR \\
\hline Fort St. Vrain Reactor & Platteville, CO & $\begin{array}{l}\text { High-temperature, } \\
\text { gas-cooled }\end{array}$ & 330 & 842 & 1989 & DECON & $D E C O N$ in progress $\mathrm{e}$ \\
\hline General Electric Testing Reactor & Pleasanton, $\mathrm{CA}$ & Tank & NE & 50 & 1977 & SAFSTOR & SAFSTOR \\
\hline Hallam Nuclear Power Facility & Hallam, NE & $\begin{array}{l}\text { Sodium-cooled, } \\
\text { graphite-moderated }\end{array}$ & 75 & 240 & 1964 & ENTOMB & ENTOMB \\
\hline $\begin{array}{l}\text { Humboldt Bay Power Plant- } \\
\text { Unit } 3\end{array}$ & Eureka, CA & Boiling-water & 65 & 242 & 1976 & SAFSTOR & SAFSTOR ${ }^{f}$ \\
\hline Indian Point Station-Unit 1 & Buchanan, NY & Pressurized-water & 265 & 615 & 1974 & SAFSTORg & SAFSTOR \\
\hline
\end{tabular}




\begin{tabular}{|c|c|c|c|c|c|c|c|}
\hline \multirow{2}{*}{ Reactor facility } & \multirow{2}{*}{ Location } & \multirow{2}{*}{ Reactor type } & \multicolumn{2}{|c|}{ Capacity rating } & \multirow{2}{*}{$\begin{array}{l}\text { Year of } \\
\text { permanent } \\
\text { shutdown }\end{array}$} & \multirow{2}{*}{$\begin{array}{l}\text { Decommissioning } \\
\text { alternative } \\
\text { selected }\end{array}$} & \multirow{2}{*}{$\begin{array}{l}\text { Present status of } \\
\text { decommissioning } \\
\text { alternative }\end{array}$} \\
\hline & & & $M W(e)$ & MW(th) & & & \\
\hline $\begin{array}{l}\text { La Crosse Nuclear Generating } \\
\text { Station }\end{array}$ & Genoa, WI & Boiling-water & 48 & 165 & 1987 & SAFSTOR & SAFSTOR ${ }^{h}$ \\
\hline Pathfinder Atomic Plant & Sioux Falls, SD & Boiling-water & 66 & 192 & 1967 & DECON & $D_{E C O N}{ }^{i}$ \\
\hline $\begin{array}{l}\text { Peach Bottom Power Station- } \\
\text { Unit } 1\end{array}$ & Peach Bottom, PA & $\begin{array}{l}\text { High-temperature, } \\
\text { gas-cooled }\end{array}$ & 40 & 115 & 1974 & SAFSTOR & SAFSTOR \\
\hline Piqua Nuclear Power Facility & Piqua, $\mathrm{OH}$ & $\begin{array}{l}\text { Organic-cooled and } \\
\text { moderated }\end{array}$ & 11 & 46 & 1966 & ENTOMB & ENTOMB \\
\hline Plum Brook Reactor & Sandusky, $\mathrm{OH}$ & Tank & $\mathrm{NE}$ & 60 & 1974 & SAFSTOR & SAFSTOR \\
\hline Rancho Seco & Clay Station, CA & Pressurized-water & 918 & 2,915 & 1989 & SAFSTOR & SAFSTOR preparation $j$ \\
\hline San Onofre-Unit 1 & San Clemente, CA & Pressurized-water & 436 & 1,347 & 1992 & SAFSTOR & SAFSTOR preparation $k$ \\
\hline $\begin{array}{l}\text { Saxton Nuclear Experimental } \\
\text { Reactor Project }\end{array}$ & Saxton, PA & Pressurized-water & 3 & 24 & 1972 & SAFSTOR & DECON in progress $l$ \\
\hline Shippingport Power Station & Shippingport, PA & Pressurized-water & 72 & 236 & 1982 & DECON & DECON completed ${ }^{\mathrm{m}}$ \\
\hline Shoreham Reactor & Brookhaven, NY & Boiling-water & 820 & 2,436 & 1989 & DECON & DECON completed ${ }^{n}$ \\
\hline Sodium Reactor Experiment & Santa Susana, CA & $\begin{array}{l}\text { Sodium-cooled, } \\
\text { graphite-moderated }\end{array}$ & 10 & 30 & 1964 & DECON & DECON completed ${ }^{\circ}$ \\
\hline $\begin{array}{l}\text { Southwest Experimental Fast } \\
\text { Oxide Reactor (SEFOR) }\end{array}$ & Strickler, AR & Sodium-cooled, fast & $\mathrm{NE}$ & 20 & 1972 & SAFSTOR & SAFSTOR \\
\hline $\begin{array}{l}\text { Three Mile Island-Unit } 2 \\
\text { Reactor }\end{array}$ & $\begin{array}{l}\text { Londonderry } \\
\text { Township, PA }\end{array}$ & Pressurized-water & 926 & 2,770 & 1979 & $\mathrm{p}$ & $\mathrm{p}$ \\
\hline Trojan-Unit 19 & Rainier, OR & Pressurized-water & 1,130 & 3,411 & 1992 & DECON & DECON \\
\hline
\end{tabular}


Table 7.1 (continued)

\begin{tabular}{|c|c|c|c|c|c|c|c|}
\hline \multirow{2}{*}{ Reactor facility } & \multirow{2}{*}{ Location } & \multirow{2}{*}{ Reactor type } & \multicolumn{2}{|c|}{ Capacity rating } & \multirow{2}{*}{$\begin{array}{l}\text { Year of } \\
\text { permanent } \\
\text { shutdown }\end{array}$} & \multirow{2}{*}{$\begin{array}{l}\text { Decommissioning } \\
\text { altemative } \\
\text { selected }\end{array}$} & \multirow{2}{*}{$\begin{array}{l}\text { Present status of } \\
\text { decommissioning } \\
\text { alternative }\end{array}$} \\
\hline & & & $M W(e)$ & $\mathrm{MW}(\mathrm{th})$ & & & \\
\hline $\begin{array}{l}\text { Vallecitos Boiling-Water } \\
\text { Reactor (VBWR) }\end{array}$ & Pleasanton, CA & Boiling-water & 5 & 33 & 1963 & SAFSTOR & SAFSTOR \\
\hline $\begin{array}{l}\text { Westinghouse Testing Reactor } \\
\text { (WTR) }\end{array}$ & Waltz Mill, PA & Tank & $\mathrm{NE}$ & 60 & 1962 & SAFSTOR & SAFSTOR \\
\hline Yankee Rowe-Unit $1^{r}$ & Rowe, MA & Pressurized-water & 167 & 600 & 1991 & SAFSTOR & SAFSTOR preparation \\
\hline
\end{tabular}

adapted from refs. 2 and 3.

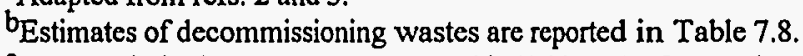

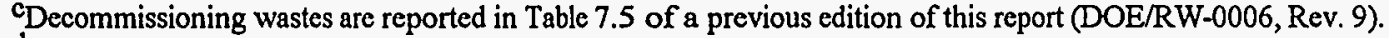

$\mathrm{d}_{\mathrm{NE}}=$ no electricity generated by reactor before it was shut down.

Estimates of decommissioning wastes are reported in Table 7.10. Actual inventories recovered are reported in Table 7.9.

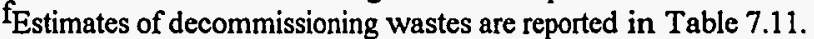

EEstimates of decommissioning wastes are reported in Table 7.12.

$\mathrm{h}_{\text {Estimates of decommissioning wastes are reported in Table 7.13. }}$

Estimates of decommissioning wastes are reported in Table 7.14 of a previous edition of this report (DOE/RW-0006, Rev. 10)

Decommissioning wastes are reported in Table 7.14.

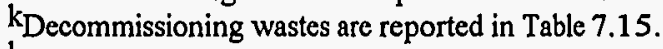

IDECON of the Saxton facility started in 1986. Estimates of decommissioning wastes are given in Table 7.16.

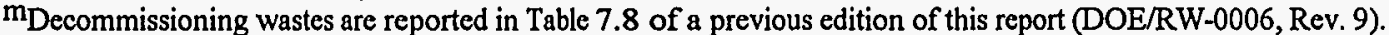

nDECON of the Shoreham plant started in 1992. Estimates of decommissioning wastes are reported in Table 7.17.

oDecommissioning wastes are reported in Table 7.6 of a previous edition of this report (DOE/RW-0006, Rev. 9).

PTMI-Unit 2 has completed defueling and decontamination in selected areas. The plant will be placed in a long-term monitored storage mode and will be decommissioned when

TMI-Unit 1 is dismantled. Inventories of decontamination wastes are reported in Table 7.18.

qDecommissioning wastes are reported in Table 7.19.

Decommissioning wastes are reported in Table 7.20. 
Table 7.2. List of U.S. nuclear fuel cycle facilities shut down or being decommissioned as of December 31, 1994ª

\begin{tabular}{|c|c|c|c|c|c|}
\hline Facility & Type of installation & Location & Rated capacity & $\begin{array}{l}\text { Period of } \\
\text { operation }\end{array}$ & $\begin{array}{l}\text { Decommissioning } \\
\text { objectives or status }\end{array}$ \\
\hline Sequoyah Fuels Corporation Plant & Nuclear fuel conversion plant & Gore, OK & 9,090 MTU/year & $1970-1993$ & $\begin{array}{l}\text { Complete dismantlement } \\
\text { by 2002-2004 }\end{array}$ \\
\hline Oak Ridge Gaseous Diffusion Plant (K-25 Site) & Uranium enrichment plant & Oak Ridge, TN & 7,700 t SWU/year & $1945-1985$ & $\mathrm{TBD}^{\mathrm{b}}$ \\
\hline Cimarron Fuel Fabrication Facility & Nuclear fuel fabrication plant ${ }^{c}$ & Crescent, OK & $300 \mathrm{MTU} /$ year & $1965-1975$ & $\begin{array}{l}\text { Anticipated completion in } \\
1995\end{array}$ \\
\hline West Valley Demonstration Project & Nuclear fuel reprocessing plant & West Valley, NY & $300 \mathrm{MTIHM} /$ year & $1966-1972$ & Proceeding ${ }^{d}$ \\
\hline
\end{tabular}

aExcludes uranium mining and milling facilities being decommissioned. These are indicated in Table 5.2 of Chapter 5.

$\mathrm{b}_{\mathrm{TBD}}=$ to be determined.

This facility was comprised of a uranium fuel fabrication plant and a mixed-oxide fuel fabrication plant. The uranium fuel fabrication plant operated from 1965 to 1975 and had a rated capacity of $300 \mathrm{MTU} / \mathrm{year}$. The mixed-oxide fuel fabrication plant operated from 1969 to 1975 and had a rated plutonium product (PuO $)$ capacity of $0.5 \mathrm{t} / \mathrm{year}$.

$\mathrm{d}_{\text {More than }} 70 \%$ of the cell surface areas of the original process building have been decontaminated and released for project reuse. 
Table 7.3. Projections of radioactive wastes from decommissioning reference commercial power reactors and fuel cycle facilities ${ }^{a}$

\begin{tabular}{|c|c|c|c|c|c|c|}
\hline \multirow{2}{*}{ Fuel cycle facility } & \multirow{2}{*}{ Capacity } & \multicolumn{2}{|c|}{ Operation } & \multirow{2}{*}{$\begin{array}{l}\text { Decommissioning } \\
\text { alternative }\end{array}$} & \multicolumn{2}{|c|}{ Waste volume, $\mathrm{m}^{3}$} \\
\hline & & Period & $\begin{array}{l}\text { Lifetime } \\
\text { (years) }\end{array}$ & & $L L W^{b}$ & GTCC $^{c}$ \\
\hline Boiling-water reactor & $1,155 \mathrm{MW}(\mathrm{e})$ & & $40^{\mathrm{d}}$ & DECON & 14,275 & 7 \\
\hline Pressurized-water reactor & $1,175 \mathrm{MW}(\mathrm{e})$ & & $40^{\mathrm{d}}$ & DECON & 6,981 & 11 \\
\hline $\begin{array}{l}\text { Uranium conversion plant } \\
\text { (solvent extraction process) }\end{array}$ & 10,000 MTIHM/year & & 40 & DECON & 1,260 & 0 \\
\hline \multicolumn{7}{|l|}{$\begin{array}{l}\text { Uranium enrichment plants } \\
\text { (gaseous diffusion plants) }\end{array}$} \\
\hline - K-25 site & $7,700,000 \mathrm{~kg}$ SWU/year & $1945-1985$ & 40 & DECON & $212,466^{e}$ & 0 \\
\hline - Paducah site & $11,300,000 \mathrm{~kg} \mathrm{SWU} /$ year & $1954-2005$ & 51 & DECON & 662,414 & 0 \\
\hline - Portsmouth site & $8,300,000 \mathrm{~kg}$ SWU/year & 1956-2005 & 49 & DECON & 630,093 & 0 \\
\hline Fuel fabrication plant & 1,000 MTIHM/year & & 40 & DECON & 1,090 & 0 \\
\hline
\end{tabular}

\footnotetext{
${ }^{a}$ Based on information reported in refs. 10-15.

bClass-A, Class-B, and Class-C LLW.

${ }^{\circ}$ Greater-than-Class-C LLW.

dReactor operations assume a $75 \%$-capacity factor.

Includes some liquids $\left(1,849 \mathrm{~m}^{3}\right)$, but mostly solids (mainly in the form of metal equipment and scrap, roofing and construction debris, sludge, and other
} materials) 
Table 7.4. Schedule of actual and projected final shutdown dates for commercial light-water reactors, ${ }^{a}, \mathrm{~b}$

\begin{tabular}{|c|c|c|c|c|c|c|}
\hline \multirow{2}{*}{$\begin{array}{l}\text { Calendar year of } \\
\text { shutdown }\end{array}$} & \multicolumn{2}{|c|}{ BWR } & \multicolumn{2}{|c|}{ PWR } & \multicolumn{2}{|c|}{ Total LWR } \\
\hline & No. & $M W(e)$ & No. & MW(e) & No. & $M W(e)$ \\
\hline 1963 & 1 & 5 & & & 1 & 5 \\
\hline 1967 & 1 & 66 & 1 & 17 & 2 & 83 \\
\hline 1968 & 2 & 39 & & & 2 & 39 \\
\hline 1972 & & & 1 & 3 & 1 & 3 \\
\hline 1974 & & & 1 & 265 & 1 & 265 \\
\hline 1976 & 1 & 65 & & & 1 & 65 \\
\hline 1978 & 1 & 200 & & & 1 & 200 \\
\hline 1979 & & & 1 & $926^{\mathrm{c}}$ & 1 & 926 \\
\hline 1982 & & & 1 & 72 & 1 & 72 \\
\hline 1987 & 1 & 48 & 1 & 48 & & \\
\hline 1989 & 1 & 820 & 1 & 918 & 2 & 1,738 \\
\hline 1991 & & & 1 & 167 & 1 & 167 \\
\hline 1992 & & & 2 & 1,566 & 2 & 1,566 \\
\hline 1994 & 0 & 0 & 0 & 0 & 0 & 0 \\
\hline $\begin{array}{l}\text { Actual totals } \\
\text { through } 1994\end{array}$ & 8 & 1,243 & 9 & 3,934 & 17 & 5,177 \\
\hline 2000 & 1 & 67 & & & 1 & 67 \\
\hline 2007 & & & 2 & 1,226 & 2 & $.1,226$ \\
\hline 2008 & 3 & 2,691 & 1 & 870 & 4 & 3,561 \\
\hline 2009 & 1 & 610 & 1 & 470 & 2 & 1,080 \\
\hline 2010 & 3 & 1,952 & 2 & 1,175 & 5 & 3,127 \\
\hline 2011 & & & 1 & 755 & 1 & 755 \\
\hline 2012 & 2 & 1,161 & 1 & 781 & 3 & 1,942 \\
\hline 2013 & 4 & 3,376 & 11 & 8,728 & 15 & 12,104 \\
\hline 2014 & 7 & 5,498 & 6 & 4,689 & 13 & 10,187 \\
\hline 2015 & & & 2 & 1,853 & 2 & 1,853 \\
\hline 2016 & 2 & 1,832 & 6 & 5,231 & 8 & 7,063 \\
\hline 2017 & & & 2 & 1,875 & 2 & 1,875 \\
\hline 2018 & 1 & 761 & 2 & 1,756 & 3 & 2,517 \\
\hline 2020 & & & 3 & 3,104 & 3 & 3,104 \\
\hline 2021 & & & 4 & 4,133 & 4 & 4,133 \\
\hline 2022 & 2 & 2,183 & & & 2 & 2,183 \\
\hline 2023 & 1 & 1,100 & 2 & 1,968 & 3 & 3,068 \\
\hline 2024 & 4 & 4,195 & 3 & 3,470 & 7 & 7,665 \\
\hline 2025 & 2 & 2,016 & 6 & 6,877 & 8 & 8,893 \\
\hline 2026 & 4 & 4,175 & 2 & 1,989 & 6 & 6,164 \\
\hline 2027 & & & 5 & 5,626 & 5 & 5,626 \\
\hline 2028 & & & 3 & 3,421 & 3 & 3,421 \\
\hline 2029 & 1 & 1,055 & 1 & 1,162 & 2 & 2,217 \\
\hline 2030 & & & 3 & 3,465 & 3 & 3,465 \\
\hline $\begin{array}{c}\text { Projected totals } \\
(1995-2030)\end{array}$ & 38 & 32,672 & 69 & 64,624 & 107 & 97,296 \\
\hline
\end{tabular}

${ }^{a}$ Data from refs. 2 and 23. Historical data (prior to 1995) are based on ref. 2. Projected shutdown dates are based on utility-projected dates for reactor retirement reported in Table 4 of ref. 23.

byears in which no reactor shutdown is expected are eliminated.

'Shutdown of Three Mile Island-Unit 2 nuclear power plant due to an accident. Upon completion of the present cleanup campaign, the plant will be placed in a monitored storage mode and will be decommissioned when TMI-Unit 1 is dismantled. 
Table 7.5. Quantities of shipped LLW from completed and ongoing reactor decommissioning projects ${ }^{\mathrm{a}}$

\begin{tabular}{|c|c|c|c|c|c|}
\hline Reactor & Type $^{b}$ & $\begin{array}{l}\text { Rated capacity } \\
\text { [MW(e)] }\end{array}$ & $\begin{array}{l}\text { Year of } \\
\text { permanent } \\
\text { shutdown }\end{array}$ & $\begin{array}{c}\text { Lifetime electrical } \\
\text { generation } \\
\text { [MW(e)-years] }\end{array}$ & $\begin{array}{c}\text { Volume of } \\
\text { LLW shipped } \\
\left(\mathrm{m}^{3}\right)\end{array}$ \\
\hline Elk River & BWR & 22 & 1968 & 58 & 3,377 \\
\hline Fort St. Vrain ${ }^{c}$ & HTGR & 330 & 1989 & 490 & 1,914 \\
\hline La Crosse & BWR & 48 & 1987 & 462 & 75 \\
\hline Pathfinder & BWR & 66 & 1967 & 16 & $562^{\mathrm{d}}$ \\
\hline Santa Susana & SCGM & 10 & 1964 & 4 & 3,863 \\
\hline Saxton & PWR & 3 & 1972 & 10 & 1,590 \\
\hline Shippingport & PWR & 72 & 1982 & 842 & 6,056 \\
\hline Three Mile Island-Unit $2^{c}$ & PWR & 926 & 1979 & 232 & 9,216 \\
\hline Yankee Rowe & PWR & 167 & 1992 & 4,030 & 503 \\
\hline Total & & 1,644 & & 6,144 & 27,156 \\
\hline
\end{tabular}

a Information as of December 31, 1994, unless indicated otherwise.

b Abbreviations used: BWR-boiling-water reactor, HTGR-high-temperature, gas-cooled reactor, SCGM-sodiumcooled, graphite-moderated reactor, and PWR-pressurized water reactor.

CDECON for this reactor is still in progress.

dVolume of LLW disposed was $303 \mathrm{~m}^{3}$. 
Table 7.6. Revised estimates of disposal volumes for radioactive wastes from decommissioning alternatives considered for reference $L W R s^{a, b}$

\begin{tabular}{|c|c|c|c|c|c|c|}
\hline \multirow{2}{*}{$\begin{array}{l}\text { Decommissioning } \\
\text { alternative }^{\mathrm{c}}\end{array}$} & \multirow{2}{*}{$\begin{array}{l}\text { Post-shutdown } \\
\text { period d }^{d} \\
\text { (years) }\end{array}$} & \multicolumn{5}{|c|}{ Estimated disposal volume, $\mathrm{m}^{3}$} \\
\hline & & Total & Class-A LLW & Class-B LLW & Class-C LLW & GTCC LLWe \\
\hline \multicolumn{7}{|c|}{ Reference boiling-water reactor [1155 MW(e)] } \\
\hline DECON & 6.3 & 14,282 & 13,903 & 332 & 40 & 7 \\
\hline SAFSTOR 1 & 60 & 1,117 & 738 & 332 & 40 & 7 \\
\hline SAFSTOR 2 & 60 & 14,282 & 13,903 & 332 & 40 & 7 \\
\hline ENTOMB 1 & 60 & 490 & 483 & 0 & 0 & 7 \\
\hline ENTOMB 2 & 60 & 1,139 & 760 & 332 & 40 & 7 \\
\hline ENTOMB 3 & 300 & 490 & 483 & 0 & 0 & 7 \\
\hline \multicolumn{7}{|c|}{ Reference pressurized-water reactor $[1175 \mathrm{MW}(e)]$} \\
\hline DECON & 8.6 & 6,992 & 6,797 & 154 & 30 & 11 \\
\hline SAFSTOR 1 & 60 & 763 & 568 & 154 & 30 & 11 \\
\hline SAFSTOR 2 & 60 & 6,992 & 6,797 & 154 & 30 & 11 \\
\hline ENTOMB I & 60 & 305 & 294 & 0 & 0 & 11 \\
\hline ENTOMB 2 & 60 & 754 & 559 & 154 & 30 & 11 \\
\hline ENTOMB 3 & 300 & 305 & 294 & 0 & 0 & 11 \\
\hline
\end{tabular}

a Adapted from refs. 10 and 11. Before final shutdown, each reactor is assumed to have operated for 40 years with a capacity factor of 0.75 .

b The classifications listed for LLW are based on the limiting concentrations of long- and short-lived radionuclides given in Tables 1 and 2 of 10 CFR Part 61.55.

${ }^{c_{A s}}$ indicated in refs. 10 and 11 , the decommissioning alternatives listed have the following major features: DECON-assumes a period of plant safe storage until the SNF inventory has sufficiently decayed and been completely removed. This is followed by dismantlement of the radioactive portions of the plant; SAFSTOR 1-assumes extended safe storage ( $<60$ years) without any fuel in the reactor storage pool, followed by decontamination and dismantlement of the radioactive portions of the plant and that only the reactor pressure vessel and BWR sacrificial shield or PWR bioshield require disposal as LLW; SAFSTOR 2-same as SAFSTOR 1 except that all radioactive materials are assumed to still exceed unrestricted release levels. No volume reduction from DECON results; ENTOMB 1-assumes no removal of reactor pressure vessel and BWR sacrificial shield or PWR bioshield as these will decay to unrestricted levels; ENTOMB 2-assumes removal of reactor pressure vessel and BWR sacrificial shield or PWR bioshield is required during preparations for entombment to assure license termination within 60 years after final shutdown; and ENTOMB 3-allows for 300 years of radioactive decay after which the reactor pressure vessel and BWR sacrificial shield or PWR bioshield will have decayed to unrestricted release levels.

${ }^{d}$ Covers the period of time starting from final reactor shutdown to the completion of plant decommissioning activities.

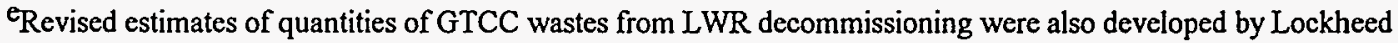
Idaho Technologies Company (now called Lockheed Martin Idaho Technologies) and are reported in ref. 22. A summary of all GTCC waste estimates reported in ref. 22 is given in Chapter 4. 
Table 7.7. Projections of cumulative volume, radioactivity, and thermal power of wastes from decommissioning commercial light-water reactors shut down during $1995-2030^{a, b, c}$

\begin{tabular}{|c|c|c|c|}
\hline Waste type & $\begin{array}{l}\text { Volume } \\
\left(\mathrm{m}^{3}\right)\end{array}$ & $\begin{array}{l}\text { Activity } \\
\text { (Ci) }\end{array}$ & $\begin{array}{l}\text { Thermal powe } \\
\text { (W) }\end{array}$ \\
\hline \multicolumn{4}{|c|}{ Boiling-water reactors } \\
\hline Class-A LLW & 393,278 & 35,559 & 209 \\
\hline Class-B LLW & 9,392 & 111,078 & 653 \\
\hline Class-C LLW & 1,132 & 299,754 & 985 \\
\hline Subtotals & 403,802 & 446,391 & 1,847 \\
\hline Greater-than-Class-C LLWd & 194 & $16,760,009$ & 80,974 \\
\hline Totals for D\&D of BWRs & 403,996 & $17,206,400$ & 82,821 \\
\hline \multicolumn{4}{|c|}{ Pressurized-water reactors } \\
\hline Class-A LLW & 373,830 & 132,374 & 700 \\
\hline Class-B LLW & 8,469 & 162,713 & 1,473 \\
\hline Class-C LLW & 1,650 & 159,331 & 1,058 \\
\hline Subtotals & 383,949 & 454,418 & 3,231 \\
\hline Greater-than-Class-C LLW & 605 & $24,628,168$ & 120,375 \\
\hline Totals for D\&D of PWRs & 384,554 & $25,082,586$ & 123,606 \\
\hline
\end{tabular}

Total light-water reactors

Total LLW

Greater-than-Class-C LLW

Totals for D\&D of LWRs

$$
787,751
$$

788,550
900,809

$41,388,177$

$42,288,986$
5,078

201,349

206,427

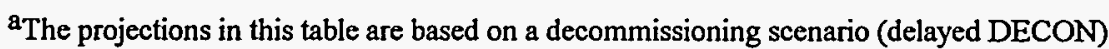
that spans a total period of 10 years following final reactor shutdown. This scenario assumes an initial 6-year period is needed for spent nuclear fuel from the reactor's last core to sufficiently cool to permit its transfer either to DOE or to a dry on-site storage facility. Active dismantlement of the reactor is assumed to begin following the completion of fuel removal and to take 4 additional years, with radioactive wastes being collected equally over each of the 4 years. In terms of numerical significance, the number of digits used to report these projections are greater than justified. However, this procedure is used for bookkeeping purposes to ensure consistency in the numerical totals reported. Since these projections are based on the reactor shutdown dates reported in ref. 23 and the source terms developed from refs. 4-11 (see Appendix C), each reported number is significant to no more than three figures.

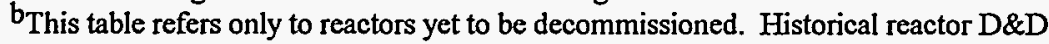
wastes are included in the institutional/industrial (I/) waste inventories reported in Chapter 4.

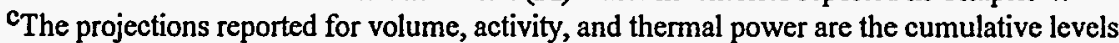
of wastes from reactor D\&D activities (delayed DECON) during the period 1994-2040. For the scenario described in footnote a, the year 2040 is the last year in which wastes are collected from reactors shut down in the year 2030 .

dincludes contributions from core shroud and other internal core components. 
Table 7.8. Projected characteristics of radioactive wastes from Dresden-Unit 1 decommissioning activities ${ }^{a, b}, c$

\begin{tabular}{|c|c|c|}
\hline Waste category & Reactor component(s) & $\begin{array}{l}\text { Volume } \\
\left(\mathrm{m}^{3}\right)\end{array}$ \\
\hline \multirow[t]{5}{*}{ Radioactive materials } & $\begin{array}{l}\text { Reactor vessel and internals: }{ }^{d} \\
\text { Reactor vessel } \\
\text { Bioshield sand and concrete } \\
\text { Thermal shield } \\
\text { Instrumentation support tubes } \\
\text { Bottom core support structure } \\
\text { Othere }\end{array}$ & $\begin{array}{r}11 \\
239 \\
2 \\
1 \\
1 \\
5\end{array}$ \\
\hline & Subtotal & 259 \\
\hline & Solidified decontamination solvents & 655 \\
\hline & Reactor station components and materials $f$ & 6,214 \\
\hline & Total & 7,128 \\
\hline \multirow[t]{2}{*}{ Radioactive hazardous materials } & $\begin{array}{l}\text { Asbestos insulation on contaminated } \\
\text { piping and components }\end{array}$ & 409 \\
\hline & Grand total & 7,537 \\
\hline \multicolumn{3}{|c|}{ 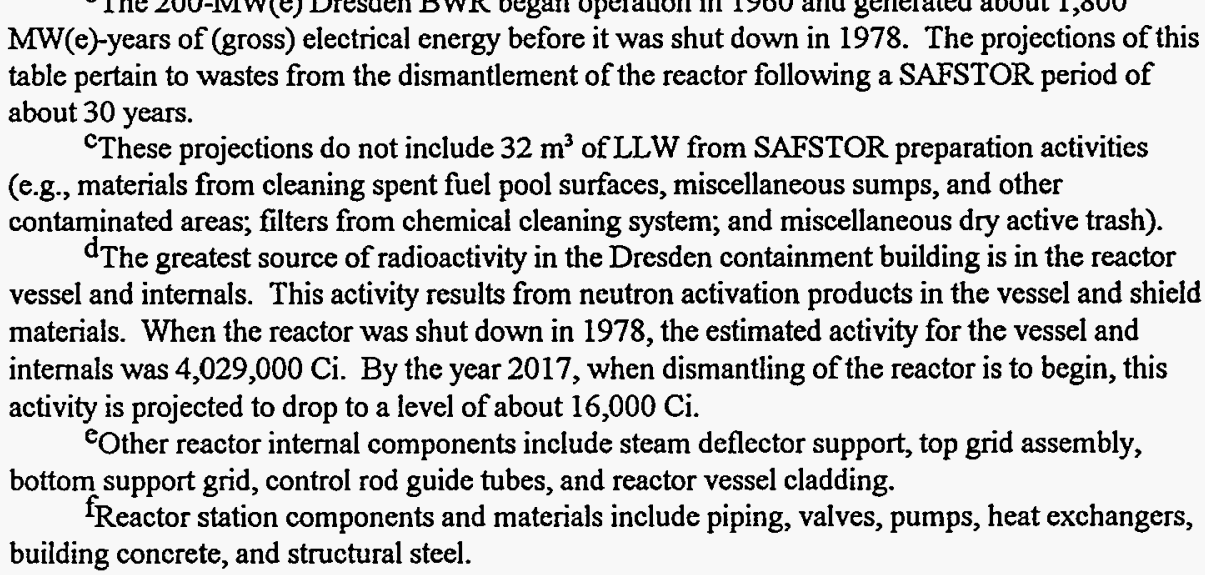 } \\
\hline
\end{tabular}


Table 7.9. Actual radioactive waste disposal from the decommissioning of the Fort St. Vrain HTGR ${ }^{a, b}$

\begin{tabular}{|c|c|c|c|c|c|c|}
\hline \multirow[b]{2}{*}{ Year } & \multicolumn{3}{|c|}{ Irradiated hardware } & \multicolumn{3}{|c|}{ Dry active waste } \\
\hline & $\begin{array}{c}\text { No. of } \\
\text { shipments }\end{array}$ & $\begin{array}{c}\text { Volume } \\
\left(\mathrm{m}^{\mathrm{3}}\right)\end{array}$ & $\begin{array}{l}\text { Activity } \\
\text { (Ci) }\end{array}$ & $\begin{array}{c}\text { No. of } \\
\text { shipments }\end{array}$ & $\begin{array}{c}\text { Volume } \\
\left(\mathrm{m}^{3}\right)\end{array}$ & $\begin{array}{l}\text { Activity } \\
\text { (Ci) }\end{array}$ \\
\hline 1991 & 6 & 29.1 & $8,083.80$ & 20 & 13.3 & 1.81 \\
\hline $1992^{d}$ & 64 & 365.8 & $32,678.49$ & 6 & 13.4 & 7.88 \\
\hline 1993 & 113 & 981.9 & $26,385.91$ & 7 & 115.1 & 8.54 \\
\hline $1994^{e}$ & 55 & 238.7 & $3,842.47$ & 14 & 156.4 & 15.28 \\
\hline Total & 238 & $1,615.5$ & $70,990.67$ & 47 & 298.2 & 33.51 \\
\hline
\end{tabular}

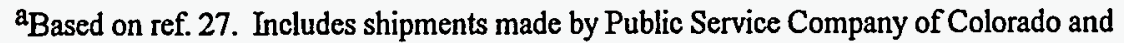
decommissioning contractor and waste processors subsequent to volume reduction.

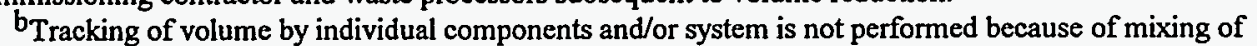
components from various waste streams, void spaces, etc.

${ }^{c}$ Actual disposal volume which therefore includes void space, filler volume, package volume, etc.

d NRC Decommissioning Order for Fort St. Vrain became effective on December 8, 1992. Before that date, decommissioning activities were considered to be early dismantlement.

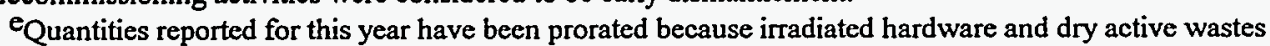
were shipped together during this time. Resins are assumed to be a dry active waste. 
Table 7.10. Projected characteristics of wastes from DECON (dismantling) of the Fort St. Vrain HTGR $^{\mathrm{a}, \mathrm{b}}$

\begin{tabular}{|c|c|c|c|}
\hline Reactor component(s)/waste & $\begin{array}{l}\text { Burial volume } \\
\qquad\left(\mathrm{m}^{3}\right)\end{array}$ & $\begin{array}{l}\text { Activity } \\
\text { (Ci) }\end{array}$ & $\begin{array}{l}\text { Projecter } \\
\text { LLW cla }\end{array}$ \\
\hline \multicolumn{4}{|c|}{ Prestressed concrete reactor vessel (PCRV) system } \\
\hline PCRV concrete & $1,174.94$ & c & A \\
\hline Control rod drives (CRDs) & 97.81 & c & A \\
\hline CRD absorber strings & 18.81 & c & C \\
\hline CRD metal clad reflector & 4.04 & c & $\mathrm{C}$ \\
\hline Boronated stainless steel rods & 845.27 & c & B \\
\hline Top cover plates & 1.59 & c & A \\
\hline Top head kaowool ${ }^{d}$ and liner & 13.32 & c & A \\
\hline Core barrel & 21.97 & c & A \\
\hline Core support blocks & 41.09 & c & A \\
\hline Core support floor kaowool, plates, and liner & 6.94 & c & A \\
\hline Metal clad reflector blocks (non-CRD) & 28.67 & c & $\mathrm{C}$ \\
\hline Dummy fuel blocks & 168.28 & $\mathrm{c}$ & A \\
\hline Graphite reflector blocks & 237.65 & $\mathrm{c}$ & $\mathrm{A}, \mathrm{B}$ \\
\hline Silica insulation blocks & 14.27 & c & A \\
\hline Large permeable reflectors & 709.32 & c & B \\
\hline Reflector keys & 0.57 & c & $\mathrm{A}$ \\
\hline Metal shell for large side reflector & 0.58 & c & A \\
\hline Radial cover plate, kaowool, and PCRV liner & 55.57 & c & A \\
\hline Region constraint devices & 1.42 & c & C \\
\hline Helium purification and regeneration system & 30.87 & c & $A$ \\
\hline Helium circulators & 4.01 & c & A \\
\hline Steam generators & 269.02 & c & $A, B$ \\
\hline PCRV system total & $3,746.01$ & $1.30 \mathrm{E}+6$ & \\
\hline
\end{tabular}

Material handling, treatment, and storage (MHTS) systems

Fuel handling machine

Fuel storage wells

Equipment storage wells Auxiliary transfer cask

Hot service facility

MHTS systems total

63.33
28.48
2.98
19.52
10.98

125.29

\begin{tabular}{cc}
$\mathrm{c}$ & $\mathrm{A}$ \\
$\mathrm{c}$ & $\mathrm{A}$ \\
$\mathrm{c}$ & $\mathrm{A}$ \\
$\mathrm{c}$ & $\mathrm{A}$ \\
$\mathrm{c}$ & $\mathrm{A}$ \\
\hline $3.88 \mathrm{E}-2$ &
\end{tabular}

A

A

A

A

Decontamination and waste (DW) systems

Decontamination

Radioactive liquid waste

Radioactive gas waste

Dry activated and other wastes

DW total

Fort St. Vrain HTGR total
9.57

9.15

32.93

153.34

204.99

$4,076.29$

$\begin{array}{ll}\text { c } & \text { A } \\ \text { c } & \text { A } \\ \text { c } & \text { A } \\ \text { c } & \text { A }\end{array}$

1.33E-4

$1.30 \mathrm{E}+6$

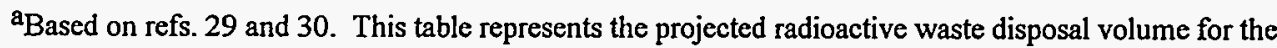
entire DECON project (projected completion in early 1996).

${ }^{b}$ The 330-MW(e) Fort St. Vrain HTGR operated from 1979 until 1989, generating about 490 MW(e)-years of (gross) electrical energy.

Information is not available.

$\mathrm{d}_{\text {Kaowool is an insulation material. }}$ 
Table 7.11. Projected burial volumes of radioactive wastes from SAFSTOR (mothballing/delayed dismantling) of Humboldt Bay-Unit 3 BWR a,b,c $^{\text {a }}$

\begin{tabular}{|c|c|}
\hline D\&D activity/reactor component & $\begin{array}{c}\text { Volume } \\
\left(\mathrm{m}^{3}\right)\end{array}$ \\
\hline Spent fuel racks & 28.3 \\
\hline \multicolumn{2}{|l|}{ Nuclear steam supply system removal } \\
\hline Reactor vessel & $66.8^{d}$ \\
\hline Reactor vessel internals & $19.1^{\mathrm{e}}$ \\
\hline Other components $\mathrm{f}^{\mathrm{f}}$ & 17.0 \\
\hline \multicolumn{2}{|l|}{ Removal of major equipment } \\
\hline Asbestos removal program & 116.1 \\
\hline Main condensers & 24.6 \\
\hline Main turbine/generator & 36.4 \\
\hline \multicolumn{2}{|l|}{ Disposal of contaminated plant systems } \\
\hline Condensate system & 65.5 \\
\hline Electrical system & 30.6 \\
\hline High-pressure steam and feedwater systems & 66.7 \\
\hline Radwaste collection and treatment systems & 49.2 \\
\hline Reactor cleanup and shutdown & 38.8 \\
\hline Turbine system & 415.3 \\
\hline Other systems 8 & 74.8 \\
\hline \multicolumn{2}{|l|}{ Decontamination of site buildings } \\
\hline Refueling building & 216.7 \\
\hline Turbine building & 21.3 \\
\hline Yard piping and soil & 211.4 \\
\hline Other ${ }^{h}$ & 14.7 \\
\hline Disposal of contaminated solid waste & 16.4 \\
\hline Process liquid waste $\mathrm{e}^{\mathrm{i}}$ & 27.3 \\
\hline $\begin{array}{l}\text { Disposal of modified plant seismic and off-gas } \\
\text { systems as a result of 1986-1991 capital } \\
\text { improvements }\end{array}$ & 20.8 \\
\hline Total & $1,577.8$ \\
\hline \multicolumn{2}{|c|}{$\begin{array}{l}\text { a Based on the information reported in ref. } 31 \text {. } \\
\text { b The 65-MW(e) Humboldt Bay-Unit } 3 \text { BWR operated from } 1963 \text { until } 1976 \text {, } \\
\text { generating } 545 \mathrm{MW}(\mathrm{e}) \text {-years of (net) electrical energy. The plant was placed in a } \\
\text { SAFSTOR mode in } 1988 \text {. The projections in this table assume delayed dismantling } \\
\text { (DECON) of the reactor begins after a SAFSTOR dormancy period of } 30 \text { years. At this } \\
\text { time (2018), the current inventory of spent nuclear fuel at the site will have been shipped to } \\
\text { a federal repository when the latter is available. } \\
\text { CExcept where noted, the volumes reported represent estimates for packaged Class-A }\end{array}$} \\
\hline $\begin{array}{l}\text { LLW. } \\
\mathrm{d}_{\text {Includes } 14.8 \mathrm{~m}^{3} \text { of Class-B LLW. }} \\
\text { 'Includes } 5.8 \mathrm{~m}^{3} \text { of Class-B LLW and } 13 \\
\mathrm{f}_{\text {Includes removed control rod drive mec }} \\
\text { gIncludes contaminated control rod drive } \\
\text { gaseous radwaste, yard piping, seismic equipm } \\
\text { conditioning equipment. } \\
\mathrm{h}_{\text {Includes contaminated buildings for hot }} \\
\text { radwaste treatment, and equipment storage. } \\
\text { iClass-B LLW. }\end{array}$ & $\begin{array}{l}\text { ation. } \\
\text { vents, } \\
\text { air- } \\
\text { uipment, }\end{array}$ \\
\hline
\end{tabular}


Table 7.12. Projected volumes of wastes from indian Point-Unit 1 PWR decommissioning activities ${ }^{a, b, c}$

\begin{tabular}{lccr}
\hline \multirow{2}{*}{ Reactor component(s) } & \multicolumn{3}{c}{ Container (type and number) } \\
\cline { 2 - 4 } & LSA boxes & Cask liners & Total \\
\hline $\begin{array}{l}\text { Contaminated piping, valves, } \\
\quad \text { equipment, and concrete }\end{array}$ & 2,440 & 0 & 2,440 \\
$\begin{array}{l}\text { Spent nuclear fuel racks } \\
\text { Reactor internals }\end{array}$ & 9 & 0 & 9 \\
Reactor vessel & 72 & 13 & 20 \\
$\quad$ Total containers & 2,508 & 13 & 52 \\
External volume $\left(\mathrm{m}^{3}\right)$ of each & 4.694 & 3.341 & 2,521 \\
$\quad$ container $($ box or liner) & 11,774 & 43 & 11,817 \\
\hline Total container volume $\left(\mathrm{m}^{3}\right)$ & & & \\
\hline
\end{tabular}

${ }^{a}$ Based on ref. 32.

${ }^{b}$ The 265-MW(e) Indian Point-Unit 1 PWR began operation in 1962 and generated about $1,440 \mathrm{MW}$ (e)-years of (gross) electrical energy before it was shut down in 1974. The projections in this table pertain to wastes from the dismantlement of the reactor following a SAFSTOR period of about 40 years.

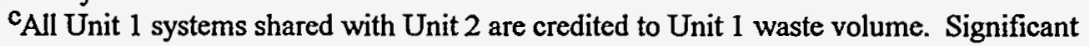
volume reductions from smelting and off-site decontamination are not factored into estimate. Total induced activity in vessel and vessel internals is approximately $25,000 \mathrm{Ci}$, projected in year 2013. About $90 \%$ of this induced activity is due to ${ }^{63} \mathrm{Ni}$ and about $9 \%$ is due to ${ }^{60} \mathrm{Co}$. 
Table 7.13. Inventories and projections of low-level radioactive wastes from La Crosse BWR decommissioning activities ${ }^{a, b}$

\begin{tabular}{|c|c|c|c|}
\hline \multirow[b]{2}{*}{ Calendar year(s) } & \multirow{2}{*}{$\begin{array}{l}\text { Decommissioning } \\
\text { mode }\end{array}$} & \multicolumn{2}{|c|}{$\begin{array}{l}\text { Quantity of waste shipped } \\
\text { to burial site } \mathbf{c}, \mathbf{d}, \mathbf{e}\end{array}$} \\
\hline & & $\begin{array}{l}\text { Volume } \\
\left(\mathrm{m}^{3}\right)\end{array}$ & $\begin{array}{l}\text { Undecayed } \\
\text { radioactivity } \\
\text { (Ci) }\end{array}$ \\
\hline \multicolumn{4}{|c|}{ Historical annual waste shipments } \\
\hline 1988 & SAFSTOR & 4.62 & 70.3 \\
\hline $1989^{f}$ & SAFSTOR & 6.74 & 32.12 \\
\hline $1990^{\mathrm{f}}$ & SAFSTOR & 4.59 & 0.74 \\
\hline $1991^{\mathrm{f}}$ & SAFSTOR & 5.46 & 0.32 \\
\hline $1992^{\mathrm{f}}$ & SAFSTOR & 2.61 & 0.44 \\
\hline $1993^{f}$ & SAFSTOR & 3.31 & 0.24 \\
\hline $1994^{\mathrm{f}}$ & SAFSTOR & 47.52 & 1.48 \\
\hline Totals (through 1994) & SAFSTOR & 74.85 & 105.64 \\
\hline \multicolumn{4}{|c|}{ Projected waste shipment quantities for certain periods } \\
\hline 1995-1998 & SAFSTOR & 0.0 & 0.0 \\
\hline $1999-2003$ & SAFSTOR & 6.5 & 13 \\
\hline 2004-2008 & SAFSTOR & 4.9 & 7 \\
\hline $2009-2013$ & SAFSTOR & 3.6 & 5 \\
\hline $2014-2020$ & SAFSTOR & 0.0 & 0.0 \\
\hline $2021-2025$ & DECON & 3,093 & 9,303 \\
\hline Projected totals (1995-2025) & SAFSTOR/DECON & 3,108 & 9,328 \\
\hline $\begin{array}{l}\text { Historical and projected totals } \\
\qquad(1988-2025)\end{array}$ & SAFSTOR/DECON & 3,183 & 9,434 \\
\hline
\end{tabular}

a Based on the information reported in ref. 33.

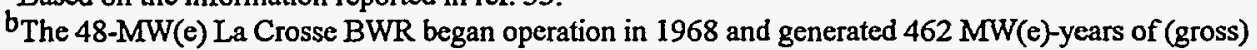
electrical energy until it was shut down in April 1987. The reactor was placed in SAFSTOR in 1991. The data in this table are based on a SAFSTOR period of 30 years.

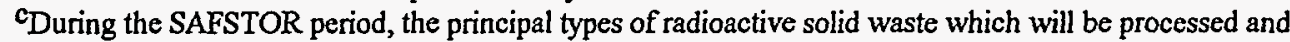
shipped to a suitable disposal facility will be low-level radioactive wastes principally with radioactivity content less than Class $C$ ( 10 CFR Part 61) wastes. These wastes will include (1) dry active wastes (DAW), normally Class A, unstable; (2) dewatered spent demineralizer resins and filtration media, normally Class A or B, stable; and (3) contaminated or irradiated plant system components, normally Class B or C, stable. estimates.

d Contributions from activated core components and structural materials are included in the 2021-2025

eValues are decayed to the time of burial.

Volume of waste for this year reflects significant reductions due to treatment. Waste shipments for this year contained DAW and contaminated metal, which were either decontaminated, supercompacted, or both by two Oak Ridge waste treatment companies [(Quadrex Recycle Center and Scientific Ecology Group (SEG), Inc]. 
Table 7.14. Projected volumes of wastes from Rancho Seco PWR decommissioning activities ${ }^{a}, \mathrm{~b}, \mathrm{c}$

\begin{tabular}{lr}
\hline \multicolumn{1}{c}{ Reactor component(s)/waste } & $\begin{array}{r}\text { Volume } \\
\left(\mathrm{m}^{3}\right)\end{array}$ \\
\hline Spent nuclear fuel racks & 359 \\
Reactor vessel & 212 \\
Reactor vessel internais & 156 \\
Primary system components and piping & 1,336 \\
\cline { 2 - 2 } Total for reactor vessel and components & 2,063 \\
Secondary and radwaste systems & \\
Contaminated structures & 2,625 \\
Processed liquid waste & 468 \\
Dry active waste & 98 \\
$\quad$ Grand total & 397 \\
\hline
\end{tabular}

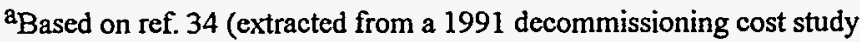
prepared by TLG Engineering, Inc.).

${ }^{b}$ The 918-MW(e) Rancho Seco-Unit 1 PWR was shut down in 1989. The reactor operated from 1974 until 1989, generating 5,277.3 MW(e)-years of (gross) electrical energy.

The projections in this table pertain to wastes from dismantlement of the reactor following a SAFSTOR period of about 20 years. 
Table 7.15. Projected volumes of wastes from San Onofre-Unit 1 PWR decommissioning activities ${ }^{\mathrm{a}, \mathrm{b}}$

\begin{tabular}{|c|c|c|c|c|c|}
\hline \multirow{2}{*}{$\begin{array}{l}\text { Activity/reactor facility component } \\
\text { or type of waste }\end{array}$} & \multicolumn{5}{|c|}{ Waste volume, $\mathrm{m}^{3}$} \\
\hline & Class A & Class B & Class $\mathrm{C}$ & GTCC & Total \\
\hline \multicolumn{6}{|l|}{ Annual SAFSTOR maintenance } \\
\hline Dry activated waste & 121.12 & & & & 121.12 \\
\hline Spent fuel racks & 14.39 & & & & 14.39 \\
\hline \multicolumn{6}{|l|}{ Nuclear steam supply system (NSSS) removal } \\
\hline Reactor coolant piping & 86.04 & & & & 86.04 \\
\hline Pressurizer quench tank & 12.94 & & & & 12.94 \\
\hline Reactor coolant system pumps and motors & 49.28 & & & & 49.28 \\
\hline Pressurizer & 56.64 & & & & 56.64 \\
\hline Steam generators & 367.54 & & & & 367.54 \\
\hline $\begin{array}{l}\text { Control rod drive mechanism and incore } \\
\text { instrumentation }\end{array}$ & 48.20 & & & & 48.20 \\
\hline Vessel internals & 22.26 & 13.06 & 30.81 & 20.25 & 86.38 \\
\hline Reactor vessel & 147.15 & 51.94 & & & 199.09 \\
\hline NSSS total & 790.05 & 65.00 & 30.81 & 20.25 & 906.11 \\
\hline \multicolumn{6}{|l|}{ Plant systems } \\
\hline Auxiliary feedwater & 92.12 & & & & 92.12 \\
\hline Containment ventilation & 42.45 & & & & 42.45 \\
\hline Contaminated electrical equipment & 79.27 & & & & 79.27 \\
\hline Feedwater sampling & 90.28 & & & & 90.28 \\
\hline Feedwater & 338.48 & & & & 338.48 \\
\hline Gaseous radwaste & 54.46 & & & & 54.46 \\
\hline Letdown demineralizer & 3.82 & & & & 3.82 \\
\hline Letdown and residual heat removal & 42.48 & & & & 42.48 \\
\hline Liquid radwaste & 37.72 & & & & 37.72 \\
\hline Post-accident sampling & 5.35 & & & & 5.35 \\
\hline Pressurizer and relief tank & 0.20 & & & & 0.20 \\
\hline Radwaste drain system & 5.21 & & & & 5.21 \\
\hline Reactor coolant pump seal water system & 13.71 & & & & 13.71 \\
\hline Reactor cool system & 3.12 & & & & 3.12 \\
\hline Reactor cool system sampling system & 13.06 & & & & 13.06 \\
\hline Safety injection & 85.70 & & & & 85.70 \\
\hline Spent fuel cooling & 11.98 & & & & 11.98 \\
\hline Chemical volume and control system & 23.93 & & & & 23.93 \\
\hline Plant systems total & 943.34 & & & & 943.34 \\
\hline \multicolumn{6}{|l|}{ Site buildings } \\
\hline Reactor sphere and enclosure & 249.27 & & & & 249.27 \\
\hline Auxiliary additions & 2.04 & & & & 2.04 \\
\hline Contaminated soil & 724.96 & & & & 724.96 \\
\hline Fuel storage & 21.69 & & & & 21.69 \\
\hline Miscellaneous contaminated buildings & 18.49 & & & & 18.49 \\
\hline Radwaste & 13.45 & & & & 13.45 \\
\hline Reactor auxiliary & 5.89 & & & & 5.89 \\
\hline Storage building & 1.42 & & & & 1.42 \\
\hline Site buildings total & $1,037.21$ & & & & $1,037.21$ \\
\hline
\end{tabular}


Table 7.15 (continued)

\begin{tabular}{|c|c|c|c|c|c|}
\hline \multirow{2}{*}{$\begin{array}{l}\text { Activity/reactor facility component } \\
\text { or type of waste }\end{array}$} & \multicolumn{5}{|c|}{ Waste volume, $\mathrm{m}^{3}$} \\
\hline & Class A & Class B & Class C & GTCC & Total \\
\hline Final waste liquid processing & & 40.24 & & & 40.24 \\
\hline Mixed waste & & 16.68 & & & 16.68 \\
\hline Totals & $2,922.79$ & 105.24 & 30.81 & 20.25 & $3,079.09$ \\
\hline
\end{tabular}

${ }^{a}$ Based on ref. 35 . Projections in this table pertain to wastes from reactor dismantlement (DECON) following a SAFSTOR period of 20 years. San Onofre-Unit 1 will be dismantled beginning in 2013.

${ }^{b}$ The 436-MW(e) San Onofre-Unit 1 PWR began operation in 1967 and generated about 6,045 MW(e)-years of (gross) electrical energy before it was shut down in 1992.

CIncludes $83.94 \mathrm{~m}^{3}$ of dry, activated waste (including protective worker clothing) generated during the latter phase of decommissioning. 
Table 7.16. Projected volumes of wastes from future Saxton PWR decommissioning activities ${ }^{a, b, c}$

\begin{tabular}{lr}
\hline \multicolumn{1}{c}{ Reactor component(s)/waste } & $\begin{array}{r}\text { Volume } \\
\left(\mathrm{m}^{3}\right)\end{array}$ \\
\hline Auxiliary system piping and valves & 28.32 \\
Containment vessel sump pumps & 0.85 \\
Containment ventilation equipment & 16.99 \\
Contaminated and activated concrete of containment vessel & 240.00 \\
Cooling heat exchangers & 16.99 \\
Demineralizers & 4.25 \\
Discharge tank drain pumps & 0.85 \\
General valves, controllers, and instrumentation & 42.48 \\
Low-level waste from disposal operations & 33.98 \\
Pressurizer & 7.26 \\
Primary coolant pump & 2.83 \\
Primary piping & 5.66 \\
Reactor vessel, head, and internals & 39.64 \\
Relief valve discharge tank & 4.25 \\
Shutdown cooling pumps & 0.85 \\
Steam generator & 8.43 \\
\multicolumn{1}{c}{ Total volume } & \\
\hline
\end{tabular}

${ }^{a}$ Based on the information reported in ref. 36.

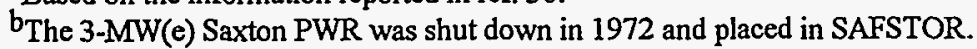
Work on dismantling the reactor site started in 1986. This facility operated from 1961 until 1971, generating 10.4 MW(e)-years of (gross) electrical energy.

${ }^{c}$ Activity data are unknown at this time. Saxton reactor decommissioning waste characteristics are still being reviewed, and additional information will be provided in this table in future reports.

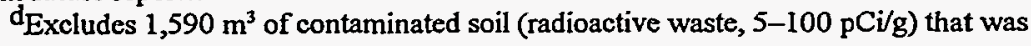
shipped to Envirocare of Utah, Inc., in 1994. 
Table 7.17. Characteristics of wastes from Shoreham BWR decommissioning activities ${ }^{a, b}$

(Unless otherwise indicated, all wastes are projected to be LLW Class A)

\begin{tabular}{|c|c|c|c|c|c|c|}
\hline \multirow{2}{*}{ Category } & \multicolumn{2}{|c|}{ Containers } & \multirow{2}{*}{$\begin{array}{l}\text { Mass } \\
(\mathrm{t})\end{array}$} & \multirow{2}{*}{$\begin{array}{l}\text { Shipment } \\
\text { volumec } \\
\left(\mathrm{m}^{3}\right)\end{array}$} & \multirow{2}{*}{$\begin{array}{l}\text { Radioactivity } \\
\text { (Ci) }\end{array}$} & \multirow{2}{*}{$\begin{array}{c}\text { Burial } \\
\text { volume } \\
\left(\mathrm{m}^{3}\right)\end{array}$} \\
\hline & Number & Type & & & & \\
\hline \multicolumn{7}{|l|}{$\begin{array}{l}\text { Items shipped to volume reduction facilities } \\
\text { for processing prior to burial }\end{array}$} \\
\hline Reactor recirculation system & e & e & e & e & e & e \\
\hline Control rod drive system $\mathrm{f}$ & $\mathrm{e}$ & e & e & $\mathrm{e}$ & e & e \\
\hline Residual heat removal system & $\mathrm{e}$ & e & e & $\mathrm{e}$ & e & e \\
\hline Core spray system & e & e & $\mathbf{e}$ & $\mathrm{e}$ & e & e \\
\hline Reactor water cleanup system & e & e & e & e & e & e \\
\hline Fuel pool cleanup system & e & e & e & e & e & e \\
\hline Condensate and demineralizer system & e & e & e & e & e & e \\
\hline Process sampling system & e & e & e & e & e & e \\
\hline SNF rack and accessories & $e$ & e & e & e & e & e \\
\hline Process and dry activated wastes & e & e & e & e & e & e \\
\hline Demineralizer system and resins/filters & e & e & e & e & e & $\mathbf{e}$ \\
\hline Liquid radwaste system & e & e & e & e & e & e \\
\hline Mirror insulation & $\mathrm{e}$ & e & $\mathbf{e}$ & $\mathbf{e}$ & e & e \\
\hline Total & 210 & $\mathbf{g}$ & $1,836.93$ & $6,329.38$ & 6.97 & 61.15 \\
\hline \multicolumn{7}{|l|}{$\begin{array}{l}\text { Items shipped directly to burial site (Barnwell) } \\
\text { Reactor internals } \\
\text { Control rod blades and drives }\end{array}$} \\
\hline Total & 51 & $\mathrm{i}$ & 96.90 & 79.62 & 675.79 & 79.62 \\
\hline Laundry (recycled)j & 588 & & 125.35 & 765.91 & 0.28 & 0 \\
\hline Miscellaneous equipment (recycled) ${ }^{k}$ & 87 & & 269.86 & 598.24 & 0.23 & 0 \\
\hline Grand total & 936 & & $2,329.06$ & $7,773.15$ & 683.27 & 140.77 \\
\hline
\end{tabular}

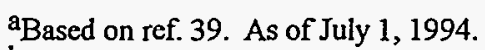

b The 820-MW(e) Shoreham BWR underwent low-power tests until 1989, when the Long Island Lighting Company agreed to sell the plant to the state of New York for decommissioning. A total of $865 \mathrm{MW}$ (e)-hours of (gross) electrical energy were generated during the low power tests.

cPrior to treatment and burial.

dVolume reduction facilities include SEG and Quadrex.

Information unavailable.

Excludes control rod blades and drives.

gContainer type: sea land containers, boxes, and high-integrity containers (HICs).

hIncludes some incore instruments, which are projected to be Class B LLW.

$\mathrm{i}_{\text {Casks and boxes. }}$

jCleaned by Interstate Nuclear Services and recycled.

$\mathrm{k}_{\mathrm{Equipment}}$ shipped to various reactor sites for salvaging. Such equipment includes traversing incore probe drives and detectors, reactor pressure vessel segments, control rod drives, steam relief valves, local power range monitors, intermediate range monitors, source range monitors, and recirculation pump components. 
Table 7.18. Characteristics of wastes from decontamination activities at the Three Mile Island-Unit 2 reactor site ${ }^{a, b}$

\begin{tabular}{|c|c|c|c|}
\hline \multirow{2}{*}{ Type of waste } & \multirow{2}{*}{$\begin{array}{l}\text { Mass } \\
\text { shipped } \\
\text { (t) }\end{array}$} & \multicolumn{2}{|c|}{$\begin{array}{l}\text { Total waste shipped from TMI } \\
\text { (August } 1979 \text { through December 1994) }\end{array}$} \\
\hline & & $\begin{array}{l}\text { Packaged volume } \\
\qquad\left(\mathrm{m}^{3}\right)\end{array}$ & $\begin{array}{l}\text { Shipment activity } \\
\text { (Ci) }\end{array}$ \\
\hline Spent nuclear fuel/core debris ${ }^{d}$ & 155.9 & 123.9 & $6,911,513$ \\
\hline \multicolumn{4}{|l|}{ Low-level and other wastes $\mathrm{e}$} \\
\hline Dry, activated waste (DAW) $\mathrm{f}$ & & $7,492.4$ & 744.7 \\
\hline Wet and solidified wasteg & & 320.3 & $7,787.5$ \\
\hline Submerged demineralizer system (SDS) & & 58.2 & $673,877.7$ \\
\hline \multicolumn{4}{|l|}{ EPICOR II system liners $\mathrm{i}$} \\
\hline First generation & & 125.7 & 77,750 \\
\hline Second generation & & 947.3 & $5,066.9$ \\
\hline Defueling water cleanup system (DWCS) & & 8.45 & $5,886.3$ \\
\hline Off-site deconable scrap & & 138.9 & 4 \\
\hline Totals & 155.9 & $9,215.5$ & $7,682,630.1$ \\
\hline
\end{tabular}

aThree Mile Island (TMI-Unit 2) is a PWR reactor with the following characteristics: rated capacity-926 MW(e); mass of fuel in core before accident-82 MTIHM; and number of fuel assemblies before accident-177. The reactor began operation in 1978 and generated $231.6 \mathrm{MW}$ (e)-years of (gross) electrical energy before being permanently shut down by an accident in March 1979.

based on information reported in ref. 40 .

CThese activities represent the cumulative sum of curies reported at the time of waste shipment. The values reported are not corrected for decay after the time of shipment.

dDefueling of the reactor started in January 1986. Fuel-debris shipments were completed in April 1990.

"Other wastes include those regarded as "abnormal" because their classification is currently uncertain.

fDry, activated wastes are dry wastes packaged in drums, boxes, and high-integrity containers.

gIncludes solidified miscellaneous liquids and miscellaneous resin liners and filters from TMI-Unit 2 systems.

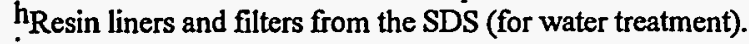

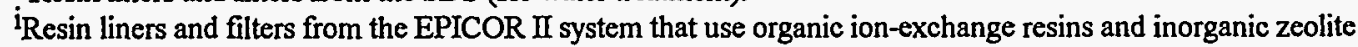
media. These include processing high-integrity containers (HICs). The EPICOR II system is a special type of filtering system used at the TMI-Unit 2 site in the final stages of decontaminating large volumes of contaminated water.

$\mathrm{j}$ Resin liners and filters from the DWCS that use inorganic zeolite media. These are primarily processing HICs. 
Table 7.19. Projected classification and volumes of wastes from Trojan PWR decommissioning activities

\begin{tabular}{|c|c|c|c|c|c|}
\hline \multirow{2}{*}{ Item } & \multicolumn{5}{|c|}{ Burial volume, $\mathrm{m}^{3}$} \\
\hline & Total & Class-A LLW & Class-B LLW & Class-C LLW & GTCC LLW \\
\hline Plant systems & $6,339.8$ & $6,150.1$ & 104.4 & 85.3 & \\
\hline Plant buildings & 637.2 & 637.2 & & & \\
\hline $\begin{array}{l}\text { Large component removal project } \\
\text { (steam generators and pressurizer) }\end{array}$ & $1,557.4$ & $1,557.4$ & & & \\
\hline Reactor vessel internals removal project & 325.8 & 267.2 & 6.5 & 42.5 & 9.6 \\
\hline Total & $8,860.2$ & $8,611.9$ & 110.9 & 127.8 & 9.6 \\
\hline
\end{tabular}

${ }^{a}$ Based on ref. 41.

b The 1,130-MW(e) Trojan PWR began operation in 1975 and generated 10,076 MW(e)-years of (gross) electrical energy before it was permanently shut down in January 1993. The projections of this table pertain to several phases of reactor dismantlement (DECON) during 1995-2001.

Table 7.20. Projected characteristics of wastes from Yankee Rowe PWR decommissioning activities ${ }^{a, b}$

\begin{tabular}{|c|c|c|}
\hline Reactor component & $\begin{array}{l}\text { Volume } \\
\left(\mathrm{m}^{3}\right)\end{array}$ & $\begin{array}{l}\text { Radioactivityc } \\
\text { (Ci) }\end{array}$ \\
\hline \multicolumn{3}{|c|}{ Component Removal Project (1993-1994) } \\
\hline Main coolant pumps (4) & 8.8 & 11 \\
\hline Pressurizer & 19.3 & 3 \\
\hline Reactor vessel internal components & 87.0 & 132,600 \\
\hline Steam generators (4) & 197.0 & 1,300 \\
\hline Miscellaneous waste & 191.0 & 370 \\
\hline Total (1993-1994) & 503.1 & 134,284 \\
\hline \multicolumn{3}{|c|}{ Final decommissioning $(1995-1997,1999)$} \\
\hline Building inventory & 410.3 & $<1$ \\
\hline General plant inventory & $1,922.8$ & $<500$ \\
\hline Reactor core baffle ${ }^{d}$ & 2.1 & $1,020,000$ \\
\hline Reactor vessel & 187.9 & 4,700 \\
\hline Soil & 707.9 & $<1$ \\
\hline Miscellaneous waste & 122.5 & $<50$ \\
\hline Total (1995-1999) & $3,353.5$ & $1,025,252$ \\
\hline Total (1993-1999) & $3,856.6$ & $1,159,536$ \\
\hline
\end{tabular}

aBased on ref. 42.

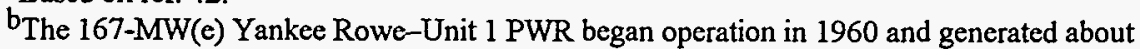
4,030 MW(e)-years of (gross) electrical energy before it was shut down in 1992.

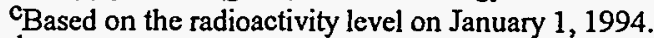

d The reactor core baffle, the component directly adjacent to the reactor core, was highly activated during plant operations. The baffle's radioactivity exceeds $10 \mathrm{CFR}$ Part 61.55 Class-C LLW requirements for near-surface disposal. DOE is responsible for the disposal of GTCC materials. 
Table 7.21. Characteristics of wastes from decommissioning activities at the Cimarron Fuel Fabrication Facility

\begin{tabular}{|c|c|c|c|c|}
\hline \multirow{2}{*}{ Project area } & \multirow{2}{*}{ Type of waste } & \multicolumn{2}{|c|}{$\begin{array}{l}\text { Total waste removed } \\
\text { from Cimarron through } \\
\text { December } 1994^{\mathrm{b}}\end{array}$} & \multirow{2}{*}{$\begin{array}{l}\text { Projected } \\
\text { waste volume } \\
\text { remaining } \\
\left(\mathrm{m}^{3}\right)\end{array}$} \\
\hline & & $\begin{array}{l}\text { Volume } \\
\left(\mathrm{m}^{3}\right)\end{array}$ & $\begin{array}{l}\text { Activity } \\
\text { (Ci) }\end{array}$ & \\
\hline Burial ground & $\operatorname{LLW}(\operatorname{LSA})^{\mathrm{d}}$ & $1,833.10$ & 5.37 & 0 \\
\hline Mixed-oxide fuel plant & $\begin{array}{l}\text { TRUW } \\
\text { LLW (LSA) }\end{array}$ & $\begin{array}{l}259.34 \\
463.88\end{array}$ & $\begin{array}{r}10.87 \\
3.25\end{array}$ & $\begin{array}{l}0 \\
0\end{array}$ \\
\hline $\begin{array}{l}\text { Uranium fuel plant areas } \\
\text { a. Uranium fuel plant } \\
\text { b. North Field }\end{array}$ & $\begin{array}{l}\text { LLW (LSA) } \\
\text { LLW (LSA) }\end{array}$ & $\begin{array}{r}2,307.30 \\
566.26\end{array}$ & $\begin{array}{l}3.65 \\
0.33\end{array}$ & $\begin{array}{c}12-24 \\
0\end{array}$ \\
\hline $\begin{array}{l}\text { Liquid process waste evaporation pond } \\
\text { a. Mixed-oxide plant pond } \\
\text { b. Uranium plant pond }\end{array}$ & $\begin{array}{l}\text { LLW (LSA) } \\
\text { LLW (LSA) }\end{array}$ & $\begin{array}{l}104.30 \\
183.73\end{array}$ & $\begin{array}{l}0.000009 \\
0.23\end{array}$ & $\begin{array}{l}0 \\
0\end{array}$ \\
\hline Sanitary lagoon piping and manholes & LLW (LSA) & $1,565.60$ & 2.93 & 0 \\
\hline Project totals & $\begin{array}{l}\text { TRUW } \\
\text { LLW (LSA) }\end{array}$ & $\begin{array}{r}259.34 \\
7,024.17\end{array}$ & $\begin{array}{l}10.87 \\
15.76\end{array}$ & $\begin{array}{c}0 \\
12-24\end{array}$ \\
\hline Total waste & & $7,283.51$ & 26.63 & $12-24$ \\
\hline
\end{tabular}

a Based on the information provided in ref. 43.

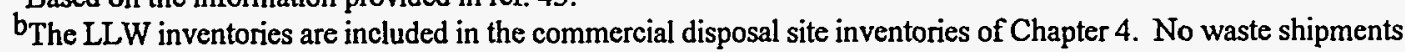
were made from the site during 1994.

CDecontamination work is anticipated to be completed during 1995. More than $95 \%$ of the estimated decontamination requirement has been completed.

$d_{\mathrm{LSA}}=$ low-specific-activity waste. 
Table 7.22. Inventories and projections of wastes from various activities at the West Valley Demonstration Project $t^{\mathrm{a}, \mathrm{b}}$

\begin{tabular}{|c|c|c|}
\hline Waste description & $\begin{array}{l}\text { Total wastes as of } \\
\text { December } 31,1994^{c}\end{array}$ & $\begin{array}{l}\text { Projected total wastes } \\
\text { upon completion } \\
\text { of the project }\end{array}$ \\
\hline \multicolumn{3}{|l|}{ Spent nuclear fuel remaining ${ }^{d}$} \\
\hline Mass, MTIHM & 27 & 27 \\
\hline Number of fuel assemblies & 125 & 125 \\
\hline \multicolumn{3}{|l|}{$\begin{array}{l}\text { High-level waste generated from reprocessing } \\
\text { operations and previtrification treatment }\end{array}$} \\
\hline Volume, $\mathrm{m}^{3}$ & $2,180^{f}$ & $240 \mathrm{~g}$ \\
\hline Activity, $\mathrm{Ci}^{\mathrm{h}}$ & $24,720,000$ & $22,400,000^{i}$ \\
\hline \multirow{2}{*}{\multicolumn{3}{|c|}{$\begin{array}{l}\text { Transuranic waste generated from presolidification } \\
\text { activities and } \mathrm{HLWW} \text { vitrification } \\
\text { Volume } \mathrm{m}^{3}\end{array}$}} \\
\hline & & \\
\hline Contact-handled & 321 & $\mathrm{j}$ \\
\hline Remote-handled & 427 & $\mathrm{j}$ \\
\hline \multicolumn{3}{|l|}{ Activity, $\mathrm{Ci}$} \\
\hline Contact-handled & $\mathbf{j}$ & j \\
\hline Remote-handled & $\vec{j}$ & $\mathrm{j}$ \\
\hline \multicolumn{3}{|c|}{$\begin{array}{l}\text { Low-level waste generated from presolidification } \\
\text { activities and HLW vitrification }\end{array}$} \\
\hline Buried waste (1982-86) volume, $\mathrm{m}^{3}$ & 5,786 & 5,786 \\
\hline Buried waste (1982-86) activity, $\mathrm{Ci}^{\text {h }}$ & 625 & j \\
\hline Stored waste volume, $\mathrm{m}^{3}$ & $9,506^{\mathrm{k}}$ & $\mathrm{j}$ \\
\hline Stored waste activity, $\mathrm{Ci}^{\mathrm{h}}$ & 1 & 1 \\
\hline \multicolumn{3}{|l|}{$\begin{array}{l}\text { Low-level waste incorporated in cement by } \\
\text { radwaste treatment system }\end{array}$} \\
\hline Stored waste volume, $\mathrm{m}^{3}$ & 4,989 & $\mathrm{j}$ \\
\hline Stored waste activity, $\mathrm{Ci}^{\mathrm{h}}$ & j & $\mathrm{j}$ \\
\hline \multicolumn{3}{|l|}{$\begin{array}{l}\text { Low-level waste from postsolidification D\&D } \\
\text { after HLW vitrification }\end{array}$} \\
\hline Volume, $\mathrm{m}^{3}$ & 0 & 4,771 \\
\hline Activity, $\mathrm{Ci}^{\mathrm{h}}$ & 0 & j \\
\hline \multicolumn{3}{|l|}{$\begin{array}{l}\text { Total low-level summary } \\
\text { (buried and stored wastes) }\end{array}$} \\
\hline Volume, $\mathrm{m}^{3}$ & 20,281 & $\mathrm{j}$ \\
\hline Activity, $\mathrm{Ci}^{\mathrm{h}}$ & j & $j$ \\
\hline
\end{tabular}

a Based on data reported in ref. 44.

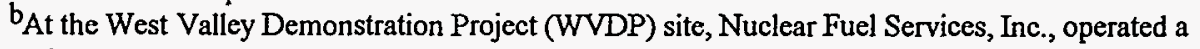
reprocessing plant with a rated capacity of 300 MTIHM/year. During its operation from 1966 to 1972 , about 640 MTHM of spent nuclear fuel were reprocessed.

CWastes generated after 1987 are regarded as stored, not buried or disposed.

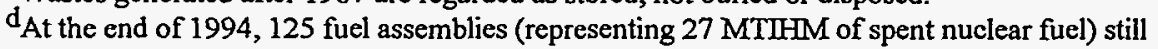
remained in storage at the WVDP. These assemblies are owned by DOE. The return shipment of all commercially owned spent fuel ( 625 fuel assemblies) to the owner utilities was completed by the end of 1986. The cursent SNF inventory at the WVDP consists of 85 BWR assemblies (11.7 MTTFMM) and 40 PWR assemblies (15.3 MTIHM). 
Table 7.22 (continued)

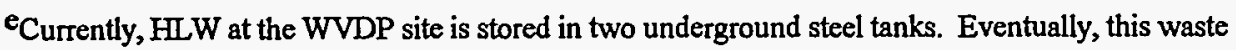
will be vitrified and about 300 canisters of glass will be produced. It is assumed that each canister will contain $0.80 \mathrm{~m}^{3}$ of glass.

W Waste form is liquid, sludge, and zeolite.

gGlass is the waste form.

${ }_{\text {h}}$ Principal nuclides include ${ }^{241} \mathrm{Am},{ }^{241} \mathrm{Pu},{ }^{137} \mathrm{Cs},{ }^{99} \mathrm{Tc},{ }^{90} \mathrm{Sr}$, and ${ }^{63} \mathrm{Ni}$.

iDecayed activity for 1998 .

junknown.

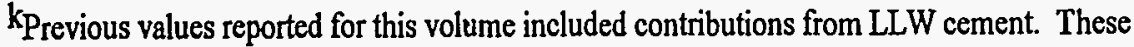
contributions are no longer included.

${ }_{1}$ Information currently unavailable. This information will be updated in future updates of this report to include estimated total activity for containerized wastes as the information becomes available.

${ }^{m}$ Comprised of Class A and Class C LLW. See Table A. 6 of Appendix A. 


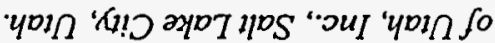

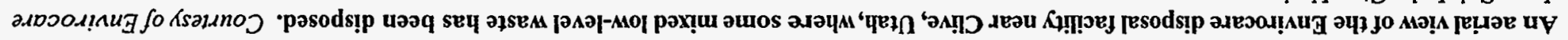

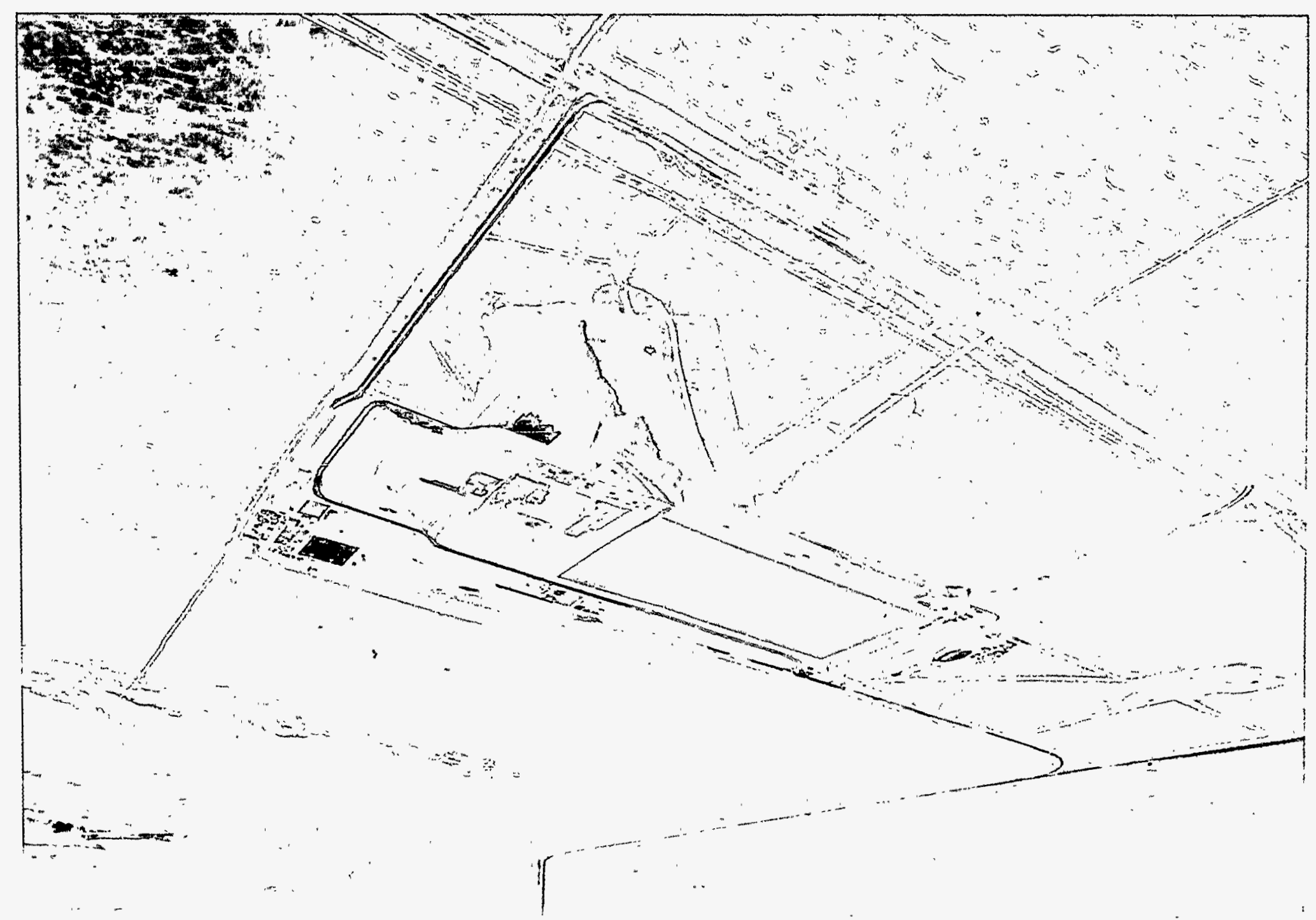

S6-SSES OIOHd INDO 


\section{MIXED LOW-LEVEL WASTE}

\subsection{INTRODUCTION}

This chapter reports estimated inventories and generation rates of mixed low-level waste (MLLW) from DOE-site and commercial operations. MLLW includes mixtures of low-level radioactive materials and (chemically and/or physically) hazardous wastes. Typically, MLLW at DOE sites includes a variety of contaminated materials, including air filters, cleaning materials, engine oils and grease, paint residues, photographic materials, soils, building materials, and plant equipment being decommissioned. Mixed wastes include other types of radioactive wastes. All high-level wastes ( $\mathrm{HLWs}$ ) are regarded as mixed and are discussed in Chapter 2. Mixed transuranic wastes (MTRUWs) are not included in this chapter, but they are included in the TRUW inventories and projections of Chapter 3.

The radioactive components of mixed wastes are subject to the Atomic Energy Act (AEA), as amended,' which, for government sources, is administered by DOE, and, for commercial sources, by NRC (unless a state has obtained agreement-state status). In general, the hazardous components of most mixed wastes are subject to either of two federal statutes that are administered by the U.S. Environmental Protection Agency (EPA) (unless a state has obtained an authorization status): (1) the Resource Conservation and Recovery Act (RCRA), as amended, ${ }^{2}$ and (2) the Toxic Substances Control Act (TSCA). ${ }^{3}$ Some mixed wastes, particularly spent engine oils, are regulated by state laws. The treatment, handling, and disposal of RCRA- and TSCA-regulated mixed wastes are subject to the regulations of the EPA ${ }^{4,5}$ and NRC (or the authorized and agreement states), or DOE. Table 8.1 (data from ref. 6) lists those states and territories designated by EPA as having mixed waste authorization.

In this report, MLLW is considered separately from the purely radioactive LLW, which is discussed in Chapter 4. This section reports MLLW inventories and projections for two major groups of $M L L W$. The first comprises wastes containing hazardous components that are RCRA- and/or state-regulated, including RCRA-/stateregulated wastes that are also subject to TSCA. The summary information reported for these RCRA-/stateregulated wastes are based on the 1995 update to the DOE Mixed Waste Inventory Report (MWIR) ${ }^{7}$ data set. At the time of preparing this IDB report, the updated MWIR data set was undergoing technical review in preparation for official release.

Wastes whose hazardous components are regulated by TSCA comprise the second group of MLLW; inventories and projections of this group are reported in this chapter. Information for the TSCA-regulated wastes was recently provided to the IDB Program by DOE sites.

Unless otherwise noted, the inventories and projections reported for MLLW are separate from those reported for strictly radioactive LLW in Chapter 4. Inventories of MLLW currently stored at DOE sites are being thoroughly characterized. As a result, the waste at some sites could require reclassification, thereby causing significant changes in the inventories that reflect current data.

The Federal Facility Compliance Act (FFCA) ${ }^{8}$ amends RCRA to require (among other things) that DOE develop a Site Treatment Plan (STP) for each site which generates, stores, or treats MLLW. Specifically, Sect. 3021(b) of RCRA, as amended by the FFCA, requires that DOE prepare STPs for developing the technologies and capacities needed to treat MLLWs according to the standards required by EPA in Sect. 3004(m) of RCRA. All STPs are required to be in place by October 1995. These STFs could significantly impact future projections of MLLWs reported in this document for DOE sites.

\subsection{WASTE CHARACTERIZATION}

MLLWs stored at DOE sites have several common features: (1) such wastes have different blends of hazardous (chemical and/or physical) and radioactive components that dictate precautionary measures; (2) several processes may be involved in generating these wastes; and (3) various methods can be used to treat and prepare these wastes for storage. In recent years, EPA has adopted new toxicity-characteristic leaching procedures 
(TCLPs) for identifying some hazardous components of these wastes. Representative data on the chemical and radionuclide compositions of MLLWs will be reported in the $1995 \mathrm{MWIR}^{7}$ and associated data set.

In this chapter, inventories and annual (1994 and projected) generation rates of MLLW are expressed in terms of physical and hazardous categories. RCRA-/stateregulated waste from DOE activities is generally classified into four physical/chemical categories: liquids, solids, specific waste forms, and final waste forms. TSCAregulated waste from DOE activities is classified into four physical categories: solid, liquid, gas, and sludge, and two hazard categories: PCB and asbestos. MLLW from commercial activities is classified by hazardous categories.

\subsection{SUMMARY OF DOE MLLW}

Cumulative mass inventories and generation rates are reported in this chapter for most of the DOE sites listed in Table C.3, Appendix C. Some generation rates may vary from current inventory additions because the generation levels reported do not reflect treatment that may take place before the waste is placed in interim storage. DOE site inventories and generation rates are given in both mass [kilograms $(\mathrm{kg})$ ] and volume [cubic meters $\left(\mathrm{m}^{3}\right)$ ] units. Most DOE sites track MLLW inventories and generation by volume based on the number of containers. However, MLLW mass information is not always available because the waste may not be weighed.

A breakdown of the MLLW volume inventory by site is graphically described in Fig. 8.1, and a breakdown of the volume-generation level by site is shown in Fig. 8.2. The current total volume inventory of MLLW at DOE sites is about $162,400 \mathrm{~m}^{3}$. About $97,100 \mathrm{~m}^{3}$ of MLLW are estimated to be generated during the period 1994 through 1998.

Summary cumulative inventories and projected cumulative generation for MLLW from DOE site operations are reported in Tables 8.2-8.5. Cumulative inventories of $\mathrm{MLLW}$ in interim storage are reported on a mass and volume basis in Tables 8.2 and 8.3 , respectively. Projected 5-year mass generation and volume generation of these wastes from DOE site activities are given in Tables 8.4 and 8.5 , respectively.

Detailed characteristics of RCRA-/state-regulated wastes from site operations are described in Tables 8.6-8.8. The MWIR documents inventories and generation levels for a wide range of categories of RCRA-Istate-regulated wastes. These categories are based on various physical and chemical groupings and are described in Table 8.6 (adapted from ref. 9). Cumulative mass and volume inventories for these physical/chemical groups are given for each site in Table 8.7 and for the DOE complex in Table 8.8. Projected cumulative 5-year generation levels for each category are given in Table 8.9.

Detailed characteristics of TSCA-regulated MLLW from DOE site operations are reported by physical category (solid, liquid, gas, and sludge) in Tables $8.10-8.13$ and by TSCA hazard category [polychlorinated biphenyls (PCBs), asbestos, and other specified materials] in Tables 8.14-8.17.

Cumulative 1994 mass inventories and volume inventories are given in the physical category tables for the TSCA-regulated wastes (Tables 8.10 and 8.11, respectively). Annual mass generation and volume generation of these wastes for 1994 are reported in Tables 8.12 and 8.13 , respectively.

The hazardous category tables for TSCA-regulated wastes include information on PCB wastes and asbestos. Other MLLWs regulated by TSCA at each site are also indicated. PCBs are a group of synthetic organic chemicals once widely used in electrical equipment, special hydraulic systems, heat-transfer systems, and other industrial products. They are currently considered a possible carcinogen. Asbestos is a group of magnesium silicate compounds that are mixed with varying amounts of calcium and iron silicates. They are fibrous, noncombustible minerals that have been previously used in the manufacture of many fireproofing and insulating materials. However, such compounds have been found to produce long-term carcinogenic effects; consequently, their use is being phased out.

Cumulative 1994 mass and volume inventories are reported in the hazardous category tables for TSCAregulated wastes (Tables 8.14 and 8.15 , respectively). Annual mass generation and volume generation of these wastes for 1994 are reported in Tables 8.16 and 8.17, respectively.

Historical and annual projections of DOE-site MLLW are reported in Tables 8.18 and 8.19. These tables report annual mass data (Table 8.18) and annual volume data (Table 8.19) on generated TSCA-regulated MLLW from DOE site activities, based on ref. 10 .

\subsection{SPECIFIC DOE SITE TSCA MLLW}

This section highlights the major ground rules and assumptions associated with the DOE site TSCA MLLW information reported in Tables 8.2-8.5 and Tables 8.7-8.19. Most of the information reported in the subsections that follow describes the TSCA MLLW inventory, generation, and projection ground rules and assumptions documented in the specific DOE site submittals of ref. 10. Corresponding site information 
associated with RCRA-/state-regulated waste data are reported in the MWIR (ref. 7). Significant changes in DOE TSCA MLLW inventory and characteristics data from that reported in the 1994 edition (1993 data) of this report (Rev. 10) are summarized in Table 8.20.

\subsubsection{Ames Laboratory (Ames)}

Materials containing radioactively contaminated asbestos comprise the TSCA MLLW inventories and projections for Ames in Tables 8.2, 8.3, 8.10, 8.11, 8.14, $8.15,8.18$, and 8.19. The inventories represent cumulative levels from 1986 .

\subsubsection{Argonne National Laboratory-East (ANL-E)}

As of the end of 1993, ANL-E had no TSCA MLLW inventory. However, $62,400 \mathrm{~kg}\left(52 \mathrm{~m}^{3}\right)$ of $\mathrm{PCB}$ wastes were generated at ANL-E during 1994. Projections reported in Tables 8.18 and 8.19 include some waste generation during 1995 .

\subsubsection{Brookhaven National Laboratory (BNL)}

Solid asbestos materials comprise the BNL TSCA MLLW inventories reported in Tables 8.2, 8.3, 8.10, 8.11, 8.14 , and 8.15 . These inventories represent cumulative levels from 1990. Projected wastes reported in Tables 8.18 and 8.19 assume that small quantities will continue to be generated as a result of maintenance activities. Decommissioning or environmental restoration activities could result in increased generation of asbestos wastes but are not anticipated at this time. PCB wastes at BNL are reported in the MWIR (ref. 7).

\subsubsection{Energy Technology Engineering Center (ETEC)}

TSCA MLLW inventories reported for ETEC in Tables 8.2, 8.3, 8.10, 8.11, 8.14, and 8.15 represent levels accumulated from 1989. Projections reported for generated mass in Table 8.18 and for generated volume in Table 8.19 are based on planned site D\&D activities. These projections include quantities of asbestos-containing floor tiles, which are scheduled to be removed during 1998-1999.

\subsubsection{Fernald Environmental Management Project (FEMP)}

Information for this site is not available.

\subsubsection{Grand Junction Projects Office (GJPO)}

GJPO TSCA MLLWs are comprised of radioactive PCBs. Tables 8.2, 8.3, 8.10, 8.11, 8.14, and 8.15 report cumulative inventories of these wastes from 1990. Projections reported in Tables 8.18 and 8.19 apply to wastes from the remediation of radioactive PCB ballasts. It is assumed that this remediation will subsequently decrease each year.

\subsubsection{Hanford Site (Hanford)}

PCB wastes comprise the TSCA MLLW inventories reported for Hanford in Tables 8.2, 8.3, 8.10, 8.11, 8.14, and 8.15 . These inventories represent cumulative levels from 1987. Site projections reported in Tables 8.18 and 8.19 are based on Hanford's 30-year solid mixed waste forecast. These projections exclude contributions from off-site generation.

\subsubsection{Idaho National Engineering Laboratory (INEL)}

Inventories of INEL PCB wastes (solids and liquids) are given in Tables 8.2, 8.3, 8.10, 8.11, 8.14, and 8.15. The earliest date from which these wastes were accumulated is presently not known. It is likely that PCB wastes will be generated at INEL in the future, however, estimates of specific quantities are not yet available. There are also inventories and projections of asbestos wastes at INEL, however, these are included in the site data for LLW in Chapter 4.

\subsubsection{K-25 Site}

For the K-25 Site, TSCA MLLW inventories are reported in Tables 8.2, 8.3, 8.10, 8.11, 8.14, and 8.15. These inventories represent cumulative levels of waste materials - some of which were produced from the startup of site operations in 1945. Projections of annual TSCA MLLW generation reported in Tables 8.18 and 8.19 are based on 1994 generation levels.

\subsubsection{Kansas City Plant (KCP)}

Currently, KCP does not have any inventories of TSCA MLLW, and no quantities of TSCA MLLW are anticipated for the future.

\subsubsection{Lawrence Berkeley Laboratory (LBL)}

LBL TSCA MLLW inventories, representing cumulative levels for 1994 only, are reported in Tables 8.2, 8.3, 8.10, 8.11, 8.14, and 8.15. Current 
inventories are comprised of PCBs. Significant quantities $(1087 \mathrm{~kg}$ ) of asbestos debris were generated during 1994 as a result of fume hood dismantling, and these were shipped to an off-site facility for treatment, storage, and disposal. Quantities reported for PCB wastes (capacitors and ballasts) are based on an assumed density of $1500 \mathrm{~kg} / \mathrm{m}^{3}$, and quantities reported for asbestos debris reflect a density of $400 \mathrm{~kg} / \mathrm{m}^{3}$. Projections of LBL generation levels reflect quantities of asbestos from glove boxes and PCBs from the replacement of PCB capacitors and ballasts.

\subsubsection{Lawrence Livermore National Laboratory (LLNL)}

Inventories of TSCA-regulated MLLW at LLNL are reported in Tables 8.2, 8.3, 8.10, 8.11, 8.14, and 8.15. These quantities represent cumulative levels from 1990. No TSCA MLLW is projected from future LLNL activities. Current inventories resulted from either the removal of PCB-containing transformers from service or from renovation/closure of buildings containing asbestos.

\subsubsection{Los Alamos National Laboratory (LANL)}

Inventories of TSCA MLLW at LANL reported in Tables 8.2, 8.3, 8.10, 8.11,8.14, and 8.15 represent cumulative levels from 1971. These reported inventories are based on limited records kept of asbestos and PCB wastes during the early operating years. A significant increase occurred during 1993 from the addition of contaminated equipment to the asbestos inventory.

\subsubsection{Mound Plant (Mound)}

TSCA MLLWs are not routinely generated at Mound. TSCA MLLW inventories reported in Tables 8.2, 8.3, $8.10,8.11,8.14$, and 8.15 are cumulative levels of asbestos from 1947. After 1994, no major generation of either PCB or asbestos wastes are anticipated. Projections reported in Tables 8.18 and 8.19 assume a generation rate of two 55-gal drums per year of radioactively contaminated asbestos pipe covers and/or floor tiles. Future generation projections will be affected by changes in site $\mathrm{D} \& \mathrm{D}$ plans and schedules.

\subsubsection{Naval Reactor (NR) Sites}

Tables $8.2,8.3,8.10,8.11,8.14$, and 8.15 report the total inventories of NR sites. These include contributions from the Bettis Atomic Power Laboratory (BAPL), the Knolls, Kesselring (KESS), and Windsor (WIND) sites of the Knolls Atomic Power Laboratory (KAPL); and six naval shipyards (Charleston, Mare Island, Norfolk, Pearl Harbor, Portsmouth, and Puget Sound). The quantities reported in these tables reflect cumulative amounts from as early as 1982. Projected wastes reported for NR sites in Tables 8.18 and 8.19 are anticipated to result from a variety of site activities, including facility D\&D, plant layup, ship inactivation, and asbestos removal/replacement.

\subsubsection{Nevada Test Site (NTS)}

No inventories of TSCA MLLW are presently found at NTS. However, current volumes of radioactive materials in storage are undergoing characterization at the site. It is not known at this time if TSCA MLLW exists among the inventory. Future volumes of TSCA MLLW may be generated from NTS D\&D and environmental restoration activities, however no estimates are available at this time.

\subsubsection{Oak Ridge National Laboratory (ORNL)}

Inventories of TSCA MLLW at ORNL reported in Tables $8.2,8.3,8.10,8.11,8.14$, and 8.15 are cumulative levels from 1977. Site-generated projections reported in Tables 8.18 and 8.19 are based on 1994 generation levels.

\subsubsection{Oak Ridge Institute of Science and Education (ORISE)}

Currently, ORISE does not have any inventories of TSCA MLLW, and no quantities of TSCA MLLW are anticipated for the future.

\subsubsection{Paducah Gaseous Diffusion Plant (PAD)}

The PAD site TSCA MLLW inventories reported in Tables 8.2, 8.3, 8.10, 8.11, 8.14, and 8.15 are cumulative levels from 1980. Projections reported for generated TSCA wastes in Tables 8.18 and 8.19 are based on the following assumptions:

- a routine constant TSCA waste generation of $608 \mathrm{~m}^{3} / \mathrm{year}$;

- the mass of a $55-\mathrm{gal}\left(0.21-\mathrm{m}^{3}\right)$ drum is $159 \mathrm{~kg}$;

- generation levels result from routine site activities, as well as environmental restoration activities, engineering project activities, and D\&D activities;

- reported quantities apply to waste solids and liquids, not sludges; and

- reported quantities exclude wastes contaminated by both PCBs and RCRA hazardous materials. 


\subsubsection{Pantex Plant (PANT)}

During $1994,4500 \mathrm{~kg}\left(3 \mathrm{~m}^{3}\right)$ of tritium-contaminated asbestos insulation was generated at PANT as a result of maintenance activities involving the removal of piping containing discharged condensate. This quantity comprises the current site TSCA MLLW inventory. No additional future generation of this waste is anticipated from this maintenance activity. The mass of this asbestos waste reported in Tables 8.2, 8.10, 8.12, 8.14, 8.16, and 8.18 is based on an assumed density of $1500 \mathrm{~kg} / \mathrm{m}^{3}$.

\subsubsection{Pinellas Plant (Pinellas)}

Pinellas currently has no inventories of TSCA MLLW. However, some projected quantities for 1995 are reported in Tables 8.18 and 8.19 as a result of the removal of ductwork containing asbestos.

\subsubsection{Portsmouth Gaseous Diffusion Plant (PORTS)}

At PORTS, TSCA-regulated MLLW is comprised of both PCBs and asbestos. Inventories of these wastes represent cumulative levels from 1954, and are reported in Tables 8.2, 8.3, 8.10, 8.11, 8.14, and 8.15. Projections of generated TSCA wastes from PORTS site activities are reported in Tables 8.18 and 8.19. These forecasts are based on annual historical data and reflect a $10 \%$ reduction/year to account for waste minimization. They do not include PCB and asbestos contributions from D\&D activities nor final removal of PCB-contaminated equipment (capacitors, transformers, impregnated gaskets, and ventilation ducts).

\subsubsection{Rocky Flats Environmental Technology Site (RFETS)}

Both radioactive PCBs and asbestos are included in the RFETS TSCA MLLW inventories reported in Tables 8.2, 8.3, 8.10, 8.11, 8.14, and 8.15. These inventories represent cumulative levels from 1987. Projections reported in Tables 8.18 and 8.19 are based on separate assumptions pertaining to PCBs and asbestos. PCB generation rates assume that historical PCB spill sites will be funded for remediation during 1995-2000, and that these sites will contain only low-level radioactivity. Asbestos generation rates assume funding for asbestos abatement projects, no significant changes in regulations affecting encapsulation and abatement, and increases in waste generation from building usage transition and $D \& D$ activities.

\subsubsection{Sandia National Laboratory/New Mexico (SNLNM)}

Radioactive asbestos comprises the SNL/NM TSCA MLLW inventories reported in Tables 8.2, 8.3, 8.10, 8.11, 8.14 , and 8.15. These inventories are levels cumulative from 1989. Future TSCA MLLWs cannot be estimated at this time. These wastes are expected to be mostly asbestos from building D\&D activities. Some older SNL/NM buildings slated for $D \& D$ are known to contain radiologically contaminated asbestos. PCBs are expected to comprise a much smaller portion of future-generated SNL/NM TSCA MLLW.

\subsubsection{Sandia National Laboratory/California (SNL/CA)}

Current inventories of TSCA MLLW at SNL/CA are based on small quantities of asbestos removed during 1994. These are reported in Tables 8.2, 8.3, and 8.10-8.17. As indicated in Tables 8.18 and 8.19 , no quantities of additional TSCA MLLW are anticipated.

\subsubsection{Savannah River Site (SRS)}

SRS TSCA MILLW inventories reported in Tables 8.2, $8.3,8.10,8.11,8.14$, and 8.15 represent cumulative levels from 1986. Most of the SRS TSCA wastes are asbestos materials whose mass was estimated from site-reported volume data using an assumed density of $500 \mathrm{~kg} / \mathrm{m}^{3}$. This density was also used to estimate the TSCA MLLW mass generation rates reported in Tables 8.12, 8.16, and 8.18. The projections reported in Tables 8.18 and 8.19 assume a reduction of $80 \%$ in levels of annual generation previously reported in this document. At SRS, programs are either being developed or under development to reduce the generation of radioactively contaminated asbestos.

\subsubsection{West Valley Demonstration Project (WVDP)}

TSCA MLLW inventories reported for WVDP in Tables 8.2, 8.3, 8.10, 8.11, 8.14, and 8.15 are cumulative quantities of asbestos from 1982. Projections reported in Tables 8.18 and 8.19 also pertain solely to asbestos and assume a $20 \%$ annual reduction through 1999. WVDP inventories and projections for radioactively contaminated PCBs are contained in the MWIR (ref. 7). The estimates reported for asbestos generation are based on actual generation levels achieved during January-April 1995. All masses reported for asbestos include contributions from asbestos, debris contaminated with asbestos, and piping still having asbestos on it. 


\subsubsection{Y-12 Plant (Y-12)}

Y-12 Plant TSCA MLLW inventories reported in Tables 8.2, 8.3, 8.10, 8.11, 8.14, and 8.15 represent cumulative levels from 1982. These wastes are PCBs regulated by TSCA. Contributions from asbestos are not reported because asbestos is considered to be a sanitary waste at the Y-12 Plant. Waste volumes reported were estimated from site-reported waste mass information using an assumed density of $1500 \mathrm{~kg} / \mathrm{m}^{3}$ for solids and $1000 \mathrm{~kg} / \mathrm{m}^{3}$ for liquids. For Tables 8.18 and 8.19 , projected TSCA MLLW generation rates for the Y-12 Plant reflect an anticipated stand-down of site operations during 1994-1996. TSCA MLLW generation is expected to increase following the anticipated resumption of site operations after 1996. However, this assumes that funding will be available for future $D \& D$ of existing site facilities.

\subsection{COMMERCIAL MLLW}

In recent years, the NRC and EPA co-sponsored a survey study to compile a national profile of the volumes, characteristics, and treatability of commercially generated MLLW. Such a profile was designed to provide the following:

- states and compacts with information to assist in planning and developing adequate disposal capacity for low-level radioactive waste, including MLLW, as mandated by the Low-Level Radioactive Waste Policy Amendments Act;"

- private developers with a clearer idea of the characteristics and volumes of mixed waste and the technical capability and capacity needed to treat this waste; and

- a reliable national data base of the volumes, characteristics, and treatability of commercial mixed waste.

In addition, the data were collected to provide a basis for possible federal actions that would effectively manage and regulate the treatment and disposal of mixed waste. Results from this investigation are documented in ref. 12 and summarized in this report.

The study identified the types and volumes of MLLW generated from five groups of facilities: nuclear utilities, medical facilities, academic institutions, industrial facilities, and NRC-licensed government facilities. The study selected a random sample of 1323 facilities out of a total target population of 2936 facilities. Data from the 1016 completed mixed waste survey questionnaires (77\% response rate) received and the use of appropriate weighting factors indicate that approximately $3950 \mathrm{~m}^{3}$ of MLLW-of which $72 \%$ was liquid scintillation fluids-were generated in the United States in 1990.

The study divided the MLLW into several hazardous stream categories, including the following:

- Liquid scintillation fluids from laboratory counting activities.

- Waste oil from various pumps, equipment, and maintenance activities.

- Chlorinated or fluorinated organics and chlorinated fluorocarbons, including sludges and contaminated filters from dry cleaning, refrigeration, degreasing, and decontamination operations. Chloroform and a number of pesticides are also included.

- Other organics, including miscellaneous solvents, reagents, expired products, and other organic compounds (or materials like rags, wipes, etc., contaminated with such) from research and manufacturing activities, experimental procedures, and laboratory and process equipment cleaning.

- Lead wastes, including lead shielding and lead solutions for research and industrial facilities.

- Mercury wastes, including equipment and debris contaminated with mercury.

- Chromate wastes, including chromium-contaminated solutions for research, maintenance, and waste treatment (ion exchange) operations.

- Cadmium wastes from decontamination activities.

- Aqueous corrosive wastes, including inorganic acids, or, in some instances, bases from cleanup and decontamination activities.

- Other hazardous materials, including materials (a) either not readily assignable to any one of the previous categories or (b) containing a number of different hazardous materials.

Summaries of estimated generation rates, amounts in storage, and amounts treated for each of the five facility categories and each of the hazardous waste stream categories are shown in Tables 8.21 and 8.22, respectively (data from ref. 12). Upper and lower bounds were also set for the volume of mixed waste that is untreatable under current technologies by making the simplifying assumption that liquid scintillation fluids, oil, nonhalogenated organics, and corrosive wastes are treatable. Deducting their total contribution from the estimated total mixed waste generation rate leaves residues of about $524 \mathrm{~m}^{3}$. This upper bound for untreatable mixed waste is about $13 \%$ of 
the estimated 1990 national generation rate of $3950 \mathrm{~m}^{3}$.

However, it was noted that the capacity to treat all of the

so-called treatable mixed waste may not be available.

\subsection{REFERENCES}

1. U.S. Congress, Atomic Energy Act of 1954, Pub. L. 83-703, Aug. 15, 1954.

2. U.S. Congress, Resource Conservation and Recovery Act of 1976, Pub. L. 94-580, Oct. 21, 1976, as amended by the Hazardous and Solid Waste Amendments Acts of 1984, Pub. L. 98-616, Nov. 9, 1984.

3. U.S. Congress, Toxic Substances Control Act of 1976, Pub. L. 94-469, Oct. 11, 1976.

4. U.S. Environmental Protection Agency, "Identification and Listing of Hazardous Waste," Code of Federal Regulations, 40 CFR Parts 260-271 (1986).

5. U.S. Environmental Protection Agency, "Subchapter R-Toxic Substances Control Act," Code of Federal Regulations, 40 CFR Parts 700-799 (1988).

6. Susan Jones, DOE Office of Program Integration, Germantown, Maryland, correspondence to Steve Storch, $\mathrm{DB}$ Program, ORNL, Oak Ridge, Tennessee, dated July 3, 1995, containing the updated respective EPA mixed waste authorization statuses for states and U.S. territories as of May 31, 1995.

7. U.S. Department of Energy, Office of Environmental Management, 1995 Mixed Waste Inventory Report (to be issued).

8. U.S. Congress, Federal Facility Compliance Act of 1992, Pub. L. 102-386, Oct. 6, 1992.

9. U.S. Department of Energy, Office of Waste Management, DOE Waste Treatability Group Guidance, DOE/LLW-217, Rev. 0 (January 1995).

10. DOE site TSCA-regulated MLLW data submittal attachments, submitted to the IDB Program during May-August 1995. The following TSCA MLLW submittals were received and reviewed by the $\mathrm{DB}$ Program before analysis and integration. Preceding each submittal is the site (in parentheses) to which it refers.

a. (Ames) Lowell K. Mathison, Ames Laboratory, Ames, Iowa, correspondence to Gaile A. Higashi, DOE Chicago Operations Office, Ames Laboratory Management Office, Argonne, Illinois, "1995 Integrated Data Base (IDB) Request for TSCA LLW," dated June 2, 1995.

b. (ANL-E) M. A. Sodaro, Argonne National Laboratory, Argonne, Illinois, correspondence to S. N. Storch, IDB Program, ORNL, Oak Ridge, Tennessee, "Department of Energy Site Toxic Substances Control Act Mixed LowLevel Waste Information Request for the 1995 Integrated Data Base Report (DOE/RW-0006, Rev. 11)," dated June 15, 1995.

c. (ANL-W) Information not available.

d. (BNL) Carson L. Nealy, DOE Brookhaven Area Office, Upton, New York, correspondence to Stephen N. Storch, IDB Program, ORNL, Oak Ridge, Tennessee, "Department of Energy (DOE) Site Toxic Substance Control Act Mixed Low-Level Waste Information Request for the 1995 Integrated Data Base Report (DOE/RW-0006, Rev. 11 )," dated June 14, 1995. 
e. (ETEC) Kevin Hartnett, DOE Oakland Operations Office, Oakland, California, correspondence to Steve Storch, IDB Program, ORNL, "Department of Energy Toxic Substance Control Act Low-Level Waste (LLW) Information Request for the 1995 Integrated Data Base Report (DOE/RW-0006, Rev. 11)," dated June 20, 1995.

f. (FNAL) Andrew E. Mravca, DOE Batavia Area Office, Batavia, Illinois, correspondence to Stephen N. Storch, IDB Program, ORNL, Oak Ridge, Tennessee, "DOE Site TSCA Mixed Low-Level Waste Information Request for the 1995 Integrated Data Base Report (DOE/RW-0006, Rev. 11)," dated Apr. 26, 1995.

g. (FEMP) Information not available.

h. (GJPO) Jolene Stelmach, DOE Grand Junction Projects Office, Grand Junction, Colorado, correspondence to Steve Storch, DB Program, ORNL, Oak Ridge, Tennessee, "Toxic Substances Control Act Mixed Low-Level Waste Information Request for the 1995 Integrated Data Base Report," dated June 14, 1995.

i. (Hanford) Mike Coony, Westinghouse Hanford Company, Richland, Washington, correspondence to Steve Storch, IDB Program, ORNL, OakRidge, Tennessee, submitting updated TSCA MLLW information for Hanford, dated Aug. 7, 1995.

j. (INEL) R. R. Piscitella, Idaho National Engineering Laboratory, Idaho Falls, Idaho, correspondence to Stephen N. Storch, IDB Program, ORNL, Oak Ridge, Tennessee, "Idaho National Engineering Laboratory (INEL) Input to Toxic Substances Control Act (TSCA) Information Request for the 1995 Integrated Data Base (IDB)," RRP-11-95, dated June 15, 1995.

k. (ITRI) Joe L. Mauderly, Lovelace Biomedical and Environmental Research Institute, Inc., Albuquerque, New Mexico, correspondence to Stephen Storch, DDB Program, ORNL, Oak Ridge, Tennessee, transmitting ITRI TSCAMLLW data attachment, dated June 12, 1995.

1. (K-25) A. L. Rivera, Lockheed Martin Energy Systems, Oak Ridge, Tennessee, correspondence to S. N. Storch, IDB Program, ORNL, Oak Ridge, Tennessee, "Integrated Database (sic) Data Call for TSCA-Rad Waste," dated July $31,1995$.

m. (KCP) Margaret E. Stockdale, DOE Kansas City Area Office, Kansas City, Missouri, personal communication with Steve Storch, IDB Program, ORNL, Oak Ridge, Tennessee, dated July 17, 1995.

n. (LANL) I-Li Chen, Los Alamos National Laboratory, Los Alamos, New Mexico, correspondence to Stephen N. Storch, IDB Program, ORNL, Oak Ridge, Tennessee, "1995 Integrated Data Base (IDB) Report," CST14-95-290, June 7, 1995.

o. (LBL) Jack Bartley, DOE Oakland Operations Office, Lawrence Berkeley Laboratory, Berkeley, California, correspondence to Stephen N. Storch, IDB Program, ORNL, Oak Ridge, Tennessee, "Lawrence Berkeley Laboratory (LBL) Integrated Data Base Report (IDB) of 1995," WM-95-123, dated June 7, 1995.

p. (LLNL) Roy Kearns, DOE San Francisco Operations Office, Oakland, California, correspondence to Steve Storch, IDB Program, ORNL, Oak Ridge, Tennessee, "Transmittal of the Lawrence Livermore National Laboratory Toxic Substances Control Act Low-Level Waste Information Request for the 1995 Integrated Data Base Report (DOE/RW-0006, Revision 11)."

q. (Mound) Robert Rothman, DOE Miamisburg Area Office, Miamisburg, Ohio, correspondence to Steve Storch, IDB Program, ORNL, Oak Ridge, Tennessee, "1995 Integrated Data Base Call for TSCA MLLW," dated June 20, 1995. 
r. (NR sites) R. B. Haemer, DOE Office of Naval Reactors (NE-60), Arlington, Virginia, correspondence to Stephen N. Storch, DB Program, ORNL, Oak Ridge, Tennessee, transmitting updated TSCA MLLW information for NR sites, dated June 12, 1995.

s. (NTS) Carl P. Gertz, DOE Nevada Operations Office, Las Vegas, Nevada, correspondence to Steve Storch, IDB Program, ORNL, Oak Ridge, Tennessee, "Toxic Substances Control Act Mixed Low-Level Waste Information Request for the 1995 Integrated Data Base (DOE/RW-006 [sic], Rev. 11)," dated May 11, 1995.

t. (ORISE) Tom Wantland, Oak Ridge Associated Universities, Oak Ridge, Tennessee, personal communication with Steve Storch, IDB Program, ORNL, Oak Ridge, Tennessee, July 17, 1995.

u. (ORNL) A. L. Rivera, Lockheed Martin Energy Systems, Oak Ridge, Tennessee, Oak Ridge, Tennessee, correspondence to S. N. Storch, DB Program, ORNL, "Integrated Database (sic) Data Call for TSCA-Rad Waste," dated July 31, 1995.

v. (PAD) Jimmie C. Hodges, DOE Paducah Site Office, Paducah, Kentucky, correspondence to Stephen N. Storch, IDB Program, ORNL, Oak Ridge, Tennessee, "Department of Energy (DOE) Site Toxic Substance Control Act (TSCA) Mixed Low-Level Waste (LLW) Information Request for the 1995 Integrated Data Base Report (DOE/RE-0006[sic], Revision 11)," dated June 14, 1995.

w. (PANT) Jerry S. Johnson, DOE Amarillo Area Office, correspondence to Steve Storch, IDB Program, ORNL, Oak Ridge, Tennessee, "Department of Energy Site Toxic Substances Control Act Mixed Low-Level Waste Information Request for 1995 Integrated Data Base Report," dated July 11, 1995.

x. (Pinellas) Gary Schmidtke, DOE Pinellas Area Office, Largo, Florida, correspondence to Steve Storch, IDB Program, ORNL, Oak Ridge, Tennessee, "Pinellas Plant TSCALLW Information Request," dated June 15, 1995.

y. (PORTS) Terry Acox, Lockheed Martin Energy Systems, Inc., Portsmouth, Ohio, correspondence to Steve Storch, IDB Program, ORNL, Oak Ridge, Tennessee, "Data Call for TSCA-Related Wastes," dated July 25, 1995.

z. (PPPL) H Allen Wrigley, DOE Princeton Area Office, Princeton, New Jersey, correspondence to Steve Storch, IDB Program, ORNL, Oak Ridge, Tennessee, "Data Call - TOSCA HW," dated May 10, 1995.

aa. (RFETS) Scott Ludwig, DOE Rocky Flats Office, Golden, Colorado, correspondence to Steve Storch, IDB Program, ORNL, Oak Ridge, Tennessee, transmitting updated TSCA MLLW information, dated Aug. 1, 1995.

ab. (SLAC) Kevin Hartnett, DOE Oakland Operations Office, Oakland, California, correspondence to Steve Storch, IDB Program, ORNL, Oak Ridge, Tennessee, "Department of Energy Toxic Substance Control Act Low-Level Waste Information Request for the 1995 Integrated Data Base Report (DOE/RW-0006, Rev. 11)," dated June $14,1995$.

ac. (SNL/CA) Sarah O'Connor, Lockheed Martin Sandia Corporation, correspondence to Stephen N. Storch, IDB Program, ORNL, Oak Ridge, Tennessee, submitting SNL/CA TSCA MLLW information, dated July 18, 1995.

ad. (SNL/NM) Maureen J. Lincoln, Sandia National Laboratory/New Mexico, Albuquerque, New Mexico, correspondence to Ted Pietrok, DOE Kirtland Area Office, Albuquerque, New Mexico, "IDB Data Base Update," dated July 15, 1994.

ae. (SRS) Paul D. Hunt, Westinghouse Savannah River Company, Aiken, South Carolina, correspondence to Steven N. Storch, IDB Program, ORNL, Oak Ridge, Tennessee, "SRS 1995 Integrated Data Base Report Input (U)," SWE-95-0303, dated June 15, 1995. 
af. (WVDP) John J. Hollinden, West Valley Nuclear Services Company, Inc., West Valley, New York, correspondence to Steven N. Storch, IDB Program, ORNL, Oak Ridge, Tennessee, "Submittal of the Department of Energy Site Toxic Substance Control Act Mixed Low-Level Waste Information for the 1995 Integrated Data Base Report (DOE/RW-0006, Rev.11)," WZ:95:0095, dated June 15, 1995.

ag. (Y-12) A. L. Rivera, Lockheed Martin Energy Systems, Inc., Oak Ridge, Tennessee, correspondence to Steve Storch, DB Program, ORNL, Oak Ridge, Tennessee, "Integrated Database (sic) Data Call for TSCA-Rad Waste," dated July 31, 1995.

11. U.S. Congress, Low-Level Radioactive Waste Policy Amendments Act of 1985, Pub. L. 99-240, Jan. $15,1986$.

12. J. A. Klein, et al., National Profile on Commercially Generated Low-Level Radioactive Mixed Waste, prepared by Oak Ridge National Laboratory, Oak Ridge, Tennessee, for U.S. Nuclear Regulatory Commission and U.S. Environmental Protection Agency, NUREG/CR-5938, Oak Ridge National Laboratory-6731 (December 1992). 
ORNL DWG 95-8847

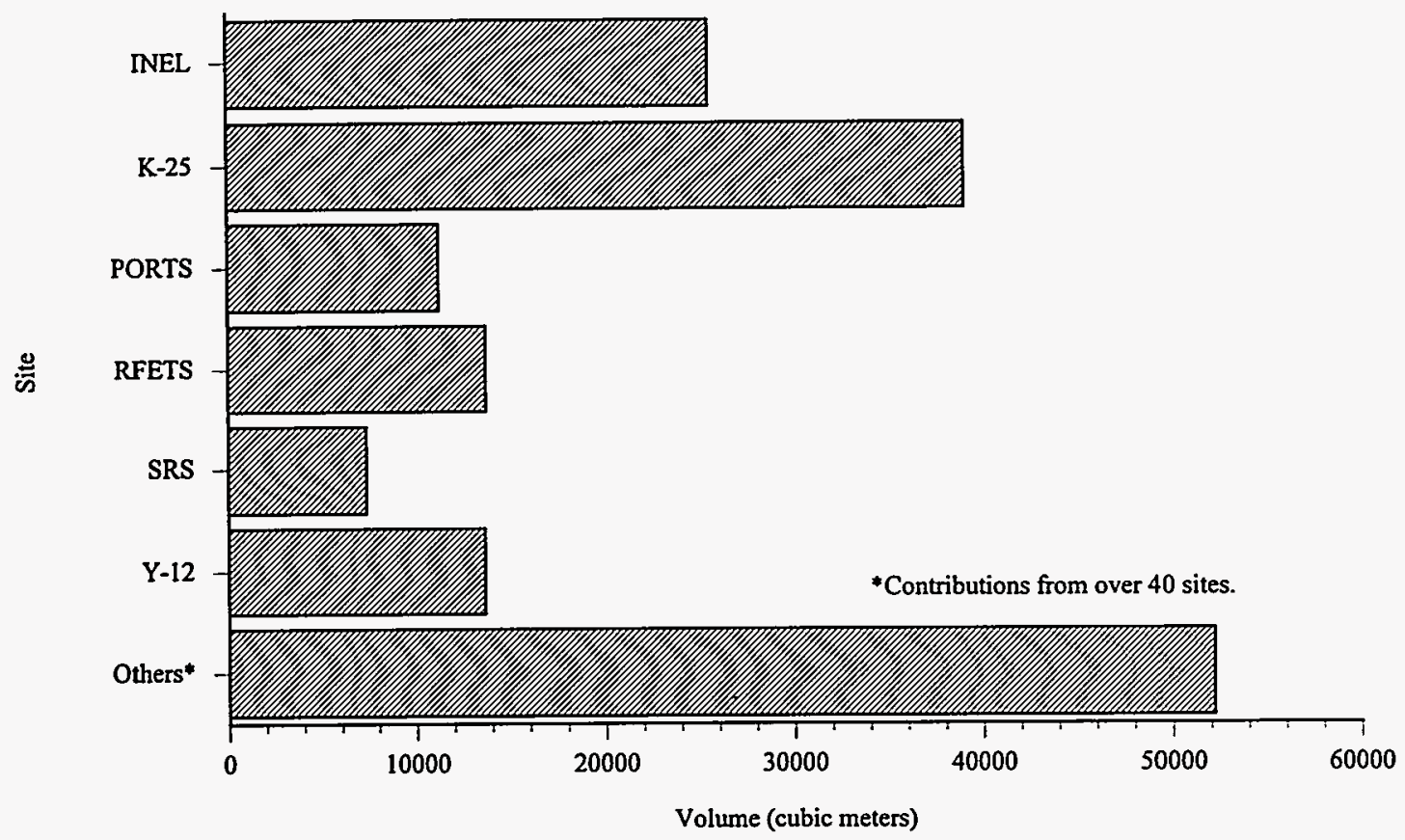

Fig. 8.1. Total volume inventory of all DOE MLLW.

ORNL DWG 95-8848

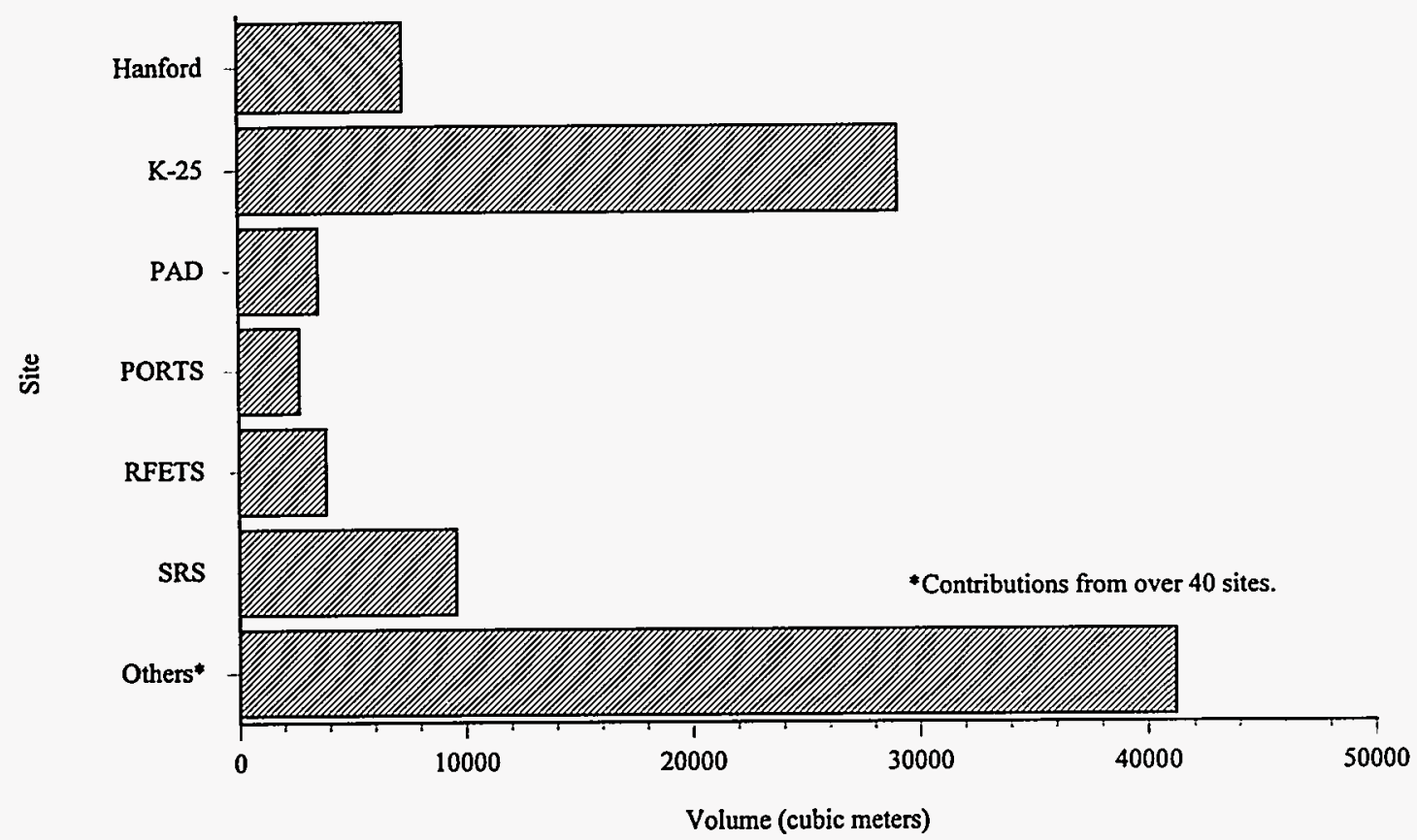

Fig. 8.2. Projected cumulative volume generation of all DOE MLLW during 1994-1998. 
Table 8.1. Thirty-eight states and territories with EPA mixed waste authorization ${ }^{\text {a }}$

\begin{tabular}{ll||lc}
\hline State/territory & Effective date & State/territory & Effective date \\
\hline Alabama & $11 / 23 / 93$ & Montana & $03 / 21 / 94$ \\
Arizona & $01 / 22 / 93$ & Nebraska & $12 / 03 / 88$ \\
Arkansas & $05 / 29 / 90$ & Nevada & $06 / 29 / 92$ \\
California & $08 / 01 / 92$ & New Hampshire & $11 / 14 / 94$ \\
Colorado & $11 / 07 / 86$ & New Mexico & $07 / 25 / 90$ \\
Connecticut & $12 / 31 / 90$ & New York & $05 / 07 / 90$ \\
Florida & $02 / 12 / 91$ & North Carolina & $11 / 21 / 89$ \\
Georgia & $09 / 26 / 88$ & North Dakota & $08 / 24 / 90$ \\
Guam & $10 / 10 / 89$ & Ohio & $06 / 30 / 89$ \\
Idaho & $04 / 09 / 90$ & Oklahoma & $11 / 27 / 90$ \\
Illinois & $04 / 30 / 90$ & Oregon & $05 / 29 / 90$ \\
Indiana & $09 / 30 / 91$ & South Carolina & $09 / 13 / 87$ \\
Kansas & $06 / 25 / 90$ & South Dakota & $06 / 17 / 91$ \\
Kentucky & $12 / 19 / 88$ & Tennessee & $08 / 11 / 87$ \\
Louisiana & $10 / 26 / 91$ & Texas & $03 / 15 / 90$ \\
Michigan & $12 / 26 / 89$ & Utah & $03 / 07 / 89$ \\
Minnesota & $06 / 23 / 89$ & Vermont & $08 / 06 / 93$ \\
Mississippi & $05 / 28 / 91$ & Washington & $11 / 23 / 87$ \\
Missouri & $03 / 12 / 93$ & Wisconsin & $04 / 24 / 92$ \\
\hline & & & \\
\hline & & &
\end{tabular}

aBased on ref. 6. Information as of May 31, 1995. 
Table 8.2. Cumulative mass (kg) inventories of MLLW from DOE site activities ${ }^{\mathrm{a}}$

\begin{tabular}{|c|c|c|c|}
\hline Site & RCRA/state ${ }^{b}$ & $\mathrm{TSCA}^{\mathrm{c}, \mathrm{d}}$ & Total \\
\hline Ames & 154 & 2,600 & 2,754 \\
\hline ANL-E & e & 62,400 & 62,400 \\
\hline ANL-W & 587,334 & $\mathfrak{e}$ & 587,334 \\
\hline $\mathrm{BNL}$ & 24,573 & 1,000 & 25,573 \\
\hline ETEC & 191 & 4,320 & 4,511 \\
\hline FEMP & e & e & e \\
\hline FNAL & e & 0 & e \\
\hline GJPO & 395 & 69,705 & 70,100 \\
\hline Hanford $f$ & 420 & 109,387 & 109,807 \\
\hline INELg & $13,153,364$ & 797 & $13,154,161$ \\
\hline ITRI & e & 0 & e \\
\hline $\mathrm{K}-25$ & $41,138,289$ & $6,381,168$ & $47,519,457$ \\
\hline $\mathrm{KCP}$ & 116 & 0 & 116 \\
\hline LANL & e & $2,504,237$ & $2,504,237$ \\
\hline LBL & e & 214 & 214 \\
\hline LLNL & 457,190 & 2,094 & 459,284 \\
\hline Mound & e & 4,113 & 4,113 \\
\hline NR sites ${ }^{h}$ & 58,843 & 10,843 & 69,686 \\
\hline NTS & 455,189 & 0 & 455,189 \\
\hline ORISE & $\mathrm{e}$ & 0 & e \\
\hline ORNL & $3,276,027$ & 18,278 & $3,294,305$ \\
\hline PAD & 515,706 & $3,001,953$ & $3,517,659$ \\
\hline PANT & 38,726 & 4,500 & 43,226 \\
\hline Pinellas & 0 & 0 & 0 \\
\hline PORTS & $7,200,966$ & $2,076,200$ & $9,277,166$ \\
\hline PPPL & e & 0 & $\mathrm{e}$ \\
\hline RFETS & $1,536,428$ & 38,610 & $1,575,038$ \\
\hline RMI & 20,354 & e & 20,354 \\
\hline SLAC & 8,072 & 0 & 8,072 \\
\hline SNL/CA & 62 & 2 & 64 \\
\hline SNL/NM & 2,738 & 68 & 2,806 \\
\hline SRS & $4,171,684$ & 70,636 & $4,242,320$ \\
\hline WVDP & 104,323 & 10,850 & 115,173 \\
\hline Y-12. & $18,235,195$ & 204,544 & $18,439,739$ \\
\hline Others $\mathrm{i}$ & 100,788 & e & 100,788 \\
\hline Total & $91,087,127^{j}$ & $14,578,519^{k}$ & $105,665,646^{k}$ \\
\hline
\end{tabular}

a Materials may be in interim storage awaiting treatment. Specific site information is provided in Sect. 8.4

$\mathrm{b}_{\text {Based on the MWIR, ref. } 7 .}$

CIn general, densities of $500 \mathrm{~kg} / \mathrm{m}^{3}$ for compressed gases, $1,000 \mathrm{~kg} / \mathrm{m}^{3}$ for liquids, and $1,500 \mathrm{~kg} / \mathrm{m}^{3}$ for solids and sludges were assumed to estimate masses when site did not report mass data.

${ }^{d}$ Based on the LOE site data submittals of ref. 10. Inventories as of December 31, 1994.

Enformation not available or unknown.

fIncludes contributions from PNL.

gIncludes contributions from the Naval Reactors Facility (NRF).

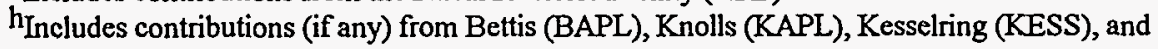
Windsor (WIND).

includes contributions from Battelle Columbus Laboratories Decommissioning Project, Colonie Interim Storage Site, General Atomics facility, Laboratory for Energy-Related Health Research, Middlesex Sampling Plant, University of Missouri, Weldon Spring Site Remedial Action Project, and 6 naval shipyards (Charleston, South Carolina; Mare Island, California; Norfolk, Virginia; Pearl Harbor, Hawaii; Portsmouth, Maine; and Puget Sound, Washington).

JMass values not reported for all streams; the values shown reflect only those which were reported.

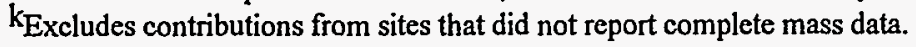


Table 8.3. Cumulative volume $\left(\mathrm{m}^{3}\right)$ inventories of $M L L W$ from DOE site activities ${ }^{a}$

\begin{tabular}{|c|c|c|c|}
\hline Site & RCRA/state ${ }^{b}$ & $\operatorname{TSCA}^{\mathrm{c}}$ & Total \\
\hline Ames & $<0.1$ & 3.4 & 3.5 \\
\hline ANL-E & 34.4 & 52.0 & 86.4 \\
\hline ANL-W & 391.5 & $\mathrm{~d}$ & 391.5 \\
\hline BNL & 26.6 & 2.1 & 28.7 \\
\hline ETEC & 1.2 & 11.3 & 12.5 \\
\hline FEMP & $2,150.6$ & d & $2,150.6$ \\
\hline FNAL & d & 0 & d \\
\hline GJPO & 0.6 & 46.5 & 47.1 \\
\hline Hanford ${ }^{\mathrm{e}}$ & $6,331.0$ & 95.6 & $6,426.6$ \\
\hline INEL f & $25,440.0$ & 1.8 & $25,441.8$ \\
\hline ITRI & 0.0 & 0 & 0.0 \\
\hline $\mathrm{K}-25$ & $29,472.9$ & $9,493.3$ & $38,966.2$ \\
\hline $\mathrm{KCP}$ & 0.2 & 0 & 0.2 \\
\hline LANL & 608.6 & $5,992.5$ & $6,601.1$ \\
\hline LBL & 6.2 & 0.1 & 6.3 \\
\hline LLNL & 457.2 & 1.7 & 458.9 \\
\hline Mound & 98.0 & 15.6 & 113.6 \\
\hline NR sitesg & 47.5 & 22.1 & 69.6 \\
\hline NTS & 297.5 & 0 & 297.5 \\
\hline ORISE & d & 0 & d \\
\hline ORNL & $3,020.1$ & 14.7 & $3,034.8$ \\
\hline PAD & $1,028.4$ & $4,359.4$ & $5,387.8$ \\
\hline PANT & 127.7 & 3.0 & 130.7 \\
\hline Pinellas & 0 & 0 & 0 \\
\hline PORTS & $7,514.5$ & $3,669.8$ & $11,184.3$ \\
\hline PPPL & 0.0 & 0 & 0.0 \\
\hline RFETS & $13,550.1$ & 112.0 & $13,662.1$ \\
\hline RMI & 23.5 & $\mathrm{~d}$ & 23.5 \\
\hline SLAC & 5.9 & 0 & 5.9 \\
\hline SNL/CA & 0.4 & 0.2 & 0.6 \\
\hline SNL/NM & 74.7 & 0.4 & 75.1 \\
\hline SRS & $7,200.2$ & 142.0 & $7,342.2$ \\
\hline WVDP & 86.3 & 43.9 & 130.2 \\
\hline$Y-12$ & $13,465.1$ & 150.2 & $13,615.3$ \\
\hline Others $^{h}$ & $26,682.6$ & d & $26,682.6$ \\
\hline Total & $138,143.6$ & $24,233.6$ & $162,377.2$ \\
\hline
\end{tabular}

${ }^{a}$ Materials may be in interim storage awaiting treatment. Specific site information is provided in Sect. 8.4.

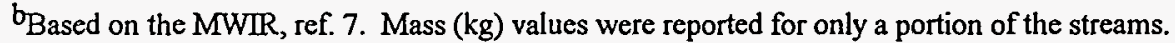

${ }^{c}$ Based on the DOE site data submittals of ref. 10. Inventories as of December 31, 1994.

dinformation not available or unknown.

EIncludes contributions from PNL.

Includes contributions from the Naval Reactors Facility (NRF).

IIncludes contributions (if any) from Bettis (BAPL), Knolls (KAPL), Kesselring (KESS), and Windsor (WIND).

hIncludes contributions from Battelle Columbus Laboratories Decommissioning Project, Colonie Interim Storage Site, General Atomics facility, Laboratory for Energy-Related Health Research, Middlesex Sampling Plant, University of Missouri, Weldon Spring Remedial Action Project, and 6 naval shipyards (Charleston, South Carolina; Mare Island, California; Norfolk, Virginia; Pearl Harbor, Hawaii; Portsmouth, Maine; and Puget Sound, Washington). 
Table 8.4. Projected 5-year (1994-1998) cumulative mass (kg) generation of MLLW from DOE site activities

\begin{tabular}{|c|c|c|c|}
\hline Site & RCRA/state ${ }^{a}$ & $\mathrm{TSCA}^{\mathrm{b}, \mathrm{c}, \mathrm{d}}$ & Total \\
\hline Ames & 220 & 100 & 320 \\
\hline ANL-E & 5,574 & 69,600 & 75,174 \\
\hline ANL-W & 10,580 & e & 10,580 \\
\hline BNL & 2,288 & 216 & 2,504 \\
\hline ETEC & e & 13,400 & 13,400 \\
\hline FEMP & e & e & e \\
\hline FNAL & $\mathrm{e}$ & 0 & e \\
\hline GJPO & 60 & 820 & 880 \\
\hline Hanford $f$ & $\mathrm{e}$ & $1,075,247$ & $1,075,247$ \\
\hline INEL $\mathrm{g}$ & $1,069,473$ & 164 & $1,069,637$ \\
\hline ITRI & 63,980 & 0 & 63,980 \\
\hline$K-25$ & $1,332,408$ & $10,829,500$ & $12,161,908$ \\
\hline $\mathrm{KCP}$ & e & 0 & $\mathrm{e}$ \\
\hline LANL & e & 201,272 & 201,272 \\
\hline LBL & e & 5,755 & 5,755 \\
\hline LLNL & e & 1,165 & 1,165 \\
\hline Mound & e & 2,414 & 2,414 \\
\hline NR sites ${ }^{h}$ & $1,105,243$ & 305,070 & $1,410,313$ \\
\hline NTS & $4,339,981$ & $d$ & $4,339,981$ \\
\hline ORISE & $\mathrm{e}$ & 0 & e \\
\hline ORNL & 685,996 & 91,390 & 777,386 \\
\hline PAD & e & $2,284,826$ & $2,284,826$ \\
\hline PANT & 719,432 & 4,500 & 723,932 \\
\hline Pinellas & e & 1,850 & 1,850 \\
\hline PORTS & e & 339,690 & 339,690 \\
\hline PPPL & e & 0 & $\mathrm{e}$ \\
\hline RFETS & e & 100,370 & 100,370 \\
\hline RMI & 5,416 & $\mathrm{e}$ & 5,416 \\
\hline SLAC & e & 0 & e \\
\hline SNL/CA & e & 2 & 2 \\
\hline SNL/NM & e & e & e \\
\hline SRS & $1,900,729$ & 170,050 & $2,070,779$ \\
\hline WVDP & 45,193 & 28,926 & 74,119 \\
\hline Y-12. & $1,829,276$ & 153,140 & $1,982,416$ \\
\hline Others $\mathrm{i}$ & $32,773,112$ & e & $32,773,112$ \\
\hline Total & $45,888,961^{j}$ & $15,679,467^{k}$ & $61,568,428^{k}$ \\
\hline
\end{tabular}

${ }^{a}$ Based on the MWIR, ref. 7. RCRA/state projections for five sites [Ames Laboratory (Ames), Argonne National Laboratory-East (ANL-E), Portsmouth Gaseous Diffusion Plant (PORTS), Reactive Metals, Inc., Titanium Extrusion Plant (RMD), and Savannah River Site (SRS)] may include contributions from DOE environmental restoration (EM-40) wastes.

${ }^{b}$ Specific site information is provided in Sect. 8.4.

CIn general, densities of $500 \mathrm{~kg} / \mathrm{m}^{3}$ for compressed gases, $1,000 \mathrm{~kg} / \mathrm{m}^{3}$ for liquids, and $1,500 \mathrm{~kg} / \mathrm{m}^{3}$ for solids and sludges were assumed to estimate masses when site did not report mass data.

${ }^{\mathrm{d}}$ Based on the DOE site data submittals of ref. 10.

Information not available or unknown.

Includes contributions from PNL.

gIncludes contributions from the Naval Reactors Facility (NRF).

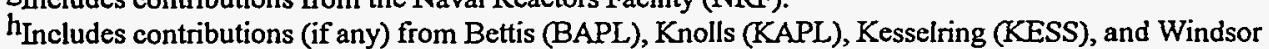
(WIND).

includes contributions (if any) from BCLDP, Colonie Interim Storage Site (CISS), General Atomics (GA) facility, LEHR, Middlesex Sampling Plant, University of Missouri (MURR), and Weldon Spring Site Remedial Action Project (WSSRAP), and 6 naval shipyards (Charleston, South Carolina; Mare Island, Califormia; Norfolk, Virginia; Pearl Harbor, Hawaii; Portsmouth, Maine; and Puget Sound, Washington).

$\mathrm{j}_{\text {Mass }}(\mathrm{kg})$ values were provided for only a limited amount of the projected waste.

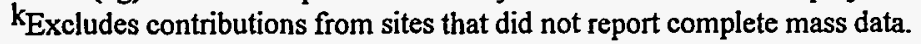


Table 8.5. Projected 5-year (1994-1998) cumulative volume $\left(\mathrm{m}^{3}\right)$ generation of MLLW from DOE site activities

\begin{tabular}{|c|c|c|c|}
\hline Site & RCRA/state ${ }^{\mathfrak{a}}$ & $\operatorname{TSCA}^{\mathrm{b}, \mathrm{c}}$ & Total \\
\hline Ames & $<0.1$ & $<0.2$ & $<0.3$ \\
\hline ANL-E & 71.6 & 58.0 & 129.6 \\
\hline ANL-W & 13.6 & d & 13.6 \\
\hline BNL & 2.1 & 1.1 & 3.2 \\
\hline ETEC & $\mathrm{d}$ & 31.1 & 31.1 \\
\hline FEMP & $d$ & d & d \\
\hline FNAL & d & 0 & d \\
\hline GJPO & $<0.1$ & 1.9 & 2.0 \\
\hline Hanford ${ }^{e}$ & $6,559.8$ & 716.8 & $7,276.6$ \\
\hline $\mathrm{INEL}^{\mathrm{f}}$ & 801.7 & 0.6 & 802.3 \\
\hline ITRI & 77.1 & 0 & 77.1 \\
\hline $\mathrm{K}-25$ & $1,076.1$ & $27,934.0$ & $29,010.1$ \\
\hline $\mathrm{KCP}$ & d & 0 & d \\
\hline LANL & 84.3 & 396.6 & 480.9 \\
\hline LBL & 5.6 & 14.0 & 19.6 \\
\hline LLNL & $1,412.0$ & 0.8 & $1,412.8$ \\
\hline Mound & d & 8.8 & 8.8 \\
\hline NR sitesg & $1,119.0$ & 851.6 & $1,970.6$ \\
\hline NTS & $2,535.0$ & d & $2,535.0$ \\
\hline ORISE & d & 0 & $d$ \\
\hline ORNL & 572.9 & 65.5 & 638.4 \\
\hline $\mathrm{PAD}$ & d & $3,509.0$ & $3,509.0$ \\
\hline PANT & 887.3 & 3.0 & 890.3 \\
\hline Pinellas & d & 3.0 & 3.0 \\
\hline PORTS & $1,648.0$ & $1,035.9$ & $2,683.9$ \\
\hline PPPL & 8.2 & 0 & 8.2 \\
\hline RFETS & $3,623.4$ & 220.0 & $3,843.4$ \\
\hline RMI & 7.7 & d & 7.7 \\
\hline SLAC & d & 0 & d \\
\hline SNL/CA & 4.1 & 0.2 & 4.3 \\
\hline SNL/NM & 28.0 & d & 28.0 \\
\hline SRS & $9,232.9$ & 340.1 & $9,573.0$ \\
\hline WVDP & 29.8 & 19.3 & 49.1 \\
\hline$Y-12$ & $1,550.9$ & 114.6 & $1,665.5$ \\
\hline Others $^{h}$ & $30,381.3$ & $\mathrm{~d}$ & $30,381.3$ \\
\hline Total & $61,732.6$ & $35,326.1$ & $97,058.7$ \\
\hline
\end{tabular}

a Based on the MWIR, ref. 7. RCRA/state projections for five sites [Ames Laboratory (Ames), Argonne National Laboratory-East (ANL-E), Portsmouth Gaseous Diffusion Plant (PORTS), Reactive Metals, Inc., Titanium Extrusion Plant (RMM), and Savannah River Site (SRS)] include contributions from DOE environmental restoration (EM-40) wastes.

${ }^{\mathrm{b}}$ Specific site information is provided in Sect. 8.4.

${ }_{\text {Based on the DOE site data submittals of ref. } 10 .}$

dinformation not available or unknown.

Tncludes contributions from PNL.

Includes contributions from the Naval Reactors Facility (NRF).

gIncludes contributions (if any) from Bettis (BAPL), Knolls (KAPL), Kesselring (KESS), and Windsor (WIND).

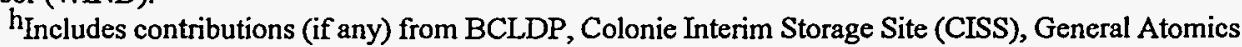
(GA) facility, LEHR, Middlesex Sampling Plant, University of Missouri (MURR), Weldon Spring Remedial Action Project (WSSRAP), and 6 naval shipyards (Charleston, South Carolina; Mare Island, California; Norfolk, Virginia; Peari Harbor, Hawaii; Portsmouth, Maine; and Puget Sound, Washington). 
Table 8.6. Treatability group matrix parameter categories used to characterize RCRAand state-regulated MLLW from DOE activities ${ }^{\mathrm{a}}$

Matrix parameter category
Aqueous liquids/slurries
Organic liquids
Unknown/other liquids
Inorganic homogeneous solids
Organic homogeneous solids
solids

\section{Soil/gravel}

Inorganic debris

Organic debris

Heterogeneous debris

Unknown/other debris

Unknown/other solids
Description

\section{Liquids $\mathrm{b}$}

Liquids and siurries containing less than $1 \%$ total organic carbon (TOC)

Liquids and slurries containing $\geq 1 \%$ TOC

Liquids and slumies which can not be categorized as aqueous liquids/slurries or organic liquids because it is not known if the TOC level is less, or greater than $1 \%$

\section{Solids ${ }^{\mathrm{C}}$}

Wastes that are at least $50 \mathrm{vol} \%$ inorganic homogeneous solids. Homogeneous solids are defined as solid waste materials, excluding soil/gravel, that do not meet the Environmental Protection Agency (EPA) land disposal restrictions (LDR) criteria for classification as debris. Inorganic homogeneous solids are further defined as those with sufficient inorganic solids content such that a minimum of approximately $20 \mathrm{wt} \%$ would remain as residue (i.e., ash/solids) following incineration

Wastes that are at least $50 \mathrm{vol} \%$ organic homogeneous solids. Homogeneous solids are defined as solid waste materials, excluding soil/gravel, that do not meet the EPA LDR criteria for classification as debris. Organic homogeneous solids are further defined as those with a base structure that is primarily organic such that a maximum of approximately $20 \mathrm{wt} \%$ would remain as residue (i.e., ash/solids) following incineration

Wastes that are at least 50 vol \% homogeneous solids, but:

- are insufficiently characterized to enable categorization as either inorganic or organic homogeneous solids, or

- do not meet the criteria for categorization as either inorganic or organic homogeneous solids

Wastes that are at least $50 \mathrm{vol} \%$ soil, including sand and silt or rock and gravel, that do not meet EPA LDR criteria for classification as debris

Wastes that are at least 80 vol \% inorganic materials that meet the EPA LDR criteria for classification as debris. Examples include scrap metal, concrete, glass, and brick

Wastes that are at least $80 \mathrm{vol} \%$ organic materials that meet the EPA LDR criteria for classification as debris. Examples include plastic, rubber, wood, paper, cloth, and biological materials

Wastes that are at least $50 \mathrm{vol} \%$ debris materials that meet the EPA LDR criteria for classification as debris but are not dominant (i.e., at least $80 \mathrm{vol} \%$ ) in either inorganic or organic debris materials

Wastes that are at least $50 \mathrm{vol} \%$ materials that meet the EPA LDR criteria for classification as debris but lack adequate characterization information to enable categorizing as inorganic, organic, or heterogeneous debris

Wastes with physically solid matrices for which insufficient characterization information exists to enable categorizing as a homogeneous solid, soil/gravel, or debris 
Table 8.6 (continued)

\begin{tabular}{|c|c|}
\hline Matrix parameter category & Description \\
\hline \multicolumn{2}{|r|}{ Specific waste forms $\mathrm{d}$} \\
\hline Lab packs & $\begin{array}{l}\text { Wastes packaged in lab pack configurations. A lab pack configuration is defined as } \\
\text { two or more waste containers packaged within a larger outer container. Typically, the } \\
\text { inner containers are surrounded by absorbent materials. If present, the absorbents can } \\
\text { be homogeneous solids or debris materials }\end{array}$ \\
\hline Reactive metals & $\begin{array}{l}\text { Reactive metal wastes that meet the criteria for classification as water-reactive or } \\
\text { ignitable-reactive per the Third Third LDR rule ( } 55 \text { FR } 22545 \text { and } 22553 \text { ). Typically, } \\
\text { this waste is sodium metal or sodium metal alloys, but can also include particulate fines } \\
\text { of aluminum, uranium, zirconium, or other pyrophoric materials }\end{array}$ \\
\hline Explosives/propellants & $\begin{array}{l}\text { Wastes consisting of substances which undergo rapid chemical transformations that } \\
\text { produce large amounts of gases and heat. The gases rapidly expand at velocities } \\
\text { exceeding the speed of sound (due to the heat of reaction), which creates a shock wave } \\
\text { and explosion. Waste that meets this definition is categorized as an } \\
\text { explosive/propellant regardless of the specific physical form }\end{array}$ \\
\hline Compressed gases/aerosols & Wastes consisting of pressurized gas cylinders or aerosol cans \\
\hline Elemental mercury & $\begin{array}{l}\text { Wastes that are bulk, pourable liquid mercury. The liquid mercury may be packaged } \\
\text { in a lab pack configuration }\end{array}$ \\
\hline Elemental hazardous metals & $\begin{array}{l}\text { Wastes that are at least } 50 \text { vol \% solid, bulk elemental hazardous metals that meet the } \\
\text { EPA LDR size criteria for classification as debris. Typical examples of solid elementa } \\
\text { hazardous metals are lead and cadmium }\end{array}$ \\
\hline Beryllium dust & $\begin{array}{l}\text { Wastes that are subject to the metal recovery treatment standard for beryllium dust as } \\
\text { specified in the Third Third LDR rule (55 FR 22545) }\end{array}$ \\
\hline Batteries & $\begin{array}{l}\text { Wastes consisting of lead acid, cadmium, or other batteries. The batteries may be } \\
\text { packaged with absorbent materials }\end{array}$ \\
\hline Unknown/other matrix & $\begin{array}{l}\text { Wastes for which insufficient characterization information is known to enable } \\
\text { categorization as a liquid or solid or as one of the specific waste forms }\end{array}$ \\
\hline \multicolumn{2}{|r|}{ Final waste forms $\mathrm{c}^{\mathrm{e}}$} \\
\hline Immobilized forms & $\begin{array}{l}\text { Wastes that have been immobilized. This includes waste considered to be either micro- } \\
\text { or macro-encapsulated }\end{array}$ \\
\hline Decontaminated solids & Waste that has been decontaminated and is ready for disposal or recycling \\
\hline Other final forms & Final waste forms other than immobilized forms and decontaminated solids \\
\hline
\end{tabular}

aBased on ref. 9.

${ }^{b}$ This category addresses wastes that are liquid, including slurries, and are packaged in bulk, free form (i.e., excludes lab packs). Slurries are defined as liquids with a total suspended/settled solids (TSS) content of $\geq 1 \%$ and $\leq 30 \%$.

"This category addresses waste with physically solid matrices, including sludges. Sludges are defined as having a TSS $>30 \%$. Certain waste with physically solid matrices are excluded from this category (see the "specific waste forms" and "final waste forms" categories above).

${ }^{d}$ This category addresses lab packs and other specific waste forms. The other specific waste forms include waste that (a) is inherently hazardous (i.e., the bulk material itself is RCRA hazardous), or (b) presents unique treatment or management concerns.

This category addresses waste that is in final form and meets applicable disposal criteria, including applicable LDR and PCB treatment standards. 
Table 8.7. Cumulative mass and volume inventories of RCRA- and state-regulated MLLW at each DOE site ${ }^{\mathrm{a}}$

\begin{tabular}{|c|c|c|c|c|}
\hline \multirow[b]{2}{*}{ Site } & \multirow[b]{2}{*}{ Category ${ }^{b}$} & \multicolumn{3}{|c|}{ Inventory } \\
\hline & & $\left.\operatorname{Date}_{(s)}\right)^{\mathrm{c}}$ & $\begin{array}{c}\text { Mass } \\
(\mathrm{kg})\end{array}$ & $\begin{array}{c}\text { Volume } \\
\left(\mathrm{m}^{3}\right)\end{array}$ \\
\hline \multirow[t]{4}{*}{ Ames } & Aqueous liquids/slurries & & 100 & 0.05 \\
\hline & Elemental hazardous metals & & 50 & 0.01 \\
\hline & Lab packs & & 4 & 0.01 \\
\hline & Ames total & $02 / 01 / 95-04 / 01 / 95$ & 154 & 0.07 \\
\hline \multirow[t]{9}{*}{ ANL-E } & Aqueous liquids/slurries & & d & 0.02 \\
\hline & Elemental hazardous metals & & d & 17.53 \\
\hline & Inorganic debris & & $\mathrm{d}$ & 0.67 \\
\hline & Inorganic homogeneous solids & & d & 5.10 \\
\hline & Lab packs & & d & 9.36 \\
\hline & Organic debris & & d & 0.79 \\
\hline & Reactive metals & & d & 0.53 \\
\hline & Soil/gravel & & d & 0.40 \\
\hline & ANL $-E$ total & 06/01/95 & d & 34.40 \\
\hline \multirow[t]{7}{*}{ ANL-W } & Aqueous liquids/slurries & & 537 & 0.42 \\
\hline & Heterogeneous debris & & 599 & 3.08 \\
\hline & Inorganic homogeneous solids & & 177 & 0.42 \\
\hline & Organic debris & & 1,799 & 0.57 \\
\hline & Organic liquids & & 42 & 0.08 \\
\hline & Reactive metals & & 584,180 & 386.99 \\
\hline & ANL $-W$ total & $04 / 30 / 95$ & 587,334 & 391.56 \\
\hline \multirow[t]{7}{*}{ BNL } & Elemental hazardous metals & & 385 & 0.44 \\
\hline & Elemental mercury & & 238 & 0.45 \\
\hline & Heterogeneous debris & & 386 & 1.03 \\
\hline & Inorganic homogeneous solids & & 7,200 & 6.20 \\
\hline & Lab packs & & 469 & 0.57 \\
\hline & Organic liquids & & 15,895 & 17.95 \\
\hline & BNL total & $06 / 01 / 95$ & 24,573 & 26.64 \\
\hline \multirow[t]{5}{*}{ ETEC } & Elemental mercury & & d & 0.10 \\
\hline & Inorganic homogeneous solids & & 159 & 0.99 \\
\hline & Organic liquids & & 32 & 0.15 \\
\hline & Reactive metals & & $\mathrm{d}$ & e \\
\hline & ETEC total & $03 / 01 / 95-06 / 01 / 95$ & 191 & 1.24 \\
\hline \multirow[t]{10}{*}{ FEMP } & Aqueous liquids/slurries & & d & 869.80 \\
\hline & Batteries & & d & 5.00 \\
\hline & Compressed gases/aerosols & & $\mathrm{d}$ & 0.40 \\
\hline & Elemental hazardous metals & & d & 2.00 \\
\hline & Elemental mercury & & d & 0.20 \\
\hline & Heterogeneous debris & & d & 26.60 \\
\hline & Immobilized forms & & d & 33.40 \\
\hline & Inorganic debris & & d & 17.40 \\
\hline & Inorganic homogeneous solids & & d & 706.20 \\
\hline & Lab packs & & $d$ & 0.40 \\
\hline
\end{tabular}


Table 8.7 (continued)

\begin{tabular}{|c|c|c|c|c|}
\hline \multirow[b]{2}{*}{ Site } & \multirow[b]{2}{*}{ Category b } & \multicolumn{3}{|c|}{ Inventory } \\
\hline & & Date $(\mathbf{s})^{\mathrm{c}}$ & $\begin{array}{c}\text { Mass } \\
(\mathrm{kg})\end{array}$ & $\begin{array}{l}\text { Volume } \\
\left(\mathrm{m}^{3}\right)\end{array}$ \\
\hline \multirow{8}{*}{$\begin{array}{l}\text { FEMP } \\
\text { (contd.) }\end{array}$} & Organic debris & & d & 21.20 \\
\hline & Organic homogeneous solids & & $\mathrm{d}$ & 78.20 \\
\hline & Organic liquids & & $\mathrm{d}$ & 374.80 \\
\hline & Reactive metals & & $\mathrm{d}$ & 2.60 \\
\hline & Soil/gravel & & $\mathrm{d}$ & 11.20 \\
\hline & Unknown/other debris & & d & 0.80 \\
\hline & Unknown/other solids & & d & 0.40 \\
\hline & FEMP total & $10 / 01 / 94$ & $\mathrm{~d}$ & $2,150.60$ \\
\hline FNAL & & $\mathrm{d}$ & d & d \\
\hline \multirow[t]{7}{*}{ GJPO } & Aqueous liquids/slurries & & 18 & 0.03 \\
\hline & Inorganic homogeneous solids & & 191 & 0.19 \\
\hline & Lab packs & & 59 & 0.22 \\
\hline & Organic debris & & 5 & 0.01 \\
\hline & Organic liquids & & 119 & 0.12 \\
\hline & Soil/gravel & & 3 & e \\
\hline & GJPO total & $05 / 01 / 94-05 / 01 / 95$ & 395 & 0.57 \\
\hline \multirow[t]{14}{*}{ Hanford $f$} & Batteries & & d & 1.65 \\
\hline & Elemental hazardous metals & & $\mathrm{d}$ & 210.81 \\
\hline & Elemental mercury & & $\mathrm{d}$ & 1.46 \\
\hline & Heterogeneous debris & & d & 629.69 \\
\hline & Inorganic debris & & d & 491.35 \\
\hline & Inorganic homogeneous solids & & $\mathrm{d}$ & $2,655.57$ \\
\hline & Lab packs & & 420 & 264.91 \\
\hline & Organic debris & & d & $1,587.26$ \\
\hline & Organic homogeneous solids & & d & 1.90 \\
\hline & Organic liquids & & d & 0.21 \\
\hline & Reactive metals & & $\mathrm{d}$ & 8.50 \\
\hline & Soil/gravel & & $\mathrm{d}$ & 459.06 \\
\hline & Unknown/other matrix & & $d$ & 18.63 \\
\hline & Hanford total & $12 / 31 / 94-05 / 01 / 95$ & 420 & $6,331.00$ \\
\hline \multirow[t]{14}{*}{ INEL $^{\mathrm{g}}$} & Aqueous liquids/slurries & & 11,050 & 12.25 \\
\hline & Elemental hazardous metals & & 871,676 & 445.5 \\
\hline & Heterogeneous debris & & $1,063,830$ & $2,954.4 S$ \\
\hline & Inorganic debris & & $3,582,300$ & $9,302.00$ \\
\hline & Inorganic homogeneous solids & & $4,040,955$ & $3,929.34$ \\
\hline & Lab packs & & 1,455 & 4.08 \\
\hline & Organic debris & & $1,817,593$ & $5,404.6$ \\
\hline & Organic homogeneous solids & & 4,244 & 6.08 \\
\hline & Organic liquids & & 10,119 & 12.86 \\
\hline & Reactive metals & & 694 & 0.93 \\
\hline & Soil/gravel & & 217,747 & 222.31 \\
\hline & Unknown/other homogeneous solids & & 200 & 0.64 \\
\hline & Unknown/other solids & & $1,531,501$ & $3,144.79$ \\
\hline & INEL total & $12 / 31 / 94-4 / 30 / 95$ & $13,153,364$ & $25,440.00$ \\
\hline
\end{tabular}


Table 8.7 (continued)

\begin{tabular}{|c|c|c|c|c|}
\hline \multirow[b]{2}{*}{ Site } & \multirow[b]{2}{*}{ Category ${ }^{b}$} & \multicolumn{3}{|c|}{ Inventory } \\
\hline & & Date(s) ${ }^{\mathbf{c}}$ & $\begin{array}{c}\text { Mass } \\
(\mathrm{kg})\end{array}$ & $\begin{array}{c}\text { Volume } \\
\left(\mathrm{m}^{3}\right)\end{array}$ \\
\hline ITRI & & $d$ & $\mathrm{~d}$ & d \\
\hline \multirow[t]{18}{*}{$\mathrm{K}-25$} & Aqueous liquids/slurries & & 445,285 & 442.19 \\
\hline & Batteries & & 24,345 & 16.23 \\
\hline & Beryllium dust & & 259 & 0.17 \\
\hline & Compressed gases/aerosols & & 6,943 & 5.76 \\
\hline & Elemental hazardous metals & & 135,551 & 90.37 \\
\hline & Elemental mercury & & 5,473 & 5.04 \\
\hline & Explosives/propellants & & 119 & 0.08 \\
\hline & Heterogeneous debris & & 153,910 & 102.99 \\
\hline & Inorganic debris & & 237,675 & 158.45 \\
\hline & Inorganic homogeneous solids & & $38,581,756$ & $27,484.14$ \\
\hline & Lab packs & & 23,273 & 21.85 \\
\hline & Organic debris & & 186,857 & 124.73 \\
\hline & Organic homogeneous solids & & 482,174 & 334.91 \\
\hline & Organic liquids & & 350,589 & 348.13 \\
\hline & Reactive metals & & 118 & 0.08 \\
\hline & Soil/gravel & & 407,451 & 271.63 \\
\hline & Unknown/other matrix & & 96,511 & 66.16 \\
\hline & $\mathrm{K}-25$ total & $12 / 31 / 94$ & $41,138,289$ & $29,472.90$ \\
\hline $\mathrm{KCP}$ & Inorganic debris & $01 / 01 / 95$ & 116 & 0.21 \\
\hline \multirow[t]{12}{*}{ LANL } & Aqueous liquids/slurries & & d & 3.34 \\
\hline & Compressed gases/aerosols & & d & 1.68 \\
\hline & Elemental hazardous metals & & d & 133.95 \\
\hline & Heterogeneous debris & & d & 6.32 \\
\hline & Inorganic homogeneous solids & & d & 269.51 \\
\hline & Organic debris & & d & 42.14 \\
\hline & Organic liquids & & d & 55.42 \\
\hline & Reactive metals & & d & 6.03 \\
\hline & Soil/gravel & & d & 49.85 \\
\hline & Unknown/other debris & & $d$ & 7.82 \\
\hline & Unknown/other matrix & & d & 32.54 \\
\hline & LANL total & 09/30/94 & d & 608.60 \\
\hline \multirow[t]{7}{*}{ LBL } & Aqueous liquids/slurries & & $d$ & 2.16 \\
\hline & Elemental hazardous metals & & d & 0.43 \\
\hline & Elemental mercury & & d & 0.11 \\
\hline & Heterogeneous debris & & d & 0.11 \\
\hline & Lab packs & & d & 0.15 \\
\hline & Organic liquids & & $d$ & 3.30 \\
\hline & LBL total & 08/01/94-05/01/95 & $d$ & 6.26 \\
\hline \multirow[t]{5}{*}{ LLNL } & Elemental mercury & & 90 & 0.09 \\
\hline & Heterogeneous debris & & 62,400 & 62.40 \\
\hline & Inorganic debris & & 19,100 & 19.10 \\
\hline & Inorganic homogeneous solids & & 253,200 & 253.20 \\
\hline & Lab packs & & 6,300 & 6.30 \\
\hline
\end{tabular}


Table 8.7 (continued)

\begin{tabular}{|c|c|c|c|c|}
\hline \multirow[b]{2}{*}{ Site } & \multirow[b]{2}{*}{ Category ${ }^{b}$} & \multicolumn{3}{|c|}{ Inventory } \\
\hline & & Date $(s)^{\mathrm{C}}$ & $\begin{array}{c}\text { Mass } \\
(\mathrm{kg})\end{array}$ & $\begin{array}{l}\text { Volume } \\
\left(\mathrm{m}^{3}\right)\end{array}$ \\
\hline \multirow{5}{*}{$\begin{array}{l}\text { LLNL } \\
\text { (contd.) }\end{array}$} & Organic homogeneous solids & & 1,200 & 1.20 \\
\hline & Organic liquids & & 92,800 & 92.80 \\
\hline & Reactive metals & & 5,400 & 5.40 \\
\hline & Soil/gravel & & 16,700 & 16.70 \\
\hline & LLNL total & $01 / 01 / 95$ & 457,190 & 457.19 \\
\hline \multirow[t]{9}{*}{ MOUND } & Batteries & & d & 0.85 \\
\hline & Elemental hazardous metals & & d & 5.00 \\
\hline & Elemental mercury & & d & 0.02 \\
\hline & Inorganic homogeneous solids & & $\mathrm{d}$ & 0.23 \\
\hline & Lab packs & & d & 43.47 \\
\hline & Organic debris & & d & 0.02 \\
\hline & Organic liquids & & $\mathrm{d}$ & 28.50 \\
\hline & Unknown/other matrix & & d & 19.90 \\
\hline & Mound total & $05 / 01 / 94$ & d & 97.98 \\
\hline \multirow[t]{13}{*}{ NR sites ${ }^{h}$} & Aqueous liquids/slurries & & 25,532 & 26.13 \\
\hline & Elemental hazardous metals & & 24,699 & 7.76 \\
\hline & Elemental mercury & & 192 & 0.02 \\
\hline & Heterogeneous debris & & 1,202 & 4.28 \\
\hline & Inorganic debris & & 180 & 1.02 \\
\hline & Inorganic homogeneous solids & & 538 & 0.92 \\
\hline & Lab packs & & 102 & 0.09 \\
\hline & Organic debris & & 72 & 0.74 \\
\hline & Organic homogeneous solids & & 4,095 & 3.57 \\
\hline & Organic liquids & & 1,000 & 1.46 \\
\hline & Reactive metals & & 205 & 0.03 \\
\hline & Soil/gravel & & 1,025 & 1.47 \\
\hline & NR sites total & $12 / 01 / 94$ & 58,843 & 47.49 \\
\hline \multirow[t]{6}{*}{ NTS } & Elemental hazardous metals & & 52,296 & 4.90 \\
\hline & Inorganic homogeneous solids & & 364,744 & 260.00 \\
\hline & Organic homogeneous solids & & 2,144 & 1.51 \\
\hline & Organic liquids & & 153 & 0.21 \\
\hline & Soil/gravel & & 35,852 & 30.91 \\
\hline & NTS total & $11 / 01 / 93-04 / 01 / 95$ & 455,189 & 297.53 \\
\hline ORISE & & d & d & $\mathrm{d}$ \\
\hline \multirow[t]{9}{*}{ ORNL } & Aqueous liquids/slurries & & $3,003,608$ & $2,799.37$ \\
\hline & Batteries & & 1,269 & 1.02 \\
\hline & Beryllium dust & & 11 & 0.01 \\
\hline & Compressed gases/aerosols & & i & d \\
\hline & Elemental hazardous metals & & 221 & 0.15 \\
\hline & Elemental mercury & & 773 & 0.76 \\
\hline & Explosives/propellants & & 14 & 0.01 \\
\hline & Heterogeneous debris & & 3,234 & 2.17 \\
\hline & Inorganic debris & & 4,107 & 2.71 \\
\hline
\end{tabular}


Table 8.7 (continued)

\begin{tabular}{|c|c|c|c|c|}
\hline \multirow[b]{2}{*}{ Site } & \multirow[b]{2}{*}{ Categoryb } & \multicolumn{3}{|c|}{ Inventory } \\
\hline & & Date(s) ${ }^{\mathrm{C}}$ & $\begin{array}{l}\text { Mass } \\
\text { (kg) }\end{array}$ & $\begin{array}{c}\text { Volume } \\
\left(\mathrm{m}^{3}\right)\end{array}$ \\
\hline \multirow{11}{*}{$\begin{array}{l}\text { ORNL } \\
\text { (contd.) }\end{array}$} & Inorganic homogeneous solids & & 102,915 & 68.93 \\
\hline & Lab packs & & 37,028 & 35.91 \\
\hline & Organic debris & & 3,529 & 2.35 \\
\hline & Organic homogeneous solids & & 475 & 0.32 \\
\hline & Organic liquids & & 61,384 & 61.21 \\
\hline & Reactive metals & & 601 & 0.40 \\
\hline & Soil/gravel & & 8,162 & 5.44 \\
\hline & Unknown/other liquids & & 2,105 & 2.10 \\
\hline & Unknown/other matrix & & 34,152 & 28.45 \\
\hline & Unknown/other solids & & 12,439 & 8.75 \\
\hline & ORNL total & $12 / 31 / 94$ & $3,276,027$ & $3,020.06$ \\
\hline \multirow[t]{16}{*}{ PAD } & Aqueous liquids/slurries & & 50,071 & 61.43 \\
\hline & Batteries & & 3,039 & 4.99 \\
\hline & Compressed gases/aerosols & & 4,951 & 15.40 \\
\hline & Elemental hazardous metals & & 5,172 & 4.25 \\
\hline & Elemental mercury & & 159 & 0.52 \\
\hline & Explosives/propellants & & 13 & 0.21 \\
\hline & Heterogeneous debris & & 1,538 & 4.02 \\
\hline & Inorganic debris & & 133,582 & 538.78 \\
\hline & Inorganic homogeneous solids & & 81,331 & 86.95 \\
\hline & Lab packs & & 4,432 & 7.70 \\
\hline & Organic debris & & 36,025 & 78.71 \\
\hline & Organic homogeneous solids & & 15,068 & 20.97 \\
\hline & Organic liquids & & 152,257 & 173.21 \\
\hline & Soil/gravel & & 5,923 & 5.01 \\
\hline & Unknown/other matrix & & 22,147 & 26.25 \\
\hline & PAD total & $12 / 31 / 94$ & 515,706 & $1,028.40$ \\
\hline \multirow[t]{11}{*}{ PANT } & Aqueous liquids/slurries & & 1,677 & 1.75 \\
\hline & Batteries & & 21 & 0.05 \\
\hline & Compressed gases/aerosols & & 1 & e \\
\hline & Elemental hazardous metals & & 22 & 0.06 \\
\hline & Heterogeneous debris & & 1,260 & 3.80 \\
\hline & Inorganic debris & & 4,230 & 11.20 \\
\hline & Inorganic homogeneous solids & & 3,675 & 18.90 \\
\hline & Lab packs & & 1,929 & 7.29 \\
\hline & Organic debris & & 24,793 & 82.01 \\
\hline & Organic liquids & & 1,118 & 2.60 \\
\hline & PANT total & $03 / 01 / 95$ & 38,726 & 127.66 \\
\hline Pinellas & & d & d & d \\
\hline \multirow[t]{8}{*}{ PORTS } & Aqueous liquids/slurries & & 76,956 & 113.33 \\
\hline & Batteries & & 91,600 & 80.00 \\
\hline & Compressed gases/aerosols & & 1,899 & 7.00 \\
\hline & Elemental mercury & & 880 & 2.20 \\
\hline & Heterogeneous debris & & 434,703 & 842.33 \\
\hline & Inorganic debris & & 63,950 & 101.70 \\
\hline & Inorganic homogeneous solids & & 535,201 & 655.75 \\
\hline & Lab packs & & 13,590 & 29.20 \\
\hline
\end{tabular}


Table 8.7 (continued)

\begin{tabular}{|c|c|c|c|c|}
\hline \multirow[b]{2}{*}{ Site } & \multirow[b]{2}{*}{ Category ${ }^{b}$} & \multicolumn{3}{|c|}{ Inventory } \\
\hline & & $\operatorname{Date}(\mathrm{s})^{\mathrm{c}}$ & $\begin{array}{c}\text { Mass } \\
(\mathrm{kg})\end{array}$ & $\begin{array}{c}\text { Volume } \\
\left(\mathrm{m}^{3}\right)\end{array}$ \\
\hline \multirow{6}{*}{$\begin{array}{l}\text { PORTS } \\
\text { (contd.) }\end{array}$} & Organic debris & & 151,104 & 280.44 \\
\hline & Organic homogeneous solids & & 213,319 & 273.60 \\
\hline & Organic liquids & & 175,164 & 250.79 \\
\hline & Reactive metals & & & 0.60 \\
\hline & Soil/gravel & & $5,442,600$ & $4,877.60$ \\
\hline & PORTS total & $12 / 01 / 94$ & $7,200,966$ & $7,514.54$ \\
\hline \multicolumn{2}{|l|}{ PPPL } & $\mathrm{d}$ & $d$ & $d$ \\
\hline \multirow[t]{11}{*}{ RFETS } & Aqueous liquids/slurries & & 293 & 533.26 \\
\hline & Beryllium dust & & 1,028 & 3.15 \\
\hline & Elemental hazardous metals & & 27,838 & 34.69 \\
\hline & Heterogeneous debris & & 1,785 & 6.66 \\
\hline & Inorganic debris & & 14,162 & 170.66 \\
\hline & Inorganic homogeneous solids & & 561,396 & $10,975.96$ \\
\hline & Lab packs & & d & 47.38 \\
\hline & Organic debris & & 588,326 & $1,241.53$ \\
\hline & Organic liquids & & d & 130.80 \\
\hline & Soil/gravel & & 341,600 & 406.05 \\
\hline & RFETS total & $10 / 01 / 94$ & $1,536,428$ & $13,550.14$ \\
\hline \multirow[t]{6}{*}{ RMI } & Aqueous liquids/slurries & & 2,221 & 2.43 \\
\hline & Inorganic debris & & 6,122 & 6.40 \\
\hline & Inorganic homogeneous solids & & 4,686 & 4.16 \\
\hline & Organic debris & & 1,308 & 3.53 \\
\hline & Organic liquids & & 6,017 & 7.00 \\
\hline & RMI total & $03 / 01 / 95$ & 20,354 & 23.52 \\
\hline \multirow[t]{3}{*}{ SLAC } & Aqueous liquids/slurries & & 12 & 0.01 \\
\hline & Elemental hazardous metals & & 8,060 & 5.90 \\
\hline & SLAC total & $11 / 01 / 94-03 / 01 / 95$ & 8,072 & 5.91 \\
\hline SNL/CA & Lab packs & $05 / 01 / 95$ & 62 & 0.40 \\
\hline \multirow[t]{11}{*}{ SNL/NM } & Aqueous liquids/slurries & & $\mathrm{d}$ & 0.02 \\
\hline & Elemental hazardous metals & & 131 & 0.04 \\
\hline & Elemental mercury & & 3 & e \\
\hline & Heterogeneous debris & & d & 4.37 \\
\hline & Inorganic debris & & 1,450 & 9.70 \\
\hline & Inorganic homogeneous solids & & 1 & 0.01 \\
\hline & Organic debris & & 1,098 & 28.60 \\
\hline & Organic liquids & & 3 & 2.91 \\
\hline & Reactive metals & & 53 & 0.06 \\
\hline & Unknown/other debris & & d & 29.00 \\
\hline & SNL/NM total & $05 / 01 / 95$ & 2,738 & 74.71 \\
\hline \multirow[t]{3}{*}{ SRS } & Aqueous liquids/slurries & & 158,140 & 157.70 \\
\hline & Elemental hazardous metals & & 129,386 & 82.20 \\
\hline & Elemental mercury & & 5,369 & 2.70 \\
\hline
\end{tabular}


Table 8.7 (continued)

\begin{tabular}{|c|c|c|c|c|}
\hline \multirow[b]{2}{*}{ Site } & \multirow[b]{2}{*}{ Category ${ }^{b}$} & \multicolumn{3}{|c|}{ Inventory } \\
\hline & & $\operatorname{Date}(s)^{c}$ & $\begin{array}{l}\text { Mass } \\
(\mathrm{kg})\end{array}$ & $\begin{array}{l}\text { Volume } \\
\left(\mathrm{m}^{3}\right)\end{array}$ \\
\hline \multirow{11}{*}{$\begin{array}{l}\text { SRS } \\
\text { (contd.) }\end{array}$} & Heterogeneous debris & & 385,250 & $3,013.60$ \\
\hline & Immobilized forms & & 2,270 & 0.30 \\
\hline & Inorganic debris & & 105,809 & 89.60 \\
\hline & Inorganic homogeneous solids & & $3,039,362$ & $2,724.20$ \\
\hline & Lab packs & & 7,476 & 8.40 \\
\hline & Organic debris & & 4,950 & 13.90 \\
\hline & Organic liquids & & 108,381 & 139.00 \\
\hline & Reactive metals & & 318 & 0.80 \\
\hline & Soil/gravel & & 23,568 & 16.80 \\
\hline & Unknown/other debris & & 201,405 & 951.00 \\
\hline & SRS total & 09/01/94-10/01/94 & $4,171,684$ & $7,200.20$ \\
\hline \multirow[t]{13}{*}{ WVDP } & Aqueous liquids/slurries & & 1,593 & 0.71 \\
\hline & Batteries & & 86 & 0.06 \\
\hline & Elemental hazardous metals & & 14,297 & 1.26 \\
\hline & Elemental mercury & & 6 & e \\
\hline & Heterogeneous debris & & 67,888 & 68.89 \\
\hline & Inorganic debris & & 2,603 & 1.74 \\
\hline & Inorganic homogeneous solids & & 966 & 0.44 \\
\hline & Lab packs & & 286 & 0.30 \\
\hline & Organic homogeneous solids & & 70 & 0.07 \\
\hline & Organic liquids & & 4,094 & 4.51 \\
\hline & Unknown/other debris & & 12,394 & 8.26 \\
\hline & Unknown/other matrix & & 39 & 0.03 \\
\hline & WVDP total & $09 / 01 / 94$ & 104,323 & 86.26 \\
\hline \multirow[t]{20}{*}{ Y-12 } & Aqueous liquids/slurries & & 40,187 & 40.13 \\
\hline & Batteries & & 6,376 & 4.25 \\
\hline & Beryllium dust & & 7 & e \\
\hline & Compressed gases/aerosols & & 831 & 0.80 \\
\hline & Elemental hazardous metals & & 7,385 & 4.92 \\
\hline & Elemental mercury & & 160 & 0.13 \\
\hline & Explosives/propellants & & 1 & e \\
\hline & Heterogeneous debris & & 49,791 & 33.72 \\
\hline & Inorganic debris & & 23,542 & 15.71 \\
\hline & Inorganic homogeneous solids & & $7,781,626$ & $6,475.92$ \\
\hline & Lab packs & & 278 & 0.26 \\
\hline & Organic debris & & 80,862 & 54.04 \\
\hline & Organic homogeneous solids & & 57,845 & 46.21 \\
\hline & Organic liquids & & 313,716 & 313.68 \\
\hline & Reactive metals & & 1,012 & 0.68 \\
\hline & Soil/gravel & & $9,793,441$ & $6,421.72$ \\
\hline & Unknown/other liquids & & 2,258 & 2.26 \\
\hline & Unknown/other matrix & & 42 & 0.03 \\
\hline & Unknown/other solids & & 75,835 & 50.62 \\
\hline & $\mathrm{Y}-12$ total & $12 / 31 / 94$ & $18,235,195$ & $13,465.07$ \\
\hline \multirow[t]{4}{*}{ Others $j$} & Aqueous liquids/slurries & & 36,040 & 43.98 \\
\hline & Batteries & & 39 & 3.50 \\
\hline & Elemental hazardous metals & & 4,118 & 28.90 \\
\hline & Elemental mercury & & d & 0.40 \\
\hline
\end{tabular}


Table 8.7 (continued)

\begin{tabular}{|c|c|c|c|c|}
\hline \multirow[b]{2}{*}{ Site } & \multirow[b]{2}{*}{ Category $^{b}$} & \multicolumn{3}{|c|}{ Inventory } \\
\hline & & $\operatorname{Date}(s)^{c}$ & $\begin{array}{c}\text { Mass } \\
(\mathrm{kg})\end{array}$ & $\begin{array}{l}\text { Volume } \\
\left(\mathrm{m}^{3}\right)\end{array}$ \\
\hline \multirow{16}{*}{$\begin{array}{l}\text { Others } \\
\text { (contd.) }\end{array}$} & Heterogeneous debris & & 21,149 & 647.03 \\
\hline & Immobilized forms & & d & 2.54 \\
\hline & Inorganic debris & & 18,017 & $1,011.87$ \\
\hline & Inorganic homogeneous solids & & 7,851 & 73.12 \\
\hline & Lab packs & & i & 4.48 \\
\hline & Organic debris & & 12,648 & 320.44 \\
\hline & Organic homogeneous solids & & $\mathrm{d}$ & 2.27 \\
\hline & Organic liquids & & 17 & 60.68 \\
\hline & Reactive metals & & $\mathrm{d}$ & 0.20 \\
\hline & Soil/gravel & & $d$ & $24,481.42$ \\
\hline & Unknown/other debris & & 100 & 0.23 \\
\hline & Unknown/other liquids & & 542 & 0.55 \\
\hline & Unknown/other matrix & & $\mathrm{d}$ & 0.21 \\
\hline & Unknown/other solids & & 267 & 0.67 \\
\hline & Others total & $03 / 01 / 94-05 / 01 / 95$ & 100,788 & $26,682.49$ \\
\hline & Grand total (DOE complex) & $11 / 01 / 93-06 / 01 / 95$ & $91,087,127$ & $138,143.60$ \\
\hline
\end{tabular}

a Based on MWIR, ref. 7.

${ }^{b}$ As described in Table 8.6.

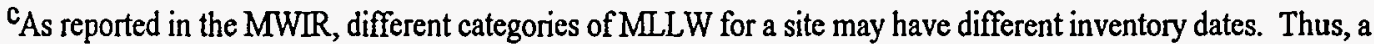
range of dates may be reported for a site's MLLW inventory. Each date is specified by the numbering system used for month/day/calendar year.

dinformation not available.

eValue $<0.005 \mathrm{~m}^{3}$.

Includes contributions from PNL.

gIncludes contributions from the Naval Reactors Facility (NRF).

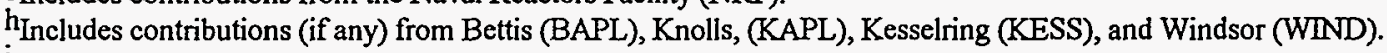

iValue $<0.5 \mathrm{~kg}$.

Jincludes contributions from Battelle Columbus Laboratories Decommissioning Project, Colonie Interim Storage Site, General Atomics facility, Laboratory for Energy-Related Health Research, Middlesex Sampling Plant, University of Missouri, Weldon Spring Site Remedial Action Project, and 6 naval shipyards (Charleston, South Carolina; Mare Island, California; Norfolk, Virginia; Pearl Harbor, Hawaii; Portsmouth, Maine; and Puget Sound, Washington). 
Table 8.8. Cumulative mass and volume inventories, by physical/chemical matrix category, of RCRA- and state-regulated MLLW from DOE site activities ${ }^{a}$

\begin{tabular}{|c|c|c|}
\hline Category $b$ & $\begin{array}{l}\text { Mass }^{c} \\
(\mathrm{~kg})\end{array}$ & $\begin{array}{l}\text { Volume } \\
\left(\mathrm{m}^{3}\right)\end{array}$ \\
\hline \multicolumn{3}{|l|}{ Liquids } \\
\hline Aqueous liquids/slurries & $3,853,320.96$ & $5,110.56$ \\
\hline Organic liquids & $1,292,901.32$ & $2,082.38$ \\
\hline Unknown/other liquids & $4,904.83$ & 4.91 \\
\hline \multicolumn{3}{|l|}{ Solids } \\
\hline Heterogeneous debris & $2,248,924.00$ & $8,417.58$ \\
\hline Inorganic debris & $4,216,943.93$ & $11,950.26$ \\
\hline Inorganic homogeneous solids & $55,367,931.09$ & $56,656.34$ \\
\hline Organic debris & $2,910,967.26$ & $9,287.65$ \\
\hline Organic homogeneous solids & $780,633.67$ & 770.81 \\
\hline Soil/gravel & $16,294,071.28$ & $37,277.58$ \\
\hline Unknown/other debris & $213,899.41$ & 997.11 \\
\hline Unknown/other homogeneous solids & 199.58 & 0.64 \\
\hline Unknown/other solids & $1,620,040.95$ & $3,205.23$ \\
\hline \multicolumn{3}{|l|}{ Specific waste forms } \\
\hline Batteries & $126,774.31$ & 117.59 \\
\hline Beryllium dust & $1,304.81$ & 3.33 \\
\hline Compressed gases/aerosols & $14,625.31$ & 31.04 \\
\hline Explosives/propellants & 146.63 & 0.30 \\
\hline Elemental hazardous metals & $1,281,286.31$ & $1,081.11$ \\
\hline Elemental mercury & $13,343.80$ & 14.21 \\
\hline Lab packs & $97,164.48$ & 492.72 \\
\hline Reactive metals & $592,582.29$ & 413.83 \\
\hline Final waste forms & $2,270.00$ & 36.24 \\
\hline Uncategorized matrix & $152,890.71$ & 192.19 \\
\hline Grand total (DOE complex) & $91,087,127$ & $138,143.6$ \\
\hline
\end{tabular}

aBased on the MWIR, ref. 7. Detailed site data for these categories are also reported in this reference.

b As described in Table 8.6.

CMass values were not reported for all streams. The values shown reflect only those that were reported. 
Table 8.9. Projected 5-year (1994-1998) cumulative mass and volume generation, by physical/chemical matrix category, of RCRAand state-regulated MLLW from DOE site activities ${ }^{\mathrm{a}}$

\begin{tabular}{|c|c|c|}
\hline Category $b$ & $\begin{array}{l}\operatorname{Mass}^{c} \\
(\mathrm{~kg})\end{array}$ & $\begin{array}{l}\text { Volume } \\
\left(\mathrm{m}^{3}\right)\end{array}$ \\
\hline \multicolumn{3}{|l|}{ Liquids } \\
\hline Aqueous liquids/slurries & $3,929,174.20$ & $8,401.97$ \\
\hline Organic liquids & $828,666.04$ & $2,100.98$ \\
\hline Unknown/other liquids & $22,440.00$ & 22.44 \\
\hline \multicolumn{3}{|l|}{ Solids } \\
\hline Heterogeneous debris & $792,545.50$ & $3,797.89$ \\
\hline Inorganic debris & $526,992.46$ & $3,616.65$ \\
\hline Inorganic homogeneous solids & $2,138,302.40$ & $4,532.64$ \\
\hline Organic debris & $488,520.48$ & $4,575.32$ \\
\hline Organic homogeneous solids & $61,008.00$ & 161.60 \\
\hline Soil/gravel & $4,405,487.00$ & $3,396.37$ \\
\hline Unknown/other debris & $49,061.02$ & 55.54 \\
\hline Unknown/other homogeneous solids & $3,440.00$ & 4.28 \\
\hline Unknown/other solids & $34,381.60$ & 26.02 \\
\hline \multicolumn{3}{|l|}{ Specific waste forms } \\
\hline Batteries & $13,579.40$ & 19.78 \\
\hline Compressed gases/aerosols & $2,220.00$ & 5.31 \\
\hline Explosives/propellants & 4.00 & d \\
\hline Elemental hazardous metals & $512,115.35$ & 407.47 \\
\hline Elemental mercury & $20,706.16$ & 8.61 \\
\hline Lab packs & $26,729.05$ & 934.27 \\
\hline Reactive metals & $10,037.92$ & 13.50 \\
\hline Final waste forms & $32,020,874.00$ & $29,603.24$ \\
\hline Uncategorized matrix & $2,676.00$ & 48.70 \\
\hline Grand total (DOE complex) & $45,888,961$ & $61,732.6$ \\
\hline
\end{tabular}

a Based on the MWIR, ref. 7. Detailed site data for these categories are also reported in this reference.

$\mathrm{b}_{\text {As described in Table 8.6. }}$

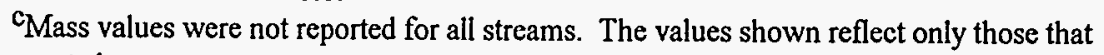
were reported.

$\mathrm{d}$ Value $<0.005 \mathrm{~m}^{3}$ 
Table 8.10. Cumulative mass (kg) inventories through 1994, by physical category, of TSCA-regulated MLLW from DOE site activities ${ }^{a, b}$

\begin{tabular}{|c|c|c|c|c|c|}
\hline Site & Solid & Liquid & $\mathrm{Gas}^{\mathrm{c}}$ & Sludge & Total \\
\hline Ames & 2,600 & 0 & 0 & 0 & 2,600 \\
\hline ANL-E & 0 & 0 & 0 & 62,400 & 62,400 \\
\hline ANL $-W$ & d & d & d & $d$ & d \\
\hline BNL & 1,000 & 0 & 0 & 0 & 1,000 \\
\hline ETEC & 4,320 & 0 & 0 & 0 & 4,320 \\
\hline FEMP & d & d & $d$ & d & d \\
\hline FNAL & 0 & 0 & 0 & 0 & 0 \\
\hline GJPO & 69,705 & 0 & 0 & 0 & 69,705 \\
\hline Hanford ${ }^{e}$ & 109,387 & 0 & 0 & 0 & 109,387 \\
\hline $\mathrm{INEL}^{\mathrm{f}}$ & 232 & 565 & 0 & 0 & 797 \\
\hline ITRI & 0 & 0 & 0 & 0 & 0 \\
\hline $\mathrm{K}-25$ & $6,231,540$ & 135,768 & 0 & 13,860 & $6,381,168$ \\
\hline $\mathrm{KCP}$ & 0 & 0 & 0 & 0 & 0 \\
\hline LANL & $2,504,237$ & 0 & 0 & 0 & $2,504,237$ \\
\hline LBL & 214 & 0 & 0 & 0 & 214 \\
\hline LLNL & 1,637 & 434 & 0 & 23 & 2,094 \\
\hline Mound & 4,113 & 0 & 0 & 0 & 4,113 \\
\hline NR sites 8 & 10,714 & 129 & 0 & 0 & 10,843 \\
\hline NTS & 0 & 0 & 0 & 0 & 0 \\
\hline ORISE & 0 & 0 & 0 & 0 & 0 \\
\hline ORNL & 10,749 & 7,473 & 0 & 56 & 18,278 \\
\hline PAD & $2,930,037$ & 71,916 & 0 & 0 & $3,001,953$ \\
\hline PANT & 4,500 & 0 & 0 & 0 & 4,500 \\
\hline Pinellas & 0 & 0 & 0 & 0 & 0 \\
\hline PORTS & $2,020,300$ & 55,900 & 0 & e & $2,076,200$ \\
\hline PPPL & 0 & 0 & 0 & 0 & 0 \\
\hline RFETS & 33,680 & 4,930 & 0 & 0 & 38,610 \\
\hline SLAC & 0 & 0 & 0 & 0 & 0 \\
\hline SNL/CA & 2 & 0 & 0 & 0 & 2 \\
\hline SNL/NM ${ }^{h}$ & 68 & 0 & 0 & 0 & 68 \\
\hline SRS & 70,636 & 0 & 0 & 0 & 70,636 \\
\hline WVDP & 10,850 & 0 & 0 & 0 & 10,850 \\
\hline$Y-12$ & 163,130 & 41,414 & 0 & e & 204,544 \\
\hline Total & $14,183,651$ & 318,529 & 0 & 76,339 & $14,578,519$ \\
\hline
\end{tabular}

a Based on the DOE site data submittals of ref. 10. Material may be in interim storage awaiting treatment. Specific site information is provided in Sect. 8.4.

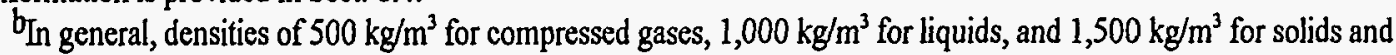
sludges were assumed to calculate masses when the site did not report mass data.

cStored in cylinders.

IInformation not available or unknown.

Includes contributions from PNL.

$\mathfrak{f}_{\text {Excludes contributions from ANL-W. }}$

GIncludes contributions (if any) from Bettis (BAPL), Knolls (KAPL), Kesselring (KESS), Windsor (WIND), and six naval shipyards.

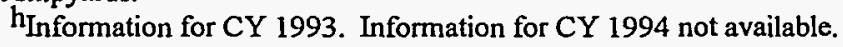


Table 8.11. Cumulative volume $\left(\mathrm{m}^{3}\right)$ inventories through 1994, by physical category, of TSCA-regulated MLLW from DOE site activities ${ }^{a}, \mathrm{~b}$

\begin{tabular}{|c|c|c|c|c|c|}
\hline Site & Solid & Liquid & $\mathrm{Gas}^{\mathrm{c}}$ & Sludge & Total \\
\hline Ames & 3.4 & 0.0 & 0.0 & 0.0 & 3.4 \\
\hline ANL-E & 0.0 & 0.0 & 0.0 & 52.0 & 52.0 \\
\hline ANL-W & d & d & d & $d$ & $d$ \\
\hline BNL & 2.1 & 0.0 & 0.0 & 0.0 & 2.1 \\
\hline ETEC & 11.3 & 0.0 & 0.0 & 0.0 & 11.3 \\
\hline FEMP & $\mathrm{d}$ & d & d & d & $d$ \\
\hline FNAL & 0.0 & 0.0 & 0.0 & 0.0 & 0.0 \\
\hline GJPO & 46.5 & 0.0 & 0.0 & 0.0 & 46.5 \\
\hline Hanforde & 95.6 & 0.0 & 0.0 & 0.0 & 95.6 \\
\hline $\mathbb{N N E L}^{\mathrm{f}}$ & 0.8 & 1.0 & 0.0 & 0.0 & 1.8 \\
\hline ITRI & 0.0 & 0.0 & 0.0 & 0.0 & 0.0 \\
\hline $\mathrm{K}-25$ & $9,340.2$ & 138.2 & 0.0 & 14.9 & $9,493.3$ \\
\hline $\mathrm{KCP}$ & 0.0 & 0.0 & 0.0 & 0.0 & 0.0 \\
\hline LANL & $5,992.5$ & 0.0 & 0.0 & 0.0 & $5,992.5$ \\
\hline LBL & 0.1 & 0.0 & 0.0 & 0.0 & 0.1 \\
\hline LLNL & 1.2 & 0.4 & 0.0 & $<0.1$ & 1.7 \\
\hline Mound & 15.6 & 0.0 & 0.0 & 0.0 & 15.6 \\
\hline NR sitesg & 21.9 & 0.2 & 0.0 & 0.0 & 22.1 \\
\hline NTS & 0.0 & 0.0 & 0.0 & 0.0 & 0.0 \\
\hline ORISE & 0.0 & 0.0 & 0.0 & 0.0 & 0.0 \\
\hline ORNL & 7.2 & 7.5 & 0.0 & -0.0 & 14.7 \\
\hline $\mathrm{PAD}$ & $4,280.3$ & 79.1 & 0.0 & 0.0 & $4,359.4$ \\
\hline PANT & 3.0 & 0.0 & 0.0 & 0.0 & 3.0 \\
\hline Pinellas & 0.0 & 0.0 & 0.0 & 0.0 & 0.0 \\
\hline PORTS & $3,574.0$ & 95.8 & 0.0 & e & $3,669.8$ \\
\hline PPPL & 0.0 & 0.0 & 0.0 & 0.0 & 0.0 \\
\hline RFETS & 104.0 & 8.0 & 0.0 & 0.0 & 112.0 \\
\hline SLAC & 0.0 & 0.0 & 0.0 & 0.0 & 0.0 \\
\hline SNL/CA & 0.2 & 0.0 & 0.0 & 0.0 & 0.2 \\
\hline SNL/NM ${ }^{h}$ & 0.4 & 0.0 & 0.0 & 0.0 & 0.4 \\
\hline SRS & 142.0 & 0.0 & 0.0 & 0.0 & 142.0 \\
\hline WVDP & 43.9 & 0.0 & 0.0 & 0.0 & 43.9 \\
\hline$Y-12$ & 108.8 & 41.4 & 0.0 & 0.0 & 150.2 \\
\hline Total & $23,795.0$ & 371.6 & 0.0 & 67.0 & $24,233.6$ \\
\hline
\end{tabular}

${ }^{a}$ Based on the DOE site data submittals of ref. 10. Material may be in interim storage awaiting treatment. Specific site information is provided in Sect. 8.4.

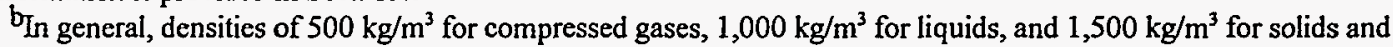
sludges were assumed to calculate masses when the site did not report mass data.

'Stored in cylinders.

${ }^{\mathrm{d}}$ Information not available or unknown.

Includes contributions from PNL.

Excludes contributions from ANL-W.

Includes contributions (if any) from Bettis (BAPL), Knolls (KAPL), Kesselring (KESS), Windsor (WIND), and six naval shipyards.

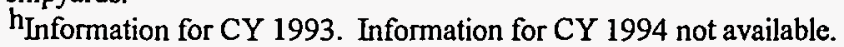


Table 8.12. 1994 mass (kg) generation, by physical category, of TSCA-regulated MLLW from DOE site activities ${ }^{\mathrm{a}, \mathrm{b}}$

\begin{tabular}{|c|c|c|c|c|c|}
\hline Site & Solid & Liquid & $\mathrm{Gas}^{\mathrm{c}}$ & Sludge & Total \\
\hline Ames & 0 & 0 & 0 & 0 & 0 \\
\hline ANL-E & 0 & 0 & 0 & 62,400 & 62,400 \\
\hline ANL-W & d & d & d & d & d \\
\hline BNL & 91 & 0 & 0 & 0 & 91 \\
\hline ETEC & 3,600 & 0 & 0 & 0 & 3,600 \\
\hline FEMP & d & d & d & d & d \\
\hline FNAL & 0 & 0 & 0 & 0 & 0 \\
\hline GJPO & 720 & 0 & 0 & 0 & 720 \\
\hline Hanford ${ }^{\mathrm{e}}$ & 4,997 & 0 & 0 & 0 & 4,997 \\
\hline $\mathrm{INEL}^{\mathrm{f}}$ & 164 & 0 & 0 & 0 & 164 \\
\hline ITRI & 0 & 0 & 0 & 0 & 0 \\
\hline $\mathrm{K}-25$ & $2,030,331$ & 125,460 & 0 & 10,109 & $2,165,900$ \\
\hline $\mathrm{KCP}$ & 0 & 0 & 0 & 0 & 0 \\
\hline LANL & 59,272 & 0 & 0 & 0 & 59,272 \\
\hline LBL & 1,151 & 0 & 0 & 0 & 1,151 \\
\hline LLNL & 1,165 & 0 & 0 & 0 & 1,165 \\
\hline Mound & 2,054 & 0 & 0 & 0 & 2,054 \\
\hline NR sitesg & 123,517 & 0 & 0 & 0 & 123,517 \\
\hline NTS & 0 & 0 & 0 & 0 & 0 \\
\hline ORISE & 0 & 0 & 0 & 0 & 0 \\
\hline ORNL & 15,625 & 2,643 & 0 & 10 & 18,278 \\
\hline PAD & 292,999 & 119,265 & 0 & 0 & 412,264 \\
\hline PANT & 4,500 & 0 & 0 & 0 & 4,500 \\
\hline Pinellas & 0 & 0 & 0 & 0 & 0 \\
\hline PORTS & 85,570 & 5,920 & 0 & 0 & 91,490 \\
\hline PPPL & 0 & 0 & 0 & 0 & 0 \\
\hline RFETS & 8,280 & 250 & 0 & 0 & 8,530 \\
\hline SLAC & 0 & 0 & 0 & 0 & 0 \\
\hline SNL/CA & 2 & 0 & 0 & 0 & 2 \\
\hline SNL/NM ${ }^{h}$ & 0 & 0 & 0 & 0 & 0 \\
\hline SRS & 6,050 & 0 & 0 & 0 & 6,050 \\
\hline WVDP & 6,726 & 0 & 0 & 0 & 6,726 \\
\hline$Y-12$ & 19,701 & 7,439 & 0 & 0 & 27,140 \\
\hline Total & $2,666,515$ & 260,977 & 0 & 72,519 & $3,000,011$ \\
\hline
\end{tabular}

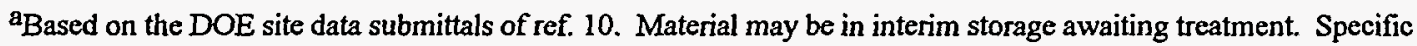
site information is provided in Sect. 8.4.

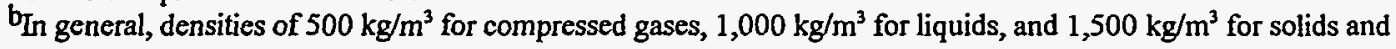
sludges were assumed to calculate masses when the site did not report mass data.

cStored in cylinders.

dinformation not available or unknown.

Includes contributions from PNL.

Excludes contributions from ANL-W.

gIncludes contributions (if any) from Bettis (BAPL), Knolls (KAPL), Kesselring (KESS), Windsor (WIND), and six naval shipyards.

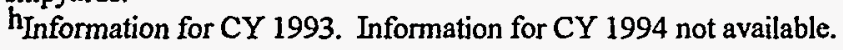


Table 8.13. 1994 volume $\left(\mathrm{m}^{3}\right)$ generation, by physical category, of TSCA-regulated MLLW from DOE site activities ${ }^{a, b}$

\begin{tabular}{|c|c|c|c|c|c|}
\hline Site & Solid & Liquid & $\mathrm{Gas}^{\mathrm{c}}$ & Sludge & Total \\
\hline Ames & 0.0 & 0.0 & 0.0 & 0.0 & 0.0 \\
\hline ANL-E & 0.0 & 0.0 & 0.0 & 52.0 & 52.0 \\
\hline ANL-W & d & d & d & $d$ & d \\
\hline $\mathrm{BNL}$ & 0.4 & 0.0 & 0.0 & 0.0 & 0.4 \\
\hline ETEC & 8.1 & 0.0 & 0.0 & 0.0 & 8.1 \\
\hline FEMP & d & d & $\mathrm{d}$ & $d$ & $d$ \\
\hline FNAL & 0.0 & 0.0 & 0.0 & 0.0 & 0.0 \\
\hline GJPO & 1.5 & 0.0 & 0.0 & 0.0 & 1.5 \\
\hline Hanford ${ }^{e}$ & 3.3 & 0.0 & 0.0 & 0.0 & 3.3 \\
\hline $\mathrm{INEL}^{\mathrm{f}}$ & 0.6 & 0.0 & 0.0 & 0.0 & 0.6 \\
\hline ITRI & 0.0 & 0.0 & 0.0 & 0.0 & 0.0 \\
\hline $\mathrm{K}-25$ & $5,438.9$ & 137.0 & 0.0 & 10.9 & $5,586.8$ \\
\hline $\mathrm{KCP}$ & 0.0 & 0.0 & 0.0 & 0.0 & 0.0 \\
\hline LANL & 166.6 & 0.0 & 0.0 & 0.0 & 166.6 \\
\hline LBL & 2.8 & 0.0 & 0.0 & 0.0 & 2.8 \\
\hline LLNL & 0.8 & 0.0 & 0.0 & 0.0 & 0.8 \\
\hline Mound & 6.8 & 0.0 & 0.0 & 0.0 & 6.8 \\
\hline NR sitesg & 344.5 & 0.0 & 0.0 & 0.0 & 344.5 \\
\hline NTS & 0.0 & 0.0 & 0.0 & 0.0 & 0.0 \\
\hline ORISE & 0.0 & 0.0 & 0.0 & 0.0 & 0.0 \\
\hline ORNL & 10.4 & 2.7 & 0.0 & -0.0 & 13.1 \\
\hline PAD & 931.7 & 129.1 & 0.0 & 0.0 & $1,060.8$ \\
\hline PANT & 3.0 & 0.0 & 0.0 & 0.0 & 3.0 \\
\hline Pinellas & 0.0 & 0.0 & 0.0 & 0.0 & 0.0 \\
\hline PORTS & 242.2 & 17.7 & 0.0 & 0.0 & 259.9 \\
\hline PPPL & 0.0 & 0.0 & 0.0 & 0.0 & 0.0 \\
\hline RFETS & 35.0 & 1.0 & 0.0 & 0.0 & 36.0 \\
\hline SLAC & 0.0 & 0.0 & 0.0 & 0.0 & 0.0 \\
\hline SNL/CA & 0.2 & 0.0 & 0.0 & 0.0 & 0.2 \\
\hline SNL/NM ${ }^{h}$ & 0.0 & 0.0 & 0.0 & 0.0 & 0.0 \\
\hline SRS & 12.1 & 0.0 & 0.0 & 0.0 & 12.1 \\
\hline WVDP & 4.5 & 0.0 & 0.0 & 0.0 & 4.5 \\
\hline$Y-12$ & 13.1 & 7.5 & 0.0 & 0.0 & 20.6 \\
\hline Total & $7,226.5$ & 295.0 & 0.0 & 62.9 & $7,584.4$ \\
\hline
\end{tabular}

a Based on the DOE site data submittals of ref. 10. Material may be in interim storage awaiting treatment. Specific site information is provided in Sect. 8.4.

bensities of $500 \mathrm{~kg} / \mathrm{m}^{3}$ for compressed gases, $1,000 \mathrm{~kg} / \mathrm{m}^{3}$ for liquids, and $1,500 \mathrm{~kg} / \mathrm{m}^{3}$ for solids and sludges were assumed to calculate masses when the site did not report mass data.

cStored in cylinders.

${ }^{d}$ Information not available or unknown.

Includes contributions from PNL.

$f_{\text {Excludes contributions from ANL-W. }}$

IIncludes contributions (if any) from Bettis (BAPL), Knolls (KAPL), Kesselring (KESS), Windsor (WIND), and six naval shipyards.

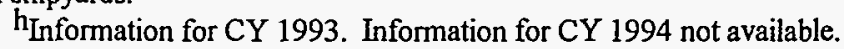


Table 8.14. Cumulative mass ( $\mathrm{kg}$ ) inventories through 1994, by hazard category, of TSCA-regulated MLLW from DOE site activities ${ }^{\mathrm{a}}$

\begin{tabular}{|c|c|c|c|c|}
\hline Site & PCB & Asbestos & Other & Total \\
\hline Ames & 0 & 2,600 & 0 & 2,600 \\
\hline ANL-E & 62,400 & 0 & 0 & 62,400 \\
\hline ANL $-W$ & b & b & b & $\mathrm{b}$ \\
\hline BNL & 0 & 1,000 & 0 & 1,000 \\
\hline ETEC & 0 & 4,320 & 0 & 4,320 \\
\hline FEMP & b & b & b & $\mathrm{b}$ \\
\hline FNAL & 0 & 0 & 0 & 0 \\
\hline GJPO & 69,705 & 0 & 0 & 69,705 \\
\hline Hanford ${ }^{\mathrm{C}}$ & 109,387 & b & b & 109,387 \\
\hline $\mathrm{INEL}^{\mathrm{d}}$ & 797 & 0 & 0 & 797 \\
\hline ITRI & 0 & 0 & 0 & 0 \\
\hline $\mathrm{K}-25$ & $6,129,696$ & 249,865 & 1,607 & $6,381,168$ \\
\hline $\mathrm{KCP}$ & 0 & 0 & 0 & 0 \\
\hline LANL & $2,010,033$ & 494,204 & 0 & $2,504,237$ \\
\hline LBL & 214 & 0 & 0 & 214 \\
\hline LLNL & 475 & 1,619 & 0 & 2,094 \\
\hline Mound & 0 & 4,113 & 0 & 4,113 \\
\hline NR sites ${ }^{\mathbf{e}}$ & 10,843 & 0 & 0 & 10,843 \\
\hline NTS & 0 & 0 & 0 & 0 \\
\hline ORISE & 0 & 0 & 0 & 0 \\
\hline ORNL & 14,897 & 3,312 & 69 & 18,278 \\
\hline PAD & $2,807,972$ & 193,981 & 0 & $3,001,953$ \\
\hline PANT & 0 & 4,500 & 0 & 4,500 \\
\hline Pinellas & 0 & 0 & 0 & 0 \\
\hline PORTS & $1,886,000$ & 190,200 & 0 & $2,076,200$ \\
\hline PPPL & 0 & 0 & 0 & 0 \\
\hline RFETS & 36,240 & 2,370 & 0 & 38,610 \\
\hline SLAC & 0 & 0 & 0 & 0 \\
\hline SNL/CA & 0 & 2 & 0 & 2 \\
\hline SNL/NM $\mathrm{f}$ & 0 & 68 & 0 & 68 \\
\hline SRS & 636 & 70,000 & 0 & 70,636 \\
\hline WVDP & 0 & 10,850 & 0 & 10,850 \\
\hline Y-12 & 204,544 & 0 & 0 & 204,544 \\
\hline Total & $13,343,839$ & $1,233,004$ & 1,676 & $14,578,519$ \\
\hline
\end{tabular}

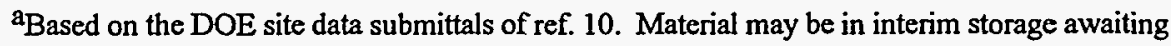
treatment. Specific site information is provided in Sect. 8.4.

Information not available or unknown.

CIncludes contributions from PNL.

${ }^{d}$ Excludes contributions from ANL-W.

'Includes contributions (if any) from Bettis (BAPL), Knolls (KAPL), Kesselring (KESS), Windsor (WIND), and six naval shipyards.

Information for CY 1993. Information for CY 1994 not available. 
Table 8.15. Cumulative volume $\left(\mathrm{m}^{3}\right)$ inventories through 1994 , by hazard category, of TSCA-regulated MLLW from DOE site activities ${ }^{a}$

\begin{tabular}{|c|c|c|c|c|}
\hline Site & PCB & Asbestos & Other & Total \\
\hline Ames & 0.0 & 3.4 & 0.0 & 3.4 \\
\hline ANL-E & 52.0 & 0.0 & 0.0 & 52.0 \\
\hline ANL-W & b & b & b & b \\
\hline $\mathrm{BNL}$ & 0.0 & 2.1 & 0.0 & 2.1 \\
\hline ETEC & 0.0 & 11.3 & 0.0 & 11.3 \\
\hline FEMP & b & $\mathrm{b}$ & b & b \\
\hline FNAL & 0.0 & 0.0 & 0.0 & 0.0 \\
\hline GJPO & 46.5 & 0.0 & 0.0 & 46.5 \\
\hline Hanford $\mathrm{d}^{\mathrm{c}}$ & 95.6 & b & b & 95.6 \\
\hline $\mathbb{N N E L}^{\mathrm{d}}$ & 1.8 & 0.0 & 0.0 & 1.8 \\
\hline ITRI & 0.0 & 0.0 & 0.0 & 0.0 \\
\hline $\mathrm{K}-25$ & $8,130.1$ & $1,356.3$ & 6.9 & $9,493.3$ \\
\hline $\mathrm{KCP}$ & 0.0 & 0.0 & 0.0 & 0.0 \\
\hline LANL & $2,100.7$ & $3,891.8$ & 0.0 & $5,992.5$ \\
\hline LBL & 0.1 & 0.0 & 0.0 & 0.1 \\
\hline LLNL & 0.5 & 1.2 & 0.0 & 1.7 \\
\hline Mound & 0.0 & 15.6 & 0.0 & 15.6 \\
\hline NR sites ${ }^{\mathrm{e}}$ & 22.1 & 0.0 & 0.0 & 22.1 \\
\hline NTS & 0.0 & 0.0 & 0.0 & 0.0 \\
\hline ORISE & 0.0 & 0.0 & 0.0 & 0.0 \\
\hline ORNL & 12.4 & 2.2 & $>0.1$ & 14.7 \\
\hline PAD & $3,952.6$ & 406.8 & 0.0 & $4,359.4$ \\
\hline PANT & 0.0 & 3.0 & 0.0 & 3.0 \\
\hline Pinellas & 0.0 & 0.0 & 0.0 & 0.0 \\
\hline PORTS & $3,507.8$ & 162.0 & 0.0 & $3,669.8$ \\
\hline PPPL & 0.0 & 0.0 & 0.0 & 0.0 \\
\hline RFETS & 71.4 & 40.6 & 0.0 & 112.0 \\
\hline SLAC & 0.0 & 0.0 & 0.0 & 0.0 \\
\hline SNL/CA & 0.0 & 0.2 & 0.0 & 0.2 \\
\hline SNLANM ${ }^{\mathrm{f}}$ & 0.0 & 0.4 & 0.0 & 0.4 \\
\hline SRS & 2.0 & 140.0 & 0.0 & 142.0 \\
\hline WVDP & 0.0 & 43.9 & 0.0 & 43.9 \\
\hline$Y-12$ & 150.2 & 0.0 & 0.0 & 150.2 \\
\hline Total & $18,145.8$ & $6,080.8$ & 7.0 & $24,233.6$ \\
\hline
\end{tabular}

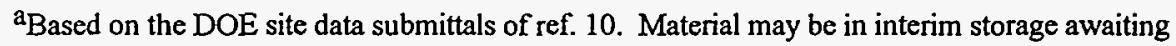
treatment. Specific site information is provided in Sect. 8.4.

Information not available or unknown.

cIncludes contributions from PNL.

${ }^{d}$ Excludes contributions from ANL-W.

Includes contributions (if any) from Bettis (BAPL), Knolls (KAPL), Kesselring (KESS), Windsor (WIND), and six naval shipyards.

Information for CY 1993. Information for CY 1994 not available. 
Table 8.16. 1994 mass (kg) generation, by hazard category, of TSCA-regulated MLLW from DOE site activities ${ }^{a}$

\begin{tabular}{|c|c|c|c|c|}
\hline Site & PCB & Asbestos & Other & Total \\
\hline Ames & 0 & 0 & 0 & 0 \\
\hline ANL-E & 62,400 & 0 & 0 & 62,400 \\
\hline ANL-W & b & b & b & b \\
\hline BNL & 0 & 91 & 0 & 91 \\
\hline ETEC & 0 & 3,600 & 0 & 3,600 \\
\hline FEMP & b & b & b & b \\
\hline FNAL & 0 & 0 & 0 & 0 \\
\hline GJPO & 174 & 546 & 0 & 720 \\
\hline Hanford ${ }^{c}$ & 4,997 & b & b & 4,997 \\
\hline $\mathbb{I N E L}^{\mathrm{d}}$ & 164 & 0 & 0 & 164 \\
\hline ITRI & 0 & 0 & 0 & 0 \\
\hline $\mathrm{K}-25$ & $2,059,870$ & 104,790 & 1,240 & $2,165,900$ \\
\hline $\mathrm{KCP}$ & 0 & 0 & 0 & 0 \\
\hline LANL & 14 & 59,258 & 0 & 59,272 \\
\hline LBL & 64 & 1,087 & 0 & 1,151 \\
\hline LLNL & 14 & 1,151 & 0 & 1,165 \\
\hline Mound & 0 & 2,054 & 0 & 2,054 \\
\hline NR sites ${ }^{\mathbb{e}}$ & 886 & 122,631 & 0 & 123,517 \\
\hline NTS & 0 & 0 & 0 & 0 \\
\hline ORISE & 0 & 0 & 0 & 0 \\
\hline ORNL & 10,580 & 5,656 & 2,042 & 18,278 \\
\hline $\mathrm{PAD}$ & 224,470 & 187,794 & 0 & 412,264 \\
\hline PANT & 0 & 4,500 & 0 & 4,500 \\
\hline Pinellas & 0 & 0 & 0 & 0 \\
\hline PORTS & 79,990 & 11,500 & 0 & 91,490 \\
\hline PPPL & 0 & 0 & 0 & 0 \\
\hline RFETS & 7,460 & 1,070 & 0 & 8,530 \\
\hline SLAC & 0 & 0 & 0 & 0 \\
\hline SNL/CA & 0 & 2 & 0 & 2 \\
\hline SNL $N M^{f}$ & 0 & 0 & 0 & 0 \\
\hline SRS & 0 & 6,050 & 0 & 6,050 \\
\hline WVDP & 0 & 6,726 & 0 & 6,726 \\
\hline $\mathrm{Y}-12$ & 27,140 & 0 & 0 & 27,140 \\
\hline Total & $2,478,223$ & 518,506 & 3,282 & $3,000,011$ \\
\hline
\end{tabular}

aBased on the DOE site data submittals of ref. 10. Material may be in interim storage awaiting treatment. Specific site information is provided in Sect. 8.4.

bInformation not available or unknown.

cIncludes contributions from PNL.

dExcludes contributions from ANL-W.

'Includes contributions (if any) from Bettis (BAPL), Knolls (KAPL), Kesselring (KESS), Windsor (WIND), and six naval shipyards.

Information for CY 1993. Information for CY 1994 not available. 
Table 8.17. 1994 volume $\left(\mathrm{m}^{3}\right)$ generation, by hazard category, of TSCA-regulated MLLW from DOE site activities ${ }^{\mathrm{a}}$

\begin{tabular}{|c|c|c|c|c|}
\hline Site & PCB & Asbestos & Other & Total \\
\hline Ames & 0.0 & 0.0 & 0.0 & 0.0 \\
\hline ANL-E & 52.0 & 0.0 & 0.0 & 52.0 \\
\hline ANL-W & b & b & $b$ & $b$ \\
\hline $\mathrm{BNL}$ & 0.0 & 0.4 & 0.0 & 0.4 \\
\hline ETEC & 0.0 & 8.1 & 0.0 & 8.1 \\
\hline FEMP & $b$ & b & $b$ & $\mathbf{b}$ \\
\hline FNAL & 0.0 & 0.0 & 0.0 & 0.0 \\
\hline GJPO & 0.1 & 1.4 & 0.0 & 1.5 \\
\hline Hanford ${ }^{c}$ & 3.3 & $\mathrm{~b}$ & $\mathrm{~b}$ & 3.3 \\
\hline $\mathrm{INEL}^{\mathrm{d}}$ & 0.6 & 0.0 & 0.0 & 0.6 \\
\hline ITRI & 0.0 & 0.0 & 0.0 & 0.0 \\
\hline$K-25$ & $5,349.0$ & 232.2 & 5.6 & $5,586.8$ \\
\hline $\mathrm{KCP}$ & 0.0 & 0.0 & 0.0 & 0.0 \\
\hline LANL & 0.2 & 166.4 & 0.0 & 166.6 \\
\hline LBL & $<0.1$ & 2.7 & 0.0 & 2.8 \\
\hline LLNL & -0 & 0.8 & 0.0 & 0.8 \\
\hline Mound & 0.0 & 6.8 & 0.0 & 6.8 \\
\hline NR sites $\mathrm{e}$ & 2.2 & 342.3 & 0.0 & 344.5 \\
\hline NTS & 0.0 & 0.0 & 0.0 & 0.0 \\
\hline ORISE & 0.0 & 0.0 & 0.0 & 0.0 \\
\hline ORNL & 7.9 & 3.8 & 1.4 & 13.1 \\
\hline $\mathrm{PAD}$ & 286.1 & 774.7 & 0.0 & $1,060.8$ \\
\hline PANT & 0.0 & 3.0 & 0.0 & 3.0 \\
\hline Pinellas & 0.0 & 0.0 & 0.0 & 0.0 \\
\hline PORTS & 250.0 & 9.9 & 0.0 & 259.9 \\
\hline PPPL & 0.0 & 0.0 & 0.0 & 0.0 \\
\hline RFETS & 31.5 & 4.5 & 0.0 & 36.0 \\
\hline SLAC & 0.0 & 0.0 & 0.0 & 0.0 \\
\hline SNL/CA & 0.0 & 0.2 & 0.0 & 0.2 \\
\hline $\mathrm{SNL} / \mathrm{NM}^{\mathrm{f}}$ & 0.0 & 0.0 & 0.0 & 0.0 \\
\hline SRS & 0.0 & 12.1 & 0.0 & 12.1 \\
\hline WVDP & 0.0 & 4.5 & 0.0 & 4.5 \\
\hline $\mathrm{Y}-12$ & 20.6 & 0.0 & 0.0 & 20.6 \\
\hline Total & $6,003.6$ & $1,573.8$ & 7.0 & $7,584.4$ \\
\hline
\end{tabular}

aBased on the DOE site data submittals of ref. 10. Material may be in interim storage awaiting treatment. Specific site information is provided in Sect. 8.4.

Information not available or unknown.

Includes contributions from PNL.

$\mathrm{d}_{\text {Excludes contributions from ANL-W. }}$

Includes contributions (if any) from Bettis (BAPL), Knolls (KAPL), Kesselring (KESS), Windsor (WIND), and six naval shipyards.

Information for CY 1993. Information for CY 1994 not available. 
Table 8.18. Historical and projected annual mass (kg) generation rates for TSCA-regulated MLLW from DOE site activities ${ }^{2}$

\begin{tabular}{|c|c|c|c|c|c|c|c|}
\hline Site & $1994^{b}$ & 1995 & 1996 & 1997 & 1998 & 1999 & $2000-2030^{c}$ \\
\hline Ames & 0 & 50 & 50 & $d$ & d & d & $d$ \\
\hline ANL-E & 62,400 & 7,200 & 0 & 0 & 0 & 0 & 0 \\
\hline psANL $-W$ & $\mathrm{~d}$ & d & $\mathrm{d}$ & $\mathrm{d}$ & d & d & d \\
\hline $\mathrm{BNL}$ & 91 & 60 & 30 & 20 & 15 & 15 & 10 \\
\hline ETEC & 3,600 & 9,000 & 0 & 0 & 800 & 1,600 & 0 \\
\hline FEMP & d & d & d & $\mathrm{d}$ & d & d & d \\
\hline FNAL & 0 & 0 & 0 & 0 & 0 & 0 & 0 \\
\hline GJPO & 720 & 50 & 20 & 20 & 10 & 10 & $<10$ \\
\hline Hanford ${ }^{e}$ & 4,997 & 255,900 & 292,800 & 252,000 & 269,550 & 418,650 & 670,100 \\
\hline $\mathrm{INEL}^{\mathrm{f}}$ & 164 & d & d & d & d & d & d \\
\hline ITRI & 0 & 0 & 0 & 0 & 0 & 0 & 0 \\
\hline $\mathrm{K}-25$ & $2,165,900$ & $2,165,900$ & $2,165,900$ & $2,165,900$ & $2,165,900$ & $2,165,900$ & $2,165,900$ \\
\hline $\mathrm{KCP}$ & 0 & 0 & 0 & 0 & 0 & 0 & 0 \\
\hline LANL & 59,272 & 37,000 & 37,000 & 37,000 & 31,000 & 28,000 & 28,000 \\
\hline LBL & 1,151 & 1,151 & 1,151 & 1,151 & 1,151 & 1,151 & 1,151 \\
\hline LLNL & 1,165 & 0 & 0 & 0 & 0 & 0 & 0 \\
\hline Mound & 2,054 & 90 & 90 & 90 & 90 & 90 & 90 \\
\hline NR sitesg & 123,517 & 46,509 & 50,878 & 42,083 & 42,083 & 41,183 & 8,055 \\
\hline NTS & 0 & 0 & $\mathrm{~d}$ & $d$ & d & $d$ & $d$ \\
\hline ORISE & 0 & 0 & 0 & 0 & 0 & 0 & 0 \\
\hline ORNL & 18,278 & 18,278 & 18,278 & 18,278 & 18,278 & 18,278 & 18,278 \\
\hline PAD & 412,264 & 469,034 & 464,934 & 467,584 & 471,010 & 463,510 & 460,810 \\
\hline PANT & 4,500 & 0 & 0 & 0 & 0 & 0 & 0 \\
\hline Pinellas & 0 & 1,850 & 0 & 0 & 0 & 0 & 0 \\
\hline PORTS & 91,490 & 72,000 & 65,000 & 58,500 & 52,700 & 47,400 & 50,000 \\
\hline PPPL & 0 & 0 & 0 & 0 & 0 & 0 & 0 \\
\hline RFETS & 8,530 & 33,640 & 19,400 & 19,400 & 19,400 & 19,400 & 32,000 \\
\hline SLAC & 0 & 0 & 0 & 0 & 0 & 0 & 0 \\
\hline SNL/CA & 2 & 0 & 0 & 0 & 0 & 0 & 0 \\
\hline SNL/NM & d & d & d & d & d & d & $\mathrm{d}$ \\
\hline SRS & 6,050 & 41,000 & 41,000 & 41,000 & 41,000 & 41,000 & 55,000 \\
\hline WVDP & 6,726 & 7,500 & 6,000 & 4,800 & 3,900 & 3,150 & 1,050 \\
\hline$Y-12$ & 27,140 & 27,000 & 27,000 & 36,000 & 36,000 & 36,000 & d \\
\hline Total & $3,000,011$ & $3,193,212$ & $3,189,531$ & $3,143,826$ & $3,152,887$ & $3,285,337$ & $3,490,454$ \\
\hline
\end{tabular}

Based on the DOE site data submittals of ref. 10. Specific site information is provided in Sect. 8.4.

$b_{\text {Actual data. }}$

${ }^{c}$ Average annual generation rate anticipated for this period.

dinformation not available or unknown.

Includes contributions from PNL.

Excludes contributions from ANL-W.

gIncludes contributions (if any) from Bettis (BAPL), Knolls, (KAPL), Kesselring (KESS), Windsor (WIND), and six naval shipyards. 
Table 8.19. Historical and projected annual volume $\left(\mathrm{m}^{3}\right)$ generation rates for TSCA-regulated MLLW from DOE site activities ${ }^{\mathrm{a}}$

\begin{tabular}{|c|c|c|c|c|c|c|c|}
\hline Site & $1994^{b}$ & 1995 & 1996 & 1997 & 1998 & 1999 & $2000-2030^{c}$ \\
\hline Ames & 0.0 & $<0.1$ & $<0.1$ & $d$ & d & d & d \\
\hline ANL-E & 52.0 & 6.0 & 0.0 & 0.0 & 0.0 & 0.0 & 0.0 \\
\hline ANL-W & d & $\mathrm{d}$ & d & d & d & d & d \\
\hline $\mathrm{BNL}$ & 0.4 & 0.3 & 0.2 & 0.1 & 0.1 & 0.1 & 0.1 \\
\hline ETEC & 8.1 & 22.0 & 0.0 & 0.0 & 1.0 & 2.0 & 0.0 \\
\hline FEMP & d & $\mathrm{d}$ & d & d & d & d & d \\
\hline FNAL & 0.0 & 0.0 & 0.0 & 0.0 & 0.0 & 0.0 & 0.0 \\
\hline GJPO & 1.5 & 0.1 & $<0.1$ & $<0.1$ & $<0.1$ & $<0.1$ & $<0.1$ \\
\hline Hanford ${ }^{e}$ & 3.3 & 170.6 & 195.2 & 168.0 & 179.7 & 279.1 & 461.0 \\
\hline $\mathrm{NNEL}^{\mathrm{f}}$ & 0.6 & $\mathrm{~d}$ & d & d & d & d & d \\
\hline ITRI & 0.0 & 0.0 & 0.0 & 0.0 & 0.0 & 0.0 & 0.0 \\
\hline $\mathrm{K}-25$ & $5,586.8$ & $5,586.8$ & $5,586.8$ & $5,586.8$ & $5,586.8$ & $5,586.8$ & $5,586.8$ \\
\hline $\mathrm{KCP}$ & 0.0 & 0.0 & 0.0 & 0.0 & 0.0 & 0.0 & 0.0 \\
\hline LANL & 166.6 & 60.0 & 60.0 & 60.0 & 50.0 & 45.0 & 45.0 \\
\hline LBL & 2.8 & 2.8 & 2.8 & 2.8 & 2.8 & 2.8 & 2.8 \\
\hline LLNL & 0.8 & 0.0 & 0.0 & 0.0 & 0.0 & 0.0 & 0.0 \\
\hline Mound & 6.8 & 0.5 & 0.5 & 0.5 & 0.5 & 0.5 & 0.5 \\
\hline NR sitesg & 344.5 & 127.2 & 154.5 & 112.7 & 112.7 & 109.7 & 17.9 \\
\hline NTS & 0.0 & 0.0 & d & $d$ & d & d & d \\
\hline ORISE & 0.0 & 0.0 & 0.0 & 0.0 & 0.0 & 0.0 & 0.0 \\
\hline ORNL & 13.1 & 13.1 & 13.1 & 13.1 & 13.1 & 13.1 & 13.1 \\
\hline $\mathrm{PAD}$ & $1,060.8$ & 612.6 & 610.3 & 611.8 & 613.5 & 609.3 & 607.8 \\
\hline PANT & 3.0 & 0.0 & 0.0 & 0.0 & 0.0 & 0.0 & 0.0 \\
\hline Pinellas & 0.0 & 3.0 & 0.0 & 0.0 & 0.0 & 0.0 & 0.0 \\
\hline PORTS & 259.9 & 225.0 & 203.0 & 183.0 & 165.0 & 149.0 & 65.0 \\
\hline PPPL & 0.0 & 0.0 & 0.0 & 0.0 & 0.0 & 0.0 & 0.0 \\
\hline RFETS & 36.0 & 76.0 & 36.0 & 36.0 & 36.0 & 36.0 & 62.0 \\
\hline SLAC & 0.0 & 0.0 & 0.0 & 0.0 & 0.0 & 0.0 & 0.0 \\
\hline SNL/CA & 0.2 & 0.0 & 0.0 & 0.0 & 0.0 & 0.0 & 0.0 \\
\hline SNL/NM & $\mathrm{d}$ & d & $\mathrm{d}$ & d & $\mathrm{d}$ & $\mathrm{d}$ & d \\
\hline SRS & 12.1 & 82.0 & 82.0 & 82.0 & 82.0 & 82.0 & 110.0 \\
\hline WVDP & 4.5 & 5.0 & 4.0 & 3.2 & 2.6 & 2.1 & 0.7 \\
\hline$Y-12$ & 20.6 & 20.0 & 20.0 & 27.0 & 27.0 & 27.0 & d \\
\hline Total & $7,584.4$ & $7,013.1$ & $6,968.6$ & $6,887.1$ & $6,872.9$ & $6,944.6$ & $6,972.8$ \\
\hline
\end{tabular}

${ }^{a}$ Based on the DOE site data submittals of ref. 10. Specific site information is provided in Sect. 8.4.

bActual data.

cAverage annual generation rate anticipated for this period.

dinformation not available or unknown.

Includes contributions from PNL.

Excludes contributions from ANL-W.

gIncludes contributions (if any) from Bettis (BAPL), Knolls, (KAPL), Kesselring (KESS), Windsor (WIND), and six naval shipyards. 
Table 8.20. Significant revisions and changes in historical inventory data for TSCA MLLW compared to inventory data reported previously

\begin{tabular}{|c|c|c|c|c|c|}
\hline Site & Waste characteristics & $\begin{array}{l}\text { Values in previous } \\
\text { report }^{\mathrm{a}}\end{array}$ & $\begin{array}{l}\text { Values in current } \\
\text { report }^{b}\end{array}$ & $\begin{array}{l}\text { Significant revisions } \\
\text { and changes }\end{array}$ & Explanation \\
\hline $\mathrm{BNL}$ & $\begin{array}{l}\text { Cumulative quantities of } \\
\text { asbestos }\end{array}$ & $\begin{array}{l}2 \mathrm{~kg} \text { (Table } 8.14 \text { ) } \\
\text { (Trace) } \mathrm{m}^{3} \text { (Table 8.15) }\end{array}$ & $\begin{array}{l}1,000 \mathrm{~kg} \text { (Table } 8.14) \\
\left.2.1 \mathrm{~m}^{3} \text { (Table } 8.15\right)\end{array}$ & $\begin{array}{l}+998 \mathrm{~kg} \\
+2.1 \mathrm{~m}^{3}\end{array}$ & $\begin{array}{l}\text { Waste was not transferred to site waste } \\
\text { management department until May } 1994 \\
\text { and thus not tracked in the waste data base } \\
\text { until that time }\end{array}$ \\
\hline PAD & $\begin{array}{l}\text { Cumulative quantities of } \\
\text { asbestos }\end{array}$ & $\begin{array}{l}45,798 \mathrm{~kg} \text { (Table } 8.14 \text { ) } \\
179.7 \mathrm{~m}^{3} \text { (Table } 8.15 \text { ) }\end{array}$ & $\begin{array}{l}193,981 \mathrm{~kg} \text { (Table 8.14) } \\
406.8 \mathrm{~m}^{3} \text { (Table 8.15) }\end{array}$ & $\begin{array}{l}+148,183 \mathrm{~kg} \\
+227.1 \mathrm{~m}^{3}\end{array}$ & $\begin{array}{l}\text { Large increase from asbestos in a special } \\
\text { area (Munter's Fill). These quantities } \\
\text { were inadvertently left off previously }\end{array}$ \\
\hline \multirow[t]{2}{*}{ PORTS } & $\begin{array}{l}\text { Cumulative quantities of } \\
\text { solids }\end{array}$ & $\begin{array}{l}1,692,401 \mathrm{~kg} \text { (Table } 8.10) \\
\left.3,318.0 \mathrm{~m}^{3} \text { (Table } 8.11\right)\end{array}$ & $\begin{array}{l}2,020,000 \mathrm{~kg} \text { (Table } 8.10) \\
\left.3,574.0 \mathrm{~m}^{3} \text { (Table } 8.11\right)\end{array}$ & $\begin{array}{l}+327,599 \mathrm{~kg} \\
+256.0 \mathrm{~m}^{3}\end{array}$ & Includes contributions from PCB sludges \\
\hline & $\begin{array}{l}\text { Cumulative quantities of } \\
\text { sludges }\end{array}$ & $\begin{array}{l}665,510 \mathrm{~kg} \text { (Table } 8.10) \\
458.0 \mathrm{~m}^{3}(\text { Table } 8.11)\end{array}$ & $\begin{array}{l}\text { Unknown (Table 8.10) } \\
\text { Unknown (Table 8.10) }\end{array}$ & $\begin{array}{l}\text { Unknown } \\
\text { Unknown }\end{array}$ & Site unable to confirm quantities of sludge \\
\hline
\end{tabular}

aDOE/RW-0006, Rev. 10 (December 1994).

bDOE/RW-0006, Rev. 11 (September 1995). 
Table 8.21. National commercially generated MLLW profile volume summary, by facility category ${ }^{\mathrm{a}}$

\begin{tabular}{lccr}
\hline & \multicolumn{3}{c}{ Waste volume, $\mathrm{m}^{3}$} \\
\cline { 2 - 4 } Facility category & $\begin{array}{c}\text { Generated } \\
\text { in } 1990\end{array}$ & $\begin{array}{c}\text { Stored as of } \\
\text { Dec. 31, 1990 }\end{array}$ & $\begin{array}{c}\text { Treated } \\
\text { in 1990 }\end{array}$ \\
\hline Academic & 820.7 & 154.2 & $1,581.9$ \\
Government & 750.4 & 78.9 & 612.5 \\
Industrial & $1,428.0$ & $1,197.3$ & $1,115.1$ \\
Medical & 563.6 & 63.1 & 466.3 \\
Nuclear power plants & 385.8 & 622.5 & 216.9 \\
\multicolumn{1}{c}{ Total $^{\mathrm{d}}$} & $3,948.5$ & $2,116.0$ & $3,992.6$ \\
\hline
\end{tabular}

aBased on ref. 12.

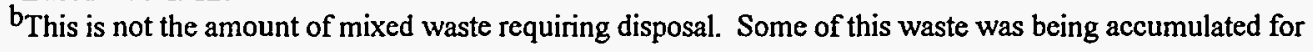
treatment.

${ }^{c}$ Treated wastes may include mixed wastes generated in years prior to 1990.

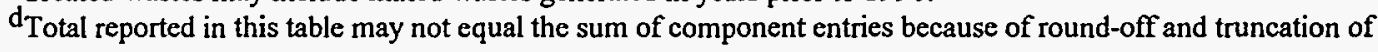
numbers. 
Table 8.22. National commercially generated MLLW profile volume summary, by hazardous waste stream ${ }^{\mathrm{a}}$

\begin{tabular}{|c|c|c|c|}
\hline \multirow[b]{2}{*}{ Hazardous stream } & \multicolumn{3}{|c|}{ Waste volume, $\mathrm{m}^{3}$} \\
\hline & $\begin{array}{l}\text { Generated } \\
\text { in } 1990\end{array}$ & $\begin{array}{l}\text { Stored as of } \\
\text { Dec. } 31,1990^{b}\end{array}$ & $\begin{array}{l}\text { Treated } \\
\text { in } 1990^{\circ}\end{array}$ \\
\hline \multicolumn{4}{|l|}{ Organics } \\
\hline Liquid scintillation fluids & $2,837.2$ & 363.4 & $3,371.8$ \\
\hline Waste oil & 148.9 & 178.1 & 139.4 \\
\hline Chlorinated organics & 70.9 & 27.0 & 23.2 \\
\hline Fluorinated organics & 0 & 3.5 & 0 \\
\hline Chlorinated fluorocarbons (CFCs) & 113.2 & 254.7 & 3.7 \\
\hline Other organics & 274.6 & 117.9 & 258.9 \\
\hline Total organics ${ }^{d}$ & $3,444.8$ & 944.6 & $3,797.0$ \\
\hline \multicolumn{4}{|l|}{ Metals } \\
\hline Lead & 81.6 & 138.7 & 6.1 \\
\hline Mercury & 12.5 & 81.1 & 1.5 \\
\hline Chromium & 28.4 & 53.3 & 3.9 \\
\hline Cadmium & 0.3 & 745.2 & 0.1 \\
\hline Total metals $\mathrm{d}$ & 122.8 & $1,018.3$ & 11.6 \\
\hline Aqueous corrosives & 80.4 & 12.2 & 2.6 \\
\hline Other hazardous materials & 300.5 & 141.0 & 181.4 \\
\hline Grand totals ${ }^{d}$ & $3,948.5$ & $2,116.0$ & $3,992.6$ \\
\hline
\end{tabular}

aBased on ref. 12.

$\mathrm{b}_{\text {This }}$ is not the amount of mixed waste requiring disposal. Some of this waste was being accumulated for treatment.

CTreated wastes may include mixed wastes generated in years prior to 1990 .

$\mathrm{d}$ Totals reported in this table may not equal the sum of component entries because of round-off and truncation of numbers. 
APPENDIX A. WASTE FLOWSHEETS, SOURCE TERMS, AND CHARACTERISTICS 


\section{APPENDIX A. WASTE FLOWSHEETS, SOURCE TERMS, AND CHARACTERISTICS}

\section{A.1 DISCUSSION}

In this report, a number of engineering estimates, assumptions, and ground rules are used to determine radioactive waste and spent nuclear fuel projections through the year 2030. Many of these involve parameters that characterize certain types of waste (e.g., see Table A.1). In other instances, estimates were made of the waste volume generated per unit of product throughput for each step in the fuel cycle. This appendix is a compilation of generic flowsheets and source terms used for making waste projections. Source terms are used to describe quantitative and qualitative characteristics of radioactive wastes. As used in this report, the source term for a particular waste is comprised of two components unique to that waste: (1) the number of curies of radioactivity expressed either per unit of facility production or per unit of waste volume or mass and (2) a listing of the relative radioactivity contributions of component radioisotopes.

The source terms used in the analysis of this report are based on reported historical data, engineering estimates, calculations, and/or experimental data. Documentation of the source terms and key waste-modeling parameters is provided in the following sets of figures and tables (based primarily on refs. 1 through 9). Detailed information on how these source terms and modeling parameters were derived is available, mainly in ref. 1 and its update (ref. 2). Figures A.1 and A.2 were adapted from refs. 3 and 4, respectively. The mass, radioactivity, and thermal power of the nuclides contained in all stored domestic commercial LWR spent nuclear fuel as of December 31, 1994, are listed in Table A.2.

Representative DOE LLW radionuclide compositions are described in Table A.3 (based on ref. 1). Average concentrations for representative radionuclides in LLW disposed of at commercial sites are given in Table A.4. This information was adapted from ref. 5. Table A.5 gives a summary of major sources and estimated characteristics of commercial greater-than-Class-C LLW (data from refs. 6 and 7). Information on the LLW to be incorporated in cement as a result of future operations by the West Valley Demonstration Project Radwaste Treatment System is presented in Table A.6, which is taken from ref. 8.

\section{A.2 REFERENCES}

1. C. W. Forsberg, W. L. Carter, and A. H. Kibbey, Flowsheets and Source Terms for Radioactive Waste Projections, ORNL/TM-8462, Oak Ridge National Laboratory, Oak Ridge, Tennessee (March 1985).

2. S. L. Loghry, A. H. Kibbey, H. W. Godbee, A. S. Icenhour, and S. M. DePaoli, Low-Level Radioactive Waste Source Terms for the 1992 Integrated Data Base, ORNL/TM-11710, Oak Ridge National Laboratory, Oak Ridge, Tennessee (January 1995).

3. R. I. Smith, Revised Analyses of Decommissioning for the Reference Boiling Water Reactor Power Station, draft report, NUREG/CR-6174, PNL-9975, Vols. 1 and 2, U.S. Nuclear Regulatory Commission, Washington, D.C. (September 1994).

4. G. J. Konzek et al., Revised Analyses of Decommissioning for the Reference Pressurized Water Reactor Power Station, draft report, NUREG/CR-5884, PNL-8742, Vols. 1 and 2, U.S. Nuclear Regulatory Commission, Washington, D.C. (October 1993). 
5. G. W. Roles, Characteristics of Low-Level Radioactive Waste Disposed During 1987 Through 1989, NUREG-1418, U.S. Nuclear Regulatory Commission, Washington, D.C. (December 1990).

6. U.S. Department of Energy, Recommendations for Management of Greater-than-Class-C Low-Level Radioactive Waste, DOE/NE-0077, Washington, D.C. (February 1987).

7. O. I. Oztunali, W. D. Pon, R. Eng, and G. W. Roles, Update of Part 61 Impacts Analysis Methodology, Vol. 2, NUREG/CR-4370, U.S. Nuclear Regulatory Commission, Washington, D.C. (January 1986).

8. John J. Hollinden, West Valley Nuclear Services Company, Inc., West Valley, New York, correspondence to Steven N. Storch, IDB Program, ORNL, Oak Ridge, Tennessee, "Submittal of the Department of Energy Site Toxic Substance Control Act Mixed Low-Level Waste Information Request for the 1995 Integrated Data Base Report (DOE/RW-0006, Rev. 11)," WZ:95:0095, dated June 15, 1995. 


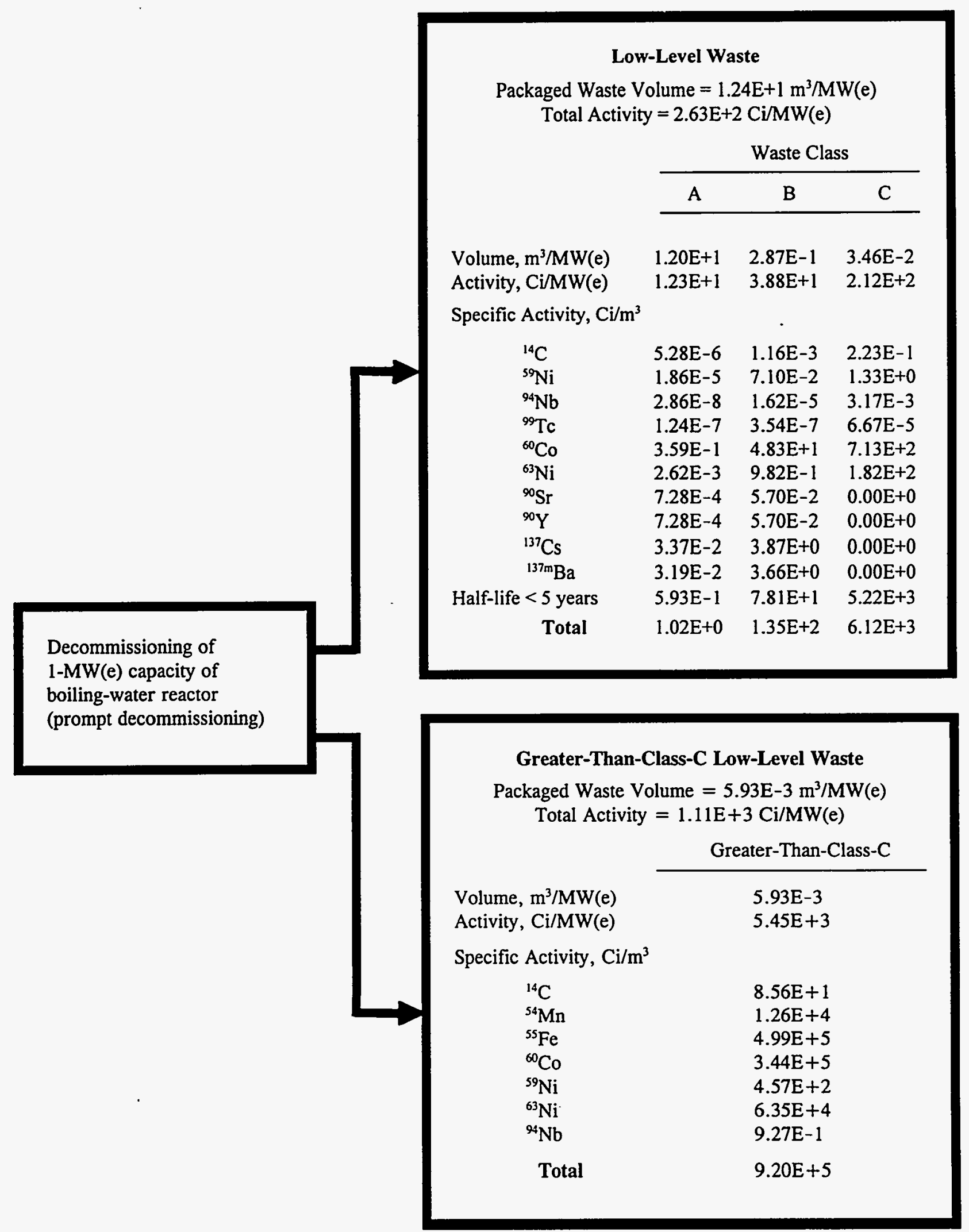

Fig. A.1. Boiling-water reactor decommissioning wastes per 1-MW(e) capacity. 
ORNL DWG 95-7625

\section{Low-Level Waste}

Packaged Waste Volume $=5.94 \mathrm{E}+0 \mathrm{~m}^{3} / \mathrm{MW}(\mathrm{e})$ Total Activity $=1.06 \mathrm{E}+2 \mathrm{Ci} / \mathrm{MW}(\mathrm{e})$

Waste Class

\begin{tabular}{lll}
\hline $\mathrm{A}$ & $\mathrm{B}$ & $\mathrm{C}$ \\
\hline
\end{tabular}

Volume, $\mathrm{m}^{3} / \mathrm{MW}(\mathrm{e})$

$5.78 \mathrm{E}+0$

1.31E-1

$2.55 \mathrm{E}-2$

Activity, $\mathrm{Ci} / \mathrm{MW}(\mathrm{e})$

$3.28 \mathrm{E}+1$

$4.40 \mathrm{E}+1$

$2.91 \mathrm{E}+1$

Specific Activity, $\mathrm{Ci} / \mathrm{m}^{3}$

$\begin{array}{llll}{ }^{14} \mathrm{C} & 0.00 \mathrm{E}+0 & 0.00 \mathrm{E}+0 & 0.00 \mathrm{E}+0 \\ { }^{59} \mathrm{Ni} & 1.25 \mathrm{E}-4 & 1.00 \mathrm{E}-2 & 3.13 \mathrm{E}-1 \\ { }^{94} \mathrm{Nb} & 6.36 \mathrm{E}-8 & 7.25 \mathrm{E}-5 & 2.30 \mathrm{E}-3 \\ { }^{99} \mathrm{Tc} & 0.00 \mathrm{E}+0 & 0.00 \mathrm{E}+0 & 0.00 \mathrm{E}+0 \\ { }^{60} \mathrm{Co} & 9.42 \mathrm{E}-1 & 1.09 \mathrm{E}+2 & 4.19 \mathrm{E}+2 \\ { }^{63} \mathrm{Ni} & 1.49 \mathrm{E}-2 & 1.61 \mathrm{E}+0 & 5.10 \mathrm{E}+1 \\ { }^{90} \mathrm{Sr} & 1.29 \mathrm{E}-4 & 2.40 \mathrm{E}-3 & 0.00 \mathrm{E}+0 \\ { }^{90} \mathrm{Y} & 1.29 \mathrm{E}-4 & 2.40 \mathrm{E}-3 & 0.00 \mathrm{E}+0 \\ { }^{137} \mathrm{Cs} & 1.42 \mathrm{E}-1 & 2.86 \mathrm{E}+0 & 0.00 \mathrm{E}+0 \\ { }^{137 m} \mathrm{Ba} & 1.35 \mathrm{E}-1 & 2.71 \mathrm{E}+0 & 0.00 \mathrm{E}+0 \\ \mathrm{e}<5 \text { years } & 4.43 \mathrm{E}+0 & 2.21 \mathrm{E}+2 & 6.69 \mathrm{E}+2 \\ \text { Total } & 5.67 \mathrm{E}+0 & 3.36 \mathrm{E}+2 & 1.14 \mathrm{E}+3\end{array}$

Decommissioning of 1-MW(e) capacity of pressurized-water reactor (prompt decommissioning)

\section{Greater-Than-Class-C Low-Level Waste}

Packaged Waste Volume $=9.36 \mathrm{E}-3 \mathrm{~m}^{3} / \mathrm{MW}(\mathrm{e})$ Total Activity $=4.07 \mathrm{E}+3 \mathrm{Ci} / \mathrm{MW}(\mathrm{e})$

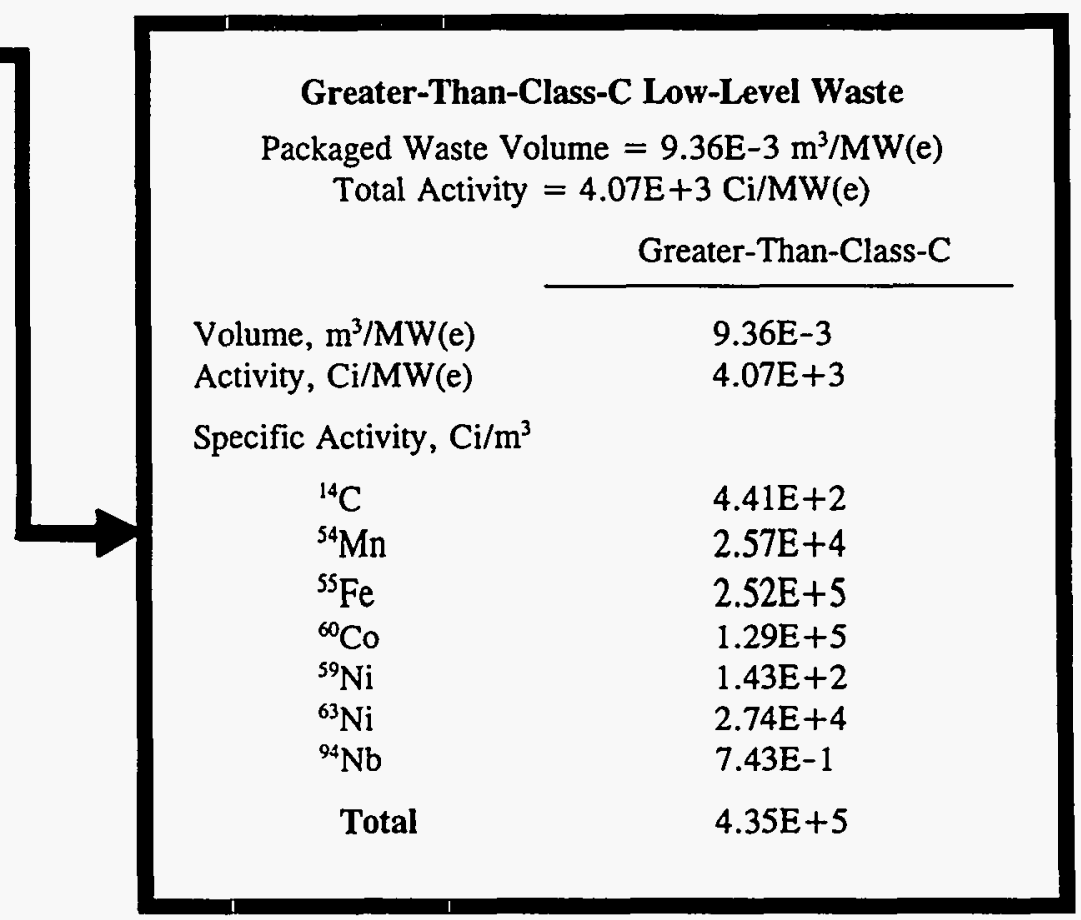

Fig. A.2. Pressurized-water reactor decommissioning wastes per 1-MW(e) capacity. 
Table A.1. Estimated representative unit activity and thermal power characteristics of various types of radioactive materials and wastes

\begin{tabular}{lcc}
\hline $\begin{array}{c}\text { Radioactive material } \\
\text { waste type }\end{array}$ & $\begin{array}{c}\text { Unit activity } \\
\left(\mathrm{Ci} / \mathrm{m}^{3}\right)\end{array}$ & $\begin{array}{c}\text { Unit thermal power } \\
\left(\mathrm{W} / \mathrm{m}^{3}\right)\end{array}$ \\
\hline $\begin{array}{l}\text { Spent nuclear fuel } \\
\text { BWR }\end{array}$ & $1,000,000-10,000,000$ & $3,500-40,000$ \\
PWR & $2,000,000-20,000,000$ & $7,500-65,000$ \\
High-level waste & $1,500-15,000$ & $5-50$ \\
Transuranic waste & & \\
Remote-handled, stored & 1,000 & $1-2$ \\
Contact-handled, stored & $25-50$ & $0.5-1.5$ \\
Buried & $0.25-0.50$ & $0.005-0.010$ \\
Low-level waste & & \\
DOE sites & $9-27$ & $0.012-0.054$ \\
Commercial sites & $\mathrm{c}$ & $0.30-1.60$ \\
Class A & $4.6-6.4$ & $0.03-0.10$ \\
Class B & $0.5-0.7$ & $14-15$ \\
Class C & $55-60$ & $0.003-115^{\mathrm{d}}$ \\
GTCC & $0.1-7,000^{\mathrm{d}}$ & $>0.003-\mathrm{No}$ limit \\
& $>0.1-\mathrm{No}$ limit & \\
Uranium mill tailings & & 0.00020 \\
\hline
\end{tabular}

a Lower-bound levels are based on cumulative spent nuclear fuel discharged; upper-bound levels are based on annual discharges.

based on 1986-1988 Solid Waste Information Management System (SWIMS) and the national Low-Level Waste Management Program (LLWMP) data access system, both of which were maintained by EG\&G, Idaho, Inc., Idaho Falls, Idaho.

CWaste classification is defined by the NRC in 10 CFR Part 61.55 on the basis of concentration of certain long- and short-lived radionuclides. The classification system is designed to minimize potential exposures in both the short and long term. The gross $\mathrm{Ci} / \mathrm{m}^{3}$ shown above are representative of typical LLW shipped to commercial disposal sites. Nuclear power plant wastes account for most of the radioactivity $(-96 \%)$ and include Class $A, B$, and $C$. Essentially all medical wastes are Class $\mathrm{A}$. Industrial wastes are largely Class $\mathrm{A}$, but they contain some $\mathrm{Class} B$ and $\mathrm{C}$.

${ }^{d}$ Maximum for ${ }^{63} \mathrm{Ni}$ in activated metal or ${ }^{90} \mathrm{Sr}$. There is no limit on concentration of ${ }^{3} \mathrm{H},{ }^{60} \mathrm{Co}$, or nuclides with half-lives $<5$ years. The maximum thermal power shown is based on the highest reported gross $\mathrm{Ci} / \mathrm{m}^{3}$ analysis for irradiated core components (1986-1988) and assumes all the activity is due to ${ }^{60} \mathrm{Co}$, which would yield the greatest heat output. If the activity is due to activation products, such as ${ }^{54} \mathrm{Mn},{ }^{58} \mathrm{Co}$, etc., the $\mathrm{Ci} / \mathrm{m}^{3}$ could be much higher for individual shipments, and the total $\mathrm{W} / \mathrm{m}^{3}$ could exceed the value shown.

In temporary storage. The concentration of actinides and ${ }^{129} \mathrm{I}$ determines the lower activity boundary. There is no limit on concentrations of ${ }^{3} \mathrm{H},{ }^{60} \mathrm{Co}$, or nuclides with half-lives $<5$ years. 
Table A.2. Mass, radioactivity, and thermal power of nuclides in domestic commercial LWR spent nuclear fuel at the end of calendar year $1994^{a}$

\begin{tabular}{|c|c|c|c|c|c|c|c|c|}
\hline \multirow{2}{*}{$\begin{array}{l}\text { Atomic } \\
\text { number }\end{array}$} & \multirow{2}{*}{ Element } & \multirow{2}{*}{$\begin{array}{l}\text { Mass number } \\
\text { of nuclide }\end{array}$} & \multicolumn{2}{|c|}{ Mass, $g$} & \multicolumn{2}{|c|}{ Radioactivity, $\mathrm{Ci}$} & \multicolumn{2}{|c|}{ Thermal power, W } \\
\hline & & & Annual & Cumulative & Annual & Cumulative & Annual & Cumulative \\
\hline \multirow[t]{2}{*}{1} & Hydrogen & Stable ${ }^{b}$ & $8.97 E+03$ & $1.41 E+05$ & $0.00 \mathrm{E}+00$ & $0.00 E+00$ & $0.00 E+00$ & $0.00 E+00$ \\
\hline & Hydrogen & 3 & $1.69 E+02$ & $1.55 \mathrm{E}+03$ & $1.63 E+06$ & $1.50 \mathrm{E}+07$ & $5.48 \mathrm{E}+01$ & $5.04 \mathrm{E}+02$ \\
\hline 2 & Helium & Stable & $5.62 \mathrm{E}+03$ & $7.15 E+04$ & $0.00 \mathrm{E}+00$ & $0.00 E+00$ & $0.00 \mathrm{E}+00$ & $0.00 \mathrm{E}+00$ \\
\hline 3 & Lithium & Stable & $2.05 E+03$ & $3.26 \mathrm{E}+04$ & $0.00 \mathrm{E}+00$ & $0.00 \mathrm{E}+00$ & $0.00 E+00$ & $0.00 \mathrm{E}+00$ \\
\hline 4 & Beryllium & Stable & $2.18 \mathrm{E}+00$ & $2.77 \mathrm{E}+01$ & $0.00 \mathrm{E}+00$ & $0.00 \mathrm{E}+00$ & $0.00 \mathrm{E}+00$ & $0.00 \mathrm{E}+00$ \\
\hline 5 & Boron & Stable & $1.90 \mathrm{E}+03$ & $2.98 \mathrm{E}+04$ & $0.00 \mathrm{E}+00$ & $0.00 \mathrm{E}+00$ & $0.00 \mathrm{E}+00$ & $0.00 \mathrm{E}+00$ \\
\hline \multirow[t]{2}{*}{6} & Carbon & Stable & $3.39 E+05$ & $5.34 E+06$ & $0.00 \mathrm{E}+00$ & $0.00 \mathrm{E}+00$ & $0.00 \mathrm{E}+00$ & $0.00 \mathrm{E}+00$ \\
\hline & Carbon & 14 & $9.54 \mathrm{E}+02$ & $1.18 E+04$ & $4.25 E+03$ & $5.27 E+04$ & $1.25 \mathrm{E}+00$ & $1.55 \mathrm{E}+01$ \\
\hline 7 & Nitrogen & Stable & $2.32 E+05$ & $3.68 E+06$ & $0.00 E+00$ & $0.00 E+00$ & $0.00 \mathrm{E}+00$ & $0.00 \mathrm{E}+00$ \\
\hline 8 & Oxygen & Stable & $2.54 \mathrm{E}+08$ & $4.02 E+09$ & $0.00 E+00$ & $0.00 E+00$ & $0.00 \mathrm{E}+00$ & $0.00 \mathrm{E}+00$ \\
\hline 9 & Fluorine & Stable & $2.01 E+04$ & $3.19 E+05$ & $0.00 \mathrm{E}+00$ & $0.00 \mathrm{E}+00$ & $0.00 \mathrm{E}+00$ & $0.00 \mathrm{E}+00$ \\
\hline 10 & Neon & Stable & $8.80 \mathrm{E}-01$ & $1.16 \mathrm{E}+01$ & $0.00 E+00$ & $0.00 \mathrm{E}+00$ & $0.00 \mathrm{E}+00$ & $0.00 \mathrm{E}+00$ \\
\hline \multirow[t]{2}{*}{11} & Sodium & Stable & $2.82 E+04$ & $4.46 E+05$ & $0.00 \mathrm{E}+00$ & $0.00 \mathrm{E}+00$ & $0.00 \mathrm{E}+00$ & $0.00 \mathrm{E}+00$ \\
\hline & Sodium & 24 & $1.66 \mathrm{E}-04$ & $1.66 \mathrm{E}-04$ & $1.45 \mathrm{E}+03$ & $1.45 E+03$ & $4.01 E+01$ & $4.01 E+01$ \\
\hline 12 & Magnesium & Stable & $3.84 E+03$ & $6.06 \mathrm{E}+04$ & $0.00 E+00$ & $0.00 E+00$ & $0.00 \mathrm{E}+00$ & $0.00 \mathrm{E}+00$ \\
\hline 13 & Aluminum & Stable & $1.54 \mathrm{E}+05$ & $2.43 \mathrm{E}+06$ & $0.00 \mathrm{E}+00$ & $0.00 \mathrm{E}+00$ & $0.00 \mathrm{E}+00$ & $0.00 \mathrm{E}+00$ \\
\hline 14 & Silicon & Stable & $9.00 \mathrm{E}+05$ & $1.43 E+07$ & $0.00 \mathrm{E}+00$ & $0.00 \mathrm{E}+00$ & $0.00 E+00$ & $0.00 \mathrm{E}+00$ \\
\hline \multirow[t]{2}{*}{15} & Phosphorus & Stable & $4.27 \mathrm{E}+05$ & $6.73 E+06$ & $0.00 \mathrm{E}+00$ & $0.00 \mathrm{E}+00$ & $0.00 \mathrm{E}+00$ & $0.00 \mathrm{E}+00$ \\
\hline & Phosphorus & 32 & $3.02 \mathrm{E}-01$ & $3.02 \mathrm{E}-01$ & $8.63 E+04$ & $8.63 E+04$ & $8.74 \mathrm{E}+02$ & $8.74 \mathrm{E}+02$ \\
\hline 16 & Sulfur & Stable & $5.02 \mathrm{E}+04$ & $7.96 \mathrm{E}+05$ & $0.00 \mathrm{E}+00$ & $0.00 E+00$ & $0.00 \mathrm{E}+00$ & $0.00 \mathrm{E}+00$ \\
\hline 17 & Chlorine & Stable & $9.21 E+03$ & $1.48 \mathrm{E}+05$ & $0.00 \mathrm{E}+00$ & $0.00 \mathrm{E}+00$ & $0.00 \mathrm{E}+00$ & $0.00 \mathrm{E}+00$ \\
\hline 18 & Argon & Stable & $8.38 \mathrm{E}+02$ & $1.11 \mathrm{E}+04$ & $0.00 \mathrm{E}+00$ & $0.00 E+00$ & $0.00 \mathrm{E}+00$ & $0.00 E+00$ \\
\hline 19 & Potassium & Stable & $3.64 \mathrm{E}+00$ & $4.79 \mathrm{E}+01$ & $0.00 \mathrm{E}+00$ & $0.00 E+00$ & $0.00 \mathrm{E}+00$ & $0.00 \mathrm{E}+00$ \\
\hline 20 & Calcium & Stable & $3.76 \mathrm{E}+03$ & $5.95 E+04$ & $0.00 \mathrm{E}+00$ & $0.00 E+00$ & $0.00 \mathrm{E}+00$ & $0.00 \mathrm{E}+00$ \\
\hline 21 & Scandium & Stable & $2.37 \mathrm{E}-01$ & $3.13 E+00$ & $0.00 \mathrm{E}+00$ & $0.00 E+00$ & $0.00 \mathrm{E}+00$ & $0.00 \mathrm{E}+00$ \\
\hline 22 & Titanium & Stable & $1.57 \mathrm{E}+05$ & $2.49 \mathrm{E}+06$ & $0.00 \mathrm{E}+00$ & $0.00 E+00$ & $0.00 \mathrm{E}+00$ & $0.00 \mathrm{E}+00$ \\
\hline \multirow[t]{2}{*}{23} & Vanadium & Stable & $3.62 \mathrm{E}+04$ & $5.10 \mathrm{E}+05$ & $0.00 \mathrm{E}+00$ & $0.00 E+00$ & $0.00 \mathrm{E}+00$ & $0.00 \mathrm{E}+00$ \\
\hline & Vanadium & 50 & $1.15 \mathrm{E}+02$ & $1.59 \mathrm{E}+03$ & $2.05 E-11$ & $2.85 E-10$ & $2.26 \mathrm{E}-13$ & $3.14 \mathrm{E}-12$ \\
\hline \multirow[t]{2}{*}{24} & Chromium & Stable & $2.06 \mathrm{E}+07$ & $3.26 E+08$ & $0.00 \mathrm{E}+00$ & $0.00 E+00$ & $0.00 \mathrm{E}+00$ & $0.00 \mathrm{E}+00$ \\
\hline & Chromium & 51 & $7.65 \mathrm{E}+01$ & $7.65 \mathrm{E}+01$ & $7.07 \mathrm{E}+06$ & $7.07 \mathrm{E}+06$ & $1.51 \mathrm{E}+03$ & $1.51 E+03$ \\
\hline \multirow[t]{2}{*}{25} & Manganese & Stable & $1.71 E+06$ & $2.72 \mathrm{E}+07$ & $0.00 \mathrm{E}+00$ & $0.00 E+00$ & $0.00 \mathrm{E}+00$ & $0.00 \mathrm{E}+00$ \\
\hline & Manganese & 54 & $1.71 \mathrm{E}+02$ & $3.30 E+02$ & $1.33 E+06$ & $2.55 E+06$ & $6.61 E+03$ & $1.27 \mathrm{E}+04$ \\
\hline \multirow[t]{3}{*}{26} & Iron & Stable & $6.26 \mathrm{E}+07$ & $9.92 \mathrm{E}+08$ & $0.00 \mathrm{E}+00$ & $0.00 \mathrm{E}+00$ & $0.00 \mathrm{E}+00$ & $0.00 \mathrm{E}+00$ \\
\hline & Iron & 55 & $4.92 \mathrm{E}+03$ & $1.99 \mathrm{E}+04$ & $1.23 \mathrm{E}+07$ & $4.98 E+07$ & $4.16 \mathrm{E}+02$ & $1.68 \mathrm{E}+03$ \\
\hline & Iron & 59 & $2.93 E+00$ & $2.94 \mathrm{E}+00$ & $1.44 \mathrm{E}+05$ & $1.45 \mathrm{E}+05$ & $1.11 E+03$ & $1.12 \mathrm{E}+03$ \\
\hline
\end{tabular}


Table A.2 (continued)

\begin{tabular}{|c|c|c|c|c|c|c|c|c|}
\hline \multirow{2}{*}{$\begin{array}{l}\text { Atomic } \\
\text { number }\end{array}$} & \multirow{2}{*}{ Element } & \multirow{2}{*}{$\begin{array}{l}\text { Mass number } \\
\text { of nuclide }\end{array}$} & \multicolumn{2}{|c|}{ Mass, $\mathrm{g}$} & \multicolumn{2}{|c|}{ Radioactivity, Ci } & \multicolumn{2}{|c|}{ Thermal power, $\mathrm{W}$} \\
\hline & & & Annual & Cumulative & Annual & Cumulative & Annual & Cumulative \\
\hline \multirow[t]{3}{*}{27} & Cobalt & Stable & $1.46 \mathrm{E}+05$ & $2.35 E+06$ & $0.00 \mathrm{E}+00$ & $0.00 \mathrm{E}+00$ & $0.00 \mathrm{E}+00$ & $0.00 \mathrm{E}+00$ \\
\hline & Cobalt & 58 & $8.52 E+01$ & $8.82 \mathrm{E}+01$ & $2.71 \mathrm{E}+06$ & $2.81 E+06$ & $1.62 E+04$ & $1.68 \mathrm{E}+04$ \\
\hline & Cobait & 60 & $1.39 \mathrm{E}+04$ & $8.73 E+04$ & $1.57 \mathrm{E}+07$ & $9.87 \mathrm{E}+07$ & $2.42 \mathrm{E}+05$ & $1.52 E+06$ \\
\hline \multirow[t]{3}{*}{28} & Nickel & Stable & $1.91 \mathrm{E}+07$ & $3.03 E+08$ & $0.00 \mathrm{E}+00$ & $0.00 \mathrm{E}+00$ & $0.00 \mathrm{E}+00$ & $0.00 \mathrm{E}+00$ \\
\hline & Nickel & 59 & $1.28 \mathrm{E}+05$ & $1.64 \mathrm{E}+06$ & $9.74 \mathrm{E}+03$ & $1.24 \mathrm{E}+05$ & $3.87 \mathrm{E}-01$ & $4.93 E+00$ \\
\hline & Nickel & 63 & $2.28 \mathrm{E}+04$ & $2.68 \mathrm{E}+05$ & $1.40 \mathrm{E}+06$ & $1.66 \mathrm{E}+07$ & $1.42 E+02$ & $1.67 \mathrm{E}+03$ \\
\hline 29 & Copper & Stable & $3.34 \mathrm{E}+04$ & $5.27 \mathrm{E}+05$ & $0.00 \mathrm{E}+00$ & $0.00 \mathrm{E}+00$ & $0.00 \mathrm{E}+00$ & $0.00 \mathrm{E}+00$ \\
\hline \multirow[t]{2}{*}{30} & Zinc & Stable & $7.58 \mathrm{E}+04$ & $1.20 E+06$ & $0.00 \mathrm{E}+00$ & $0.00 \mathrm{E}+00$ & $0.00 \mathrm{E}+00$ & $0.00 \mathrm{E}+00$ \\
\hline & Zinc & 65 & $1.98 \mathrm{E}+01$ & $3.27 \mathrm{E}+01$ & $1.63 \mathrm{E}+05$ & $2.69 \mathrm{E}+05$ & $5.72 E+02$ & $9.42 E+02$ \\
\hline 31 & Gallium & Stable & $6.96 E+01$ & $9.19 \mathrm{E}+02$ & $0.00 \mathrm{E}+00$ & $0.00 \mathrm{E}+00$ & $0.00 \mathrm{E}+00$ & $0.00 \mathrm{E}+00$ \\
\hline 32 & Germanium & Stable & $1.33 E+03$ & $1.65 E+04$ & $0.00 \mathrm{E}+00$ & $0.00 \mathrm{E}+00$ & $0.00 \mathrm{E}+00$ & $0.00 E+00$ \\
\hline 33 & Arsenic & Stable & $4.13 \mathrm{E}+02$ & $5.20 \mathrm{E}+03$ & $0.00 \mathrm{E}+00$ & $0.00 \mathrm{E}+00$ & $0.00 E+00$ & $0.00 \mathrm{E}+00$ \\
\hline \multirow[t]{2}{*}{34} & Selenium & Stable & $1.04 \mathrm{E}+05$ & $1.28 \mathrm{E}+06$ & $0.00 \mathrm{E}+00$ & $0.00 \mathrm{E}+00$ & $0.00 \mathrm{E}+00$ & $0.00 \mathrm{E}+00$ \\
\hline & Selenium & 79 & $1.24 \mathrm{E}+04$ & $1.54 \mathrm{E}+05$ & $8.66 \mathrm{E}+02$ & $1.07 E+04$ & $2.16 \mathrm{E}-01$ & $2.66 \mathrm{E}+00$ \\
\hline 35 & Bromine & Stable & $4.52 \mathrm{E}+04$ & $5.59 \mathrm{E}+05$ & $0.00 \mathrm{E}+00$ & $0.00 \mathrm{E}+00$ & $0.00 \mathrm{E}+00$ & $0.00 \mathrm{E}+00$ \\
\hline \multirow[t]{3}{*}{36} & Krypton & Stable & $7.16 \mathrm{E}+05$ & $8.75 E+06$ & $0.00 \mathrm{E}+00$ & $0.00 \mathrm{E}+00$ & $0.00 \mathrm{E}+00$ & $0.00 E+00$ \\
\hline & Krypton & 81 & $5.09 \mathrm{E}-02$ & $5.97 \mathrm{E}-01$ & $1.07 \mathrm{E}-03$ & $1.26 \mathrm{E}-02$ & $1.32 \mathrm{E}-07$ & $1.55 E-06$ \\
\hline & Krypton & 85 & $4.85 E+04$ & $4.05 E+05$ & $1.90 \mathrm{E}+07$ & $1.59 \mathrm{E}+08$ & $2.85 \mathrm{E}+04$ & $2.38 \mathrm{E}+05$ \\
\hline \multirow[t]{3}{*}{37} & Rubidium & Stable & $2.09 \mathrm{E}+05$ & $2.55 E+06$ & $0.00 \mathrm{E}+00$ & $0.00 \mathrm{E}+00$ & $0.00 \mathrm{E}+00$ & $0.00 E+00$ \\
\hline & Rubidium & 86 & $3.50 \mathrm{E}+00$ & $3.50 \mathrm{E}+00$ & $2.85 \mathrm{E}+05$ & $2.85 \mathrm{E}+05$ & $1.29 \mathrm{E}+03$ & $1.29 E+03$ \\
\hline & Rubidium & 87 & $5.19 E+05$ & $6.34 E+06$ & $4.54 \mathrm{E}-02$ & $5.55 \mathrm{E}-01$ & $3.79 \mathrm{E}-05$ & $4.64 \mathrm{E}-04$ \\
\hline \multirow[t]{3}{*}{38} & Strontium & Stable & $7.43 E+05$ & $9.07 \mathrm{E}+06$ & $0.00 \mathrm{E}+00$ & $0.00 \mathrm{E}+00$ & $0.00 \mathrm{E}+00$ & $0.00 \mathrm{E}+00$ \\
\hline & Strontium & 89 & $9.47 E+03$ & $9.54 \mathrm{E}+03$ & $2.75 E+08$ & $2.77 \mathrm{E}+08$ & $9.51 E+05$ & $9.59 E+05$ \\
\hline & Strontium & 90 & $1.12 E+06$ & $1.17 \mathrm{E}+07$ & $1.52 \mathrm{E}+08$ & $1.60 \mathrm{E}+09$ & $1.77 \mathrm{E}+05$ & $1.85 E+06$ \\
\hline \multirow[t]{3}{*}{39} & Yttrium & Stable & $9.22 \mathrm{E}+05$ & $1.11 \mathrm{E}+07$ & $0.00 \mathrm{E}+00$ & $0.00 \mathrm{E}+00$ & $0.00 E+00$ & $0.00 \mathrm{E}+00$ \\
\hline & Yttrium & 90 & $2.83 \mathrm{E}+02$ & $2.94 E+03$ & $1.54 \mathrm{E}+08$ & $1.60 \mathrm{E}+09$ & $8.55 E+05$ & $8.87 \mathrm{E}+06$ \\
\hline & Yttrium & 91 & $1.72 \mathrm{E}+04$ & $1.74 \mathrm{E}+04$ & $4.21 E+08$ & $4.28 \mathrm{E}+08$ & $1.51 E+06$ & $1.54 E+06$ \\
\hline \multirow[t]{3}{*}{40} & Zirconium & Stable & $6.55 \mathrm{E}+08$ & $1.04 E+10$ & $0.00 \mathrm{E}+00$ & $0.00 \mathrm{E}+00$ & $0.00 \mathrm{E}+00$ & $0.00 \mathrm{E}+00$ \\
\hline & Zirconium & 93 & $1.77 \mathrm{E}+06$ & $2.17 E+07$ & $4.45 \mathrm{E}+03$ & $5.45 E+04$ & $5.17 \mathrm{E}-01$ & $6.33 E+00$ \\
\hline & Zirconium & 95 & $3.25 E+04$ & $3.33 \mathrm{E}+04$ & $6.99 \mathrm{E}+08$ & $7.16 \mathrm{E}+08$ & $3.54 \mathrm{E}+06$ & $3.62 \mathrm{E}+06$ \\
\hline \multirow[t]{5}{*}{41} & Niobium & Stable & $9.67 \mathrm{E}+05$ & $1.53 \mathrm{E}+07$ & $0.00 \mathrm{E}+00$ & $0.00 \mathrm{E}+00$ & $0.00 E+00$ & $0.00 \mathrm{E}+00$ \\
\hline & Niobium & $93 m$ & $1.56 \mathrm{E}+00$ & $6.31 E+01$ & $4.42 \mathrm{E}+02$ & $1.78 \mathrm{E}+04$ & $7.84 \mathrm{E}-02$ & $3.16 \mathrm{E}+00$ \\
\hline & Niobium & 94 & $1.36 \mathrm{E}+04$ & $1.69 \mathrm{E}+05$ & $2.55 E+03$ & $3.17 E+04$ & $2.59 \mathrm{E}+01$ & $3.23 E+02$ \\
\hline & Niobium & 95 & $2.76 \mathrm{E}+04$ & $2.86 \mathrm{E}+04$ & $1.08 \mathrm{E}+09$ & $1.12 \mathrm{E}+09$ & $5.19 \mathrm{E}+06$ & $5.36 \mathrm{E}+06$ \\
\hline & Niobium & $95 m$ & $1.41 E+01$ & $1.44 E+01$ & $5.38 E+06$ & $5.50 E+06$ & $7.48 \mathrm{E}+03$ & $7.64 E+03$ \\
\hline 42 & Molybdenum & Stable & $7.57 \mathrm{E}+06$ & $9.43 E+07$ & $0.00 \mathrm{E}+00$ & $0.00 \mathrm{E}+00$ & $0.00 \mathrm{E}+00$ & $0.00 \mathrm{E}+00$ \\
\hline 43 & Technetium & 99 & $1.63 E+06$ & $2.04 E+07$ & $2.76 E+04$ & $3.45 \mathrm{E}+05$ & $1.38 \mathrm{E}+01$ & $1.73 E+02$ \\
\hline
\end{tabular}


Table A.2 (continued)

\begin{tabular}{|c|c|c|c|c|c|c|c|c|}
\hline \multirow{2}{*}{$\begin{array}{l}\text { Atomic } \\
\text { number }\end{array}$} & \multirow{2}{*}{ Element } & \multirow{2}{*}{$\begin{array}{l}\text { Mass number } \\
\text { of nuclide }\end{array}$} & \multicolumn{2}{|c|}{ Mass, $\mathrm{g}$} & \multicolumn{2}{|c|}{ Radioactivity, Ci } & \multicolumn{2}{|c|}{ Thermal power, W } \\
\hline & & & Annual & Cumulative & Annual & Cumulative & Annual & Cumulative \\
\hline \multirow[t]{3}{*}{44} & Ruthenium & Stable & $4.63 E+06$ & $5.69 E+07$ & $0.00 \mathrm{E}+00$ & $0.00 \mathrm{E}+00$ & $0.00 \mathrm{E}+00$ & $0.00 \mathrm{E}+00$ \\
\hline & Ruthenium & 103 & $1.31 \mathrm{E}+04$ & $1.31 \mathrm{E}+04$ & $4.23 E+08$ & $4.24 \mathrm{E}+08$ & $1.42 E+06$ & $1.42 E+06$ \\
\hline & Ruthenium & 106 & $2.37 \mathrm{E}+05$ & $5.06 \mathrm{E}+05$ & $7.94 E+08$ & $1.69 \mathrm{E}+09$ & $4.72 \mathrm{E}+04$ & $1.01 \mathrm{E}+05$ \\
\hline \multirow[t]{3}{*}{45} & Rhodium & Stable & $8.57 \mathrm{E}+05$ & $1.12 \mathrm{E}+07$ & $0.00 \mathrm{E}+00$ & $0.00 \mathrm{E}+00$ & $0.00 \mathrm{E}+00$ & $0.00 E+00$ \\
\hline & Rhodium & $103 m$ & $1.17 \mathrm{E}+01$ & $1.18 \mathrm{E}+01$ & $3.82 \mathrm{E}+08$ & $3.83 \mathrm{E}+08$ & $8.79 E+04$ & $8.81 E+04$ \\
\hline & Rhodium & 106 & $2.23 E-01$ & $4.76 \mathrm{E}-01$ & $7.94 \mathrm{E}+08$ & $1.69 \mathrm{E}+09$ & $7.62 \mathrm{E}+06$ & $1.62 \mathrm{E}+07$ \\
\hline \multirow[t]{2}{*}{46} & Palladium & Stable & $2.22 E+06$ & $2.64 \mathrm{E}+07$ & $0.00 \mathrm{E}+00$ & $0.00 \mathrm{E}+00$ & $0.00 \mathrm{E}+00$ & $0.00 \mathrm{E}+00$ \\
\hline & Palladium & 107 & $4.71 E+05$ & $5.88 \mathrm{E}+06$ & $2.42 \mathrm{E}+02$ & $3.03 E+03$ & $1.44 \mathrm{E}-02$ & $1.79 E-01$ \\
\hline \multirow[t]{6}{*}{47} & Silver & Stable & $1.59 \mathrm{E}+05$ & $2.04 E+06$ & $0.00 \mathrm{E}+00$ & $0.00 \mathrm{E}+00$ & $0.00 E+00$ & $0.00 E+00$ \\
\hline & Silver & 108 & $3.11 \mathrm{E}-09$ & $2.51 \mathrm{E}-08$ & $2.29 \mathrm{E}+00$ & $1.84 \mathrm{E}+01$ & $8.52 E-03$ & $6.87 \mathrm{E}-02$ \\
\hline & Silver & $108 m$ & $9.86 E-01$ & $7.95 E+00$ & $2.57 \mathrm{E}+01$ & $2.07 \mathrm{E}+02$ & $2.49 \mathrm{E}-01$ & $2.01 E+00$ \\
\hline & Silver & 110 & $1.87 \mathrm{E}-05$ & $3.05 \mathrm{E}-05$ & $7.82 E+04$ & $1.27 \mathrm{E}+05$ & $5.61 \mathrm{E}+02$ & $9.15 E+02$ \\
\hline & Silver & $110 m$ & $1.24 E+03$ & $2.02 \mathrm{E}+03$ & $5.88 E+06$ & $9.58 \mathrm{E}+06$ & $9.81 E+04$ & $1.60 E+05$ \\
\hline & Silver & 111 & $2.51 E+01$ & $2.51 \mathrm{E}+\mathrm{O} 1$ & $3.97 \mathrm{E}+06$ & $3.97 \mathrm{E}+06$ & $8.89 E+03$ & $8.89 E+03$ \\
\hline \multirow[t]{4}{*}{48} & Cadmium & Stable & $2.81 E+05$ & $3.58 \mathrm{E}+06$ & $0.00 \mathrm{E}+00$ & $0.00 \mathrm{E}+00$ & $0.00 \mathrm{E}+00$ & $0.00 \mathrm{E}+00$ \\
\hline & Cadmium & 109 & $6.98 E-01$ & $1.78 \mathrm{E}+00$ & $1.80 \mathrm{E}+03$ & $4.60 \mathrm{E}+03$ & $2.09 E-01$ & $5.35 E-01$ \\
\hline & Cadmium & $113 m$ & $5.38 \mathrm{E}+02$ & $4.93 E+03$ & $1.17 E+05$ & $1.07 \mathrm{E}+06$ & $1.96 \mathrm{E}+02$ & $1.80 \mathrm{E}+03$ \\
\hline & Cadmium & $115 m$ & $2.22 \mathrm{E}+01$ & $2.23 E+01$ & $5.65 \mathrm{E}+05$ & $5.68 \mathrm{E}+05$ & $2.11 \mathrm{E}+03$ & $2.12 E+03$ \\
\hline \multirow[t]{5}{*}{49} & Indium & Stable & $2.56 \mathrm{E}+03$ & $3.25 \mathrm{E}+04$ & $0.00 \mathrm{E}+00$ & $0.00 \mathrm{E}+00$ & $0.00 \mathrm{E}+00$ & $0.00 \mathrm{E}+00$ \\
\hline & Indium & 114 & $8.09 \mathrm{E}-05$ & $8.14 \mathrm{E}-05$ & $1.11 E+05$ & $1.12 \mathrm{E}+05$ & $5.30 \mathrm{E}+02$ & $5.34 \mathrm{E}+02$ \\
\hline & Indium & $114 m$ & $5.03 E+00$ & $5.06 \mathrm{E}+00$ & $1.16 E+05$ & $1.17 \mathrm{E}+05$ & $1.64 \mathrm{E}+02$ & $1.65 E+02$ \\
\hline & Indium & 115 & $4.38 \mathrm{E}+03$ & $6.74 E+04$ & $2.72 \mathrm{E}-08$ & $4.19 E-07$ & $3.91 \mathrm{E}-11$ & $6.02 \mathrm{E}-10$ \\
\hline & Indium & $115 m$ & $4.36 \mathrm{E}-02$ & $4.36 \mathrm{E}-02$ & $2.76 \mathrm{E}+05$ & $2.76 \mathrm{E}+05$ & $5.51 E+02$ & $5.51 E+02$ \\
\hline \multirow[t]{7}{*}{50} & Tin & Stable & $1.07 \mathrm{E}+07$ & $1.70 \mathrm{E}+08$ & $0.00 \mathrm{E}+00$ & $0.00 \mathrm{E}+00$ & $0.00 E+00$ & $0.00 \mathrm{E}+00$ \\
\hline & Tin & $117 m$ & $1.51 \mathrm{E}+01$ & $1.51 E+01$ & $1.20 \mathrm{E}+06$ & $1.20 \mathrm{E}+06$ & $2.23 E+03$ & $2.23 E+03$ \\
\hline & Tin & $119 m$ & $3.08 \mathrm{E}+03$ & $4.97 E+03$ & $1.38 \mathrm{E}+07$ & $2.22 E+07$ & $7.14 \mathrm{E}+03$ & $1.15 \mathrm{E}+04$ \\
\hline & Tin & $121 m$ & $5.18 \mathrm{E}+01$ & $5.68 \mathrm{E}+02$ & $3.06 \mathrm{E}+03$ & $3.36 E+04$ & $6.14 E+00$ & $6.73 E+01$ \\
\hline & Tin & 123 & $4.00 \mathrm{E}+02$ & $4.78 \mathrm{E}+\mathrm{O} 2$ & $3.29 E+06$ & $3.93 E+06$ & $1.03 E+04$ & $1.23 E+04$ \\
\hline & Tin & 125 & $1.06 \mathrm{E}+01$ & $1.06 \mathrm{E}+01$ & $1.15 \mathrm{E}+06$ & $1.15 E+06$ & $7.62 E+03$ & $7.62 E+03$ \\
\hline & $\operatorname{Tin}$ & 126 & $5.70 E+04$ & $7.20 \mathrm{E}+05$ & $1.62 \mathrm{E}+03$ & $2.04 E+04$ & $2.02 E+00$ & $2.55 \mathrm{E}+01$ \\
\hline \multirow[t]{5}{*}{51} & Antimony & Stable & $4.38 \mathrm{E}+04$ & $5.58 \mathrm{E}+05$ & $0.00 \mathrm{E}+00$ & $0.00 \mathrm{E}+00$ & $0.00 \mathrm{E}+00$ & $0.00 \mathrm{E}+00$ \\
\hline & Antimony & 124 & $3.73 E+01$ & $3.80 \mathrm{E}+01$ & $6.53 \mathrm{E}+05$ & $6.64 E+05$ & $8.67 \mathrm{E}+03$ & $8.82 E+03$ \\
\hline & Antimony & 125 & $3.03 E+04$ & $1.29 \mathrm{E}+05$ & $3.13 E+07$ & $1.34 E+08$ & $9.77 \mathrm{E}+04$ & $4.18 E+05$ \\
\hline & Antimony & 126 & $1.34 E+00$ & $1.34 \mathrm{E}+00$ & $1.12 \mathrm{E}+05$ & $1.12 E+05$ & $2.07 \mathrm{E}+03$ & $2.07 \mathrm{E}+03$ \\
\hline & Antimony & 127 & $1.09 \mathrm{E}+01$ & $1.09 \mathrm{E}+01$ & $2.92 E+06$ & $2.92 \mathrm{E}+06$ & $1.73 E+04$ & $1.73 E+04$ \\
\hline \multirow[t]{2}{*}{52} & Tellurium & Stable & $9.90 \mathrm{E}+05$ & $1.23 E+07$ & $0.00 E+00$ & $0.00 \mathrm{E}+00$ & $0.00 \mathrm{E}+00$ & $0.00 E+00$ \\
\hline & Tellerium & 123 & $2.83 E+01$ & $3.06 \mathrm{E}+02$ & $8.23 E-09$ & $8.90 E-08$ & $8.34 E-13$ & $9.02 E-12$ \\
\hline
\end{tabular}


Table A.2 (continued)

\begin{tabular}{|c|c|c|c|c|c|c|c|c|}
\hline \multirow{2}{*}{$\begin{array}{l}\text { Atomic } \\
\text { number }\end{array}$} & \multirow{2}{*}{ Element } & \multirow{2}{*}{$\begin{array}{c}\text { Mass number } \\
\text { of nuclide }\end{array}$} & \multicolumn{2}{|c|}{ Mass, $\mathbf{g}$} & \multicolumn{2}{|c|}{ Radioactivity, Ci } & \multicolumn{2}{|c|}{ Thermal power, $\mathrm{W}$} \\
\hline & & & Annual & Cumulative & Annual & Cumulative & Annual & Cumulative \\
\hline & Tellurium & $123 m$ & $2.72 E+00$ & $3.12 \mathrm{E}+00$ & $2.41 \mathrm{E}+04$ & $2.76 \mathrm{E}+04$ & $3.51 E+01$ & $4.03 E+01$ \\
\hline & Tellurium & $125 m$ & $4.10 \mathrm{E}+02$ & $1.80 \mathrm{E}+03$ & $7.39 E+06$ & $3.24 \mathrm{E}+07$ & $6.21 E+03$ & $2.72 E+04$ \\
\hline & Tellurium & 127 & $4.90 \mathrm{E}+00$ & $5.40 \mathrm{E}+00$ & $1.29 \mathrm{E}+07$ & $1.42 E+07$ & $1.75 E+04$ & $1.92 E+04$ \\
\hline & Tellurium & $127 m$ & $1.10 \mathrm{E}+03$ & $1.24 \mathrm{E}+03$ & $1.03 E+07$ & $1.17 \mathrm{E}+07$ & $5.56 \mathrm{E}+03$ & $6.29 E+03$ \\
\hline & Tellurium & 129 & $3.52 \mathrm{E}-01$ & $3.52 \mathrm{E}-01$ & $7.38 \mathrm{E}+06$ & $7.38 \mathrm{E}+06$ & $2.64 \mathrm{E}+04$ & $2.64 E+04$ \\
\hline & Tellurium & $129 m$ & $3.63 \mathrm{E}+02$ & $3.63 E+02$ & $1.09 \mathrm{E}+07$ & $1.09 \mathrm{E}+07$ & $1.92 \mathrm{E}+04$ & $1.92 E+04$ \\
\hline & Tellurium & 132 & $9.84 E+01$ & $9.84 \mathrm{E}+01$ & $2.99 \mathrm{E}+07$ & $2.99 E+07$ & $5.92 E+04$ & $5.92 E+04$ \\
\hline \multirow[t]{3}{*}{53} & Iodine & Stable & $1.12 E+05$ & $1.41 E+06$ & $0.00 \mathrm{E}+00$ & $0.00 \mathrm{E}+00$ & $0.00 \mathrm{E}+00$ & $0.00 \mathrm{E}+00$ \\
\hline & Iodine & 129 & $3.77 \mathrm{E}+05$ & $4.74 E+06$ & $6.65 E+01$ & $8.37 E+02$ & $3.08 \mathrm{E}-02$ & $3.87 E-01$ \\
\hline & Iodine & 131 & $4.35 E+02$ & $4.35 \mathrm{E}+02$ & $5.40 \mathrm{E}+07$ & $5.40 \mathrm{E}+07$ & $1.83 \mathrm{E}+05$ & $1.83 E+05$ \\
\hline \multirow[t]{4}{*}{54} & Xenon & Stable & $1.10 \mathrm{E}+07$ & $1.36 \mathrm{E}+08$ & $0.00 \mathrm{E}+00$ & $0.00 \mathrm{E}+00$ & $0.00 \mathrm{E}+00$ & $0.00 \mathrm{E}+00$ \\
\hline & Xenon & $129 m$ & $3.74 \mathrm{E}-03$ & $3.74 \mathrm{E}-03$ & $4.73 E+02$ & $4.73 E+02$ & $6.62 E-01$ & $6.62 E-01$ \\
\hline & Xenon & $131 \mathrm{~m}$ & $1.75 \mathrm{E}+01$ & $1.75 \mathrm{E}+01$ & $1.47 \mathrm{E}+06$ & $1.47 E+06$ & $1.41 \mathrm{E}+03$ & $1.41 E+03$ \\
\hline & Xenon & 133 & $4.31 \mathrm{E}+02$ & $4.31 E+02$ & $8.06 E+07$ & $8.06 E+07$ & $8.64 \mathrm{E}+04$ & $8.64 \mathrm{E}+04$ \\
\hline \multirow[t]{5}{*}{55} & Cesium & Stable & $2.34 \mathrm{E}+06$ & $2.94 E+07$ & $0.00 \mathrm{E}+00$ & $0.00 \mathrm{E}+00$ & $0.00 \mathrm{E}+00$ & $0.00 \mathrm{E}+00$ \\
\hline & Cesium & 134 & $2.26 \mathrm{E}+05$ & $7.45 \mathrm{E}+05$ & $2.93 E+08$ & $9.65 E+08$ & $2.98 \mathrm{E}+06$ & $9.82 E+06$ \\
\hline & Cesium & 135 & $7.31 \mathrm{E}+05$ & $8.36 E+06$ & $8.42 \mathrm{E}+02$ & $9.63 E+03$ & $2.81 \mathrm{E}-01$ & $3.21 \mathrm{E}+00$ \\
\hline & Cesium & 136 & $6.96 E+01$ & $6.96 \mathrm{E}+01$ & $5.10 \mathrm{E}+06$ & $5.10 E+06$ & $6.96 \mathrm{E}+04$ & $6.96 \mathrm{E}+04$ \\
\hline & Cesium & 137 & $2.51 \mathrm{E}+06$ & $2.66 \mathrm{E}+07$ & $2.18 \mathrm{E}+08$ & $2.31 \mathrm{E}+09$ & $2.42 \mathrm{E}+05$ & $2.56 \mathrm{E}+06$ \\
\hline \multirow[t]{4}{*}{56} & Barium & Stable & $2.95 \mathrm{E}+06$ & $3.56 \mathrm{E}+07$ & $0.00 E+00$ & $0.00 \mathrm{E}+00$ & $0.00 \mathrm{E}+00$ & $0.00 \mathrm{E}+00$ \\
\hline & Barium & $136 m$ & $3.12 \mathrm{E}-06$ & $3.12 \mathrm{E}-06$ & $8.41 E+05$ & $8.41 E+05$ & $1.02 E+04$ & $1.02 E+04$ \\
\hline & Barium & $137 m$ & $3.84 \mathrm{E}-01$ & $4.07 \mathrm{E}+00$ & $2.07 E+08$ & $2.19 E+09$ & $8.12 E+05$ & $8.60 E+06$ \\
\hline & Barium & 140 & $1.94 \mathrm{E}+03$ & $1.94 \mathrm{E}+03$ & $1.41 E+08$ & $1.41 \mathrm{E}+08$ & $3.94 E+05$ & $3.94 \mathrm{E}+05$ \\
\hline \multirow[t]{3}{*}{57} & Lanthanum & Stable & $2.59 \mathrm{E}+06$ & $3.19 \mathrm{E}+07$ & $0.00 \mathrm{E}+00$ & $0.00 \mathrm{E}+00$ & $0.00 \mathrm{E}+00$ & $0.00 \mathrm{E}+00$ \\
\hline & Lanthanum & 138 & $1.15 \mathrm{E}+01$ & $1.48 \mathrm{E}+02$ & $2.21 E-07$ & $2.84 E-06$ & $1.62 \mathrm{E}-09$ & $2.08 \mathrm{E}-08$ \\
\hline & Lanthanum & 140 & $2.94 \mathrm{E}+02$ & $2.94 \mathrm{E}+02$ & $1.64 \mathrm{E}+08$ & $1.64 \mathrm{E}+08$ & $2.74 \mathrm{E}+06$ & $2.74 \mathrm{E}+06$ \\
\hline \multirow[t]{4}{*}{58} & Cerium & Stable & $2.59 \mathrm{E}+06$ & $3.16 \mathrm{E}+07$ & $0.00 \mathrm{E}+00$ & $0.00 \mathrm{E}+00$ & $0.00 \mathrm{E}+00$ & $0.00 \mathrm{E}+00$ \\
\hline & Cerium & 141 & $1.22 E+04$ & $1.22 E+04$ & $3.47 E+08$ & $3.48 \mathrm{E}+08$ & $5.09 E+05$ & $5.09 E+05$ \\
\hline & Cerium & 142 & $2.40 \mathrm{E}+06$ & $2.95 \mathrm{E}+07$ & $5.77 \mathrm{E}-02$ & $7.08 \mathrm{E}-01$ & $0.00 \mathrm{E}+00$ & $0.00 \mathrm{E}+00$ \\
\hline & Cerium & 144 & $4.54 E+05$ & $8.22 \mathrm{E}+05$ & $1.45 E+09$ & $2.62 E+09$ & $9.61 \mathrm{E}+05$ & $1.74 E+06$ \\
\hline \multirow[t]{4}{*}{59} & Praseodymium & Stable & $2.30 \mathrm{E}+06$ & $2.81 E+07$ & $0.00 \mathrm{E}+00$ & $0.00 \mathrm{E}+00$ & $0.00 \mathrm{E}+00$ & $0.00 \mathrm{E}+00$ \\
\hline & Praseodymium & 143 & $2.10 E+03$ & $2.10 E+03$ & $1.41 E+08$ & $1.41 E+08$ & $2.64 E+05$ & $2.64 E+05$ \\
\hline & Praseodymium & 144 & $1.92 \mathrm{E}+01$ & $3.47 \mathrm{E}+01$ & $1.45 \mathrm{E}+09$ & $2.62 E+09$ & $1.06 \mathrm{E}+07$ & $1.93 \mathrm{E}+07$ \\
\hline & Praseodymium & $144 m$ & $9.58 \mathrm{E}-02$ & $1.73 \mathrm{E}-01$ & $1.74 \mathrm{E}+07$ & $3.15 E+07$ & $5.95 E+03$ & $1.08 \mathrm{E}+04$ \\
\hline \multirow[t]{3}{*}{60} & Neodymium & Stable & $5.63 E+06$ & $7.01 E+07$ & $0.00 \mathrm{E}+00$ & $0.00 \mathrm{E}+00$ & $0.00 \mathrm{E}+00$ & $0.00 \mathrm{E}+00$ \\
\hline & Neodymium & 144 & $2.44 E+06$ & $3.36 \mathrm{E}+07$ & $2.89 E-06$ & $3.97 \mathrm{E}-05$ & $0.00 \mathrm{E}+00$ & $0.00 \mathrm{E}+00$ \\
\hline & Neodymium & 147 & $5.80 \mathrm{E}+02$ & $5.80 \mathrm{E}+02$ & $4.66 E+07$ & $4.66 E+07$ & $1.13 E+05$ & $1.13 E+05$ \\
\hline
\end{tabular}


Table A.2 (continued)

\begin{tabular}{|c|c|c|c|c|c|c|c|c|}
\hline \multirow{2}{*}{$\begin{array}{l}\text { Atomic } \\
\text { number }\end{array}$} & \multirow{2}{*}{ Element } & \multirow{2}{*}{$\begin{array}{l}\text { Mass number } \\
\text { of nuclide }\end{array}$} & \multicolumn{2}{|c|}{ Mass, 8} & \multicolumn{2}{|c|}{ Radioactivity, Ci } & \multicolumn{2}{|c|}{ Thermal power, $W$} \\
\hline & & & Annual & Cumulative & Annual & Cumulative & Annual & Cumulative \\
\hline \multirow[t]{3}{*}{61} & Promethium & 147 & $2.39 \mathrm{E}+05$ & $1.03 E+06$ & $2.22 E+08$ & $9.54 E+08$ & $7.95 E+04$ & $3.42 \mathrm{E}+05$ \\
\hline & Promethium & 148 & $6.34 \mathrm{E}+01$ & $6.34 \mathrm{E}+01$ & $1.04 \mathrm{E}+07$ & $1.04 \mathrm{E}+07$ & $8.03 E+04$ & $8.03 E+04$ \\
\hline & Promethium & $148 m$ & $4.00 \mathrm{E}+02$ & $4.01 E+02$ & $8.55 E+06$ & $8.57 \mathrm{E}+06$ & $1.08 \mathrm{E}+05$ & $1.09 \mathrm{E}+05$ \\
\hline \multirow[t]{5}{*}{62} & Samarium & Stable & $9.00 \mathrm{E}+05$ & $1.14 E+07$ & $0.00 E+00$ & $0.00 \mathrm{E}+00$ & $0.00 \mathrm{E}+00$ & $0.00 \mathrm{E}+00$ \\
\hline & Samarium & 147 & $1.66 E+05$ & $4.48 \mathrm{E}+06$ & $3.78 E-03$ & $1.02 E-01$ & $5.18 \mathrm{E}-05$ & $1.40 \mathrm{E}-03$ \\
\hline & Samarium & 148 & $3.86 \mathrm{E}+05$ & $4.34 \mathrm{E}+06$ & $1.17 \mathrm{E}-07$ & $1.31 \mathrm{E}-06$ & $1.39 \mathrm{E}-09$ & $1.56 \mathrm{E}-08$ \\
\hline & Samarium & 149 & $5.41 E+03$ & $8.25 E+04$ & $1.30 \mathrm{E}-09$ & $1.98 \mathrm{E}-08$ & $0.00 \mathrm{E}+00$ & $0.00 \mathrm{E}+00$ \\
\hline & Samarium & 151 & $2.54 \mathrm{E}+04$ & $3.38 E+05$ & $6.68 \mathrm{E}+05$ & $8.90 \mathrm{E}+06$ & $7.83 \mathrm{E}+01$ & $1.04 \mathrm{E}+03$ \\
\hline \multirow[t]{5}{*}{63} & Europium & Stable & $2.28 \mathrm{E}+05$ & $2.81 \mathrm{E}+06$ & $0.00 \mathrm{E}+00$ & $0.00 E+00$ & $0.00 \mathrm{E}+00$ & $0.00 \mathrm{E}+00$ \\
\hline & Europium & 152 & $6.90 \mathrm{E}+01$ & $6.29 \mathrm{E}+02$ & $1.19 \mathrm{E}+04$ & $1.09 E+05$ & $9.03 E+01$ & $8.23 E+02$ \\
\hline & Europium & 154 & $8.49 \mathrm{E}+04$ & $6.14 \mathrm{E}+05$ & $2.29 E+07$ & $1.66 \mathrm{E}+08$ & $2.05 \mathrm{E}+05$ & $1.48 \mathrm{E}+06$ \\
\hline & Europium & 155 & $2.94 E+04$ & $1.67 E+05$ & $1.37 \mathrm{E}+07$ & $7.78 \mathrm{E}+07$ & $9.95 E+03$ & $5.66 \mathrm{E}+04$ \\
\hline & Europium & 156 & $4.79 E+02$ & $4.79 \mathrm{E}+02$ & $2.64 \mathrm{E}+07$ & $2.64 \mathrm{E}+07$ & $2.73 E+05$ & $2.73 E+05$ \\
\hline \multirow[t]{3}{*}{64} & Gadolinium & Stable & $1.24 \mathrm{E}+06$ & $1.89 \mathrm{E}+07$ & $0.00 \mathrm{E}+00$ & $0.00 \mathrm{E}+00$ & $0.00 \mathrm{E}+00$ & $0.00 \mathrm{E}+00$ \\
\hline & Gadolinium & 152 & $6.31 E+01$ & $2.46 \mathrm{E}+03$ & $1.38 \mathrm{E}-09$ & $5.36 \mathrm{E}-08$ & $1.79 \mathrm{E}-11$ & $6.99 \mathrm{E}-10$ \\
\hline & Gadolinium & 153 & $1.42 \mathrm{E}+02$ & $2.38 \mathrm{E}+02$ & $5.01 \mathrm{E}+05$ & $8.40 \mathrm{E}+05$ & $4.52 \mathrm{E}+02$ & $7.59 \mathrm{E}+02$ \\
\hline \multirow[t]{3}{*}{65} & Terbium & Stable & $2.26 E+04$ & $2.89 E+05$ & $0.00 E+00$ & $0.00 \mathrm{E}+00$ & $0.00 \mathrm{E}+00$ & $0.00 E+00$ \\
\hline & Terbium & 160 & $1.88 \mathrm{E}+02$ & $1.94 \mathrm{E}+02$ & $2.12 \mathrm{E}+06$ & $2.19 E+06$ & $1.73 E+04$ & $1.79 E+04$ \\
\hline & Terbium & 161 & $1.38 \mathrm{E}+00$ & $1.38 \mathrm{E}+00$ & $1.62 \mathrm{E}+05$ & $1.62 \mathrm{E}+05$ & $3.24 \mathrm{E}+02$ & $3.24 \mathrm{E}+02$ \\
\hline \multirow[t]{5}{*}{81} & Thallium & Stable & $2.30 \mathrm{E}-09$ & $2.51 \mathrm{E}-08$ & $0.00 \mathrm{E}+00$ & $0.00 \mathrm{E}+00$ & $0.00 \mathrm{E}+00$ & $0.00 \mathrm{E}+00$ \\
\hline & Thallium & 206 & $3.45 \mathrm{E}-22$ & $3.45 \mathrm{E}-22$ & $7.53 E-14$ & $7.53 \mathrm{E}-14$ & $6.80 \mathrm{E}-16$ & $6.80 E-16$ \\
\hline & Thallium & 207 & $7.06 \mathrm{E}-12$ & $4.55 E-10$ & $1.35 \mathrm{E}-03$ & $8.66 \mathrm{E}-02$ & $3.95 \mathrm{E}-06$ & $2.54 E-04$ \\
\hline & Thallium & 208 & $1.04 \mathrm{E}-08$ & $5.29 \mathrm{E}-07$ & $3.06 \mathrm{E}+00$ & $1.56 \mathrm{E}+02$ & $7.20 E-02$ & $3.67 \mathrm{E}+00$ \\
\hline & Thallium & 209 & $2.08 \mathrm{E}-14$ & $1.57 \mathrm{E}-13$ & $8.49 E-06$ & $6.44 E-05$ & $1.41 \mathrm{E}-07$ & $1.07 \mathrm{E}-06$ \\
\hline \multirow[t]{8}{*}{82} & Lead & Stable & $1.86 \mathrm{E}+03$ & $2.94 E+04$ & $0.00 E+00$ & $0.00 \mathrm{E}+00$ & $0.00 \mathrm{E}+00$ & $0.00 \mathrm{E}+00$ \\
\hline & Lead & 204 & $2.59 \mathrm{E}+01$ & $4.10 \mathrm{E}+02$ & $3.24 \mathrm{E}-13$ & $5.13 E-12$ & $4.99 \mathrm{E}-15$ & $7.91 \mathrm{E}-14$ \\
\hline & Lead & 205 & $6.81 \mathrm{E}-02$ & $8.99 \mathrm{E}-01$ & $3.96 \mathrm{E}-06$ & $5.23 E-05$ & $1.15 \mathrm{E}-10$ & $1.52 \mathrm{E}-09$ \\
\hline & Lead & 209 & $2.04 \mathrm{E}-10$ & $7.73 E-10$ & $9.28 \mathrm{E}-04$ & $3.51 E-03$ & $1.07 \mathrm{E}-06$ & $4.04 E-06$ \\
\hline & Lead & 210 & $1.06 \mathrm{E}-07$ & $1.75 \mathrm{E}-05$ & $8.10 E-06$ & $1.34 \mathrm{E}-03$ & $1.88 \mathrm{E}-09$ & $3.09 \mathrm{E}-07$ \\
\hline & Lead & 211 & $5.47 \mathrm{E}-11$ & $3.52 E-09$ & $1.35 \mathrm{E}-03$ & $8.69 \mathrm{E}-02$ & $4.04 \mathrm{E}-06$ & $2.60 \mathrm{E}-04$ \\
\hline & Lead & 212 & $6.12 \mathrm{E}-06$ & $3.12 \mathrm{E}-04$ & $8.51 E+00$ & $4.33 E+02$ & $1.62 \mathrm{E}-02$ & $8.25 \mathrm{E}-01$ \\
\hline & Lead & 214 & $1.79 \mathrm{E}-12$ & $2.72 \mathrm{E}-10$ & $5.87 \mathrm{E}-05$ & $8.92 \mathrm{E}-03$ & $1.87 \mathrm{E}-07$ & $2.84 \mathrm{E}-05$ \\
\hline \multirow[t]{5}{*}{83} & Bismuth & Stable & $7.53 E+02$ & $1.19 \mathrm{E}+04$ & $0.00 \mathrm{E}+00$ & $0.00 \mathrm{E}+00$ & $0.00 \mathrm{E}+00$ & $0.00 \mathrm{E}+00$ \\
\hline & Bismuth & 208 & $1.09 \mathrm{E}-02$ & $1.44 \mathrm{E}-01$ & $5.08 E-05$ & $6.74 \mathrm{E}-04$ & $8.00 E-07$ & $1.06 \mathrm{E}-05$ \\
\hline & Bismuth & 210 & $1.31 E-05$ & $1.31 \mathrm{E}-05$ & $1.63 \mathrm{E}+00$ & $1.63 E+00$ & $3.75 \mathrm{E}-03$ & $3.75 \mathrm{E}-03$ \\
\hline & Bismuth & $210 m$ & $6.47 \mathrm{E}-02$ & $8.54 \mathrm{E}-01$ & $3.67 \mathrm{E}-05$ & $4.85 \mathrm{E}-04$ & $1.15 \mathrm{E}-06$ & $1.52 \mathrm{E}-05$ \\
\hline & Bismuth & 211 & $3.22 \mathrm{E}-12$ & $2.08 \mathrm{E}-10$ & $1.35 \mathrm{E}-03$ & $8.69 \mathrm{E}-02$ & $5.38 \mathrm{E}-05$ & $3.46 \mathrm{E}-03$ \\
\hline
\end{tabular}


Table A.2 (continued)

\begin{tabular}{|c|c|c|c|c|c|c|c|c|}
\hline \multirow{2}{*}{$\begin{array}{l}\text { Atomic } \\
\text { number }\end{array}$} & \multirow{2}{*}{ Element } & \multirow{2}{*}{$\begin{array}{c}\text { Mass number } \\
\text { of nuclide }\end{array}$} & \multicolumn{2}{|c|}{ Mass, $\mathrm{g}$} & \multicolumn{2}{|c|}{ Radioactivity, Ci } & \multicolumn{2}{|c|}{ Thermal power, W } \\
\hline & & & Annual & Cumulative & Annual & Cumulative & Annual & Cumulative \\
\hline & Bismuth & 212 & $5.81 E-07$ & $2.96 \mathrm{E}-05$ & $8.51 E+00$ & $4.33 E+02$ & $1.45 E-01$ & $7.37 \mathrm{E}+00$ \\
\hline & Bismuth & 213 & $2.03 E-11$ & $1.54 \mathrm{E}-10$ & $3.93 E-04$ & $2.98 E-03$ & $1.65 E-06$ & $1.25 \mathrm{E}-05$ \\
\hline & Bismuth & 214 & $1.33 E-12$ & $2.02 \mathrm{E}-10$ & $5.87 \mathrm{E}-05$ & $8.92 \mathrm{E}-03$ & $7.53 \mathrm{E}-07$ & $1.14 \mathrm{E}-04$ \\
\hline \multirow[t]{8}{*}{84} & Polonium & 210 & $8.19 E-03$ & $1.01 \mathrm{E}-02$ & $3.68 E+01$ & 4.53E+01 & $1.18 E+00$ & $1.45 \mathrm{E}+00$ \\
\hline & Polonium & 211 & $3.96 \mathrm{E}-17$ & $2.55 \mathrm{E}-15$ & $3.78 E-06$ & $2.43 E-04$ & $1.70 E-07$ & $1.09 E-05$ \\
\hline & Polonium & 212 & $3.07 E-17$ & $1.57 \mathrm{E}-15$ & $5.45 \mathrm{E}+00$ & $2.78 \mathrm{E}+02$ & $2.89 \mathrm{E}-01$ & $1.47 \mathrm{E}+01$ \\
\hline & Polonium & 213 & $3.05 E-20$ & $2.31 E-19$ & $3.85 E-04$ & $2.92 E-03$ & $1.95 E-05$ & $1.48 \mathrm{E}-04$ \\
\hline & Polonium & 214 & $2.35 \mathrm{E}-19$ & $2.78 \mathrm{E}-17$ & $7.56 \mathrm{E}-05$ & $8.93 E-03$ & $3.51 E-06$ & $4.15 E-04$ \\
\hline & Polonium & 215 & $4.58 \mathrm{E}-17$ & $2.95 \mathrm{E}-15$ & $1.35 E-03$ & $8.69 \mathrm{E}-02$ & $6.02 E-05$ & $3.88 \mathrm{E}-03$ \\
\hline & Polonium & 216 & $2.44 E-11$ & $1.24 \mathrm{E}-09$ & $8.50 E+00$ & $4.33 E+02$ & $3.48 \mathrm{E}-01$ & $1.77 \mathrm{E}+01$ \\
\hline & Polonium & 218 & $2.08 \mathrm{E}-13$ & $3.15 \mathrm{E}-11$ & $5.88 \mathrm{E}-05$ & $8.92 \mathrm{E}-03$ & 2.13E-06 & $3.23 E-04$ \\
\hline 85 & Astatine & 217 & $2.44 \mathrm{E}-16$ & $1.85 \mathrm{E}-15$ & $3.93 E-04$ & $2.98 \mathrm{E}-03$ & $1.68 \mathrm{E}-05$ & $1.27 \mathrm{E}-04$ \\
\hline \multirow[t]{4}{*}{86} & Radon & 218 & $1.14 E-17$ & $1.14 \mathrm{E}-17$ & $1.68 \mathrm{E}-05$ & $1.68 \mathrm{E}-05$ & $7.24 \mathrm{E}-07$ & $7.24 \mathrm{E}-07$ \\
\hline & Radon & 219 & $1.04 \mathrm{E}-13$ & $6.67 \mathrm{E}-12$ & $1.35 \mathrm{E}-03$ & $8.69 \mathrm{E}-02$ & $5.60 \mathrm{E}-05$ & $3.60 E-03$ \\
\hline & Radon & 220 & $9.21 E-09$ & $4.70 \mathrm{E}-07$ & $8.50 \mathrm{E}+00$ & $4.33 E+02$ & $3.23 \mathrm{E}-01$ & $1.65 \mathrm{E}+01$ \\
\hline & Radon & 222 & $3.82 E-10$ & $5.80 E-08$ & $5.88 E-05$ & $8.92 E-03$ & $1.95 E-06$ & $2.96 \mathrm{E}-04$ \\
\hline \multirow[t]{2}{*}{87} & Francium & 221 & $2.22 E-12$ & $1.68 \mathrm{E}-11$ & $3.93 \mathrm{E}-04$ & $2.98 \mathrm{E}-03$ & $1.52 \mathrm{E}-05$ & $1.15 \mathrm{E}-04$ \\
\hline & Francium & 223 & $5.19 \mathrm{E}-13$ & $3.14 \mathrm{E}-11$ & $2.01 \mathrm{E}-05$ & $1.22 \mathrm{E}-03$ & $5.22 E-08$ & $3.16 \mathrm{E}-06$ \\
\hline \multirow[t]{6}{*}{88} & Radium & 222 & $1.26 \mathrm{E}-14$ & $1.26 \mathrm{E}-14$ & $1.68 \mathrm{E}-05$ & $1.68 \mathrm{E}-05$ & $6.66 \mathrm{E}-07$ & $6.66 \mathrm{E}-07$ \\
\hline & Radium & 223 & $2.63 E-08$ & $1.70 \mathrm{E}-06$ & $1.35 \mathrm{E}-03$ & $8.69 E-02$ & $4.81 E-05$ & $3.09 \mathrm{E}-03$ \\
\hline & Radium & 224 & $5.33 \mathrm{E}-05$ & $2.72 E-03$ & $8.50 E+00$ & $4.33 E+02$ & $2.92 E-01$ & $1.49 \mathrm{E}+01$ \\
\hline & Radium & 225 & $9.19 E-09$ & $7.52 \mathrm{E}-08$ & $3.61 \mathrm{E}-04$ & $2.95 \mathrm{E}-03$ & $2.53 E-07$ & $2.07 \mathrm{E}-06$ \\
\hline & Radium & 226 & $5.98 E-05$ & $9.04 E-03$ & $5.92 \mathrm{E}-05$ & $8.94 \mathrm{E}-03$ & $1.71 E-06$ & $2.58 \mathrm{E}-04$ \\
\hline & Radium & 228 & $3.45 E-11$ & $5.30 \mathrm{E}-09$ & 8.07E-09 & $1.24 \mathrm{E}-06$ & $6.22 E-13$ & $9.56 \mathrm{E}-11$ \\
\hline \multirow[t]{3}{*}{89} & Actinium & 225 & $6.77 E-09$ & $5.13 E-08$ & $3.93 E-04$ & $2.98 \mathrm{E}-03$ & $1.37 \mathrm{E}-05$ & $1.04 \mathrm{E}-04$ \\
\hline & Actinium & 227 & $2.01 E-05$ & $1.22 \mathrm{E}-03$ & $1.46 \mathrm{E}-03$ & $8.82 \mathrm{E}-02$ & $7.05 E-07$ & $4.27 \mathrm{E}-05$ \\
\hline & Actinium & 228 & $5.94 \mathrm{E}-12$ & $6.49 E-12$ & $1.33 E-05$ & $1.46 \mathrm{E}-05$ & $1.15 \mathrm{E}-07$ & $1.26 \mathrm{E}-07$ \\
\hline \multirow[t]{9}{*}{90} & Thorium & 226 & $6.27 E-13$ & $6.27 \mathrm{E}-13$ & $1.68 \mathrm{E}-05$ & $1.68 \mathrm{E}-05$ & $6.43 E-07$ & $6.43 E-07$ \\
\hline & Thorium & 227 & $4.45 \mathrm{E}-08$ & $2.80 \mathrm{E}-06$ & 1.37E-03 & $8.61 E-02$ & $5.00 \mathrm{E}-05$ & $3.14 \mathrm{E}-03$ \\
\hline & Thorium & 228 & $1.02 E-02$ & $5.25 E-01$ & $8.39 E+00$ & $4.31 E+02$ & $2.74 \mathrm{E}-01$ & $1.41 \mathrm{E}+01$ \\
\hline & Thorium & 229 & $1.47 E-03$ & $1.36 \mathrm{E}-02$ & $3.12 \mathrm{E}-04$ & $2.90 \mathrm{E}-03$ & $9.56 \mathrm{E}-06$ & $8.89 E-05$ \\
\hline & Thorium & 230 & $3.31 E+00$ & $1.51 \mathrm{E}+02$ & $6.69 \mathrm{E}-02$ & $3.04 E+00$ & $1.89 \mathrm{E}-03$ & 8.61E-02 \\
\hline & Thorium & 231 & $6.93 E-05$ & $1.01 \mathrm{E}-03$ & $3.68 \mathrm{E}+01$ & $5.35 \mathrm{E}+02$ & $2.07 \mathrm{E}-02$ & $3.00 \mathrm{E}-01$ \\
\hline & Thorium & 232 & $5.75 \mathrm{E}-01$ & $2.72 \mathrm{E}+01$ & $6.31 \mathrm{E}-08$ & $2.99 \mathrm{E}-06$ & $1.53 \mathrm{E}-09$ & $7.23 \mathrm{E}-08$ \\
\hline & Thorium & 233 & $9.99 \mathrm{E}-12$ & $9.99 \mathrm{E}-12$ & $3.65 E-04$ & $3.65 \mathrm{E}-04$ & $9.23 \mathrm{E}-07$ & $9.23 \mathrm{E}-07$ \\
\hline & Thorium & 234 & $2.57 \mathrm{E}-02$ & $4.11 \mathrm{E}-01$ & $5.95 \mathrm{E}+02$ & $9.52 \mathrm{E}+03$ & $2.41 \mathrm{E}-01$ & $3.86 \mathrm{E}+00$ \\
\hline
\end{tabular}


Table A.2 (continued)

\begin{tabular}{|c|c|c|c|c|c|c|c|c|}
\hline \multirow{2}{*}{$\begin{array}{l}\text { Atomic } \\
\text { number }\end{array}$} & \multirow{2}{*}{ Element } & \multirow{2}{*}{$\begin{array}{l}\text { Mass number } \\
\text { of nuclide }\end{array}$} & \multicolumn{2}{|c|}{ Mass, $\mathrm{g}$} & \multicolumn{2}{|c|}{ Radioactivity, Ci } & \multicolumn{2}{|c|}{ Thermal power, $\mathrm{W}$} \\
\hline & & & Annual & Cumulative & Annual & Cumulative & Annual & Cumulative \\
\hline \multirow[t]{6}{*}{91} & Protactinium & 231 & $6.96 \mathrm{E}-01$ & $9.22 \mathrm{E}+00$ & $3.29 E-02$ & $4.36 E-01$ & $9.91 E-04$ & $1.31 E-02$ \\
\hline & Protactinium & 232 & $1.26 \mathrm{E}-05$ & $1.26 \mathrm{E}-05$ & $5.43 E+00$ & $5.43 E+00$ & $3.55 \mathrm{E}-02$ & $3.55 \mathrm{E}-02$ \\
\hline & Protactinium & 233 & $3.35 \mathrm{E}-02$ & $3.84 E-01$ & $6.96 \mathrm{E}+02$ & $7.97 \mathrm{E}+03$ & $1.58 \mathrm{E}+00$ & $1.81 \mathrm{E}+01$ \\
\hline & Protactinium & 234 & $3.92 \mathrm{E}-07$ & $6.19 E-06$ & $7.83 E-01$ & $1.24 \mathrm{E}+01$ & $1.13 E-02$ & $1.78 \mathrm{E}-01$ \\
\hline & Protactinium & $234 m$ & $8.65 E-07$ & $1.39 \mathrm{E}-05$ & $5.95 \mathrm{E}+02$ & $9.52 E+03$ & $2.94 \mathrm{E}+00$ & $4.70 \mathrm{E}+01$ \\
\hline & Protactinium & 235 & $6.54 \mathrm{E}-17$ & $6.54 \mathrm{E}-17$ & $2.17 \mathrm{E}-09$ & $2.17 \mathrm{E}-09$ & $6.06 E-12$ & $6.06 \mathrm{E}-12$ \\
\hline \multirow[t]{11}{*}{92} & Uranium & 230 & $6.15 E-10$ & $6.15 E-10$ & $1.68 \mathrm{E}-05$ & $1.68 \mathrm{E}-05$ & $5.97 \mathrm{E}-07$ & $5.97 \mathrm{E}-07$ \\
\hline & Uranium & 231 & $5.88 \mathrm{E}-09$ & $5.88 \mathrm{E}-09$ & $7.91 E-04$ & $7.91 \mathrm{E}-04$ & $6.54 \mathrm{E}-07$ & $6.54 \mathrm{E}-07$ \\
\hline & Uranium & 232 & $1.34 \mathrm{E}+00$ & $2.65 E+01$ & $2.87 \mathrm{E}+01$ & $5.68 \mathrm{E}+02$ & $9.20 \mathrm{E}-01$ & $1.82 E+01$ \\
\hline & Uranium & 233 & $2.75 E+00$ & $5.92 \mathrm{E}+01$ & $2.67 \mathrm{E}-02$ & $5.74 E-01$ & $7.75 E-04$ & $1.67 \mathrm{E}-02$ \\
\hline & Uranium & 234 & $3.48 \mathrm{E}+05$ & $5.17 E+06$ & $2.18 \mathrm{E}+03$ & $3.23 E+04$ & $6.27 \mathrm{E}+01$ & $9.30 \mathrm{E}+02$ \\
\hline & Uranium & 235 & $1.40 \mathrm{E}+07$ & $2.44 E+08$ & $3.04 \mathrm{E}+01$ & $5.29 \mathrm{E}+02$ & $7.96 \mathrm{E}-01$ & $1.38 \mathrm{E}+01$ \\
\hline & Uranium & 236 & $8.30 \mathrm{E}+06$ & $1.02 E+08$ & $5.37 \mathrm{E}+02$ & $6.62 E+03$ & $1.46 \mathrm{E}+01$ & $1.79 \mathrm{E}+02$ \\
\hline & Uranium & 237 & $5.39 E+02$ & $5.40 \mathrm{E}+02$ & $4.41 E+07$ & $4.41 \mathrm{E}+07$ & $8.34 \mathrm{E}+04$ & $8.35 E+04$ \\
\hline & Uranium & 238 & $1.77 \mathrm{E}+09$ & $2.83 E+10$ & $5.95 \mathrm{E}+02$ & $9.52 E+03$ & $1.51 \mathrm{E}+01$ & $2.41 E+02$ \\
\hline & Uranium & 239 & $1.44 \mathrm{E}-02$ & $1.44 \mathrm{E}-02$ & $4.80 \mathrm{E}+05$ & $4.80 E+05$ & $1.29 \mathrm{E}+03$ & $1.29 \mathrm{E}+03$ \\
\hline & Uranium & 240 & $2.25 \mathrm{E}-04$ & $2.25 E-04$ & $2.08 \mathrm{E}+02$ & $2.08 \mathrm{E}+02$ & $1.71 \mathrm{E}-01$ & $1.71 \mathrm{E}-01$ \\
\hline \multirow[t]{9}{*}{93} & Neptunium & 235 & $6.97 \mathrm{E}-03$ & $1.50 \mathrm{E}-02$ & $9.78 \mathrm{E}+00$ & $2.10 E+01$ & $5.68 \mathrm{E}-04$ & $1.22 \mathrm{E}-03$ \\
\hline & Neptunium & 236 & $8.77 \mathrm{E}-01$ & $9.60 \mathrm{E}+00$ & $1.16 \mathrm{E}-02$ & $1.27 E-01$ & $2.33 \mathrm{E}-05$ & $2.55 \mathrm{E}-04$ \\
\hline & Neptunium & $236 m$ & $1.02 \mathrm{E}-04$ & $1.02 E-04$ & $6.00 \mathrm{E}+01$ & $6.00 \mathrm{E}+01$ & $4.74 \mathrm{E}-02$ & $4.74 \mathrm{E}-02$ \\
\hline & Neptunium & 237 & $9.89 \mathrm{E}+05$ & $1.13 \mathrm{E}+07$ & $6.97 \mathrm{E}+02$ & $7.97 E+03$ & $2.13 E+01$ & $2.44 \mathrm{E}+02$ \\
\hline & Neptunium & 238 & $2.33 \mathrm{E}+01$ & $2.33 E+01$ & $6.04 E+06$ & $6.04 \mathrm{E}+06$ & $2.89 \mathrm{E}+04$ & $2.89 E+04$ \\
\hline & Neptunium & 239 & $1.44 E+03$ & $1.44 \mathrm{E}+03$ & $3.35 \mathrm{E}+08$ & $3.35 \mathrm{E}+08$ & $8.09 E+05$ & $8.10 \mathrm{E}+05$ \\
\hline & Neptunium & 240 & $3.41 \mathrm{E}-04$ & $3.41 \mathrm{E}-04$ & $4.11 E+03$ & $4.11 E+03$ & $4.36 \mathrm{E}+01$ & $4.36 \mathrm{E}+01$ \\
\hline & Neptunium & $240 m$ & $2.00 \mathrm{E}-06$ & $2.00 \mathrm{E}-06$ & $2.11 E+02$ & $2.11 E+02$ & $1.22 \mathrm{E}+00$ & $1.22 \mathrm{E}+00$ \\
\hline & Neptunium & 241 & $1.11 \mathrm{E}-13$ & $1.11 \mathrm{E}-13$ & $5.42 \mathrm{E}-06$ & $5.42 E-06$ & $1.51 \mathrm{E}-08$ & $1.51 \mathrm{E}-08$ \\
\hline \multirow[t]{11}{*}{94} & Plutonium & 236 & $2.34 \mathrm{E}+00$ & $9.23 E+00$ & $1.24 \mathrm{E}+03$ & $4.91 E+03$ & $4.33 E+01$ & $1.71 E+02$ \\
\hline & Plutonium & 237 & $1.96 \mathrm{E}-02$ & $1.97 \mathrm{E}-02$ & $2.37 \mathrm{E}+02$ & $2.38 E+02$ & $8.75 E-02$ & $8.79 \mathrm{E}-02$ \\
\hline & Plutonium & 238 & $3.36 \mathrm{E}+05$ & $3.43 E+06$ & $5.76 \mathrm{E}+06$ & $5.87 \mathrm{E}+07$ & $1.91 E+05$ & $1.94 \mathrm{E}+06$ \\
\hline & Plutonium & 239 & $9.41 E+06$ & $1.45 \mathrm{E}+08$ & $5.85 \mathrm{E}+05$ & $9.00 \mathrm{E}+06$ & $1.80 \mathrm{E}+04$ & $2.77 \mathrm{E}+05$ \\
\hline & Plutonium & 240 & $4.53 \mathrm{E}+06$ & $6.14 E+07$ & $1.03 E+06$ & $1.40 \mathrm{E}+07$ & $3.21 E+04$ & $4.36 E+05$ \\
\hline & Plutonium & 241 & $2.42 E+06$ & $2.42 \mathrm{E}+07$ & $2.50 \mathrm{E}+08$ & $2.49 E+09$ & $7.74 E+03$ & $7.72 E+04$ \\
\hline & Plutonium & 242 & $1.03 E+06$ & $1.23 E+07$ & $3.95 E+03$ & $4.69 E+04$ & $1.17 \mathrm{E}+02$ & $1.39 \mathrm{E}+03$ \\
\hline & Plutonium & 243 & $2.07 \mathrm{E}-01$ & $2.07 \mathrm{E}-01$ & $5.39 \mathrm{E}+05$ & $5.39 E+05$ & $6.22 E+02$ & $6.22 \mathrm{E}+02$ \\
\hline & Plutonium & 244 & $6.50 \mathrm{E}+01$ & $6.68 \mathrm{E}+02$ & $1.15 \mathrm{E}-03$ & $1.19 \mathrm{E}-02$ & $3.34 \mathrm{E}-05$ & $3.44 \mathrm{E}-0.4$ \\
\hline & Plutonium & 245 & $2.46 \mathrm{E}-06$ & $2.46 \mathrm{E}-06$ & $2.97 \mathrm{E}+00$ & $2.97 \mathrm{E}+00$ & $7.05 \mathrm{E}-03$ & $7.05 \mathrm{E}-03$ \\
\hline & Plutonium & 246 & $5.42 \mathrm{E}-07$ & $5.42 \mathrm{E}-07$ & $2.65 \mathrm{E}-02$ & $2.65 E-02$ & $2.23 \mathrm{E}-05$ & $2.23 \mathrm{E}-05$ \\
\hline
\end{tabular}


Table A.2 (continued)

\begin{tabular}{|c|c|c|c|c|c|c|c|c|}
\hline \multirow{2}{*}{$\begin{array}{l}\text { Atomic } \\
\text { number }\end{array}$} & \multirow{2}{*}{ Element } & \multirow{2}{*}{$\begin{array}{l}\text { Mass number } \\
\text { of nuclide }\end{array}$} & \multicolumn{2}{|c|}{ Mass, $\mathrm{g}$} & \multicolumn{2}{|c|}{ Radioactivity, $\mathrm{Ci}$} & \multicolumn{2}{|c|}{ Thermal power, W } \\
\hline & & & Annual & Cumulative & Annual & Cumulative & Annual & Cumulative \\
\hline \multirow[t]{10}{*}{95} & Americium & 239 & $3.71 \mathrm{E}-09$ & 3.71E-09 & 4.09E-03 & $4.09 \mathrm{E}-03$ & $9.90 \mathrm{E}-06$ & $9.90 \mathrm{E}-06$ \\
\hline & Americium & 240 & $1.63 E-05$ & $1.63 \mathrm{E}-05$ & $4.20 \mathrm{E}+00$ & $4.20 \mathrm{E}+00$ & $2.75 \mathrm{E}-02$ & $2.75 E-02$ \\
\hline & Americium & 241 & $1.29 \mathrm{E}+05$ & $1.07 E+07$ & $4.43 E+05$ & $3.69 E+07$ & $1.47 \mathrm{E}+04$ & $1.23 E+06$ \\
\hline & Americium & 242 & $5.47 \mathrm{E}-01$ & $7.38 \mathrm{E}-01$ & $4.42 E+05$ & $5.97 \mathrm{E}+05$ & $5.02 \mathrm{E}+02$ & $6.78 \mathrm{E}+02$ \\
\hline & Americium & $242 m$ & $1.54 \mathrm{E}+03$ & $1.76 \mathrm{E}+04$ & $1.50 \mathrm{E}+04$ & $1.71 \mathrm{E}+05$ & $5.91 E+00$ & $6.75 E+01$ \\
\hline & Americium & 243 & $2.22 E+05$ & $2.38 \mathrm{E}+06$ & $4.43 E+04$ & $4.75 E+05$ & $1.43 E+03$ & $1.53 E+04$ \\
\hline & Americium & 244 & $1.57 \mathrm{E}-02$ & $1.57 \mathrm{E}-02$ & $1.99 E+04$ & $1.99 E+04$ & $1.04 E+02$ & $1.04 \mathrm{E}+02$ \\
\hline & Americium & $244 m$ & $1.39 \mathrm{E}-04$ & $1.39 E-04$ & $4.12 E+03$ & $4.12 E+03$ & $1.25 \mathrm{E}+01$ & $1.25 E+01$ \\
\hline & Americium & 245 & $5.80 \mathrm{E}-07$ & $5.80 \mathrm{E}-07$ & $3.58 \mathrm{E}+00$ & $3.58 \mathrm{E}+00$ & $6.65 \mathrm{E}-03$ & $6.65 \mathrm{E}-03$ \\
\hline & Americium & 246 & $8.68 \mathrm{E}-10$ & $8.68 \mathrm{E}-10$ & $2.66 \mathrm{E}-02$ & $2.66 \mathrm{E}-02$ & $2.14 \mathrm{E}-04$ & $2.14 \mathrm{E}-04$ \\
\hline \multirow[t]{8}{*}{96} & Curium & 242 & $1.47 \mathrm{E}+04$ & $1.91 \mathrm{E}+04$ & $4.86 \mathrm{E}+07$ & $6.32 E+07$ & $1.79 E+06$ & $2.33 E+06$ \\
\hline & Curium & 243 & $8.87 \mathrm{E}+02$ & $7.81 \mathrm{E}+03$ & $4.58 \mathrm{E}+04$ & $4.03 E+05$ & $1.68 E+03$ & $1.48 \mathrm{E}+04$ \\
\hline & Curium & 244 & $7.10 E+04$ & $5.55 E+05$ & $5.74 \mathrm{E}+06$ & $4.49 E+07$ & $2.01 E+05$ & $1.57 \mathrm{E}+06$ \\
\hline & Curium & 245 & $2.84 \mathrm{E}+03$ & $2.63 \mathrm{E}+04$ & $4.88 \mathrm{E}+02$ & $4.51 E+03$ & $1.62 E+01$ & $1.50 \mathrm{E}+02$ \\
\hline & Curium & 246 & $4.12 E+02$ & $3.48 \mathrm{E}+03$ & $1.27 \mathrm{E}+02$ & $1.07 \mathrm{E}+03$ & $4.14 \mathrm{E}+00$ & $3.51 \mathrm{E}+01$ \\
\hline & Curium & 247 & $4.40 \mathrm{E}+00$ & $3.48 \mathrm{E}+01$ & $4.08 E-04$ & $3.23 E-03$ & $1.30 \mathrm{E}-05$ & $1.03 E-04$ \\
\hline & Curium & 248 & $2.63 E-01$ & $1.95 \mathrm{E}+00$ & $1.12 \mathrm{E}-03$ & $8.30 \mathrm{E}-03$ & $1.39 \mathrm{E}-04$ & $1.03 E-03$ \\
\hline & Totals & & $2.90 \mathrm{E}+09$ & $4.60 \mathrm{E}+10$ & $1.10 \mathrm{E}+10$ & $2.67 E+10$ & $4.61 E+07$ & $1.00 E+08$ \\
\hline
\end{tabular}

aIncludes contributions from nuclides in the fuel, cladding, and fuel assembly structural material.

b The term stable represents a group of nonradioactive nuclides of a particular element. 
Table A.3. Representative DOE LLW radionuclide composition by percent activity ${ }^{a}$

\begin{tabular}{|c|c|c|c|c|c|c|c|c|c|}
\hline \multicolumn{2}{|c|}{ Uranium/thorium } & \multicolumn{2}{|c|}{ Fission product } & \multicolumn{2}{|c|}{ Induced activity } & \multicolumn{2}{|c|}{ Alpha, $<100 \mathrm{nCi} / \mathrm{g}$} & \multicolumn{2}{|c|}{ Other } \\
\hline Nuclide & Composition & Nuclide & Composition & Nuclide & Composition & Nuclide & Composition & Nuclide & Composition \\
\hline${ }^{208} \mathrm{Tl}$ & 0.0017 & ${ }^{60} \mathrm{Co}$ & 0.08 & ${ }^{51} \mathrm{Cr}$ & 4.95 & ${ }^{238} \mathrm{Pu}$ & 2.62 & ${ }^{3} \mathrm{H}$ & 1.22 \\
\hline${ }^{212} \mathrm{~Pb}$ & 0.0045 & ${ }^{90} \mathrm{Sr}$ & 7.77 & ${ }^{54} \mathrm{Mn}$ & 38.10 & ${ }^{239} \mathrm{Pu}$ & 0.20 & ${ }^{14} \mathrm{C}$ & 0.06 \\
\hline${ }^{212} \mathrm{Bi}$ & 0.0045 & ${ }^{90} \mathrm{Y}$ & 7.77 & ${ }^{88} \mathrm{Co}$ & 55.40 & ${ }^{240} \mathrm{Pu}$ & 0.70 & ${ }^{54} \mathrm{Mn}$ & 6.76 \\
\hline${ }^{212} \mathrm{Po}$ & 0.0029 & ${ }^{95} \mathrm{Zr}$ & 1.27 & ${ }^{59} \mathrm{Fe}$ & 0.49 & ${ }^{241} \mathrm{Pu}$ & 96.4 & ${ }^{38} \mathrm{Co}$ & 6.24 \\
\hline${ }^{216} \mathrm{Po}$ & 0.0045 & ${ }^{95} \mathrm{Nb}$ & 2.83 & ${ }^{60} \mathrm{Co}$ & 0.87 & ${ }^{241} \mathrm{Am}$ & 0.004 & ${ }^{60} \mathrm{Co}$ & 18.03 \\
\hline${ }^{224} \mathrm{Ra}$ & 0.0045 & ${ }^{99} \mathrm{Tc}$ & 0.02 & ${ }^{65} \mathrm{Zn}$ & 0.19 & ${ }^{242} \mathrm{Cm}$ & 0.056 & ${ }^{90} \mathrm{Sr}$ & 8.48 \\
\hline${ }^{228} \mathrm{Ra}$ & 0.0269 & ${ }^{125} \mathrm{Sb}$ & 2.93 & & $\longrightarrow$ & ${ }^{244} \mathrm{Cm}$ & 0.020 & ${ }^{90} \mathrm{Y}$ & 8.48 \\
\hline${ }^{228} \mathrm{Ac}$ & 0.0269 & ${ }^{125 \mathrm{~m}} \mathrm{Te}$ & 0.73 & & 100.00 & & $\longrightarrow$ & ${ }^{99} \mathrm{Tc}$ & 0.12 \\
\hline${ }^{228} \mathrm{Th}$ & 0.0045 & ${ }^{106} \mathrm{Ru}$ & 6.39 & & & & 100.000 & ${ }^{134} \mathrm{Cs}$ & 13.98 \\
\hline${ }^{231} \mathrm{Th}$ & 0.0259 & ${ }^{106} \mathrm{Rh}$ & 6.39 & & & & & ${ }^{137} \mathrm{Cs}$ & 18.45 \\
\hline${ }^{232} \mathrm{Th}$ & 0.273 & ${ }^{134} \mathrm{Cs}$ & 0.38 & & & & & ${ }^{137} \mathrm{ma}$ & 17.45 \\
\hline${ }^{234} \mathrm{Th}$ & 33.197 & ${ }^{137} \mathrm{Cs}$ & 17.31 & & & & & ${ }^{238} \mathrm{U}$ & 0.73 \\
\hline${ }^{234} \mathrm{~m} \mathrm{~Pa}$ & 33.197 & ${ }^{137} \mathrm{~m} \mathrm{Ba}$ & 16.38 & & & & & & $\longrightarrow$ \\
\hline${ }^{234} \mathrm{~Pa}$ & 0.0034 & ${ }^{144} \mathrm{Ce}$ & 14.67 & & & & & & 100.00 \\
\hline${ }^{235} \mathrm{U}$ & 0.0258 & ${ }^{144} \mathrm{Pr}$ & 14.67 & & & & & & \\
\hline \multirow[t]{6}{*}{${ }^{238} \mathrm{U}$} & 33.197 & ${ }^{147} \mathrm{Pm}$ & 0.06 & & & & & & \\
\hline & & ${ }^{151} \mathrm{Sm}$ & 0.11 & & & & & & \\
\hline & 100.0000 & ${ }^{152} \mathrm{Eu}$ & 0.09 & & & & & & \\
\hline & & ${ }^{154} \mathrm{Eu}$ & 0.09 & & & & & & \\
\hline & & ${ }^{15 s} \mathrm{Eu}$ & 0.06 & & & & & & \\
\hline & & & 100.00 & & & & & & \\
\hline
\end{tabular}

${ }^{\mathrm{a}}$ Based on ref. 1. 
Table A.4. Average concentrations for representative radionuclides in LLW at commercial disposal sites ${ }^{a}$

\begin{tabular}{|c|c|c|}
\hline Radionuclide & Half-life ${ }^{b}$ & $\begin{array}{l}\text { Concentration } \\
\left(\mathrm{Ci} / \mathrm{m}^{3}\right)\end{array}$ \\
\hline${ }^{3} \mathrm{H}$ & $1.228 E+01 y$ & $1.083 \mathrm{E}+00$ \\
\hline${ }^{14} \mathrm{C}$ & $5.730 E+03 y$ & $5.079 \mathrm{E}-03$ \\
\hline${ }^{26} \mathrm{Al}$ & $7.300 E+05 y$ & $2.980 \mathrm{E}-10$ \\
\hline${ }^{32} \mathrm{Si}$ & $1.000 E+02 y$ & $3.725 \mathrm{E}-11$ \\
\hline${ }^{32} \mathrm{P}$ & $1.428 E+01 d$ & $9.292 \mathrm{E}-04$ \\
\hline${ }^{35} \mathrm{~S}$ & $8.751 E+01 d$ & $2.208 \mathrm{E}-03$ \\
\hline${ }^{36} \mathrm{Cl}$ & $3.010 E+05 y$ & $6.143 \mathrm{E}-06$ \\
\hline${ }^{40} \mathrm{~K}$ & $1.280 E+09 y$ & $1.766 \mathrm{E}-07$ \\
\hline${ }^{\mathrm{s1}} \mathrm{Cr}$ & $2.770 E+01 d$ & $7.137 \mathrm{E}-02$ \\
\hline${ }^{54} \mathrm{Mn}$ & $3.122 E+02 d$ & $3.895 \mathrm{E}-01$ \\
\hline${ }^{55} \mathrm{Fe}$ & $2.730 E+00 y$ & $3.112 E+00$ \\
\hline${ }^{99} \mathrm{Fe}$ & $4.445 E+01 d$ & $5.081 E-03$ \\
\hline${ }^{58} \mathrm{Co}$ & $7.092 \mathrm{E}+01 \mathrm{~d}$ & $2.047 \mathrm{E}-01$ \\
\hline${ }^{60} \mathrm{Co}$ & $5.271 E+00 y$ & $2.242 E+00$ \\
\hline${ }^{s 9} \mathrm{Ni}$ & $7.500 E+04 y$ & $1.364 \mathrm{E}-03$ \\
\hline${ }^{63} \mathrm{Ni}$ & $1.001 E+02 y$ & $2.692 \mathrm{E}-01$ \\
\hline${ }^{65} \mathrm{Zn}$ & $2.441 E+02 d$ & $1.174 \mathrm{E}-01$ \\
\hline${ }^{85} \mathrm{Kr}$ & $1.072 E+01 y$ & $8.147 \mathrm{E}-04$ \\
\hline${ }^{89} \mathrm{Sr}$ & $5.055 E+01 d$ & $6.032 \mathrm{E}-03$ \\
\hline${ }^{90} \mathrm{Sr}$ & $2.850 E+01 y$ & $6.987 \mathrm{E}-02$ \\
\hline${ }^{90} \mathrm{Y}$ & $2.671 E+01 d$ & $6.987 \mathrm{E}-02$ \\
\hline${ }^{91} \mathrm{Y}$ & $5.851 E+01 d$ & $8.859 E-03$ \\
\hline${ }^{95} \mathrm{Zr}$ & $6.402 E+01 d$ & $1.036 \mathrm{E}-02$ \\
\hline${ }^{24} \mathrm{Nb}$ & $2.030 E+04 y$ & $1.659 \mathrm{E}-05$ \\
\hline${ }^{95} \mathrm{Nb}$ & $3.497 E+01 d$ & $1.916 \mathrm{E}-02$ \\
\hline${ }^{93} \mathrm{Mo}$ & $3.500 E+03 y$ & $9.273 \mathrm{E}-12$ \\
\hline${ }^{99} \mathrm{Tc}$ & $2.130 E+05 y$ & $1.949 \mathrm{E}-04$ \\
\hline${ }^{103} \mathrm{Ru}$ & $3.925 \mathrm{E}+01 \mathrm{~d}$ & $5.900 \mathrm{E}-04$ \\
\hline${ }^{108 \mathrm{~m}} \mathrm{Ag}$ & $1.300 \mathrm{E}+02 \mathrm{y}$ & $5.534 E-06$ \\
\hline${ }^{110 \mathrm{~m}} \mathrm{Ag}$ & $2.498 E+02 d$ & $3.600 \mathrm{E}-02$ \\
\hline${ }^{113} \mathrm{Cd}$ & $9.000 \mathrm{E}+15 \mathrm{y}$ & $4.223 E-12$ \\
\hline${ }^{124} \mathrm{Sb}$ & $6.020 E+00 d$ & $2.621 \mathrm{E}-03$ \\
\hline${ }^{125} \mathrm{Sb}$ & $2.730 E+00 y$ & $1.901 \mathrm{E}-02$ \\
\hline${ }^{123} \mathrm{Te}$ & $1.300 E+13 y$ & $5.710 \mathrm{E}-07$ \\
\hline${ }^{125} \mathrm{I}$ & $6.014 E+00 d$ & $4.570 \mathrm{E}-04$ \\
\hline${ }^{129} \mathrm{~T}$ & $1.570 E+07 y$ & $2.101 E-05$ \\
\hline${ }^{131} \mathrm{I}$ & $8.040 E+00 d$ & $5.299 \mathrm{E}-03$ \\
\hline${ }^{134} \mathrm{Cs}$ & $2.062 E+00 y$ & $8.661 \mathrm{E}-02$ \\
\hline${ }^{135} \mathrm{Cs}$ & $3.000 E+06 y$ & $1.105 E-05$ \\
\hline${ }^{137} \mathrm{Cs}$ & $3.017 E+01 y$ & $2.431 \mathrm{E}-01$ \\
\hline${ }^{137 m} \mathrm{Ba}$ & $2.552 \mathrm{E}+00 \mathrm{~min}$ & $2.300 \mathrm{E}-01$ \\
\hline${ }^{141} \mathrm{Ce}$ & $3.250 \mathrm{E}+01 \mathrm{~d}$ & $1.649 \mathrm{E}-03$ \\
\hline${ }^{144} \mathrm{Ce}$ & $2.849 \mathrm{E}+02 \mathrm{~d}$ & $1.463 \mathrm{E}-02$ \\
\hline
\end{tabular}


Table A.4 (continued)

\begin{tabular}{ccc}
\hline Radionuclide & Half-life ${ }^{\mathrm{b}}$ & $\begin{array}{c}\text { Concentration } \\
\left(\mathrm{Ci} / \mathrm{m}^{3}\right)\end{array}$ \\
\hline${ }^{144} \mathrm{Pr}$ & $1.728 \mathrm{E}+01 \mathrm{~min}$ & $1.463 \mathrm{E}-02$ \\
${ }^{144} \mathrm{Nd}$ & $2.100 \mathrm{E}+15 \mathrm{y}$ & $1.689 \mathrm{E}-10$ \\
${ }^{147} \mathrm{Pm}$ & $2.623 \mathrm{E}+00 \mathrm{y}$ & $1.317 \mathrm{E}-02$ \\
${ }^{157} \mathrm{~Tb}$ & $1.100 \mathrm{E}+02 \mathrm{y}$ & $1.012 \mathrm{E}-10$ \\
${ }^{158} \mathrm{~Tb}$ & $1.800 \mathrm{E}+02 \mathrm{y}$ & $3.768 \mathrm{E}-10$ \\
${ }^{175} \mathrm{Hf}$ & $7.000 \mathrm{E}+01 \mathrm{~d}$ & $1.427 \mathrm{E}-03$ \\
${ }^{181} \mathrm{Hf}$ & $4.240 \mathrm{E}+01 \mathrm{~d}$ & $3.235 \mathrm{E}-03$ \\
${ }^{187} \mathrm{Re}$ & $4.100 \mathrm{E}+10 \mathrm{y}$ & $1.772 \mathrm{E}-11$ \\
${ }^{209} \mathrm{Po}$ & $3.253 \mathrm{E}+00 \mathrm{~h}$ & $1.284 \mathrm{E}-10$ \\
${ }^{226} \mathrm{Ra}$ & $1.600 \mathrm{E}+03 \mathrm{y}$ & $2.852 \mathrm{E}-04$ \\
${ }^{229} \mathrm{Th}$ & $7.340 \mathrm{E}+03 \mathrm{y}$ & $1.310 \mathrm{E}-10$ \\
${ }^{230} \mathrm{Th}$ & $7.540 \mathrm{E}+04 \mathrm{y}$ & $1.721 \mathrm{E}-08$ \\
${ }^{232} \mathrm{Th}$ & $1.405 \mathrm{E}+10 \mathrm{y}$ & $8.482 \mathrm{E}-03$ \\
${ }^{231} \mathrm{~Pa}$ & $3.276 \mathrm{E}+04 \mathrm{y}$ & $1.016 \mathrm{E}-10$ \\
${ }^{233} \mathrm{U}$ & $1.592 \mathrm{E}+05 \mathrm{y}$ & $2.308 \mathrm{E}-07$ \\
${ }^{234} \mathrm{U}$ & $2.454 \mathrm{E}+05 \mathrm{y}$ & $5.368 \mathrm{E}-05$ \\
${ }^{235} \mathrm{U}$ & $7.037 \mathrm{E}+08 \mathrm{y}$ & $3.179 \mathrm{E}-05$ \\
${ }^{236} \mathrm{U}$ & $2.432 \mathrm{E}+07 \mathrm{y}$ & $7.886 \mathrm{E}-07$ \\
${ }^{238} \mathrm{U}$ & $4.468 \mathrm{E}+09 \mathrm{y}$ & $9.970 \mathrm{E}-03$ \\
${ }^{237} \mathrm{~Np}$ & $2.140 \mathrm{E}+06 \mathrm{y}$ & $2.210 \mathrm{E}-07$ \\
${ }^{239} \mathrm{Pu}$ & $2.413 \mathrm{E}+04 \mathrm{y}$ & $1.021 \mathrm{E}-05^{\mathrm{c}}$ \\
${ }^{240} \mathrm{Pu}$ & $6.563 \mathrm{E}+03 \mathrm{y}$ & $2.504 \mathrm{E}-06 \mathrm{c}$ \\
${ }^{242} \mathrm{Pu}$ & $3.763 \mathrm{E}+05 \mathrm{y}$ & $6.148 \mathrm{E}-07 \mathrm{c}$ \\
${ }^{241} \mathrm{Am}$ & $4.322 \mathrm{E}+02 \mathrm{y}$ & $4.053 \mathrm{E}-05$ \\
${ }^{243} \mathrm{Am}$ & $7.380 \mathrm{E}+03 \mathrm{y}$ & $1.398 \mathrm{E}-08$ \\
${ }^{248} \mathrm{Cm}$ & $3.400 \mathrm{E}+05 \mathrm{y}$ & $6.220 \mathrm{E}-07$ \\
\hline & & $8.380 \mathrm{E}+00$ \\
\hline
\end{tabular}

${ }^{a}$ Adapted from ref. 5.

$b_{y}=$ years; $d=$ days; $h=$ hours; $\min =$ minutes; and $s=$ seconds.

Isotopes of plutonium are omitted when this list is applied to waste disposed at Barnwell, South Carolina, because this site has not permitted disposal of plutonium (even though traces of plutonium could have entered with other wastes). 
Table A.5. Estimated sources and characteristics of commercial Greater-Than-Class-C LLWa

\begin{tabular}{|c|c|c|}
\hline Waste source & Physical form & $\begin{array}{l}\text { Primary isotopes of concern } \\
\text { for disposal }\end{array}$ \\
\hline \multicolumn{3}{|r|}{ 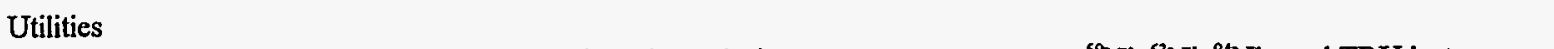 } \\
\hline Reactor operations & $\begin{array}{l}\text { Activated metals, instruments, } \\
\text { filters, ion-exchange resins, sludges }\end{array}$ & ${ }^{59} \mathrm{Ni},{ }^{63} \mathrm{Ni},{ }^{94} \mathrm{Nb}$, and TRU isotopes \\
\hline Reactor decommissioning & Activated metals & ${ }^{59} \mathrm{Ni},{ }^{63} \mathrm{Ni}$, and ${ }^{94} \mathrm{Nb}$ \\
\hline \multicolumn{3}{|l|}{ Fuel testing labs } \\
\hline Burnup lab operation & $\begin{array}{l}\text { Solidified liquids, metal cuttings, } \\
\text { glassware, equipment, ion-exchange } \\
\text { resins }\end{array}$ & ${ }^{90} \mathrm{Sr}$ and TRU isotopes \\
\hline Burnup lab decommissioning & $\begin{array}{l}\text { Solidified liquids, metals, glassware, } \\
\text { equipment }\end{array}$ & ${ }^{90} \mathrm{Sr}$ and TRU isotopes \\
\hline \multicolumn{3}{|l|}{ Sealed sources } \\
\hline Manufacturer operations & Trash, metal, foils & ${ }^{14} \mathrm{C},{ }^{90} \mathrm{Sr},{ }^{137} \mathrm{Cs},{ }^{241} \mathrm{Am}$, and $\mathrm{Pu}$ isotopes \\
\hline Manufacturer decommissioning & Trąsh, metal, foils & ${ }^{14} \mathrm{C},{ }^{90} \mathrm{Sr},{ }^{137} \mathrm{Cs},{ }^{241} \mathrm{Am}$, and $\mathrm{Pu}$ isotopes \\
\hline Sources designated as waste & Sealed sources & ${ }^{137} \mathrm{Cs},{ }^{238} \mathrm{Pu},{ }^{239} \mathrm{Pu}$, and ${ }^{241} \mathrm{Am}$ \\
\hline \multicolumn{3}{|l|}{ Other } \\
\hline${ }^{14} \mathrm{C}$ users & Solidified process liquids & ${ }^{14} \mathrm{C}$ \\
\hline Test and research reactors & Activated metals & ${ }^{39} \mathrm{Ni},{ }^{94} \mathrm{Nb}$, and TRU isotopes \\
\hline Other & Soil, trash & ${ }^{241} \mathrm{Am}$ \\
\hline
\end{tabular}

a Gleaned from information given in refs. 6 and 7. 
Table A.6. Historical and projected number and volume of drums and classes of $\mathrm{LLW}$ incorporated in cement to be generated in the WVDP Low-Level Radwaste Treatment System ${ }^{a, b}, c, d$

\begin{tabular}{|c|c|c|c|c|}
\hline \multirow{2}{*}{$\begin{array}{l}\text { End of } \\
\text { calendar } \\
\text { year }\end{array}$} & \multicolumn{3}{|c|}{ Number of drums } & \multirow{2}{*}{$\begin{array}{l}\text { Total volume } \\
\text { of drums } \\
\left(\mathrm{m}^{3}\right)\end{array}$} \\
\hline & Class $\mathrm{A}^{\mathrm{e}}$ & Class B & Class $\mathrm{C}^{\mathrm{e}}$ & \\
\hline 1987 & $726^{f}$ & $g$ & - & 189 \\
\hline 1988 & - & $\mathrm{g}$ & 2,009 & 523 \\
\hline 1989 & - & $\mathrm{g}$ & 4,523 & 1,177 \\
\hline $1990^{\mathrm{h}}$ & - & $\mathrm{g}$ & 3,862 & 1,055 \\
\hline 1991 & - & $\mathrm{g}$ & 0 & 0 \\
\hline 1992 & 1,268 & $g$ & 268 & 399 \\
\hline 1993 & 2,387 & $\mathrm{~g}$ & 1,298 & 959 \\
\hline 1994 & - & $\mathrm{g}$ & 2,641 & 687 \\
\hline 1995 & - & $\mathrm{g}$ & 1,632 & 425 \\
\hline Total number & 4,381 & $\mathrm{~g}$ & 16,238 & \\
\hline Total volume, $\mathrm{m}^{3}$ & 1,140 & $\mathrm{~g}$ & 4,225 & 5,414 \\
\hline
\end{tabular}

aThe so-called square drums used are parallelepipeds of square cross sections $(-0.6 \mathrm{~m} \times 0.6 \mathrm{~m} \times 0.8 \mathrm{~m})$ with a volume of $71 \mathrm{gal}\left(0.27 \mathrm{~m}^{3}\right)$.

${ }^{b}$ The classes are in accordance with the Classes $(A, B$, or $C)$ as set by requirements of the NRC in 10 CFR Part 61.55.

Taken from ref. 8.

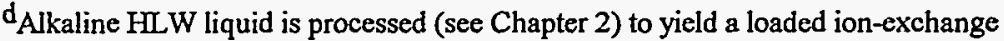
material (zeolite), which is $\mathrm{HLW}$, and an effluent, which is LLW. This effluent is solidified with cement.

'Stored in a shielded drum cell.

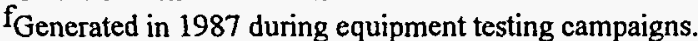

gNo Class-B LLW is expected to be generated with the effluent mentioned in footnote d.

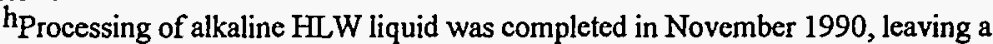
$1,090-\mathrm{m}^{3}$ heel of liquid in the alkaline HLW waste (liquid plus sludge) storage tank. 


\section{APPENDIX B. CHARACTERISTICS OF IMPORTANT RADIONUCLIDES}




\section{APPENDIX B. CHARACTERISTICS OF IMPORTANT RADIONUCLMES}

\section{B.1 DISCUSSION}

The following Table B.1 lists radionuclides whose characteristics are most often referenced in the variety of studies and evaluations discussed in Chapters 1-7. It includes isotopes for HLW, TRUW, LLW, and UMT as defined by EPA, ${ }^{1}$ NRC, ${ }^{2,3}$ and DOE. ${ }^{4,5}$ The data in Table B.1 were obtained from refs. 6-9.

\section{B.2 REFERENCES}

1. U.S. Environmental Protection Agency, "Environmental Radiation Protection Standards for Management and Disposal of Spent Nuclear Fuel, High-Level and Transuranic Radioactive Wastes," Code of Federal Regulations, 40 CFR Part 191 (July 1, 1995).

2. U.S. Nuclear Regulatory Commission, "Licensing Requirements for Land Disposal of Radioactive Waste," Code of Federal Regulations, 10 CFR Part 61 (Jan. 1, 1995).

3. U.S. Nuclear Regulatory Commission, "Biomedical Waste Disposal," Fed. Regist. 46(47), 16230-16234 (Mar. 11, 1981).

4. U.S. Department of Energy, DOE Order 5820.2A, Radioactive Waste Management, Washington, D.C., Sept. 26, 1988.

5. U.S. Department of Energy, Energy Information Administration, Domestic Uranium Mining and Milling Industry 1992-Viability Assessment, DOE/EIA-0477(92), Washington, D.C. (December 1993).

6. D. C. Kocher, Radioactive Decay Data Tables, DOE/TIC-11026, U.S. Department of Energy, Washington, D.C. (1981).

7. D. C. Kocher, A Radionuclide Decay Data Base-Index and Summary Table, NUREG/CR-1413, ORNL/NUREG-70, Oak Ridge National Laboratory, Oak Ridge, Tennessee (May 1980).

8. E. Browne and R. B. Firestone, Table of Radioactive Isotopes, ed. V. S. Shirley, John Wiley and Sons, Inc., New York (1986).

9. A. G. Croff, ORIGEN2-A Revised and Updated Version of the Oak Ridge Isotope Generation and Depletion Code, ORNL-5621, Oak Ridge National Laboratory, Oak Ridge, Tennessee (July 1980). 
Table B.1. Characteristics of important radionuclides ${ }^{\mathrm{a}}$

\begin{tabular}{|c|c|c|c|c|c|c|c|c|c|c|}
\hline \multirow{2}{*}{ Nuclide } & \multirow{2}{*}{$\begin{array}{l}\text { Atomic } \\
\text { number }\end{array}$} & \multirow{2}{*}{ Half-life ${ }^{b}$} & \multirow{2}{*}{$\begin{array}{l}\text { Principal } \\
\text { mode(s) of } \\
\text { decayc }\end{array}$} & \multicolumn{3}{|c|}{$\begin{array}{l}\text { Major radiation energies }{ }^{d} \\
(\mathrm{MeV} / \mathrm{dis})\end{array}$} & \multicolumn{2}{|c|}{ "Q" value } & \multirow{2}{*}{$\begin{array}{c}\text { Specific } \\
\text { activity } \\
(\mathrm{Ci} / \mathrm{g})\end{array}$} & \multirow{2}{*}{ Daughter(s) } \\
\hline & & & & $\alpha$ & $\mathbf{E}$ & $\gamma$ and $X$ & $(\mathrm{MeV} / \mathrm{dis})$ & $(\mathrm{W} / \mathrm{Ci})$ & & \\
\hline${ }^{3} \mathrm{H}$ & 1 & $1.233 \mathrm{E}+01 \mathrm{y}$ & $\beta$ & & 0.00568 & & $5.68 \mathrm{E}-03$ & $3.37 \mathrm{E}-05$ & $9.650 \mathrm{E}+03$ & ${ }^{3} \mathrm{He}$ \\
\hline${ }^{14} \mathrm{C}$ & 6 & $5.730 \mathrm{E}+03 \mathrm{y}$ & $\beta$ & & 0.0495 & & $4.95 \mathrm{E}-02$ & $2.93 \mathrm{E}-04$ & 4.457 & ${ }^{14} \mathrm{~N}$ \\
\hline${ }^{26} \mathrm{Al}$ & 13 & $7.2 \mathrm{E}+05 \mathrm{y}$ & $\mathrm{EC}$ & & 0.4451 & 2.6758 & 3.079 & $1.825 \mathrm{E}-02$ & $1.91 \mathrm{E}-02$ & ${ }^{26} \mathrm{Mg}$ \\
\hline${ }^{32} \mathrm{Si}$ & 14 & $650 y$ & $\beta$ & & 0.0647 & & $2.10 \mathrm{E}-01$ & $1.245 E-03$ & $1.719 \mathrm{E}+01$ & ${ }^{32} \mathrm{P}$ \\
\hline${ }^{32} \mathrm{P}$ & 15 & $14.282 \mathrm{~d}$ & $\beta$ & & 0.6947 & & $6.95 E-01$ & $4.12 E-03$ & $2.853 \mathrm{E}+05$ & ${ }^{32} \mathrm{~S}$ \\
\hline${ }^{35} \mathrm{~S}$ & 16 & $87.51 \mathrm{~d}$ & $\beta$ & & 0.0486 & & $4.86 \mathrm{E}-02$ & $2.88 \mathrm{E}-04$ & $4.263 E+04$ & ${ }^{35} \mathrm{Cl}$ \\
\hline${ }^{36} \mathrm{Cl}$ & 17 & $3.01 E+05 y$ & $\begin{array}{l}\beta(98.1 \%) \\
\operatorname{EC}(1.9 \%)\end{array}$ & & 0.2460 & & $2.460 \mathrm{E}-01$ & $1.458 \mathrm{E}-03$ & $3.299 \mathrm{E}-02$ & ${ }^{36} \mathrm{Ar}$ \\
\hline${ }^{40} \mathrm{~K}$ & 19 & $1.277 \mathrm{E}+09 \mathrm{y}$ & $\begin{array}{l}\beta(89.33 \%): \\
\operatorname{EC}(10.67 \%)\end{array}$ & & 0.4545 & 0.1559 & $6.104 E-01$ & $3.62 \mathrm{E}-03$ & $6.983 \mathrm{E}-06$ & $\begin{array}{l}{ }^{40} \mathrm{Ca} \\
{ }^{40} \mathrm{Ar}\end{array}$ \\
\hline${ }^{45} \mathrm{Ca}$ & 20 & $163.8 \mathrm{~d}$ & $\beta$ & & 0.0770 & & $7.70 \mathrm{E}-02$ & $4.56 \mathrm{E}-04$ & $1.780 \mathrm{E}+04$ & ${ }^{45} \mathrm{Sc}$ \\
\hline${ }^{46} \mathrm{Sc}$ & 21 & $83.83 \mathrm{~d}$ & $\beta$ & 0.1120 & 2.0095 & & 2.122 & $1.257 \mathrm{E}-02$ & $3.381 E+04$ & ${ }^{46} \mathrm{Ti}$ \\
\hline${ }^{\mathrm{s1}} \mathrm{Cr}$ & 24 & $27.704 \mathrm{~d}$ & $\mathrm{EC}$ & & 0.0031 & 0.0325 & $3.56 \mathrm{E}-02$ & $2.11 \mathrm{E}-04$ & $9.240 \mathrm{E}+04$ & ${ }^{s 1} \mathrm{~V}$ \\
\hline${ }^{54} \mathrm{Mn}$ & 25 & $312.20 \mathrm{~d}$ & $\mathrm{EC}$ & & 0.0034 & 0.8360 & $8.394 \mathrm{E}-01$ & $4.975 \mathrm{E}-03$ & $7.738 \mathrm{E}+03$ & ${ }^{54} \mathrm{Cr}$ \\
\hline${ }^{s 5} \mathrm{Fe}$ & 26 & $2.73 y$ & $\mathrm{EC}$ & & 0.0038 & 0.0016 & $5.4 \mathrm{E}-03$ & $3.2 \mathrm{E}-05$ & $2.500 \mathrm{E}+03$ & ${ }^{55} \mathrm{Mn}$ \\
\hline${ }^{59} \mathrm{Fe}$ & 26 & $44.496 \mathrm{~d}$ & $\beta$ & & 0.1174 & 1.1882 & 1.3056 & $7.741 E-03$ & $4.918 E+04$ & ${ }^{59} \mathrm{Co}$ \\
\hline${ }^{57} \mathrm{Co}$ & 27 & $271.77 \mathrm{~d}$ & $\mathrm{EC}$ & & 0.0176 & 0.1252 & $1.428 \mathrm{E}-01$ & $8.464 \mathrm{E}-01$ & $8.456 \mathrm{E}+03$ & ${ }^{37} \mathrm{Fe}$ \\
\hline${ }^{58} \mathrm{Co}$ & 27 & $70.92 \mathrm{~d}$ & $\mathrm{EC}$ & & 0.0336 & 0.9758 & 1.0094 & $5.99 \mathrm{E}-03$ & $3.181 E+04$ & ${ }^{38} \mathrm{Fe}$ \\
\hline${ }^{60} \mathrm{Co}$ & 27 & $5.271 \mathrm{y}$ & $\beta$ & & 0.0958 & 2.5058 & 2.6016 & $1.541 \mathrm{E}-02$ & $1.131 E+03$ & ${ }^{60} \mathrm{Ni}$ \\
\hline${ }^{60 \pi} \mathrm{Co}$ & 27 & $10.47 \mathrm{~min}$ & $\begin{array}{l}\text { IT }(99.75 \%) \\
\beta(0.25 \%)\end{array}$ & & 0.0536 & 0.0066 & $6.02 \mathrm{E}-02$ & $3.57 \mathrm{E}-04$ & $2.993 E+08$ & $\begin{array}{l}{ }^{60} \mathrm{Co} ; \\
{ }^{60} \mathrm{Ni}\end{array}$ \\
\hline${ }^{59} \mathrm{Ni}$ & 28 & $7.5 E+04$ y & EC & & 0.0043 & 0.0024 & $6.72 E-03$ & $3.98 \mathrm{E}-05$ & $8.079 \mathrm{E}-02$ & ${ }^{59} \mathrm{Co}$ \\
\hline${ }^{63} \mathrm{Ni}$ & 28 & $1.001 \mathrm{E}+02 \mathrm{y}$ & $\beta$ & & 0.0171 & & $1.71 \mathrm{E}-02$ & $1.01 \mathrm{E}-04$ & $6.168 E+01$ & ${ }^{63} \mathrm{Cu}$ \\
\hline
\end{tabular}


Table B.1 (continued)

\begin{tabular}{|c|c|c|c|c|c|c|c|c|c|c|}
\hline \multirow{2}{*}{ Nuclide } & \multirow{2}{*}{$\begin{array}{l}\text { Atomic } \\
\text { number }\end{array}$} & \multirow{2}{*}{ Half-life ${ }^{b}$} & \multirow{2}{*}{$\begin{array}{l}\text { Principal } \\
\text { mode(s) of } \\
\text { decayc }\end{array}$} & \multicolumn{3}{|c|}{$\begin{array}{l}\text { Major radiation energies }{ }^{d} \\
\text { (MeV/dis) }\end{array}$} & \multicolumn{2}{|c|}{ "Q" value e } & \multirow{2}{*}{$\begin{array}{c}\text { Specific } \\
\text { activity } \\
(\mathrm{Ci} / \mathrm{g})\end{array}$} & \multirow{2}{*}{ Daughter(s) } \\
\hline & & & & $\alpha$ & $\epsilon$ & $\gamma$ and $X$ & (MeV/dis) & (W/Ci) & & \\
\hline${ }^{65} \mathrm{Zn}$ & 30 & $244.1 \mathrm{~d}$ & $\mathrm{EC}$ & & 0.0066 & 0.5838 & $5.90 \mathrm{E}-01$ & $3.51 \mathrm{E}-03$ & $8.237 \mathrm{E}+03$ & ${ }^{65} \mathrm{Cu}$ \\
\hline${ }^{67} \mathrm{Ga}$ & 31 & $3.261 \mathrm{~d}$ & $\mathrm{EC}$ & & 0.0333 & 0.1549 & $1.882 \mathrm{E}-01$ & $1.115 \mathrm{E}-03$ & $5.975 \mathrm{E}+05$ & ${ }^{67} \mathrm{Zn}$ \\
\hline${ }^{75} \mathrm{Se}$ & 34 & $119.77 d$ & $\mathrm{EC}$ & & 0.0134 & 0.3924 & $4.06 \mathrm{E}-01$ & $2.41 E-03$ & $1.453 E+04$ & ${ }^{75}$ As \\
\hline${ }^{79} \mathrm{Se}$ & 34 & $<6.5 \mathrm{E}+04 \mathrm{y}$ & & & 0.0529 & & $5.29 \mathrm{E}-02$ & $3.13 E-04$ & $6.966 \mathrm{E}-02$ & ${ }^{79} \mathrm{Br}$ \\
\hline${ }^{85} \mathrm{Kr}$ & 36 & $1.072 \mathrm{E}+01 \mathrm{y}$ & $\beta$ & & 0.2505 & 0.0022 & $2.53 \mathrm{E}-01$ & $1.50 \mathrm{E}-03$ & $3.923 E+02$ & ${ }^{85} \mathrm{Rb}$ \\
\hline${ }^{86} \mathrm{Rb}$ & 37 & $18.66 \mathrm{~d}$ & $\beta$ & & 0.6670 & 0.0945 & $7.62 \mathrm{E}-01$ & $4.52 E-03$ & $8.138 E+04$ & ${ }^{86} \mathrm{Sr}$ \\
\hline${ }^{89} \mathrm{Sr}$ & 38 & $50.55 \mathrm{~d}$ & $\beta$ & & 0.5829 & 0.0001 & $5.83 \mathrm{E}-01$ & $3.46 \mathrm{E}-03$ & $2.905 E+04$ & ${ }^{89} \mathrm{Y}$ \\
\hline${ }^{90} \mathrm{Sr}$ & 38 & $2.85 E+01 y$ & $\beta$ & & 0.1958 & & $1.96 \mathrm{E}-01$ & $1.16 \mathrm{E}-03$ & $1.364 E+02$ & ${ }^{90} \mathrm{Y}$ \\
\hline${ }^{90} Y$ & 39 & $2.671 \mathrm{~d}$ & $\beta$ & & 0.9332 & & $9.33 E-01$ & $5.54 \mathrm{E}-03$ & $5.441 E+05$ & $90 \mathrm{Zr}$ \\
\hline${ }^{91} \mathrm{Y}$ & 39 & $58.51 \mathrm{~d}$ & $\beta$ & & 0.6039 & 0.0036 & $6.07 \mathrm{E}-01$ & $3.60 \mathrm{E}-03$ & $2.452 E+04$ & ${ }^{91} \mathrm{Zr}$ \\
\hline${ }^{93} \mathrm{Zr}$ & 40 & $1.53 E+06 y$ & $\beta$ & & 0.0471 & 0.0018 & $4.89 \mathrm{E}-02$ & $2.90 \mathrm{E}-04$ & $2.513 E-03$ & ${ }^{93} \mathrm{Nb}$ \\
\hline${ }^{95} \mathrm{Zr}$ & 40 & $64.02 \mathrm{~d}$ & $\beta$ & & 0.1200 & 0.7337 & 8.54E-01 & $5.06 \mathrm{E}-03$ & $2.148 E+04$ & ${ }^{95} \mathrm{Nb}$ \\
\hline${ }^{93 \mathrm{~m}} \mathrm{Nb}$ & 41 & $1.36 \mathrm{E}+01 \mathrm{y}$ & IT & & 0.0281 & 0.0018 & $2.99 \mathrm{E}-02$ & $1.77 \mathrm{E}-04$ & $2.826 \mathrm{E}+02$ & ${ }^{93} \mathrm{Nb}$ \\
\hline${ }^{94} \mathrm{Nb}$ & 41 & $2.03 E+04 y$ & $\beta$ & & 0.1454 & 1.5715 & 1.7169 & $1.018 \mathrm{E}-02$ & $1.873 \mathrm{E}-01$ & ${ }^{94} \mathrm{Mo}$ \\
\hline${ }^{95} \mathrm{Nb}$ & 41 & $34.97 \mathrm{~d}$ & $\beta$ & & 0.0435 & 0.7643 & $8.078 \mathrm{E}-01$ & $4.788 \mathrm{E}-03$ & $3.910 \mathrm{E} \div 04$ & ${ }^{95} \mathrm{Mo}$ \\
\hline${ }^{93} \mathrm{Mo}$ & 42 & $3500 y$ & $\mathrm{EC}$ & & 0.0051 & 0.0107 & $1.58 \mathrm{E}-02$ & $9.37 \mathrm{E}-05$ & 1.10 & ${ }^{93} \mathrm{Nb}$ \\
\hline${ }^{99} \mathrm{Mo}$ & 42 & $2.748 \mathrm{~d}$ & $\beta$ & & 0.4076 & 0.2723 & $6.799 \mathrm{E}-01$ & $4.028 \mathrm{E}-03$ & $4.796 \mathrm{E}+05$ & ${ }^{99} \mathrm{Tc}$ \\
\hline${ }^{99} \mathrm{Tc}$ & 43 & $2.13 E+05 y$ & $\beta$ & & 0.0846 & & $8.46 \mathrm{E}-02$ & $5.01 \mathrm{E}-04$ & $1.695 \mathrm{E}-02$ & ${ }^{99} \mathrm{Ru}$ \\
\hline${ }^{9 m} \mathrm{Te}$ & 43 & $6.006 \mathrm{~h}$ & IT & & 0.0142 & 0.1240 & $1.382 \mathrm{E}-01$ & $8.186 E-04$ & $5.271 \mathrm{E}+06$ & ${ }^{99} \mathrm{Tc}$ \\
\hline${ }^{103} \mathrm{Ru}$ & 44 & $39.254 \mathrm{~d}$ & $\beta$ & & 0.1105 & 0.4851 & $5.96 \mathrm{E}-01$ & $3.53 \mathrm{E}-03$ & $3.227 \mathrm{E}+04$ & ${ }^{103} \mathrm{Rh}$ \\
\hline${ }^{106} \mathrm{Ru}$ & 44 & $1.020 \mathrm{y}$ & $\beta$ & & 0.1004 & & $1.004 \mathrm{E}-01$ & $5.951 \mathrm{E}-04$ & $3.346 \mathrm{E}+03$ & ${ }^{106} \mathrm{Rh}$ \\
\hline${ }^{103 m} \mathrm{Rh}$ & 45 & $56.12 \mathrm{~min}$ & IT & & 0.0375 & 0.0017 & $3.92 \mathrm{E}-02$ & $2.32 \mathrm{E}-04$ & $3.253 E+07$ & ${ }^{103} \mathrm{Rh}$ \\
\hline${ }^{106} \mathrm{Rh}$ & 45 & $2.17 \mathrm{~h}$ & $\beta$ & & 0.3144 & 2.8826 & 3.197 & $1.894 \mathrm{E}-02$ & $3.560 \mathrm{E}+09$ & ${ }^{106} \mathrm{Pd}$ \\
\hline
\end{tabular}


Table B.1 (continued)

\begin{tabular}{|c|c|c|c|c|c|c|c|c|c|c|}
\hline \multirow{2}{*}{ Nuclide } & \multirow{2}{*}{$\begin{array}{l}\text { Atomic } \\
\text { number }\end{array}$} & \multirow{2}{*}{ Half-life ${ }^{b}$} & \multirow{2}{*}{$\begin{array}{l}\text { Principal } \\
\text { mode(s) of } \\
\text { decayc }\end{array}$} & \multicolumn{3}{|c|}{$\begin{array}{l}\text { Major radiation energies }{ }^{d} \\
\text { (MeV/dis) }\end{array}$} & \multicolumn{2}{|c|}{ "Q" value } & \multirow{2}{*}{$\begin{array}{l}\text { Specific } \\
\text { activity } \\
(\mathrm{Ci} / \mathrm{g})\end{array}$} & \multirow{2}{*}{ Daughter(s) } \\
\hline & & & & $\alpha$ & $\epsilon$ & $\gamma$ and $X$ & (MeV/dis) & $(\mathrm{W} / \mathrm{Ci})$ & & \\
\hline${ }^{107} \mathrm{Pd}$ & 46 & $6.5 E+06 y$ & $\beta$ & & & 0.0093 & $9.3 E-03$ & $5.5 \mathrm{E}-0.5$ & $5.143 E-04$ & ${ }^{107} \mathrm{Ag}$ \\
\hline${ }^{110} \mathrm{Ag}$ & 47 & $24.6 \mathrm{~s}$ & $\begin{array}{l}\beta(99.70 \%) \\
\operatorname{EC}(0.30 \%)\end{array}$ & & 1.1842 & 0.0316 & 1.216 & $7.208 \mathrm{E}-03$ & $4.169 \mathrm{E}+09$ & $\begin{array}{l}{ }^{110} \mathrm{Cd} ; \\
{ }^{110} \mathrm{Pd}\end{array}$ \\
\hline${ }^{110+n} \mathrm{Ag}$ & 47 & $249.76 \mathrm{~d}$ & $\begin{array}{l}\beta(98.64 \%) \\
\text { IT }(1.36 \%)\end{array}$ & & 0.0755 & 2.7392 & 2.815 & $1.669 E-02$ & $4.750 \mathrm{E}+03$ & $\begin{array}{l}{ }^{110} \mathrm{Cd} ; \\
{ }^{110} \mathrm{Ag}\end{array}$ \\
\hline${ }^{113} \mathrm{Cd}$ & 48 & $9.3 \mathrm{E}+15 \mathrm{y}$ & $\beta$ & & 0.0933 & & $9.13 E-02$ & $5.412 \mathrm{E}-04$ & $3.402 \mathrm{E}-13$ & ${ }^{113} \mathrm{In}$ \\
\hline${ }^{113 m} \mathrm{Cd}$ & 48 & $1.37 \mathrm{E}+01 \mathrm{y}$ & $\begin{array}{l}\beta(99.9 \%) \\
\text { IT }(0.1 \%)\end{array}$ & & & 0.1834 & $1.83 E-01$ & $1.08 \mathrm{E}-03$ & $2.168 \mathrm{E}+02$ & $\begin{array}{l}{ }^{113} \mathrm{In} ; \\
{ }^{113} \mathrm{Cd}\end{array}$ \\
\hline${ }^{115 m} \mathrm{Cd}$ & 48 & $44.6 \mathrm{~d}$ & $\beta$ & & 0.6029 & 0.0329 & $6.36 \mathrm{E}-01$ & $3.76 \mathrm{E}-03$ & $2.546 \mathrm{E}+04$ & ${ }^{115}$ In \\
\hline${ }^{111}$ In & 49 & $2.807 d$ & $\mathrm{EC}$ & & 0.0340 & 0.4053 & $4.393 E-01$ & $2.604 E-03$ & $4.157 \mathrm{E}+05$ & ${ }^{111} \mathrm{Cd}$ \\
\hline $113 m$ In & 49 & $1.658 \mathrm{~h}$ & IT & & 0.1340 & 0.2555 & $3.89 \mathrm{E}-01$ & $2.31 E-03$ & $1.673 E+07$ & ${ }^{113}$ In \\
\hline 1144m In & 49 & $49.51 \mathrm{~d}$ & $\begin{array}{l}\text { IT }(95.7 \%) \\
\text { EC }(4.3 \%)\end{array}$ & & 0.1431 & 0.0943 & $2.37 \mathrm{E}-01$ & $1.40 \mathrm{E}-03$ & $2.313 E+04$ & $\begin{array}{l}{ }^{114} \mathrm{In} ; \\
{ }^{114} \mathrm{Cd}\end{array}$ \\
\hline${ }^{113} \mathrm{Sn}$ & 50 & $115.09 \mathrm{~d}$ & $\mathrm{EC}$ & & 0.1394 & 0.2808 & $4.20 \mathrm{E}-01$ & $2.48 E-03$ & $1.004 \mathrm{E}+04$ & ${ }^{113}$ In \\
\hline${ }^{117 m} S n$ & 50 & $13.61 \mathrm{~d}$ & IT & & 0.1613 & 0.1580 & $3.19 E-01$ & $1.89 E-03$ & $7.969 E+04$ & ${ }^{117} \mathrm{Sn}$ \\
\hline${ }^{119 m} \mathrm{Sn}$ & 50 & $293.0 \mathrm{~d}$ & IT & & 0.0783 & 0.0114 & $8.97 \mathrm{E}-02$ & $5.32 \mathrm{E}-04$ & $4.478 E+03$ & ${ }^{119} \mathrm{Sn}$ \\
\hline${ }^{121 m} \mathrm{Sn}$ & 50 & $5.5 \mathrm{E}+01 \mathrm{y}$ & $\begin{array}{l}\text { IT }(77.6 \%) \\
\beta(22.4 \%)\end{array}$ & & 0.0352 & 0.0050 & $4.02 \mathrm{E}-02$ & $2.43 \mathrm{E}-04$ & $5.912 \mathrm{E}+01$ & $\begin{array}{l}{ }^{121} \mathrm{Sn} ; \\
{ }^{121} \mathrm{Sb}\end{array}$ \\
\hline${ }^{123} \mathrm{Sn}$ & 50 & $129.2 \mathrm{~d}$ & $\beta$ & & 0.5222 & 0.0069 & $5.29 E-01$ & $3.14 E-03$ & $8.219 E+03$ & ${ }^{123} \mathrm{Sb}$ \\
\hline${ }^{125} \mathrm{Sn}$ & 50 & $9.64 \mathrm{~d}$ & $\beta$ & & 0.8110 & 0.3124 & 1.123 & $6.656 \mathrm{E}-03$ & $1.084 \mathrm{E}+05$ & ${ }^{125} \mathrm{Sb}$ \\
\hline${ }^{126} \mathrm{Sn}$ & 50 & $\sim 1 E+05 y$ & $\beta$ & & 0.1249 & 0.0573 & $1.82 \mathrm{E}-01$ & $1.08 \mathrm{E}-03$ & $2.837 \mathrm{E}-02$ & ${ }^{126} \mathrm{Sb}$ \\
\hline${ }^{124} \mathrm{Sb}$ & 51 & $60.20 \mathrm{~d}$ & $\beta$ & & 0.3897 & 1.8523 & 2.242 & $1.329 \mathrm{E}-02$ & $1.749 \mathrm{E}+04$ & ${ }^{124} \mathrm{Te}$ \\
\hline${ }^{125} \mathrm{Sb}$ & 51 & $2.73 \mathrm{y}$ & $\beta$ & & 0.1257 & 0.4434 & $5.69 \mathrm{E}-01$ & $3.37 \mathrm{E}-03$ & $1.032 E+03$ & ${ }^{125} \mathrm{Te}$ \\
\hline${ }^{126} \mathrm{Sb}$ & 51 & $12.4 \mathrm{~d}$ & $\beta$ & & 0.3527 & 2.7496 & 3.102 & $1.839 \mathrm{E}-02$ & $8.360 \mathrm{E}+04$ & ${ }^{126} \mathrm{Te}$ \\
\hline${ }^{126 m} \mathrm{Sb}$ & 51 & $19.0 \mathrm{~min}$ & $\begin{array}{l}\beta(86 \%) ; \\
\text { IT }(14 \%)\end{array}$ & & 0.6323 & 1.5484 & 2.181 & $1.292 \mathrm{E}-02$ & $7.854 \mathrm{E}+07$ & $\begin{array}{l}{ }^{126} \mathrm{Te} \\
{ }^{126} \mathrm{Sb}\end{array}$ \\
\hline${ }^{123 \mathrm{~m}} \mathrm{Te}$ & 52 & $119.7 \mathrm{~d}$ & IT & & 0.1020 & 0.1482 & $2.502 E-01$ & $1.482 \mathrm{E}-03$ & $8.870 \mathrm{E}+03$ & ${ }^{123} \mathrm{Te}$ \\
\hline${ }^{125 \mathrm{~m}} \mathrm{Te}$ & 52 & $58 \mathrm{~d}$ & IT & & 0.1106 & 0.0361 & $1.467 \mathrm{E}-01$ & $8.690 \mathrm{E}-04$ & $1.801 \mathrm{E}+04$ & ${ }^{125} \mathrm{Te}$ \\
\hline${ }^{127} \mathrm{Te}$ & 52 & $9.35 \mathrm{~h}$ & $\beta$ & & 0.2248 & 0.0048 & $2.30 \mathrm{E}-01$ & $1.36 E-03$ & $2.639 E+06$ & ${ }^{127} \mathrm{I}$ \\
\hline
\end{tabular}


Table B.1 (continued)

\begin{tabular}{|c|c|c|c|c|c|c|c|c|c|c|}
\hline \multirow{2}{*}{ Nuclide } & \multirow{2}{*}{$\begin{array}{l}\text { Atomic } \\
\text { number }\end{array}$} & \multirow{2}{*}{ Half-life $^{b}$} & \multirow{2}{*}{$\begin{array}{c}\text { Principal } \\
\text { mode(s) of } \\
\text { decayc }\end{array}$} & \multicolumn{3}{|c|}{$\begin{array}{l}\text { Major radiation energies }{ }^{d} \\
\text { (MeV/dis) }\end{array}$} & \multicolumn{2}{|c|}{ "Q" value e } & \multirow{2}{*}{$\begin{array}{c}\text { Specific } \\
\text { activity } \\
(\mathrm{Ci} / \mathrm{g})\end{array}$} & \multirow{2}{*}{ Daughter(s) } \\
\hline & & & & $\alpha$ & $\epsilon$ & $\gamma$ and $X$ & (MeV/dis) & $(\mathrm{W} / \mathrm{Ci})$ & & \\
\hline${ }^{127 \mathrm{~m}} \mathrm{Te}$ & 52 & $109 \mathrm{~d}$ & $\begin{array}{l}\text { IT (97.6\%); } \\
\beta(2.4 \%)\end{array}$ & & 0.0821 & 0.0111 & $9.32 \mathrm{E}-02$ & $5.52 \mathrm{E}-04$ & $9.432 E+03$ & ${ }^{127} \mathrm{Te} ;$ \\
\hline${ }^{129} \mathrm{Te}$ & 52 & $1.160 \mathrm{~h}$ & $\beta$ & & 0.5422 & 0.0624 & $6.05 \mathrm{E}-01$ & $3.58 \mathrm{E}-03$ & $2.094 E+07$ & ${ }^{129} \mathrm{I}$ \\
\hline${ }^{129 \mathrm{~m} T \mathrm{Te}}$ & 52 & $33.6 \mathrm{~d}$ & $\begin{array}{l}\text { IT }(64 \%) \\
\beta(36 \%)\end{array}$ & & 0.2663 & 0.0370 & $3.03 \mathrm{E}-01$ & $1.80 \mathrm{E}-03$ & $3.013 E+04$ & ${ }^{129} \mathrm{Te}$ \\
\hline${ }^{123} \mathrm{I}$ & 53 & $13.2 \mathrm{~h}$ & EC & & 0.0276 & 0.1729 & $2.005 E-01$ & $1.188 E-03$ & $1.940 E \div 06$ & ${ }^{123} \mathrm{Te}$ \\
\hline $125 \mathrm{I}$ & 53 & $60.14 d$ & $\mathrm{EC}$ & & 0.0179 & 0.0423 & $6.02 \mathrm{E}-02$ & $3.57 E-04$ & $1.737 E+04$ & ${ }^{125} \mathrm{Te}$ \\
\hline${ }^{129} \mathrm{I}$ & 53 & $1.57 \mathrm{E}+07 y$ & $\beta$ & & 0.0556 & 0.0248 & $8.04 \mathrm{E}-02$ & 4.77E-04 & $1.765 \mathrm{E}-04$ & ${ }^{129} \mathrm{Xe}$ \\
\hline${ }^{131} \mathrm{I}$ & 53 & $8.040 \mathrm{~d}$ & $\beta$ & & 0.1913 & 0.3826 & $5.74 \mathrm{E}-01$ & $3.40 \mathrm{E}-03$ & $1.240 \mathrm{E}+05$ & ${ }^{231} \mathrm{Xe}$ \\
\hline${ }^{133} \mathrm{Xe}$ & 54 & $5.245 \mathrm{~d}$ & $\beta$ & & 0.1363 & 0.0459 & $1.82 \mathrm{E}-01$ & $1.08 \mathrm{E}-03$ & $1.872 \mathrm{E}+05$ & ${ }^{133} \mathrm{Cs}$ \\
\hline${ }^{134} \mathrm{Cs}$ & 55 & $2.062 \mathrm{y}$ & $\beta$ & & 0.1639 & 1.5555 & 1.719 & $1.019 \mathrm{E}-02$ & $1.294 E+03$ & ${ }^{134} \mathrm{Ba}$ \\
\hline${ }^{135} \mathrm{Cs}$ & 55 & $3.0 \mathrm{E}+06 \mathrm{y}$ & $\beta$ & & 0.0563 & & $5.63 \mathrm{E}-02$ & $3.32 \mathrm{E}-04$ & $1.151 E-03$ & ${ }^{135} \mathrm{Ba}$ \\
\hline${ }^{137} \mathrm{Cs}$ & 55 & $3.017 \mathrm{E}+01 \mathrm{y}$ & $\begin{array}{l}\beta(94.6 \%) \\
\beta(5.4 \%)\end{array}$ & & & 0.1708 & $1.71 \mathrm{E}-01$ & $1.01 \mathrm{E}-03$ & $8.698 \mathrm{E}+01$ & $\begin{array}{l}{ }^{137 m} \mathrm{Ba} ; \\
{ }^{137} \mathrm{Ba}\end{array}$ \\
\hline${ }^{133} \mathrm{Ba}$ & 56 & $1.054 E+01 y$ & $\mathrm{EC}$ & & 0.0547 & 0.4045 & $4.592 \mathrm{E}-01$ & $2.722 \mathrm{E}-03$ & $2.500 \mathrm{E}+02$ & ${ }^{133} \mathrm{Cs}$ \\
\hline${ }^{137 m} \mathrm{Ba}$ & 56 & $2.552 \mathrm{~min}$ & IT & & 0.0652 & 0.5991 & $6.64 \mathrm{E}-01$ & $3.94 \mathrm{E}-03$ & $5.379 \mathrm{E}+08$ & ${ }^{137} \mathrm{Ba}$ \\
\hline${ }^{141} \mathrm{Ce}$ & 58 & $32.50 \mathrm{~d}$ & $\beta$ & & 0.1707 & 0.0770 & $2.48 \mathrm{E}-01$ & $1.47 \mathrm{E}-03$ & $2.848 \mathrm{E}+04$ & ${ }^{141} \mathrm{Pr}$ \\
\hline${ }^{144} \mathrm{Ce}$ & 58 & $284.9 \mathrm{~d}$ & $\beta$ & & 0.0918 & 0.0192 & $1.11 \mathrm{E}-01$ & $6.58 \mathrm{E}-04$ & $3.190 E+03$ & ${ }^{144} \mathrm{Pr}$ \\
\hline${ }^{143} \operatorname{Pr}$ & 59 & $13.58 \mathrm{~d}$ & $\beta$ & & 0.3156 & & $3.16 \mathrm{E}-01$ & $1.87 \mathrm{E}-03$ & $6.731 E+04$ & ${ }^{143} \mathrm{Nd}$ \\
\hline${ }^{144} \mathrm{Pr}$ & 59 & $17.28 \mathrm{~min}$ & $\beta$ & & 1.2091 & 0.0289 & 1.238 & $7.338 \mathrm{E}-03$ & $7.555 E+07$ & ${ }^{144} \mathrm{Nd}$ \\
\hline${ }^{144 m} \mathrm{Pr}$ & 59 & $7.2 \mathrm{~min}$ & $\begin{array}{l}\text { IT }(99.96 \%) \\
\beta(0.04 \%)\end{array}$ & & 0.0464 & 0.0121 & $5.85 \mathrm{E}-02$ & $3.43 E-04$ & $1.814 \mathrm{E}+08$ & ${ }^{144} \mathrm{Pr}$ \\
\hline${ }^{146} \mathrm{Pm}$ & 61 & $5.53 \mathrm{y}$ & $\begin{array}{l}\text { EC }(66.1 \%) \\
\beta(33.9 \%)\end{array}$ & & 0.0928 & 0.7542 & $8.47 \mathrm{E}-01$ & $5.02 \mathrm{E}-03$ & $4.428 \mathrm{E}+02$ & $\begin{array}{l}{ }^{146} \mathrm{Nd} ; \\
{ }^{146} \mathrm{Sm}\end{array}$ \\
\hline${ }^{147} \mathrm{Pm}$ & 61 & $2.6234 \mathrm{y}$ & $\beta$ & & 0.6196 & & $6.20 \mathrm{E}-02$ & $3.67 \mathrm{E}-04$ & $9.270 \mathrm{E}+02$ & ${ }^{147} \mathrm{Sm}$ \\
\hline${ }^{148} \mathrm{Pm}$ & 61 & $5.370 \mathrm{~d}$ & $\beta$ & & 0.7235 & 0.5747 & 1.298 & $7.691 \mathrm{E}-03$ & $1.643 E+05$ & ${ }^{148} \mathrm{Sm}$ \\
\hline${ }^{148 m} \mathrm{Pm}$ & 61 & $41.29 d$ & $\begin{array}{l}\beta(95.4 \%) \\
\text { IT }(4.6 \%)\end{array}$ & & 0.1695 & 1.9861 & 2.156 & $1.278 \mathrm{E}-02$ & $2.136 \mathrm{E}+04$ & $\begin{array}{l}{ }^{148} \mathrm{Sm} \\
{ }^{148} \mathrm{Pm}\end{array}$ \\
\hline
\end{tabular}


Table B.1 (continued)

\begin{tabular}{|c|c|c|c|c|c|c|c|c|c|c|}
\hline \multirow{2}{*}{ Nuclide } & \multirow{2}{*}{$\begin{array}{l}\text { Atomic } \\
\text { number }\end{array}$} & \multirow{2}{*}{ Half-life ${ }^{b}$} & \multirow{2}{*}{$\begin{array}{l}\text { Principal } \\
\text { mode(s) of } \\
\text { decayc }\end{array}$} & \multicolumn{3}{|c|}{$\begin{array}{c}\text { Major radiation energies } \\
\text { (McV/dis) }\end{array}$} & \multicolumn{2}{|c|}{ "Q" value } & \multirow{2}{*}{$\begin{array}{r}\text { Specific } \\
\text { activity } \\
(\mathrm{Ci} / \mathrm{g})\end{array}$} & \multirow{2}{*}{ Daughter(s) } \\
\hline & & & & $\alpha$ & $\epsilon$ & $\gamma$ and $X$ & (MeV/dis) & $(\mathrm{W} / \mathrm{Ci})$ & & \\
\hline${ }^{151} \mathrm{Sm}$ & 62 & $9.0 \mathrm{E}+01 \mathrm{y}$ & $\beta$ & & 0.1251 & & $1.25 \mathrm{E}-01$ & $7.41 \mathrm{E}-04$ & $2.631 \mathrm{E}+01$ & ${ }^{151} \mathrm{Eu}$ \\
\hline${ }^{152} \mathrm{Eu}$ & 63 & $1.333 \mathrm{E}+01 \mathrm{y}$ & $\begin{array}{l}\text { EC }(72.08 \%) \\
\beta(27.92 \%)\end{array}$ & & 0.1275 & 1.1628 & 1.290 & $7.646 \mathrm{E}-03$ & $1.729 E+02$ & $\begin{array}{l}{ }^{152} \mathrm{Sm} \\
{ }^{152} \mathrm{Gd}\end{array}$ \\
\hline${ }^{154} \mathrm{Eu}$ & 63 & $8.8 \mathrm{y}$ & $\beta$ & & 0.2794 & 1.2531 & 1.532 & $9.081 E-03$ & $2.699 E+02$ & ${ }^{154} \mathrm{Gd}$ \\
\hline${ }^{13 s} \mathrm{Eu}$ & 63 & $4.96 \mathrm{y}$ & $\beta$ & & 0.0650 & 0.0633 & $1.28 \mathrm{E}-01$ & $7.59 \mathrm{E}-04$ & $4.651 \mathrm{E}+02$ & ${ }^{155} \mathrm{Gd}$ \\
\hline${ }^{153} \mathrm{Gd}$ & 64 & $241.6 \mathrm{~d}$ & $\mathrm{EC}$ & & 0.0399 & 0.1015 & $1.414 \mathrm{E}-01$ & $8.381 E-04$ & $3.526 \mathrm{E}+03$ & ${ }^{153} \mathrm{Eu}$ \\
\hline${ }^{157} \mathrm{~Tb}$ & 65 & $150 y$ & $\mathrm{EC}$ & & 0.0031 & 0.0050 & $8.10 \mathrm{E}-03$ & $4.802 \mathrm{E}-05$ & $1.519 \mathrm{E}+01$ & ${ }^{157} \mathrm{Gd}$ \\
\hline${ }^{158} \mathrm{~Tb}$ & 65 & $150 y$ & $\begin{array}{l}E C(82 \%) \\
\beta(18 \%)\end{array}$ & & & & $9.02 \mathrm{E}-01$ & $5.347 \mathrm{E}-03$ & $1.508 \mathrm{E}+01$ & ${ }^{158} \mathrm{Gd}$ \\
\hline${ }^{160} \mathrm{~Tb}$ & 65 & $72.3 \mathrm{~d}$ & $\beta$ & & 0.2535 & 1.1271 & 1.381 & $8.186 \mathrm{E}-03$ & $1.129 E+04$ & ${ }^{160}$ Dy \\
\hline${ }^{169} \mathrm{Yb}$ & 70 & $32.02 \mathrm{~d}$ & $\mathrm{EC}$ & & 0.1117 & 0.3121 & $4.238 \mathrm{E}-01$ & $2.512 \mathrm{E}-03$ & $2.414 \mathrm{E}+04$ & ${ }^{169} \mathrm{Tm}$ \\
\hline${ }^{175} \mathrm{Hf}$ & 72 & $70.0 \mathrm{~d}$ & EC & & 0.0439 & 0.3646 & $4.085 \mathrm{E}-01$ & $2.422 \mathrm{E}-03$ & $1.066 \mathrm{E}+04$ & ${ }^{175} \mathrm{Lu}$ \\
\hline${ }^{181} \mathrm{Hf}$ & 72 & $42.39 \mathrm{~d}$ & $\beta$ & & 0.1943 & 0.5441 & $7.54 \mathrm{E}-01$ & $4.47 \mathrm{E}-03$ & $1.702 \mathrm{E}+04$ & ${ }^{181} \mathrm{Ta}$ \\
\hline${ }^{182} \mathrm{Ta}$ & 73 & $115.0 \mathrm{~d}$ & $\beta$ & & 0.2073 & 1.3011 & 1.508 & $8.940 \mathrm{E}-03$ & $6.253 E+03$ & ${ }^{182} \mathrm{~W}$ \\
\hline${ }^{187} \mathrm{Re}$ & 75 & $4.6 \mathrm{E}+10 \mathrm{y}$ & $\beta$ & & 0.0007 & & 2.59 & $1.535 \mathrm{E}-02$ & $3.823 E-08$ & ${ }^{187} \mathrm{Os}$ \\
\hline${ }^{192} \mathrm{Ir}$ & 77 & $73.831 \mathrm{~d}$ & $\begin{array}{l}\beta(95.4 \%) \\
\operatorname{EC}(4.6 \%)\end{array}$ & & 0.2162 & 0.8137 & 1.030 & $6.105 E-03$ & $9.211 \mathrm{E}+03$ & $\begin{array}{l}{ }^{192} \mathrm{Pt} \text {; } \\
{ }^{192} \mathrm{Os}\end{array}$ \\
\hline${ }^{201} \mathrm{TI}$ & 81 & $3.046 \mathrm{~d}$ & $\mathrm{EC}$ & & 0.0481 & 0.0924 & $1.40 E-01$ & $8.30 \mathrm{E}-04$ & $2.132 \mathrm{E}+05$ & ${ }^{201} \mathrm{Hg}$ \\
\hline${ }^{207} \mathrm{Tl}$ & 81 & $4.77 \mathrm{~min}$ & $\beta$ & & 0.4931 & 0.0022 & $4.95 \mathrm{E}-01$ & $2.93 E-03$ & $1.904 \mathrm{E}+08$ & ${ }^{207} \mathrm{~Pb}$ \\
\hline${ }^{208} \mathrm{Tl}$ & 81 & $3.053 \mathrm{~min}$ & $\beta$ & & 0.5979 & 3.3742 & 3.972 & $2.354 \mathrm{E}-02$ & $2.945 E+08$ & ${ }^{208} \mathrm{~Pb}$ \\
\hline${ }^{209} \mathrm{~Pb}$ & 82 & $3.253 \mathrm{~h}$ & $\beta$ & & 0.1980 & & $1.98 \mathrm{E}-01$ & $1.17 \mathrm{E}-03$ & $4.544 \mathrm{E}+06$ & ${ }^{209} \mathrm{Bi}$ \\
\hline${ }^{211} \mathrm{~Pb}$ & 82 & $36.1 \mathrm{~min}$ & $\beta$ & & 0.4523 & 0.0678 & $5.20 \mathrm{E}-01$ & $3.083 E-03$ & $2.468 E+07$ & ${ }^{211} \mathrm{Bi}$ \\
\hline${ }^{212} \mathrm{~Pb}$ & 82 & $10.64 \mathrm{~h}$ & $\beta$ & & 0.1752 & 0.1453 & $3.20 \mathrm{E}-01$ & $1.90 \mathrm{E}-03$ & $1.389 E+06$ & ${ }^{212} \mathrm{Bi}$ \\
\hline${ }^{211} \mathrm{Bi}$ & 83 & $2.14 \mathrm{~min}$ & $\begin{array}{l}\alpha(99.727 \%) \\
\beta(0.273 \%)\end{array}$ & 6.5505 & 0.0099 & 0.0467 & 6.607 & $3.916 \mathrm{E}-02$ & $4.184 \mathrm{E}+08$ & $\begin{array}{l}{ }^{207} \mathrm{Tl} \\
{ }^{211} \mathrm{Po}\end{array}$ \\
\hline
\end{tabular}


Table B.1 (continued)

\begin{tabular}{|c|c|c|c|c|c|c|c|c|c|c|}
\hline \multirow{2}{*}{ Nuclide } & \multirow{2}{*}{$\begin{array}{l}\text { Atomic } \\
\text { number }\end{array}$} & \multirow{2}{*}{ Half-life ${ }^{b}$} & \multirow{2}{*}{$\begin{array}{c}\text { Principal } \\
\text { mode(s) of } \\
\text { decayc }\end{array}$} & \multicolumn{3}{|c|}{$\begin{array}{l}\text { Major radiation energies }{ }^{d} \\
\text { (MeV/dis) }\end{array}$} & \multicolumn{2}{|c|}{ " $Q$ " value } & \multirow{2}{*}{$\begin{array}{c}\text { Specific } \\
\text { activity } \\
(\mathrm{Ci} / \mathrm{g})\end{array}$} & \multirow{2}{*}{ Daughter(s) } \\
\hline & & & & $\alpha$ & $\epsilon$ & $\gamma$ and $X$ & (MeV/dis) & $(\mathrm{W} / \mathrm{Ci})$ & & \\
\hline${ }^{212} \mathrm{Bi}$ & 83 & $1.0092 \mathrm{~h}$ & $\begin{array}{l}\alpha(35.94 \%) \\
\beta(64.06 \%)\end{array}$ & 2.1740 & 0.5025 & 0.1061 & 2.783 & $1.649 \mathrm{E}-02$ & $1.465 E+07$ & $\begin{array}{l}{ }^{208} \mathrm{Tl} ; \\
{ }^{212} \mathrm{Po}\end{array}$ \\
\hline${ }^{213} \mathrm{Bi}$ & 83 & $45.59 \mathrm{~min}$ & $\begin{array}{l}\alpha(2.16 \%) \\
\beta(97.84 \%)\end{array}$ & 0.1268 & 0.4563 & 0.0825 & $6.66 \mathrm{E}-01$ & $3.95 \mathrm{E}-03$ & $1.934 E+07$ & $\begin{array}{l}{ }^{209} \mathrm{Tl} ; \\
{ }^{213} \mathrm{Po}\end{array}$ \\
\hline${ }^{209} \mathrm{Po}$ & 84 & $102 y$ & $\begin{array}{l}\alpha(99.74 \%) \\
\mathrm{EC}(0.26 \%)\end{array}$ & & & & 4.9645 & $2.943 \mathrm{E}-01$ & $1.68 \mathrm{E}+01$ & ${ }^{205} \mathrm{~Pb}$ \\
\hline${ }^{212} \mathrm{Po}$ & 84 & $2.98 \mathrm{E}-07 \mathrm{~s}$ & $\alpha$ & 8.7844 & & & 8.784 & $5.207 \mathrm{E}-02$ & $1.774 E+17$ & ${ }^{208} \mathrm{~Pb}$ \\
\hline${ }^{213} \mathrm{Po}$ & 84 & $4.2 \mathrm{E}-06 \mathrm{~s}$ & $\alpha$ & 8.3757 & & & 8.375 & $4.964 \mathrm{E}-02$ & $1.261 E+16$ & ${ }^{209} \mathrm{~Pb}$ \\
\hline${ }^{215} \mathrm{Po}$ & 84 & $1.780 \mathrm{E}-03 \mathrm{~s}$ & $\alpha$ & 7.3864 & & & 7.386 & $4.378 \mathrm{E}-02$ & $2.948 E+13$ & ${ }^{211} \mathrm{~Pb}$ \\
\hline${ }^{216} \mathrm{Po}$ & 84 & $1.50 \mathrm{E}-02 \mathrm{~s}$ & $\alpha$ & 6.7785 & & & 6.779 & $4.018 \mathrm{E}-02$ & $3.482 \mathrm{E}+11$ & ${ }^{212} \mathrm{~Pb}$ \\
\hline${ }^{217} \mathrm{At}$ & 85 & $3.23 \mathrm{E}-02 \mathrm{~s}$ & $\alpha$ & 7.0657 & & 0.0002 & 7.066 & $4.189 \mathrm{E}-02$ & $1.610 \mathrm{E}+12$ & ${ }^{213} \mathrm{Bi}$ \\
\hline${ }^{219} \mathrm{Rn}$ & 86 & $3.96 \mathrm{~s}$ & $\alpha$ & 6.8122 & 0.0064 & 0.0560 & 6.875 & $4.076 \mathrm{E}-02$ & $1.301 E+10$ & ${ }^{215}$ Po \\
\hline${ }^{220} \mathrm{Rn}$ & 86 & $55.6 \mathrm{~s}$ & $\alpha$ & 6.2878 & & 0.0005 & 6.288 & $3.727 \mathrm{E}-02$ & $9.223 E+08$ & ${ }^{216} \mathrm{Po}$ \\
\hline${ }^{222} \mathrm{Rn}$ & 86 & $3.825 \mathrm{~d}$ & $\alpha$ & 5.4892 & & 0.0004 & 5.490 & $3.255 \mathrm{E}-02$ & $1.538 \mathrm{E}+05$ & ${ }^{218} \mathrm{Po}$ \\
\hline${ }^{221} \mathrm{Fr}$ & 87 & $4.9 \mathrm{~min}$ & $\alpha$ & 6.3571 & 0.0084 & 0.0277 & 6.393 & $3.789 \mathrm{E}-02$ & $1.772 E+08$ & ${ }^{217}$ At \\
\hline${ }^{223} \mathrm{Fr}$ & 87 & $21.8 \mathrm{~min}$ & $\beta$ & & 0.3805 & 0.0542 & $4.35 \mathrm{E}-01$ & $2.85 \mathrm{E}-03$ & $3.868 \mathrm{E}+07$ & ${ }^{223} \mathrm{Ra}$ \\
\hline${ }^{223} \mathrm{Ra}$ & 88 & $11.43 \mathrm{~d}$ & $\alpha$ & 5.6972 & 0.0731 & 0.1348 & 5.905 & $3.500 \mathrm{E}-02$ & $5.121 E+04$ & ${ }^{219} \mathrm{Rn}$ \\
\hline${ }^{224} \mathrm{Ra}$ & 88 & $3.66 \mathrm{~d}$ & $\alpha$ & 5.6751 & 0.0022 & 0.0103 & 5.688 & $3.372 \mathrm{E}-02$ & $1.593 E+05$ & ${ }^{220} \mathrm{Rn}$ \\
\hline${ }^{225} \mathrm{Ra}$ & 88 & $14.2 \mathrm{~d}$ & $\beta$ & & 0.1057 & 0.0137 & $1.19 \mathrm{E}-01$ & $7.08 \mathrm{E}-04$ & $3.920 \mathrm{E}+04$ & ${ }^{225} \mathrm{Ac}$ \\
\hline${ }^{226} \mathrm{Ra}$ & 88 & $1.600 E+03 y$ & $\alpha$ & 4.7741 & 0.0035 & 0.0067 & 4.784 & $2.836 \mathrm{E}-02$ & $9.887 \mathrm{E}-01$ & ${ }^{222} \mathrm{Rn}$ \\
\hline${ }^{228} \mathrm{Ra}$ & 88 & $5.75 \mathrm{y}$ & $\beta$ & & 0.0116 & & $1.16 \mathrm{E}-02$ & $6.88 \mathrm{E}-05$ & $2.340 \mathrm{E}+02$ & ${ }^{228} \mathrm{Ac}$ \\
\hline${ }^{225} \mathrm{Ac}$ & 89 & $10.0 \mathrm{~d}$ & $\alpha$ & 5.7501 & 0.0257 & 0.0176 & 5.793 & $3.434 \mathrm{E}-02$ & $5.803 E+04$ & ${ }^{221} \mathrm{Fr}$ \\
\hline${ }^{227} \mathrm{Ac}$ & 89 & $2.177 E+01 y$ & $\begin{array}{l}\beta(98.62 \%) ; \\
\alpha(1.38 \%)\end{array}$ & 0.0673 & 0.0125 & 0.0002 & $8.00 \mathrm{E}-02$ & $4.74 \mathrm{E}-04$ & $7.233 E+01$ & ${ }^{227} \mathrm{Th} ;$ \\
\hline${ }^{228} \mathrm{Ac}$ & 89 & $6.13 \mathrm{~h}$ & $\beta$ & & 0.4292 & 0.9269 & 1.356 & $8.038 \mathrm{E}-03$ & $2.242 E+06$ & ${ }^{228} \mathrm{Th}$ \\
\hline${ }^{227} \mathrm{Th}$ & 90 & $18.718 \mathrm{~d}$ & $\alpha$ & 5.9022 & 0.0543 & 0.1113 & 6.068 & $3.597 \mathrm{E}-02$ & $3.073 E+04$ & ${ }^{223} \mathrm{Ra}$ \\
\hline${ }^{228} \mathrm{Th}$ & 90 & $1.913 \mathrm{y}$ & $\alpha$ & 5.3992 & 0.0201 & 0.0034 & 5.423 & $3.214 \mathrm{E}-02$ & $8.196 E+02$ & ${ }^{224} \mathrm{Ra}$ \\
\hline${ }^{229} \mathrm{Th}$ & 90 & $7.340 \mathrm{E}+03 y$ & $\alpha$ & 4.8620 & & 0.0343 & 4.896 & $2.902 \mathrm{E}-02$ & $2.127 E-01$ & ${ }^{225} \mathrm{Ra}$ \\
\hline${ }^{230} \mathrm{Th}$ & 90 & $7.54 \mathrm{E}+04 \mathrm{y}$ & $\alpha$ & 4.6651 & & 0.0004 & 4.665 & $2.765 E-02$ & $2.109 \mathrm{E}-02$ & ${ }^{226} \mathrm{Ra}$ \\
\hline
\end{tabular}


Table B.1 (continued)

\begin{tabular}{|c|c|c|c|c|c|c|c|c|c|c|}
\hline \multirow{2}{*}{ Nuclide } & \multirow{2}{*}{$\begin{array}{l}\text { Atomic } \\
\text { number }\end{array}$} & \multirow{2}{*}{ Half-life ${ }^{b}$} & \multirow{2}{*}{$\begin{array}{l}\text { Principal } \\
\text { mode(s) of } \\
\text { decayc }\end{array}$} & \multicolumn{3}{|c|}{$\begin{array}{c}\text { Major radiation energies }{ }^{d} \\
(\mathrm{MeV} / \mathrm{dis})\end{array}$} & \multicolumn{2}{|c|}{ "Q" value } & \multirow{2}{*}{$\begin{array}{c}\text { Specific } \\
\text { activity } \\
(\mathrm{Ci} / \mathrm{g})\end{array}$} & \multirow{2}{*}{ Daughter(s) } \\
\hline & & & & $\alpha$ & $\epsilon$ & $\gamma$ and $X$ & (MeV/dis) & $(\mathrm{W} / \mathrm{Ci})$ & & \\
\hline${ }^{231} \mathrm{Th}$ & 90 & $1.0633 \mathrm{~d}$ & $\beta$ & 0.1732 & & 0.0295 & $2.03 E-01$ & $1.21 \mathrm{E}-03$ & $5.316 E+05$ & ${ }^{231} \mathrm{~Pa}$ \\
\hline${ }^{232} \mathrm{Th}$ & 90 & $1.405 E+10 y$ & $\alpha$ & 4.0056 & & 0.0002 & 4.006 & $2.375 \mathrm{E}-02$ & $1.097 \mathrm{E}-07$ & ${ }^{228} \mathrm{Ra}$ \\
\hline${ }^{234} \mathrm{Th}$ & 90 & $24.10 \mathrm{~d}$ & $\beta$ & & 0.0158 & 0.0094 & $2.52 E-02$ & $1.49 \mathrm{E}-04$ & $2.316 \mathrm{E}+04$ & ${ }^{234} \mathrm{~Pa}$ \\
\hline${ }^{231} \mathrm{~Pa}$ & 91 & $3.276 E+04 y$ & $\alpha$ & 4.9230 & 0.0483 & 0.0399 & 5.011 & $2.970 \mathrm{E}-02$ & $4.723 E-02$ & ${ }^{227} \mathrm{Ac}$ \\
\hline${ }^{233} \mathrm{~Pa}$ & 91 & $27.0 \mathrm{~d}$ & $\beta$ & & 0.1941 & 0.2042 & $3.98 E-01$ & $2.36 \mathrm{E}-03$ & $2.075 E+04$ & ${ }^{233} \mathrm{U}$ \\
\hline${ }^{234 \mathrm{~m}} \mathrm{~Pa}$ & 91 & $1.17 \mathrm{~min}$ & $\begin{array}{l}\beta(99.87 \%) \\
\text { IT }(0.13 \%)\end{array}$ & & 0.8227 & 0.0121 & $8.35 \mathrm{E}-01$ & $4.95 \mathrm{E}-03$ & $6.868 \mathrm{E}+08$ & ${ }^{234} \mathrm{U}$ \\
\hline${ }^{232} \mathrm{U}$ & 92 & $6.89 \mathrm{E}+01 \mathrm{y}$ & $\alpha$ & 5.3065 & & 0.0002 & 5.307 & $3.146 \mathrm{E}-02$ & $2.140 E+01$ & ${ }^{228} \mathrm{Th}$ \\
\hline${ }^{233} \mathrm{U}$ & 92 & $1.592 E+05 y$ & $\alpha$ & 4.8141 & 0.0055 & 0.0013 & 4.821 & $2.857 \mathrm{E}-02$ & $9.680 E-03$ & ${ }^{229} \mathrm{Th}$ \\
\hline${ }^{234} \mathrm{U}$ & 92 & $2.454 \mathrm{E}+05 \mathrm{y}$ & $\alpha$ & 4.7732 & & 0.0001 & 4.773 & $2.829 \mathrm{E}-02$ & $6.248 E-03$ & ${ }^{230} \mathrm{Th}$ \\
\hline${ }^{235} \mathrm{U}$ & 92 & $7.037 E+08 y$ & $\alpha$ & 4.3785 & 0.0426 & 0.1561 & 4.577 & $2.713 \mathrm{E}-02$ & $2.161 \mathrm{E}-06$ & ${ }^{231} \mathrm{Th}$ \\
\hline${ }^{236} \mathrm{U}$ & 92 & $2.342 E+07 y$ & $\alpha$ & 4.4793 & 0.0108 & 0.0015 & 4.492 & $2.662 \mathrm{E}-02$ & $6.469 \mathrm{E}-05$ & ${ }^{232} \mathrm{Th}$ \\
\hline${ }^{238} \mathrm{U}$ & 92 & $4.468 \mathrm{E}+09 \mathrm{y}$ & $\alpha$ & 4.1945 & 0.0095 & 0.0013 & 4.205 & $2.492 \mathrm{E}-02$ & $3.362 \mathrm{E}-07$ & ${ }^{234} \mathrm{Th}$ \\
\hline${ }^{236} \mathrm{~Np}$ & 93 & $1.550 \mathrm{E}+05 \mathrm{y}$ & $\begin{array}{l}\text { EC (91\%); } \\
\beta(8.9 \%) \\
\alpha(0.20 \%)\end{array}$ & & 0.1967 & 0.1411 & $3.38 \mathrm{E}-01$ & $2.00 \mathrm{E}-03$ & $1.317 \mathrm{E}-02$ & $\begin{array}{l}{ }^{236} \mathrm{U} ; \\
{ }^{236} \mathrm{Pu} ; \\
{ }^{232} \mathrm{~Pa}\end{array}$ \\
\hline${ }^{237} \mathrm{~Np}$ & 93 & $2.140 E+06 y$ & $\alpha$ & 4.7604 & 0.0640 & 0.0327 & 4.857 & $2.879 E-02$ & $7.049 \mathrm{E}-04$ & ${ }^{233} \mathrm{~Pa}$ \\
\hline${ }^{239} \mathrm{~Np}$ & 93 & $2.355 \mathrm{~d}$ & $\beta$ & & 0.2521 & 0.1740 & $4.26 \mathrm{E}-01$ & $2.53 \mathrm{E}-03$ & $2.320 \mathrm{E}+05$ & ${ }^{239} \mathrm{Pu}$ \\
\hline${ }^{236} \mathrm{Pu}$ & 94 & $2.851 \mathrm{y}$ & $\alpha$ & 5.7521 & 0.0126 & 0.0020 & 5.767 & $3.418 \mathrm{E}-02$ & $5.313 E+02$ & ${ }^{232} \mathrm{U}$ \\
\hline${ }^{238} \mathrm{Pu}$ & 94 & $8.774 E+01 y$ & $\alpha$ & 5.4871 & 0.0099 & 0.0018 & 5.499 & 3.2593E-02 & $1.712 \mathrm{E}+01$ & ${ }^{234} \mathrm{U}$ \\
\hline${ }^{239} \mathrm{Pu}$ & 94 & $2.411 \mathrm{E}+04 \mathrm{y}$ & $\alpha$ & 5.1011 & & 0.0001 & 5.101 & $3.024 \mathrm{E}-02$ & $6.216 \mathrm{E}-02$ & ${ }^{235} U$ \\
\hline${ }^{240} \mathrm{Pu}$ & 94 & $6.563 E+03 y$ & $\alpha$ & 5.1549 & & & 5.155 & $3.056 \mathrm{E}-02$ & $2.279 \mathrm{E}-01$ & ${ }^{236} \mathrm{U}$ \\
\hline${ }^{241} \mathrm{Pu}$ & 94 & $1.44 \mathrm{E}+01 \mathrm{y}$ & $\beta$ & 0.0001 & & 0.0052 & $5.3 \mathrm{E}-03$ & $3.2 E-05$ & $1.030 \mathrm{E}+02$ & ${ }^{241} \mathrm{Am}$ \\
\hline${ }^{242} \mathrm{Pu}$ & 94 & $3.763 E+05 y$ & $\alpha$ & 4.8901 & 0.0081 & 0.0014 & 4.900 & $2.904 \mathrm{E}-02$ & $3.818 \mathrm{E}-03$ & ${ }^{238} \mathrm{U}$ \\
\hline${ }^{244} \mathrm{Pu}$ & 94 & $8.26 \mathrm{E}+07 \mathrm{y}$ & $\begin{array}{l}\alpha(99.875 \%) \\
\operatorname{SPF}(0.125 \%)\end{array}$ & 4.5751 & 0.0007 & 0.0001 & 4.576 & $2.712 \mathrm{E}-02$ & $1.774 \mathrm{E}-05$ & $\begin{array}{l}{ }^{240} \mathrm{U} ; \\
\text { (fission } \\
\text { products) }\end{array}$ \\
\hline${ }^{241} \mathrm{Am}$ & 95 & $4.327 E+02 y$ & $\alpha$ & 5.4801 & 0.0304 & 0.0287 & 5.539 & $3.283 \mathrm{E}-02$ & 3.432 & ${ }^{237} \mathrm{~Np}$ \\
\hline${ }^{242} \mathrm{Am}$ & 95 & $16.01 \mathrm{~h}$ & $\begin{array}{l}\beta(82.7 \%) ; \\
\operatorname{EC}(17.3 \%)\end{array}$ & & 0.1781 & 0.0180 & $1.96 \mathrm{E}-01$ & $1.16 \mathrm{E}-03$ & $8.084 E+05$ & $\begin{array}{l}{ }^{242} \mathrm{Cm} \\
{ }^{242} \mathrm{Pu}\end{array}$ \\
\hline
\end{tabular}


Table B.1 (continued)

\begin{tabular}{|c|c|c|c|c|c|c|c|c|c|c|}
\hline \multirow{2}{*}{ Nuclide } & \multirow{2}{*}{$\begin{array}{l}\text { Atomic } \\
\text { number }\end{array}$} & \multirow{2}{*}{ Half-life ${ }^{b}$} & \multirow{2}{*}{$\begin{array}{l}\text { Principal } \\
\text { mode(s) of } \\
\text { decayc }\end{array}$} & \multicolumn{3}{|c|}{$\begin{array}{l}\text { Major radiation energies } \\
\text { (MeV/dis) }\end{array}$} & \multicolumn{2}{|c|}{ "Q" value e } & \multirow{2}{*}{$\begin{array}{c}\text { Specific } \\
\text { activity } \\
(\mathrm{Ci} / \mathrm{g})\end{array}$} & \multirow{2}{*}{ Daughter(s) } \\
\hline & & & & $\alpha$ & $\epsilon$ & $\gamma$ and $X$ & (MeV/dis) & $(\mathrm{W} / \mathrm{Ci})$ & & \\
\hline${ }^{242 m} \mathrm{Am}$ & 95 & $1.41 \mathrm{E}+02 \mathrm{y}$ & $\begin{array}{l}\text { IT }(99.55 \%) \\
\alpha(0.45 \%)\end{array}$ & 0.0232 & 0.0403 & 0.0049 & $6.84 \mathrm{E}-02$ & $4.05 E-04$ & 9.718 & $\begin{array}{l}{ }^{242} \mathrm{Am} \\
{ }^{238} \mathrm{~Np}\end{array}$ \\
\hline${ }^{243} \mathrm{Am}$ & 95 & $7.380 E+03 y$ & $\alpha$ & 5.2656 & & 0.0481 & 5.3137 & $3.1496 \mathrm{E}-02$ & $1.993 \mathrm{E}-01$ & ${ }^{239} \mathrm{~Np}$ \\
\hline${ }^{242} \mathrm{Cm}$ & 96 & $162.94 \mathrm{~d}$ & $\alpha$ & 6.0434 & 0.0090 & 0.0018 & 6.0542 & $3.5886 \mathrm{E}-02$ & $3.306 \mathrm{E}+03$ & ${ }^{238} \mathrm{Pu}$ \\
\hline${ }^{243} \mathrm{Cm}$ & 96 & $2.85 E+01 y$ & $\begin{array}{l}\alpha(99.76 \%) \\
\operatorname{EC}(0.24 \%)\end{array}$ & 5.8380 & 0.1129 & 0.1316 & 6.083 & $3.605 \mathrm{E}-02$ & $5.162 \mathrm{E}+01$ & $\begin{array}{l}{ }^{239} \mathrm{Pu} ; \\
{ }^{243} \mathrm{Am}\end{array}$ \\
\hline${ }^{244} \mathrm{Cm}$ & 96 & $1.811 \mathrm{E}+01 \mathrm{y}$ & $\alpha$ & 5.7965 & & 0.0016 & 5.798 & $3.437 \mathrm{E}-02$ & $8.090 \mathrm{E}+01$ & ${ }^{240} \mathrm{Pu}$ \\
\hline${ }^{245} \mathrm{Cm}$ & 96 & $8.5 E+03$ y & $\alpha$ & 5.3631 & 0.1342 & 0.1178 & 5.615 & $3.329 E-02$ & $1.717 \mathrm{E}-01$ & ${ }^{241} \mathrm{Pu}$ \\
\hline${ }^{246} \mathrm{Cm}$ & 96 & $4.73 E+03 y$ & $\alpha$ & 5.3764 & 0.0072 & 0.0014 & 5.385 & $3.192 \mathrm{E}-02$ & $3.072 E-01$ & ${ }^{242} \mathrm{Pu}$ \\
\hline${ }^{247} \mathrm{Cm}$ & 96 & $1.56 \mathrm{E}+07 \mathrm{y}$ & $\alpha$ & 4.9475 & & 0.3152 & 5.263 & $3.119 E-02$ & $9.278 E-05$ & ${ }^{243} \mathrm{Pu}$ \\
\hline${ }^{248} \mathrm{Cm}$ & 96 & $3.40 \mathrm{E}+05 \mathrm{y}$ & $\begin{array}{l}\alpha(91.74 \%) \\
\operatorname{SPF}(8.26 \%)\end{array}$ & 4.6524 & & & 4.6524 & $2.7577 \mathrm{E}-02$ & $4.251 \mathrm{E}-03$ & $\begin{array}{l}{ }^{244} \mathrm{Pu} \text {; } \\
\text { (fission } \\
\text { products) }\end{array}$ \\
\hline${ }^{252} \mathrm{Cf}$ & 98 & $2.645 \mathrm{y}$ & $\begin{array}{l}\alpha(96.908 \%) \\
\operatorname{SPF}(3.092 \%)\end{array}$ & 5.9308 & 0.0051 & 0.0011 & 5.9370 & $3.5191 E-02$ & $5.378 \mathrm{E}+02$ & $\begin{array}{l}{ }^{248} \mathrm{Cm} ; \\
\text { (fission } \\
\text { products) }\end{array}$ \\
\hline
\end{tabular}

a Based on refs. 6-9.

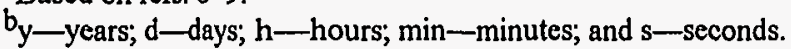

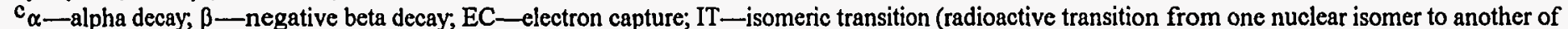
lower energy); and SPF-spontaneous fission.

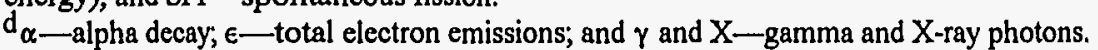

${ }^{2}$ The sum of the average energies for different radiation types in $\mathrm{MeV} /$ disintegration or W/Ci includes alphe and beta particles, discrete electrons, and photons). The "value" indicates the amount of energy (heat) that could be deposited in a radioactive material from each decay event if none of the radiation escaped from the material. Neutrinos are not included. 


-


APPENDIX C. REFERENCE SITES AND FACUITIES 


\section{APPENDIX C. REFERENCE SITES AND FACILITIES}

This appendix provides information on the major DOE and commercial sites and facilities discussed in this report. The DOE operations and special site offices are identified in Table C.1, along with the sites for which they have responsibility. This is followed by Table C.2, which lists DOE Naval Reactors Program (NE-60) offices and sites. Table C.3 lists major DOE sites and facilities referred to in this report, and major commercial radioactive waste disposal sites are given in Table C.4. For each site or facility listed in Tables C.3 and C.4, additional information is provided, including reference symbol or label, location, operations contractor, and, for DOE sites, the supervisory DOE operations and area office. 
Table C.1. DOE operations and special site offices

\begin{tabular}{|c|c|c|c|}
\hline DOE office & Symbol/label & $\begin{array}{l}\text { General mailing address } \\
\text { (Phone number) }{ }^{\mathrm{a}}\end{array}$ & $\begin{array}{c}\text { Radioactive waste sites for which DOE } \\
\text { office has responsibility }\end{array}$ \\
\hline $\begin{array}{l}\text { Albuquerque Operations } \\
\text { Office }^{b}\end{array}$ & DOE/AL & $\begin{array}{l}\text { P.O. Box } 5400 \\
\text { Albuquerque, NM } 87115-5400 \\
(505 / 845-4154)\end{array}$ & $\begin{array}{l}\text { Grand Junction Projects Office sites } \\
\text { Inhalation Toxicology Research Institute } \\
\text { Kansas City Plant } \\
\text { Los Alamos National Laboratory } \\
\text { Pantex Plant } \\
\text { Pinellas Plant } \\
\text { Sandia National Laboratories/California } \\
\text { Sandia National Laboratories/New Mexico } \\
\text { Uranium Mill Tailings Remedial Action Program sites } \\
\text { Waste Isolation Pilot Plant }\end{array}$ \\
\hline Chicago Operations Office ${ }^{c}$ & $\mathrm{DOE} / \mathrm{CH}$ & $\begin{array}{l}\text { Building } 201 \\
9800 \text { South Cass Avenue } \\
\text { Argonne, 프 } 60439 \\
(708 / 252-2001)\end{array}$ & $\begin{array}{l}\text { Ames Laboratory } \\
\text { Argonne National Laboratory-East } \\
\text { Argonne National Laboratory-West } \\
\text { Battelle Columbus Laboratories Decommissioning Project } \\
\text { Brookhaven National Laboratory } \\
\text { Fermi National Accelerator Laboratory } \\
\text { Princeton Plasma Physics Laboratory } \\
\text { Reactive Metals, Inc., Extrusion Plant }\end{array}$ \\
\hline Idaho Operations Office & DOE/ID & $\begin{array}{l}785 \text { DOE Place } \\
\text { Idaho Falls, ID } 83402 \\
(208 / 526-0111)\end{array}$ & Idaho National Engineering Laboratory \\
\hline Nevada Operations Office & DOE/NV & $\begin{array}{l}\text { P.O. Box } 98518 \\
\text { Las Vegas, Nevada } 89193-8518 \\
(702 / 295-1212)\end{array}$ & Nevada Test Site \\
\hline $\begin{array}{l}\text { Oakland Operations } \\
\text { Office }\end{array}$ & DOE/OAK & $\begin{array}{l}1301 \text { Clay Street } \\
\text { Oakland, CA 94612-5208 } \\
(510 / 273-6383)\end{array}$ & $\begin{array}{l}\text { Energy Technology Engineering Center (Santa Susana Field } \\
\text { Laboratory) } \\
\text { Laboratory for Energy-Related Health Research (Institute of } \\
\text { Toxicology and Environmental Health) } \\
\text { Lawrence Berkeley Laboratory } \\
\text { Lawrence Livermore National Laboratory } \\
\text { Stanford Linear Accelerator Center }\end{array}$ \\
\hline
\end{tabular}


Table C.1 (continued)

\begin{tabular}{|c|c|c|c|}
\hline DOE office & Symbol/label & $\begin{array}{l}\text { General mailing address } \\
{\text { (Phone number })^{\mathrm{a}}}\end{array}$ & $\begin{array}{l}\text { Radioactive waste sites for which DOE } \\
\text { office has responsibility }\end{array}$ \\
\hline $\begin{array}{l}\text { Oak Ridge Operations } \\
\text { Office }^{d}\end{array}$ & DOE/OR & $\begin{array}{l}\text { P.O. Box } 2001 \\
\text { Oak Ridge, TN } 37831 \\
(423 / 576-5454)\end{array}$ & $\begin{array}{l}\text { Formerly Utilized Sites Remedial Action Program (FUSRAP) sites } \\
\text { Oak Ridge Institute for Science and Education } \\
\text { Oak Ridge K-25 Site } \\
\text { Oak Ridge National Laboratory } \\
\text { Oak Ridge Y-12 Plant } \\
\text { Paducah Gaseous Diffusion Plant } \\
\text { Portsmouth Gaseous Diffusion Plant } \\
\text { Weldon Spring Site Remedial Action Project }\end{array}$ \\
\hline Ohio Field Office & $\mathrm{DOE} / \mathrm{OH}$ & $\begin{array}{l}\text { P.O. Box } 3020 \\
\text { Miamisburg, OH } 45343-3020 \\
(513 / 865-3977)\end{array}$ & $\begin{array}{l}\text { Fernald Environmental Management Project } \\
\text { Mound Plant } \\
\text { West Valley Demonstration Project }\end{array}$ \\
\hline Richland Operations Office & $\mathrm{DOE} / \mathrm{RL}$ & $\begin{array}{l}\text { P.O. Box } 550 \\
\text { 825 Jadwin Avenue } \\
\text { Richland, WA } 99352 \\
(509 / 376-7411)\end{array}$ & $\begin{array}{l}\text { Hanford Site } \\
\text { Pacific Northwest Laboratory }\end{array}$ \\
\hline Rocky Flats Office & $\mathrm{DOE} / \mathrm{RF}$ & $\begin{array}{l}\text { P.O. Box } 928 \\
\text { Golden, CO } 80401-0928 \\
(303 / 966-7000)\end{array}$ & Rocky Flats Environmental Technology Site \\
\hline $\begin{array}{l}\text { Savannah River Operations } \\
\text { Office }\end{array}$ & $\mathrm{DOE} / \mathrm{SR}$ & $\begin{array}{l}\text { P.O. Box A } \\
\text { Aiken, SC } 29802 \\
(803 / 725-6211)\end{array}$ & Savannah River Site \\
\hline
\end{tabular}

\footnotetext{
${ }^{a}$ Access to main organizations.

b The Albuquerque Operations Office also has the following area offices (monitoring activities of the sites indicated) under its purview: Amarillo (Pantex Plant), Grand Junction (Grand Junction Projects Office), Kansas City (Kansas City Plant), Los Alamos (Los Alamos National Laboratory), and Pinellas (Pinellas Plant).

${ }^{\mathbf{C}}$ The Chicago Operations Office has the following area offices (monitoring activities of the sites indicated) under its purview: Argonne (Argonne National Laboratory-East), Batavia (Fermi National Accelerator Laboratory), Upton (Brookhaven National Laboratory), and Princeton (Princeton Plasma Physics Laboratory).

${ }^{d}$ The Oak Ridge Operations Office has a separate site office located at the following: Oak Ridge K-25 Site, Oak Ridge National Laboratory, Oak Ridge Y-12

Plant, Paducah Gaseous Diffusion Plant, Portsmouth Gaseous Diffusion Plant, and the Weldon Spring Site Remedial Action Project.
} 
Table C.2. DOE Naval Reactors Program offices and sites

\begin{tabular}{|c|c|c|c|}
\hline DOE office & Symbol/label & $\begin{array}{l}\text { General mailing address } \\
\text { (Phone number) }^{\mathrm{a}}\end{array}$ & $\begin{array}{l}\text { Radioactive waste sites for which DOE } \\
\text { office has responsibility }\end{array}$ \\
\hline $\mathrm{DOE} / \mathrm{HQ}$, Office of Naval Reactors & $\mathrm{DOE} / \mathrm{HQ} / \mathrm{NE}-60$ & $\begin{array}{l}\text { Route Symbol NE-60 } \\
2521 \text { Jefferson Davis Hwy } \\
\text { Arlington, VA 22202 } \\
(703 / 603-7321)\end{array}$ & $\begin{array}{l}\text { Oversees Pittsburgh and Schenectady area offices } \\
\text { and their sites; oversees the following shipyards: } \\
\text { - Charleston Naval Shipyard, Charleston, SC } \\
\text { - Mare Island Naval Shipyard, Mare Island, CA } \\
\text { - Norfolk Naval Shipyard, Norfolk, VA } \\
\text { - Pearl Harbor Naval Shipyard, Pearl Harbor, HI } \\
\text { - Portsmouth Naval Shipyard, Portsmouth, ME } \\
\text { - Puget Sound Naval Shipyard, Puget Sound, WA }\end{array}$ \\
\hline Pittsburgh Naval Reactors Offices & DOE/PNRO & $\begin{array}{l}\text { P.O. Box } 109 \\
\text { West Mifflin, PA 15122-0109 } \\
(412 / 476-5000)\end{array}$ & $\begin{array}{l}\text { Bettis Atomic Power Laboratory } \\
\text { Naval Reactors Facility (Idaho Falls, ID) }\end{array}$ \\
\hline Schenectady Naval Reactors Office & DOE/SNRO & $\begin{array}{l}\text { P.O. Box } 1069 \\
\text { Schenectady, NY 12301-1069 } \\
(518 / 395-4000)\end{array}$ & Knolls Atomic Power Laboratory \\
\hline
\end{tabular}

${ }^{\mathrm{a} A c c e s s}$ to main organizations. 
Table C.3. Major DOE sites and facilities referred to in this report

\begin{tabular}{|c|c|c|c|}
\hline Site/facility & Symbol/label & $\begin{array}{l}\text { Principal contractor(s) for site } \\
\text { operations and mailing address } \\
\text { (Phone number) }{ }^{\mathrm{a}}\end{array}$ & $\begin{array}{l}\text { DOE operations office } \\
\text { (Phone number) }^{\mathrm{a}}\end{array}$ \\
\hline Ames Laboratory & Ames & $\begin{array}{l}\text { Iowa State University } \\
\text { Spedding Hall } \\
\text { Pammel Drive } \\
\text { Ames, IA 50011-3020 } \\
(515 / 294-2680)\end{array}$ & $\begin{array}{l}\text { Chicago } \\
(708 / 252-2001)\end{array}$ \\
\hline Argonne National Laboratory-East & ANL-E & $\begin{array}{l}\text { University of Chicago } \\
9700 \text { South Cass Avenue } \\
\text { Argonne, II } 60439 \\
(708 / 252-2000)\end{array}$ & $\begin{array}{l}\text { Chicago } \\
\text { Argonne Area Office } \\
(708 / 252-2001)\end{array}$ \\
\hline Argonne National Laboratory-West ${ }^{b}$ & ANL-W & $\begin{array}{l}\text { University of Chicago } \\
\text { Idaho Site } \\
\text { P.O. Box } 2528 \\
\text { Idaho Falls, ID } 83403-2528 \\
(208 / 533-7000)\end{array}$ & $\begin{array}{l}\text { Chicago } \\
(708 / 252-2001)\end{array}$ \\
\hline $\begin{array}{l}\text { Battelle Columbus Laboratories } \\
\text { Decommissioning Project }\end{array}$ & BCLDP & $\begin{array}{l}\text { Battelle Memorial Institute } \\
505 \text { King Avenue } \\
\text { Columbus, OH } 43201-2693 \\
(614 / 424-3989)\end{array}$ & $\begin{array}{l}\text { Chicago } \\
(708 / 252-2001)\end{array}$ \\
\hline Brookhaven National Laboratory & $\mathrm{BNL}$ & $\begin{array}{l}\text { Associated Universities, Inc. } \\
16 \text { South Railroad Street } \\
\text { Upton, NY 11973-2310 } \\
(516 / 282-2123)\end{array}$ & $\begin{array}{l}\text { Chicago } \\
\text { Brookhaven Area Office } \\
(516 / 282-3427)\end{array}$ \\
\hline Colonie Interim Storage Site & CISS & $\begin{array}{l}\text { Bechtel National, Inc. } \\
\text { P.O. Box } 5169 \\
\text { Albany, NY } 12205 \\
(518 / 482-0237)\end{array}$ & $\begin{array}{l}\text { Oak Ridge } \\
(423 / 576-5454)\end{array}$ \\
\hline
\end{tabular}


Table C.3 (continued)

\begin{tabular}{|c|c|c|c|}
\hline Site/facility & Symbol/label & $\begin{array}{l}\text { Principal contractor(s) for site } \\
\text { operations and mailing address } \\
\text { (Phone number) }{ }^{\mathrm{a}}\end{array}$ & $\begin{array}{l}\text { DOE operations office } \\
{\text { (Phone number })^{\mathrm{a}}}\end{array}$ \\
\hline $\begin{array}{l}\text { Energy Technology Engineering Center } \\
\text { (Santa Susana Field Laboratory) }\end{array}$ & $\begin{array}{l}\text { ETEC } \\
\text { (SSFL) }\end{array}$ & $\begin{array}{l}\text { Rockwell International } \\
\text { Rocketdyne Division } \\
6633 \text { Canoga Avenue } \\
\text { P.O. Box } 1449 \\
\text { Canoga Park, CA } 91304 \\
(818 / 586-5326)\end{array}$ & $\begin{array}{l}\text { Oakland } \\
(510 / 273-6383)\end{array}$ \\
\hline Fermi National Accelerator Laboratory & FNAL & $\begin{array}{l}\text { University Research Association } \\
\text { P.O. Box } 500 \\
\text { Batavia, II } 60510 \\
(708 / 840-3000)\end{array}$ & $\begin{array}{l}\text { Chicago } \\
\text { Batavia Area Office } \\
(708 / 840-3281)\end{array}$ \\
\hline Fernald Environmental Management Project & FEMP & $\begin{array}{l}\text { Fluor Daniels } \\
\text { Fernald Environmental Restoration } \\
\text { Management Corporation } \\
\text { P.O. Box } 398704 \\
7400 \text { Wiley Road } \\
\text { Cincinnati, OH 45239-8704 } \\
(513 / 738-6200)\end{array}$ & $\begin{array}{l}\text { Ohio Field Office } \\
\text { Fernald Area Office } \\
(513 / 648-3119)\end{array}$ \\
\hline Grand Junction Projects Office & GJPO & $\begin{array}{l}\text { Chem-Nuclear Geotech, Inc. } \\
\text { P.O. Box } 14000 \\
\text { Grand Junction, CO } 81502-5504 \\
(303 / 248-6200)\end{array}$ & $\begin{array}{l}\text { Nlbuquerque } \\
(505 / 845-4154)\end{array}$ \\
\hline Hanford Site & Hanford & $\begin{array}{l}\text { Westinghouse Hanford Company, Inc. } \\
\text { P.O. Box } 1970 \\
\text { Richland, WA } 99352 \\
(509 / 376-7411)\end{array}$ & $\begin{array}{l}\text { Richland } \\
(509 / 376-7411)\end{array}$ \\
\hline Idaho National Engineering Laboratory & NNEL & $\begin{array}{l}\text { Lockheed Martin Idaho Technologies } \\
\text { P.O. Box } 1625 \\
\text { Idaho Falls, D } 83415-4201 \\
(208 / 526-0111)\end{array}$ & $\begin{array}{l}\text { Idaho } \\
(208 / 526-0111)\end{array}$ \\
\hline
\end{tabular}


Table C.3 (continued)

\begin{tabular}{|c|c|c|c|}
\hline Site/facility & Symbol/label & $\begin{array}{l}\text { Principal contractor(s) for site } \\
\text { operations and mailing address } \\
\text { (Phone number) }\end{array}$ & $\begin{array}{l}\text { DOE operations office } \\
\text { (Phone number) }^{\mathrm{a}}\end{array}$ \\
\hline Inhalation Toxicology Research Institute & ITRI & $\begin{array}{l}\text { Lovelace Biomedical and Environmental } \\
\text { Research Institute, Inc. } \\
\text { P.O. Box } 5890 \\
\text { Albuquerque, NM } 87185 \\
\text { (505/845-1037) }\end{array}$ & $\begin{array}{l}\text { Albuquerque } \\
(505 / 845-4154)\end{array}$ \\
\hline Kansas City Plant & $\mathrm{KCP}$ & $\begin{array}{l}\text { Allied-Signal Acrospace Company } \\
\text { Kansas City Division } \\
\text { Bannister Federal Complex } \\
\text { Kansas City, MO 64141 } \\
(816 / 997-2000)\end{array}$ & $\begin{array}{l}\text { Albuquerque } \\
\text { Kansas City Area Office } \\
(816 / 997-3348)\end{array}$ \\
\hline $\begin{array}{l}\text { Laboratory for Energy-Related Health } \\
\text { Research (Institute of Toxicology and } \\
\text { Environmental Health) }\end{array}$ & $\begin{array}{l}\text { LEHR } \\
\text { (ITEH) }\end{array}$ & $\begin{array}{l}\text { University of California-Davis } \\
\text { Old Davis Road } \\
\text { Davis, CA 95616-8615 } \\
(916 / 752-1340)\end{array}$ & $\begin{array}{l}\text { Oakland } \\
(510 / 273-6383)\end{array}$ \\
\hline Lawrence Berkeley Laboratory & LBL & $\begin{array}{l}\text { University of California } \\
\text { One Cyclotron Road } \\
\text { Berkeley, CA } 94720 \\
(510 / 486-4000)\end{array}$ & $\begin{array}{l}\text { Oakland } \\
\text { Lawrence Berkeley Laboratory } \\
\text { Site Office } \\
(510 / 486-4363)\end{array}$ \\
\hline Lawrence Livermore National Laboratory & LLNL & $\begin{array}{l}\text { University of California } \\
7000 \text { East Avenue } \\
\text { P.O. Box 808, L-1 } \\
\text { Livermore, CA } 94550 \\
(510 / 422-1100)\end{array}$ & $\begin{array}{l}\text { Oakland } \\
(510 / 273-6383)\end{array}$ \\
\hline Los Alamos National Laboratory & LANL & $\begin{array}{l}\text { University of California } \\
\text { P.O. Box 1663 } \\
\text { Los Alamos, NM } 87545 \\
(505 / 667-5061)\end{array}$ & $\begin{array}{l}\text { Albuquerque } \\
\text { Los Alamos Area Office } \\
(505 / 667-5061)\end{array}$ \\
\hline
\end{tabular}


Table C.3 (continued)

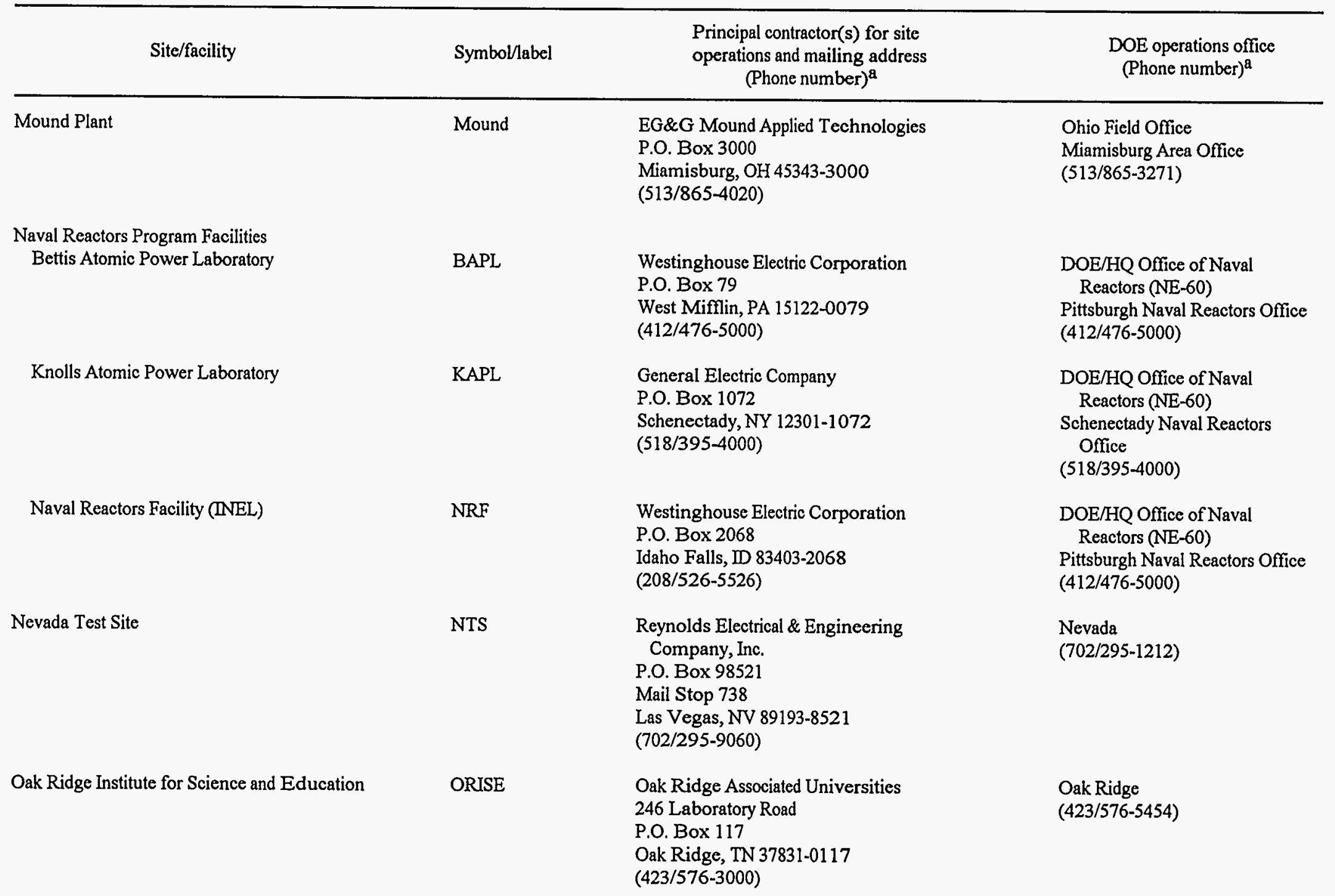


Table C.3 (continued)

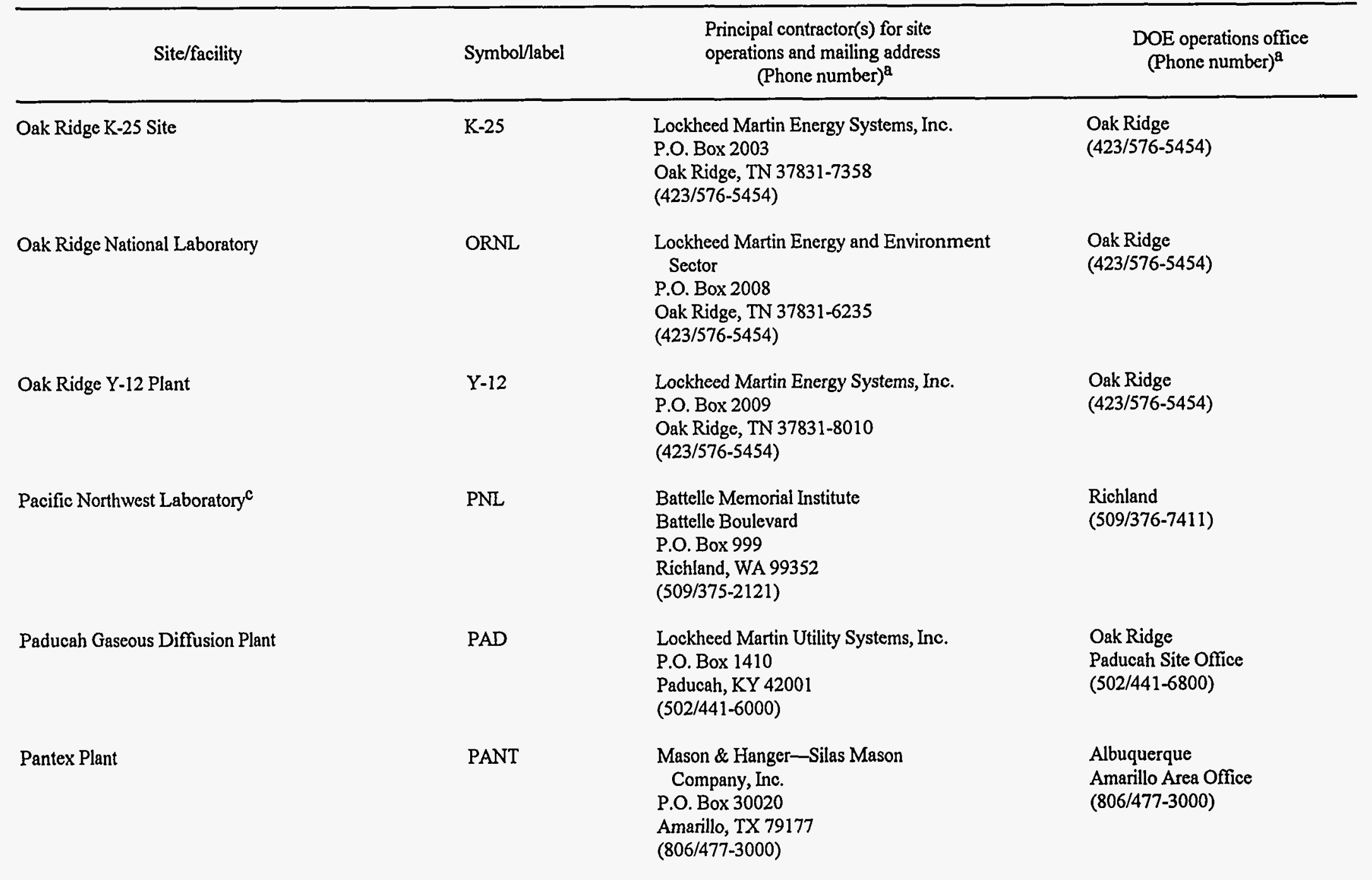


Table C.3 (continued)

\begin{tabular}{|c|c|c|c|}
\hline Site/facility & Symbol/label & $\begin{array}{l}\text { Principal contractor(s) for site } \\
\text { operations and mailing address } \\
\text { (Phone number) }\end{array}$ & $\begin{array}{l}\text { DOE operations office } \\
\text { (Phone number) }\end{array}$ \\
\hline Pinellas Plant & Pinellas & $\begin{array}{l}\text { Lockheed Martin Specialty Components, Inc. } \\
\text { P.O. Box } 2908 \\
\text { Largo, FL 34649-2908 } \\
(813 / 541-8001)\end{array}$ & $\begin{array}{l}\text { Albuquerque } \\
\text { Pinellas Area Office } \\
(813 / 541-8196)\end{array}$ \\
\hline Portsmouth Gaseous Diffusion Plant & PORTS & $\begin{array}{l}\text { Lockheed Martin Utility Systems, Inc. } \\
\text { P.O. Box } 628 \\
\text { Piketon, OH } 45661 \\
(614 / 897-2331)\end{array}$ & $\begin{array}{l}\text { Oak Ridge } \\
\text { Portsmouth Site Office } \\
(614 / 897-2331)\end{array}$ \\
\hline Princeton Plasma Physics Laboratory & PPPL & $\begin{array}{l}\text { Princeton University } \\
\text { P.O. Box } 451 \\
\text { Princeton, NJ } 08543 \\
(609 / 243-2000)\end{array}$ & $\begin{array}{l}\text { Chicago } \\
\text { Princeton Area Office } \\
(609 / 243-3700)\end{array}$ \\
\hline Reactive Metals, Inc., Extrusion Plant & RMI & $\begin{array}{l}\text { RMI Titanium Company } \\
\text { P.O. Box } 579 \\
\text { Ashtabula, OH } 44004 \\
(216 / 992-7442)\end{array}$ & $\begin{array}{l}\text { Chicago } \\
\text { RMI Decommissioning Project } \\
\text { Office } \\
(216 / 992-7442)\end{array}$ \\
\hline Rocky Flats Environmental Technology Site & RFETS & $\begin{array}{l}\text { EG\&G Rocky Flats, Inc. } \\
\text { P.O. Box } 464 \\
\text { Golden, CO 80401-0464 } \\
(303 / 966-7000)\end{array}$ & $\begin{array}{l}\text { Rocky Flats Office } \\
(303 / 966-7000)\end{array}$ \\
\hline \multicolumn{4}{|l|}{ Sandia National Laboratories } \\
\hline California & $\mathrm{SNL} / \mathrm{CA}$ & $\begin{array}{l}\text { Lockheed Martin Sandia Corporation } \\
\text { P.O. Box } 969 \\
\text { Livermore, CA } 94551-0969 \\
(510 / 294-3000)\end{array}$ & $\begin{array}{l}\text { Albuquerque } \\
(505 / 845-4154)\end{array}$ \\
\hline New Mexico & $\mathrm{SNL} / \mathrm{NM}$ & $\begin{array}{l}\text { Lockheed Martin Sandia Corporation } \\
\text { P.O. Box } 5800 \\
\text { Albuquerque, NM } 87185-5800 \\
(505 / 844-5678)\end{array}$ & $\begin{array}{l}\text { Albuquerque } \\
(505 / 845-4154)\end{array}$ \\
\hline
\end{tabular}


Table C.3 (continued)

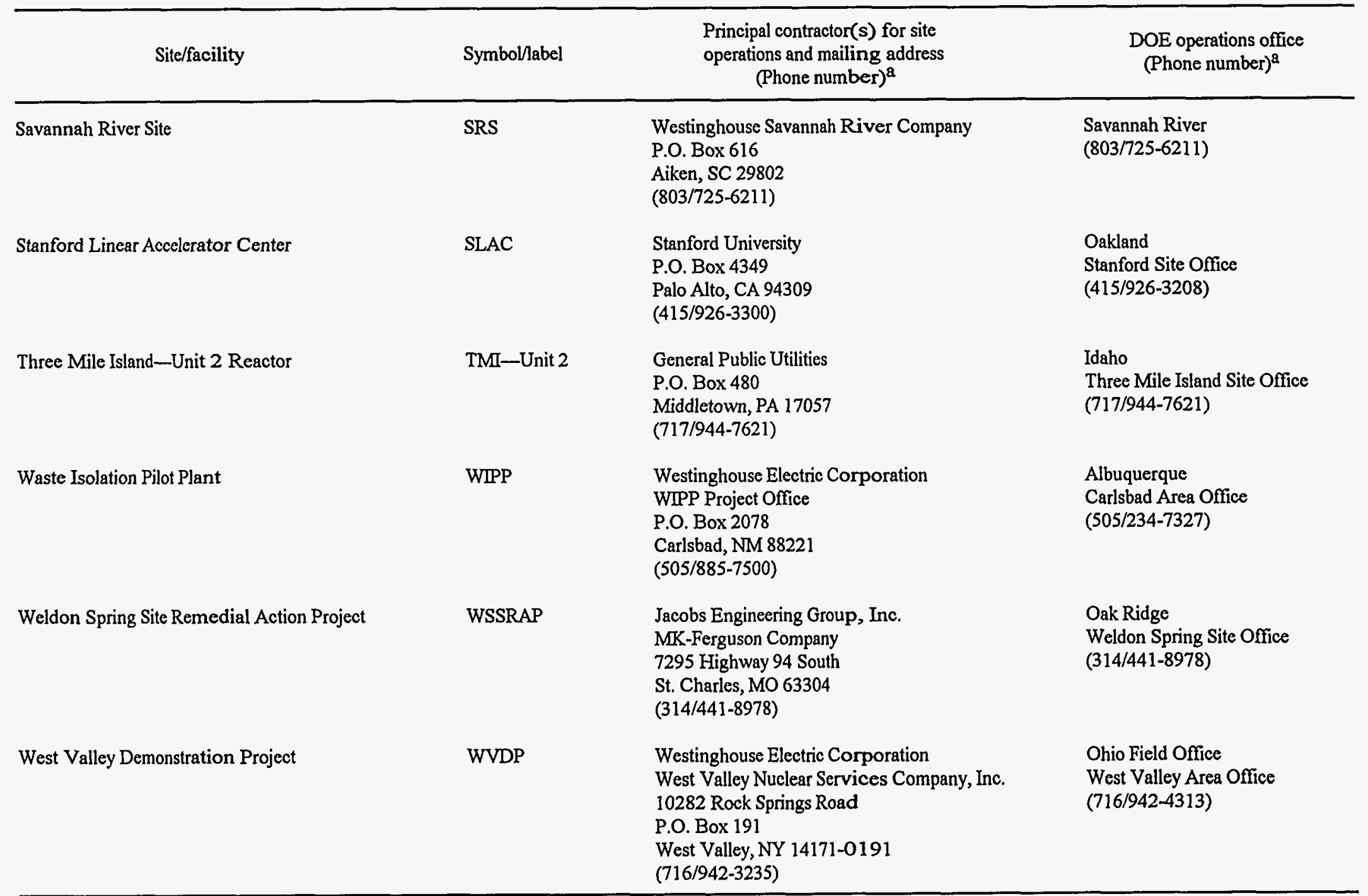

aphone number for access to main organization.

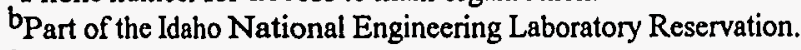

cPart of the Hanford Site. 
Table C.4. Major commercial radioactive waste disposal sites included in this report ${ }^{a}$

\begin{tabular}{|c|c|c|}
\hline Site & Symbol/label & $\begin{array}{c}\text { Principal contractor(s) for site operations } \\
\text { and mailing address } \\
\text { (Phone number) }{ }^{b}\end{array}$ \\
\hline Barnwell & BARN & $\begin{array}{l}\text { Chem-Nuclear Systems, Inc. } \\
140 \text { Stoneridge Drive } \\
\text { Columbia, SC } 29210 \\
(803 / 256-0450)\end{array}$ \\
\hline Beatty & BETY & $\begin{array}{l}\text { U.S. Ecology, Nuclear } \\
\text { P.O. Box } 578 \\
\text { Beatty, NV } 89003 \\
(702 / 553-2203)\end{array}$ \\
\hline Envirocare $^{c}$ & ENVR & $\begin{array}{l}\text { Envirocare of Utah, Inc. } \\
46 \text { West Broadway } \\
\text { Suite } 240 \\
\text { Salt Lake City, Utah } 84101 \\
(801 / 532-1330)\end{array}$ \\
\hline Maxey Flats & MFKY & $\begin{array}{l}\text { Commonwealth of Kentucky } \\
\text { Department of Environmental Protection/Superfund Branch } \\
\text { Maxey Flats Project } \\
\text { 14 Reilly Road } \\
\text { Frankfort, KY 40601-1190 } \\
(502 / 564-6716)\end{array}$ \\
\hline & & $\begin{array}{l}\text { Site address: } \\
\text { Maxey Flats Project } \\
\text { Route } 2 \\
\text { P.O. Box 238A } \\
\text { Hillsboro, KY 41049 } \\
(606 / 784-6612)\end{array}$ \\
\hline Richland & RICH & $\begin{array}{l}\text { U.S. Ecology, Nuclear } \\
\text { P.O. Box } 638 \\
\text { Richland, WA } 99352 \\
(509 / 377-2411)\end{array}$ \\
\hline Sheffield & SHEF & $\begin{array}{l}\text { U.S. Ecology, Nuclear } \\
\text { P.O. Box } 158 \\
\text { Sheffield, IL. } 61361 \\
(815 / 454-2342)\end{array}$ \\
\hline West Valley & WVNY & $\begin{array}{l}\text { Westinghouse Electric Corporation } \\
\text { West Valley Nuclear Services Company, Inc. } \\
\text { 10282 Rock Springs Road } \\
\text { P.O. Box } 191 \\
\text { West Valley, NY 14171-0191 } \\
\text { (716/942-3235) }\end{array}$ \\
\hline
\end{tabular}


Table C.4 (contrinued)

\begin{tabular}{lll}
\hline \multicolumn{1}{c}{ Site } & Symbol/label & \multicolumn{1}{c}{$\begin{array}{c}\text { Principal contractor(s) for site operations } \\
\text { and mailing address } \\
\text { (Phone number) }^{\mathrm{b}}\end{array}$} \\
\hline $\begin{array}{l}\text { West Valley } \\
\text { (contd.) }\end{array}$ & WVNY & $\begin{array}{l}\text { New York State Energy Research and Development Authority } \\
\text { 2 Rockefeller Plaza } \\
\text { Albany, NY 12223 } \\
(518 / 465-6251)\end{array}$ \\
\hline
\end{tabular}

aDoes not include uranium mill tailings sites. See Table 5.2.

bPhone number for access to main organizations.

cAlso contains disposed wastes from DOE site activities.

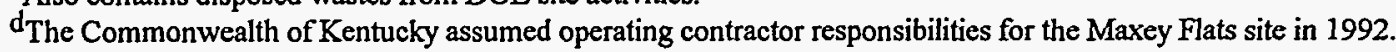


APPENDIX D. INTEGRATED DATA BASE DOCUMENT ACCESS ON THE INTERNET 


\section{APPENDIX D. INTEGRATED DATA BASE DOCUMENT ACCESS ON THE INTERNET}

A copy of this document (IDB Rev. 11) will be accessible via the Internet on the DOE Office of Environmental Management World Wide Web (EM WWW) Server at the Universal Resource Locator (URL) via the following path: http://www.em.doe.gov/idb95 .

This document may also be accessed from the EM WWW home page at http://www.em.doe.gov . From the home page menu, select Environmental Information. Then from the Environmental Information menu, select Waste Management. The IDB reports are listed on the Waste Management menu.

The EM WWW Server provides a collection of documents and other information and data relating to DOE/EM's mission. It was established as an EM-wide information resource available to DOE employees, other federal agencies, Congress, state and tribal governments, and the general public. The EM WWW Server is updated and maintained to provide historical and current DOE/EM information. 
APPENDIX E. INTEGRATED DATA BASE READER COMMENT FORM 


\section{APPENDIX E. INTEGRATED DATA BASE READER COMMENT FORM}

To maintain an updated distribution list for this report, the Integrated Data Base (IDB) is asking its readership to supply the information requested on the Reader Comment Form provided in the front of this report. When filling out this form, please respond to the questions in Items $1-11$ (note that some require two answers). Item 12 requests some personal information (please type or print your complete name and mailing address). To be eligible for future updates of this report, please fold, attach stamp, and mail the completed Reader Comment Form to the Integrated Data Base Program at the mailing address given on the back of the form (and listed below) at your earliest convenience. Also, please notify the IDB Program of any corrections or future changes in your mailing address. Your cooperation and assistance are greatly appreciated.

Integrated Data Base Program

Oak Ridge National Laboratory

105 Mitchell Road, Mail Stop 6495

P.O. Box 2008

Oak Ridge, TN 37831-6495

Phone(s): 423/574-6823

$423 / 576-7575$

Fax(es): $423 / 576-0327$

423/574-3431 


\section{GLOSSARY OF TERMS}

This glossary gives definitions of some terms commonly used in the main body of this report. A more detailed glossary of waste terms, applicable to the DOE complex, is being developed by the DOE Office of Environmental Management and will be issued for use by DOE and DOE contractors in the near future.

Actinides: Elements with atomic numbers from 89 (actinium) or 90 (thorium) to 103 (lawrencium) inclusive.

Activation product: A radioactive material produced by bombardment with neutrons, protons, or other nuclear particles.

Agreement State: A state that has entered into an agreement with the U.S. Nuclear Regulatory Commission (as specified by the Atomic Energy Act of 1954) and has authority to regulate the disposal of low-level radioactive waste under such an agreement. This term is used in the Low-Level Radioactive Waste Policy Act (Public Law 99-240).

Alpha decay: Radioactive decay in which an alpha particle ( ${ }^{4} \mathrm{He}$ nucleus) is emitted.

Beta decay: Radioactive decay in which a beta particle (negative or positive electron) is emitted.

Borosilicate glass: A type of glass containing at least 5\% boric oxide. It is used in glassware that resists heat and is a leading candidate for use in high-level waste immobilization and disposal.

Branching ratio: In branching radioactive decay, the fraction of nuclei that disintegrates in a specific way. (It is usually expressed as a percentage.)

Burnup, specific: The total energy released per initial unit mass of reactor fuel as a result of the fission process occurring. The unit commonly used for specific burnup is megawatt-days per metric ton of initial heavy metal, MWd/MTIHM.

By-product material: (1) Any radioactive material (except special nuclear material) yielded in, or made radioactive by, exposure to the radiation incident to the process of producing or utilizing special nuclear material. For purposes of determining the applicability of the Resource Conservation and Recovery Act of 1976 to any radioactive waste, the term "any radioactive material" refers only to the actual radionuclides dispersed or suspended in the waste substance. The nonradioactive hazardous waste component of the waste substance will be subject to regulation under the Resource Conservation and Recovery Act; (2) the tailings or waste produced by the extraction or concentration of uranium or thorium from any ore processed primarily for its source material content. Ore bodies depleted by uranium solution extraction operations and which remain underground do not constitute by-product material.

Calcine: A form of high-level waste produced from defense reactor fuel reprocessing waste (at the Idaho Chemical Processing Plant) by heating to a temperature below the melting point to bring about loss of moisture and nonradioactive volatile oxides, thus producing a chemically stable granular powder.

Canister: A metal container used for the storage or disposal of heat-producing, solid, high-level radioactive waste.

Capacity factor, plant: The ratio of the electrical energy actually supplied by a power plant in a given time interval to the electrical energy that could have been produced at continuous full-power operation during the same time period.

Capsules: Encapsulated strontium and cesium high-level wastes produced from defense reactor fuel reprocessing at the Hanford Site. For purposes of this report, inventories of encapsulated strontium and cesium are included in a separate table in the high-level waste section (Chapter 2). 
Cladding: A corrosion-resistant tube (commonly aluminum, zirconium alloy, or stainless steel) surrounding the reactor fuel pellets which provides protection from a chemically reactive environment and containment of fission products.

Code of Federal Regulations: A documentation of the general rules by the executive departments of the federal government. The code is divided into 50 titles that represent broad areas subject to federal regulation. Each title is divided into chapters that usually bear the name of the issuing agency. Each chapter is further subdivided into parts covering specific regulatory areas.

Control rod: A movable part of the reactor core that is adjusted to regulate the degree of fuel fissioning in the core.

Conversion, fuel: Chemical treatment of yellowcake $\left(\mathrm{U}_{3} \mathrm{O}_{8}\right)$ to uranium hexafluoride $\left(\mathrm{UF}_{6}\right)$ in preparation for enrichment.

Core, nuclear reactor: That part of the reactor which contains the nuclear fuel and in which most or all of the nuclear fissions occur.

Daughter product(s): The nuclide(s) formed by the radioactive decay of the parent radionuclide.

Decay, radioactive: The transition of a nucleus from one energy state to a lower one, usually involving the emission of a photon, electron, neutron, or alpha particle.

Decay chain, radioactive: A series of nuclides in which each member decays to the next member of the chain through radioactive decay until a stable nuclide has been formed.

Decommissioning: Activities taken to reduce the potential health and safety impacts of commercial and DOE-contaminated facilities, including removing a unit from operation, decontamination, entombment, dismantlement, or conversion of the site to another use.

Decommissioning wastes: Wastes (generally low-level) collected or resulting from facility decommissioning activities.

Decontamination: Activities taken to remove unwanted (typically radioactive) material from facilities, soils, or equipment by washing, chemical action, mechanical cleaning, or other (treatment) techniques.
Deep bed plant: A boiling-water reactor facility using a demineralizer vessel for water purification which contains an ion-exchange resin that is $3 \mathrm{ft}$ or more deep.

Disintegration energy (Q-value): The amount of energy released in a particular nuclear disintegration. This is usually expressed in $\mathrm{MeV} /$ disintegration.

DOE waste: Radioactive waste produced from activities supported by the Department of Energy and/or U.S. government defense programs.

Double-shell tank wastes: High-level wastes, generated from defense reactor fuel reprocessing at Hanford, which are stored in double-shelled tanks. These wastes consist of a mixture of liquid and suspended solids referred to as slurry. See also "single-shell tank wastes."

Electron capture: Radioactive decay in which an orbital electron is captured by the nucleus of the radionuclide.

Enrichment, fuel: A nuclear fuel cycle process which increases the concentration of fissionable uranium (i.e., ${ }^{235} \mathrm{U}$ ) in uranium ore above its natural level of $0.71 \%$. (The method currently used in the United States is gaseous diffusion.)

Environmental Impact Statement: A report that documents the information required to evaluate the environmental impact of a project. Such a report informs decision-makers and the public of the reasonable alternatives which would avoid or minimize adverse impacts or enhance the quality of the environment.

Environmental restoration: Cleanup and restoration of sites contaminated with radioactive and/or hazardous substances during past production, accidental releases, or disposal activities.

Equilibrium cycle: An assumed nuclear fuel cycle in which the feed and waste materials of a facility have constant compositions. In a reactor this condition typically results after the third or fourth fuel-loading schedule.

Fabrication, fuel: Conversion of enriched uranium hexafluoride $\left(\mathrm{UF}_{6}\right)$ into pellets of ceramic uranium dioxide $\left(\mathrm{UO}_{2}\right)$. These pellets are then sealed into corrosion-resistant tubes of zirconium alloy or stainless steel. The loaded tubes, called fuel elements or rods, are then mounted into special assemblies for loading into the reactor core. 
Fertile nuclide: A nuclide capable of being transformed into a fissile nuclide by neutron capture.

Filter/demineralizer plant: A facility that combines filtration and ion-exchange processing using nonregenerable powered resins.

Fissile nuclide: A nuclide capable of undergoing nuclear fission with thermal neutrons.

Fission, nuclear: The division of a heavy atomic nucleus into two or more isotopes, usually accompanied by the emission of neutrons and gamma radiation.

Fission products: Nuclides produced either by fission or by the subsequent decay of the nuclides thus formed.

Fission, spontaneous: Nuclear fission that occurs without the addition of particles or energy to the nucleus.

Formerly utilized site: A site contaminated with radioactive wastes which was previously used for supporting nuclear activities of the DOE's predecessor agencies, the Manhattan Engineer District (Manhattan Project) and the Atomic Energy Commission.

Fuel assembly: A grouping of nuclear fuel rods that remains integral during the charging and discharging of a reactor core.

Fuel cycle, nuclear: The complete series of steps involved in supplying fuel for nuclear reactors. It includes mining, refining, $\mathrm{UF}_{6}$ conversion, enrichment, fabrication of fuel elements, use in a reactor, and management of radioactive waste. It may also involve chemical processing to recover the fissionable material remaining in the spent nuclear fuel, reenrichment of the fuel material, and/or refabrication of new fuel elements.

Generation (electricity): The process of producing electric energy from other forms of energy; also, the amount of electric energy produced, commonly expressed in kilowatt-hours (kWh) or megawatt-years [MW(e)-years].

Generation (gross): The total amount of electric energy produced by the generating units in a generating station or stations, measured at the generator terminals.

Generation (net): Gross generation less the electric energy consumed at the generating station for station use.
Generation (waste): The origination of new wastes from various facility operations (including production, rework, decontamination and decommissioning, and environmental restoration), including the recovery of pre-1970 transuranic-produced wastes, should their recovery be determined necessary.

Glass frit: A fusible ceramic mixture used to make glass for use in the immobilization and disposal of high-level wastes.

Greater-than-Class-C low-level waste: Waste from commercial sources containing concentrations of radionuclides that exceed U.S. Nuclear Regulatory Commission limits for Class $\mathrm{C}$ low-level radioactive waste, as defined in 10 CFR Part 61.55.

Grout: A mortar or cement mixture used to immobilize radioactive wastes.

Half-life, radioactive: For a single radioactive decay process, the time required for the activity to decrease to one-half of its initial value by that process.

Hazardous waste: Nonradioactive waste that has at least one of the following characteristics: (1) is listed as a hazardous waste in Subpart D of 40 CFR Part 261, (2) exhibits any of the characteristics identified in Subpart C of 40 CFR Part 261, or (3) contains PCBcontaining wastes subject to regulation under the Toxic Substances Control Act and 40 CFR Parts 702-799.

High-level waste: As defined by the Nuclear Waste Policy Act, high-level waste is (1) the highly radioactive material resulting from the reprocessing of spent nuclear fuel, including the liquid waste produced directly in reprocessing and any solid material derived from such liquid waste that contains fission products in sufficient concentrations and (2) other highly radioactive material that the U.S. Nuclear Regulatory Commission, consistent with existing law, determines by rule to require permanent isolation.

Hydrofracture: A process formerly used for permanent disposal of low-level (approximately $0.25 \mathrm{Ci} / \mathrm{L}$ ) liquid waste at the Oak Ridge National Laboratory. The process involved mixing the waste with a blend of cement and other additives with the resulting grout being injected into shale at a depth of 200 to $300 \mathrm{~m}$. The injected grout hardened into thin, horizontal sheets several hundred meters wide. 
Industrial waste: Commercial low-level waste resulting from nonnuclear fuel cycle sources. These include the commercial producers of radiochemicals and radiopharmaceuticals, luminous dial manufacturers, and instruments that incorporate sealed source components (e.g., smoke detectors).

Institutional waste: Commercial low-level waste resulting from bioresearch, medical, and certain nonbioresearch sources. Bioresearch wastes include wastes from animal studies at universities. Medical wastes include those generated from diagnostic and therapeutic procedures on humans at hospitals. Nonbioresearch wastes include research reactor wastes; small-volume, sealed radiation sources; and accelerator targets.

Leaching: The process of removal or separation of soluble components from a solid by percolating water or other liquids through the solid.

Low-level waste: As specified in the Low-Level Radioactive Waste Policy Amendments Act of 1985 (Public Law 99-240), radioactive waste not classified as high-level waste, spent nuclear fuel, or by-product material specified as uranium or thorium tailings and waste.

Mill tailings, uranium: Earthen residues that remain after the extraction of uranium from ores. Tailings may also contain other minerals or metals not extracted in the process.

Mixed low-level waste: Waste that satisfies the definition of low-level radioactive waste (LLW) in the Low-Level Radioactive Waste Policy Amendments Act of 1985 and that contains hazardous waste that has at least one of the following characteristics: (1) is listed as a hazardous waste in Subpart D of 40 CFR Part 261, (2) exhibits any of the hazardous waste characteristics identified in Subpart C of 40 CFR Part 261, or (3) contains PCBcontaining wastes subject to regulation under the Toxic Substances Control Act and 40 CFR Parts 702-799.

Mixed-oxide fuel: Nuclear reactor fuel composed of plutonium and uranium in oxide form.

Mixed waste: Waste that includes concentrations of both radionuclides and hazardous chemicals.

Moderator: A material used to reduce neutron energy (for fissioning if in a reactor) by elastic scattering.
MRS facility: A proposed facility for the monitored retrievable storage of spent nuclear fuel from commercial power plants. Such a facility would permit continuous monitoring, management, and maintenance of these wastes and provide for their ready retrieval for further processing or disposal.

Naturally occurring or accelerator-produced radioactive material: Any radioactive material that can be considered naturally occurring and is not source, special nuclear, or by-product material or that is produced in a charged particle accelerator.

Neutron activation: The process of irradiating a material with neutrons so that the material itself is transformed into a radioactive nuclide.

Nonfuel components: Nuclear reactor core parts and hardware, excluding the nuclear fuel itself. Such components include shrouds, control rods, fuel channels, in-core chambers, support tubes, and dummy fuel rods.

Parent: A radionuclide that upon decay yields a specified nuclide (the daughter) either directly or as a later member of a radioactive decay series.

Pressure vessel, reactor: A strong-walled container housing the core of most types of power reactors. It usually also contains other core components such as the moderator and control rods.

PUREX ${ }^{\mathrm{TM}}$ process: A solvent extraction process that may be used in the reprocessing of uranium/plutoniumbased nuclear fuels.

Radioactivity: The property possessed by certain nuclides of spontaneously emitting alpha or beta particles and, sometimes, gamma radiation by the disintegration of atomic nuclei. The units for the rate of decay of radioactive nuclides are the curie, $\mathrm{Ci}\left(3.7 \times 10^{10}\right.$ disintegrations per second exactly), or, in SI units, the becquerel, $\mathrm{Bq}$ (1 disintegration per second).

Reactor, boiling-water: A light-water reactor in which water, used as both coolant and moderator, is allowed to boil in the core. The resulting steam is used directly to drive a turbine.

Reactor, breeder: A reactor that produces more fissionable fuel than it consumes. The new fissionable material is created by a process (breeding) in which fission neutrons are captured in fertile materials. 
Reactor, fast flux: A reactor in which fission is induced predominantly by fast neutrons.

Reactor, high-temperature, gas-cooled: A nuclear reactor that uses an inert gas (helium) as the primary coolant and graphite as the moderator.

Reactor, light-water: A nuclear reactor that uses light water $\left(\mathrm{H}_{2} \mathrm{O}\right)$ as the primary coolant and moderator and slightly enriched uranium as the fuel. There are two types of commercial light-water reactors: boiling-water and pressurized-water.

Reactor, naval propulsion: A reactor used to power a U.S. Navy vessel.

Reactor, pressurized-water: A light-water reactor in which heat is transferred from the core to a heat exchanger via water kept under high pressure, so that high temperatures can be maintained in the primary coolant system without boiling the water. Steam is generated in a secondary circuit.

Reactor, production: A reactor whose primary purpose is to produce fissile or other materials or to perform irradiations on an industrial scale. Unless otherwise specified, the term usually refers to either a tritium- or plutonium-production facility used to produce materials for nuclear weapons.

Reactor, research: A reactor whose nuclear radiations are used primarily as a tool for basic or applied research. Typically, it has a thermal power of $10 \mathrm{MW}(t)$ or less and may include facilities for testing reactor materials.

Reactor, test: A reactor associated with an engineering-scale test program conducted to develop basic design information or demonstrate safety characteristics of nuclear reactor systems.

Reinserted fuel: Irradiated reactor fuel that is discharged in one cycle and inserted into the same reactor during a subsequent refueling. In a few cases, fuel discharged from one reactor has been used to fuel a different reactor.

Repository, geologic: A facility that has an excavated subsurface system for the permanent disposal of spent nuclear fuel and high-level waste.

Reprocessing, fuel: The chemical/mechanical processing of irradiated nuclear reactor fuel to remove fission products and recover fissile and fertile material.
Salt cake: A salt form of high-level waste stored in tanks, which is produced from neutralizing acidic liquid waste from defense reactor fuel reprocessing with an alkaline agent (caustic soda).

Saltstone: A low-level waste by-product from the solidification of high-level waste at the Savannah River Site. Saltstone is retained in trenches at the Savannah River Site.

Sea-bed disposal: Placement of waste packages in deep ocean sediments.

Sea dumping (disposal): The practice of periodically dumping shiploads of drummed, solidified waste into the ocean at specified locations. (No longer performed.)

Separative work unit: The standard measure of enrichment services. The separative work unit (SWU) is expressed as a unit of mass. For example, 1 kilogram of separative work is expressed as $1 \mathrm{~kg}$ SWU.

Single-shell tank wastes: High-level wastes, generated from defense reactor fuel reprocessing at Hanford, which are stored in single-shelled tanks that contain liquid, sludge, and salt cake. See also "double-shell tank wastes."

Slurry, high-level waste: A watery mixture of highly radioactive, insoluble matter.

Solvent extraction: The separation of materials of different chemical types by exploiting the relative chelating ability of different chemicals which preferentially dissolve in one of the two phases. In spent nuclear fuel reprocessing, liquid-liquid contact of two immiscible solvents (one aqueous, one organic) permits recovery and separation of uranium and plutonium in one phase and fission products in the other phase.

Source term (DB Program usage): A set of qualitative and quantitative features used to describe the origin and concentration of radioactive waste. The qualitative features include a flowchart of waste streams generated by a facility or an activity. Quantitative features include (1) the number of curies of radioactivity expressed either per unit of facility production or per unit of waste volume or mass and (2) a listing of the relative concentrations of component radioisotopes per curie of waste activity.

Special nuclear material: Plutonium or uranium enriched to a higher-than-natural assay. 
Spent nuclear fuel: Nuclear fuel that has been permanently discharged from a reactor after it has been irradiated. Typically, spent nuclear fuel is measured in terms of either the number of discharged fuel assemblies or the quantity of discharged fuel mass. The latter is measured either in metric tons of heavy metal (i.e., only the heavy-metal content of the spent nuclear fuel is considered) or in metric tons of initial heavy metal (essentially, the initial heavy-metal mass of the fuel before irradiation). The difference between these two quantities is the weight of the fission products produced during irradiation.

Thermal power: A measure of the rate of heat-energy emission that results from the radioactive decay of a material. A unit of thermal power commonly used is the watt (W).

THOREX process: A solvent extraction process developed to reprocess thorium-based nuclear fuels.

Transuranic waste: As defined and used by the U.S. Department of Energy (DOE Order 5820.2A), radioactive waste that, at the time of assay, contains more than $100 \mathrm{nCi} / \mathrm{g}$ of alpha-emitting isotopes with atomic numbers greater than 92 and half-lives greater than 20 years.

Transuranic waste acceptance criteria: A set of requirements/criteria that must be satisfied prior to transport to and emplacement in the Waste Isolation Pilot Plant for disposal.
Transuranic waste certification: The process for verifying that waste meets the applicable requirements/criteria for transport to and emplacement in a repository for disposal.

Transuranic waste, contact-handled: Transuranic waste with a surface dose rate of less than $200 \mathrm{mrem} / \mathrm{h}$.

Transuranic waste nondestructive assay/ nondestructive examination: Nondestructive test procedures performed on suspect transuranic wastes to determine their transuranic isotope concentration. From these tests such wastes can be properly classified (certified) as transuranic or low-level.

Transuranic waste, remote-handled: Transuranic waste with a surface dose rate of greater than $200 \mathrm{mrem} / \mathrm{h}$.

Vitrification: The conversion of high-level waste materials into a glassy or noncrystalline solid for subsequent disposal.

Waste Isolation Pilot Plant: A facility, located near Carlsbad, New Mexico, to be used for demonstrating the safe disposal of transuranic wastes from DOE defenserelated activities.

Yellowcake: A uranium oxide concentrate that results from milling (concentrating) uranium ore. It typically contains 80 to $90 \% \mathrm{U}_{3} \mathrm{O}_{8}$. 


\section{INDEX}

Agreement state, 209

Ames Laboratory (Ames), 12, 289

LLW at, 17-19, 116-17, 122, 128-31

MLLW at, $17-18,211,221-24,227,237-46$

TRUW at, 17-19, 88, 90-91, 93-94, 96-98, 101-7

Argonne National Laboratory

Argonne, Illinois (ANL-E), 12, 289

LLW at, 17-19, 128-31

MLLW at, 17-18, 211, 221-24, 227, 237-46

SNF at, 41

TRUW at, 17-19, 88, 90-91, 93-94, 96-99, 102-7

Idaho Falls, Idaho (ANL-W), 12, 289

LLW at, 17-19

MLLW at, 17-18, 221-24, 227, 237-46

SNF at, 41

TRUW at, 17-19, 88, 90-91, 93-94, 96-98, 101-7

Atomic Energy Act (AEA), 3, 8, 157, 160, 168, 171

Barnwell (South Carolina) commercial waste site, 296

LLW at, $116,118,138-39$

Baseline Environmental Management Report (BEMR), 158

Battelle Columbus Laboratories Decommissioning Project (BCLDP), 289

Beatty (Nevada) commercial waste site, 296

LLW at, 116, 118, 138-39

Boiling-water reactor (BWR), 6, 14-16, 23-24, 29-30, 32-33, 36, 38-39, 141, 175-78, 183-85, 187-92, 195, 197, 202, 206, 255, 257

Borosilicate glass (see High-level waste)

Brookhaven National Laboratory (BNL), 12, 289

LLW at, $17-19,116-17,122,128-31$

MLLW at, 17-18, 211, 221-24, 227, 237-47

SNF at, 41

Burnup, SNF

distribution in BWRs, 39

distribution in PWRs, 40
By-product material, 3, 109

mill tailings, $145-46$

$11(\mathrm{e}) 2,16,20,160,165-72$

Calcine (see High-level waste)

Cape Henry (HEN) LLW, 117, 134

Ceramic (see High-level waste)

Classification of LLW, 109

Code of Federal Regulations (CFR)

10 CFR Part 61, 111, 197, 324

40 CFR Part 191, 3, 78

40 CFR Parts 260-271, 209

40 CFR Parts 702-799, 209

Colonie Interim Storage Site (CISS), 164, 289

Comprehensive Environmental Response, Compensation, and Liability Act (CERCLA) of 1980,157

Decommissioning of reactors

NRC alternatives, 175

reactor shutdown dates, 188

waste volumes from alternatives, 190

Decommissioning wastes, commercial, 8, 175-207

alternatives for (DECON, SAFSTOR, ENTOMB), 175

characteristics of, $175-76$

from BWRs, 190-91

from PWRs, 190-91

from specific facilities and sites

Cimarron Fuel Fabrication Facility, 178, 186, 205

Dresden Unit-1 Reactor, 177, 183, 192

Elk River Reactor, 176, 183, 189

Fort St. Vrain Reactor, 177, 183, 189, 193-94

Humboldt Bay-Unit 3 Reactor, 177, 183, 195

Indian Point-Unit 1 Reactor, 177, 183, 196

La Crosse Reactor, 177, 184, 189, 197

Oak Ridge Gaseous Diffusion Plant (K-25), 186-87

Paducah Gaseous Diffusion Plant, 187 
Pathfinder Reactor, 184, 189

Peach Bottom Unit-1 Reactor (HTGR), 184

Portsmouth Gaseous Diffusion Plant, 187

Rancho Seco Reactor, 177, 184, 198

San Onofre-Unit 1 Reactor, 177, 184, 199-200

Santa Susana Sodium Reactor Experiment, 176, 184,189

Saxton Reactor, 177-78, 189, 201

Sequoyah Fuels Corporation Plant, 186

Shippingport Station Decommissioning Project, 176,189

Shoreham Reactor, 178, 202

Three Mile Island-Unit 2 Reactor, 178, 189, 203

Trojan Reactor, 178, 204

West Valley Demonstration Project, 178, 206-7

Yankee Rowe Reactor, 178, 189, 204

Decommissioning wastes, submarine reactor compartments, 178-79

Defense Waste Processing Facility (DWPF), 6, 14, 49,56

Department of Energy (DOE), 1-2, 19, 45-47, 75-78, 109-10, 157-61, 178-79, 209-11, $253,273,285-95,301$

Energy Information Administration (ELA), 5-6, $23-24,145-46$

Naval Reactors Program, 288

Office of Environmental Restoration, 7-8, 76, 157-59

Field Offices for, 158

Office of Waste Management, 75-76, 158, 161

Office of Facility Transition, 161

Office of Technology Development, 158

Operations and Area Offices, 162, 166-71, 283-95

Order 5820.2A, 3, 75, 109, 160

Disintegration energy (see Q-value)

Double-shell tank waste (see High-level waste)

Energy Technology Engineering Center (ETEC), 12, 290

MLLW at, 17-18, 211, 221-24, 227, 237-46

TRUW at, 17-19, 88, 90-91, 93-94, 96-98, 101-107

Envirocare Facility, 20, 111, 160, 172, 296

Environmental Impact Statement (EIS)

Hanford defense waste, 48

West Valley, 110

Environmental Protection Agency (EPA), 158, 209, 273

Environmental restoration program (DOE), 7-8, $157-72$

contaminated debris, 159-61, 169-71 contaminated soils, 159-61, 166-68

decommissioning activities, $8,157-58$

FUSRAP activities, $158-60$

contaminated media from, 160

locations of, 164

site status, 164

RA activities, 8, 157, 160-61

site status, 8

UMTRAP activities, 158, 160

contaminated media from, 166, 169

locations of, 163

site status, 163

wastes disposed of at the Envirocare Facility, 172

Fabrication (nuclear fuel), 109, 187

Farallon Islands (FIS) LLW, 117, 134

Fast Flux Test Facility (FFTF), 41-42

Federal Facility Compliance Act (FFCA), 4, 46, 209

Fermi National Accelerator Laboratory (FNAL), 12, 290

LLW at, 17-19, 128-31

MLLW at, $17-18,211,221-24,228,237-46$

Fernald Environmental Management Project (FEMP), 12, 290

LLW at, 17-19, 116-117, 122, 130

MLLW at, $17-18,211,221-24,227-28,237-46$

Foreign Research Reactors (FRRs), 41-42

Fort St. Vrain Reactor (FSVR)

decommissioning of, $177,183,189,193-94$

SNF from, 24, 37, 41-42

Formerly Utilized Site Remedial Action Program

(FUSRAP), 158-60, 164

Fuel assemblies

projected for LWRs, 36

reference characteristics of, 38

General Atomics (GA)

environmental restoration at, 167,170

Grand Junction Projects Office (GJPO), 290

MLLW at, 17-18, 211, 221-24, 228, 237-46

Greater-than-Class-C (GTCC) low-level waste, 111 from LWR decommissioning, 16, 111, 141, 190-91, 199, 204

sources and characteristics, 111, 141

Hanford Site (Hanford), 12, 290

environmental restoration at, 167,170

HLW at, $15,17-18,47-49,52-55,57-60,62-64$, $66-67,71-72$

canisters, $60-64,66-67,69-70$ 
chemical composition of, 72

radionuclide composition of, 67

treatment methods for, 55

LLW at, 17-19, 116-17, 122, 128-29, 132-33

MLLW at, 17-18, 211, 221-24, 227, 237-46

SNF at, 41

TRUW at, 17-19, 88, 90-91, 93-94, 96-99, 102-7

Hanford Waste Vitrification Plant (HWVP), 14, 48

High-level waste (HLW), 1-3, 6-7, 11, 14-18, 45-73

agreements and interfaces, 46

calcine, $47-49,68$

canisters, 60-64, 66-67, 69-70

capsules, 47-49, 55, 66-67

characterization, 46

chemical composition of future glass, 72-73

inventories and projections, $57-60,62-64,66-67$

liquid forms of, 45-46, 52, 66-67

locations of, 52

precipitate, $48,66-67$

salt cake, $48,66-67$

sludge, $47-48,66-67$

solid forms of, 47-48, 66-67

solidification for disposal, 48-49

treatment methods for, 55-56

zeolite, 48, 66-67

Hydrofracture, 110, 134

Idaho Chemical Processing Plant (ICPP), 13

HLW at, 14, 17-18, 55, 57-70, 72

chemical composition of future glass, 72

canisters, $60-64,66-67,69-70$

radionuclide composition of, 68

treatment methods for, 55

Idaho National Engineering Laboratory (INEL) [includes Idaho Chemical Processing Plant (ICPP)], 12, 290

environmental restoration at, 166,169

HLW at, $14,17-18,55,57-70,72$

chemical composition of future glass, 72

radionuclide composition of, 68

treatment methods for, 55

LLW at, $17-19,116-17,122,128-33$

MLLW at, 17-18, 211, 221-24, 228, 237-46

SNF at, $41-42$

TRUW at, 17-19, 88, 90-91, 93-94, 96-99, 102-7

Inhalation Toxicology Research Institute (ITRI), 12, 291

LLW at, 17-19, 128-32

MLLW at, 17-18, 221-24, 228, 237-46

Integrated Data Base (MB) Program, 1-2

Reader comment form, 305

Report assumptions, 5, 14
Report access on the Internet, 301

Summary waste data, 15-19

K-25 Site (Oak Ridge), 12, 293

LLW at, $17-19,116-17,122,128-31$

MLLW at, 17-18, 211, 221-24, 229, 237-46

Kansas City Plant (KCP), 12, 291

LLW at, 17-19, 128-32

MLLW at, 17-18, 211, 221-24, 228, 237-46

Knolls Atomic Power Laboratory (KAPL), 12, 292

TRUW at, 17-19, 88, 90-91, 93-94, 96-98, 102-7

Laboratory for Energy-Related Health Research (LEHR), 291

Lawrence Berkeley Laboratory (LBL), 12, 291

LLW at, 17-19, 128-31

MLLW at, 17-18, 211, 221-24, 228, 237-46

TRUW at, 17-19, 88, 90-91, 93-94, 96-98, 101-7

Lawrence Livermore National Laboratory (LLNL), 12,291

LLW at, 17-19, 116-17, 122, 128-31

MLLW at, 17-18, 212, 221-24, 229-30, 237-46

TRUW at, 17-19, 88, 90-91, 93-94, 96-99, 102-7

Light-water reactor (LWR)

LLW (see Low-level waste)

radionuclide characteristics of SNF, 258-65

Los Alamos National Laboratory (LANL), 12, 291

LLW at, 17-19, 116-17, 122, 128-29

MLLW at, $17-18,211,221-24,229,237-46$

SNF at, $41-42$

TRUW at, 17-19, 88, 90-91, 93-94, 96-99, 102-7

Low-Level Radioactive Waste Policy Act (LLRWPA), 109

Low-Level Radioactive Waste Policy Amendments Act (LLRWPAA), 3, 111

Low-level waste (LLW)

commercial, 109-12, 116, 118-19, 121, 138-42

greater-than-Class-C (GTCC) wastes, 3, 111, 141

inventories, 119, 138-39

locations of disposal sites, 118

projections, 121

state shipments to disposal sites, 140

DOE, 17-18, 109-10, 116-17, 119-20, 122-37

Cape Henry, at [see Cape Henry (HEN) LLW]

inventories and projections, 119,122

disposed wastes, 122, 127, 132-33

generated wastes, $125,128-29$

stored wastes, 126, 130-31

locations of disposal sites, 117

physical forms of, 123-24

saltstone (at SRS), 110, 137 
sea disposal of, 110,134

inventory, significant revisions of, 135

Low-Level Waste Management Program

(LLWMP), 257

Massachusetts Bay wastes, 117, 134

Material not categorized as waste (MNCAW), 1

Maxey Flats (Kentucky) commercial waste site, 296

LLW at, $110,116,118,138-39$

Midwest Fuel Recovery Plant (MFRP) SNF, 2,23

Mixed waste authorization (EPA), 220

Mixed Waste Inventory Report (MWIR), 4, 209-10

MLLW, 3-4, 8, 17-18, 209-49

commercial, 214-15, 248-49

characteristics of, 214-15

generation, 248-49

by facility category, 248

by hazardous waste stream, 249

DOE, 17-18, 209-47

characteristics of, 209-10, 219, 221-44

RCRA-/state-regulated wastes, $8,17-18,209-10$, $221-36$

generation, 17, 223-24, 236

inventories, 18, 221-22, 227-34

treatability group categories, $225-26$

TSCA-regulated wastes, 8, 17-18, 209-14, 221-24, 237-47

generation, 17, 223-24, 239-40, 243-46

inventories, 18, 221-22, 237-38, 241-42

significant inventory revisions, 247

wastes at specific sites, 210-14

Mound Plant (Mound), 12, 292

LLW at, $17-19,128-31$

MLLW at, 17-18, 212, 221-24, 230, 237-46

TRUW at, 17-19, 88, 90-91, 93-94, 96-98, 100, 102-7

National Environmental Policy Act (NEPA), 157

Naturally occurring or accelerator-produced radioactive material (NARM), 1, 4

National Priorities List (EPA), 157

Naval Reactors Program Facilities (DOE/HQ, NE-60), 12, 288

LLW at, 17-19, 128-29

MLLW at, 17-18, 212, 221-24, 230, 237-46

Neutron activation products, 175-76, 269

Nevada Test Site, 12, 292

LLW at, $17-19,116-17,122,128-33$

MLLW at, 17-18, 212, 221-24, 230, 237-46

TRUW at, 17-19, 88, 90-91, 93-94, 96-98, 100, 102-7
Nuclear power reactors in U.S., 2, 5-6, 14-16, 23-24, $110-11,255-56$

locations of, 26

Nuclear Regulatory Commission (NRC), 3, 110, $175-76,209,214,273$

Oak Ridge Institute for Science and Education (ORISE), 12, 292

MLLW at, 17-18, 212, 221-24, 230, 237-46

Oak Ridge National Laboratory (ORNL), 12, 293

environmental restoration at, 167,170

LLW at, 17-19, 116-17, 122, 128-33

MLLW at, 17-18, 212, 221-24, 230-31, 237-46

SNF at, 42

TRUW at, 17-19, 88, 90-91, 93-94, 96-98, 100, 102-7

ORIGEN2 computer code, 5-6

Pacific Northwest Laboratory (PNL), 13, 293

Paducah (PAD) Gaseous Diffusion Plant, 12, 293 LLW at, $17-19,116-17,122,130$

MLLW at, 17-18, 212, 221-24, 231, 237-47

TRUW at, 17-19, 88, 90-91, 93-94, 96-98, 101-7

Pantex (PANT) Plant, 12, 293

LLW at, 17-19, 116-17, 122, 128-31

MLLW at, 17-18, 213, 221-24, 231, 237-46

Pinellas Plant (Pinellas), 12, 294

LLW at, 17-19, 128-31

MLLW at, $17-18,213,221-24,231,237-46$

Portsmouth (PORTS) Gaseous Diffusion Plant, 12, 294

LLW at, $17-19,116-17,122,130$

MLLW at, 17-18, 213, 221-24, 231-32, 237-47

Pressurized-water reactor (PWR), 6, 14-16, 23-24, 29-30, 33-34, 36, 38, 40, 141, 175, 177-78, $183-85,187-91,196,198-201,203-4,206$, 256-57

Princeton Plasma Physics Laboratory (PPPL), 12, 294

LLW at, 17-19, 128-31

MLLW at, 17-18, 221-24, 232, 237-46

Projection scenarios (DOE/EIA), commercial LWR, $5-6,23-24,27-28,30-36,146$

Low Case

for electric power generating capacity, 14,30

for discharged SNF, 16, 31-36

Q-value, 274-81 
Radionuclide characteristics, 273-81

Radioactive Decay and Accumulation (RADAC) computer code, 77

Reactive Metals, Incorporated (RMI), Extrusion Plant, 12, 294

MLLW at, $17-18,221-24,232,237-46$

Remedial action projects, 7-8, 157-61

MLLW from, 17-18, 221-24, 227-28, 231-34, $237-47$

Repository, geologic, 2, 6, 23-24, 47-49, 75, 111

Reprocessing, fuel, $2,5,14,23-24,45-49,75,178$, $186,206-7$

Resource Conservation and Recovery Act (RCRA) of 1976

wastes regulated by, 3, 8, 157-58, 160-61, 209-11, 221-36

Richland (Washington) commercial waste site, 296

LLW at, $110,116,118,138-39$

Rocky Flats Environmental Technology Site (RFETS), 12, 294

LLW at, 17-19, 128-29

MLLW at, $17-18,213,221-24,232,237-46$

TRUW at, 17-19, 88, 90-91, 93-94, 96-98, 100, 102-7

Salt cake (see High-level waste)

Saltstone (see Low-level waste)

Sandia National Laboratory, California (SNL/CA), 12, 294

LLW at, 17-19, 128-31

MLLW at, 17-18, 213, 221-24, 232, 237-46

Sandia National Laboratory, New Mexico (SNL/NM), 13, 294

LLW at, 17-19, 116-17, 122

MLLW at, 17-18, 213, 221-24, 232, 237-46

SNF at, 42

TRUW at, 17-19, 88, 90-91, 93-94, 96-98, 101-7

San Diego (SDG) LLW, 117, 134

Santa Cruz Basin (SCB) LLW, 117, 134

Santa Susana Field Laboratory (SSFL) [see Energy Technology Engineering Center (ETEC)]

Savannah River Site (SRS), 13, 295

Defense Waste Processing Facility (DWPF), 6, 14, 49,56

environmental restoration at, 167,170

HLW at, $14-18,45-49,52-53,56-67,69,73$

canisters, $60-65,70$

chemical composition of future glass, 73

radionuclide composition of, 69

treatment methods for, 56

LLW at, 17-19, 116-17, 122, 128-33

saltstone, 137
MLLW at, 17-18, 213, 221-24, 232-33, 237-46

SNF at, 42

TRUW at, 17-19, 88, 90-91, 93-94, 96-98, 100, 102-7

Sea-bed disposal, 110, 117, 134

Sheffield (mlinois) commercial waste site, 296

LLW at, $110,116,118,138-39$

Single-shell tank waste (see High-level waste)

Site treatment plans (STPs), 46, 209

Slurry (see High-level waste)

Source terms, waste, $8,253,255-56,266-68$

for commercial LLW disposal sites, 267-68

for DOE LLW disposal sites, 266

for wastes from D\&D of LWRs, 255-56

Specific activity, 274-81

Spent nuclear fuel (SNF), 2, 6-7, 11-16, 23-42, $257-65$

commercial, 2, 6, 11, 14-16, 23-40

disposal of, 2,24

DOE, 7, 24, 41-42

radionuclide inventory, 258-65

reference LWR characteristics, 38

Stanford Linear Accelerator Center (SLAC), 13, 295

LLW at, 17-19, 128-31

MLLW at, 17-18, 221-24, 232, 237-246

Tailings (see Uranium or Thorium mill tailings)

Thorium mill tailings, 109

Three Mile Island-Unit 2 Reactor, 2, 295

wastes from, 178, 189, 203

Toxic Substances Control Act (TSCA)

wastes regulated by, 3-4, 8, 158, 160, 209-14, $221-24,237-47$

Transuranic waste (TRUW), 3, 7, 11, 15-19, 75-107, $160,165-71,206-7,209$

buried, $7,15-16,19,75,78,106$

composition of, 99-105

contact-handled (CH) stored, 3, 17-18, 75-78, 84-95, 99-102, 104

isotopic content of, 102, 104

debris contaminated by, $160,165,169-71$

decay calculations, 77-78, 102-6

disposal in WIPP, 75, 78

generated, 75-76

generator/storage sites, 83

inventories and projections of, 76, 78, 88-101, 106

mixed transuranic waste (MTRUW), 75, 85-87, 90-93, 97

remote-handled (RH) stored, 3, 17-18, 75-78, 84-90, 96-101, 103, 105

isotopic content of, 103, 105

site data submittals, 77 
soil contaminated by, $160,165-68$

Uranium mill tailings (UMT), 3, 7, 15-16, 109, $145-55,160$

characteristics of, $145-46,155$

generated, 154

historical and projected volume of, 150

locations and status of uranium mills, 148, 151-53

Uranium Mill Tailings Remedial Action Program (UMTRAP), 158, 160, 163, 166, 169

Vitrification, 7, 14, 48-49, 54-56, 60-73

Waste form characterization, 2-6

generated, treated, stored, and disposed wastes, 4

Waste Isolation Pilot Plant (WIPP), 295

TRUW to be placed in, 75, 78

Waste Isolation Pilot Plant Transuranic Waste

Baseline Inventory Report (WTWBIR), 76-77, 88-102, 104-6

Waste sites, 12-13, 289-97

Weldon Spring Site Remedial Action Project (WSSRAP), 295

wastes at, 18, 130, 168, 171

West Valley Demonstration Project (WVDP), 13, 295
HLW at, $13-15,17-18,45-49,52-53$, $56-67,70-71,73,206-7$

canisters, $60-67,70$

chemical composition of future glass, 73

inventory, significant revisions of, 71

radionuclide composition of, 70

treatment methods for, 56

zeolite resin, 48-49

LLW at, 17-19, 128-31, 206-7, 270

Low-Level Radwaste Treatment System, 253, 270

MLLW at, 17-18, 213, 221-24, 233, 237-46

SNF at, 42, 206

TRUW at, $17-19,89-90,92-93,95-98,101-7$, 206-7

West Valley (New York) commercial waste site, 296-97

LLW at, $110,116,118,138-39$

Yellowcake, 7

Yucca Mountain, Nevada

potential repository site characterization, 24

Y-12 Plant (Oak Ridge), 13

LLW at, $17-19,116-17,122,128-31$

MLLW at, 17-18, 214, 221-24, 233, 237-46

Zeolite (see High-level waste) 


\section{DISTRIBUTION}

\section{DOE Headquarters}

Energy Information Administration, Office of Coal, Nuclear, Electric, and Alternate Fuels, Route Symbol EI-53, $1707 \mathrm{H}$ Street, N.W., Washington, DC 20585-0001

Office of Civilian Radioactive Waste Management, Waste Acceptance Division, Route Symbol RW-44, 1000 Independence Ave., S.W., Washington, DC 20585-0001

Office of Environment, Safety, and Health, Route Symbol EH-20, 1000 Independence Ave., S.W., Washington, DC 20585-0001

Office of Environmental Management, Office of Program Integration, Technical Analysis Division, Route Symbol EM-332, Trevion II, Washington, DC 20585-0002

Office of Environmental Management, Office of Spent Fuel Management, Route Symbol EM-37, 1000 Independence Ave., S.W., Washington, DC 20585-0001

Office of Environmental Management, Office of Environmental Restoration, Route Symbol EM-432, Trevion II, Washington, DC 20585-0002

Office of Environmental Management, Office of Technology Development, Route Symbol EM-50, Trevion II, Washington, DC 20585-0002

Office of Environmental Management, Office of Facility Transition and Management, Route Symbol EM-60, Trevion II, Washington, DC 20585-0002

Office of Naval Reactors, Route Symbol NE-60, 2531 Jefferson Davis Highway, Arlington, VA 22242-5160

\section{DOE Operations Offices}

Albuquerque Operations Office, P.O. Box 5400, Albuquerque, NM 87185-5400

Chicago Operations Office, Building 201, 9800 South Cass Ave., Argonne, IL 60439

Idaho Operations Office, 785 DOE Place, Idaho Falls, ID 83402

Nevada Operations Office, P.O. Box 98518, Las Vegas, NV 89193-8518

Oakland Operations Office, 1301 Clay St., Oakland, CA 94612-5208

Oak Ridge Operations Office, P.O. Box 2001, Oak Ridge, TN 37831-8501

Richland Operations Office, P.O. Box 550, 825 Jadwin Ave., Richland, WA 99352

Savannah River Operations Office, P.O. Box A, Aiken, SC 29802 


\section{DOE Sites, Contractors, Special Site/Area Offices, and Special Program Offices}

Ames Laboratory, Iowa State University, Spedding Hall, Pammel Drive, Ames, IA 50011-3020

Argonne National Laboratory, University of Chicago, 9700 South Cass Ave., Argonne, Il 60439

Brookhaven National Laboratory:

Associated Universities, Inc., 16 South Railroad Street, Upton, NY 11973-2310

DOE Brookhaven Area Office, 53 Bell Ave., Upton, NY 11973-2311

DOE Office of Scientific and Technical Information, P.O. Box 62, Oak Ridge, TN 37831

Fermi National Accelerator Laboratory:

DOE Batavia Area Office, P.O. Box 2000, Batavia, IL 60510

University Research Association, P.O. Box 500, Batavia, IL 60510

Fernald Environmental Management Project:

DOE Fernald Area Office, P.O. Box 398705, 7400 Wiley Road, Cincinnati, OH 45239-8705

Fluor Daniels, Fernald Environmental Restoration Management Corporation, P.O. Box 398704, 7400 Wiley Road, Cincinnati, OH 45239-8704

Formerly Utilized Sites Remedial Action Program:

Bechtel National, Inc., 151 Lafayette Drive, P.O. Box 350, Oak Ridge, TN 37831-0350

DOE Oak Ridge Operations Office, P.O. Box 2001, Oak Ridge, TN 37831-8723

Grand Junction Remedial Action Project:

Department of Energy, Grand Junction Projects Office, P.O. Box 2567, Grand Junction, CO 81502

Hanford Site, Westinghouse Hanford Company, P.O. Box 1970, Richland, WA 99352

Hazardous Waste Remedial Actions Program, Tri-County Mall, P.O. Box 2003, Oak Ridge, TN 37831-7606

Idaho National Engineering Laboratory:

Argonne National Laboratory-West, P.O. Box 2528, Idaho Falls, ID 83401-2528

Idaho Chemical Processing Plant, Lockheed Martin Idaho Technologies, P.O. Box 4000, Idaho Falls, ID 83404

Lockheed Martin Idaho Technologies, P.O. Box 1625, Idaho Falls, ID 83415-4201

Naval Reactors Facility, P.O. Box 2068, Idaho Falls, ID 83403-2068

Inhalation Toxicology Research Institute:

DOE Kirtland Area Office, P.O. Box 5890, Kirtland Air Force Base, Albuquerque, NM 87185

Lovelace Biomedical and Environmental Research Institute, Inc., P.O. Box 5890, Albuquerque, NM 87185 


\section{Kansas City Plant:}

Allied-Signal Inc., Kansas City Division, Bannister Federal Complex, Kansas City, MO 64141

DOE Kansas City Area Office, P.O. Box 410202, Kansas City, MO 64141-0202

Lawrence Berkeley Laboratory, University of California, One Cyclotron Road, Berkeley, CA 94720

Lawrence Livermore National Laboratory, P.O. Box 808, L-1, 7000 East Ave., Livermore, CA 94550

Los Alamos National Laboratory:

DOE Los Alamos Area Office, 528 35th Street, Los Alamos, NM 87544

University of California, P.O. Box 1663, Los Alamos, NM 87545

Mound Plant:

DOE Miamisburg Area Office, P.O. Box 66, Miamisburg, OH 45343-0066

EG\&G Mound Applied Technologies, Inc., P.O. Box 3000, Miamisburg, OH 45343-3000

Naval Reactors Program (DOE/HQ, NE-60) Facilities:

Bettis Atomic Power Laboratory, Westinghouse Electric Corporation, P.O. Box 79, West Mifflin, PA 15122-0079

DOE Pittsburgh Naval Reactors Office, P.O. Box 109, West Mifflin, PA 15122-0109

DOE Schenectady Naval Reactors Office, P.O. Box 1069, Schenectady, NY 12301-1069

Knolls Atomic Power Laboratory, General Electric Company, P.O. Box 1072, Schenectady, NY 12301-1072

Naval Reactors Facility (see Idaho National Engineering Laboratory)

Nevada Test Site:

Reynolds Electric and Engineering Company, P.O. Box 98521, Mail Stop 738, Las Vegas, NV 89193-8521

Oak Ridge Reservation:

Oak Ridge Institute of Science and Education, Oak Ridge Associated Universities, 246 Laboratory Road, P.O. Box 117, Oak Ridge, TN 37831-0117

Oak Ridge K-25 Site, Lockheed Martin Energy Systems, P.O. Box 2003, Oak Ridge, TN 37831-7358

Oak Ridge National Laboratory, P.O. Box 2008, Oak Ridge, TN 37831-6235

Oak Ridge Y-12 Plant, Lockheed Martin Energy Systems, P.O. Box 2009, Oak Ridge, TN 37831-8010

Pacific Northwest Laboratory, Battelle Memorial Institute, Battelle Boulevard, P.O. Box 999, Richland, WA 99352

Paducah Gaseous Diffusion Plant, Lockheed Martin Utility Systems, P.O. Box 1410, Paducah, KY 42001

Pantex Plant:

DOE Amarillo Area Office, P.O. Box 30030, Amarillo, TX 79120-0030

Mason and Hanger-Silas Mason Co., P.O. Box 30020, Amarillo, TX 79177 
Pinellas Plant:

DOE Pinellas Area Office, P.O. Box 2900, Largo, FL 34649

Lockheed Martin Specialty Components, P.O. Box 2908, Largo, FL 34649-2908

Portsmouth Gaseous Diffusion Plant, Lockheed Martin Utility Systems, P.O. Box 628, Piketon, OH 45661

Princeton Plasma Physics Laboratory, Princeton University, P.O. Box 451, Princeton, NJ 08543

Reactive Materials Incorporated (RMI) Extrusion Plant, P.O. Box 579, Ashtabula, OH 44004

Rocky Flats Environmental Technology Site:

DOE Rocky Flats Office, P.O. Box 928, Golden, CO 80401-0928

EG\&G Rocky Flats, Inc., P.O. Box 464, Golden, CO 80401-0464

Sandia National Laboratories/California, Lockheed Martin Sandia Corporation, P.O. Box 969, Livermore, CA 94551-0969

Sandia National Laboratories/New Mexico, Lockheed Martin Sandia Corporation, P.O. Box 5800, Albuquerque, NM $87185-5800$

Savannah River Site, Westinghouse Savannah River Company, P.O. Box 616, Aiken, SC 29802

Stanford Linear Accelerator Center, P.O. Box 4349, Palo Alto, CA 94309

Uranium Mill Tailings Remedial Action Program (UMTRAP):

DOE Albuquerque Operations Office, UMTRA Project Office, P.O. Box 5400, Albuquerque, NM 87115

Jacobs Engineering Group, Inc., Suite 1700, 5301 Central Ave., NE, Albuquerque, NM 87108

Waste Isolation Pilot Plant:

Department of Energy, Waste Isolation Pilot Plant Project Office, P.O. Box 3090, Carlsbad, NM 88221

Westinghouse Electric Corporation, Waste Isolation Pilot Plant Project Office, P.O. Box 2078, Carlsbad, NM 88221

West Valley Demonstration Project:

DOE West Valley Area Office, 10282 Rock Springs Road, P.O. Box 191, West Valley, NY 14171-0191

West Valley Nuclear Services Company, Inc., 10282 Rock Springs Road, P.O. Box 191, West Valley, NY 14171-0191

\section{Federal (non-DOE) Agencies}

Congressional General Accounting Office, P.O. Box 321, Richland, WA 99352

Congressional Office of Technology Assessment, 600 Pennsylvania Ave., S.E., Washington, DC 20510-8025

Environmental Protection Agency, 401 M St., S.W., Washington, DC 20460

Nuclear Regulatory Commission, 1717 H St., N.W., Washington, DC 20555

Extra copies for DOE/OSTI to fill orders (200). 
For additional information regarding the distribution of this report, contact

U.S. Department of Energy

Office of Civilian Radioactive Waste Management 1000 Independence Avenue, SW

Route Symbol RW-44

Washington, DC 20585-0001

Phones: (202) 586-5684;

(202) $586-9867$

U.S. Department of Energy

Office of Environmental Management

Route Symbol 432

Cloverleaf Building

Germantown, MD 20874

Phone: (301) 903-8121
U.S. Department of Energy

Office of Environmental Management

Route Symbol EM-332

Trevion II

Washington, DC 20585-0002

Phone: (301) 903-7126

Oak Ridge National Laboratory

Integrated Data Base Program

P.O. Box 2008, Mail Stop 6495

105 Mitchell Road

Oak Ridge, TN 37831-6495

Phones: (423) 574-6823;

(423) $576-7575$ 


\section{INTEGRATED DATA BASE (IDB) Program Reader Comment Form for This Report (DOE/RW-0006, Rev. 11)}

1. Have you received a copy of the last report (DB Rev, 10)? Yes _ No If yes, please answer questions 2-4. If no, please go on to question 5.

2. How many times did you use IDB Rev. 10 ?

$20+$ times 11-20 times 4-10 times 1-3 times Not at all

3. How often did you find the information you needed in IDB Rev. 10 ? Almost always (75-100\%) Most of the time (50-74\%)

4. Was the information in IDB Rev. 10 easy to find and clearly presented? (Please check one answer in each column.) Easy to find Not easy to find Not clearly presented

5. How much time have you spent looking over or reviewing this report (IDB Rev. 11)? None Less than 1 hour 1-3 hours More than 3 hours (specify how many)

6. Does the information that you anticipate that you will need appear in IDB Rev. 11 ? Almost always (75-100\%) Most of the time (50-74\%)

7. Is the information in IDB Rev. 11 easy to find and clearly presented? (Please check one answer in each column.) Easy to find

Not easy to find

\section{Clearly presented}

Not clearly presented

8. What information would you like to see added or expanded in the next report (IDB Rev. 12)?

9. What other improvements of this IDB report do you suggest? Please attach additional pages if necessary.

10. Would you like to receive the next report (IDB Rev. 12)? Yes No

11. What organization sponsors your work?

A. DOE organization Office of Civilian Radioactive Waste Management (RW)

- Office of Waste Operations (EM-30)

__ Office of Environmental Restoration (EM-40)

B. Other organizations Other DOE office (please specify):

State and local governments
Private
Other

12. Submitted by:

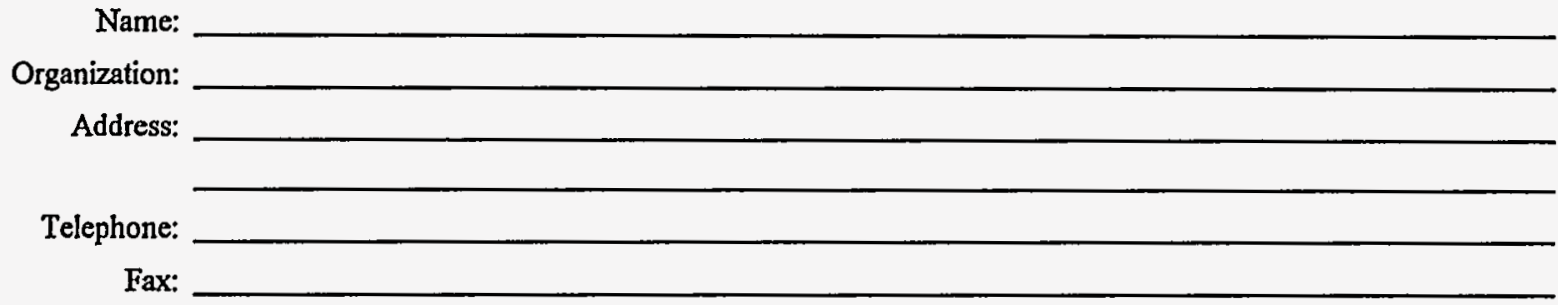

